\title{
Calculation of Dosimetry Parameters for Fast Neutron Radiotherapy
}

\author{
Alan Harvey Wells*
}

"Nuclear Assurance Corporation, 24 Executive Park West, Atlanta, GA 30329. 


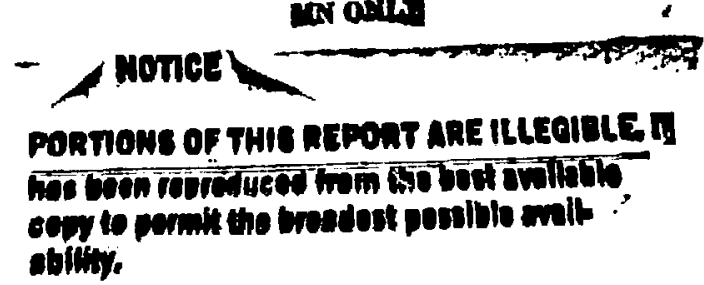

TABLE OF CONTENTS

Chapter

Page

List of Tables . . . . . . . . . . . . . . . . . iv

List of Figures . . . . . . . . . . . . . . . . . . . v

Abstract. . . . . . . . . . . . . . . . . vii

I. Introduction . . . . . . . . . . . . . . . . 1

II. Dosimetry Parameters. . . . . . . . . . . . . . 3

III. Source Spectrum and Phantom Geometry . . . . . . . 11

IV. Computer Simulation Models . . . . . . . . . . . 19

V. Charged Particle Transport and Kerma Analysis . . . 31

VI. Validation of Computer Programs . . . . . . . . . 36

VII. Results and Discussion. . . . . . . . . . . . 47

VIII. Conclusions . . . . . . . . . . . . . . . . . 70

References. . . . . . . . . . . . . . . . . . . . . . . .

Appendix A. . . . . . . . . . . . . . . . . . . . . . 78

Appendix B. . . . . . . . . . . . . . . . . . . . . . 80

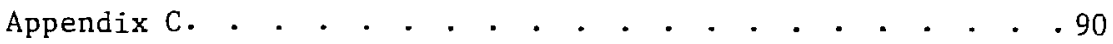

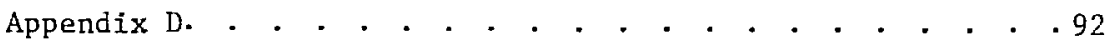

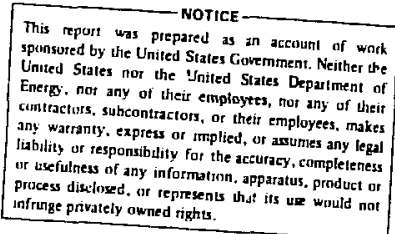


l Energy Dependences of W. . . . . . . . . . . . . .8

2 Material Cumpositions . . . . . . . . . . 17

3 D-T Spectrum Parameters. . . . . . . . . . . . 37

$450 \mathrm{MeV} \mathrm{d}^{+}$on Be Neutron Spectrum Dosimetry. . . . . 48 Parameters

$550 \mathrm{MeV} \mathrm{d}^{+}$on Be Neutron Spectrum Relative . . . . 51 Dose Contributions

$\because \mathrm{YeV} \mathrm{\textrm {d } ^ { + }}$ on Be Neutron Spectrum Dose. . . . . . . 52 Comparisons

$750 \mathrm{NieV} \mathrm{d}$ on Be Neutron Spectrum Recoil . . . . . . 58 Yuclei

$842 \mathrm{MeV}{ }^{+}$on Be Neutron Spectrum Dosimetry. . . . 62 Parameters

$942 \mathrm{MeV} \mathrm{P}^{+}$on Be Neutron Spectrum Reiative : . . . . 64 Dose Contributions

$10 \angle 2 \mathrm{MeV} \mathrm{P}^{+}$on Be Neutron Spectrum Recoil . . . . . . 69 Nuclei

11 Recommended Dosimetry Parameters. . . . . . . 71

12 Energy Loss Parameters for Shonka A-150 Plastic . . 82

13 Energy Loss Parameters for ICRU Muscle Tissue . . . 83

14 Elemental Kerma Values. . . . . . . . . . . 90 
$150 \mathrm{MeV} \mathrm{d}^{+}$on Be Neutron Spectrum . . . . . . . . . . 12

2 In-Air Scan. . . . . . . . . . . . . . . 13

3 Source Geometry . . . . . . . . . . . . . 15

$442 \mathrm{MeV} \mathrm{p}^{+}$on Be Neutron Spectrum . . . . . . . . 18

5 Progl Flow Diagram. . . . . . . . . . 20

6 Hydrogen Cross Section. . . . . . . . . . . . 26

7 Carbon-12 Cross Section . . . . . . . . . . . . 27

8 Nitrogen-14 Cross Section. . . . . . . . . . 28

9 Oxygen-16 Cross Section. . . . . . . . . . . . . 29

10 D-T Neutron Spectrum at $1 \mathrm{~cm}$ Depth. . . . . . . 38

11 D-T Neutron Spectrum at $10 \mathrm{~cm}$ Depth . . . . . . . 39

12 D-T Neutron Spectrum at $25 \mathrm{~cm}$ Depth . . . . . . . . 40

13 Hydrogen Kerma . . . . . . . . . . . . . 42

14 Carbon Kerma . . . . . . . . . . . . . . 43

15 Nitrogen Kerma . . . . . . . . . . . . . 44

16 Oxygen Kerma . . . . . . . . . . . . . . . 45

$1750 \mathrm{MeV} \mathrm{d}^{+}$on Be Neutron Spectrum at $1 \mathrm{~cm}$. . . . . 54

$1850 \mathrm{MeV} \mathrm{d}^{+}$on Be Neutron Spectrum at $10 \mathrm{~cm}$. . . . . 55

$1950 \mathrm{MeV} \mathrm{d}^{+}$on Be Neutron Spectrum at $25 \mathrm{~cm}$. . . . . 56 
$2042 \mathrm{MeV} \mathrm{p}^{+}$on Be Neutron Spectrum at $1 \mathrm{~cm} . . . . .966$

$2142 \mathrm{MeV} \mathrm{p}^{+}$on Be Neutron Spectrum at $10 \mathrm{~cm}$. . . . . .67

$2242 \mathrm{MeV} \mathrm{p}{ }^{+}$on Be Neutron Spectrum at $25 \mathrm{~cm}$. . . . . . 68

$23 \xi(\mathrm{E})$ for $\mathrm{C}^{12}$. . . . . . . . . . . . . . . . . 84

$24 \xi(E)$ for $0^{16}$. . . . . . . . . . . . . . . . . . 85

$25 \bar{\mu}$ (E) for $\mathrm{C}^{12}$. . . . . . . . . . . . . . . . 86

$26 \bar{F}(E)$ for $0^{16}$. . . . . . . . . . . . . . . . . . 87

27 Proton Slowing Down Spectrum at $6 \mathrm{~cm}$. . . . . . . . . 92

28 Deuteron Slowing Down Spectrum at $6 \mathrm{~cm}$. . . . . . . . 93

29 Triton Slowing Down Spectrum at $6 \mathrm{~cm}$. . . . . . . . 94

30 Alpha Slowing Down Spectrum at $6 \mathrm{~cm}$. . . . . . . . 95 


\section{ABSTRACT}

Calculation of Dosinetry Parameters

for Fast Neutron Radiotherapy. (May 1978)

Alan Harvey Wells, B.S., Stevens Institute of Technology;

M.S., Texas A\&M University

Co-Chairmen of Advisory Committee: Dr. J. B. Smathers

Dr. D. G. Foster, Jr.

A computer simulation of the interactions of $50 \mathrm{MeV} \mathrm{d}^{+}$on Be and $42 \mathrm{MeV} \mathrm{p}^{+}$on Be neutron spectra with ICRU muscle tissue and Shonka A-150 tissue equivalent plastic was performed to allow computation of the charged particle spectra that result. Nuclear data were obtained from the Evaluated Nuclear Data File (ENDF) whenever possible and from the Intranuclear Cascade and Evaporation models otherwise. The dosimetry parameters calculated are:

the kerma ratio, $\mathrm{K}_{\mathrm{A}-150} / \mathrm{K}_{\text {tissue }}$ the energy required to form an ion parr, $W$; and the stopping power ratio, $S_{B}^{W}$.

$50 \mathrm{MeV} \mathrm{d}^{+}$on Be Neutron Sfectrum

$\begin{array}{llllll}\begin{array}{c}\text { Depth } \\ (\mathrm{cm})\end{array} & \frac{\mathrm{K}_{\mathrm{A}-150}}{\mathrm{~K}_{\text {tissue }}} & \begin{array}{c}\mathrm{W}_{\mathrm{TE} \text { gas }} \\ \text { (eV/ion } \\ \text { pair) }\end{array} & \mathrm{S}_{\mathrm{TE} \text { gas }}^{\mathrm{W}} & \begin{array}{c}\mathrm{W}_{\mathrm{Air}} \\ \text { (eV/ion } \\ \text { pair) }\end{array} & \mathrm{S}_{\mathrm{Air}}^{\mathrm{W}} \\ \text { in Air } & 1.09 & 30.13 & 1.011 & 34.05 & 1.189 \\ 1.00 & 1.07 & 30.13 & 1.011 & 34.05 & 1.189 \\ 10.0 & 1.04 & 30.13 & 1.011 & 34.05 & 1.189 \\ 25.0 & 1.00 & 30.13 & 1.011 & 34.05 & 1.189\end{array}$


$42 \mathrm{MeV} \mathrm{p}^{+}$on Be Neutron Spectrum

( $6 \mathrm{~cm}$ polyethylene Eiltration)

$\begin{array}{llllll}\begin{array}{c}\text { Depth } \\ \text { (cin) }\end{array} & \frac{\mathrm{K}_{\mathrm{A}-150}}{\mathrm{~K}_{\text {tissue }}} & \begin{array}{c}\mathrm{W}_{\mathrm{TE} \text { gas }} \\ \text { (eV/ion } \\ \text { pair) }\end{array} & \begin{array}{c}\mathrm{S}^{\mathrm{W}} \\ \mathrm{TE} \text { gas }\end{array} & \begin{array}{c}\mathrm{W}_{\text {Air }} \\ \text { (eV/ion } \\ \text { pair) }\end{array} & \mathrm{S}^{\mathrm{W}} \\ \text { in Air } & 1.10 & 30.12 & 1.010 & 34.03 & 1.188 \\ 1.00 & 1.08 & 30.12 & 1.010 & 34.03 & 1.188 \\ 10.0 & 1.05 & 30.12 & 1.010 & 34.03 & 1.188 \\ 25.0 & 1.01 & 30.12 & 1.010 & 34.03 & 1.188\end{array}$

The values listed above for the kerma in air

require an ionization chamber wall thickness of $0.5 \mathrm{~cm}$

to ensure that charged partic'e spectrum equilibrium

Uiij occur.

Doses given at the Texas A\&M Variable Energy Cyclotron (TAWVC) using currently accepted dosimetry parameters agree within $4 \%$ at depths of $1 \mathrm{~cm}$ or more with doses calculated with the parameters listed above.

Calculated values of $\mathrm{s}_{\mathrm{g}}^{\mathrm{W}}$ and $\mathrm{W}$ for the $50 \mathrm{MeV} \mathrm{d}^{+}$on $\mathrm{Be}$ and the $42 \mathrm{MeV} \mathrm{p}^{+}$on Be neutron spectra are the same, within $0.1 \%$ The kerma ratio for the $42 \mathrm{MeV} \mathrm{p}^{+}$on Be neutron spectrum is about $1 \%$ greater than the kerma ratio for the $50 \mathrm{MeV} \mathrm{d}+$ on Be neutron spectrum. 


\section{CHAPTER I}

\section{INTRODUCTION}

Medium energy neutrons have been useci at TAMVEC and other facilities in recent years for cancer radiotherapy. Proper treatment requires a knowledge of the precise dose delivered to a tissue volume by the neutron bearn. The energy range of the source neutron spectra used at early facilities did not exceed $20 \mathrm{MeV}$, thus permitting the use of the Evaluated ivclear Data File (ENDF) to perform reasonably accurate computations for the dosimetry parameters of interest. Later facilities, such as TAMVEC, used higher energy reactions which resulted in source neutron spectra with energies extending to $54 \mathrm{MeV}^{3}$ Experimental nuclear data for neutron energies greater than $20 \mathrm{MeV}$ are sparse and computations exceedingly expensive, thus initial dosimetry constants for this enerby region were necessarily based upon extrapolations or approximations using available 0 to $20 \mathrm{MeV}$ data., ${ }^{4}$ Possible errors present in these approximations could have a significant effect, if radiotherap:

This dissertation follows the format and style of Radiation Research. 
protocols developed for the $50 \mathrm{MeV} \mathrm{d}^{+}$on Be neutron beam at TAMVEC are used with a beam which has a different neutron spectrum. This is because the constants required to compute the dose delivered to tissue are dependent on the neutron spectrum and the charged particle spectrum the bean produces. If the approximate constants differ appreciably from the actual physical constants, a dose selected to achieve a given therapeutic effect with one neutron bean will give different results with a different beam. Also, comparisons between different types of radiotherapy, such as pion, neutron, proton, and heavy ion therapy, are made more difficult by dosimetry uncertainties. Thus the purpose of this research is to develop better values for the required dosimetry constants, via a unified system of computer programs and evaluated experimental data, for the $50 \mathrm{MeV} \mathrm{d}^{+}$on Be neutron therapy beam and for the $42 \mathrm{MeV} \mathrm{p}^{+}$on Be neutron spectrum. As previously mentioned, the sparsity of data for neutron energies greater than $20 \mathrm{MeV}$ and the calculational complexity required have prevented the accomplishment of such an evaluation prior to this work. 


\section{DOS IMETRY PARAMETERS}

The dose to tissue is computed using the free charge produced in the gas filling an ionization chamber, which is related to the dose to the chamber wall (made of Tissue Equivalent plastic ${ }^{4}$ ) via the Bragg-Gray principle: ${ }^{6,7}$

$$
\begin{aligned}
& 100(\mathrm{~W} / \mathrm{e}) \mathrm{s}_{\text {gas }}^{\text {wall }} Q \quad\left(\mathrm{C}_{\mathrm{TPC}}\right) \quad\left(\mathrm{C}_{\text {attn }}\right) \\
& \mathrm{D}_{\text {chaipber }}=-\cdots
\end{aligned}
$$

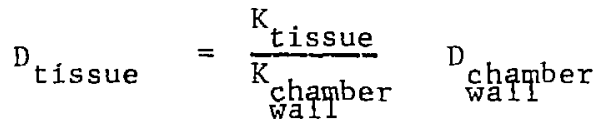

$$
\begin{aligned}
& \text { where: } D_{\text {tissue }}=\text { dose to tissue in rads } \\
& \mathrm{D}_{\text {chapher }}=\text { dose to the chamber wall in rads } \\
& \mathrm{W} / \mathrm{e}=\text { energy per ion pair, eV } / \mathrm{C} \\
& \text { ? = charge coliected, } \mathrm{C} \\
& \mathrm{m}=\text { mass of gas in the chamber, } \mathrm{kg} \\
& \mathrm{s}_{\text {gas }}^{\text {wa11 }}=\text { stopping power ratio (wall to gas, } s_{g}^{w} \text { ) } \\
& \mathrm{C}_{\mathrm{TPC}}=\text { correction to standard temperature }
\end{aligned}
$$

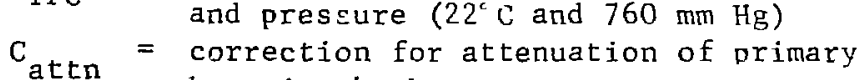

$$
\begin{aligned}
& \text { beam in chamber } \\
& \mathrm{K}_{\text {tissue }}=\text { kerma value for tissue }\left(\mathrm{K}_{\mathrm{t}}\right) \text {, rad } \\
& \underset{\text { chaqber }}{=} \text { kerma value for the chamber wall }\left(\mathrm{K}_{\mathrm{w}}\right) \text {, rad }
\end{aligned}
$$


The values for $W, K_{t}, K_{w}$ and $S_{g}^{W}$ are particle and energy dependent, and thus vary as the neutron spectrum and its gamma component change. The kerma values and hence the kerma ratio, $\mathrm{K}_{\mathrm{t}} / \mathrm{K}_{\mathrm{w}}$, may be obtained from the initial charged particle spectrum produced by neutrons in tissue and in T.E. plastic, respectively. 8,9 The energy of these initial charged particles (including recoil nuclei) produced in a small mass element of the material being considered is the kerma ${ }^{6}$ :

$$
\mathrm{k}=\Delta \mathrm{E}_{\mathrm{K}} / \Delta \mathrm{m}
$$

where $\Delta \mathrm{E}_{\mathrm{K}}$ is the sum of the initial kinetic energies of all charged particles, including recoil nuclei, produced by indirectly ionizing radiation in a volume element of a specified material with mass $\Delta \mathrm{m}$.

The stopping power ratio, $s_{g}^{W}$, is obtained from the equilibrium charged particle distribution. For each charged particia of the $j^{\text {th }}$ type and particle energy $E, S_{j}(E)$ is the energy lost per unit path length. $S_{j}$ (E) may be obtained via the continuous slowing down approximation:

$$
S_{j}(E)=\frac{4 \pi e^{4}}{m_{e} \frac{v_{j}^{2}}{v_{j}}} \sum_{i} \quad N_{i} Z_{i} B_{i}
$$




$$
\begin{aligned}
& \text { where } B_{i}=\left[\ln \frac{2 \mathrm{~m}_{\mathrm{e}} \mathrm{v}^{2}}{\mathrm{I}_{i}}-\ln \left(1-\varepsilon^{2}\right)-\mathrm{s}^{2}\right] \\
& \text { and } I_{i}=\text { jonization potential for the } i^{\text {th }} \text { element, eV } \\
& \mathrm{N}_{i}=\text { number of atoms of } i^{\text {th }} \text { type/unit volume } \\
& \text { with atomic number } z_{i} \\
& m_{e}=\text { mass of the electron, } \mathrm{kg} \\
& \text { e = charge of the electron, coul } \\
& v_{j}=\text { velocity of the } j^{\text {th }} \text { charged particle, } m / s \\
& \beta=v_{j} / c \text {, where } c \text { is the speed of } 1 \text { ight }
\end{aligned}
$$

This expression for $S_{j}(E)$ may also be corrected for various effects, including shell corrections, and these corrections have been made fer tabulated values ${ }^{10}$ of the stopping power for protons, $s_{P}(E)$, for various media. For heavy ions: ${ }^{11}$

$$
s_{j}(E)=\left[\frac{z_{j}^{*}}{z_{p}^{*}}\right]^{2} s_{p}(E)
$$

where

$$
z_{j}^{*}=z_{j}\left[\begin{array}{lll}
1-\exp \left(-125 v_{j} / c z_{j}^{2 / 3}\right)
\end{array}\right]
$$

is the effective charge of a charged particle of the $j^{\text {th }}$ type. $z_{\mathrm{P}}^{*}$, the effective charge for protons, is also computed with this expression.

For deuterons and tritons, whose charge is the same as the proton's: 


$$
s_{j}(E)=s_{P}\left(i^{*}\right) \text {, where } E^{*}=\frac{m_{P}}{m_{j}} E \text {. }
$$

The average stopping power, $\overline{\mathrm{s}}$, when more than one type of charged particle is present, is:

$$
\overline{\mathrm{S}}=\sum_{i} \frac{\int \mathrm{N}_{j}(\mathrm{E}) \mathrm{S}_{j}(\mathrm{E}) \mathrm{dE}}{\mathrm{N}}
$$

where

$$
\begin{aligned}
& N_{j}(E) \text { is the number of the } j^{\text {th }} \text { type charged } \\
& \text { particle at energy } E \text {, and } N \text { is the total } \\
& \text { number of charged particles per unit volum?. }
\end{aligned}
$$

The stopping power ratio, $\mathrm{S}_{\mathrm{g}}^{\mathrm{w}}$, is the ratio of $\overline{\mathrm{S}}$ for the chamber wall material to $\overline{\mathrm{S}}$ for the gas filling the chamber. It has been customary in the past to approximate $\mathrm{s}_{\mathrm{g}}^{\mathrm{W}}$ by averaging ${ }^{12}$ the stopping power of charged particles over the initial (also called the primary or source) charged particle spectrum, rather than the slowing down spectrum as was done in this work. The initial charged particle spectrum is the energy spectrum of the charged particles created by the interaction of radiation with nuclei, which have not been transported away from the interaction site. A Monte Carlo program was written for this work to transport these charged particles and compute the slowing down spectrum, which is the physical spectrum actually present within irradiated media. 
$\bar{W}$, the average energy required to produce an ion pair in the chanber gas, is obtained from the equilibrium charged particle distribution:

$$
\bar{W}=\frac{\sum_{j} \quad \int W_{j}(E) N_{j}(E) d E}{\sum_{j} \int N_{j}(E) d E},
$$

where $W_{j}$ (E) is the energy per ion pair produced for a particle of type $j$ and energy $E$.

Experimental data for $W_{j}(E)$ are sparse, although data for protons and deuterons incident on argon and T.E. gas are available. 13

A theoretical model for calculating $W(E)$ has been developed. 14

Recent absolute measurements of $\mathrm{W}$ for T.E. gas made at Brookhaven National Laboratory ${ }^{15}$ were used in this work. A consistent set of $W$ values as a function of energy for air is not available. It is possible $\mathrm{e}^{16}$ to approximate the enery dependence of $\mathrm{W}$ for air by using the BNL energy dependence of $W$ for T.E. gas and the electron $W$ value for air. ${ }^{17}$ A similar synthesis of $W$ for nitrogen agrees quite well with measured values, ${ }^{18}$ thus lending, edence to the approximation for air, which is $75 \%$ nitrogen by weight. The expressions for $W$ for T.E. gas, air, and nitrogen are listed in Table 1.

The stopping power ratio for Shonka A-150 plastic to air may be obtained from the slowing down charged particle spectrum 


\section{TABLE 1}

Energy Dependences of $\mathrm{W}$

Material

TE gas

Air

Nitrogen

\section{Function}

$W_{P}(E)=29.91+0.57 E^{-1 / 2}$

$W_{\alpha}(E)=29.79+3.64 E^{-1 / 2}$

$W_{P}(E)=33.73+0.64 E^{-1 / 2}$

$W_{Q}(E)=33.73+4.12 E^{-1 / 2}$

$W_{P}(E)=36.4+0.69 E^{-1 / 2}$

$W_{\alpha}(E)=36.4+4.45 \mathrm{E}^{-1 / 2}$ 
as described above. However, because of the large differences in composition of A-150 plastic and air and the 3-10\% uncertainties in stopping powers, ${ }^{19}$ the resulting uncertainty in $\mathrm{s}_{\mathrm{Air}}^{\mathrm{w}}$ is $15 \%$ or more. A more accurate method for determining $\mathrm{S}_{\mathrm{A} \text { ir }}^{\mathrm{W}}$ is to perform a measurement of a fixed dose with a specific ionization chamber with both air and T.E. gas fillings. Since the dose is equal for both measurements, and given the $W$ for air and T.E. gas and the $S_{T E}^{W}$ gas, the value of $S_{\text {Air }}^{W}$ may be obtained as follows:

$$
\mathrm{D}_{\mathrm{TE} \text { gas }}=\mathrm{D}_{\mathrm{Air}}
$$

or, upon inserting the Bragg Gray expression:

$$
\frac{100\left(\mathrm{~W} / \mathrm{e}_{\mathrm{TE}} \mathrm{s}_{\mathrm{TE} \text { gas }}^{\mathrm{W}} \mathrm{Q}_{\mathrm{TE}}\left(\mathrm{C}_{\mathrm{TPC})} \dot{\mathrm{C}}_{\text {attn }}\right)\right.}{\mathrm{m}_{\mathrm{TE} \text { gas }}}=
$$

$$
\mathrm{m}_{\text {Air }}
$$

Eliminating common factors and inserting measured ${ }^{5}$ values for the charges collected and the chamber masses yields:

$$
\mathrm{S}_{\mathrm{Air}}^{\mathrm{W}}=\frac{\mathrm{S}_{\mathrm{TE}}^{\mathrm{W}} \text { gas } \mathrm{W}_{\mathrm{TE} \text { gas }}(1.32937)}{\mathrm{W}_{\mathrm{A} i \mathrm{r}}}
$$

Both the above expression and the slowing down spectrum were 
used to obtain $\mathrm{S}_{\mathrm{Air}}^{\mathrm{W}}$ in this work, for purposes of comparison. The atove expression may be rewritten to obtain a derived value of $W_{A i r}$ given the value of $\mathrm{S}_{\mathrm{Air}}^{\mathrm{W}}$ obtained from the slowing down spectrum:

$$
\mathrm{W}_{\mathrm{Air}}=\frac{\mathrm{W}_{\mathrm{TE} \text { gas }} \mathrm{S}_{\mathrm{TE} \text { gas }}^{\mathrm{W}}(1.329)}{\mathrm{S}_{\mathrm{Air}}^{\mathrm{W}}}
$$

This value was also calculated for purposes of comparison.

Differences in the stopping power for a mixture of elements between the solid and gas phases ${ }^{20}$ were neglected in this work because their magnitude is less than the uncertainties in the stopping power itself. 


\section{SOURCE SPECTRUM AND PHANTON GEOMETRY}

The source energy spectrum for $50 \mathrm{Mev} \mathrm{d}^{+}$on beryllium neutrons is presented in Figure 1 as measured at TANVEC. The evaluated neutron spectrum, shown in FIgure 1 , was entered in one $\mathrm{MeV}$ increments from 0 to $54 \mathrm{MeV}$, into a source subroutine for computer processing. The source subroutine linearly interpolates between the 55 data points entered to reproduce the source energy spectrum. The angular dependence of the source spectrum intensity was determined for an 11 by $11 \mathrm{~cm}$ square field at $140 \mathrm{~cm}$ surface to skin distance, SSD, from an in-air scan made at TAMVEC ${ }^{5}$, that is presented in Figure 2. This scan was made with a polyethylene flattening filter in place, $j$ ust as patients are normally treated at TANVEC. The scan was actually made at $142 \mathrm{~cm}$ because of equipment limitations, and then normalized to $140 \mathrm{~cm}$. The normalized scan was inserted into the source subroutine as a rectangular distribution, depicted in Figure 2 as a dashed line. A precise representation of the in-air scan vas not considered necessary beca'se the scan is different for each of the more than 30 fields that can be treated at TAMVEC. Thus, the neutrons are considered to "illuminate" evenly an 11 by 


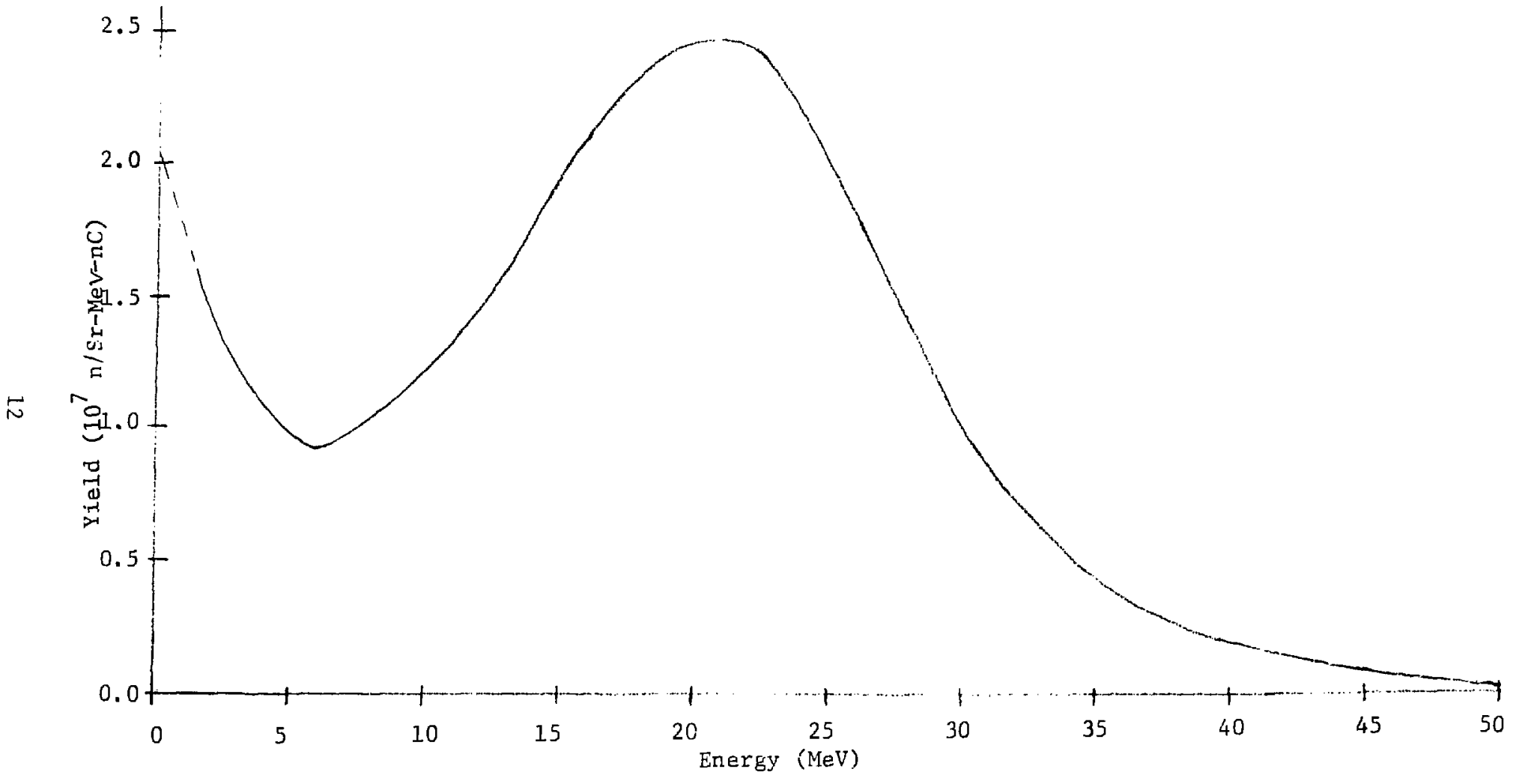

FIGURE I

$50 \mathrm{MeV} \mathrm{d}^{+}$on Be Ncutron Spectrum 


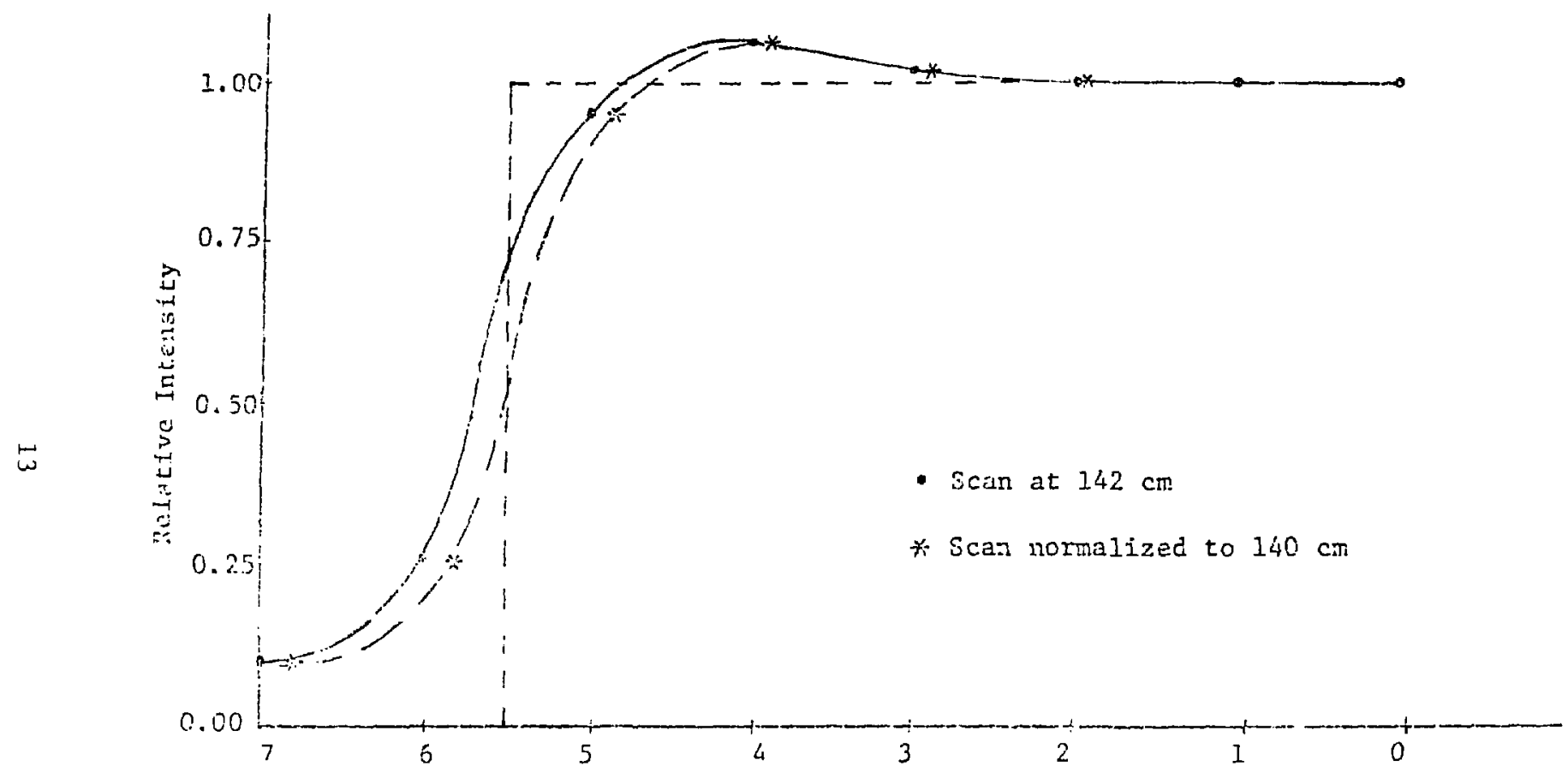

FIGURE 2

Distance from Bean Line Canter (cm)

Ir-AjI Scan 
11 field at $140 \mathrm{~cm}$ SSD on the face of a cubical phantom that is $40 \mathrm{~cm}$ on an edge. The $40 \mathrm{~cm}$ cubical phantom is effectively infinite when effects near the entrance face are considered. 21 The beryllium target is considered as a point source of neutrons. This geometry is depicted in Figure 3. The neutron spectrum as a function of energy is also angle dependent; but for the small angular range ( $2 \frac{1}{4}$ degrees about beam line center) of this problem the neutron energy spectrum is essentially constant..$^{22}$ The $0^{\circ}$ (beam line center) energy spectrum was therefore used for this work at all angles. Within the cubical phantom, planes perpendicular to beam line center were established at various depths, chosen by trial and error to provide finer resolution in the equilibrium or slowing down charged particle spectrum near the entry face of the phantom. Ir this region, the dose builds up rapidly, reaching a maximum value at $1 \mathrm{~cm}$, and changes in the dosimetry parameters occur more rapid1: than in the rest of the phantom. The depths used were $0.00,0.05,0.10,0.15,0.20,0.40$, $0.60,1.00,2.00,4.00,6.00,10.0$, and $14.0 \mathrm{~cm}$. The dosimetry parameters $W$ and $S_{g}^{W}$ were evaluated at these depths, and kerma values were calculated iur volume elements bounded by these $11 \mathrm{~cm}$ by $11 \mathrm{~cm}$ planes.

Flane geometry was used throughout this work partly because 


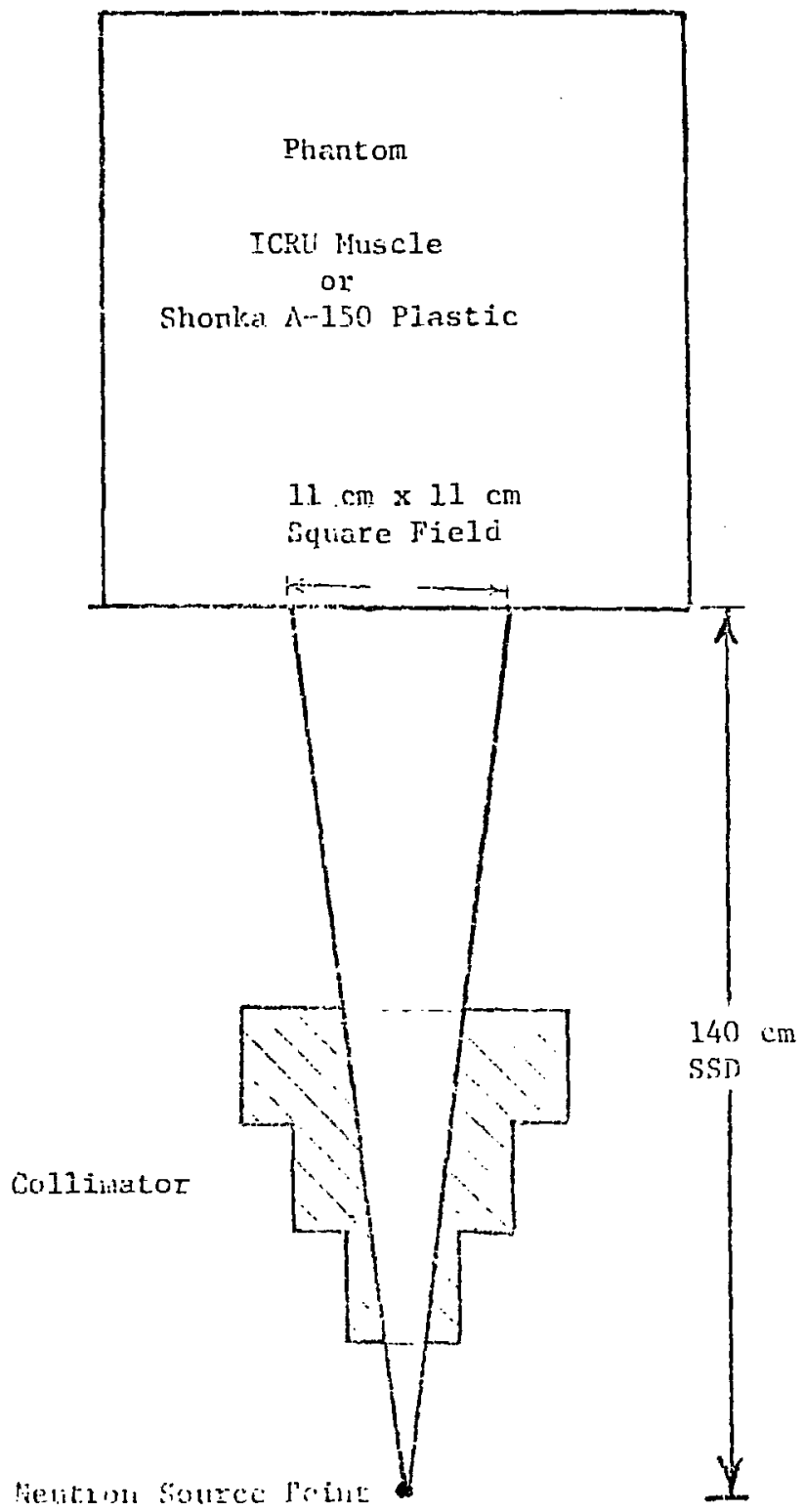

FIGURE 3

Somee Geometry 
of the simplicity it allows, but more importantly because of the practice of irradiating patients with abdominal tumors, witil the TANVEC horizontal beam, in a restraint that reduces the patient's effective thickness and also establishes plane geometry on the patient's surface. For a cyclotron with an isocentric head, the patient would be treated in the supine position, with similar results on the geometry of the region to be treated. Plane geometry is also more appropriate for the chest area. The limbs and head are better simulated as cylinders and a sphere, but the additional computing cost to simulate these geometries as well was considered unwarranted.

The composition of the phantom was Shonka A-150 tissue equivalent plastic for a series of computer runs used to determine $W$ and $S_{g}^{W}$ for tissue equivalent gas and air. An identical series of computer runs with ICRU muscle tissue for the phantom composition was made to allow the evaluation of the kerma ratio, Shonka A-150 plastic to ICRU muscle tissue. The compositions of these four materials are given in Table 2.

Dosimetry parameters were also calculated for the $42 \mathrm{MeV} \mathrm{p}^{+}$on Be neutron spectrum with polyethylene filtration depicted in Figure 4. This neutron source spectrum has been selected for neutron therapy at $M$. D. Anderson Hospital and Tumor Institute, Houston, Texas. 
TABLE 2

Material Compositions

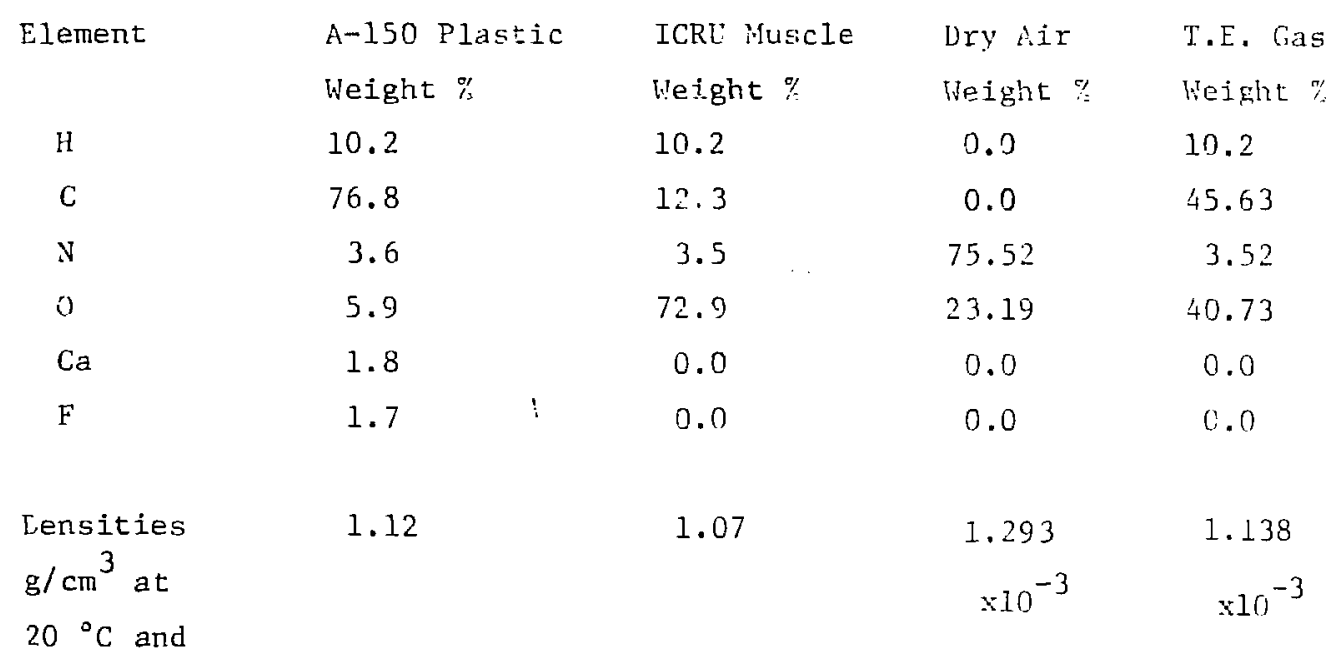

$760 \mathrm{~mm} \mathrm{Hg}$ 


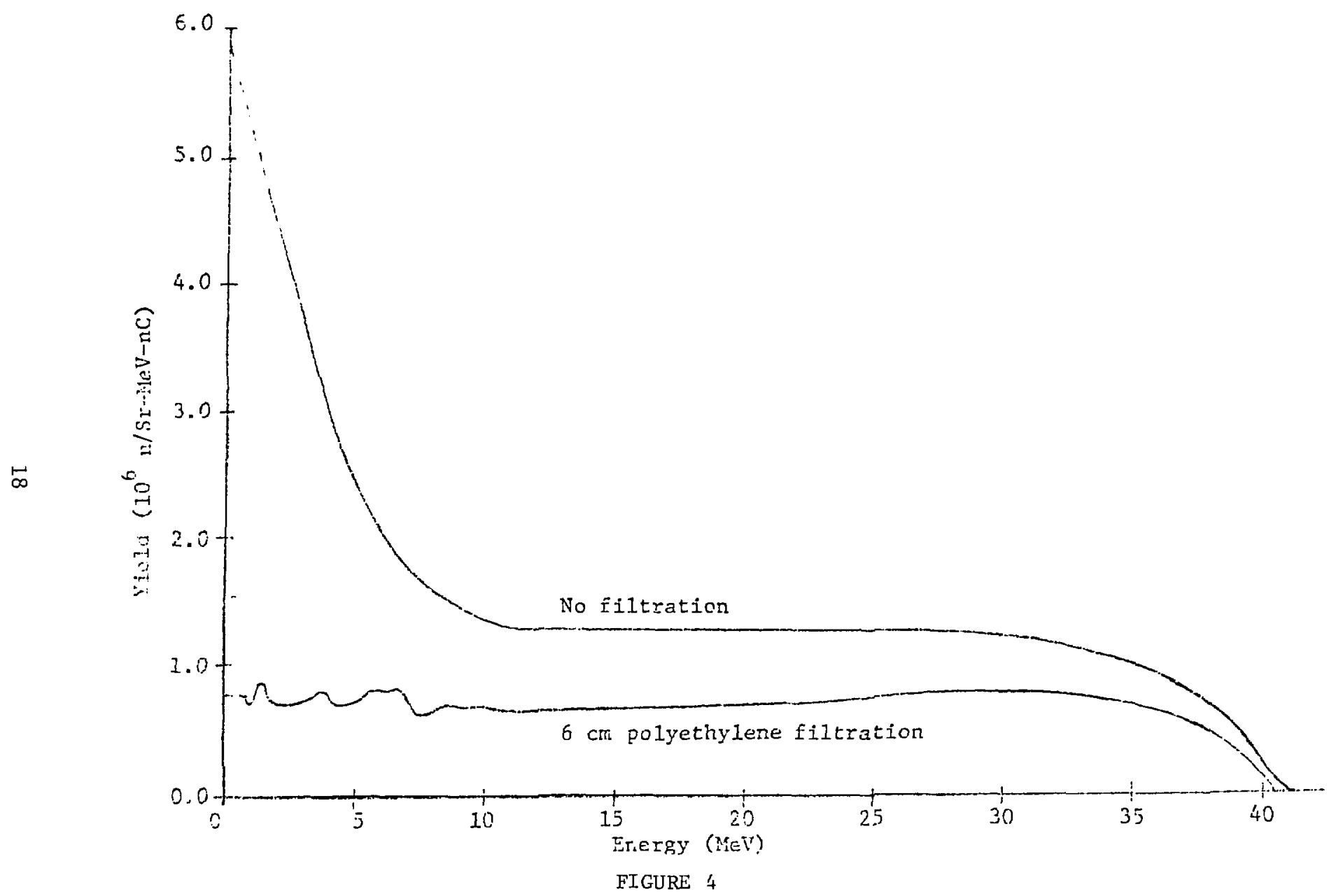

$42 \mathrm{MeV} \mathrm{P}^{+}$on Be Neutron Spectrum 
COMPUTER SIMULATION MODELS

To generate the desired charged particle spectra and the kerma, neutron interactions with the ICRU muscle and A-150 plastic media must first be simulated. The simulation is performed via a two-phase Monte Carlo method, using a LASL code, MCNP, and an ORNL code, NITC. Figure 5 is a flowchart of the program system used. The Nucleon-Meson Iransport Code (NMTC) performs the nucleonnucleus interaction simulation by using the Intranuclear Cascade plus Evaporation (ICE) model, and neglecting elastic scattering with nuclei other than hydrogen. The energy range treated by NMTC is 20 to $50 \mathrm{MeV}$. Source neutrons and secondary neutrons and protons of energy greater than $20 \mathrm{MeV}$ are transportad by NMTC. Neutrons of energy less than or equal to $20 \mathrm{MeV}$ are treated by the Monte Carlo Neutron-Photon code (MCNP) by using the Evaluated Nuclear Data File (ENDF) cross sections to determine elastic and nonelastic interactions. The energy of recoil protons is determined by non-relativistic kinematics, and the energies of chargea particles produced by nuclear interactions are determined via the evaporation model.

The Intranuclear Cascade model used by MMTC is based upon 


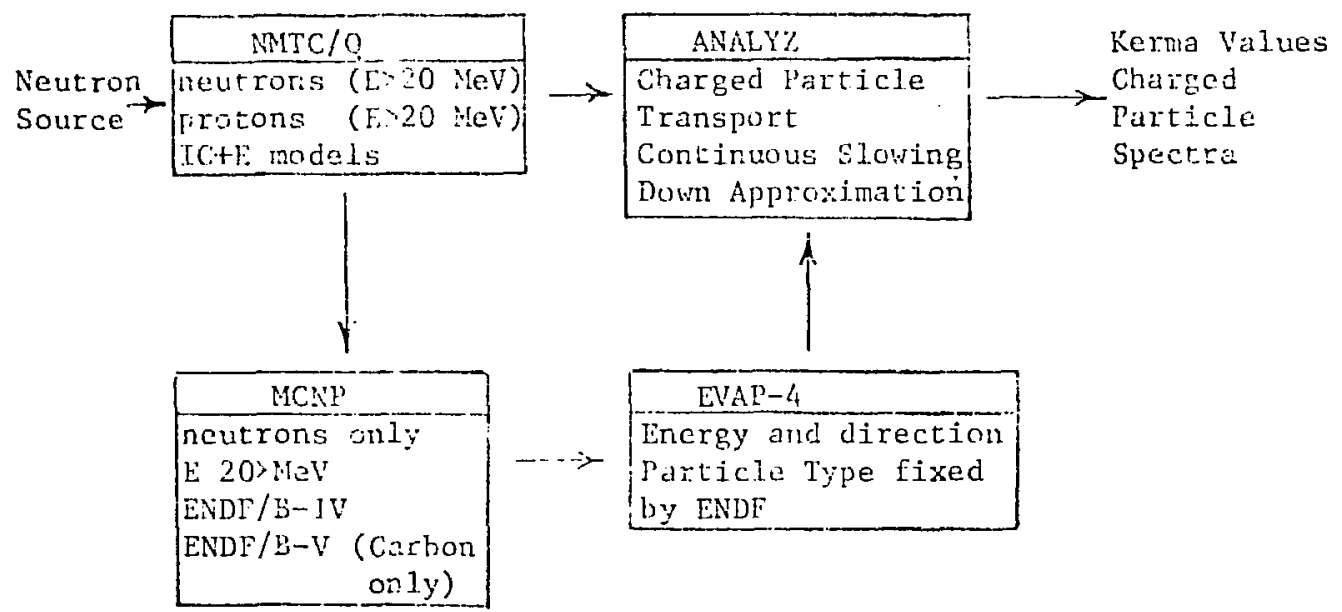

ICtE: Intranuclear Cascado plus Evaporation

ENDF: Evoluated Nuclcar Data File

FIGUR: 5

Program Flow Diagrau 
the Serber model ${ }^{23}$ of nucleon-nucleus interactions. When incidentnucleon wavelengths are short relative to internucleon distances within the nucleus, which is true for energies over about $100 \mathrm{Mev}$, the interaction may be regarded approximately as a scattering process between nucleons in free space. Differences in the free scattering cross sections for neutrons and protons caused by their electric charge difference are very small and are neglected. For energies below about $100 \mathrm{MeV}$, the nucleon wavelengths become greater than internucleon distances and the free-space Serber approximation does not strictly hold. However, recent work of Metropolis et al ${ }^{24}$ and Alsmiller and Hermann $^{25}$ have shown that, while not strictly correct, the cascade model does predict cross sections and secondary spectra quite well down to $20 \mathrm{MeV}$. The Intranuclear Cascade model is so named because an incident nucleon may be considered to strike a nucleon in the nucleus, and it and the struck nucleon may strike others, and so forth, thereby forming a cascade of particles moving within or leaving the nucleus. As particles are knocked out of the nucleus, the atomic mass and atomic number of the nucleus are reduced. However, this change is not included in the Intranuclear Cascade model because, for an energetic nucleon, the transit time of the nucleus is short relative to the time needed to alter the Fermi distribution of nucleons within the nucleus. This means 
that the probability of striking a given nucleon is not altered by the removal of other nucleons from the nucleus.

The Intranuclear Cascade model has been implemented as a computer program by various groups. Three such programs are due to Barashenkov and Eliseev ${ }^{26}$ and Barashenkov et al ${ }^{27-29}$ at the Joint Institute of Nuclear Research (JINR), Bertini ${ }^{30,31}$ at the Oak Ridge National Laboratory (ORNL), and Chen et al. ${ }^{32}$ at the Brookhaven National Laboratory and Columbia University (BNL-CU). These implementations of the cascade model have been compared by Barashenkov et a1.., Bertini ${ }^{34}$, and Blann. These comparisons show that all three versions generate basically the same results. The BNL-CU version and ORNL version are both available at LASL, but only the ORNL version was used for this work. This was in spite of the fact that the BNL-CU version treats the refraction and reflection processes in the nucleus, which the ORNL version neglects. It appears that the BNL-CU version does not yet properly handle the refraction and reflection processes, since the cross sections and spallation spectra it produces at low energies are not quite as good as the ORNL version. 35 Work is continuing on this failing of the BNL-CU version. The BNL-CU version does seem to have a better description of pion interactions and stopping ${ }^{36}$, but that 
is not relevant to this work.

The evaporation phase of the ICE model occurs because the nucleus is left in an excited and/or unstable state after the intranuclear cascade. The eveporation model is so called because the form of the statistical expression which represents the probability of emission of an elementary particle from an excited or unstable nucleus is simi?ar to that for the evaporation of a microscopic particle from a macroscopic droplet at low temperature. 37 The evaporation model was incorporated into a Monte Carlo code by Dostrovsky et al. . $^{38}$ and later modified by Dresner ${ }^{39}$ and Guthrie. 40 The current version of the evaporation model is called EVAn-4 In NMTC, EVAP-4 first computes the probability of emission of particles, including protons, deuterons, tritons, helium-3, and alphas. The program chooses one of these via a Monte Carlo process and then computes the energy and direction of emission. In MCNP, the evaporation model was incorporated to determine the energy of the evaporated particle(s), the particle type having been determined by the ENDF cross-section data. MCNP does not incorporate the intranuclear cascade process, but a neutron-nucleus interaction may still leave an excited and/or unstable nucleus which can aeexcite by the emission of a particle or y:a gamma emission. Gamma emission is neglected in both NMTC and MCNP, both because the 
probability of emission of a particle is on the order of $10^{5}$ times greater than gamma emission, if the excitation energy is greater than the particle threshold, and because recent work of $\mathrm{Ito}^{41}$ indicates that the dose to tissue due to gammas is about $1.5 \%$ of the total biological dose for the $50 \mathrm{MeV} \mathrm{d}+$ on Be neutron beam, as described in Appendix $\mathrm{A}$.

The Intranuclear Cascade nodel used in NMTC at LASL is the version of Bertini without modification. However, this code sometimes generates residual nuclei with negative excitation energy, an obviously unphysical result. In the LASL version of NMTC, this condition is noted and the evaporation phase bypassed. 42 A more appropriate response to a negative excitation energy has been implemented at LASL in a Monte Carlo cross-section code, CROTX. This program also uses Bertini's cascade model (MECC-2) ${ }^{19}$ In CROIX, if the magnitude of the negative excitation energy is more than a specified fraction of the incident particle energy, not to exceed $5 \mathrm{MeV}$, the interaction is discarded and the MECC-2 subroutine called again. If the magnitude of the negative excitation energy is less than the limit, the energies of the cascade particles are renormalized by a constant chosen so that the resulting excitation is zero. The evaporation phase is then 
entered with zero excitation energy because the residual nucleus may be unstable and capable of emitting particles. This renormalization violates the conservation of momentum slightly, but is a small effect and does not justify reusing the computationally expensive MECC-2 subroutine. This technique was inserted into the modified LASL version of NMTC, called NNTC/Q.

CROIX was also built with an improved nuclear mass-excess table and a corrected computation of the direction cosines of evaporated particles. These improvements were also included in the modified version of NMTC.

The cross sections used in this work are presented in Figures 6-9 for hydrogen, carbon, nitrogen, and oxygen. For carbon, the ENDF/B-IV library used for $\mathrm{H}, \mathrm{N}$, and $\mathrm{O}$ was found to be inadequate, since it contains no evaluated data between 15 and $20 \mathrm{MeV}$, and also lacks $(n, d)$ and $(n, t)$ cross sections. A preliminary version of the EINDF/B Version $V$ cross section data library was used instead, and both versions appear in Figure 7 for comparison. The ICE cross sections that appear in these figures were produced by. CROIX ${ }^{43}$, and are the same as those produced by the modified NMTC. Inspection of these figures shows good agreement between 


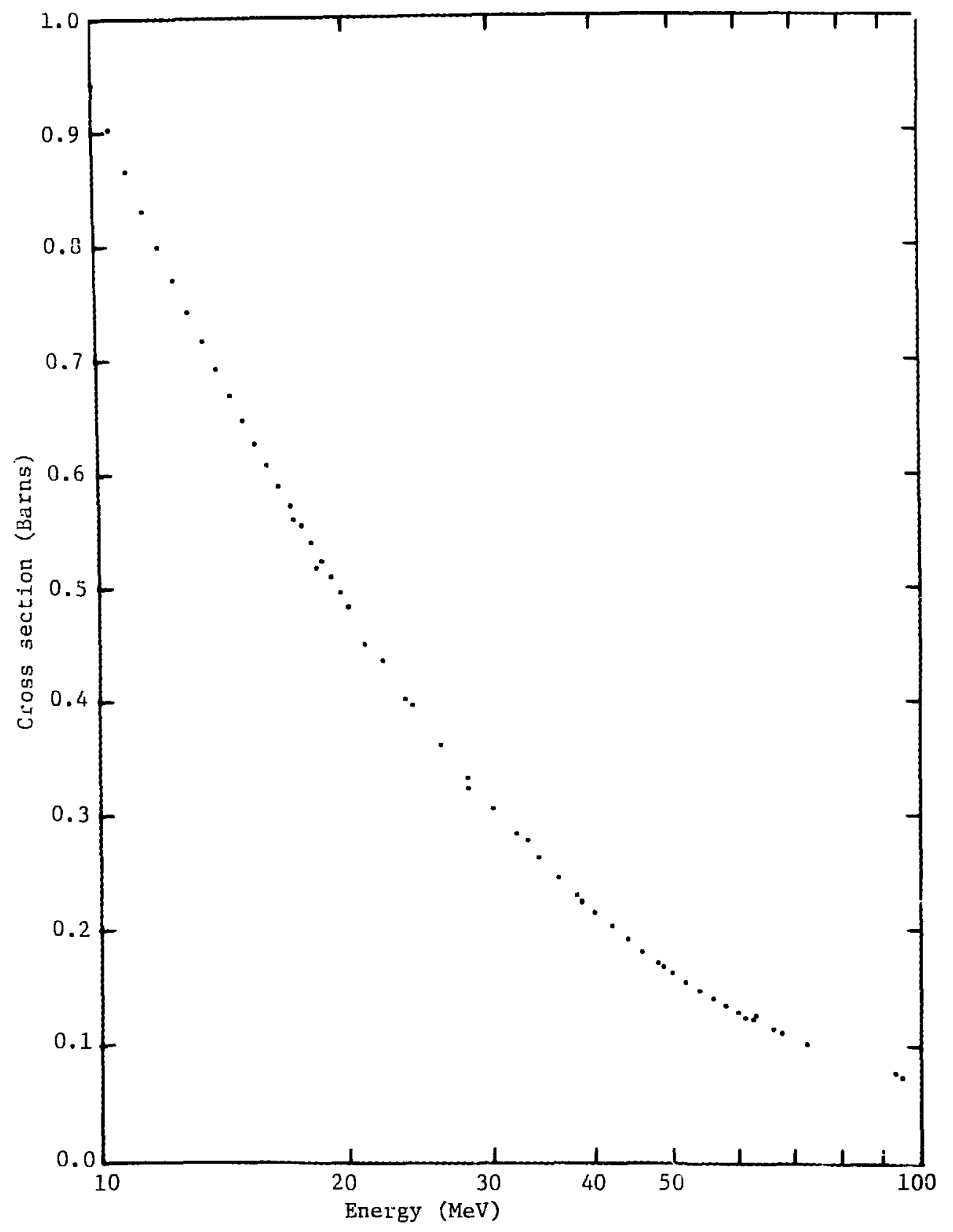

FIGURE 6

Hydrogen Cross Section 


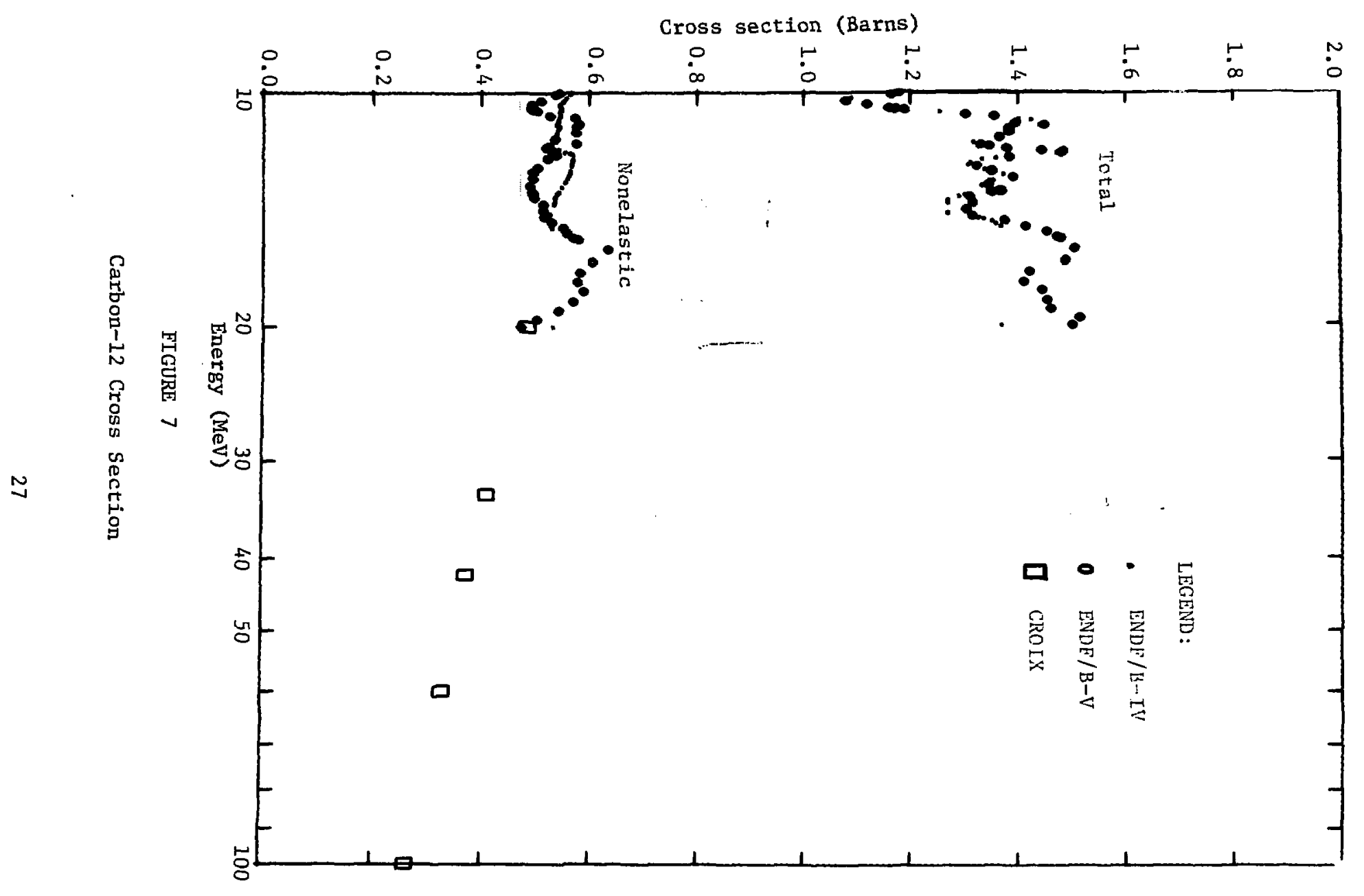




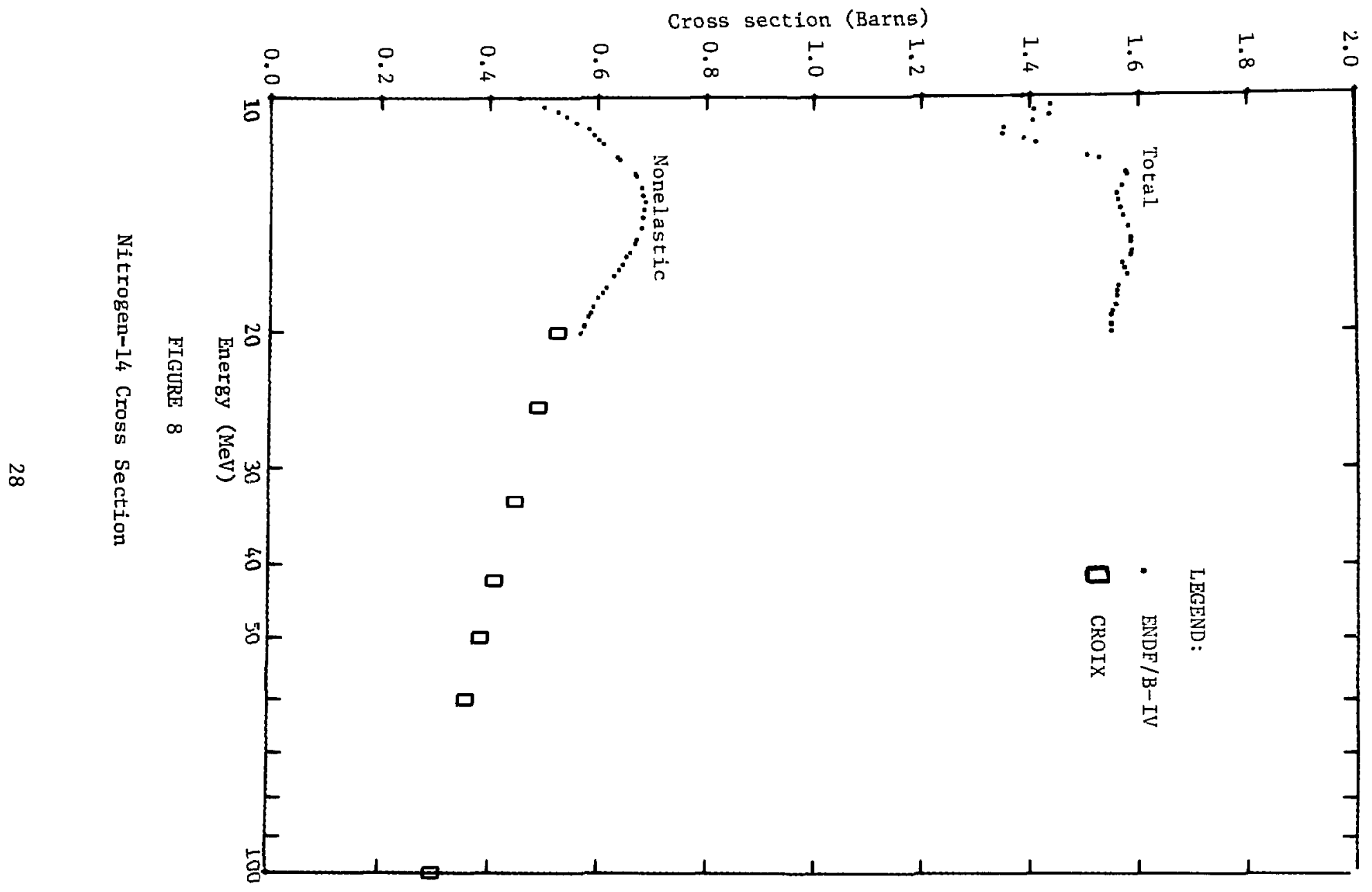




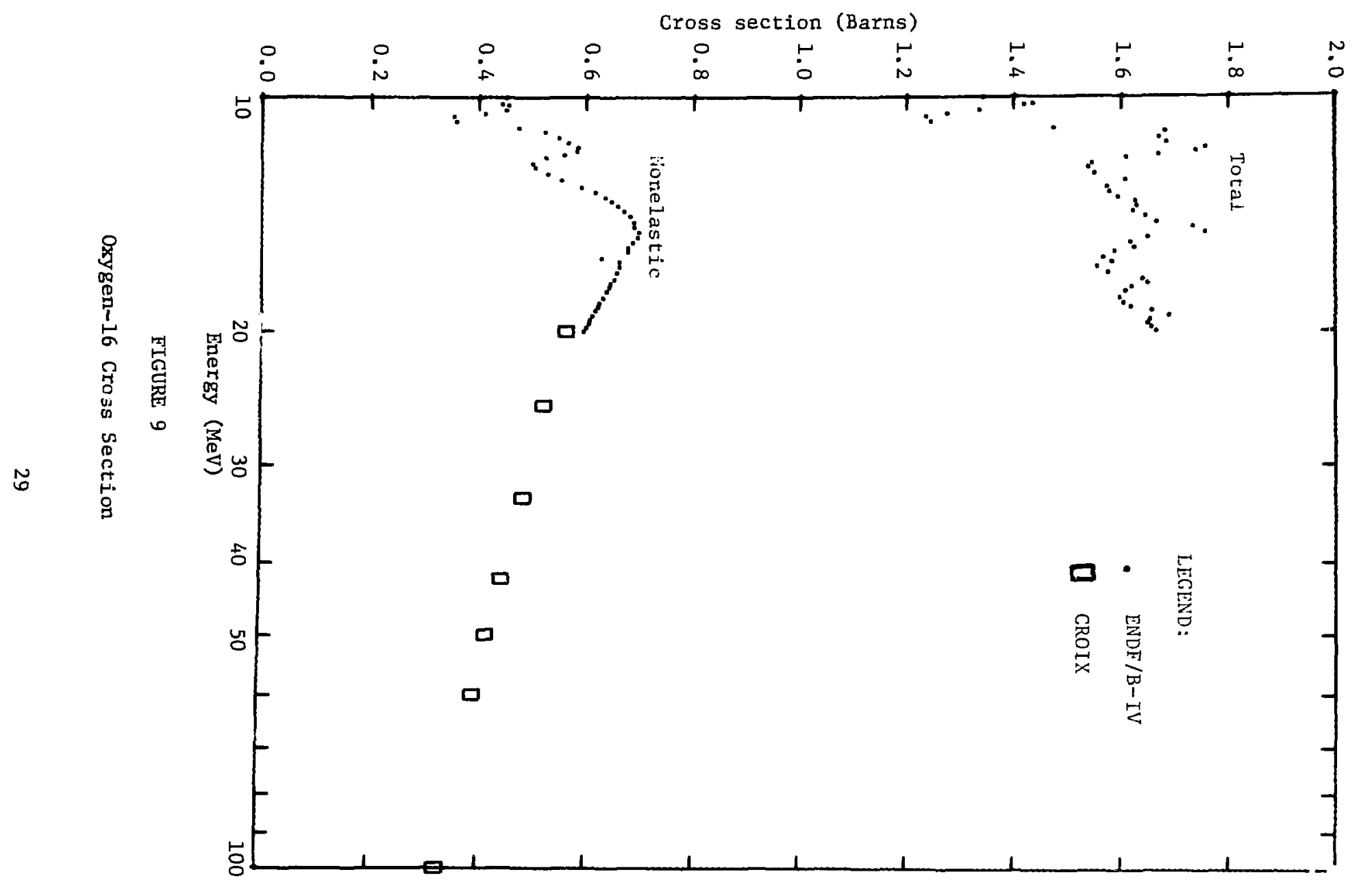


the ICE and ENDF cross sections at the $20 \mathrm{MeV}$ crossover point between ENDF and the ICE model. Both the total and ronelastic cross sections are plotted for the ENDF data, since MCNP treats both elastic and nonelastic collisions. The LASL version of NMTC does not treat elastic collisions with nuclei other than hydrogen, although the Oak Ridge version does. Thus elastic collisions with nuclei other than hydrogen are neglected for energies over $20 \mathrm{MeV}$. If included, these elastic collisions would contribute little to the energy loss of a nucleon in this range and would not produce light charged particles, as described in Appendix B. 
CHAPTER V

GA:RGED PARTICLE TRANSPORT AND KERMLA ANALYSIS

NMTC and MCNP are the source programs for charged particles, but these particles must also be transported to obtain the equilibrium or slowing down spectrum needed to calculate the desired dosimetry parameters. The charged particle transport below $20 \mathrm{MeV}$ is accomplished via the Continuous Sloving Down Approximation (CSDA), in which the discrete nature of charged particle collisions is neglected and particle slowing down is treated as a continuous process. Coulomb scattering is also neglected in this approach. Although this approximation would be inadequate for electrons in the energy range of this work ( $50 \mathrm{keV}-50 \mathrm{MeV})$, it suffices for the more massive protons and heavier charged particles.

The CSDA was implemented in a computer program in two ways. The method used initially was to transport each charged particle created by NMTC or MCNP through the ICRU muscle or Shonka A-150 plastic medium until its energy dropped below $50 \mathrm{keV}$. However, because of the short range of charged particles in these media, relative to the spacing of the planes at which the dosimetry parameters were to be calculated, it was found that the majority of the charged particles generated by NMTC and MCNP did not con- 
tribute to the slowing down spectra at the desired planes. This caused the statistics of the resulting spectra to be very poor even for large samples of neutron source particles. An alternative method was therefore substituted. This method does not transport each generated charged particle individually; instead, each particle's weight is added to the charged particle source spectrum within a volume element. The charged particle source spectra for each depth are divided into 10 equal direction cosine intervals, relative to the z-axis or depth, for each of 90 energy intervals. The energy intervals selected are those used by Williamson et al. 44 to describe the ranges and stopping powers of the elements, since these intervals are appropriate to both $\mathrm{S}$ and $\mathrm{W}$ calculations. Equal direction cosine intervals were selected since they are equiprobable intervals for isotropic particle emission. All charged particles generated by MMTC and MCNP will contribute to the charged particle source spectra, thus improving the statistics of the resulting planar slowing down spectra for a given computing cost. Each segment of the source spectrum may be transported as a single particle whose weight is the sum of all particle weights that contributed to it. This "pseudoparticle" is then transported through the medium according to the following scheme:

1) First, for a given energy, the range of the pseudoparticle 
is found from the appropriate range-energy table.

2) Next, the pseudoparticle weight is normalized by the fraction of the source volume which can contribute to the spectrum at the plane, which is the range divided by the source volume thickness.

3) The pseudoparticle weight and the contributing subvolume are divided by twice the number of the energy interval, but not less than ten, of the pseudoparticle. This is to insure that the pseudoparticle has the opportunity to contribute to each energy interval of the planar spectrum. The pseudoparticle has now been split into many fragments, each with correspondingly reduced weight.

4) The pseudopartic] = fragments are individually transported from the center of their respective subvolumes to the plane, and the weight of each fragment is added to the proper energy interval of the planar slowing down spectrum. If the fragment possesses enough energy after crossing the plane to reach a succeeding plane, it is transported to that plane as well.

5) The process is repeated for each of the ten direction cosine intervals.

6) The process is repeated for all energies. The actual implementation of the CSDA is very simple. First 
a range-energy table is constructed for the medium from Janni's stopping power tables? For a particle of a given energy (E), z-axis direction cosine (W), and distance to a plane (x):

1) First, compute the actual distance the particle must travel to reach the plane, $d$, where: $d=x / w$

2) Find the particle range, $R$, from the range-energy tables.

3) Compute the particle's remaining range, $R^{\prime}$, after it reaches the plane: $R^{\prime}=R-d$

4) Find the particle's remaining energy from the range-energy tables, given $\mathrm{R}^{\prime}$.

The range-energy tables are linearly interpolated between energy and range values.

Because of computer storage limitations and the complexity of MCNP, charged particle histories are not actually generated by MCNP. Instead, MCNP is used to generate a collision history describing all collisions of neutrons with energy less than or equal to $20 \mathrm{MeV}$ with nuclei. The collision history is used to drive the EVAP-4 evaporation module described in Chapter 4. MCNP normally sums all absorption cross sections and disregards individual charged particle production mechanisms. Rather than modify this, a collision history dependent upon the total absorption cross section was accepted. The relative strengths of the 
( $n, a 1$ pha), (n,proton), ( $n$,deuteron), and ( $n$,triton) cross sections from the Evaluated Nuclear Data File are used to determine the relative production probability of each charged particle. Thus, every collision is used to contribute to the source spectrum for every allowable charged particle. To further improve the statistics of the energy and direction cosine dependence generated by the EVAP- 4 module, up to four particles of the most probable type or types are generated with correspondingly reduced weights. These techniques, with proper normalization, cause up to 13 charged particles to be generated from a single collision, with relative probabilities and hence weights dictated by ENDF, and energy and direction cosine selected by EVAP-4. This particle splitting technique is used because the computer costs of the neutron transport process are an order of magnitude greater than the charged particle generation and transport costs; hence, any improvement in the charged particle generation is a good investment, since it results in a marked improvement in the accuracy of the charged particle spectrum with a small increase in cost. 


\section{VALIDATION OF CUIPUTER PROGRAMS}

The computer programs involved in this work are very large and complex, and it was therefore considered desirable to perform various numerical experiments the results of which could be compared to available data. Two such tests were performed: the first was a simulation of dosimetry parameters for a deuterium-tritium (D-T) spectrum and the second was a series of calculations to obtain kerma values for monoenergetic neutrons at various energies in $H, C, N$, and $O$.

The D-T spectrum simulation results are presented in Table 3 with values accepted by various therapy groups. 45 The simulated dosimetry parameters do agree quite well with the accepted values. Neutron spectra at 1,10 , and $25 \mathrm{~cm}$ deep in phantom were calculated as we1l. Comparison with measurements made with time-of-flight techniques ${ }^{46}$ shows reasonable agreement, even though the time-of-flight measurements do not include any neutrons with paths that do not intersect the detector, which subtends a very small solid angle. The results calculated here include neutrons with any direction. The calculated neutron spectra are presented in Figures 10-12. 
TABLE 3

\begin{tabular}{llll}
\multicolumn{3}{c}{ D-T Spectrum Parameters } & \\
& $\begin{array}{l}\text { ENDIP } \\
\text { Average } \\
\text { Value }\end{array}$ & Calculated & Difference \\
$\mathrm{W}_{\mathrm{TE} \text { gas }}$ & 30.6 & 30.3 & 1 percent \\
$\mathrm{S}_{\mathrm{TE} \text { gas }}^{\mathrm{A} 150}$ & 1.023 & 1.021 & .2 percent \\
$\mathrm{K}_{\mathrm{A}-150}$ & 1.05 & 1.03 & 2 percent \\
$\mathrm{K}_{\text {tissue }}$ & & &
\end{tabular}

*European Neutron Dosimetry Intercomparison Program 


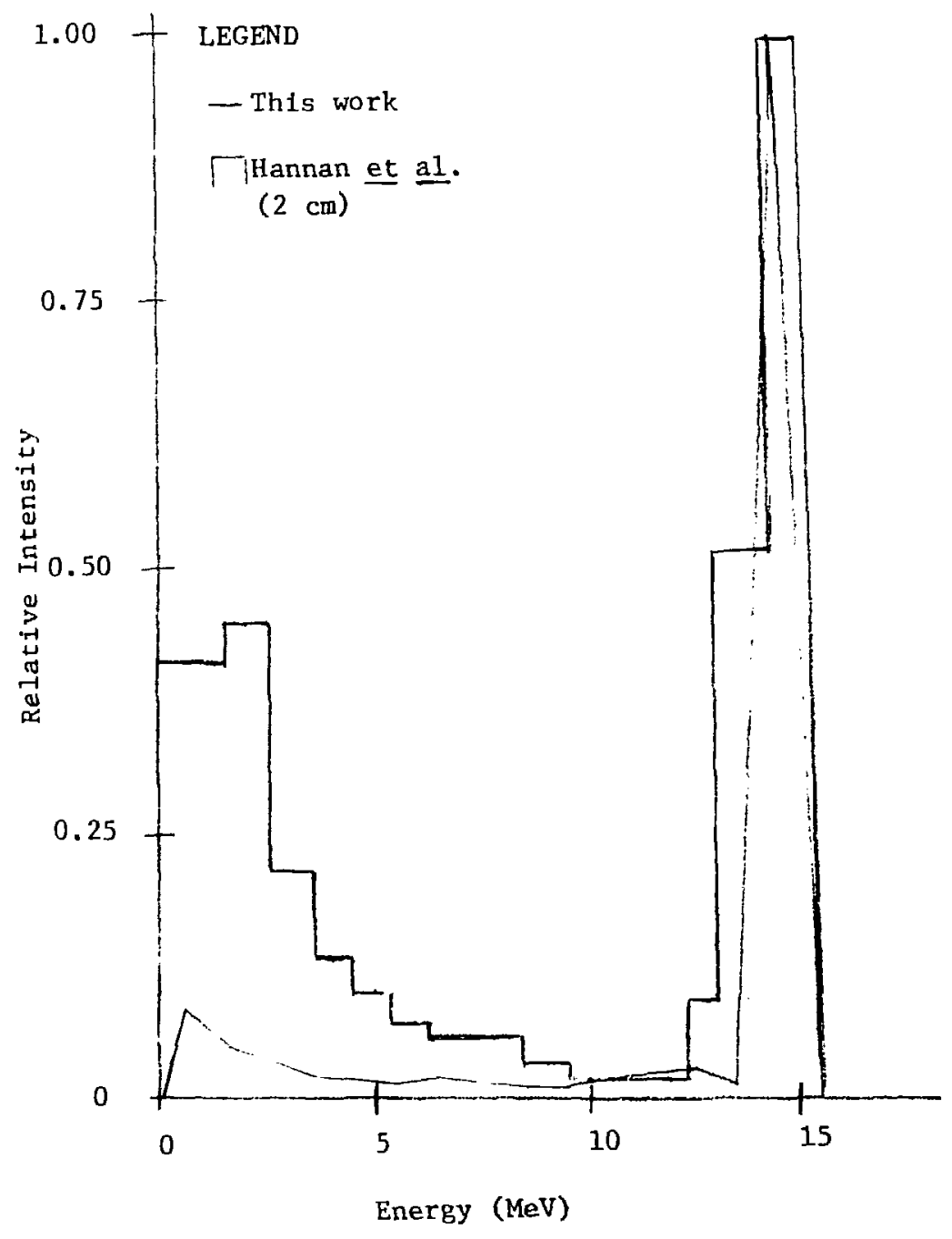

FIGURE 10

D-T Neut ron Spectrum at $1 \mathrm{~cm}$ Depth 


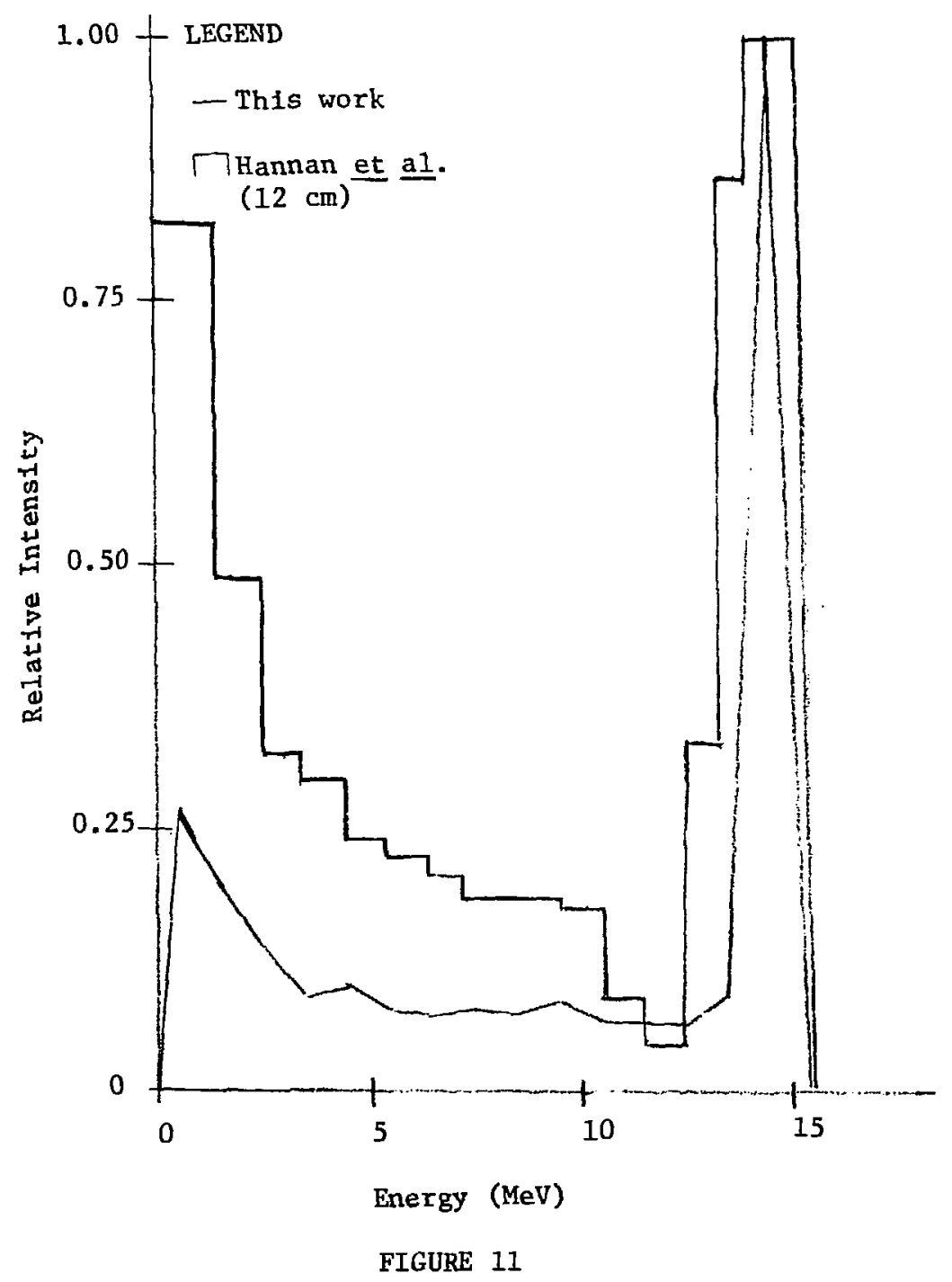

D-T Neutron Spectrum at $10 \mathrm{~cm}$ Depth 


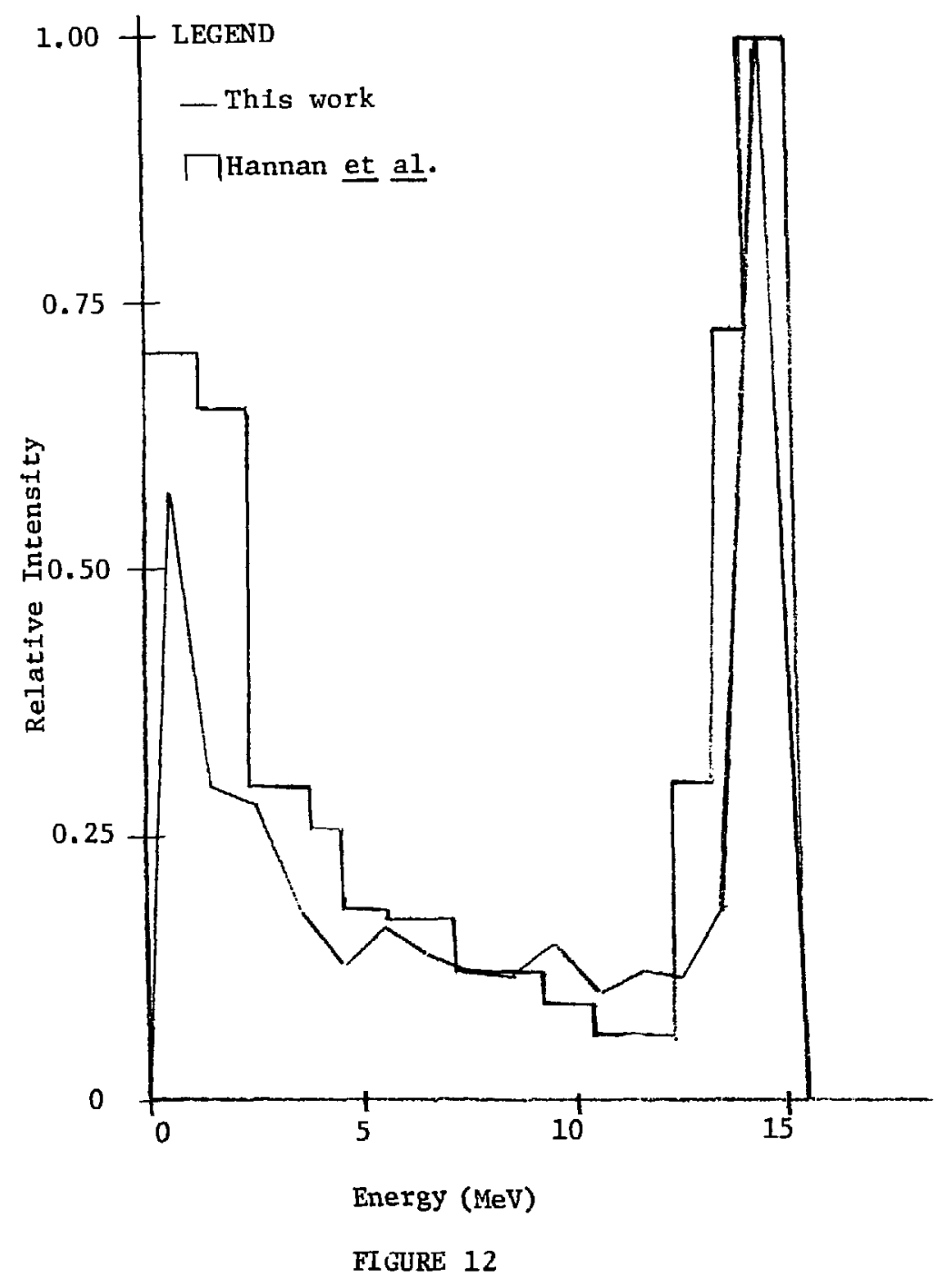

D-T Neutron Spectrum at $25 \mathrm{~cm}$ depth 
Kerma values for elemental materials can be obtained from NMTC/Q and MCNP by allowing monoenergetic neutrons to enter a phantom of the desired element and requiring that only the first collision of each neutron be calculated. Kermas for $\mathrm{H}, \mathrm{C}, \mathrm{N}$, and 0 were computed using this technique, and the results are presented in Figures 13-16 with the kerma values obtained by Caswe11 and Coyne $^{47}$ and Alsmiller and Barish ${ }^{48}$ included for comparison. At energies less than or equal to $20 \mathrm{MeV}$, a third source of kerma values is available at LASL in the ACE (A Compact ENDF) data files used by MCNP and other Monte Carlo programs. The ACE values may be considered to be an upper bound to the kerma because they can contain a contribution from gamma rays. The kerma may be expressed as the product of the average energy released by charged particles per interaction and the macroscopic cross section. Thus, the value of the cross section used to calculate the kerma is very importane. For energies less than $20 \mathrm{MeV}$, the cross sections from ENDF were used. Above $20 \mathrm{MeV}$, evaluations of the nonelastic cross sections for $\mathrm{C}, \mathrm{N}$, and 0 were made. For carbon, measured cross section values ${ }^{49}$ were used in the evaluation. For nitrogen and oxygen, measured data are not available above $20 \mathrm{MeV}$. Both Intranuclear Cascade plus Evaporation and Optical model ${ }^{50}$ calculations were made, and the evaluations are simply the ICE 


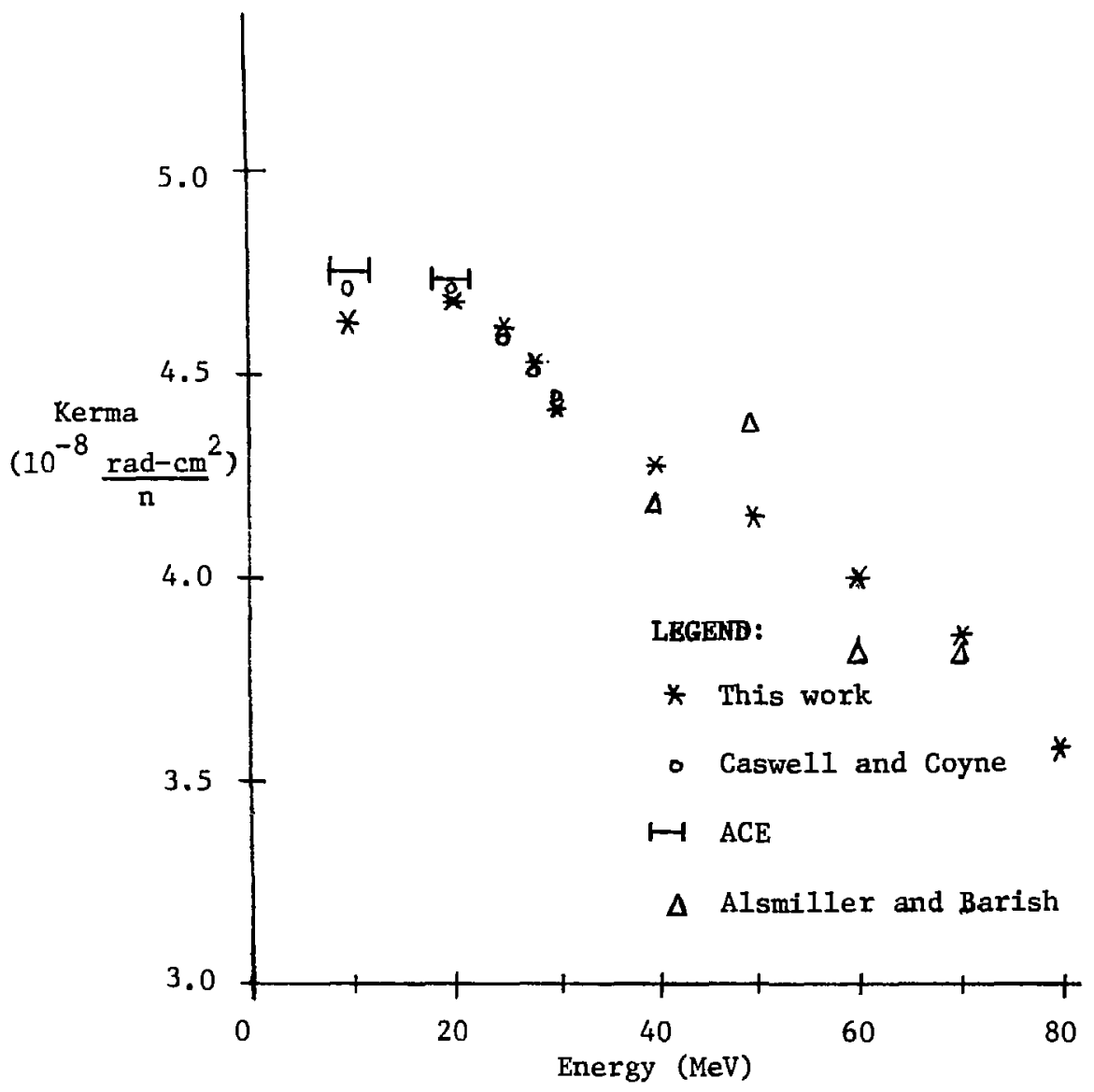

FIGURE 13

Hydrogen Kerma 


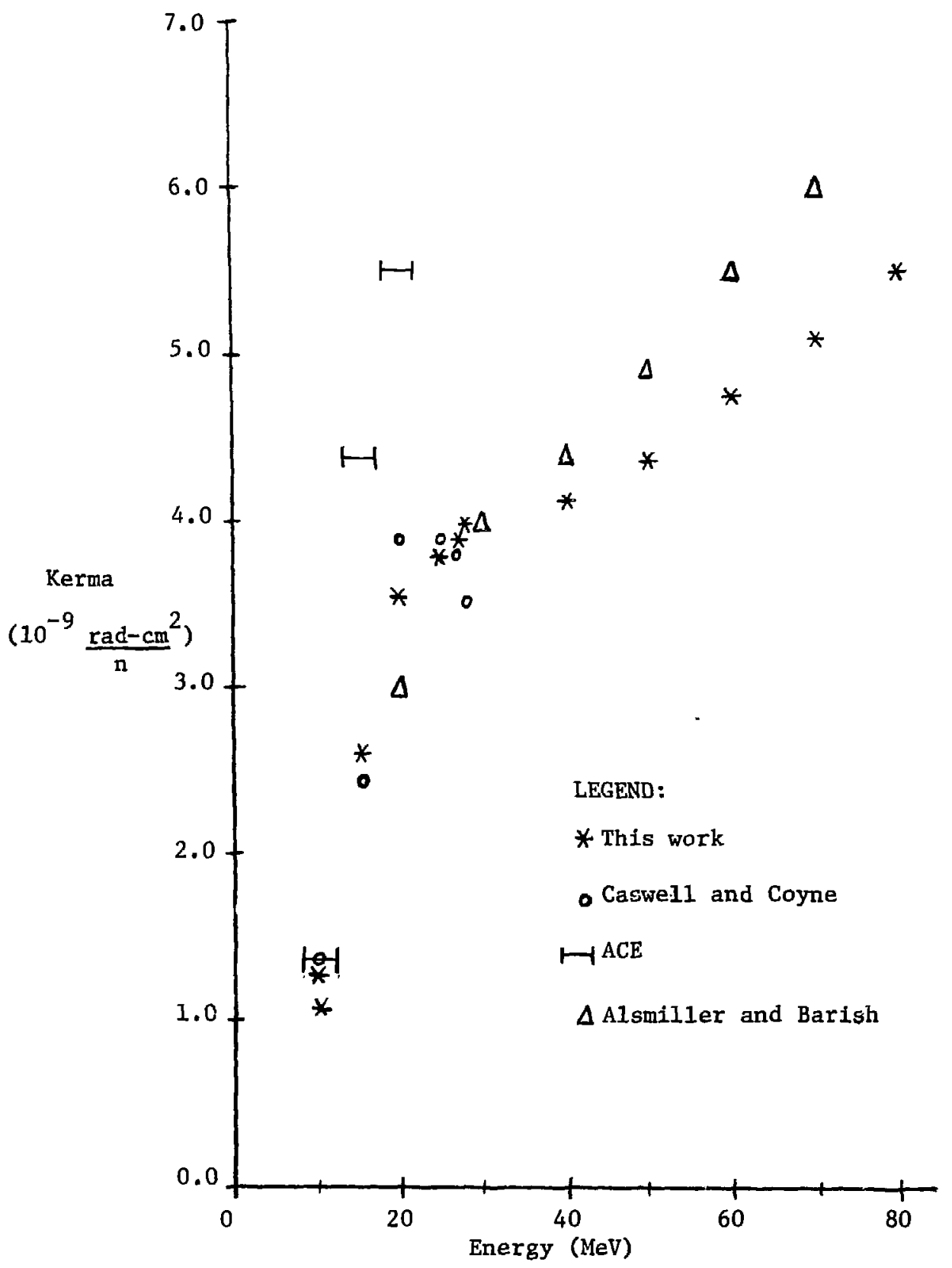

FIGURE 14

Carbon Kerma 


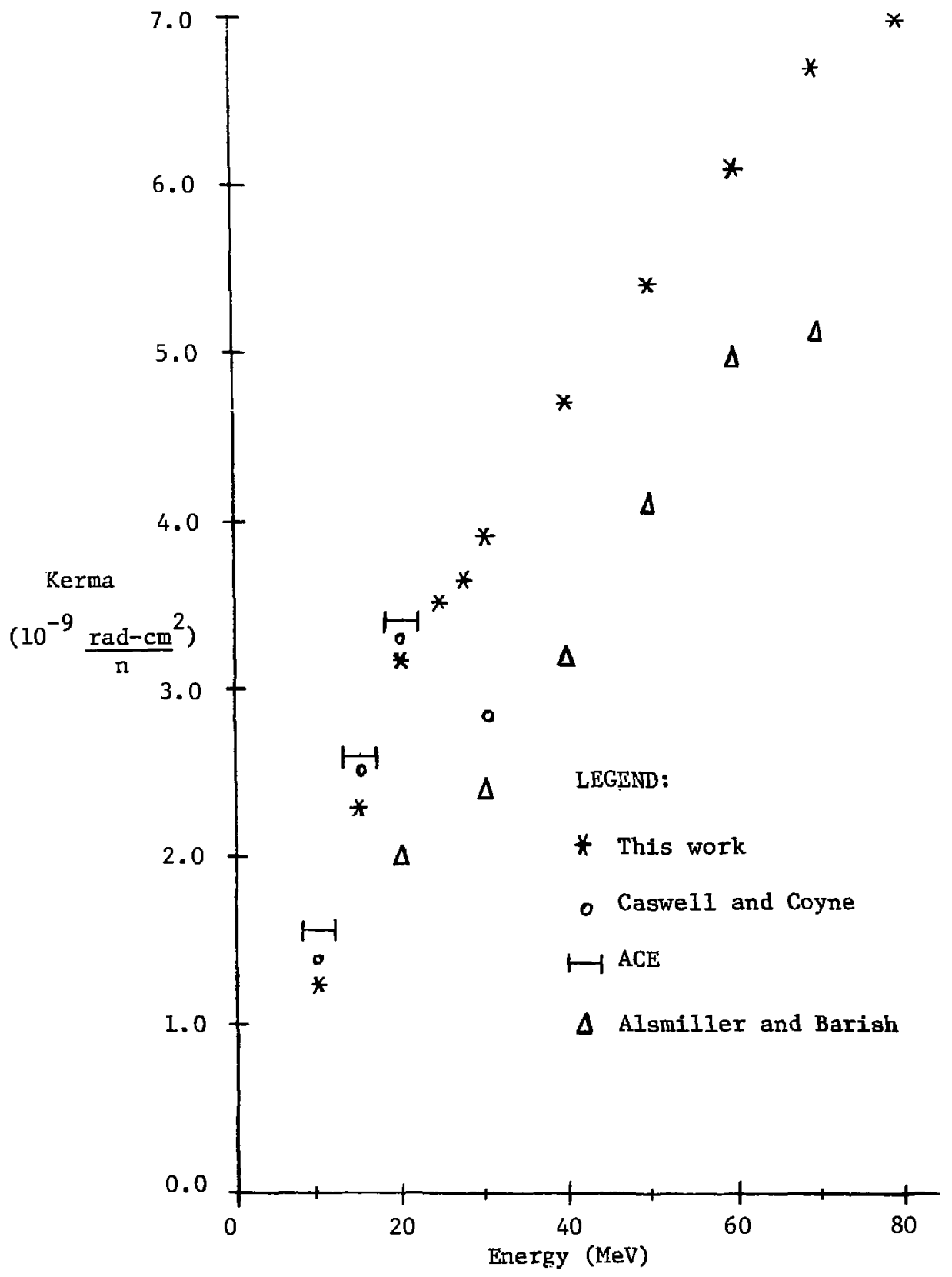

FIGURE 15

Nitrogen Kerma 


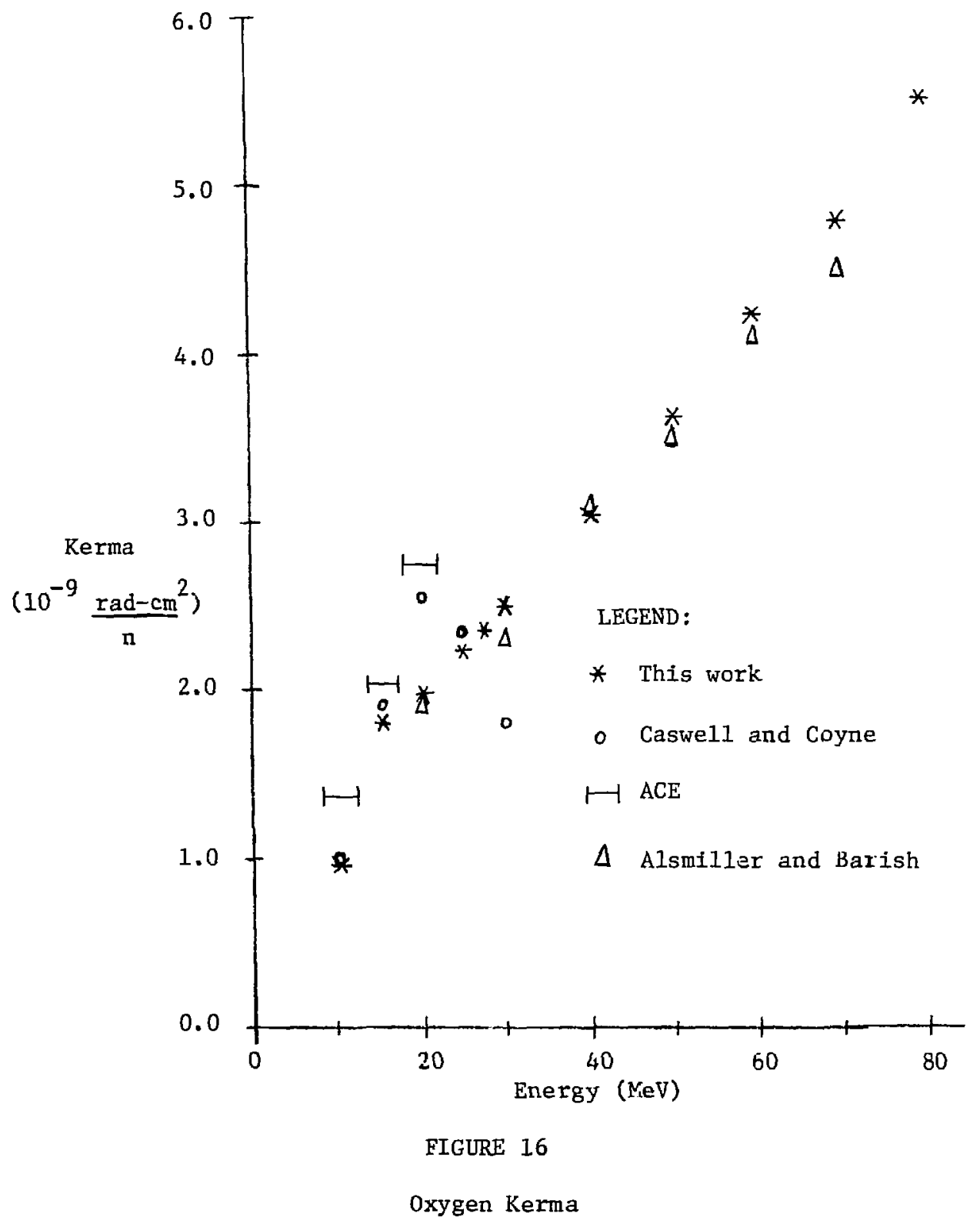


values fitted ro the ENDF cross sections at $20 \mathrm{MeV}$. The kerma values obtained from NMTC/Q were normalized to agree with the MCNP values at $20 \mathrm{MeV}$ so that a consistent set of values would result. Inspection of Figures $13-16$ indicates that the kerma values are comparable to those calculated by others. The tests described above allowed several technical and programming errors in this work to be discovered, and result in more credibility in the computational methods used here. 
The dosimetry parameters calculated in this work are tabulated at various depths in phantom in Table 4. Inspection of this table indicates that the values of $W_{A i r}$ derived from the value of $\mathrm{S}_{\mathrm{Air}}^{\mathrm{W}}$ obtained from the slowing down spectrum are unphysical, since they are lower than the $W$ value for electrons in air, $33.73 \mathrm{ev} /$ ion pair. This is due to the large differences in composition for Shonka A-150 plastic and air and the 5-10 percent uncertainties in stopping power values. The values of $W_{\text {Air }}$ calculated with this method are all within $6 \%$ of the electron lower limit, and the stopping power uncertainties account for the unphysical results. The results for $\mathrm{s}_{\mathrm{Air}}^{\mathrm{W}}$ obtained from the slowing down spectrum and the results for $\mathrm{W}_{\mathrm{Air}}$ derived from them were discarded. Instead, the values of $\mathrm{W}_{\mathrm{Air}}$ obtained using the synthesized energy dependence as described in Chapter II, and the $\mathrm{s}_{\mathrm{A} \text { ir }}^{\mathrm{W}}$ derived from them, Table 4 indicates that $\mathrm{s}_{\mathrm{g}}^{\mathrm{W}}$ and $\mathrm{H}$ are larger in the buildup region, from 0 to $1 \mathrm{~cm}$ deep, than at greater depths. This is due to the reduction of the recoil proton component of the 


\section{TABLE 4}

$50 \mathrm{MeV} \mathrm{d}^{+}$on Be Neutron Spectrum Dosinetry Parameters

\begin{tabular}{|c|c|c|c|c|c|c|}
\hline $\begin{array}{r}\text { Depth } \\
(\mathrm{cm})\end{array}$ & $\mathrm{S}_{\mathrm{TE}}^{\mathrm{W}}$ gas & ${ }^{\mathrm{T}} \mathrm{TE}$ gas & $\begin{array}{l}\mathrm{S}_{\mathrm{Air}}^{\mathrm{W}} \\
\text { (synthe } \\
\mathrm{W}_{\mathrm{Air}}\end{array}$ & $\begin{array}{c}\text { WAir } \\
\text { sized } \\
\text { ) }\end{array}$ & $\begin{array}{l}\mathrm{S}_{\text {Air }}^{W *} \\
\text { (slowing } \\
\text { spectra }\end{array}$ & $\begin{array}{l}w_{\text {Air }}^{*} \\
\text { down } \\
\left.S_{\text {Air }}\right)\end{array}$ \\
\hline 0.00 & 1.034 & 30.77 & 1.216 & 34.77 & 1.340 & 31.55 \\
\hline 0.05 & 1.015 & 30.39 & 1.194 & 34.34 & 1.262 & 32.50 \\
\hline 0.10 & 1.016 & 30.34 & 1.196 & 34.29 & 1.263 & 32.45 \\
\hline 0.15 & 1.011 & 30.27 & 1.189 & 34.20 & 1.236 & 32.91 \\
\hline 0.20 & 1.011 & 30.18 & 1.189 & 34.10 & 1.224 & 33.12 \\
\hline 0.40 & 1.011 & 30.19 & 1.189 & 34.12 & 1.227 & 33.07 \\
\hline 0.60 & 1.010 & 30.15 & 1.188 & 34.07 & 1.218 & 33.22 \\
\hline 1.00 & 1.010 & 30.12 & 1.188 & 34.04 & 1.214 & 33.30 \\
\hline 2.00 & 1.010 & 30.13 & 1.188 & 34.05 & 1.212 & 33.23 \\
\hline 4.00 & 1.011 & 30.13 & 1.189 & 34.05 & 1.220 & 33.18 \\
\hline 6.00 & 1.011 & 30.13 & 1.189 & 34.05 & 1.221 & 33.16 \\
\hline 10.0 & 1.011 & 30.13 & 1.189 & 34.05 & 1.220 & 33.18 \\
\hline 14.0 & 1.010 & 30.13 & 1.188 & 34.05 & 1.217 & 33.23 \\
\hline
\end{tabular}

* Values for $\mathrm{S}_{\mathrm{W}}^{\mathrm{W}}$ and $\mathrm{W}_{\mathrm{Air}}$ obtained from the slowing down spectrum are subjectite large uncertainties, and were discarded. 
TABLE 4

\section{(continued)}

\begin{tabular}{|c|c|c|}
\hline $\begin{array}{l}\text { Depth } \\
(\mathrm{cm})\end{array}$ & 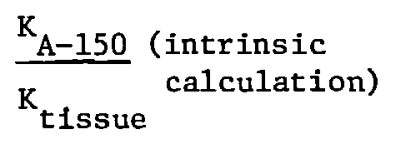 & 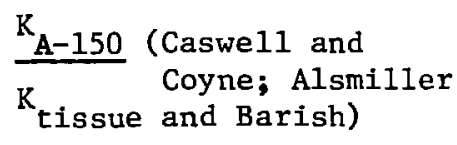 \\
\hline 1.00 & 1.08 & 1.07 \\
\hline 10.0 & 1.05 & 1.03 \\
\hline 25.0 & 1.00 & 1.00 \\
\hline $\ln \operatorname{Air}^{* *}$ & -- & 1.09 \\
\hline
\end{tabular}


slowing down spectrum near the surface of the phantom, which is caused by the forward peaking of the recoil proton scattering angle. 51 This effect may be seen in Table 5, which lists the contribution to the dose from protons and alphas as a function of depth. Deuterons and tritons are neglected in this table because they contribute less than $0.1 \%$ at all depths. The average contribution of alphas for depths greater than $1 \mathrm{~cm}$ is $9.7 \%$, which agrees very well with the $9 \%$ estimated by Bewley.

The kerma ratios listed in Table 4 include values calculated "intrinsically" by directly summing the Initial kinetic energy of all charged particles, including recoil nuclei, by the charged particle transport programs, and also include values obtained by using the neutron spectra calculated at 1,10 , and $25 \mathrm{~cm}$ to welght the kerma values of Caswell and Coyne 47 for energles of $30 \mathrm{MeV}$ or less and the values of Alsmiller and Barish ${ }^{48}$ for energies of more than $30 \mathrm{MeV}$. Values obtained via these two methods differ by only $2 \%$ or less.

If a fixed charge is collected in an ionizstion chamber, doses may be calculated using the dosimetry parameters developed In this work and those currently used at TAMVEC. 53 The differences between these doses were calculated with kerma ratios bbtained with both methods described above, and are tabulated in Table 6 . 


\section{TABLE 5}

$50 \mathrm{MeV} \mathrm{d}^{+}$on Be Neutron Spectrum Relative Dose Contributions

$\begin{array}{llc}\begin{array}{l}\text { Depth } \\ (\mathrm{cm})\end{array} & \begin{array}{l}\text { Protons } \\ \text { (percent) }\end{array} & \begin{array}{c}\text { Alphas } \\ \text { (percent) }\end{array} \\ 0.00 & 83.08 & 16.86 \\ 0.05 & 89.41 & 10.58 \\ 0.10 & 88.06 & 11.92 \\ 0.15 & 91.20 & 8.73 \\ 0.20 & 87.60 & 12.37 \\ 0.40 & 88.50 & 11.48 \\ 0.60 & 90.80 & 9.17 \\ 1.00 & 90.76 & 9.18 \\ 2.00 & 89.10 & 10.87 \\ 4.00 & 90.24 & 9.73 \\ 6.00 & 90.11 & 9.84 \\ 10.0 & 91.19 & 8.75 \\ 14.0 & 90.26 & 9.69\end{array}$


TABLE 6

$50 \mathrm{MeV} \mathrm{d}^{+}$on Be Neutron Spectrum Dose Comparisons

$\begin{array}{lll}\begin{array}{l}\text { Depth } \\ (\mathrm{cm})\end{array} & \begin{array}{l}\text { Difference } \\ \text { Using "intrinsic" } \\ \text { kerma ratio }\end{array} & \begin{array}{l}\text { Difference } \\ \text { Using neutron } \\ \text { spectrum kerma } \\ \text { ratio }\end{array} \\ 1.00 & -4.3 \% \text { (relative to } & -3.9 \% \\ 10.0 & -1.9 \% \quad \text { TAMVEC) } & -0.9 \% \\ 25.0 & +2.6 \% & +2.1 \%\end{array}$


The result that the dosimetry: parameters change with depth In approximately the first centimeter of the phantom is due to the shift in the neutron energy spectrum that occurs: The average neutron energy in the source spectrum is $20 \mathrm{MeV}$, but this is decreased to only about $14 \mathrm{MeV}$ by the first centimeter of phantom. This shift in the energy spectrum is not as strong at depths greater than $1 \mathrm{~cm}$, as indicated by comparison of Figures 17-19, which depict the neutron spectra at 1,10 , and $25 \mathrm{~cm}$ depth. No depth dependence was noted for the $\mathrm{D}-\mathrm{T}$ spectrum for the dosimetry parameters, described in Chapter 6. This is because of the overwhelming predominance of the energy deposition of $14.7 \mathrm{MeV}$ source neutrons. Dosimetry parameters that are a function of depth may be characteristic of neutron source spectra that are continuous functions of energy.

The energy of heavy recoil nuclei is regarded as deposited locally in this work. The average energies of the predominant recoil species, $0^{16}$ in ICRU muscle tissue and $\mathrm{C}^{12}$ in Shonka A-150 plastic, are 0.30 and $0.38 \mathrm{MeV}$ respectively. With such large masses and high effective charges, the ranges of these particles in tissue and A-150 plastic are about $2 \times 10^{-4} \mathrm{~mm}$ The ranges of protons and alphas, by comparison, are 


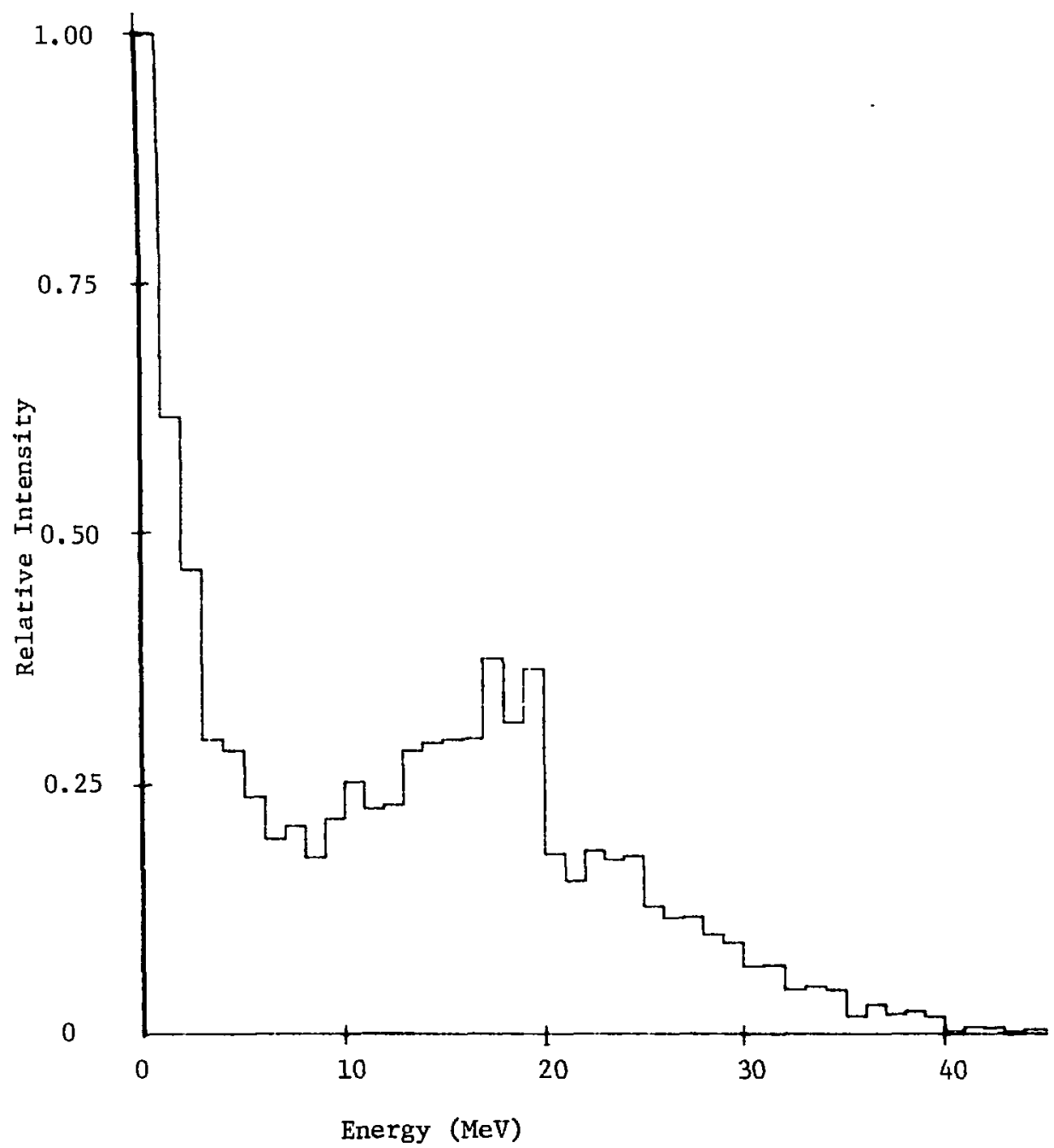

FIGURE 17

$50 \mathrm{Mev} \mathrm{d}^{+}$on Be Neutron Spectrum at $1 \mathrm{~cm}$ 


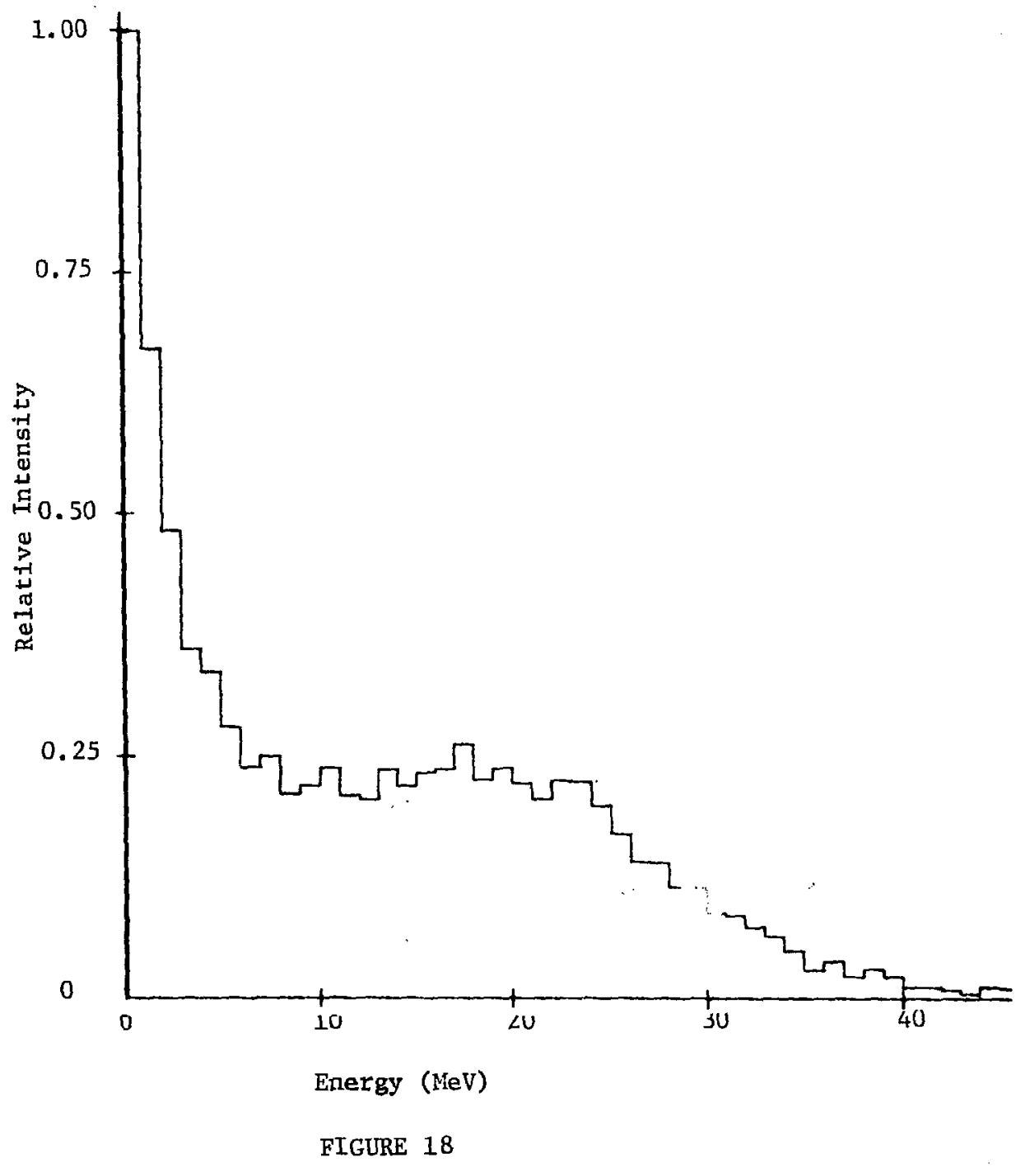

$50 \mathrm{MeV} \mathrm{d}{ }^{+}$on Be Neutron Spectrum at $10 \mathrm{~cm}$ 


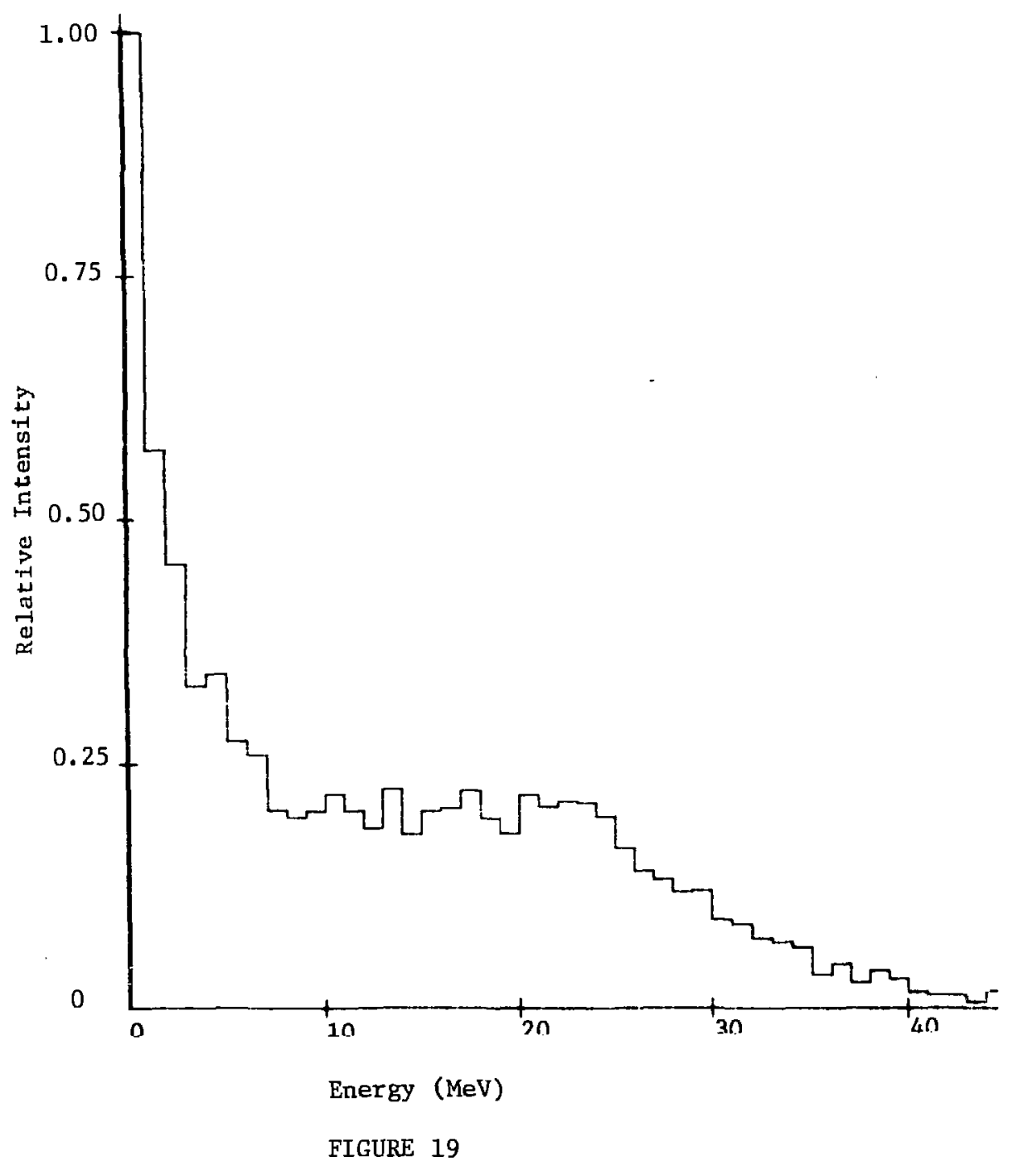

$50 \mathrm{MeV} \mathrm{d}^{+}$on Be Neutron Spectrum at $25 \mathrm{~cm}$ 
about $1 \mathrm{~mm}$ and $0.02 \mathrm{~mm}$. Recoll nuclel may therefore be regarded as stopplng locally, with a very small contribution to the slowIng down spectrum and hence to the $\mathrm{W}$.and $\mathrm{s}_{5}^{\mathrm{W}}$ parameters, although they must be Included in the kerma (see Table 7).

The use of Monte Carlo programs to determine the charged particle spectra Introduces statistical uncertainties into the resulting dosimetry parameters. An estimate of the size of these uncertainties may be obtained by processing a fixed number of source neutrons and calculating the desired dosimetry parameters. This process may be repeated a number of times, and the variance and standard deviation computed for $s_{g}^{W}, W$, $\mathrm{x}_{\mathrm{A}-150}, \mathrm{~K}_{\mathrm{tissue}}$, and the ratio $\mathrm{K}_{\mathrm{A}-150} / \mathrm{K}_{\text {tissue }}$. For this work, groups of 8,000 source neutrons were processed until a total of 56,000 source neutrons had been processed, generating over 300,000 charged particles, for A-150 plastic and ICRU muscle tissue. Each 1,000 source neutrons required approximately one minute of CDC 7600 computer time.

Statistical uncertainties in the dosimetry parameters are sma11, being less than $0.1 \%$ for $W$ and $s_{g}^{W}$ and about $2 \%$ for $t t_{3}=$ kerma values, for a $1 \%$ uncertainty in the kerma ratios. The sources of uncertaInty due to error in measurement of experi- 
$50 \mathrm{MeV} \mathrm{d}{ }^{+}$on Be Neutron Spectrum Recoil Nuclei

\begin{tabular}{|c|c|c|c|c|}
\hline $\begin{array}{l}\text { Recoil } \\
\text { Nucleus }\end{array}$ & $\begin{array}{l}\text { ICRU Muscle } \\
\text { Abundance } \\
\text { (percent) }\end{array}$ & $\begin{array}{l}\text { Tissue } \\
\text { Average } \\
\text { Energy } \\
(\mathrm{MeV})\end{array}$ & $\begin{array}{l}\text { Shonka A-150 } \\
\text { Abundance } \\
\text { (percent) }\end{array}$ & $\begin{array}{l}\text { Plastic } \\
\text { Average } \\
\text { Energy } \\
(\mathrm{MeV})\end{array}$ \\
\hline $\mathrm{Li}{ }^{7}$ & 0.00 & -- & 0.05 & 2.29 \\
\hline $\mathrm{Be}^{9}$ & 0.04 & 2.36 & 2.33 & 2.39 \\
\hline $\mathrm{Be} e^{10}$ & 0.00 & -- & 0.02 & 1.62 \\
\hline $\mathrm{Be}^{11}$ & 0.01 & 1.30 & 0.04 & 1.44 \\
\hline $\mathrm{B}^{10}$ & 0.05 & 2.00 & 0.20 & 2.19 \\
\hline $\mathrm{B}^{11}$ & 0.56 & 1.51 & 2.61 & 1.31 \\
\hline $\mathrm{B}^{12}$ & 0.22 & 0.98 & 1.36 & 0.93 \\
\hline $\mathrm{c}^{11}$ & 0.05 & 0.89 & 0.34 & 1.22 \\
\hline$c^{12}$ & 19.34 & 0.78 & 83.35 & 0.38 \\
\hline$c^{13}$ & 2.52 & 1.58 & 0.53 & 1.27 \\
\hline$c^{14}$ & 0.18 & 0.63 & 0.15 & 0.61 \\
\hline$c^{15}$ & 0.07 & 0.86 & 0.00 & 0.78 \\
\hline $\mathrm{N}^{13}$ & 0.02 & 0.62 & 0.01 & 0.50 \\
\hline $\mathrm{N}^{14}$ & 3.41 & 0.60 & 2.99 & 0.39 \\
\hline $\mathrm{N}^{15}$ & 4.57 & 1.04 & 0.40 & 1.07 \\
\hline $\mathrm{N}^{16}$ & 1.18 & 0.68 & 0.12 & 0.99 \\
\hline $0^{15}$ & 0.36 & 0.85 & 0.03 & 0.77 \\
\hline $0^{16}$ & 67.27 & $0.3 n$ & 5.44 & 0.28 \\
\hline
\end{tabular}


mental data used to obtain the dosimetry parameters may be evaluated, but the uncertainty due to use of the Intra-Nuclear Cascade and Evaporation models is difficult to estimate. The very close agreement between the kerma ratios calculated via the ICE model and the optical model of CasweIl and Coyne 47 indicates that the kerma ratio is not strongly affected by choice of model. The small changes of $\mathrm{W}$ and $\mathrm{s}_{\mathrm{g}}^{\mathrm{W}}$ with depth indicate that these values are not strong functions of the charged particle spectra, which were generated partly via the models.

The uncertainty in $\mathrm{W}_{\mathrm{TE}}$ gas is about $1 \%$, the accuracy claimed for the experimental data. Because the compositions of TE gas and Shonka A-150 plastic are very similar, absolute error in the stopping powers is less apparent in the stopping power ratio. To determine if the choice of stopping power tables, Janni's? or the tables of Williamson et $a 1^{44}$ had any effect on the stopping power ratios, calculations were made with both. The change in $\mathrm{S}_{\mathrm{TE}}^{\mathrm{W}}$ gas was about $0.1 \%$, but the change in $\mathrm{s}_{\mathrm{Air}}^{\mathrm{W}}$ was about $3 \%$. This demonstrates the importance of similar compositions for wall material and gas to reduce the uncertainty in $\mathrm{s}_{\mathrm{g}}^{\mathrm{W}}$. Because of the small change in $\mathrm{S}_{\mathrm{TE}}^{\mathrm{W}}$ gas incurred by changing stopping power tables, and because of the relatively high (about $11 \mathrm{MeV}$ ) 
average energy of the protons in the slowing down spectra, an overall accuracy of about $1 \%$ will be attributed to $\mathrm{S}_{\mathrm{TE}}^{\mathrm{W}}$ gas The uncertainty of the stopping power decreases with increasing particle energy, from about $10 \%$ at $50 \mathrm{keV}$ to about $2 \%$ at 10 $\mathrm{MeV}^{7}$, so that a more energetic charged particle spectrum results in a smaller uncertainty.

Since the kerma ratios calculated by both methods described above agree within $2 \%$ and other sources of error are less than $1 \%$, a $3 \%$ uncertainty will be attributed to the kerma ratio. A more precise estimate of the uncertainly is difficult to obtain because the kerna ratio is obtained via both experimental data and models. Even below $20 \mathrm{MeV}$ where ENDF is available, a model is still required to describe (n,charged particle) reactions because the residual excitation of the nucleus after charged particle emtssion is unknown. An additional source of error for the kerma ratio is the approximately $10 \%$ uncertainty in the measured source neutron spectrum. However, the $30 \%$ change in mean neurron energy in the first $\mathrm{cm}$ of the phantom results in only a $2 \%$ change in the kerma ratio, so the source spectrum uncertainty does not affect the kerma ratio more than about $2 / 3 \%$. The overall accuracy in a computed dose is therefore about $3.5 \%$. 
As stated in Chapter III, the $42 \mathrm{MeV} \mathrm{p}^{+}$on Be bean has been selected for neutron radiotherapy and is therefore of interest. A Monte Carlo simulation of the $42 \mathrm{MeV} \mathrm{p}^{+}$on Be neutron spectrum interactions with ICRU muscle tissue and Shonka A-150 tissue equivalent plastic was performed in exactly the same manner as for the $50 \mathrm{MeV} \mathrm{d}^{+}$on Be neutron spectrum. Since this neutron beam is not currently used for radiotherapy, no comparison of doses computed with accepted dosimetry parameters and the values calculated here was made. Table 8 lists the dosimetry parameters calculated for the $42 \mathrm{MeV} \mathrm{p}^{+}$on Be neutron beam. The dosimetry parameters are essentially constant for depths greater than $0.4 \mathrm{~cm}$, indicating that charged particle equilibrium is achieved at a depth of approximately $0.5 \mathrm{~cm}$, as for the $50 \mathrm{MeV} \mathrm{d}^{+}$on $\mathrm{Be}$ neutron beam. The values of $\mathrm{S}_{\mathrm{g}}^{\mathrm{W}}$ and $\mathrm{W}$ are equal to those calculated for the $50 \mathrm{MeV} \mathrm{d}^{+}$on Be neutron spectrum, within the statistical uncertainty quoted above. The kerma ratios are about $1 \%$ higher at all depths than those calculated for the $50 \mathrm{MeV} \mathrm{d}^{-1}$ on Be spectrum.

Table 9 lists the relative dose contributions from protons and alphas for the $42 \mathrm{MeV} \mathrm{p}^{+}$on be neutron beam. As for the $50 \mathrm{MeV} \mathrm{d}^{+}$on Be sp:strum, recoil protons are the primary dose component except at depths less than about $0.2 \mathrm{~cm}$. 


\section{TABLE 8}

$42 \mathrm{MeV}{ }^{+}$on Be Neutron Spectrum Dosimetry Parameters

\begin{tabular}{|c|c|c|c|c|}
\hline $\begin{array}{l}\text { Depth } \\
(\mathrm{cm})\end{array}$ & $S_{T E}^{W}$ gas & $\mathrm{W}_{\mathrm{TE}}$ gas & $\begin{array}{l}\mathrm{S}_{\text {Air }}^{\mathrm{W}} \\
\text { (synthesized } \\
\mathrm{W}_{\text {Air }}(\mathrm{E})\end{array}$ & $\mathrm{W}_{\mathrm{Air}}$ \\
\hline 0.00 & 1.026 & 30.70 & 1.207 & 34.69 \\
\hline 0.05 & 1.015 & 30.37 & 1.194 & 34.32 \\
\hline 0.10 & 1.014 & 30.29 & 1.193 & 34.23 \\
\hline 0.15 & 1.011 & 30.26 & 1.189 & 34.20 \\
\hline 0.20 & 1.014 & 30.18 & 1.193 & 34.10 \\
\hline 0.40 & 1.009 & 30.17 & 1.187 & 34.09 \\
\hline 0.60 & 1.009 & 30.14 & 1.187 & 34.06 \\
\hline 1.00 & 1.010 & 30.11 & 1.187 & 34.03 \\
\hline 2.00 & 1.010 & 30.11 & 1.188 & 34.03 \\
\hline 4.00 & 1.010 & 30.12 & 1.188 & 34.03 \\
\hline 6.00 & 1.010 & 30.12 & 1.188 & 34.03 \\
\hline 10.0 & 1.010 & 30.11 & 1.188 & 34.03 \\
\hline 14.0 & 1.010 & 30.11 & 1.188 & 34.03 \\
\hline
\end{tabular}




\section{TABLE 8}

\section{(continued)}

\begin{tabular}{|c|c|c|}
\hline $\begin{array}{c}\text { Depth } \\
(\mathrm{cm})\end{array}$ & 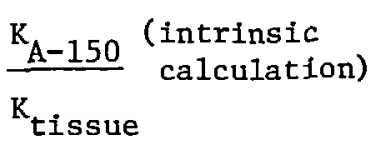 & $\frac{\mathrm{K}_{\mathrm{A}-150}}{\mathrm{~K}_{\text {tissue }}} \begin{array}{l}\text { (Caswe } 11 \text { and } \\
\text { Coyne; Alsmiller }\end{array}$ \\
\hline 1.00 & 1.07 & 1.085 \\
\hline 10.0 & 1.04 & 1.05 \\
\hline 25.0 & 1.00 & 1.01 \\
\hline in $\operatorname{Air}^{*}$ & - & 1.10 \\
\hline
\end{tabular}


TABLE 9

$42 \mathrm{MeV} \mathrm{P}^{+}$on Be Neutron Spectrum Relative Dose Contributions

$\begin{array}{ccc}\begin{array}{c}\text { Depth } \\ (\mathrm{cm})\end{array} & \begin{array}{l}\text { Protons } \\ \text { (percent) }\end{array} & \begin{array}{l}\text { A1phas } \\ \text { (percent) }\end{array} \\ 0.00 & 85.52 & 14.41 \\ 0.05 & 85.10 & 14.89 \\ 0.10 & 86.18 & 13.78 \\ 0.15 & 96.47 & 3.34 \\ 0.20 & 88.88 & 11.08 \\ 0.40 & 86.85 & 13.14 \\ 0.60 & 87.54 & 12.41 \\ 1.00 & 91.57 & 8.36 \\ 2.00 & 89.98 & 9.98 \\ 4.00 & 89.33 & 10.55 \\ 6.00 & 90.95 & 8.99 \\ 10.0 & 89.93 & 10.02 \\ 14.0 & 90.21 & 9.75\end{array}$


Figures 20-22 depict the $42 \mathrm{MeV}^{+}$on Be neutron spectra at 1,10 , and $25 \mathrm{~cm}$, respectively. Inspection of these figures indicates that the shift in neutrons in the first centimeter of the phantom is not as great as for the $50 \mathrm{MeV} \mathrm{d}^{+}$on $\mathrm{Be}$ neutron spectrum. The degradation of neutron energies for depths greater than $1 \mathrm{~cm}$ is similar to the degradation of the $50 \mathrm{MeV} \mathrm{\textrm {d } ^ { + }}$ on Be neutron spectrum. This is also indicated by the constant $1 \%$ difference between the kerma ratios, listed in Tables 4 and 8 .

Table 10 lists the recoil nuclei and associated average energies for the $42 \mathrm{MeV} \mathrm{P}^{+}$on Be neutron beam. As stated above for the $50 \mathrm{MeV} \mathrm{d}^{+}$on Be neutron beam, the energy of recoil nuclei nay be considered to ba locally deposited. 


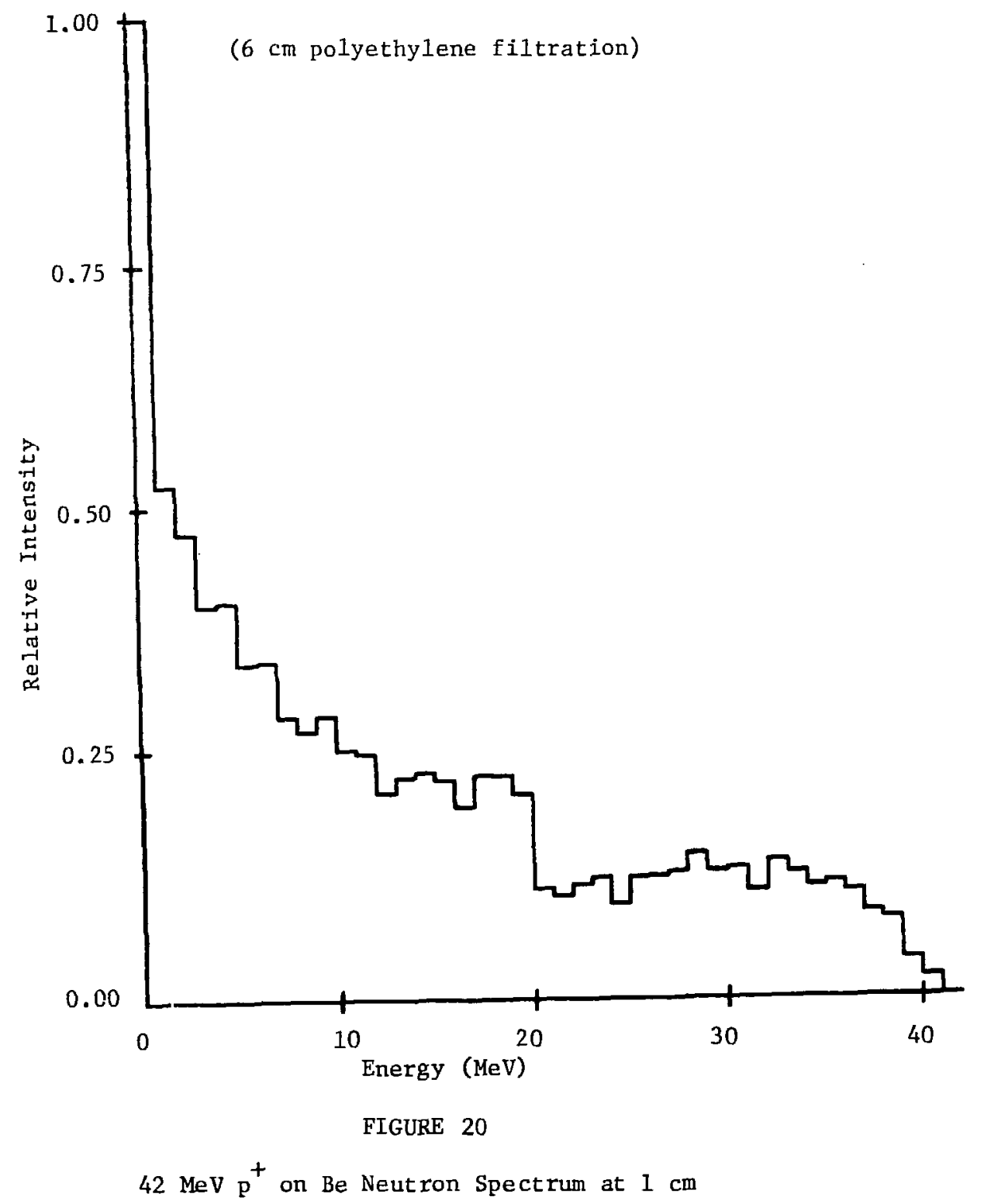




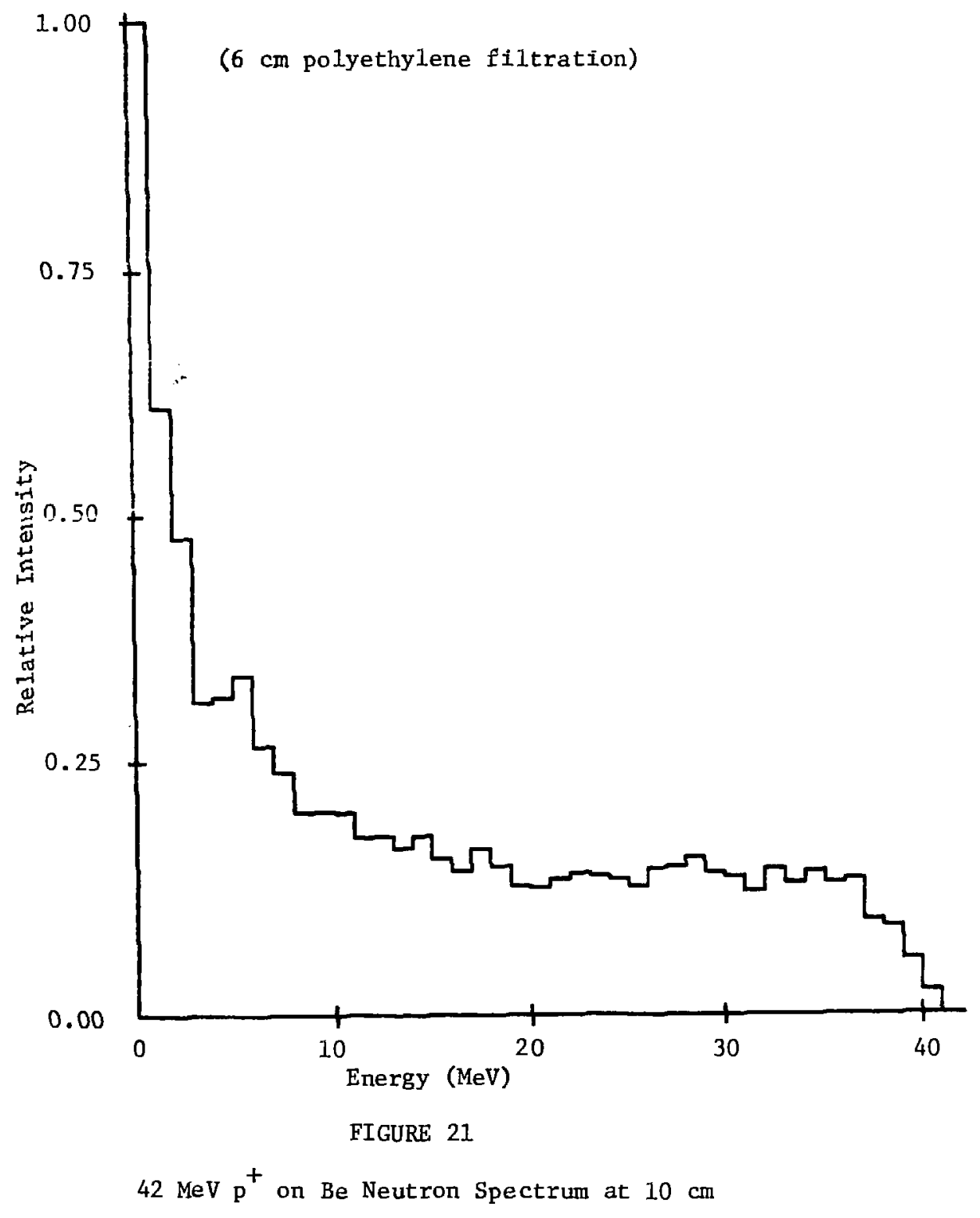




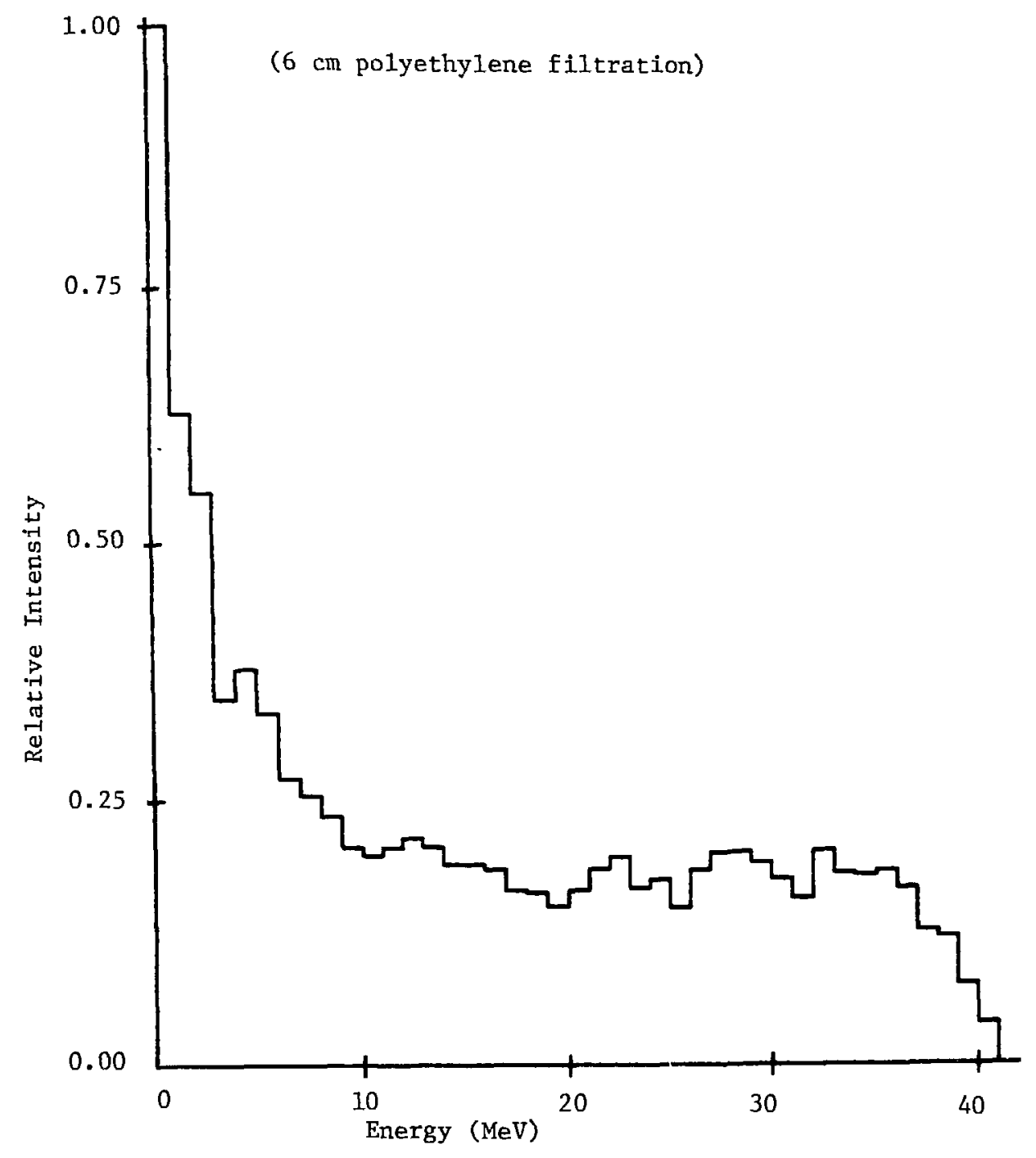

FIGURE 22

$42 \mathrm{MeV} \mathrm{p}^{+}$an Be Neutron Spectrum at $25 \mathrm{~cm}$ 
TABLE 10

$42 \mathrm{MeV}^{+}$on Be Neutron Spectrum Recoil Nuclei

(6 cm polyethylene filtration)

\begin{tabular}{|c|c|c|c|c|}
\hline $\begin{array}{l}\text { Recoil } \\
\text { Nucleus }\end{array}$ & $\begin{array}{l}\text { ICRU Muscle } \\
\text { Abundance } \\
\text { (percent) }\end{array}$ & $\begin{array}{l}\text { Tissue } \\
\text { Average } \\
\text { Energy } \\
(\mathrm{MeV})\end{array}$ & $\begin{array}{l}\text { Shonka A-1 } \\
\text { Abundance } \\
\text { (percent) }\end{array}$ & $\begin{array}{l}0 \text { Plastic } \\
\text { Average } \\
\text { Energy } \\
(\mathrm{MeV})\end{array}$ \\
\hline $\mathrm{Li}^{7}$ & 0.04 & 2.29 & 0.12 & 2.31 \\
\hline $\mathrm{B} e^{9}$ & 0.35 & 2.20 & 2.04 & 2.22 \\
\hline $\mathrm{B}^{10}$ & 0.07 & 2.32 & 0.33 & 2.02 \\
\hline $\mathrm{B}^{11}$ & 0.74 & 1.48 & 3.38 & 1.39 \\
\hline $\mathrm{B}^{12}$ & 0.21 & 0.85 & 1.30 & 0.94 \\
\hline$c^{11}$ & 0.08 & 1.43 & 0.53 & 1.15 \\
\hline $\mathrm{c}^{12}$ & 19.03 & 0.74 & 82.83 & 0.36 \\
\hline$c^{13}$ & 2.26 & 1.54 & 0.48 & 1.11 \\
\hline $\mathrm{N}^{14}$ & 3.95 & 0.72 & 2.84 & 0.38 \\
\hline $\mathrm{N}^{15}$ & 4.76 & 1.06 & 0.41 & 1.14 \\
\hline $\mathrm{N}^{16}$ & 1.19 & 0.64 & - & -- \\
\hline $0^{15}$ & 0.55 & 0.92 & 0.05 & 0.56 \\
\hline $0^{16}$ & 66.48 & 0.28 & 5.30 & 0.28 \\
\hline
\end{tabular}


CHAPTER VIII

CONCLUSIONS

A recommended set of dosimetry parameters is presented in Table 11. The values of $\mathrm{S}_{\mathrm{g}}^{\mathrm{W}}$ and $\mathrm{W}$ are constant for depths greater than or equal to $\frac{1}{2} \mathrm{~cm}$, but the kerma ratio varies enough with depth that a constant value should not be used. Doses calculated by using the TAMVEC parameters for tumors at a depth of several centimeters or more agree to within about $1 \%$ with doses calculated with the parameters recommended here. Tumor volumes extending outward from the normal body contours, with resulting thicknesses of $2 \mathrm{~cm}$ or less, may be under-dosed by as much as $3.5 \%$ relative to doses calculated with the parameters recommended here. Dose accuracies previously obtained at TAMVEC are therefore within the $5 \%$ accuracy that was expected, and are in fact within $4 \%$. The calculated values of $\mathrm{s}_{\mathrm{g}}^{\mathrm{W}}$ and $\mathrm{W}$ are the same, within $0.1 \%$, for the $50 \mathrm{MeV} \mathrm{d}^{+}$on $\mathrm{Be}$ and the $42 \mathrm{MeV} \mathrm{P}^{+}$on Be neutron spectra. The kerma ratio for the $42 \mathrm{MeV} \mathrm{P}^{+}$on Be neutron spectrum is about $1 \%$ higher than the kerma ratio for the $50 \mathrm{MeV} \mathrm{d}^{+}$on Be spectrum. 
TABLE 11

Recommended Dosimetry Parameters

$50 \mathrm{MeV} \mathrm{\textrm {a } ^ { + }}$ on Be Neutron Spectrum

$\begin{array}{llllll}\begin{array}{c}\text { Depth } \\ (\mathrm{cm})\end{array} & \frac{\mathrm{K}_{\mathrm{A}-150}}{\mathrm{~K}_{\text {tissue }}} & \begin{array}{c}\mathrm{W}_{\mathrm{TE}} \text { gas } \\ \text { (eV/ion } \\ \text { pair) }\end{array} & \mathrm{S}_{\mathrm{TE} \text { gas }}^{\mathrm{W}} & \begin{array}{c}\mathrm{W}_{\text {Air }} \\ \text { (eV/ion } \\ \text { pair) }\end{array} & \mathrm{s}_{\text {Air }}^{\mathrm{W}} \\ \text { in Air } & 1.09 & 30.13 & 1.011 & 34.05 & 1.189 \\ 1.00 & 1.07 & 30.13 & 1.011 & 34.05 & 1.189 \\ 10.0 & 1.04 & 30.13 & 1.011 & 34.05 & 1.189 \\ 25.0 & 1.00 & 30.13 & 1.011 & 34.05 & 1.189\end{array}$

42

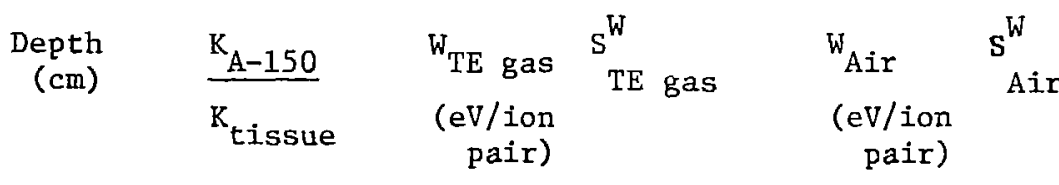

$\begin{array}{llllll}\text { in Air } & \text { 1.10 } & 30.12 & 1.010 & 34.03 & 1.188 \\ 1.00 & 1.08 & 30.12 & 1.010 & 34.03 & 1.188 \\ 10.0 & 1.05 & 30.12 & 1.010 & 34.03 & 1.188 \\ 25.0 & 1.01 & 30.12 & 1.010 & 34.03 & 1.188\end{array}$

* A chamber wall thickness of $0.5 \mathrm{~cm}$ is required to ensure that charged particle spectrum equilibrium will occur. 
ACKNOWLEDGMENTS

I wish to express my appreciation to:

James B. Smathers and D. Graham Foster, without

whom this dissertation would have been impossible;

W. B. Wilson, R. J. Barrett, and R. G. Graves, who answered many questions and provided friendly guidance. 
REFERENCES

1) Hussey, D.H., Parker, R.G., and Rogers, C.C., Intermational Journal of Radiation Oncology, Vo1. 3, 255 (1977)

2) Bewley, D.K., British Journal of Radiology, Vo1. 36, No. $422,81(1963)$

3) Personal Communication froun Dr. R. G. Graves, Division of Bioengineering, Industrial Engineering Department, Texas A\&M University, College Station, Texas.

4) Smathers, J.R., Otte, V.A., Smith, A.R., Almond, P., Attix, F.H., Spokas, J.J., Quain, W.M., Goodman, L.J., Medical Physics, Vol. 4, No. 1, 74 (1977)

5) Personal Communication from Dr. J. B. Smathers, Head, Div. of Bioengineering, Industrial Engineering Department, Texas A\&M University, College Station, Texas.

6) Morgan, K.Z., and Turner, J.E., Principles of Radiation Protection, Krieger Publishing Co., New York (1973)

7) Price, W.J., Nuclear Radiation Detertion, McGraw-Hill, New York (1964)

8) Caswe11, R.S. and Coyne, J.J., Proc. Workshop on Physical Data for Neutron Dosimetry, Rijswijk, The Netherlands (1976)

9) Alsmiller, R.G., and Barish, J., ORNL-TM-5702.

10) Janni, J.F., AFWL-TR-65-150 (1966)

11) Wilson, J.W., NASA TN D-8381 (1977) 
12) Attix, F.H., Roesch, W.C., and Tochilin, L., Radiation Dosimetry, Vol. 1, Academic Press, New York (1968)

13) Chemtob, M., Lavigne, B., Chary, J., Nguyen, D., Parmentier, N., Noel, J.P., Fiche, C., Physics of Medical Biology, Vol. 22, No. 2, 208 (1977)

14) Bichsel, H., Inokuti, M., ANL-76-88, Part I, Radiological and Environk.ental Research Division Annual Report (1976)

15) Rohrig, N. and Colvett, R., BNL-23691 (1978)

16) Personal communication from Dr. N. Rohrig, Medical Dept., Brookhaven National Laboratory, Upton, New York.

17) Guiho, J.P. and Simoen, J.P., International Journal of Applied Radiation and Isutopes, Vol. 26, 714 (1975)

18) Lowry, R.A. and Miller, G.H., Physical Review, Vol. 109, No. 3,826 (1958)

19) Bichsel, H., Proceedings of an International Workshop on Particle Radiation Therapy, sponsored by the American College of Radiology, Key Biscayne, Florida (Oct 1975)

20) Bichsel, H., AAFM Charged Particle Task Group Workshop, Jos Alamos, N.M. (Mar 1978)

21) Eenmaa, J., University of Washington Internal Report No. 75.3, (Jun 1975)

22) Meulders, J.P., Leleux, R., Macq, P.C., and Pirart, C., Physics of Medical Biology, Vol. 20, No. 2, 235 (1975) 
23) Serber, R., Physical Review, Vo1. 72, 1114 (Dec 1947)

24) Metropolis, N., Bivins, R., Storm, M., Turkevitch, A., Miller, J.M., and Friedl.znder, G., Physical Review, Vol. 110 (Apr 1958)

25) Alsmiller, R.G. Jr., and Hermann, O.W., Nuclear Science and Engineering, Vol. 40, 254 (1970)

26) Barashenkov, V.S. and Eliseev, S.M., JINR Preprint P-1678, Dubna (1964)

27) Barashenkov, V.S., Maltsev, V.M., and Toneev, V.D., JINR Preprint P-1969, Dubna (1965)

28) Barashenkov, V.S., Maltsev, V.M., and Toneev, V.D., JINR Preprint P-1978, Dubna (1965)

29) Barashenkov, V.S., Maltsev, V.M., and Toneev, V.D., JINR Preprint P-1970, Dubna (1965)

30) Bertini, H.W., ORNL-3383 (1963)

31) Bertini, H.W., Physical Review, Vol. 131, 1801 (Aug 1963)

32) Chen, K., Fraenkel, Z., Friedlander, G., Grover, J.R., Miller, J.M., and Shimamoto, Y., Physical Review, Vo1. 166, 949 (Feb 1968)

33) Barashenkov, V.S., Bertini, H.W., Chen, K., Friedlander, G., Harp, G.D., Iljinov, A.S., Miller, J.M., and Toneev, V.D., Nuclear Physies A187, 531 (1972) 
34) Bertini, H.W., ORNL 66-7-54 (1966)

35) Blann, Annual Review of Nuclear Science, Vol. 25, 128, (1975)

36) Personal Communication from J. Ginnochio, Los Alamos Sclentific Laboratory, N.M.

37) Weisskopf, V., Physical Review, Vol. 52, 295 (Aug 1937)

38) Dostrovsky, I., Fraenkel, Z. and Friedlander, G., Physical Review, Vo1. 116, 683 (1959)

39) Dresner, L., ORNL-TM-196 (Apr 1962)

40) Guthrie, M.P., ORNL-TM-3119 (Sep 1970)

41) Personal Communication from A. Ito, Cyclotron Unit, Institute of Medical Science, University of Tokyo (1977)

42) Personal Communication from D.G. Foster, Jr., Los Alamos Scientific Laboratury, N.M.

43) Wilson, W.B., PhD. Dissertation, Texas A\&M University, Texas (Jun 1977)

44) Williamson, C.F., Boujot, J.P., Picard, J., Report CEA-R-3042 (Ju1 1966)

45) European Neutron Dosimetry Intercomparison Project, to appear

46) Hannan, W.J., Porter, D., Lawson, R.C., Railton, R., Physics of Medical Biology, Vol. 18, No. 6, 808 (1973)

47) Caswe11, R., and Coyne, J., Procedural Workshop on Physical Data for Neutron Dosimetry, Rijswijk, The Netherlands (May 1976) 
48) Alsmiller, R.G., Jr. and Barish, J., ORNL/TM-5702 (1976)

49) Del Guerra, A., Nuclear Instruments and Methods, Vol. 135, 337 (1976)

50) Perey, C.M., and Perey, F.G., Atomic Data and Nuclear Data Tables, Vol. 17, 1 (1976)

51) Bewley, D.K., and McNally, N.J., and Page, B.C., Radiation Research, Vol. 58, 111 (1974)

52) Bewley, D.k., British Journal of Radiology, Vol. 36, No. 422, 81 (Feb 1963)

53) Smith, A.R., Almond, P.R., Smathers, J.B., Otte, V.A., Attix, F.H., Theus, R.B., Wooten, P., Bischel, H., Eenmaa, J., Williams, D., Bewley, D.K., and Parne11, C.J., Medical Physics, Vo1. 2, No. 4 (Ju1 75)

54) Bewley, D.K., Symposium on Pre-theraputic Experiments with the Fast Neutron Beam from the MRC Cyclotron at the Annual Congress of the British Institute of Radiology (Apr 1962)

55) Personal Communication from A.R. Smith, University of New Mexico, N.M. 
APPENDIX A

Effect of Gamma Rays on the Dosimetry Parameters

The effect of gamma radiation produced by the interactions of neutrons with tissue may be estimated by simple methods, although its precise calculation is much more difficult. The biological dose from gammas is approximately $1.5 \%^{41}$ to $2.0 \%^{54}$ of the total dose, and with a Relative Biological Effectiveness (RBE) of about 3 , this produces a physical dose in rads due to gammas that is about $5 \%$ of the total dose in rads.

The average energy of gammas may be estimated to be about $2 \mathrm{MeV}^{55}$, with the average energy of Compton eliectrons about 1.6 $\mathrm{MeV}$. The stopping power in water, which approximates tissue, is thus $2 \mathrm{MeV}-\mathrm{cm}^{2} / \mathrm{gm}$, and in air, $1.8 \mathrm{MeV}-\mathrm{cm}^{2} / \mathrm{gm}$. The average stopping power of protons in ICRU muscle tissue at $10 \mathrm{~cm}$ is $37.29 \mathrm{MeV}-\mathrm{cm}^{2} / \mathrm{gm}$ and it is $31.38 \mathrm{MeV}-\mathrm{cm}^{2} / \mathrm{gm}$ for air. Thus we can write:

$$
\begin{aligned}
& s^{W}=0.95(37.29)+0.05(2.0)=35.53 \\
& s^{\text {Air }}=0.95(31.38)+0.05(1.8)=29.90 \\
& \text { and } s_{\text {Air }}^{W}=s^{W} / s^{\text {Air }}=1.18884
\end{aligned}
$$

The value calculated for $\mathrm{s}_{\mathrm{Air}}^{\mathrm{W}}$ by neglecting gammas is 1.18859 , so including gammas has caused a change of only $0.015 \%$. 
The $W$ value for air at $10 \mathrm{~cm}$ due to charged particles is $34.03 \mathrm{eV}$ for each ion pair formed, and the $\mathrm{W}$ value for air do to the average Compton electron is 33.8 . Thus we can write:

$$
\begin{aligned}
W_{\text {Air }} & =0.95 \times 34.03+0.05 \times 33.8 \\
\text { and } W_{\text {Air }} & =34.02
\end{aligned}
$$

The value for $\mathrm{W}_{\mathrm{Air}}$ has changed by less than $0.03 \%$ because of the addition of gammas.

The $0.015 \%$ change in $\mathrm{S}_{\text {Air }}^{\mathrm{W}}$ and the $0.03 \%$ change in $\mathrm{W}_{\text {Air }}$ due to the addition of gammas are both less than the $0.1 \%$ statistical uncertainty of $\mathrm{S}_{\mathrm{g}}^{\mathrm{W}}$ and $\mathrm{W}$. Thus the effect of gammas upon the dosimetry parameters may be neglected. 
APPENDIX B

Effects of Elastic Collisions of Neutrons

For energies greater than $20 \mathrm{MeV}$, neutron elastic collisions with nuclei other than hydrogen are neglected in the LASL version of NMTC. An estimate of the effect of this simplification of the neutron transport must be made for the Shonka A-150 plastic and ICRU muscle tissue materials used in this work, because they contain light nuclei other than hydrogen.

To simplify this estimate, calcium and fluorine are neglected. This causes a slight overestimate of elastic collision effects, since the mean logarithmic energy decrement per collision, $\boldsymbol{\xi}$, decreases with increasing atomic weight.

The basis of this estimate is to consider a hypothetical collision containing elastic and nonelastic mechanisms that are averaged over all nuclei. This average collision has an energy loss $\overrightarrow{\Delta \mathrm{E}}$ that can be written:

$$
\widehat{\Delta E}=\sum_{i=1}^{3} P_{i} \widehat{\Delta E}_{i}
$$

where the subscript $i$ includes elastic collisions with hydrogen, elastic collisions with $C, N$, and 0 , and nonelastic collisions with $C, N$, and $0, P_{i}$ is tine probability of 
$i^{\text {th }}$ type of collision.

The relative probability, $\mathrm{P}_{i}$, is just:

$$
P_{i}=\frac{\sigma_{i}}{\sum_{i=1}^{3} \sigma_{i}}
$$

where $\sigma_{i}$ is the macroscopic cross section for the $i^{\text {th }}$

type of collision.

For elastic collisions with $C, N$, and $O$, the average energy

loss per collision is:

$$
\overline{\Delta E}=E_{0}-E_{0} e^{-\xi}
$$

and the average for a mixture is

$$
\vec{\xi}_{\text {Mix }}=\frac{\sum_{j} e_{j} \boldsymbol{\xi}_{j}}{\sum_{j} \sigma_{j}}
$$

where $\sigma_{e_{j}}$ is the macroscopic elastic cross section for the $j^{\text {th }}$ nuclide and $\xi_{j}$ is the mean logarithmic energy decrement per collision for the $j^{\text {th }}$ nuclide.

For elastic collisions with hydrogen, the average energy loss per collision is available from NMTC/Q. For nonelastic collisions with $C, N$, and 0 , NMTC/Q provides the needed information to compute $\overline{\Delta E}$ in terms of the average energies and multiplicities of secondary particles produced by the cascade 
and evaporation processes.

It must be noted that only $40 \%$ of all source ileutrons have energy greater than $20 \mathrm{MeV}$, so that the overall effect of the omission of elastic collisions with $\mathrm{C}, \mathrm{N}$, and 0 is lessened.

Table 12 contains the parameters required to estimate the effect of the omission of elastic collisions of neutrons with energies over $20 \mathrm{MeV}$ with $\mathrm{C}, \mathrm{N}$, and 0 for Shonka A-150 plastic, and Table 13 contains the same parameters for ICRU muscle tissue. The energy dependence of $\xi$ for $C$ and $O$ is depicted in Figures 22 and 24 , respectively. Ingpection of these figures indicates that $\boldsymbol{\xi}$ decreases with increasing energy, so that $20 \mathrm{MeV}$ is the "worst case" energy for this estimate.

The energy dependence of $\bar{\mu}$, the average direction cosine after elastic scattering with $C$ and $O$, is presented in Figures 25 and 26 , respectively. Inspection of these figures indicates that the scattering angle diminishes with increasing: energy; i.e., scattering is more forward as the energy increases above $20 \mathrm{MeV}$. At $20 \mathrm{MeV}$, for $\mathrm{C}$ and $\mathrm{O}$, the average scattering angle is 41 degrees. This too is a "worst case" at $20 \mathrm{MeV}$.

As the energy of the neutron increases over $20 \mathrm{MeV}$, the elastic and nonelastic cross sections both decrease, but 
Energy Loss Parameters for Shonka A-150 Plastic

$\begin{array}{lccc}\text { Nuclide } & \sigma_{\mathrm{e}}^{\left(\mathrm{cm}^{-1}\right)} & \sigma_{\mathrm{ne}}\left(\mathrm{cm}^{-1}\right) & \xi(20 \mathrm{MeV}) \\ \mathrm{H} & 3.37 \times 10^{-2} & - & - \\ \mathrm{C} & 4.46 \times 10^{-2} & 2.01 \times 10^{-2} & 0.04145 \\ \mathrm{O} & 1.72 \times 10^{-3} & 9.74 \times 10^{-4} & 0.02952 \\ \mathrm{~N} & 2.67 \times 10^{-3} & 1.48 \times 10^{-3} & 0.03545 \\ \vec{\xi}_{\mathrm{C}, \mathrm{N}, \mathrm{O}=0.0406} & & & \\ & & & \\ \text { Collision Type } & \overline{\Delta E}_{\mathrm{j}}(\mathrm{MeV}) & \mathrm{P}_{\mathrm{j}} & \mathrm{P}_{\mathrm{j}} \overline{\Delta \mathrm{E}}_{\mathrm{j}}(\mathrm{MeV}) \\ \text { Elastic (C,N,0) } & 0.80 & 0.4655 & 0.37 \quad(4.3 \%) \\ \text { Elastic (H) } & 12.9 & 0.3202 & 4.13 \quad(48.5 \%) \\ \text { Nonelastic (C,N,0) } & 18.7 & 0.2143 & 4.01 \quad(47.2 \%)\end{array}$


Energy Loss Parameters for ICRU muscle tissue

$\begin{array}{cccl}\text { Nuclide } & \sigma_{\mathrm{e}}\left(\mathrm{cm}^{-1}\right) & \sigma_{\mathrm{ne}}\left(\mathrm{cm}^{-1}\right) & \xi(20 \mathrm{MeV}) \\ \mathrm{H} & 3.22 \times 10^{-2} & -- & - \\ \mathrm{C} & 6.25 \times 10^{-3} & 2.82 \times 10^{-3} & 0.04145 \\ \mathrm{O} & 3.15 \times 10^{-2} & 1.75 \times 10^{-2} & 0.02952 \\ \mathrm{~N} & 1.59 \times 10^{-3} & 9.02 \times 10^{-4} & 0.03545 \\ \bar{\xi}_{\mathrm{C}, \mathrm{N}, \mathrm{O}}=0.03166 & & \\ \text { Col1ision Type } \overline{\Delta \mathrm{E}}(\mathrm{MeV}) & \mathrm{P}_{\mathrm{j}} & \\ \text { Elastic (C,N,0) } 0.62 & 0.4241 & \mathrm{P}_{\mathrm{j}} \overline{\Delta \mathrm{E}}_{\mathrm{j}}(\mathrm{MeV}) \\ \text { Elastic (H) } & 12.8 & 0.3471 & 0.263(2.8 \%) \\ \text { Nonelastic }(\mathrm{C}, \mathrm{N}, 0) 18.5 & 0.2288 & 4.43 \quad(49.7 \%)\end{array}$




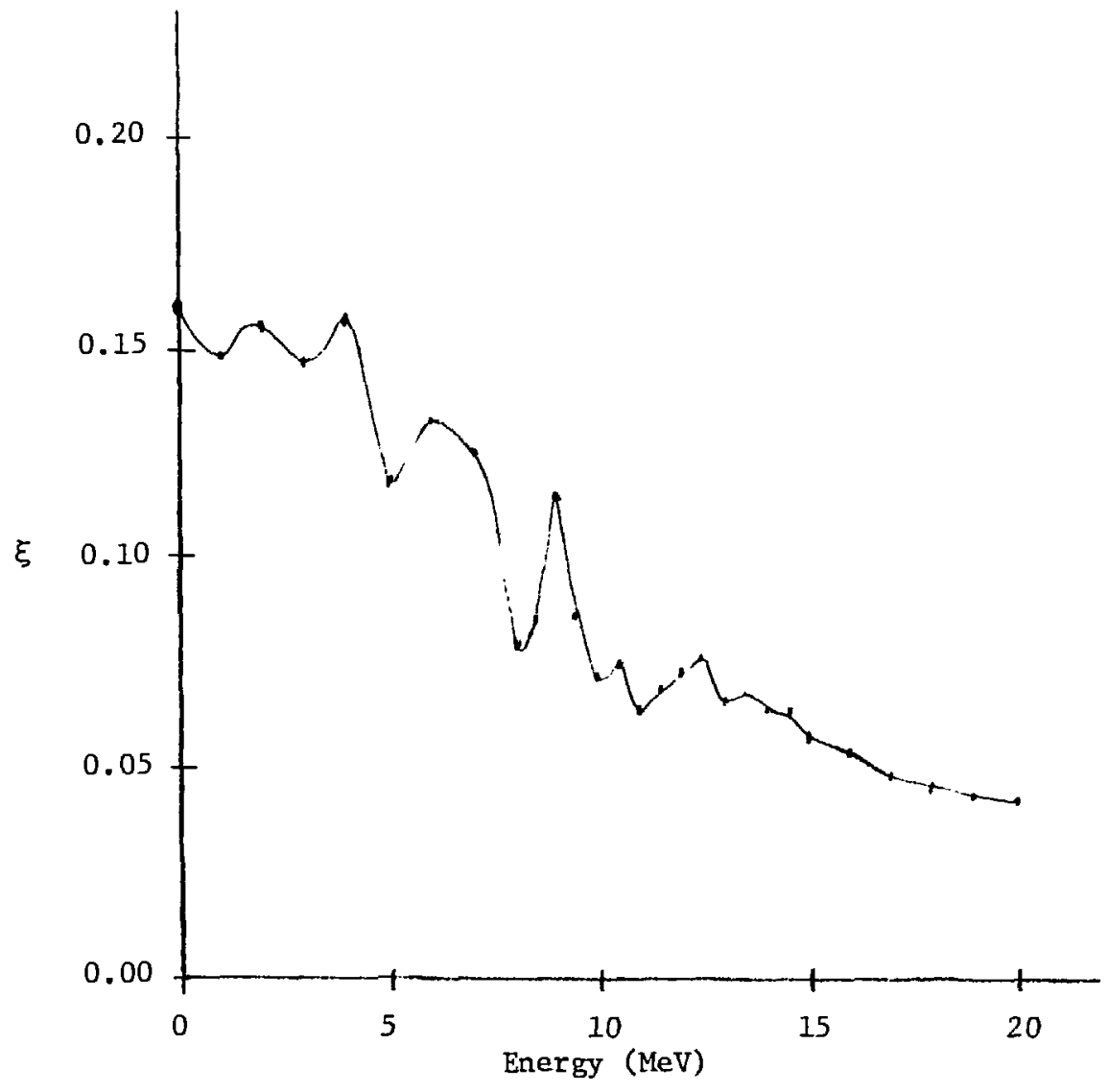

FIGURE 23

$\xi$ (E) for $C^{12}$ 
$0.20 \vdots$

$0.15-$
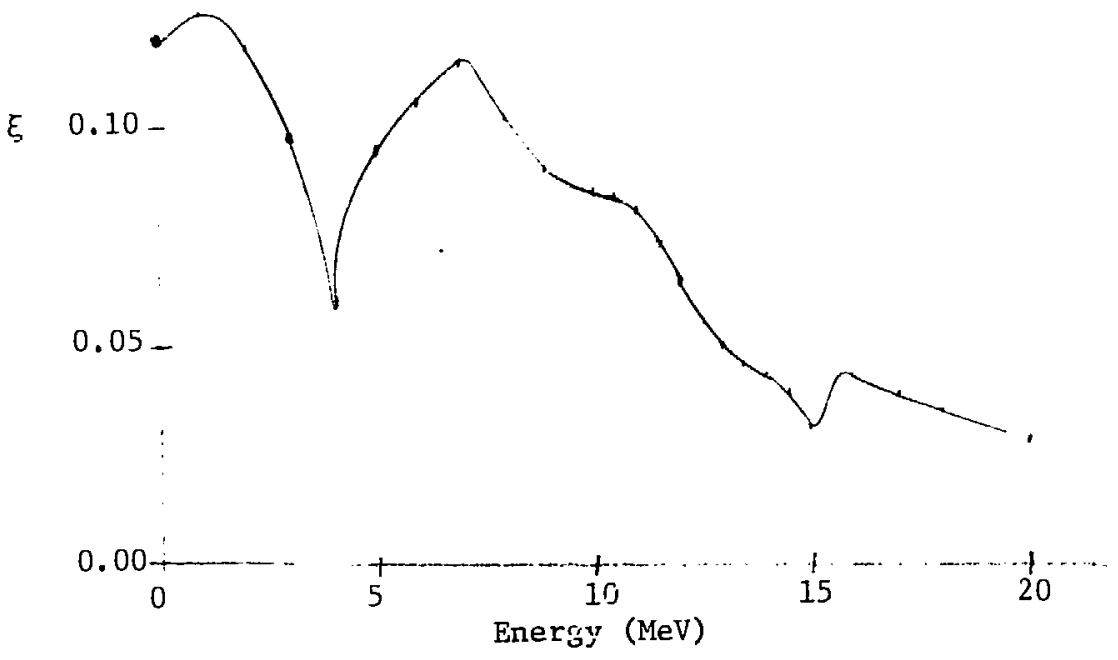

FIGURE 24

$\xi(E)$ for $0^{16}$ 


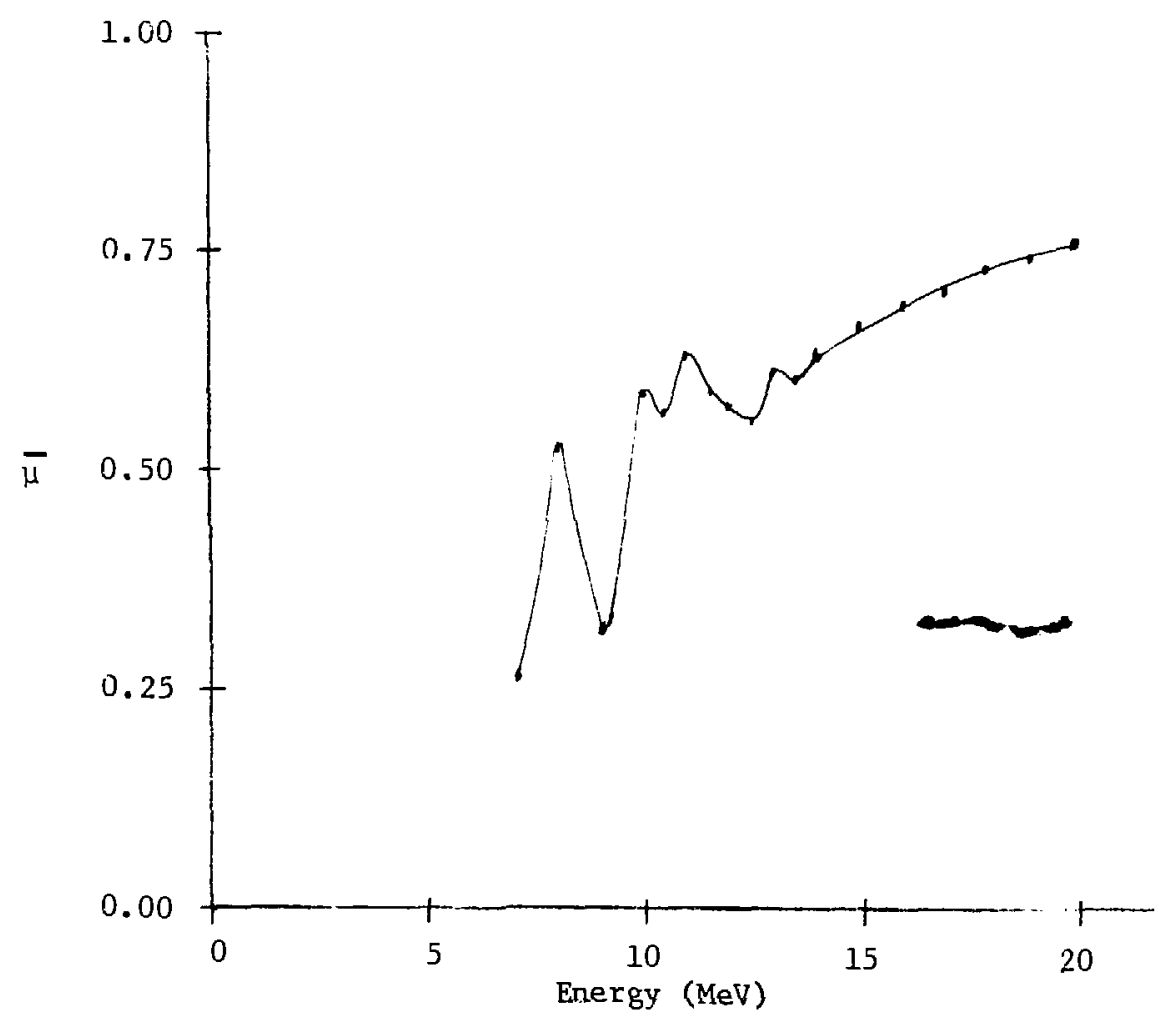

FIGURE 25

$\bar{\mu}(E)$ for $C^{12}$ 


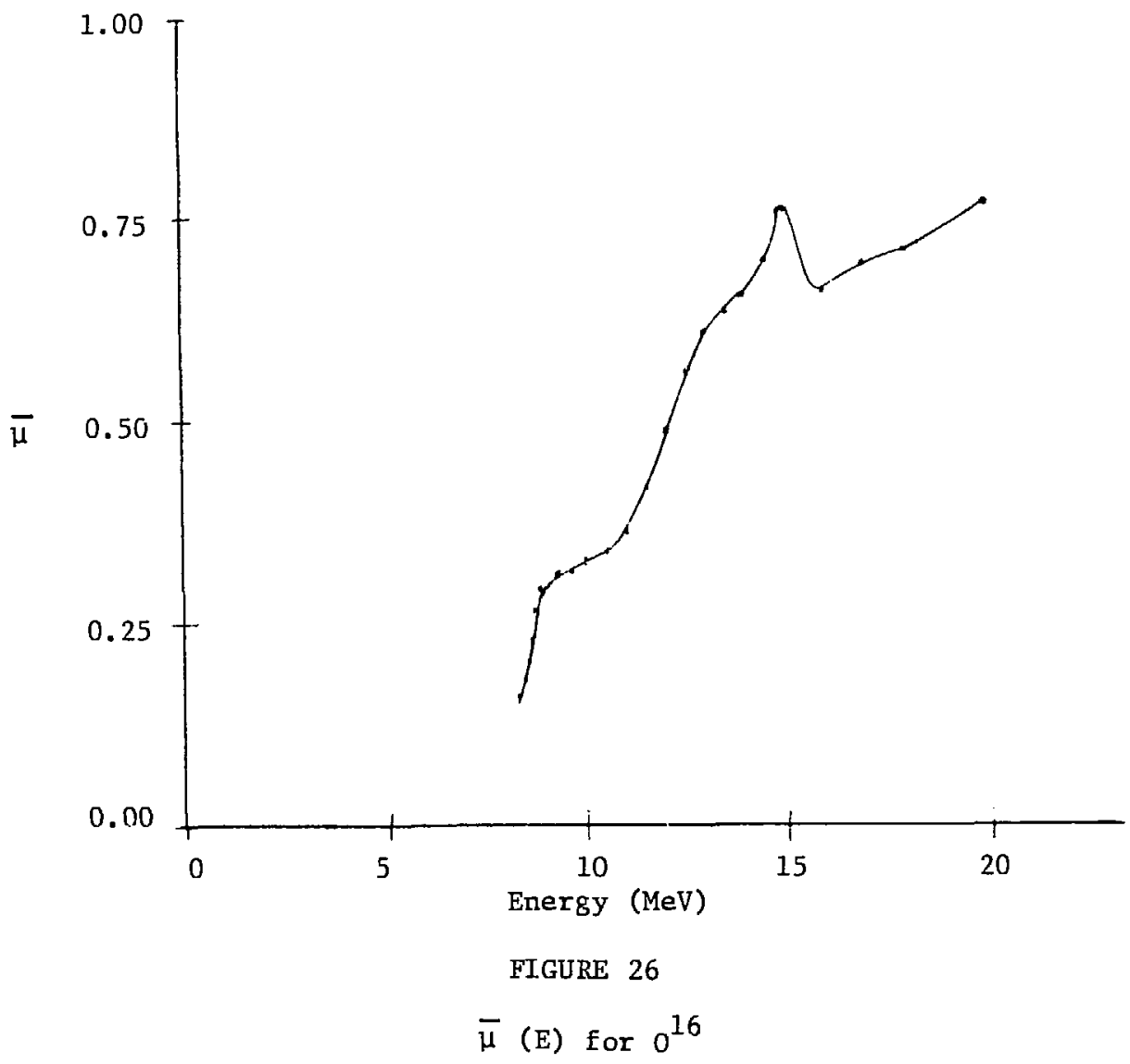


their ratio, elastic/nonelastic, decreases towards unity. The relative probability of elastic scatter then decreases toward the probability of nonelastic interaction. Thus, this is also a "worst case" at $20 \mathrm{MeV}$.

The estimates performed here at $20 \mathrm{MeV}$ are therefore overestimates of the effect of the omission of elastic collisions with nuclei other than hydrogen.

After making these estimates, one may reach several conclusions:

1) Only $40 \%$ of al1 source neutrons are involved.

2) The mean elastic scattering angle is less than

41 degrees.

3) Elastic scattering with $\mathrm{C}, \mathrm{N}$, and 0 accounts for less than $4.3 \%$ of the energy loss of neutrons with energy greater than $20 \mathrm{MeV}$ in Shonka A-150 plastic, and less than $2.8 \%$ in ICRU muscle tissue.

4) Omission of elastic collisions of neutrons with energy greater than $20 \mathrm{MeV}$ with nuclei other than hydrogen may be neglected for this work. 
APPENDIX C

\section{Elemental Kerma Values}

A method of obtaining kerma values for various incident neutron energies for specified elements was descri'nd in Chapter VI. The elemental kerma values which were calculated are listed in Table 14. 


\section{TABLE 14}

Elemental Kerma Values

$\begin{array}{ccccc}\begin{array}{c}\text { Energy } \\ (\mathrm{MeV})\end{array} & \begin{array}{l}\text { Hydrogen } \\ \left(10^{-9}\right.\end{array} & \begin{array}{c}\text { Carbon } \\ \left.\mathrm{rad}-\mathrm{cm}^{2} / \mathrm{n}\right)\end{array} & \text { Nitrogen } & \text { Oxygen } \\ 10 & 45.6 & 1.13 & 1.24 & 0.99 \\ 15 & 46.1 & 2.57 & 2.28 & 1.78 \\ 20 & 46.9 & 3.55 & 3.15 & 1.96 \\ 25 & 46.0 & 3.77 & 3.46 & 2.22 \\ 28 & 45.0 & 3.90 & 3.66 & 2.45 \\ 30 & 44.1 & 3.98 & 3.90 & 2.53 \\ 40 & 41.7 & 4.13 & 4.67 & 3.02 \\ 50 & 41.5 & 4.35 & 5.41 & 3.62 \\ 60 & 39.9 & 4.72 & 6.10 & 4.21 \\ 70 & 38.5 & 5.10 & 6.72 & 4.78 \\ 80 & 35.8 & 5.52 & 6.98 & 5.49\end{array}$


APPENDIX D

Charged Particle Spectra

Figures 27-30 are examples of computer generated graphs of charged particle spectra. Figures $27-30$ are slowing down spectra for protons, deuterons, tritons, and alphas, respectively. A complete set of slowing down and initjal spectra are included in this report for depths of 0.00 to $14.0 \mathrm{am}$ in Shonka A-150 plastic and ICRU muscle tissue, for the $50 \mathrm{MeV} \mathrm{d}^{+}$on $\mathrm{Be}, 42 \mathrm{MeV} \mathrm{p}^{+}$on $\mathrm{Be}$, and D-T neutron spectra. A complete set of computer generated graphs of these spectra may be found on the microfiche located in a pocket on the inside cover of this report. Printed tabulations of the same spectra may be found immediately following Figure 30 in this appendix. 


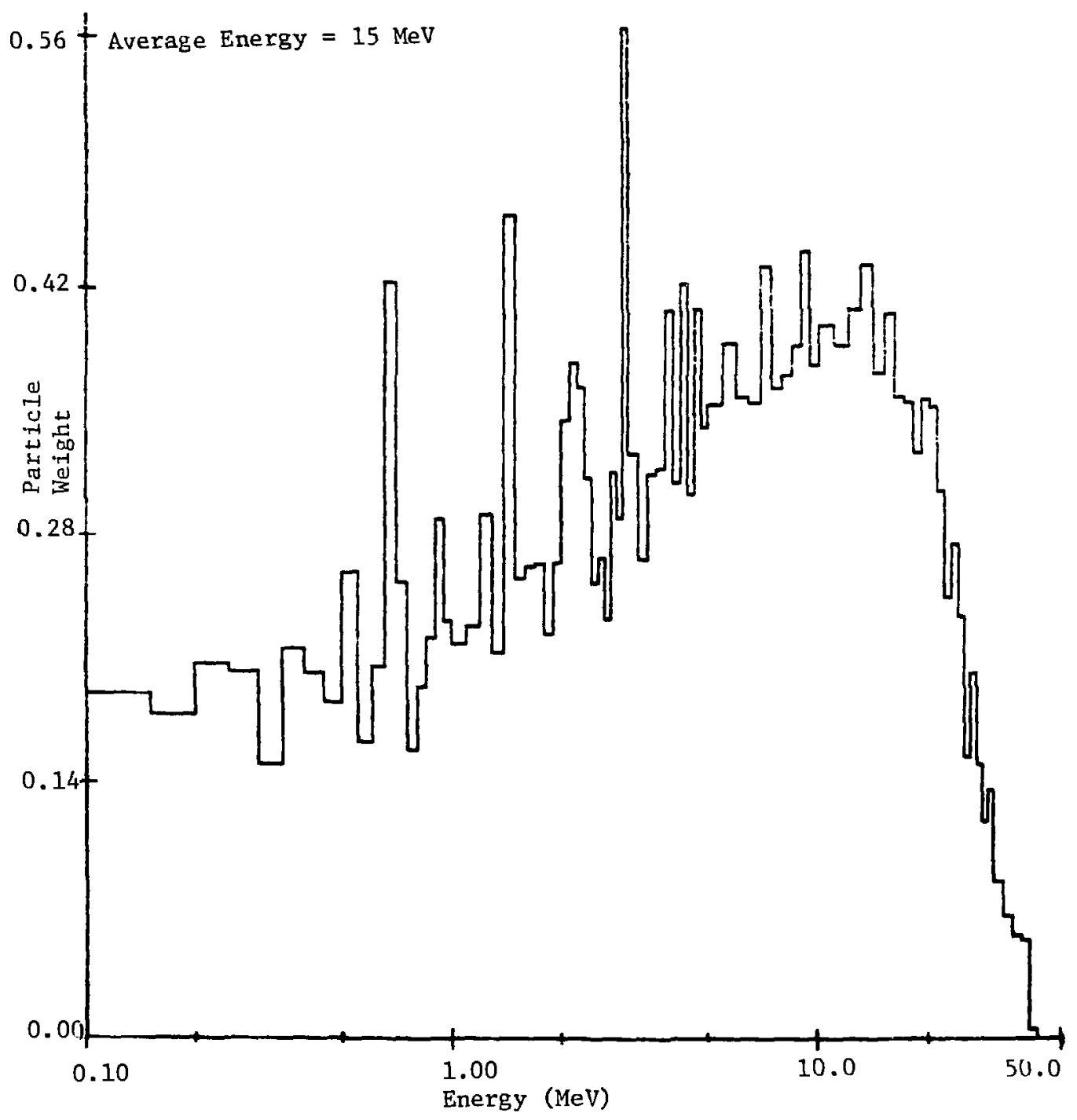

FIGURE 27

Proton Slowing Down Spectrum at $6 \mathrm{~cm}$ (42 $\mathrm{MeV}^{+}$on Be Neutron Spectrum) 


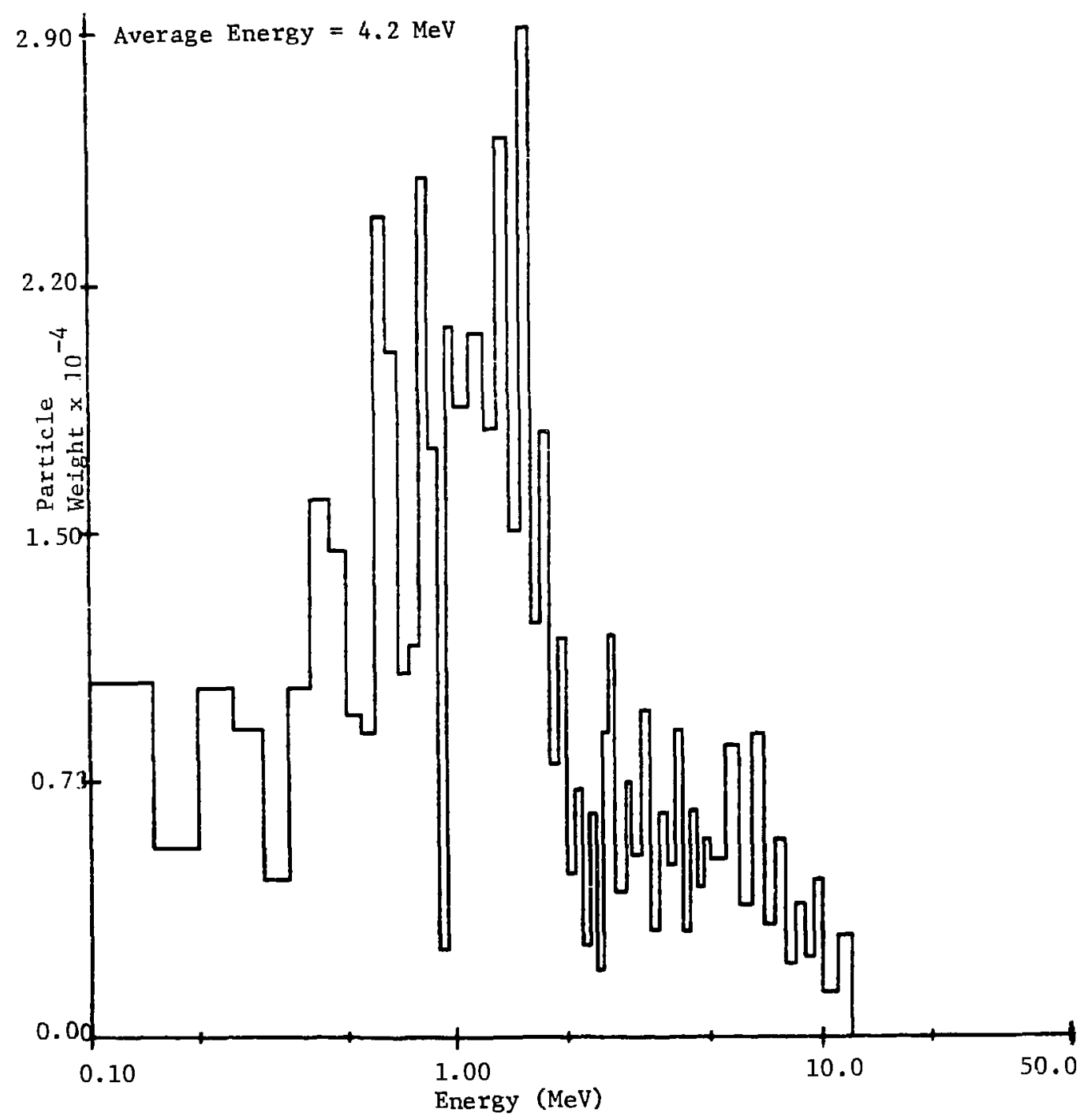

FIGURE 28

Deuteron Slowing Down Spectrum at $6 \mathrm{~cm}$

(42 $\mathrm{MeV}^{+}$on Be Neutron Spectrum) 


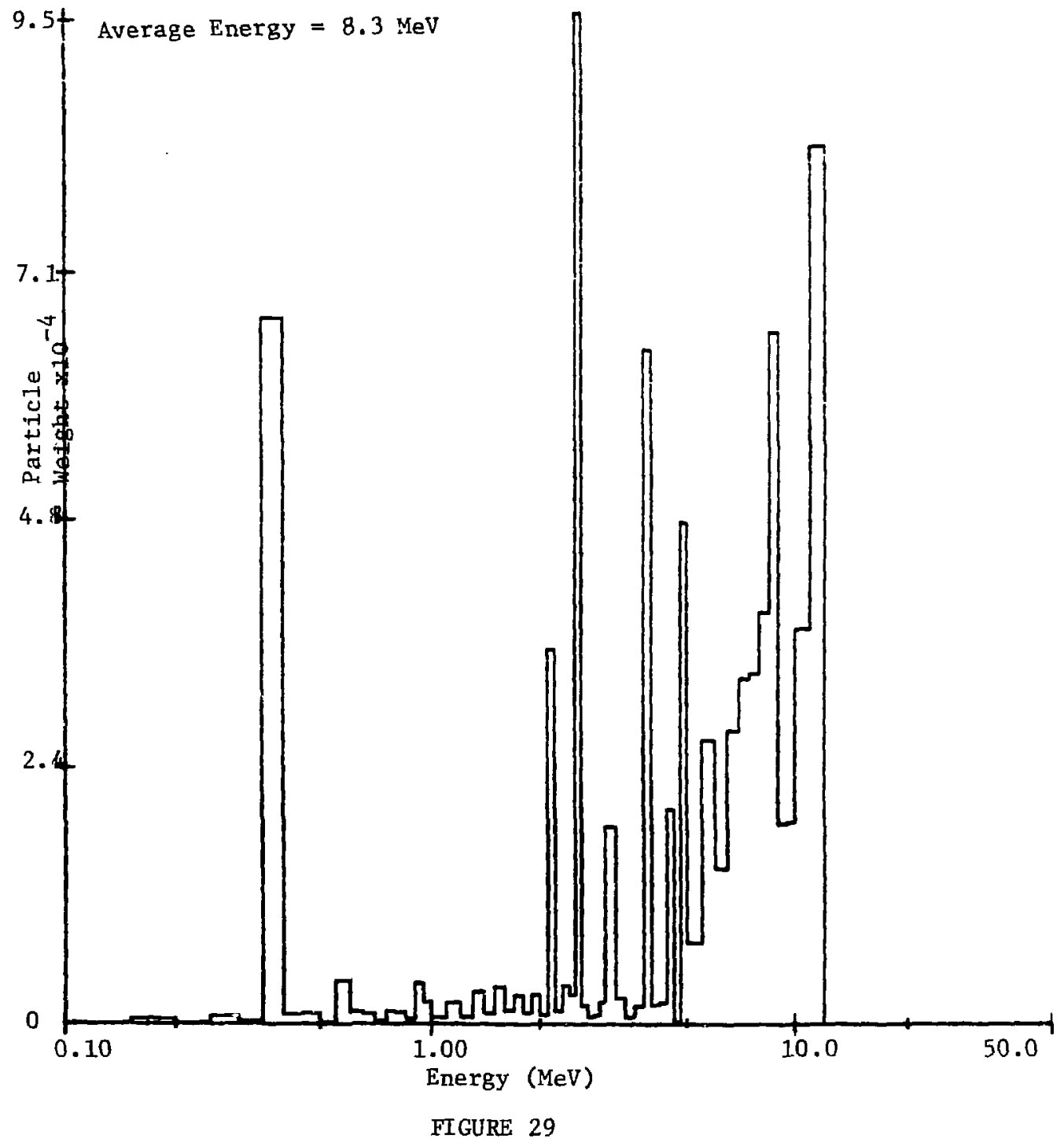

Triton Slowing Down Spectrum at $6 \mathrm{~cm}$

(42 $\mathrm{MeV}^{+}$on Be Neutron Spectrum) 


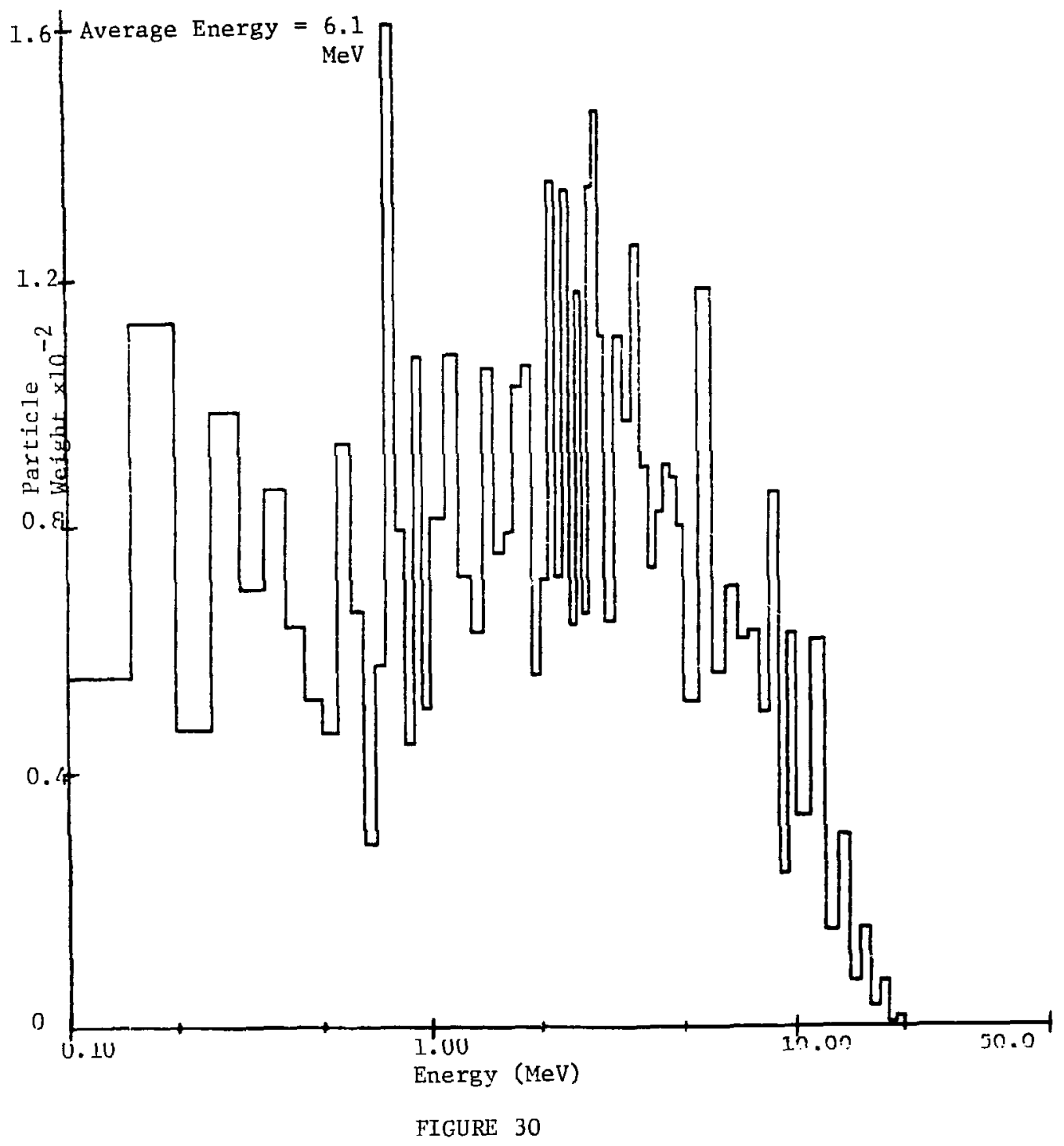

Alpha Slowing Down Spectrum at $6 \mathrm{~cm}$ (42 $\mathrm{MeV} \mathrm{p}^{+}$on Be Neutron Spectrum) 
ISLOWING DOWN SPECTRUM AT D, OG CM. FOR SHONKA A-15G PLASTIC 50 MEV D+ ON BE NELITROIN SOFCTRIJM

\section{ENERGY}

D. DS MEV

0.10 MEV

0.15 MEV

0.2 I MEV

0.25 MEV

0.30 MEV

0.35 MEV

0.40 MEV

$0.45 \mathrm{MEV}$

0.50 MEV

0.55 MEV

0.63 MEV

$0.65 \mathrm{MEV}$

$0.70 \mathrm{MEV}$

0.75 MEV

0.80 MEV

0.85 MEV

0.90 MEV

0.95 MEV

¿.DO MEV

1.10 MEV

1.20 MEV

1.3D MEV

1.40 MFV

$1.50 \mathrm{MEV}$

1.60 MEV

1.70 MEV

1.80 MEV

$1.90 \mathrm{MEV}$

2.06 MEV

2.10 MEV

2.20 MEV

$2.36 \mathrm{MEV}$

2. 40 MEV

2.50 MEV

$2.60 \mathrm{MEV}$

$2.70 \mathrm{MEV}$

2. BD MEV

$2.90 \mathrm{MEV}$

3.00 MEV

$3.20 \mathrm{MEV}$

3.40 MEV

3.60 MEV

3,80 MEV

4.DO MEV
PROTONS

O.

8.19E+DO

5. $48 E-D 1$

7. $11 E-D 1$

1. $19 E+J 0$

1.7. $7 E+09$

1. $2 B E+D G$

2. $62 E+110$

$1.79 E+\Delta A$

1. $5 B E+413$

1. $1 B E+B A$

$2.66 E+D B$

$2.39 E+30$

1.67E+A

1. $74 E+b A$

7. GTE-DI

4. $10 E+190$

7. 4 IE- IT 1

1. $\theta E+B$

8. $24 E-31$

9.11E-U1

4. D2E-O1

7. $87 E-31$

1. $45 E+00$

4. 65E-dI

$8.11 E-01$

3.40E- 21

1.56E-O1

$1.35 E-d 1$

4. 35E-

$8,57 \mathrm{E}-01$

$6,74 E-42$

$6.7 \pi E-02$

1. $10 E-O 1$

3. $28 E-D 1$

$6.94 E-12$

2. $98 E-D 1$

$4.79 E-\square 2$

$4.73 E-02$

$6,31 E-02$

$9.9 B E-U 2$

$2.65 E=D 2$

7.8GE-J3

1. B3E- DZ

$9.68 E-43$
DEUTERONS

0.

o. $58 E-k^{3}$

1. $49 E=P 4$

1. $25 E-\not 24$

$1.65 E-44$

2. $55 E=4$

9.7 BE- $\triangle 5$

1. $54 E-04$

$5.567 .-185$

7. J5E-AS

4. $45 E-25$

5.2BE- .5

$3.55 E-15$

7.9 $3 E-95$

$4.13 E-195$

8. 1 OF- 65

B. 1 UE $=06$

$1.99 \mathrm{E}-115$

1. 1BE-D5

4. 4 AE $=15$

$3.64 E=04$

$4,13 E=15$

8. $1 \mathrm{UE}-116$

1. $62 F-05$

8. $75 E=95$

$1.68 E-94$

$\theta$

0.

a.

$9.13 E-05$

0.

0.

3. $9 \mathrm{JE}=04$

3.18E- 25

9.

$1.63 E=04$

a.

B. 52E-05

6. $12 E-96$

ค. $52 E-D S$

2. $11 E=14$

2. $\triangle 9 E-04$

1. 9 QDE $=95$

1. $53 E=94$

$3.87 F-B 5$
TRITUNS

is.

4. $12 E-W 7$

4. I ZE-UT

$4.12 E=07$

$4.12 E=b 7$

$4.12 E=97$

B. $24 E-N T$

3. $28 E-B$

$7.31 E-1) 6$

$1.24 E-\operatorname{Ti} 0$

1. $24 E-46$

3. $3 A E-D h$

$3,275=14$

$\therefore$.

त. 4 QE $=6$

i.

9.

I.

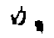

8. $49 E-16$

$\therefore$

6,

4. $25 E=06$

4. $25 E-36$

1. $84 E-D 5$

$4.25 E=110$

4.

4. $25 E-46$

$4,25 E-06$

$4.25 E-36$

4. $25 E-D 6$

b.

4. $25 E=166$

2. $27 E-\Delta 5$

$4.25 E-46$

$8,49 E=06$

$4.25 E-\Delta b$

$4.25 E-46$

$4.25 E-06$

2. $69 \mathrm{E}-155$

6.37E-26

6. $49 E=06$

2. $19 E-155$

2.76F-105

$9.21 E-06$
ALPHAS

4.

4. पWE-WS

$6.24 E-13$

$5.74 E-113$

7. $8 U E-13$

$5.20 E-H 3$

2. $32 E=13$

$5.34 E-13$

$0.24 E-13$

2. पUE-A3

$3.16 E-O 3$

$5,74 E-13$

$3,44 F_{-}-13$

4. 66E. IS

$3.31 E-d 3$

1. 36E-H2

$3,29 E=13$

$0.37 E-U S$

6. HIE-WS

1,1 UE -102

$6.37 E-\Delta 3$

$5.84 E-43$

$5,5 b E-D 3$

b. $2 b E-b 3$

6.) 4 E=B 3$

$5.89 E=183$

$3.50 E \cdot b 3$

$4.85 E=63$

$4.41 E-H 3$

8. $54 \mathrm{E}-\mathrm{G} 3$

$5.54 E-13$

$5.01 E-k 3$

$3.04 E=103$

$0,77 E-23$

$3.15 E-13$

$5,29 E-B 3$

$4.92 E-W 3$

2. $41 E-03$

$0,65 E-1) 3$

$1,8 \Delta E=-3$

$2,28 E-113$

$5.20 E-33$

$4.95 E-133$

$5.71 t-03$

$3,54 E-103$ 


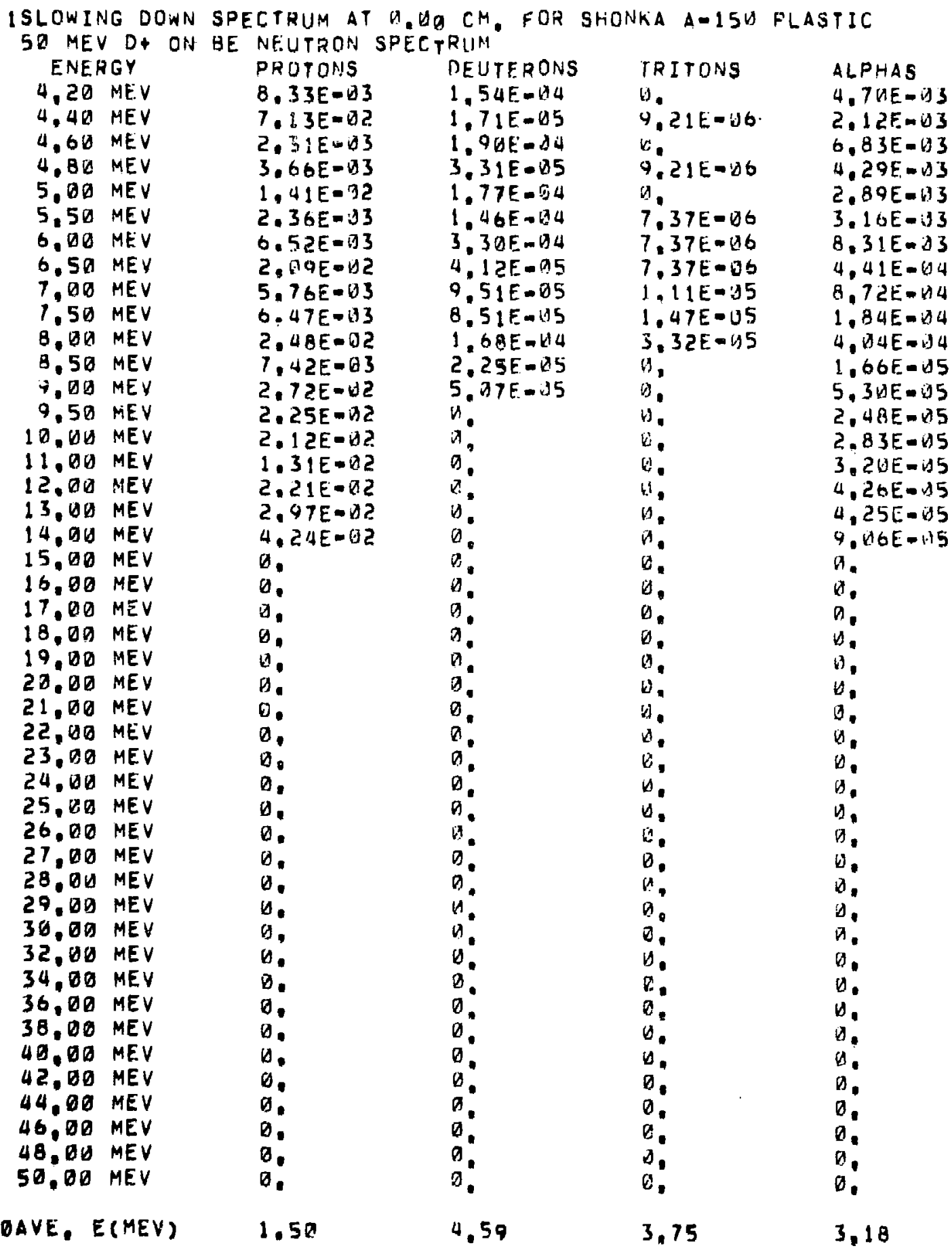


ISLOKING DOWN SPECTRUH AT H. D5 CHI, FOR SHONKA A-150 PLASTIC 50 MEV D+ ON BE NEUTRON SPECTRUM

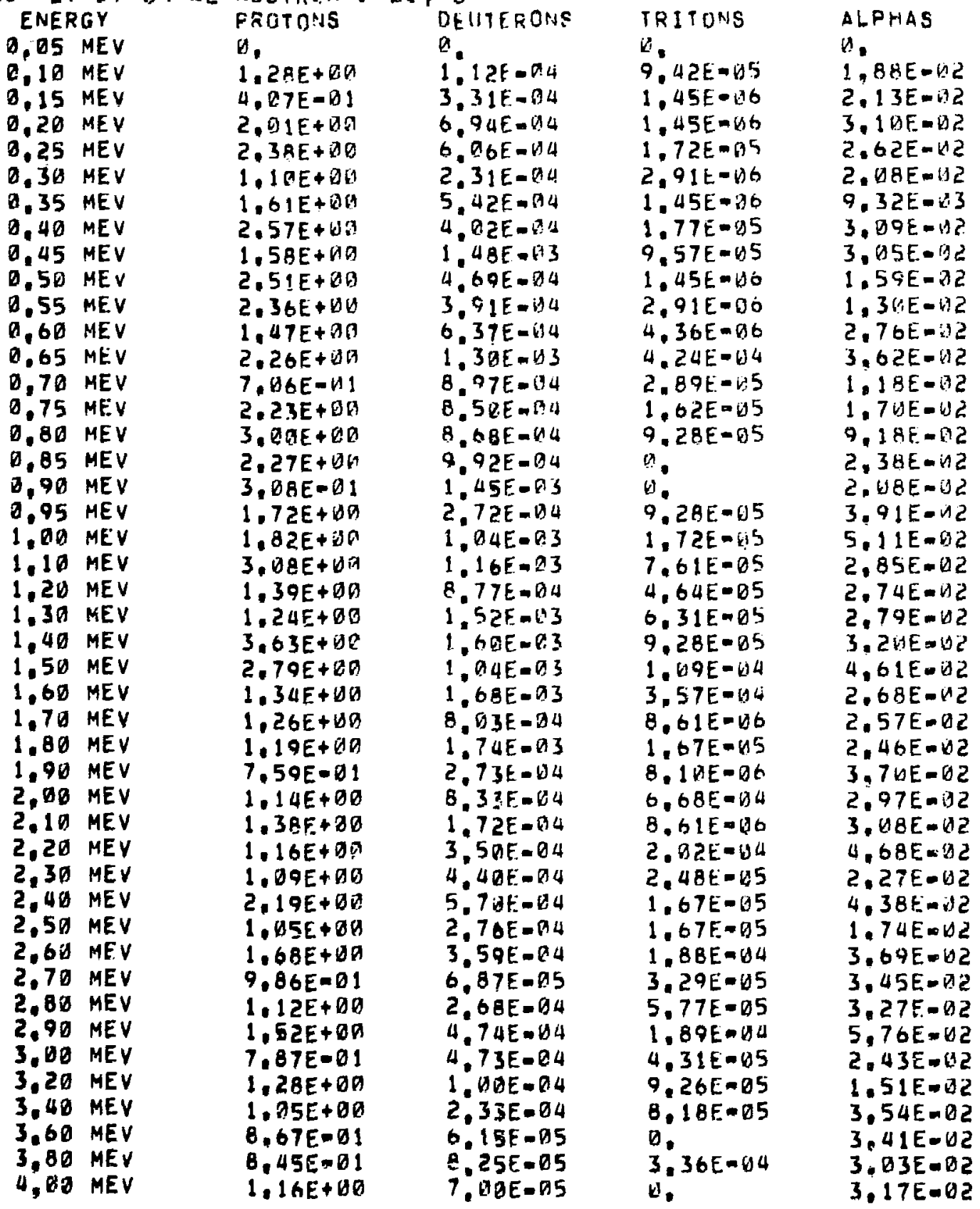


1SLUWING DOWN SPECTRUM AT D. TE CM. FOR SHONKA A-15H PLASTJC 50 MEV D+ ON BE NEUTRON SPECTPUIM

\section{ENERGY}

$4,20 \mathrm{MEV}$

4,46 MEV

$4,60 \mathrm{MEV}$

4.80 MEV

5.00 MEV

5,50 MEV

6,00 MEV

$6.50 \mathrm{MEV}$

7,0 M MEV

$7.50 \mathrm{MEV}$

8. DO MEV

8.50 MEV

9.00 MEV

$9.50 \mathrm{MEV}$

10.00 MEV

11.00 MEV

12.00 MEV

13.00 MEV

$14,00 \mathrm{MEV}$

$15,00 \mathrm{MEV}$

16.00 MEV

$17.00 \mathrm{MEV}$

18. OU MFV

19,00 MEV

$20,00 \mathrm{MEV}$

21. DU MEV

22, OU MEV

23,00 MEV

24,00 MEV

25,00 MEV

26, DU MEV

27. DO MEV

$28.00 \mathrm{MEV}$

$29.00 \mathrm{MEV}$

$30.00 \mathrm{MEV}$

$32,00 \mathrm{MEV}$

$34.00 \mathrm{MEV}$

$36.00 \mathrm{MEV}$

$38.00 \mathrm{MEV}$

40,UD MEV

42,00 MEV

$44.00 \mathrm{MEV}$

46,00 MEV

48,00 MEV

$50.00 \mathrm{MEV}$

\begin{abstract}
PROTONS
\end{abstract}
1. $15 E+D 0$

$1, B 1 E+00$

$1.17 F+\theta H$

8. SALEDI

$B, 2 \cap E-B 1$

$6.94 E-61$

$0.2\lceil E-\Delta 1$

$3.73 E-B 1$

$3,47 E-D 1$

4. D5E- $D 1$

$2.92 E-01$

$9.76 E-02$

2.3BE- 111

$6.75 E-02$

$4.73 E-02$

$4.45 E-Q 2$

$4.26 E-192$

$4.55 E-02$

$5.3 B E=D 2$

1. 8 AD- -22

$3,37 E=B 2$

2. $4 B E-172$

$\theta$.

$8.03 E-13$

$1.8 A E-02$

$\theta$.

0.

$8.13 E-02$

$1,8 \cap E=\square 2$

b.

ט.

$6,81 E=03$

0.

0.

0.

D.

0.

0.

$\theta$.

0.

0.

0.

0.

$\theta$

3.611
DEUTERONS

1. OUFEAL

6. BTE-DS

$5.12 E-A S$

1. $28 \mathrm{E}-64$

$4.98 E-A 5$

$9.14 E-185$

1. 19E-OA

$1,31 E=04$

$2,79 E-A 4$

$9.1 \triangle E-05$

1. $56 \mathrm{E}=\mathrm{A4}$

5. DOE-O5

1. $\triangle B E=04$

5. $92 F-05$

$1.38 \mathrm{E}=24$

a.

a.

D.

a.

i.

0

:

a.

a

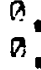

9.

0.

$a$.

$n$

0 .

0.

0.

0

$a$

0

$0:$

0

$B$

0

9.

2.75
TRITONS

h.

1. $\triangle B F=-65$

b.

1. $38 \mathrm{E}=115$

$3.26 E-114$

$4,32 E-B 6$

$1,39 E-B 4$

$8.64 E-016$

$1,35 E=04$

$1.43 E=V 4$

$1,39 E-64$

$1.48 E-114$

$1.69 t=(1) 4$

$2,60 E=04$

$5.21 E-B 4$

0.

$1.64 E-115$

o.

0

(A.

b.

0.

$\forall$.

b.

a,

0.

b.

b.

$b$.

0.

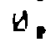

$\theta$

$\theta$

$\theta$

0.

0.

0.

0.

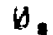

$\theta$.

0.

0.

0.

0.

0

6.51
ALPHAS

A. 6hEMC

$2.62 E=02$

$3.84 \mathrm{~F} .62$

$2.53 E-12$

$2.05 E-1) 2$

$1.82 \mathrm{E}-\mathrm{DZ}$

$3.87 E-B 2$

$1.31 E-02$

$2.26 E-12$

$1.29 E-B 2$

$1.56 E-62$

$8.57 E-03$

$1.98 \mathrm{E}-02$

2. $29 E=03$

$4.94 E-133$

$2.73 E-13$

4. $11 E-113$

$3,55 E=113$

$5.94 E-03$

2,6 EE- 03

$7,14 E=03$

1. $25 E-\forall 5$

$1.2 S E-W 5$

$1.66 E-155$

$3.74 E-155$

b.

0.

n.

ด.

0.

a.

$\theta$

$\theta$

0.

$D$

".

$\theta$.

D.

b.

0.

0.

9.

0

9.

0.

4.98 
1SLOWING DOWN SPECTRUM AT 21 CM, FUR SHONKA A-15U PLASTIC 50 MEV D+ ON BE NEUTRON SPECTRUM

\section{ENERGY}

D. D5 MEV

0.10 MEV

0.15 MEV

$0,20 \mathrm{MEV}$

0.25 MEV

0.30 MEV

0.35 MEV

$0.40 \mathrm{MEV}$

0.45 MEV

$0.50 \mathrm{MEV}$

0,55 MEV

$0,60 \mathrm{MEV}$

0.65 MEV

$0.70 \mathrm{MEV}$

0.75 MEV

$0.80 \mathrm{MEV}$

$0.85 \mathrm{MEV}$

$0,90 \mathrm{MEV}$

$0,95 \mathrm{MEV}$

$1,00 \mathrm{MEV}$

$1,10 \mathrm{MEV}$

1,20 MEV

$1,30 \mathrm{MEV}$

1.40 MEV

1,50 MEV

1,60 MEV

1,70 MEV

1.80 MEV

1.90 ME.

2.DO MEV

$2.10 \mathrm{MEV}$

$2.20 \mathrm{MEV}$

$2.30 \mathrm{MEV}$

$2.40 \mathrm{MEV}$

2.50 MEV

2,60 MEV

$2,70 \mathrm{MEV}$

$2.80 \mathrm{MEV}$

$2,90 \quad M E V$

3, $00 \mathrm{MEV}$

$3.20 \mathrm{MEV}$

3.40 MEV

3,60 MEV

3.80 MEV

$4.00 \mathrm{MEV}$
PROTONS

1.

$2.55 i+20$

$5.52 E-01$

1. $38 E+D G$

$9.72 E-01$

$1,71 E+\theta 13$

1. $\triangle A E+Q D Q$

$1.72 E+6 B$

$1.30 E+B A$

2. $05 E+00$

$3.35 E+80$

$2.63 E+D 4$

$3.39 E+D O$

$2.2 a E+69$

$3,54 E+U B$

$1.11 E+15$

$2.23 E+80$

$1.51 E+00$

9. BTE-OI

$1,35 E+\theta(1)$

2. $27 E+a 1$

$1, B 1 E+B 1$

$1.15 E+D a$

$1.12 E+D E$

3. $08 E+\triangle Q$

3. $D 2 E+Q D$

$1.16 E+00$

$1.12 E+B \theta$

$1.31 E+00$

1. HOE+BO

1. $54 E+00$

Q.15E-01

1. $54 E+E D$

$1.05 E+00$

8. $12 E-91$

$9.34 E-01$

1. $11 E+00$

$7.58 E-\square !$

$1.81 E+D \theta$

$2.03 E+E D$

$9.53 E-B 1$

$1.21 E+01$

$9.72 E-11$

$7.00 E-01$

$B, 34 E-B 1$
DEUTERONS

D.

$7.94 E-05$

2. TIE-RI 4

2.OIE- II 4

2. $91 E=O 4$

1. 6BE- (X) 4

$1.89 E-194$

2. $44 E-114$

$5.59 E-(34$

$1.99 E-114$

$7.01 E-65$

$1.51 E-C 4$

$3.5 \triangle E-04$

2. $44 E-94$

$1,1+E-D 4$

4. BIE-DA

2. BIE-OA

$4.22 E-04$

$7.92 E-D 5$

$6.28 E-24$

$3.4 Q E=14$

$7.73 E-34$

3. $\triangle S E-D 4$

5. BRE-BA

$2.93 E=04$

$4.51 E-114$

$1,93 E=04$

2. 2BE-DU

2.03EnOA

3. $45 E-12$

5. BIE-BS

2, 4DE-94

$1.11 E-64$

8. 4 QE- 95

$6.57 E-05$

$1,31 E-04$

$1.69 E=04$

$2.63 E-D 4$

o.

a.

$2.14 E-04$

$1.87 E-105$

$9.58 E=016$

$2,25 E-0.5$

1. ATE-OS
TRITONS

$\theta$

b.

b.

4,

(1).

$\theta$.

0.

0.

2. $\triangle 2 E-B 4$

$1,76 E-64$

$\theta$.

n.

4.

$2.02 E-04$

$9,84 E-66$

$1,76 E=04$

a.

a.

$a$

6.

$3.79 E=05$

$0.79 E-05$

3.

$8,79 E-05$

4.

$8.79 E-45$

$9.28 E-H 5$

$8,795=-15$

$B, 79 E=0.5$

$1.76 E-V .4$

$1.76 E=04$

$3,51 E-P 4$

i.

$7.75 E-65$

6.

$\theta$

$\theta$.

0.

$4.92 E=06$

H.

$\theta$.

$8,43 E-95$

$3.63 E-95$

$2,46 E=(1) 6$

s.
ALPHAS

$D$.

$1,56 E-12$

$1, B Q E=H E$

$2,78 E-B ?$

$1,93 \mathrm{E}-\mathrm{d}$ ?

2.57E- 32

$9,31 E$ II

2. $68 \mathrm{BE}: 12$

$1,4 \theta 6 .-92$

$1.24 E-\Delta 2$

$1.14 E-\Delta 2$

$1.76 E-62$

$2.7 \mathrm{bE}-3 \mathrm{Z}$

1. $\triangle 4 E-V I 2$

$1,25 \mathrm{E}-\mathrm{DC}$

5,3 GE- 12

$1.79 E-\triangle 2$

$1,3(1 E-D 2$

$1.96 E-\Delta 2$

$1.47 \mathrm{E}-4 \mathrm{~V}$

2. 44E-IDZ

2. $12 E=112$

$1.92 E-B z$

2.73E- U2

$2.48 E-42$

1, GSE 92

$2.17 E-112$

$2.34 E=02$

$1.91 E-62$

$1,75 E-\Delta 2$

$1.88 E-02$

$3.37 E-1] 2$

$1.28 E-62$

2. $4 U E=U 2$

$1.5\{E-B) 2$

$3,13 E-1) 2$

$1,3 D E=\Delta 2$

$1,9 b E-V) 2$

$3.69 E-12$

$1.61 E-12$

$1,58 E=02$

2. 36E -142

$2.05 E-02$

2. 3 $315=192$

$1,56 E-42$ 
ISLUWING DONN SPECTRIIM AT H,IQ RM. FOR SHONKA A-15D PLASTIC 5马 MEV D+ ON HE NEUTRON SPECTRUM

\section{ENERGY}

4.2D MEV

4.40 MEV

4.6D MEV

4, BD MEV

5. 90 MEV

5.5W MEV

6. DO MEV

6.50 MEV

7. $\triangle D$ MEV

$7,50 \mathrm{MEV}$

8. $\triangle D$ MEV

8.50 MEV

9. DS MEV

9.5 $\triangle$ MEV

10. NO MEVV

11. DD MEV

12., 00 MEV

$13,00 \mathrm{MEV}$

$14.00 \mathrm{MEV}$

15.00 MEV

16.90 MEV

$17.00 \mathrm{MEV}$

18,00 MEV

$19.00 \mathrm{MEV}$

20.0 MEV

21. OU MEV

$22 . \Delta 0 \mathrm{MEV}$

23. DO MEV

24.00 MEV

25.00 MEV

26.00 MEV

$27.00 \mathrm{MEV}$

28.00 MEV

29. UD MEV

$30.00 \mathrm{MEV}$

32,00 MEV

34.00 MEV

36. DO MEV

38. DO MEV

40.00 MEV

42. OU MEV

44.00 MEV

46.00 $4 E V$

48,00 MEV

5D. Dด MEV

BAVE. E(MEV)
PROTONS

9. 26E $=01$

$1,16 E+100$

$1,17 E+B G$

1. $17 E+00$

7. QOUE-O1

$1.1 A E+D A$

$9.63 E-31$

$8.96 E-41$

$4,13 E-101$

$1,41 E-01$

$2,13 E-01$

$1.55 E-11$

$1.39 E-01$

4. 52E- 02

2. $02 E-01$

2. $\triangle 8 E-01$

1. $1 E-01$

2. $14 E-D 2$

1. $28 E-61$

2. $U 1 E-01$

0.

$3,3 B E-63$

$7.66 E-02$

1. $04 E-11$

$5,65 E-42$

0.

1. $52 E-\triangle 1$

5.17E-D2

5. $72 F-43$

0.

$1.33 E-22$

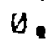

i.

0.

$2.49 E-12$

0

a.

$v$.

0.

a.

9.

0.

0

0

4.65
DEUTFEOINS

2. 83E-DS

2. MAE $\triangle 14$

2. $83 E$ G 15

3.? IE- 05

1.87F. 15

1. $19 E=14$

$1.53 E-04$

9. $18 E-116$

8. $73 F=05$

$1.65 \mathrm{E}-\mathrm{H} 4$

$1.1 D E=94$

$1.52 F_{-}=04$

$3.05 E-94$

(3)

(1).

b.

0

a.

0

0.

a.

a.

b.

a.

0.

0

a.

a.

$\theta$

0

a.

0

a.

b

a.

a.

0

a.

ด.

a.

3.89
TRITDNS

2. $46 E-06$

3. $88 E-145$

b.

2. $46 E=016$

2. $46 E-66$

$1.65 E-155$

$1.75 E-05$

$1.85 E-\square 5$

3. $89 E-05$

2. $90 E=05$

$5,81 E=05$

:

a.

ㄴ.

$\theta$

$D$.

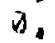

3

3.

b.

b.

0.

6.

a.

0.

i.

0.

).

b.

u.

(s)

a

$\Delta$.

$v$

0.

i).

$b$

0

a.

0

b.

b.

3.36
ALPHAS

2. 30E-02

1. $\triangle 9 E-\triangle 2$

1. $55 E-12$

$1,13 E-1.2$

$1.57 E-62$

6. $39 E-A 3$

$1.24 E-62$

$5,87 E-W 3$

$9.7 U E=B 3$

$7,20 E M U 3$

1. $V I E=\square 2$

7. $02 E-13$

$1.29 E \mathrm{mBC}$

$3.36 E-\triangle 3$

$6,21 E-b 3$

$3.24 E-03$

b. $56 E=03$

1. $19 \mathrm{E}=03$

$1.52 E-43$

2. $02 E-13$

$4,62 E-03$

$\Delta$.

$D$

U.

$\theta$.

o.

b.

0

G.

a.

a.

0.

$\theta$

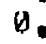

a.

$\Delta$.

0.

D.

0.

$\theta$.

U.

$\theta$

0.

0

0.

5.00 
ISLOWING DOWN SPECTRUM AT O. 15 CM. FOR SHONKA A-15D PLASTIC 5\% MEV O+ ON BE NEUTRMIV SPECTRUM

\section{ENERGY}

0. 45 MEV

0.10 MEV

0.15 MEV

0.20 MEV

D. 25 MEV

0.3D MEV

0.35 MEV

0.40 MEV

$0.45 \mathrm{MFV}$

$0.50 \mathrm{MEV}$

$0.55 \mathrm{MEV}$

$0.60 \mathrm{MEV}$

0,65 MEV

0.7 MEV

$0.75 \mathrm{MEV}$

0.80 MEV

0.85 MEV

0.90 MEV

$0.95 \mathrm{MEV}$

1. DU MEV

1.10 MEV

1.20 MEV

1.30 MEV

1.40 MEV

$1.50 \mathrm{MEV}$

$1.60 \mathrm{MEV}$

1.70 MEV

$1,80 \mathrm{MEV}$

1.90 MEV

2.00 MEV

2.1D MEV

2.20 MEV

2.30 MEV

2.40 MEV

$2.50 \mathrm{MEV}$

2.60 MEV

$2.70 \mathrm{MEV}$

2.BO MEV

2.90 MEV

$3.00 \mathrm{MEV}$

3.20 MEV

3. 40 MEV

3.60 MEV

3.8G MEV

4.DO MEV
PROTONS

b.

5.75E-U1

5. .? $7 E-Q 1$

$6.50 E-b 1$

8.10E-B1

$5,34 E-W 1$

$B, 73 E=1$

$2.48 E+B A$

$3.91 E-31$

$1.89 E+O C$

$6.1 D E-1$

$1.45 E+10 Q$

1. ดBE + Oด

9. MSE-61

1. $31 E+0$.

9.8OE-OI

$1.06 E+100$

$1,42 E+\square B$

4. 1SE+BO

$9.19 E-W 1$

1.4TE+ DO

3. $82 E+\square G$

$1.43 E+\square U$

1. $90 E+O Q$

$1.38 E+190$

$1.53 E+D A$

$1.96 E+60$

$1,67 E+D(1)$

$2.47 E+60$

1. $29 E+06$

$2.23 E+B O$

2. $3 B E+9 Q$

$2.52 E+100$

2. उBE $+0 Q$

1. $23 E+01$

2. $D 1 E+00$

1. $37 E+D$

2. $10 E+\square 0$

$1.15 E+Q Q$

$2.77 E+60$

1. $91 E+B C$

$1.67 E+\angle Q$

$2.79 E+0 B$

1. $42 E+00$

$1.17 E+Q 0$
DEUTERONS

a.

1. 3UE- 14

3. $44 E-P: 4$

7. B उE- 4

4. $31 \mathrm{E}-14$

3. 3AE-A 4

$4.32 E=4$

3. 39F $=04$

1. $2 Q E=k 3$

6. $26 E-044$

$3.76 E-04$

$9.04 E-04$

1. $13 E=03$

1. $55 E-93$

2. $87 \mathrm{E}-\mathrm{DU}$

7. 21E-64

3. BटE-DA

$1.52 F=03$

3. BOE-O 4

$9.51 E-D 4$

1. DAE-OB

1. $32 \mathrm{E}-113$

1. $83 \mathrm{~F}-03$

1. $85 E-83$

4. 2BE-DU

1. GGE-A3

3. $45 E-144$

5. 22E-D 4

3. BQE-R? 4

5. I UEMU

1. $\triangle 1 E-O A$

1. $32 E-33$

1. $82 E-174$

5. ODE-D 4

$1.37 F-04$

$5, D 7 F-35$

9.45E-6 4

1. $44 E-05$

3. 35E-A.1

1. $A E-A 3$

1. DBE-04

$7.95 E-155$

$1.19 E-54$

2. $91 E-04$

1. $47 E-Q 4$
TRI TONS

G.

b.

B. 32E- 115

5.97E- 16

1.)

1.

4!.

(1)

$5,97 E=06$

2. 1 AE $=05$

B. $32 E=05$

$2.41 t-05$

$5.97 E=06$

1.

2. D2E-Eय

$5.97 E-06$

6. 32E- 15

$b$.

$3 . D Q E-05$

1. $05 E-\mathrm{D} U$

$1.51 E-104$

$4.46 E-105$

$1.95 E-04$

5. $55 E-05$

1. $16 E-114$

2. $98 E-16$

3. $28 E-05$

2. $9 B E-16$

1.8RE= A

2. 15E-154

1. $8 A E-15$

$1.65 E-04$

2. 1 AE-15

$1.33 E-D 4$

2. $28 E-15$

1. $39 E-U 4$

1. $28 E-144$

2. $29 E-65$

1. $24 E-B 4$

2.68E-OA

1. 1 BE $=04$

$1.52 E-04$

$8.60 E-05$

$6.66 E-05$

$6.86 E-05$
ALPHAS

$D$.

$1.34 E-\pi 2$

1. 18E- $\triangle 2$

$2,56 E-k 2$

1. $31 \mathrm{E}=\mathrm{A} \mathrm{C}$

1.92E- 12

$1.09 E-1) 2$

$2.51 E-02$

$1,15 F=12$

1. $16 E=112$

$7.99 E=193$

$2.4 M E-B 2$

1. $47 E-A 2$

3.61E-O3

1. $43 E-\square 2$

2. $32 E-12$

1. $15 F-B 2$

$1.52 E-D 2$

$1.73 E-U 2$

8. $29 E-\Delta 3$

1. $60 E-W 2$

$1.71 E-12$

1. 39E-UZ

$1,39 E-12$

2. BUE-Uट

1.25E-W

$1,35 E-12$

$1.93 E-62$

$1 . B 2 E-92$

$1.79 E-132$

$9,95 E-103$

$2.85 E-62$

$1.39 E-132$

$2.76 E-182$

$1.93 E-62$

2. 45E- $\triangle 2$

$2.17 E-02$

$3.95 E-02$

$1.71 E-U 2$

1.78E- 92

1.24E- 62

1.98E- 12

1. $84 E-62$

$1.88 E-\square 2$

$1.42 E-02$ 
1SLOWING DOWN SPECTRUM AT D, 15 CH. FOR SHONKA A-1SH PLASTIC 50 MEV D+ ON BE NEUTRON SPECTRUMM

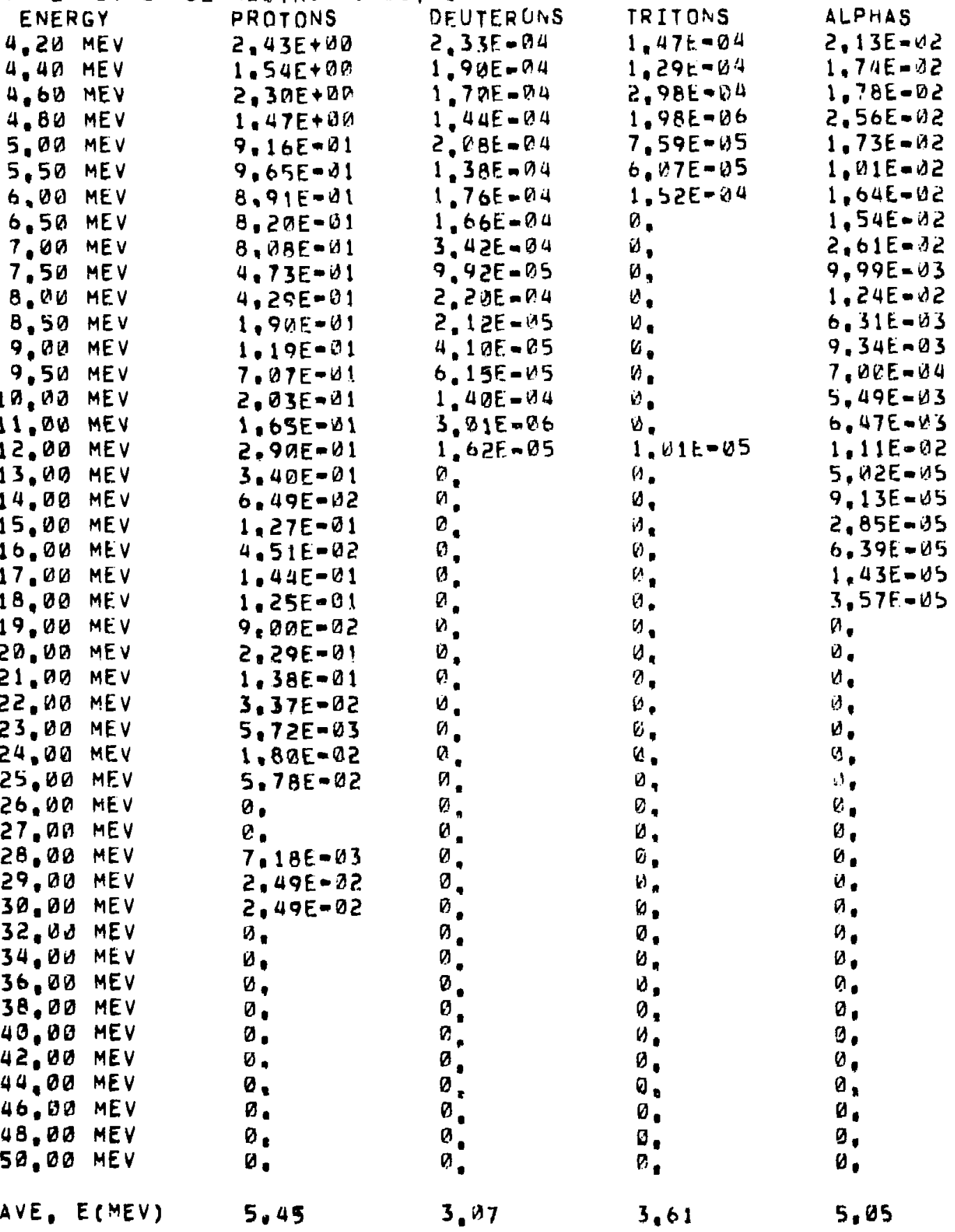


ISLOWING DONN SPECTRUM AT B. 2 G CM. FOR SHONKA A-15" PLASTIC 50 MEV D+ ON BE NEUTRON SPFCTRIJM

\section{ENERGY}

D. $05 \mathrm{MEV}$

$0,10 \mathrm{MEV}$

0.15 MEV

$9.20 \mathrm{MEV}$

$0.25 \mathrm{MEV}$

0. 30 MEEV

$0,35 \mathrm{MEV}$

D. 40 MEV

0.45 MEV

$0.50 \mathrm{MEV}$

0,55 MEV

0.60 MEV

0.65 MEV

0.70 MEV

0.75 MEV

$0.80 \mathrm{MEV}$

Q.85 MEV

0.90 MEV

0.95 MEV

$1.00 \mathrm{MEV}$

$1.10 \mathrm{MEV}$

1.20 MEV

1,30 MEV

$1,40 \mathrm{MEV}$

1,50 MEV

$1,60 \mathrm{MEV}$

1,70 MEV

$1.80 \mathrm{MEV}$

1.90 MEV

2.00 MEV

2.10 MEV

2.20 MEV

$2.30 \mathrm{MEV}$

2.40 MEV

2,50 MEV

2,60 MEV

2,70 MEV

$2,80 \mathrm{MEV}$

2.90 MEV

3, (1.) MEV

$3,20 \mathrm{MEV}$

3.40 MEV

3,60 MEV

$3,80 \mathrm{MEV}$

$4,00 \mathrm{MEV}$
PROTONS

D.

$9,83 E-01$

3. $26 E-D 1$

2. 5TEM 1

$4.6 B E-D 1$

$4,22 E=01$

$3.83 E-01$

$1.28 E+0 \theta$

$4.64 E-D 1$

$5.49 E-B 1$

3. QUE $-\triangle 1$

3. 3BE-DI

$6,36 E-a 1$

$1.34 E+D Q$

$9,87 E-D 1$

$7.14 E-A 1$

$6.89 E-11$

$7.36 E-21$

$7,32 E-01$

$1.04 E+B 0$

$1.75 E+\partial B$

$1.17 E+O B$

$9,55 E-\Delta 1$

$1.23 E+00$

$1.37 E+B$ B

$7.75 E-B 1$

$8,7 D E-01$

$9.90 E-i] 1$

$6.26 E=1$

1. $99 E+00$

$8,96 E-D 1$

$7,5 ? E-D 1$

$1,31 E+00$

$0.64 E=01$

$1.78 E+O A$

5, $88 E-01$

$3, O B E+O D$

$6,44 E=B 1$

7.7BE-DI

$9.24 E-D 1$

$1.37 E+D 0$

$1.14 E+00$

$1.16 E+00$

$9.41 E-41$

$1,08 E+00$

\section{DEUTERONS}

a.

$1.10 F-104$

$3,92 E=114$

$1.83 E-04$

1.?DE-A3

3. $04 \mathrm{E}-\mathrm{B}+\mathrm{Ci}$

$4.97 E-144$

$5.93 E-O 4$

$5.50 E-04$

4. KBE-24

$7.98 F-14$

$4.52 E-94$

$1.35 E-193$

$5.81 E-B^{3} 4$

$9.62 E-04$

$7.065-134$

$1.345-93$

1. $\triangle 5 E-73$

1.9GE-D 3

$1.78 \mathrm{E}-\mathrm{DS}$

$1.16 E=03$

$1.96 E-(1) 3$

$9.13 E-174$

1. 3PE-V3

$9.19 E-\pi 4$

$1.52 E=03$

$1.64 E=13.3$

$1.59 E=13$

$4.82 E-A 4$

$9,6 B E-04$

$1.72 E-04$

$3.63 E=04$

1. DLE-OS

$8.97 E=0.4$

$8.83 E=05$

1.30E-OA

$8,55 E-4) 4$

3. $46 E-105$

$2.87 E-05$

$8.70 E-04$

$5,72 E=$ W 4

$4.56 E-04$

$6.72 E-04$

$5.09 E-D 4$

$8,7 D E-04$
TRITONS

U.

vi.

1, 33E- Bt

b. $32 E-V 5$

0.

4.

$1,33 E-06$

2, U6E-34

4.

$1.72 E-116$

$1,33 E-36$

$2.87 E-W 5$

$5.43 t-06$

$8,7 \forall t-t=5$

i).

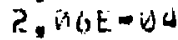

3. O1E-US

2. $45 E-15$

$3.65 E-46$

$7,15 E=0$

$1,20 E-B 4$

$7,22 E-D 6$

$1,72 E=94$

$6.45 E-45$

2. $12 E=-134$

$1,46 E-134$

$1,61 E-D 4$

3. VTE-V4

$4,13 E-60$

$1.57 E=04$

1. $33 E-46$

$0.13 E-05$

$3.9 B E-B 6$

$4.91 E-15$

2. GAE-U15

$9.53 E-45$

$1.62 E-615$

$5,59 E-05$

$1,81 E=04$

$1.92 E-64$

B. $\triangle Q E-D 6$

$7,30 E-105$

3. $44 E-05$

$5.76 E-\forall 5$

$7,136 E-05$
ALPHAS

D.

$1.48 E-132$

$1,84 t=42$

$2.48 E-112$

$1.87 E=12$

$1.94 \mathrm{E}-\Delta 2$

$7,14 E-13$

2. $49 E-152$

$2,38 E-12$

$1.43 \mathrm{E} D 2$

$9,61 E=\square 3$

$2.42 E-12$

2. 49E-A2

$1.05 E-92$

1. $4, J E=B 2$

$4.11 E-\Delta 2$

$1,62 E-U 2$

$1.57 E-112$

1. B8E-DI

1.89E-W2

2. $53 E-112$

C. DQE-WZ

1. TUE-H2

$2.39 E-6)$

$2.44 E-02$

$1.74 \mathrm{E}-192$

1. $27 E-62$

$1,76 E-192$

$2.87 E-\Delta 2$

$1.54 E=02$

$1.73 E=\square 2$

$3.20 E=102$

$9.85 E-03$

$2.24 E-42$

$1.7 M E=U 2$

$3.16 E-02$

$9.85 E-113$

$4, B G E=E 2$

$2.45 E-22$

$1.95 E-02$

$1.53 E-02$

$1.79 E-02$

$1.04 E-02$

$1.53 E-(1) 2$

$1,05 E-02$ 
ISLOWING DOWN SPECTRUM AT M.ZA CM. FDH 50 MEV D+ ON BE NEUTRON SPECTRUM

\section{ENERGY}

4.20 MEV

4.40 MEV

4.60 MEV

4.80 MEY

5. OA MEV

5.50 MEV

6.00 MEV

$6.5 \mathrm{O}$ MEV

7. DD MEV

$7.5 U$ MEV

8. DO MEV

8. $50 \mathrm{MEV}$

9.00 MEV

$9.50 \mathrm{MEV}$

$10.00 \mathrm{MEV}$

$11.00 \mathrm{MEV}$

$12,0 V M E V$

$13,00 \mathrm{MEV}$

14. DO MEV

$15,00 \mathrm{MEV}$

$16,00 \mathrm{MEV}$

$17.00 \mathrm{MEV}$

$18,00 \mathrm{MEV}$

19.00 MEV

$20.00 \mathrm{MEV}$

$21.00 \mathrm{MEV}$

$22.00 \mathrm{MEV}$

23.00 MEV

24. 00 MEV

25.00 MEV

26.0U MEV

$27.00 \mathrm{MEV}$

$28.00 \mathrm{MEV}$

$29.00 \mathrm{MEV}$

$30.00 \mathrm{MEV}$

32,00 MEV

34.0D MEV

$36.00 \mathrm{MEV}$

$38.00 \mathrm{MEV}$

40.00 MEV

$42.00 \mathrm{MEV}$

44.00 MEV

46.00 MEV

$48.00 \mathrm{MEV}$

$50,00 \mathrm{MEV}$

GAVE, ES MEV)

\section{PROTONS}

1. $15 E+D A$

1.70E+ 16

$7.45 E-11$

1. $25 E+(1)$

1. $78 E+b)$

1. $47 E+00$

1. $H 7 E+D \theta$

1. $22 E \div B U$

$7.89 E-11$

1. $23 E+0 A$

1. $45 E+4$

$1.11 E+0 B$

1. $39 E+3 B$

1. $] B E+B G$

$1.16 E+0 \theta$

$1.27 E+0.4$

1. $43 E+00$

1. $40 E+O B$

7.86E- 01

3. 33E-DI

$2.62 E=D 1$

1. $1 B F=01$

3. 35E- iो?

$3.87 F-B 1$

1. $2 B E-\square I$

0.

1. $\triangle 7 E-12$

2. $4 R E-B$ ?

1.77E-OI

$1.91 E=0 ?$

$1.12 E-152$

B. $21 E-02$

$3.21 E-62$

ट. $49 E-02$

B.73E- 02

ด.

Y.

$5.3 Z E-b 3$

6.

0.

,

$\theta$.

$\theta$

0.

B.

8.41

\section{DEUTERONS}

1. 3DE-US

1. $72 E-B A$

2. $B 6 E=114$

8. $55 E-95$

5.7 AE-OA

1. $62 \mathrm{~F}-114$

1.96E- 44

3. $10 E-194$

2. $93 E-1) 4$

$4.75 E-14+$

4. $72 E$. 4

$2.89 F_{-194}$

$3.72 E-D 4$

5. UIE-A5

2. $88 \mathrm{E}-\mathrm{BH}$

?. 7 OEDU

4. $49 F-04$

a.

a.

$n$

a

a

$a$

a

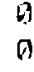

0

0

0

0

i.

$B$

i.

$a$

$a$

b)

0

a.

$a$

0

9.

0

0.

4.64
NHKÁ A-15U PLASTIC

TRITONS

b. $49 E=$ i 5

5. UAE-U 5

1. $32 E-116$

$6.95 E-45$

$5.17 E-05$

4. 9 ME $=15$

5. $3 h E=05$

4. $63 \mathrm{E}=05$

$4.33 E=65$

$6,8 D E-65$

1. $D 1 E-(1) 4$

$0.36 E=115$

1. $30 E-04$

1. $28 E-104$

$3.13 E-64$

$1.66 E-115$

2. $50 E-05$

$3.33 E-\square 5$

$7.91 E-05$

0

a.

,

i)

a.

H.

i.

b.

bi

a.

$\theta$

$\theta$

3

0.

$\pi$

$\theta$.

$b$

$\theta$

v.

a.

a.

D.

b.

u.

3.

7.00
ALPHAS

$1.59 E-D 2$

$6.21 E-\| 3$

$1.16 E=w 2$

$1.79 E-192$

$9.43 E-13$

$6.67 E=3$

$9,97 E-63$

1. ब1E $=$ तC

1.11E-U2

$9,46 E=13$

$1,13 E-A ?$

6. $D U E-U 3$

B. $27 E-03$

$1,66 E-D S$

7.49E-U 3

7. $85 E=H 3$

1. $40 E-D Z$

$4.25 E-U 4$

$9.57 E=B 4$

4. $48 E=B 5$

$9.24 E-65$

2. $21 E-15$

$5,18 E=135$

8. $93 E-196$

2.175065

a.

4.

a.

0

0.

H.

U.

$D$.

D.

D.

a.

s.

B.

b.

A.

D.

$\Delta$.

a.

8.

0

5,07 
1SLOWING DOWN SPECTRUM AT A, 4 O CM. FLIR SHOMKA A-15H FLASTIC 50 MEV D+ ON BE NEUTRON SPECTRUM

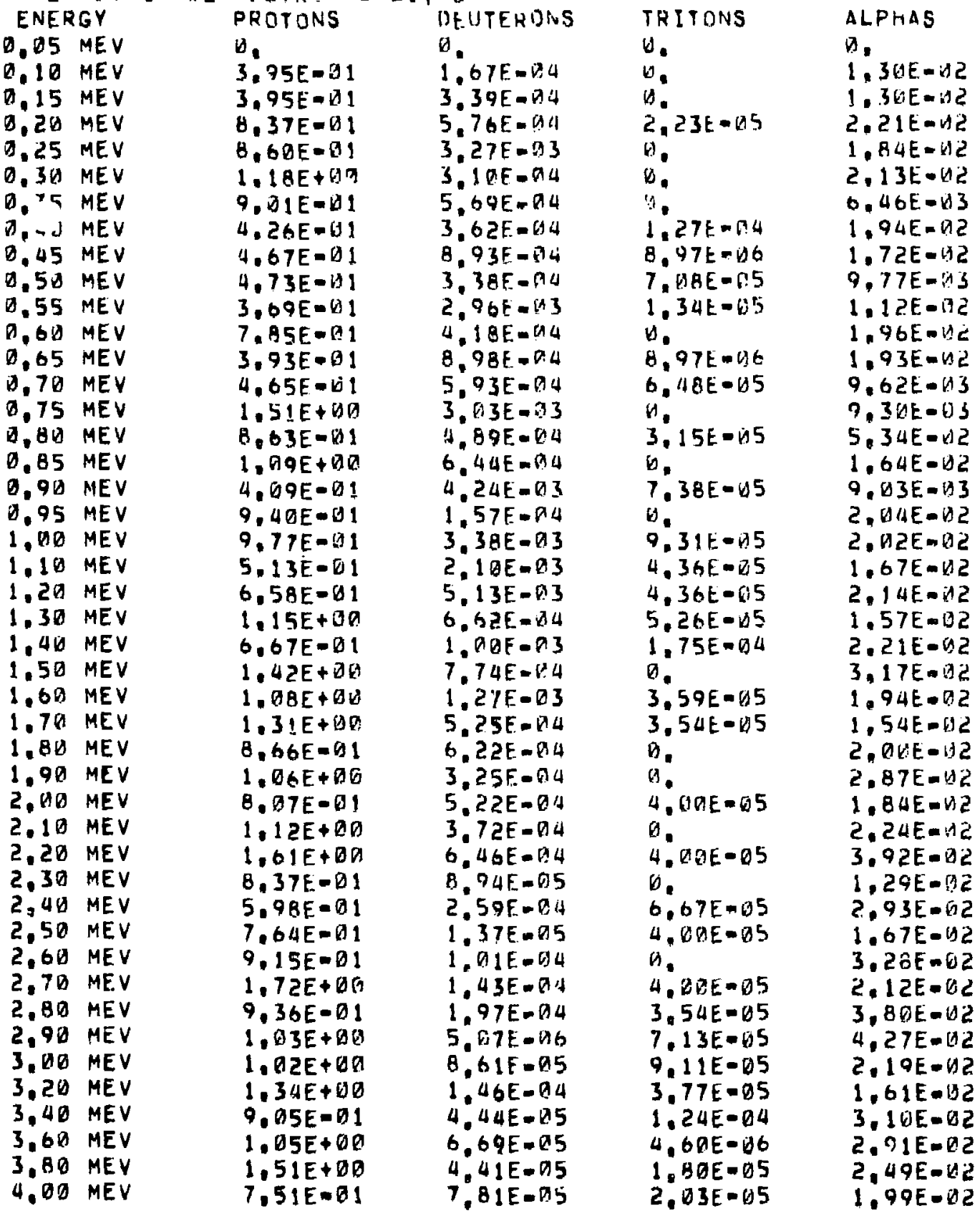


ISLOWING DONN SPECTRIIM AT H.4G CM. FOR SHUNKA A-15G PLASTIC 50 MEV D+ ON BE NEUTROII SPECTRUH

\section{ENERGY}

4.20 MEV

4. 4U MEV

4.60 MEV

$4.80 \mathrm{MEV}$

5. OO MEV

5.50 MEV

6. QV MEV

6.50 MEV

7. DG MEV

7.50 MEV

8. $D U$ MEV

8.50 MEV

$9.00 \mathrm{MEV}$

$9,50 \mathrm{MEV}$

$10.06 \mathrm{MEV}$

11.01 MEV

12. HV MEV

13. DO MEV

$14.0 \mathrm{MU}$ MEV

15. $\triangle 0 \mathrm{MEV}$

16. DO MEV

17. DD MEV

18.00 MEV

19. DD MEV

$20.00 \mathrm{MEV}$

21. RG MEV

22. QD MEV

23. 00 MEV

24. 9 MEV

$25.00 \mathrm{MEV}$

$26.00 \mathrm{MEV}$

$27.00 \mathrm{MEV}$

$28.00 \mathrm{MEV}$

29.01 AAEV

30.00 MEV

32.00 MEV

34.00 MEV

$36.00 \mathrm{MEV}$

38. DO MEV

4D. DO MEV

42. DO MEV

44, OO MEV

46. OO MEV

4B. 0 MEV

$50,00 \mathrm{MEV}$

\section{PROTONS}

$9.85 E-$ is 1

1. 4BE $+B Q$

1. $33 E+60$

1. $D 7 E+B D$

1. $51 E+00:$

1. $3 E+\triangle 0$

1.1AE +30

1. $\triangle \angle E+Q Q$

$9.26 E=01$

1. $15 E+0 P$

1. $1 A E+D O$

$1.12 E+00$

1. $07 E+00$

$1.61 \mathrm{E}+\mathrm{aB}$

1. $13 E+D \theta$

$1.12 E+B Q$

$1.93 E+130$

7. $43 E-01$

$5.79 E-01$

$6.75 E-01$

$7.46 E-V 2$

4. 32E-D2

$2.32 E-01$

8. 37E-日2

3. D5E-A2

3. $24 E-12$

1. $98 E-01$

$9.86[-6]$

$4.66 E-02$

$9.51[-02$

$6.67 E-03$

$9.88 E-03$

0.

$\theta$.

0.

$5.32 E-03$

a.

$\theta$

0.

$4.94 E-03$

0.

0.

0.

0.

0.

7.96

\section{DEUTERONS}

9. $21 E-65$

9. DIF-PS

1. $13 F=\square 4$

1. $29 E-\frac{\pi}{4}$

$2.93 E-94$

2. B $3 E-125$

3. 36E $=5$

$4.99 E-05$

4. $42 E-0 S$

8. OYE-95

1. $10 E-b$ i

3. $84 E-105$

5. $56 E-05$

1. $16 E-05$

$4.66 F-05$

$4.51 E-05$

6. $22 E=15$

a.

a

$a$

9

a.

is.

$\lambda$

0

$a$

$n$

$a$

a

a

a.

0

$\theta$

a.

$\theta$

$\theta$

0

0

a.

$B$

2.18
THITONS

2.39E- 16

2. $E 3 E-05$

?. D $3 E-155$

$3.59 E-0 S$

$7.94 t=15$

$7.36 E-0106$

$1.66 t-05$

1. $12 E-05$

10

$1,12 E-115$

b.

$1.12 E-145$

$1,12 E=V S$

i.

$1.12 E=15$

$1.67 E-65$

$2,77 E-15$

,

is

is

i)

b.

a.

i.

i).

b.

a.

i.

v.

0.

6.

$\theta$.

(5)

b.

0

b.

D.

b.

U.

0.

a.

0.

$b$

0.

0.

4.74
ALPHAE

Z. 37E-U2

2. $16 E-162$

2.67E-VI2

2. $40 E-02$

?. $23 E-12$

1. $29 E-\triangle Z$

$2.5 H E-W 2$

1. $30 E=192$

2. $2 B E-D C$

1. $21 E-B 2$

$1,45 E-42$

$0.32 E-93$

$1.53 E=U E$

$3.47 E=D 3$

$B, B 2 E=V B$

7. DAE-D 3

$1.23 E-D 2$

1. $77 \mathrm{E}=03$

$3,34 E=13$

9. DLE-EUU

9.2LE-E4

$8.87 E=04$

2. $66 \mathrm{E}-\mathrm{E}^{\circ} \mathrm{S}$

(i).

a ,

E.

A.

$\therefore$

v.

$\theta$.

b.

$\theta$

B.

b.

D.

0

a.

$\theta$

0

0

b

a.

0.

1.

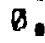

5.49 
ISLOWING DOWN SPECTRUM AT A,60 CM. FOR SHONKA A-150 PLASTIC. 50 MEV D+ ON BE NEUTRON SPECTRUM

\section{ENERGY}

D.B5 MEV

$0.10 \mathrm{MEV}$

$0.15 \mathrm{MEV}$

0.20 MEV

0.25 MEV

$0.30 \mathrm{MEV}$

0.35 MEV

0.40 MEV

$0.45 \mathrm{MEV}$

$0.50 \mathrm{MEV}$

$0.55 \mathrm{MEV}$

$0.60 \mathrm{MEV}$

$0.65 \mathrm{MEV}$

0.70 MEV

0.75 MEV

$0,80 \mathrm{MEV}$

0.85 MEV

$0.90 \mathrm{MEV}$

0.95 MEV

1.00 MEV

$1,10 \mathrm{MEV}$

1.20 MEV

$1.30 \mathrm{MEV}$

1.40 MEV

$1.50 \mathrm{MEV}$

1.60 MEV

1.70 MEV

$1,810 \mathrm{MEV}$

1.90 MEV

2. 00 MEV

2.10 MEV

2.20 MEV

2.30 MEV

2.40 MEV

2.50 MEV

2,60 MEV

$2,70 \mathrm{MEV}$

$2.80 \mathrm{MEV}$

$2.90 \mathrm{MEV}$

3. DO MEV

3,20 MEV

3.40 MEV

$3.60 \mathrm{MEV}$

$3.80 \mathrm{MEV}$

4.00 MEV
PROTONS

$\theta$.

$4.14 E=01$

$3.62 E-01$

$3,30 E=01$

$3,49 E-01$

$4.79 E-01$

3. B3E-D 1

3. $A 1 E-Q 1$

3. $26 E=01$

5. 3QE-B1

3. $46 E-01$

$4,07 E-01$

4. UTE-DI

$6.77 \mathrm{E}$ O

$3.92 E=01$

$4,44 E=1$

$1.45 E+D 0$

$5,1 B E=01$

$4.4 B E-01$

$6.72 E-11$

5. $77 E-01$

$5,56 E-01$

$5,23 E=01$

$7,82 E=D 1$

$1.05 E+B D$

$5.22 E-11$

$8.87 E-(1)$

$8.73 E-91$

3. $82 E-01$

$9,81 E-01$

$6,56 E=01$

$9,54 E-01$

$8.43 E-01$

$9,37 E-01$

$7,30 E-01$

$6,01 E-01$

$1,09 E+B D$

$1,26 E+00$

$6.62 E-01$

$9,11 E-01$

$7.35 E-01$

$7.57 E-01$

$8.84 E-01$

$1.17 E+B D$

B. 29E-51

\section{DEUTERONS}

$\theta$.

1.79E- 34

$3.48 E-04$

6. $40 E-174$

$5.41 E=04$

2. $24 E=04$

$4.44 E-04$

$2.25 E-04$

$1.33 E-03$

$3.91 E=14$

$4.63 E-04$

$3.77 E=A$

1. $19 E-B 3$

$7.47 E-Q 4$

$3.46 E=94$

$7.29 E-04$

$1.06 E-03$

$1.67 E-03$

$3,32 E-04$

$1.28 \mathrm{E}-63$

$1,21 E-83$

$9.95 E=04$

$1.2 \mid E=03$

$1.39 E-23$

1. $96 \mathrm{E}-03$

$1.91 E-B 3$

6. QZE-DL

1. $1 \mathrm{BE}-03$

$4.50 E=04$

$1.15 E-Q 3$

2,59E-DL

$4,62 E=04$

$2.93 E-104$

$2.90 E-04$

$\because .03 E=04$

$4,60 E-04$

$1,1 \triangle E-Q 4$

2.96E-04

2.94E-05

$2,37 E-04$

$1.70 E-24$

$3.93 E=04$

9.DIE-05

3. D3E.04

1. $43 E-65$
TRITONS

a,

$2.63 E-45$

$1,30 E-06$

$9.24 E=Q B$

4.

$1.30 E-06$

$2.61 E-56$

$1,25 E-85$

$1.12 E=\square 5$

0.

$7.93 E-116$

$3,49 E-636$

1. $12 E-115$

$1.59 E-04$

19.

$3,42 E-65$

$1,83 E-65$

$0.60 E-196$

1. $47 E-65$

1. $\triangle D E-04$

$1.66 E-45$

$1,35 E-05$

1. $05 E-154$

$3.81 E-05$

2. $1 \mathrm{DE}-15$

$0,93 E-05$

$8,39 E-05$

3. $07 E-66$

$9.61 E-0.6$

$1,51 E-04$

$9.61 E=06$

$8,33 E=05$

B. $02 E-B 5$

$9.89 E-25$

$2.76 E-05$

$1,59 E-104$

$1,70 E-05$

1. $44 E=04$

$1.62 E-64$

$2.42 E-04$

$3.94 E-05$

$1,47 E-64$

$1.92 E-05$

$3.00 E-05$

$6,61 E-06$
ALPHAS

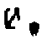

$1.34 E-112$

$1.72 E-12$

2.37E-G2

2. DIE- II

2.55E- ज2

$9.23 E=103$

$2.8 \cup E-02$

$1.74 E=192$

$1.13 E=1$ ?

$1.24 E=42$

2. $44 E=152$

2. $44 E-112$

$7.9 \mathrm{hE}=03$

1. $12 E-\Delta 2$

$5.13 E-U C$

$2.29 E-112$

$4.05 E-133$

$2.45 E=12$

$2.86 E=192$

2. $18 E-02$

3. OUE 132

1. B3E-UZ

$1.95 E-\theta 2$

$3.32 E-42$

$1.61 \mathrm{E}-12$

$1,85 E-02$

$2,60 E-\triangle 2$

$2.86 E-122$

2. $46 E-92$

2. $13 E-\square 2$

4. $21 E M B 2$

1.7 TE= $=2$

$3.33 E-\Delta 2$

$1.91 E=12$

$3,62 E=02$

$2.56 E=\Delta 2$

$2,68 E-112$

$4,20 E=92$

$1.96 E-02$

$1.73 E-02$

$3.13 E-02$

2. 99E-02

$2.60 E-02$

$2.10 E-B 2$ 
ISLOWING DOWN SPECTRUM AT M,6B EM. FOR SHONKA A-15U PLASTIC 50 MEY D+ ON BE NEUTTRON SPECFRLIN

\section{ENERGY}

4.20 MEV

4.40 MEV

$4.60 \mathrm{MEV}$

4.80 MEV

5. 00 MEV

5.50 MEV

6,00 MEV

6.50 MEV

7. OU MEV

$7.50 \mathrm{MEV}$

8. $D Q M E V$

8.50 MEV

$9.00 \mathrm{MEV}$

9.50 MEV

$10.00 \mathrm{MEV}$

$11.00 \mathrm{MEV}$

$12.00 \mathrm{MEV}$

13,00 MEV

14.00 MEV

$15.00 \mathrm{MEV}$

16.00 MEV

$17.00 \mathrm{MEV}$

18. OD MEV

19.00 MEV

$20.00 \mathrm{MEV}$

$21.00 \mathrm{MEV}$

22.00 MEV

23.00 MEV

24.00 MEV

25.0D MEV

26. 00 MEV

$27.00 \mathrm{NICV}$

$28.00 \mathrm{MEV}$

29.DO MEV

30,00 MEV

32.00 MEV

$34.00 \mathrm{MEV}$

36,00 MEV

38.00 MEV

40. 00 MEV

42.00 MEV

44.00 MEV

46.0D MEV

48,00 MEV

$50.00 \mathrm{MEV}$
PROTONS

8. B6E-OI

$7.73 E-31$

$1.14 E+05$

1. $A 1 E+06$

$1.33 E+00$

8.66E-O1

$9.35 E-01$

$8.64 F-01$

1. $12 E+2 B$

1. $10 E+00$

1. $D 2 E+0 D$

$1.29 E+00$

$9.93 E-61$

$1.15 E+00$

$8.90 E-01$

$9.17 E-B 1$

1. $44 E+0 B$

$0,36 E-B 1$

$9.79 E-Q 1$

B. 2 AE-01

7.8GE-O1

$6.38 E-A 1$

$5,93 E-21$

7. $2.2 E-01$

5.55E-01

$4.63 E-01$

2. $14 E=01$

3. $\triangle 9 E-02$

a.

$3.95 E=03$

2. 2BE=02

$4.48 E-02$

$1.15 E-02$

0.

0.

$1.10 E-B 3$

1. $21 E-03$

4. $18 E-03$

$1.07 E-02$

v.

$b$

$5.74 E=93$

0.

0

a.
DEUTERONS

$3.55 E=25$

$4.33 E=05$

$5.84 E=05$

3. $44 E-95$

1. $56 E=04$

4. $21 E-05$

1. 3EE-D 4

$1.67 E-65$

7 . $1 E=05$

$7.83 E-05$

B. BSE-OS

8. $13 E-O S$

1. AGE DOA

1. 19E-D 4

2. $21 F-04$

2. $19 E D O 5$

5. $75 E=05$

0

A.

a

0

a

0

0

0

0

a.

$a$

0

0.

a.

0.

0

$\theta$

0

0

0

0

0

$b$.

0

a.

2.98
TRITUNS

3. $49 E-106$

1. $27 E-95$

$1,13 E-65$

4. DQE -96

b.

$4.93 E=06$

$4.93 E-166$

$4.93 E=06$

B. $25 E=06$

$9.85 E=06$

2. $13 E-05$

b.

U.

a.

b.

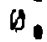

0

b.

a.

$b$

0

a.

$\theta$

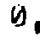

i.

b.

0.

0

B.

$\theta$.

$\emptyset$.

a.

b.

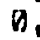

0

ט.

0

0.

0

b.

$b$

a.

a.

2.87
ALPHAS

2.89E-G2

$2,40 E-\triangle 2$

$2.77 E-D 2$

$2 ., \triangle B E-112$

$2,18 E-D Z$

1. 26E- D2

$2,60 E-02$

$1.21 E-U 2$

2. $41 E-02$

1. $02 E-D 2$

1. $5 U E-\triangle 12$

$7.49 E-U 3$

$1,27 E=02$

$2.77 E-03$

$6,89 E=03$

$4.62 E-103$

$9.73 E=183$

$7,17 E=04$

$1.76 E=13$

1.85E-05

$4.63 E=185$

0.

b.

0.

a.

$\theta$.

$\forall$.

b.

9.

0.

$\theta$.

D.

a.

b.

0.

0

0

b.

$\theta$

D.

0.

0.

a.

0

10.31

4.76 
1SLOWING DOWN SPECTRUM AT 1, BA CM. FOR SHOHKA A-15Q PLASTIC 50 MEV D+ ON BE NEUITRON SPECTRLIM

ENERGY
$0.05 \mathrm{MEV}$
$0.10 \mathrm{MEV}$
$0.15 \mathrm{MEV}$
$0.20 \mathrm{MEV}$
$0.25 \mathrm{MEV}$
$0.30 \mathrm{MEV}$
$0.35 \mathrm{MEV}$
$0.40 \mathrm{MEV}$
$0.45 \mathrm{MEV}$
$0.50 \mathrm{MEV}$
$0.55 \mathrm{MEV}$
$0.60 \mathrm{MEV}$
$0.65 \mathrm{MEV}$
$0.70 \mathrm{MEV}$
$0.75 \mathrm{MEV}$
$0.80 \mathrm{MEV}$
$0.85 \mathrm{MEV}$
$0.90 \mathrm{MEV}$
$0.95 \mathrm{MEV}$
$1.00 \mathrm{MEV}$
$1.10 \mathrm{MEV}$
$1.20 \mathrm{MEV}$
$1.30 \mathrm{MEV}$
$1.40 \mathrm{MEV}$
$1.50 \mathrm{MEV}$
$1.60 \mathrm{MEV}$
$1.70 \mathrm{MEV}$
$1.80 \mathrm{MEV}$
$1.90 \mathrm{MEV}$
$2.00 \mathrm{MEV}$
$2.10 \mathrm{MEV}$
$2.20 \mathrm{MEV}$
$2.30 \mathrm{MEV}$
$2.40 \mathrm{MEV}$
$2.50 \mathrm{MEV}$
$2.60 \mathrm{MEV}$
$2.70 \mathrm{MEV}$
$2.80 \mathrm{MEV}$
$2.90 \mathrm{MEV}$
$3.00 \mathrm{MEV}$
$3.20 \mathrm{MEV}$
$3.40 \mathrm{MEV}$
$3.60 \mathrm{MEV}$
$3.80 \mathrm{MEV}$
$4.00 \mathrm{MEV}$

\begin{abstract}
PROTONS
\end{abstract}
O.

6.06E-01

3. $40 E=B 1$

3.52E-OI

3. $01 E=91$

3. $53 E=01$

3. $76 E=91$

3. $85 E-Q 1$

4. $21 E=01$

4.68E-31

3. $35 E=\mid A 1$

3.9BE-DI

$4.33 E-D 1$

4. $42 E-U I$

$4.64 E=01$

$4.33 E-D 1$

6.01E-O1

3. $22 E=01$

6.6BE-DI

$6.12 E=01$

$4.34 E-01$

5.65E-QI

5.18E-D 1

$3.64 E-21$

8.15E-01

3.89E-G1

$6.93 E-01$

$7.65 E-01$

$3.56 E=01$

$6.21 E=01$

$5.25 E-01$

$5.50 E=91$

$9.91 E=01$

$4.84 E-01$

$4.27 E=01$

$4.6 B E=01$

$7.53 E-O 1$

$4.43 E=01$

$7.75 E-01$

1. $31 E+O 0$

$7.46 E=01$

$5.17 E-01$

$6.13 E \cap D I$

9. BAEDOI

6. $38 E=01$
DEUTERONS

a.

$1.03 E-04$

2. $26 E-U 4$

$4.27 E=\mathrm{H} 4$

$6.92 E-14$

2. Q7E-OU

3. $20 E=04$

3. $Q 1 E=94$

9.7 DEDA

$3.42 \mathrm{~F}=14$

$5.33 E=Q 4$

$4.12 E=14$

8. $12 E=04$

$7.55 E=04$

4.65E-OA

$5.74 E-04$

6.78E- -14

1.25E- D3

3. $82 E=04$

$9.05 E-A 4$

$7.93 E=04$

1. DBE $=03$

$7.26 E-O 4$

$1.01 E-03$

$5.66 E=P A$

$1.14 E=03$

4.99E-D4

$7.12 E=04$

4. OGE-Q 4

9.31E-Q4

$1.76 E=04$

3.78E=04

2.07E.04

$4.68 F=04$

B.7BE-05

$2.15 E=04$

$3.21 E=04$

1.75E-04

$1.46 E=04$

$2.95 E=04$

2.98E:DA

2.95E-04

$2.53 E=R A$

$5.65 E=84$

?.45E-04
IRITONS

Q.

$1.67 E-135$

$1,12 E=05$

$3.88 E-05$

$1.23 E=05$

$1.11 E-06$

$1.20 E-15$

$1.95 E-14$

$4.69 E-186$

$B, 39 E-155$

$4.61 \varepsilon-06$

$4.99 E-05$

$2,82 E=125$

$4.04 E=05$

$1.78 E=15$

$1.60 E-05$

3. $B 8 E-105$

5. $47 E-05$

1. $8 B E=05$

1. $Q A E=\square 4$

$9,60 E-05$

$3.83 E=05$

$5,21 E=15$

$0.41 E=05$

$0.04 E=15$

$1,21 E-$ is 4

$1.52 E=04$

$8.13 E=05$

$5,89 E-05$

$1,30 \mathrm{EOA}$

1. BIE-DS

$1,25 E-04$

$0.63 E-05$

8.19E-D5

3.94E-05

1. 36E- $\triangle 4$

$1.42 E-04$

$1,03 E-04$

1. B1E-OU

1.2TE- TU 4

$7.27 E-B 5$

१. $\triangle O E-O 4$

$7, \triangle 9 E=05$

$1.63 E=04$

$6,33 E=05$
ALPHAS

Q

$1,33 E=02$

1.2BE-ด2

$2.45 E-B 2$

$1.35 E=92$

2.07E-02

$6.17 E-03$

1.5BF-02

1.5UE-O2

$1.10 E-1 D 2$

1. 1ZE- 12

1.59E-D2

2. $28 E-02$

9.5DE-D 3

$9.11 E=\square 3$

4.1UE-UZ

1. $\triangle D E=B 2$

1. DBE-OZ

$1.64 E=62$

1. BBE-G2

$1.45 E-02$

$2.98 E=02$

$1.3 \triangle E=\triangle 2$

$1.84 E-U 2$

$2.18 E=02$

$1.19 E-02$

$1.42 E=02$

2. OAEDQZ

$2.17 \mathrm{E}=12$

1. 6BE-D2

$1.93 E 002$

3. D2E D?

1. $\triangle 9 E=02$

$1.94 E-B 2$

$1.24 E=02$

$2,67 E-02$

$1.52 E=02$

$1.71 E-02$

3. OBE-02

$1 . \triangle B E=02$

$1.39 E=\square 2$

$2.66 E-02$

$1.9 B E=02$

2.07E-OZ2

$1.81 E-0 ?$ 
1SLOWING DOWN SPECTRUM AT 1, BH CM. FOR SHDNKA A-15G PLASTIC 50 MEV D+ ON BE NEUTRON SPECYRUM

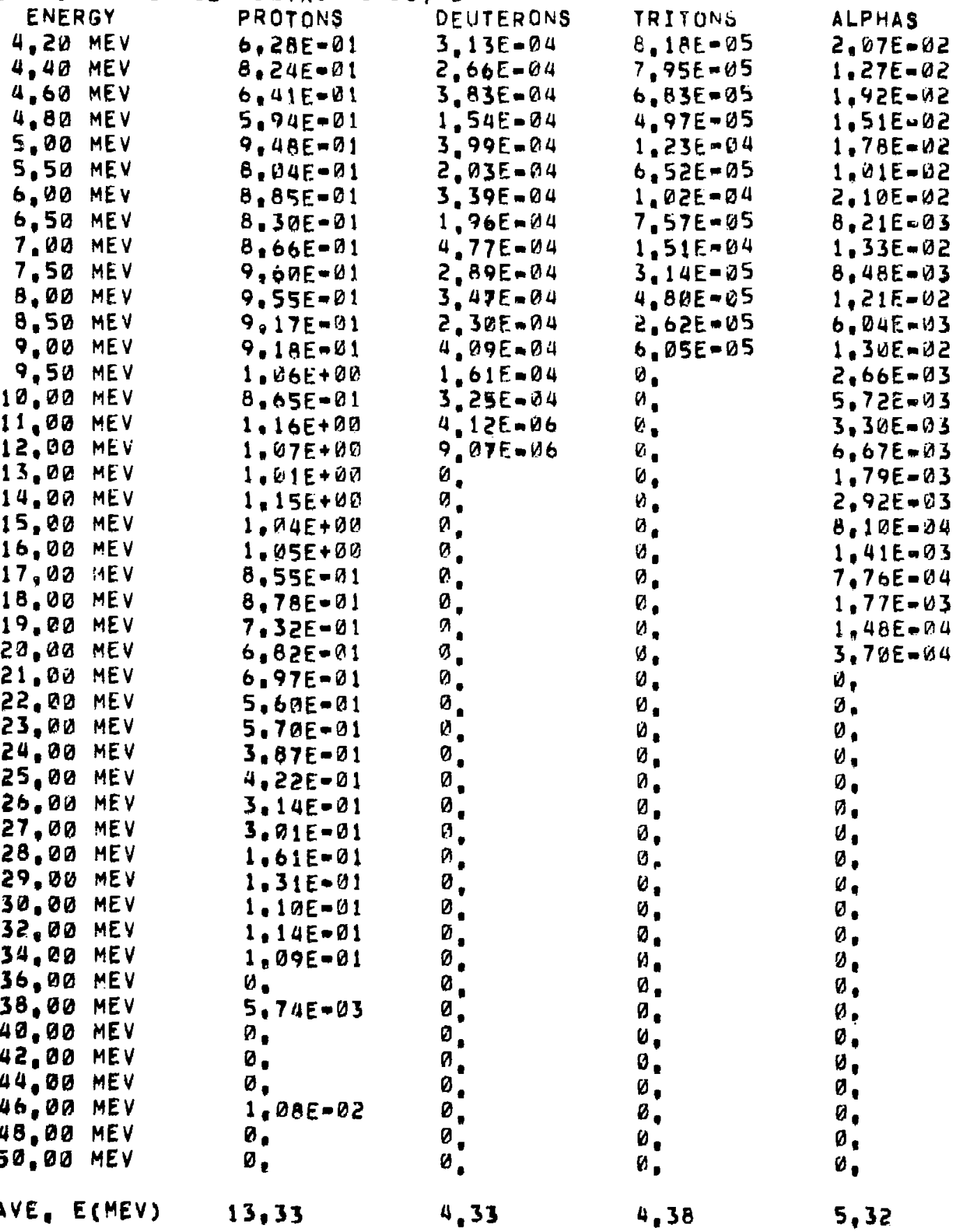


1SLOWING DOHN SPECTRIIIA AT 2. VA CH. FUR SHONKA A-15L PLASTIC 52 MEV D+ ON HE NEUTROH SPECTRLM

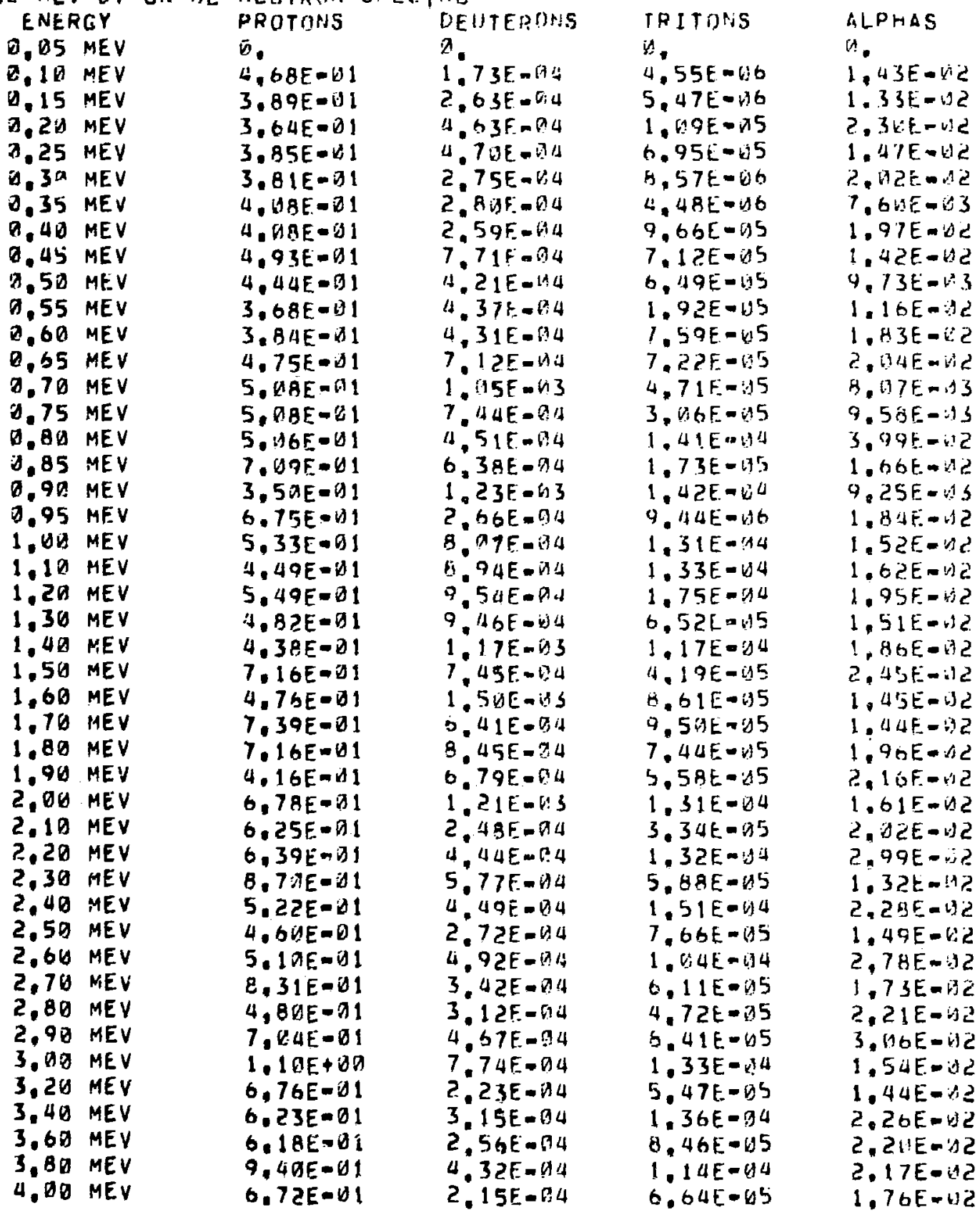


1SLOWING DONN SPECTRUM AT 2, HA CM. FOH SHOHKA A-15H PLASTIC SO MEV D+ ON HE NEUTRON SPECTRLIM

ENERGY

4.20 MEV

4.40 MEV

4.60 MEV

$4,86 \mathrm{MEV}$

S.QD MEV

$5,50 \mathrm{MEV}$

$6,00 \mathrm{MEV}$

$6,5 B$ MEV

$7.00 \mathrm{MEV}$

$7,56 \mathrm{MEV}$

B. OV MEV

8.50 MEV

9 , i) 1 MEV

$9,50 \mathrm{MEV}$

19, 90 MEV

$11.0 \mathrm{ON}$ MEV

12. OIS MEV

$13.00 \mathrm{MEV}$

14, $\triangle B$ MEV

15. HU MEV

16. OO MEV

17. Dg MEV

18. DU MEV

19.00 HEV

20.00 MEV

21. DU MEV

22,00 MEV

23,06 MEV

24.00 MEV

25. OU MEV

26.00 MEV

27. DD MEV

28.00 MEV

$29.00 \mathrm{MEV}$

30.00 MEV

32.00 MEV

34.00 MEV

36.00 MEV

$38,00 \mathrm{MEV}$

$40,00 \mathrm{MLV}$

$42,00 \mathrm{MEV}$

44. 06 MEV

46.00 MEV

$48,00 \mathrm{MEV}$

$50.00 \mathrm{MEV}$
FRTTONS

G. 1 BE $-U 1$

$7.99 E-91$

$7.57 E=611$

$6.5 a E-41$

$8.29 E=(7)$

7. $45 E-61$

$9,17 E-U 1$

8.5QE- 1

$8.59 F-111$

$9.9(E-V) 1$

9. $\$ 4[E-1]$

9. $\triangle 9 E=7$

9. 2UE-D1

$9.13 E-31$

9. $17 E-11$

1. $\triangle 5 E+40$

Q. $31 E=01$

$8.75[-71$

8. 2OE-OI

8. DQE-DI

$7.35 E-i d 1$

6. $ठ 1 E-H 1$

$6,31 E-d !$

5. 49E- 11

$5.15 E-D 1$

$4.74 E-D 1$

$3.34 E-i) 1$

$3,44 E-01$

$2, D 3 E=\Delta 1$

$1.89 E-B 1$

$1.66 E-01$

$1,17 E-31$

1. $24 E-01$

$6.57 E-\theta 2$

$7.07 E=02$

$1.13 E-01$

1. $\triangle 5 E-01$

$3,23 E-\Delta 2$

$2.05 E-\Delta 2$

$9.89 E-13$

$1,64 \mathrm{E}-102$

$2,23 E-\lambda 2$

0 .

0.
DEUTFEIUS

Z. $G B E=-24$

2. $49 E-ก 4$

$3.59 E=64$

$1.71 E-64$

$4.33 E-B A$

$2.64 \mathrm{~F}-34$

$4.39 E-14$

$3: 49 F-14$

$7.75 E-04$

$1.98 E=04$

$2,79 E-114$

1.3GE-B:

2. $27 E-B 4$

$1,12 E-D 4$

2. $45 E-44$

$5,23 E-55$

9.3 IE-OS

2. $11 E-35$

$4.26 E-A 5$

$3.99 E-65$

1.66E-35

n.

a.

$\pi$

14

9

n.

$a$

a

19

$a$

D.

9

a

a

a.

a.

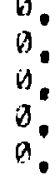

4.41
TRITON:S

$1.93 \mathrm{E}-194$

$7.35 i-65$

$5.82 E-415$

$4.46 E=145$

$1,25 E-74$

b. $26 E-U 5$

1. $14 E-64$

$5,73 E=125$

$1.13 E-614$

5.50E-US

$9,5 O E=115$

2. 2 OIE-WS

3. OOE. - 15

3. VGE- UI 5

$0.621-H 5$

1. A $1 E-45$

$1.51 E-05$

$6.38 E-20$

1. $58 E-65$

$\because$

n.

u.

$n$

9.

0

$d$

i).

6.

$n$.

v.

0

0

v.

19

a.

w.

is.

$D$.

0.

9.

$\theta$.

H.

$\theta$.

0.

$y$.

5.69
AL PHAS

2. 115E-132

1. ?SE-UC

$1.76 E-192$

$1.72 E-12$

1.47E-OL

$9.64 E-113$

$1.9 B F-112$

$7.92 E-113$

$1,50 \mathrm{E}-1 \mathrm{C}$

7.61E-3

1. $69 \mathrm{E}-\mathrm{HL}$

4. $51 \mathrm{E}-\mathrm{J} 3$

B. $5 B t-v 3$

3. $16 E-A S$

$1,35 E-13$

2. $19 E-13$

3. $59 E-113$

$1.49 \mathrm{~F}-\mathrm{BS}$

$3.1 \mathrm{E}-\mathrm{U}$

$5.30 E-H 4$

$1.23 E \mathrm{mWS}$

$6.14 E-96$

1. SUIE- biS

$3.90 E-10$

$b, 12 E-136$

in.

0.

b.

a.

.

a.

is.

ต.

.

a.

i.

D.

d.

B.

$\theta$

0.

B.

a.

$s$

u.

4.78 
1SLOWING DONN SPECTRUM AT M. MA CM. FOH SHCNKA A-1SH PLASTIC 5D MEV D+ ON AE NEUTRON SPECTRIJM

\section{ENERG}

D. 05 MEV

0.10 MEE

0.15 MEV

2. 2 П $M E V$

9.25 MEV

0.30 MEV

$0.35 \mathrm{MEV}$

0. 40 HEV

D. 45 MEV

$0.50 \mathrm{MEV}$

$0.55 \mathrm{MEV}$

$0.60 \mathrm{MEV}$

0.65 NEV

$0.76 \mathrm{MEV}$

0.75 MEV

Q.8U MEV

ग.05 MEV

$0.90 \mathrm{MEV}$

0.95 MEV

1. HO MEV

1.1 1 MEV

1.20 MEV

1.30 MEV

1. $\triangle V$ MEV

1.5O MEV

$1.66 \mathrm{MEV}$

1.70 MEV

1. BV MEV

$1.90 \mathrm{MEV}$

2.00 MEV

2.10 AEV

$2.20 \mathrm{MEV}$

$2,3 H$ MEV

2.40 MEV

$2,50 \mathrm{MEV}$

2.60 MEV

$2.70 \mathrm{MEV}$

2.80 MEV

2.90 MEV

3. 00 MEV

3.2U MEV

3. $40 \mathrm{MEV}$

$3.60 \mathrm{MEV}$

3.80 MEV

4. DO MEV
PROTONS

औ.

$6.57 E-31$

3. $6\{E-(1)$

$3.03 E-11$

$3.61 E-11$

$3.51 E-01$

$3,45 E=01$

$3,79 E-01$

4. USE $A-B 1$

$4.86 E-M 1$

$3.26 E-91$

$3.815=1$

$4.27 E-1$

$4.93 E-11$

$4,55 E-A 1$

$4.44[-41]$

$6,88 \mathrm{E}-\mathrm{J} 1$

$3,54 E-B 1$

5. $26 E-U 1$

$5.63 E-01$

4. UAE $-A 1$

5. 3AE-U1

$4.94 E-B 1$

$4.23 E=1$

$7.72 F-101$

$4.74 E-B 1$

6. $84 E-D$

6. 4 UE $B 1$

$4.18 E-31$

$6.40 E-V 1$

$6.45 E-B 1$

$5,39 E-01$

7. $30 E=D 1$

$4.91 E-11$

5. $25 E-61$

$4.92 E-11$

7. $56 E-31$

4. $69 E-91$

$6.2 Q E=\square$ !

$1.04 E+O W$

5.9 $\triangle E-D 1$

5.77E-OD

5. 59E-O 1

$8.45 E-01$

$6.57 E=01$
DEUTERONS

i1.

$1.25 F-194$

1. $36 F-94$

3. 4 DE DI 4

$3.52 F=174$

1.91E-G14

2. $54 E=04$

2. $425=174$

1. GIAE- 3

?. $690-114$

1. 47E-A3

2. $711-64$

7. $28 E-B^{7}+4$

5. 2 o $\mathrm{m} \mathrm{K} \mathrm{k}^{2}$

3. $5 \Delta E \mathrm{EH} M$

4. 1 उE-P 4

1. $4 D F-A 3$

9. $28 E m b \omega$

1. $57 \mathrm{E}-\mathrm{H} 4$

6. $72 E-04$

E. $20 E-\square$

7.34E-DL

1. $27 \mathrm{E}-13$

$1.35 F-43$

$6.67 E=0.4$

1. 3uE $=03$

5. $50 E=04$

1. $15 \mathrm{E}=?^{13}$

$1.32 E-3$

$5.93 E-44$

$4.62 E-1) 4$

5. $97 E=04$

$1.87 E-34$

1. 35E-A3

$3.95 E=194$

1. $15 \mathrm{~F}=03$

7. 2 QE $=$ OA

3. $17 E=14$

1.87E-94

7. $69 E-61$

$5.46 E-144$

$5.38 E-04$

$7.53 E-D 4$

1. $22 E=03$

$1.74 E-04$
TRITINS

त)

1. $15 E-05$

7. $26 E=07$

$1.89 E-65$

$1.95 E=66$

1. $35 E-05$

$2.56 E=616$

4. WPE-W 5

$9,15 E-616$

$2.57 E-35$

$1.14 E-105$

4. 39E-U5

1. $28 E=05$

4. $\triangle 1 E-05$

1. $43 E=0.5$

2. $52 E-45$

3. $27 E-15$

2. $39 E=135$

1. 7 AE-U 5

4. DAE- I5

$3.55 E-65$

$4.03 E-05$

$3.41 E-15$

4. 84E-05

2. DTE- 155

य. 76E- 95

$0.56 E-0.5$

$3.69 E-05$

2. $14 E-15$

$5.62 F-135$

$2,50 E=15$

$6,23 E-15$

3. $21 E-05$

$7.14 E=45$

$3,82 t=05$

$0.57 E-05$

$3.43 E=05$

$4.91 E-0.5$

$6.45 E-115$

$6.19 E=05$

$3.90 E-05$

5. 10E-OBS

$2.93 E-05$

5. $83 E=05$

$2.66 E=65$
ALPHAS

i.

$1.45 \mathrm{E} \cdot 11 \mathrm{C}$

1. $31 E=12$

$2.46 E=142$

1. 54E-V2

$2.03 E-12$

$8,11 E=13$

$1.87 t-1) 2$

$1.66 E-B 2$

1. U3E-UL

9. คRE- 3

$1.7 B E-U 2$

1. $99 E=112$

$7.97 E-13$

1. $155-1.12$

$4.47 E-A 2$

$1.65 E-0 ?$

$1, U 4 E-12$

1. B1E-U2

1. $71 \mathrm{E}=112$

1. 5 旦 -12

2. ULE-A2

1. $49 \mathrm{E}=\mathrm{WL}$

1.91E-AC

$2.61 \mathrm{~F}-4 \mathrm{C}$

1. $4 B E-42$

1. $79 E-12$

2. $12 E=12$

$2.38 E-12$

$1.71 E=U_{C}$

$2.45 t-02$

3. $43 E-6 i$.

1. $16 E-142$

$2,41 E-62$

$1.65 E-112$.

$2.94 E=U 2$

1.74E- 192

2. 7 AE 42

$3.47 E=U 2$

1. $78 \mathrm{E}-02$

1. 39E-O2

$2,55 E-02$

$2,34 E-\Delta Z$

2. $\triangle 2 E=\square 2$

1. $9 W E=D 2$ 
ISLOWING DONN SPECTRIIM AT D, RG CM, FOR SHOHAA AOISH PLASTIC. 50 MEV D+ ON BE MEUTROIN SUERTHUM

\section{ENERGY}

4. 2 UE $M E$

4. 40 MEV

4. OD MEV

4.BQ MEV

5. QU MEV

5.50 MEV

6.010 MEV

$6,50 \mathrm{MEV}$

$7.0 B$ MEV

7.50 MEV

8. $\triangle A$ MEV

8.5U MEV

9.1 (i) HEV

9.5 M MEV

$10.0 D$ MEV

11. DI MEV

$12.00 \mathrm{MEV}$

13. $O A$ MEV

$14.00 \mathrm{MEV}$

15,00 HEV

16.01 MEV

17. 90 MEV

$18.0 A$ MEV

$19,0 B$ MEV

2月. $A$ ME MEV

$21.0 \triangle \mathrm{MEV}$

22. DO MEV

23.00 MEV

24. 00 MEV

$25.00 \mathrm{MEV}$

$26.00 \mathrm{MEV}$

27. 13 MEV

2B. DO MEV

29. DQ MEV

30, OO MEV

$32, \triangle D M E V$

34. OO MEV

36. $\triangle Q$ MEV

$38.00 \mathrm{MEV}$

$40,00 \mathrm{MEV}$

42.DO MEV

44.DO MEV

46.DO MEV

48. DU MEV

50. DO MEV

gAVE, E(MEV)

\section{PROTONS}

5.71E- 11

$7.37 E-B 1$

$6.98 E-01$

$6.09 E-11$

$7.95 \mathrm{E}=\mathrm{d} 1$

$6.89 E=B 1$

8. 47E-61

$0.43 E=61$

B. 25F-01

$8.64 E-1$

8. $16 E-01$

Q.5 IE-1

B. $19 \mathrm{~F}-\mathrm{kI}$

B. $2 B E-W 1$

B. UP.E

8.5山E- औI

$8.29[-2]$

7. $54 \mathrm{E}-\mathrm{B} 1$

B. $1 R E-U 1$

7. $\triangle 2 E-W 1$

$0.95 E-11$

b. $72 E-B 1$

$0.12 E-01$

$4.95 E-01$

$5.30 E-B 1$

5. $07 E-1$

$5.06 E-01$

3. $52 E=01$

3. UUE-BI

$2,37 E-\cap 1$

2. $15 E-B 1$

2. 36E-UI

$1.87 \mathrm{E}-\mathrm{B} 1$

1. $52 E-11$

B.78E-U2.

$7, U L E=\square 2$

2. $82 E-\eta 2$

2, $11 E-B 2$

1.59E- 132

8.13E- 13

1. $56 E-62$

$1.32 E-132$

$B$.

b.

0.

12.73

\section{DEUTEROAS}

?. $28 F-04$

3. UAE- $A 4$

$5.37 E-114$

1. $16 \mathrm{E}=11 \mathrm{H}$

2.69F.m.

$1.36 E-194$

$2.43 E=14$

1. $18 E-x_{4}$

?. $475-m 4$

1. $11 E-B 4$

1. $42 F-14$

$8.73 E=6.5$

1. $56 E=b^{2} 4$

4. $94 E-155$

1. $15 \mathrm{~F} .114$

2. $16 E-1.15$

3. $58 \mathrm{E}=\mathrm{M}$

B. $R 2 E=00$

2. $17 E=V 5$

1. $D_{6} E-170$

2. 39F-17O

औ.

$n$

.

a.

a.

a.

a.

w)

n

$a$

a.

is.

$a$

0.

$P$

a

a.

$a$

$a$

$a$

a.

a.

a.

b.

$3.4 !$
TRITUIS

$4.45 \mathrm{t}-0.5$

4. 7 Ut $-b 5$

$3,5 B E-65$

1. $79 \mathrm{t}=\$ 15$

$4.44 t=05$

$2.91 E-0.5$

J. DAE -05

3. 60E-6.5

D. EIE-VIS

2. $24 E-15$

2.91E- 15

1. $39 E-15$

2. $96 E-65$

1. SAE- H.

3. $46 E-0 h$

4. 31AE-W6

7. DQE- 26

2. $\triangle B E-10$

$4.17 E=66$

i.

b.

$r$

it.

b.

11.

in

in.

$\ddot{v}$.

i).

i)

b.

b.

u.

b.

a.

a.

4.

0

is.

is.

B.

D.

4.

i)

U.

4.83
ALPHAS

2. $2 B E-B_{2}$

$1,56 E=2$

$2,16 E=: 2$

$2,11 E=12$

$1.92 E-1.2$

$1 ., 17 E=42$

C. $2 H E-i j 2$

9. BuIE-(i) 3

$1.74 E-1)$

$9.71 E-13$

$1.22 E-12$

6. 9 U. E- 143

$1,23 F=12$

3. $44 \mathrm{E}=1 \mathrm{t}$

B. ज2E $=x^{13}$

$4.19 E=13$

$1.28 E-13$

2. VUE -63

$3.56 E-43$

7. BAE $=14$

1. $55 \mathrm{f}-13$

$3.14 E-14$

$7.19 E-B 14$

4. $8 C E=115$

$1.21 E-134$

b.

H.

(1)

P.

a.

$\theta$

4.

a.

0

a.

$b$

$\theta$

b.

$a$

0.

0.

$\theta$.

D.

bs

0

5.22 
1SLONING DOWN SPECTRUM AT 6, TI CM. FOR SHONKA A-1SK PLASTIC 50 MEV D+ ON BE NEUTRON SPECTRLIM

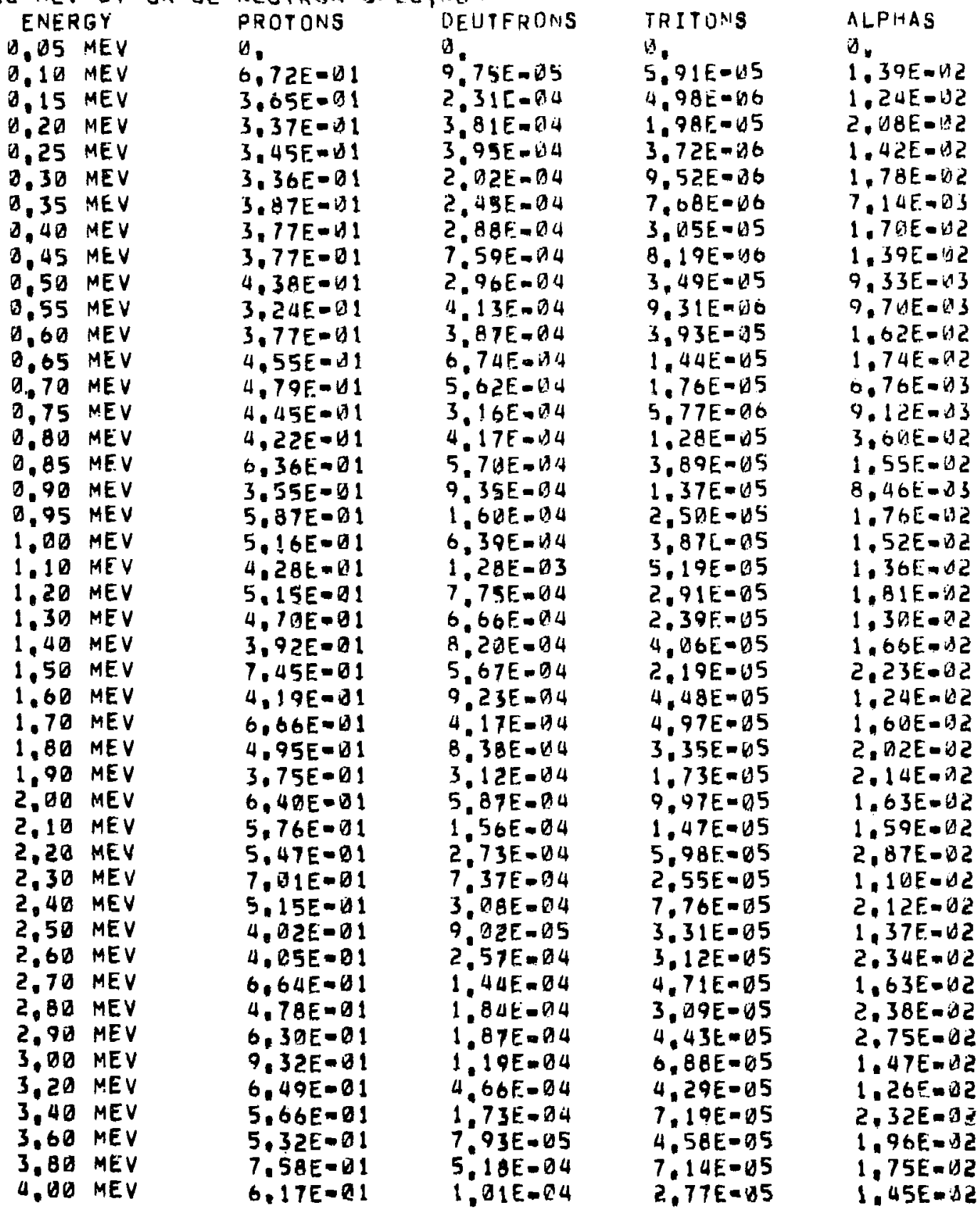


1SLOWING DOWN SEECTRUH AT 6. MA CM. FUR SHONKA A-15O PLASTIC 50 MEV O+ ON HE INEUTRON SPECYRUM

\section{ENERGY}

4. 2 i] $M E V$

4. 10 MEV

4.69 MEV

4.8ด MEV

5.013 MEV

$5.56 \mathrm{MEV}$

$6,00 M E V$

$6.50 \mathrm{MEV}$

7.100 MEV

7.50 MEV

8. $D B$ MEV

8,5D MEV

9. $\triangle O$ MEV

$9.50 \mathrm{MEV}$

10.0 MEV

11.00 MEV

12.0D MEV

13. HD MEV

14. W MEV

15. DO MEV

$10.50 \mathrm{MEV}$

17. 月ด MEV

$18.0 \mathrm{MEV}$

19.0 MEV

$23 . D O$ MEV

$21, D D M E V$

22.00 MEV

$23.00 \mathrm{MEV}$

$24.00 \mathrm{MEV}$

25.0O MEV

26.0D MEV

$27.00 \mathrm{MEV}$

$28.00 \mathrm{MEV}$

$29.00 \mathrm{MEV}$

30.00 MEV

$32.00 \mathrm{MEV}$

$34.00 \mathrm{MEV}$

$36.00 \mathrm{MEV}$

33.00 MEV

48,00 MEV

42,00 MEV

4U,OD MEV

¿S. OO MEV

49.00 MEV

$5 \partial, \partial 0$ MEV

G)

\begin{abstract}
PROTONS
\end{abstract}
G. IOE-DI

$6,77 E-01$

$7.42 E-111$

$5.96 E-01$

8. $12 E-U 1$

$0.62 E-01$

$7.65 \mathrm{E}=01$

$7.76 E-D I$

$7.19 E=U 1$

7. 7AE $=1$

$7.29 E-B I$

$7.78 E-01$

$7.39 E-01$

$7.75 E-O 1$

$7.30 E-01$

$7.66 E-d 1$

$7.68 E=01$

$6.49 E-31$

$7.72 F=\mathrm{JI}$

6.SAE-DI

$6.45 E-31$

$6.2 A F-11$

5. $22 E-101$

4. 5OE-U1

4. $33 E-31$

4. $13 E-31$

$3.53 E-01$

2. $865-B 1$

3. $11 E-D 1$

$2.32 E-\square 1$

2. $29 E-B !$

2. $B$ DE $=1$

1. $\triangle 3 E-D 1$

1. $17 E-11$

1. $\triangle 7 E-\triangle 1$

7. 32E- 02

$5.12 E-92$

4. $26 E-02$

1. B1E-OC

4. $93 E-03$

5. $29 E-03$

$1.31 E-B 2$

$\theta$

0.

$a$.

12,66
CEUTERONS

4. 2RE- $\triangle 4$

$1.89 E-114$

$4.53 E-04$

$1.13 E-64$

5. 4BE-OU

$3.65 E-144$

7. B $9 E-U 4$

1. $45 E-104$

3. DJE-D 4

1. $22 E-B L$

$1.51 E-B 4$

8. $91 E-15$

1. $59 E-1 D 4$

$3.65 E-05$

$8.77 E-65$

$3.41 E-05$

$5.99 E-115$

$6.79 E-136$

$1.53 E$ - HS

ด.

a.

a.

a.

$a$

0

(1)

a.

a.

2

0

0

U.

in

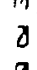

0

0.

9

0

0

0

4.07
TRITONS

$4.96 E=35$

$3.65 E-65$

$4.99 E-105$

$2 . \triangle 1 E=05$

$6.97 E-65$

$3,56 E-115$

$6.41 E-45$

$3.01 E=95$

6. $11 E-55$

$4.30 E-05$

$3.66 F=05$

2. 54E-125

4. $58 E-05$

2. $38 E-15$

$5,54 E-115$

$7.69 E=06$

$1.58 E-05$

$5,92 E=106$

1. 5 Q -45

a.

1.

i.

$\theta$

a.

v.

$\theta$

$b$

a.

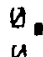

b.

0.

a.

$\theta$

i.

$n$

a.

b.

(A.

$\theta$

a.

0.

b.

b.

a.

5,89
ALPHAS

$1.69 E-02$

$1,28 E-D 2$

$1,62 E-62$

$1,55 E-102$

$1.45 E-92$

$8,68 E=103$

$1.75 E-\Delta 2$

$7.79 E-03$

$1.43 E-12$

$7.5 D E-\triangle 3$

1. $\triangle D E-O 2$

$5,06 E-13$

9. $\triangle 3 E=63$

$2.45 E-13$

$5.72 E-03$

$3.49 E=153$

$6.42 E-13$

$1.22 E-\square 3$

$1,82 E-d 3$

$7,16 E=01$

$1.55 E-03$

2. 3UE- П4

$5,54 E=04$

$1.59 E-104$

$3.18 E=24$

0

0.

a.

n.

D.

0

$b$

a.

0

0

0.

d.

$b$

D.

D.

$a$

$\theta$

0

0

5,08 
1SLOWING DOWN SPECTRUM AT 1 H. O CM. FOR SHONKA A-150 PLASTIC 50 MEV D+ ON BE NEUTRON SPECTRUIM

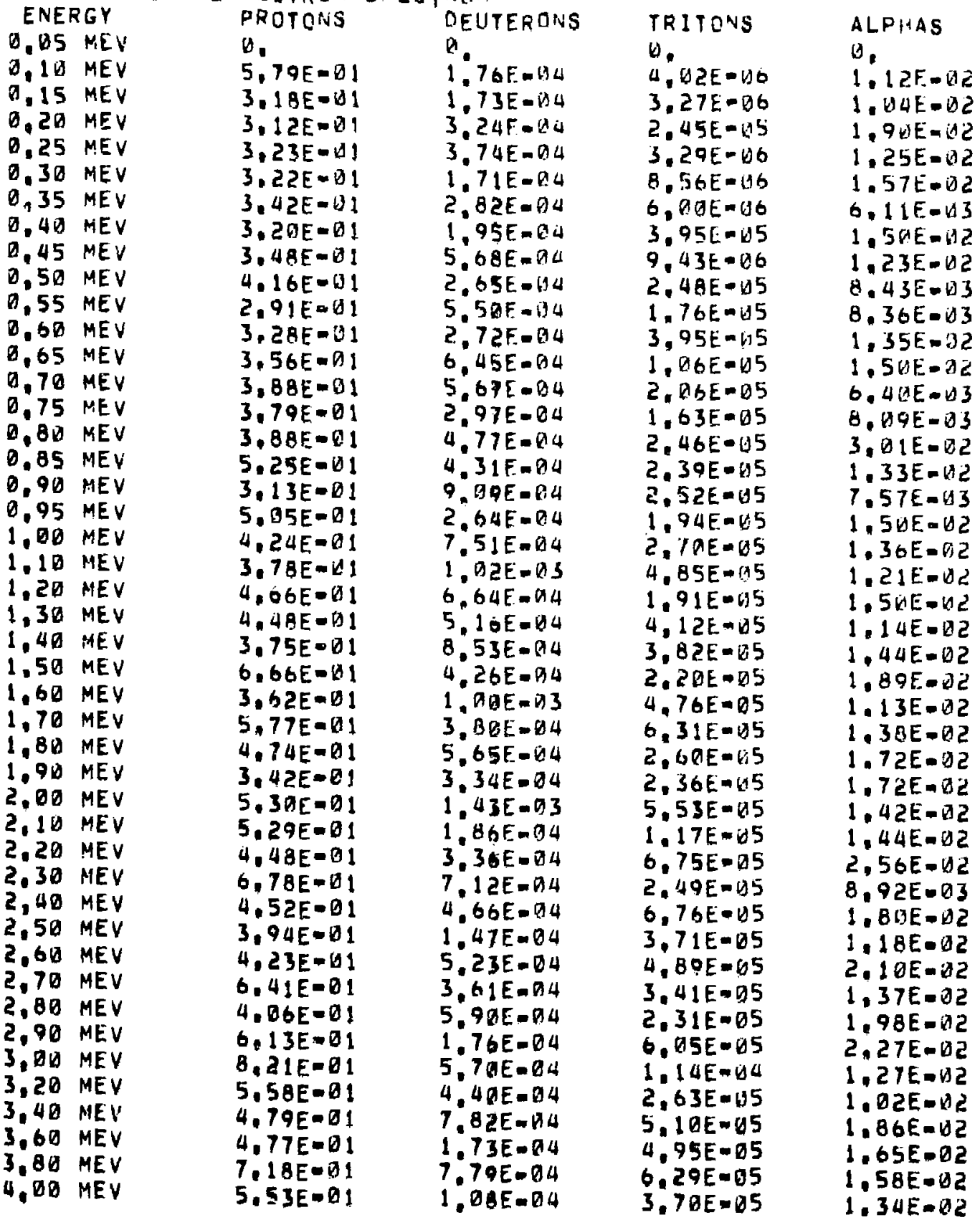


1SLOWING DOWN SPECTRUM AT 10, CM. FOR SHONKA A-15: PLASTIC 50 MEV D+ ON BE NEUTRON SPECTRUM

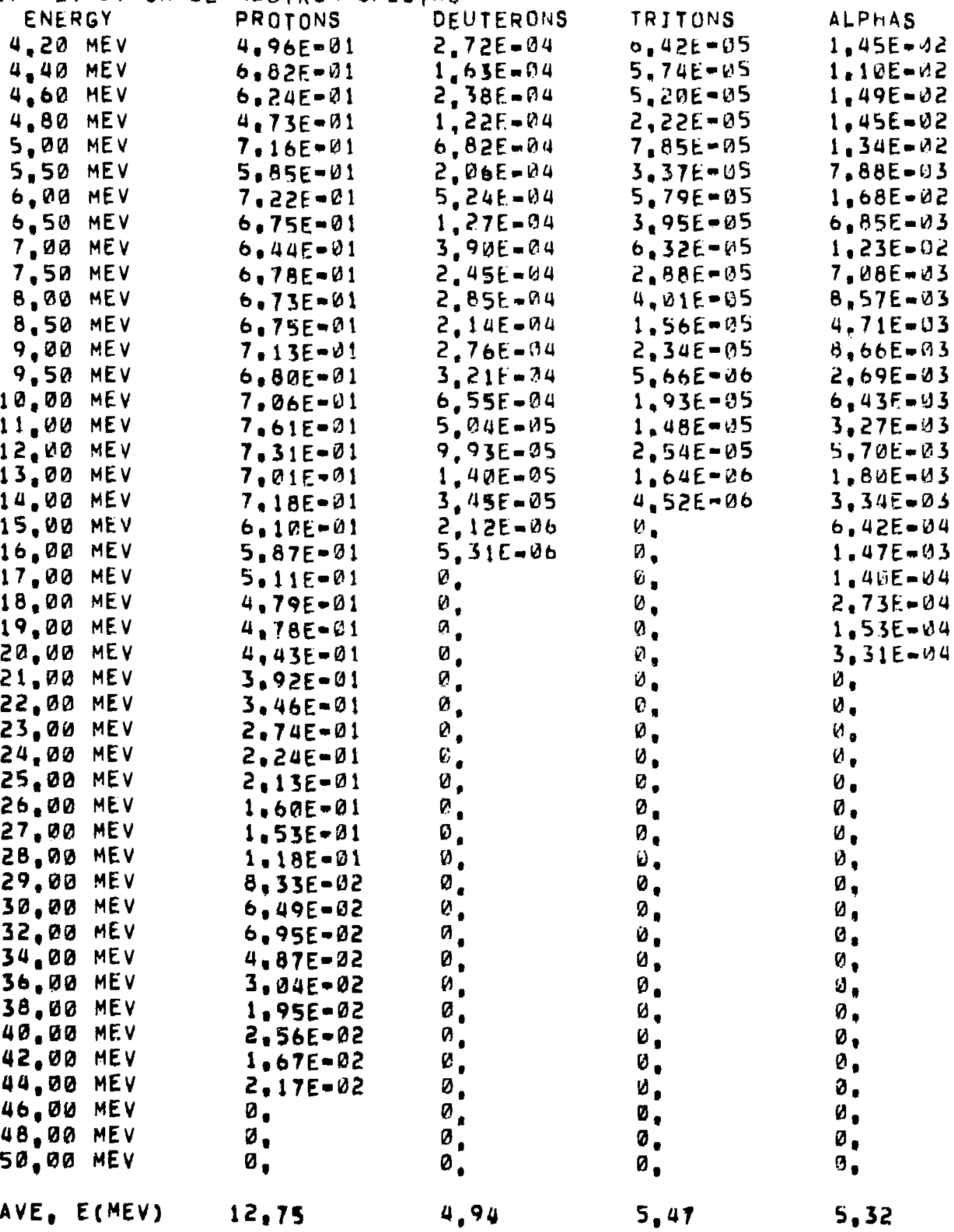


ISLOWING DOWN SPECTRUM AT 14, D CM, FOR SHONKA A-15O PLASTIC 50 MEV D+ ON BE NEUTRON SPECTRUM

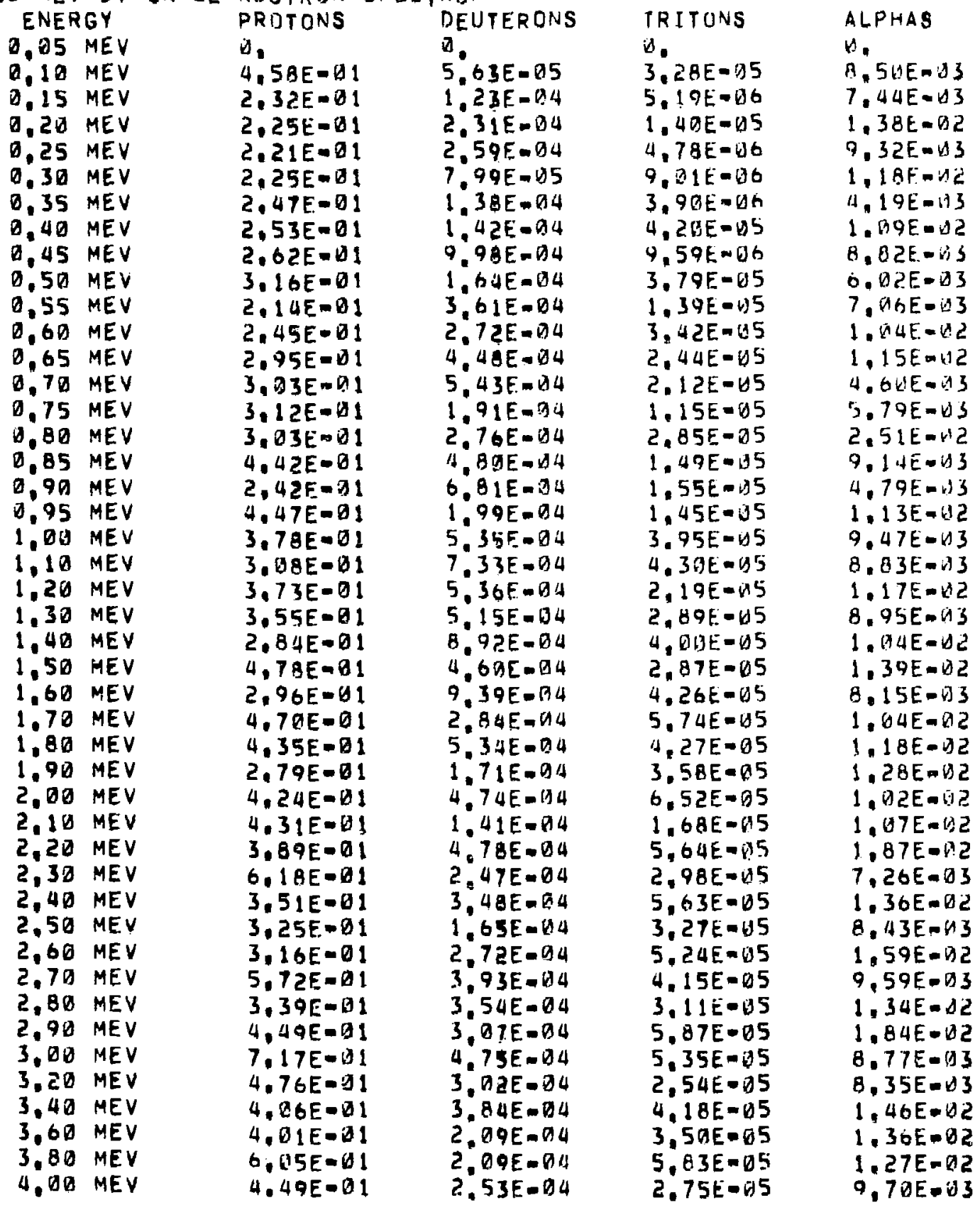


1SLOWING DOWN SPECTRUM AT 14.3 CM. FOR SHONKA A-15R PLASTIC 50 MEV D+ ON BE NEUTRON SPECTRUM

\section{ENERGY}

4. 20 MEV

4.40 MEV

4. 60 MEV

4.8D MEV

5. DO MEV

5.50 MEV

$6.00 \mathrm{MEV}$

$6.50 \mathrm{MEV}$

7. $U D$ MEV

$7.59 \mathrm{MEV}$

B. 80 MEV

8.50 MEV

9.90 MEV

$9,50 \mathrm{MEV}$

$10.00 \mathrm{MEV}$

$11.00 \mathrm{MEV}$

12.00 MEV

13. DO MEV

14.00 MEV

15.06 MEV

$16.00 \mathrm{MEV}$

$17.00 \mathrm{MEV}$

$18,00 \mathrm{MEV}$

19.0 MEV

20,00 MEV

21. OO MEV

22.00 MEV

23,00 MEV

$24.00 \mathrm{MEV}$

25. OQ MEV

26. DV MEY

27.00 MEV

$28.00 \mathrm{MEV}$

29.00 MEV

$3 \%, 00$ MEV

32,00 MEV

$34.00 \mathrm{MEV}$

$36.00 \mathrm{MEV}$

$30.00 \mathrm{MEV}$

$40.00 \mathrm{MEV}$

42.DD MEY

44,00 MEV

46.00 MEV

48.00 MEV

$50.00 \mathrm{MEV}$

QAYE. E.(MEV)

\section{PHOTONS}

4. 36E-01

5. $44 E-91$

$5.54 E-U 1$

$4.17 E-01$

5.90E-D1

$4.95 E-91$

$6.24 E-101$

$5,80 E-91$

$5.61 E-B 1$

$5.77 \mathrm{~F}-\mathrm{UI}$

$5,97 E-U 1$

$5,77 E-01$

$6, \triangle 3 E-B 1$

$5,94 E-101$

$5.78 E-B 1$

6. $75 E=01$

O. $\triangle O E-01$

5. B1E-DI

$0,03 E=01$

$5,03 E-01$

$5.34 E-B 1$

4. $47 E-01$

4. 2.3E- П1

$3.72 E-01$

3.9ดE-И1

$3.65 E-81$

2. $98 E-11$

$2.41 E=\square 1$

2. $03 E-01$

1.79E-G1

1. $49 E=U 1$

1. $42 E=61$

$9,10 E=02$

$6.91 E-12$

$7.96 E-142$

$6.38 \mathrm{E}-\mathrm{QD}$

$5.335-42$

3. $46 E-112$

2. 51E-02

2, 06E-12

1. $29 E-02$

$6.55 E-03$

$1.53 E-23$

$4,59 E-D 3$

b.

12.97

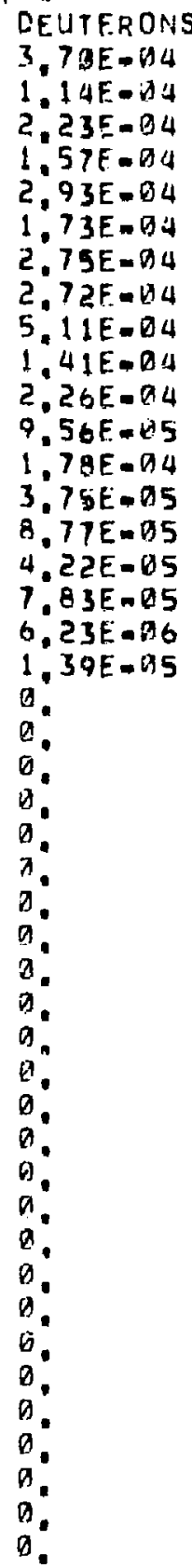

4.38
TRITONS

4. 4DE-65

4. $14 E-05$

3. $02 E=135$

2. $40 E-15$

$0.67 E-155$

2. $B O E-\triangle 5$

5. DOEE-DS

$3,03-05$

$5,66.05$

2. D2E- D 5

3. $45 E-05$

$1.33 E-05$

2. $72 E-05$

$8,65 E-06$

2. DQE-D 5

$5.67 E-46$

$1,23 E-05$

2. 9 月E -166

$7.46 E=06$

0.

1

i.

(1).

0.

0.

0.

v.

v.

i.

a.

$\theta$.

b.

$\theta$.

$\theta$.

$\theta$.

$b$.

$\theta$

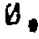

0.

$\Delta$.

0.

D.

0.

V.

5.27
ALPHAS

1.22E $=02$

7. BSE-D 3

1. $\triangle Q E-D 2$

$1.01 E-42$

$1.03 E=02$

$5.77 \mathrm{E}-\mathrm{B}) \mathrm{S}$

$1.25 E-62$

5. $98 E-103$

$8.93 E m b 3$

$5.32 E 003$

$7,61 E-113$

3. ? 2E- DI

$6.30 E-\square 3$

2. 49E-OB

$5,39 E-13$

$1,88 E-103$

$3.48 E=03$

7.60E-DA

$1,36 E=03$

$3,36 E=04$

$7,3 G E D O A$

$8.51 E-05$

2. $02 E-04$

1. $05 E-06$

$2,29 E-136$

b.

0

0

$\theta$.

$\theta$.

0

0.

B.

b.

$\theta$.

$D$.

$\theta$.

$D$.

(1)

0.

$b$.

b.

$\theta$

D.

0.

5.0? 
1SLOWING DOWN SPECTRUH AT O. OQ CM. FOK ICRU MUSCLE TISSUE 50 MEV D+ ON BE NEUTRON SPECTRUM

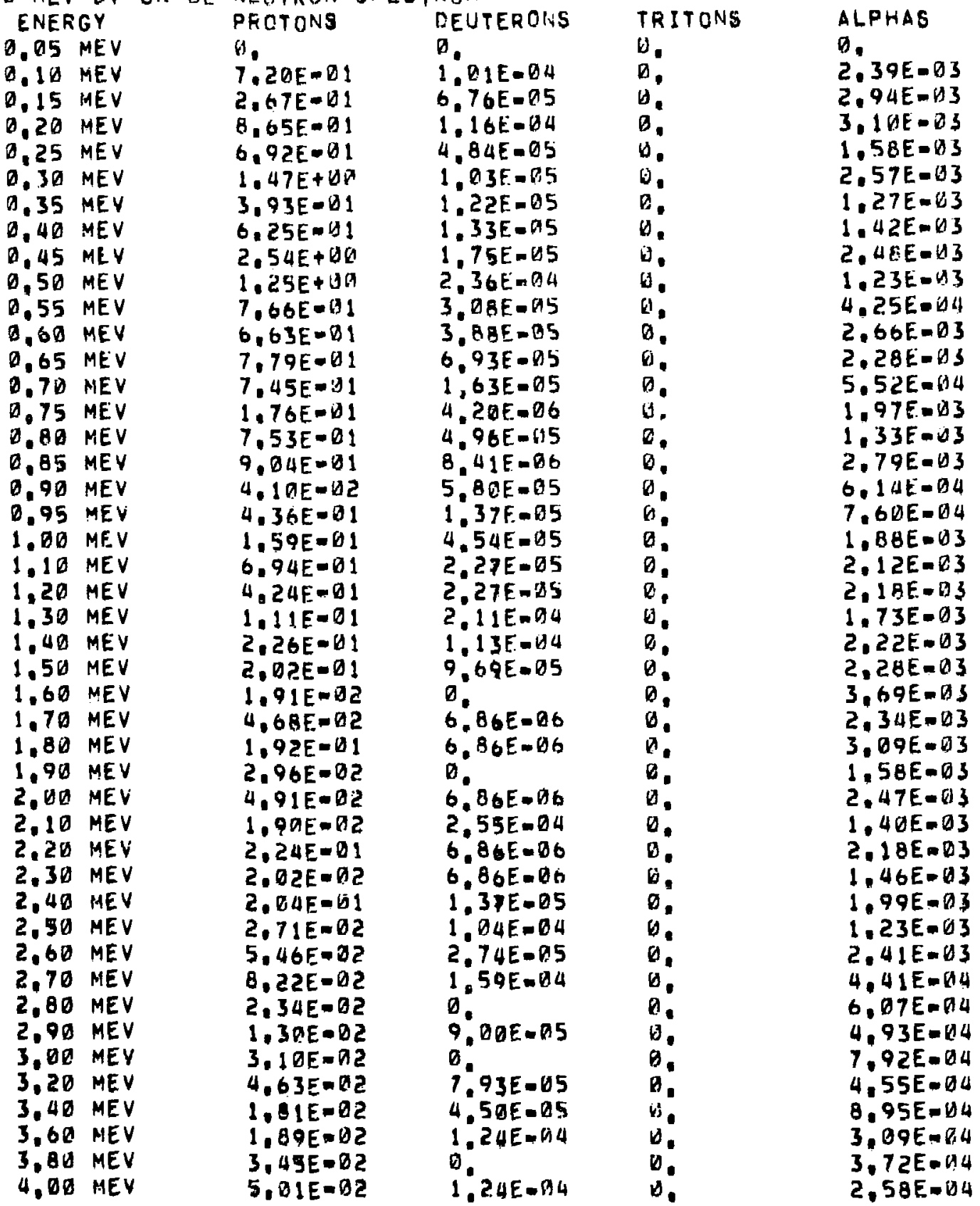


1SLOWING DOWN SPECTRUM AT D, AD CM, FOR ICRU MUSCLE TISSUE 5D MEV D+ ON BE NEUTRON SPECTRUM

\section{ENERGY}

4.20 MEV

4.40 MEV

$4,60 \mathrm{MEV}$

$4,80 \mathrm{MEV}$

5,00 MEV

$5,50 \mathrm{MEV}$

$6,00 \mathrm{MEV}$

$6,50 \mathrm{MEV}$

7. $\triangle 0$ MEV

T.50 MEV

B. DO MEV

$8.50 \mathrm{MEV}$

$9.00 \mathrm{MEV}$

$9.50 \mathrm{MEV}$

10,00 MEV

$11.0 Q \mathrm{MEV}$

$12,00 \mathrm{MEV}$

13.0O MEV

14.00 MEV

15.00 MEV

$16,00 \mathrm{MEV}$

$17.00 \mathrm{MEV}$

$18.00 \mathrm{MEV}$

$19.00 \mathrm{MEV}$

$20.00 \mathrm{MEV}$

21, DD MEV

22.00 MEV

23,DD MEV

24.00 MEV

25, OD MEV

26.DO MEV

27.00 MEV

28, 10 MEV

29.DO MEV

30,00 MEV

32,00 MEV

$34.00 \mathrm{MEV}$

36. DO MEV

$38,00 \mathrm{MEV}$

$40,00 \mathrm{MEV}$

42. DE MEV

$44,00 \mathrm{MEV}$

46.00 MEV

48,00 MEV

$50.00 \mathrm{MEV}$

GAVE, E(MEV)

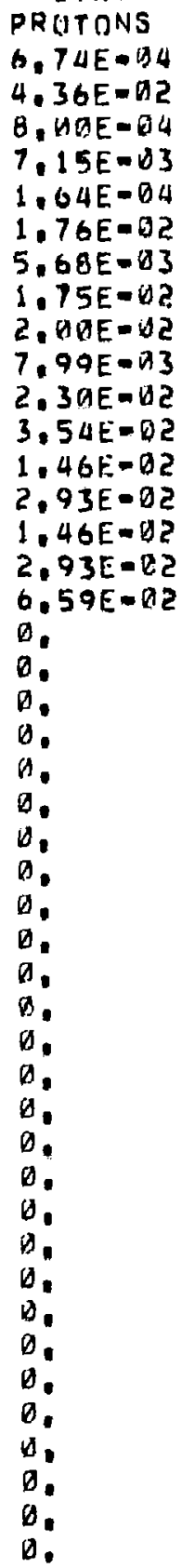

2.23
DEUTF.RONS

$4.5 Q E-V 5$

1. $24 F=(1) 4$

$1.24 E-04$

$4.50 E=05$

$1.24 E-14$

$1.35 E-A 14$

$2.75 E-\theta 4$

1. 27E-OS

2. $35 E-0 / 4$

Q.

D.

i:

0

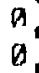

a

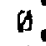

9.

0.

0

a.

9

0

0

0

$\theta$

a.

0.

0

0

a.

0

$a$

0.

.

$\theta$

$\theta$

0.

0.

0

0

4.63
TRITONS

ALPHAS

B.

$\theta$

0

$0:$

a.

a.

b.

$\Delta$

0.

i.

$\theta$

$\theta$.

B.

0.

0.

n.

$\theta$.

D.

:

a.

0.

0.

.

i.

D.

$D$.

0.

$\theta$

b.

$\theta$

9.

0.

0.

0.

$\theta$

0.

8.

b.

0.

0
$2,72 E-94$

$2.78 E-D 4$

$3.62 E=144$

1.76E-DIS

$1.59 E-14$

$1.15 E-74$

$2.26 E-04$

$1.13 E=014$

$2.14 E=194$

$5,55 E-35$

$1,27 E-34$

$1,05 E-05$

$2,15 E-\triangle 5$

$2.43 E=00$

$7,32 \mathrm{~F}=110$

$1.37 E-06$

$3,93 E=00$

$\theta$.

$\theta$.

a.

0.

0.

0.

a,

$\theta$.

0.

D.

0.

n.

$\theta$.

0

a.

0.

$\theta$.

$\theta_{0}$

0.

$B$.

$\theta$.

$\theta$.

$\theta$

$\theta$.

0.

0.

$\theta$.

2.01 
1SLOWING DOWN SPECTRUM AT M. O5 CM. FRR ICRU MUSCLE TISSLE 59 MEV O+ ON BE NEUTRON SPECTRUM

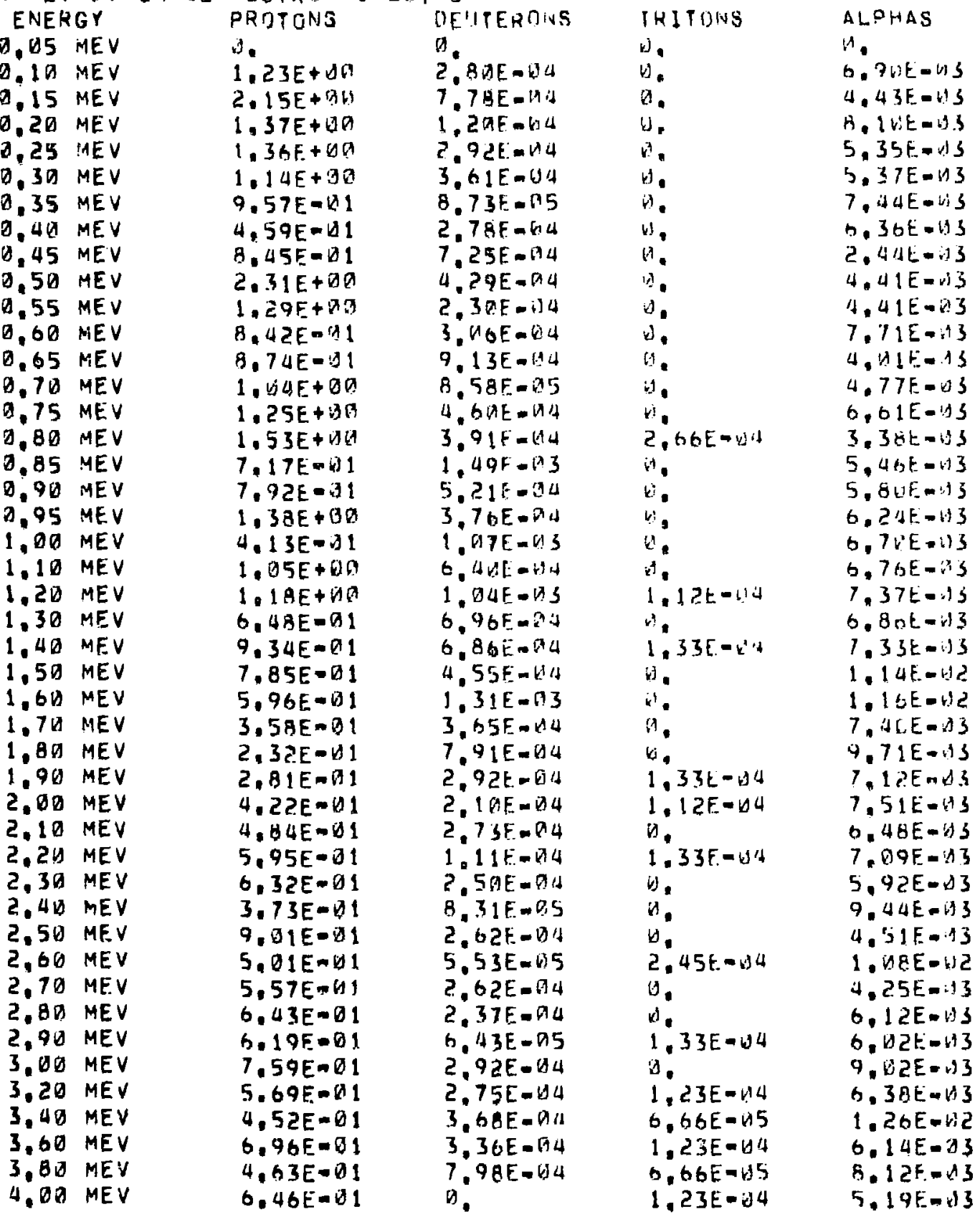




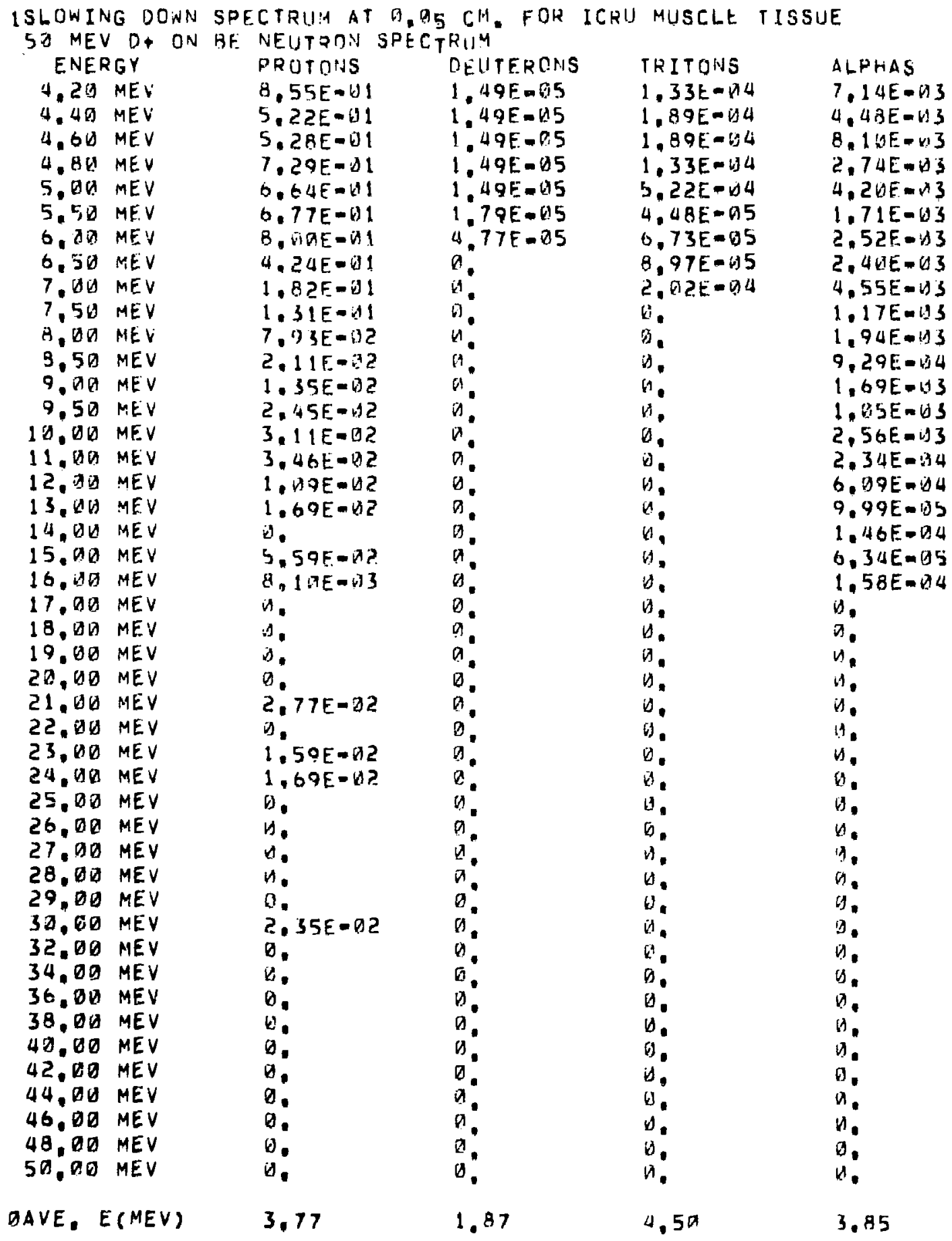


1SLOWING DOWN SPECTRUM AT O. 1 O CH. FOR ICRU MUSCLE TISSUE 50 MEV D+ ON BE NEUTRON SPECTRUM

\section{ENERGY}

D. US MEV

0.10 MEV

0.15 MEV

$8.20 \mathrm{MEV}$

0.25 MEV

$3.30 \mathrm{MEV}$

0.35 MEV

0.40 MEV

$0.45 \mathrm{MEY}$

ग.50 MEV

$0.55 \mathrm{MEV}$

$0.60 \mathrm{MEV}$

$0.65 \mathrm{MEV}$

$0.76 \mathrm{MEV}$

$0.75 \mathrm{MEV}$

D. BU MEV

$\triangle . B 5$ MEV

0.90 MEV

0.75 MEV

I. IS MEV

1.10 MEV

$1.20 \mathrm{MEV}$

$1.30 \mathrm{MEV}$

$1.40 \mathrm{MEV}$

1.52 MEV

1.60 MEV

1.76 MEV

$1.80 \mathrm{MEV}$

$1.90 \mathrm{MEV}$

2,OQ MEV

2.18 MEV

2.20 MEV

$2.30 \mathrm{MEV}$

2.4D MEV

2.50 MEV

$2.60 \mathrm{MEV}$

2,70 MEV

2.80 MEV

2,90 MEV

3. OE MEV

3.20 MEV

3.4 MEV

3.60 MEV

$3.80 \mathrm{MEV}$

$4,00 \mathrm{MEV}$

\section{PROTONS}

H.

1. O2F+ 19

$3,35 E=01$

$7.18 E-61$

$9.19 E-11$

$6,20 E-01$

$5,49 E-(1)$

8. 9RE-DI

$1.53 E+a A$

$2,03 E+14$

$1.31 E+B D$

1 .6) $E+13$

$9.52 E-D 1$

$9.79 E-D 1$

$1.81 E+39$

6. $2 \geq E=01$

5. HAE - D I

8.91E-OI

1.2UE+JG

$9.66 F-D !$

$1.59 E+00$

8. $\angle B E-B 1$

1. $21 E+\partial Q$

$1,17 E+1)$

$7.33 E-D 1$

$6,55 E-\lambda 1$

5. 1 BE -1

$6,62 E=1$

8. उ9E-D1

$3.33 E=01$

$4.31 E-\theta 1$

6.2 GE. BI

$1.16 E+\theta 0$

$7.92 E-41$

$3.71 E-1$

7. $42 E-B 1$

$7.23 E-B 1$

8. $3 B E=01$

$6.06 E-B 1$

$9.79 E-1$

$7.38 E-D 1$

$6.35 E-B 1$

4. 90E-DI

$4.14 E-B !$

7. 9 OE $=01$
DEUTFRDNS

a.

4. RTE-OS

2. $02 F-114$

$5.65 E-.95$

1. $12 E-B A$

8. $18[-615$

6. $\triangle 9 E-1.5$

1. $535 .-14$

$4.325-34$

2. $75 E-011$

$7.43 E=145$

1.19E-0.4

4. 145.04

$9.60 E-195$

$1.22 t-H 4$

4. AUE-75

5.53E- 14

1.18E-.14

$6.37 E-55$

3. 9QE-BA

$2.70 E-D 4$

$3.33 E-D 4$

2. $5 B E=4$

$4.39 F-D A$

3. $196 E-44$

6. B8E-AI

1. $41 E=04$

$1.46 E=014$

8. $14 E-05$

$1.99 E-0.14$

5. DIE-VIS

$5.64 E-05$

$6.55 E-0.5$

$1.26 E-94$

$6.86 E=P 5$

4. 9QE-D 5

$8.72 E-15$

$1.21 E-014$

$4.65 E-05$

4. $94 F-25$

$5.13 E-0.5$

$1.39 E-D 4$

1. $B 65 .-A 5$

2. 29E- 35

$1.35 E-B b$
IRITONS

V.

a.

is:

n.

i.

$n$.

4.

v.

v.

v.

4 ,

n.

a.

B.

B.

$\nabla$

$\pi$

a.

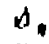

$\theta$

.

$\theta$

a.

6.

$\theta$

vi.

b.

i.

b.

9.

0.

0.

$b$.

b.

b.

G.

b.

U.

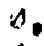

s.

v.

$v$.

is.

V. alprias

B.

$5.47 E-13$

$5,39 E=03$

3. $4 S E-113$

b. $79 E=0 S$

$2.91 E-W 3$

7. $2.4 E-13$

$4.69 E-D 3$

$5.13 \mathrm{E}-13$

$3.57 \mathrm{Em} B \mathrm{~S}$

$4.32 E-U 3$

B. $B Q D-193$

3. $40 E-D S$

$3.44 E-\triangle 3$

$5,91 E-43$

$4.25 E-D 3$

$7.65 E-113$

$9.78 E-133$

$1.14 E-$ in

$9.53 E$-US

$3.44 E=63$

$4.42 E-143$

$2,92 E-D 3$

4, iT $2 E-B 3$

$6.11 E-103$

$5,19 E=\square 3$

5. DUE-B3

$4.77 E-D 3$

6. $21 E-173$

$5.24 E-\triangle 5$

$3.83 E-13$

$4.94 E-13$

$4.92 E-133$

$3.85 E-13$

1. 2UE-W2

$3,84 E=03$

$5,70 E=03$

$5.7 b E=113$

$4,914-V 3$

$7,84 E-63$

$4.21 E-D 3$

Q. $U U E=-33$

7. $39 E-113$

$7.13 E-A 3$

$9.93 E-\Delta) 3$ 
ISLOWING DOWN SPECTRUA AT $\pi, 19$ CH. FOR ICRU MIJSCLE TISSUE 50 MEV D\& ON BE HEUTRON SPECTPUM

ENEKGY

4. 2 O MEV

4. $\triangle \square$ MEV

4.60 MEV

4. 80 MEV

5. QU MEV

5.50 MEV

$6, D O M E V$

$6.50 \mathrm{MEV}$

7. DO MEV

$7.50 \mathrm{MEV}$

B. OO MEV

8.50 MEV

$9.00 \mathrm{MEV}$

$9.50 \mathrm{MEV}$

$10,00 \mathrm{MEV}$

11.00 MEV

12. OD MEV

13. $90 \mathrm{MEV}$

14.00 MEV

15. 50 MEV

16, DO MEV

17. $\triangle Q$ MEV

18.Dด MEV

$19.00 \mathrm{MEV}$

20.0 MEY

21.00 MEV

22, DO MEV

23,00 MEV

24.00 MEV

25. DD MEV

26.00 MEV

27. DO MEV

28.00 MEV

29, DO MEV

$30.00 \mathrm{MEV}$

$32.00 \mathrm{MEV}$

$34.00 \mathrm{MEV}$

$36.00 \mathrm{MEV}$

38,00 MEV

40.DB MEV

42,90 MEV

$44.00 \mathrm{MEV}$

46, DO MEV

$48,00 \mathrm{MEV}$

50. DO MEV

GAVE, E(MEV)

\section{PROTONS}

$4.71 E-D 1$

$5.10 E-B 1$

$4.91 E=B 1$

B. $26 E-11$

$3.93 E-\Delta 1$

5.30E-Q1

4. $20 E-D 1$

2. $26 E-01$

$2.33 E-61$

1. $99 E-B 1$

$1.22 E-11$

7. B5E- U?

3. $46 E=\sqrt{2}$

$9.21 E-102$

$7.62 E=92$

$6.39 E-02$

$1.935-02$

6. $04 E-02$

5. $46 E-d 2$

$6.72 E-32$

$2.64 E-13$

$3.63 E-02$

$5.29 E=03$

$3,44 E-D ?$

2. $77 \mathrm{E}-02$

1. $23 E-1$ ?

$1.59 E-B Z$

$1.67 E-D 2$

(1)

0.

0.

0.

0.

$4.33 E-02$

0.

0.

b.

$b$.

ด.

0.

0.

8.

0

0.

D.

4.29

\section{DEUITERONS TRITINS}

2. 7DE- :DO

2.7 OE $=0.6$

2. $75 E-1) 6$

2. 7 QE- BO

$9.44 E-1) 6$

क.

b.

a.

ด.

i.

a.

G.

(1)

$\theta$

a.

b.

a.

a.

a.

a.

a.

a

a.

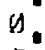

a.

a.

$a$

0

$\theta$

a.

9

0.

0

9

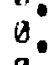

0

6.

1.48 b.

bi.

a.

a.

i.

H.

is.

b.

b.

b.

9.

9.

6.

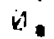

a.

v)

in.

10

(1.

U,

v.

0.

$\Delta$.

A.

is.

$n$

v.

$\theta$

6

v.

a.

b.

b.

$H$.

0

U.

$v$

$\forall$.

U.

b.

$\theta$

H.

b.

b.

4.50
ALOHAS

$9,09 E-13$

2. $45 E-43$

$3.46 E-43$

$3,22 E-13$

2. 2WE-113

$4.51 E-113$

$7.13 E=113$

2. 3 BE. -13

$2.93 t .133$

$1.05 E-13$

2. $81 \mathrm{E}-43$

C. ARE- 113

$3.44 E-13$

3. WटE- 13

5. $42 E-13$

$1.62 E-03$

$2.61 E=43$

$2.97 \mathrm{E}-\mathrm{US}$

7. AUE-U3

$a$

b.

.

b.

H.

0.

(i).

G.

i)

$y$.

$\underline{v}$,

i).

0

1.

U.

B.

H.

b.

U.

$n$.

i)

k.

a.

a

b.

$b$.

0.41 
1SLONING DOWN SPECTRIIN AT M.15 CM, FOF ICRIJ HUSCLE TISSUE 50 MEV D+ ON BE NEUTRON SPECTRUM

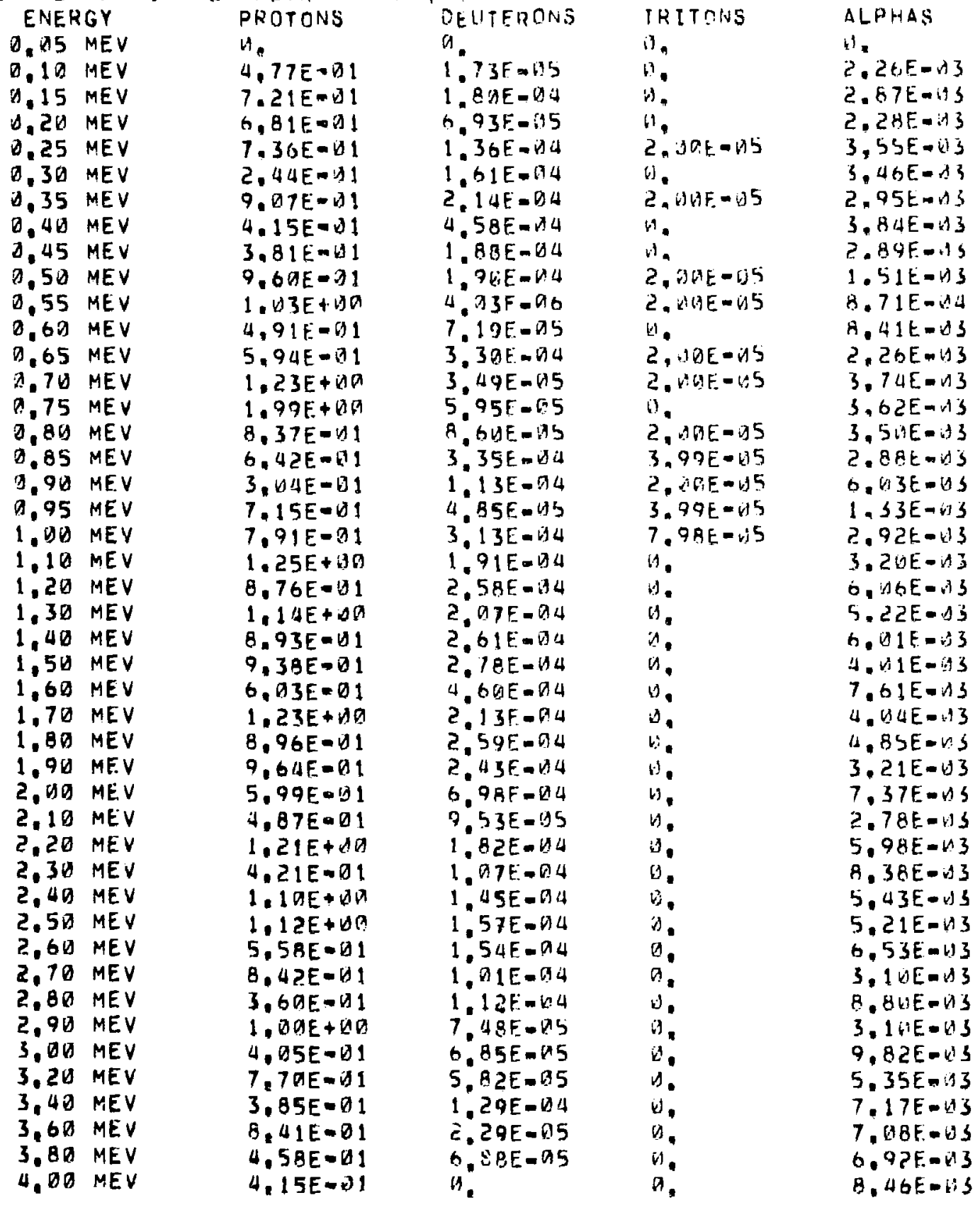




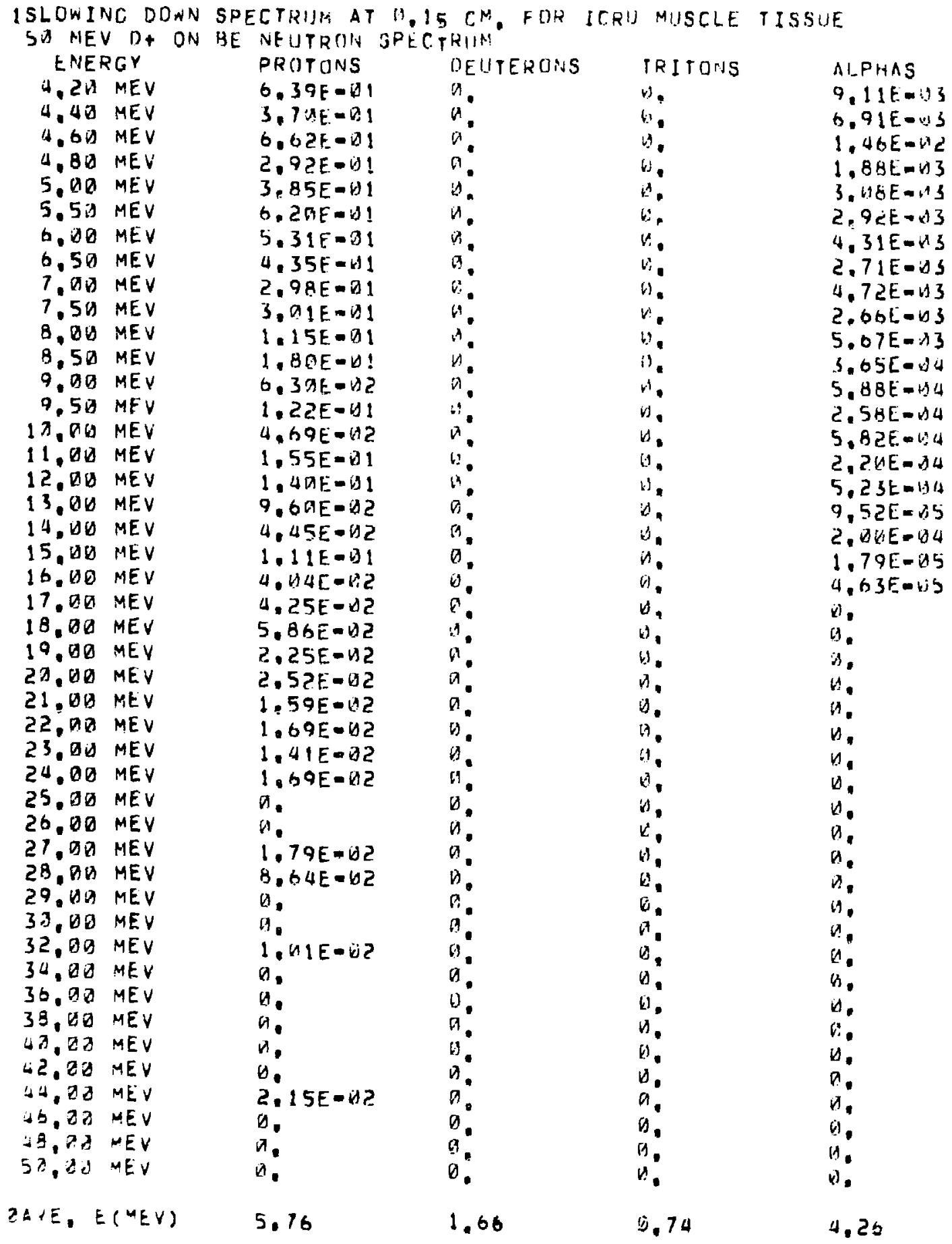


1SLOMING DOWN SPECTRIIM AT R. ZA CM. FOR JCRU MUSCLE TISSUE SO MEY D+ ON BE PIEUTROIN SPEC.TRIJ!"

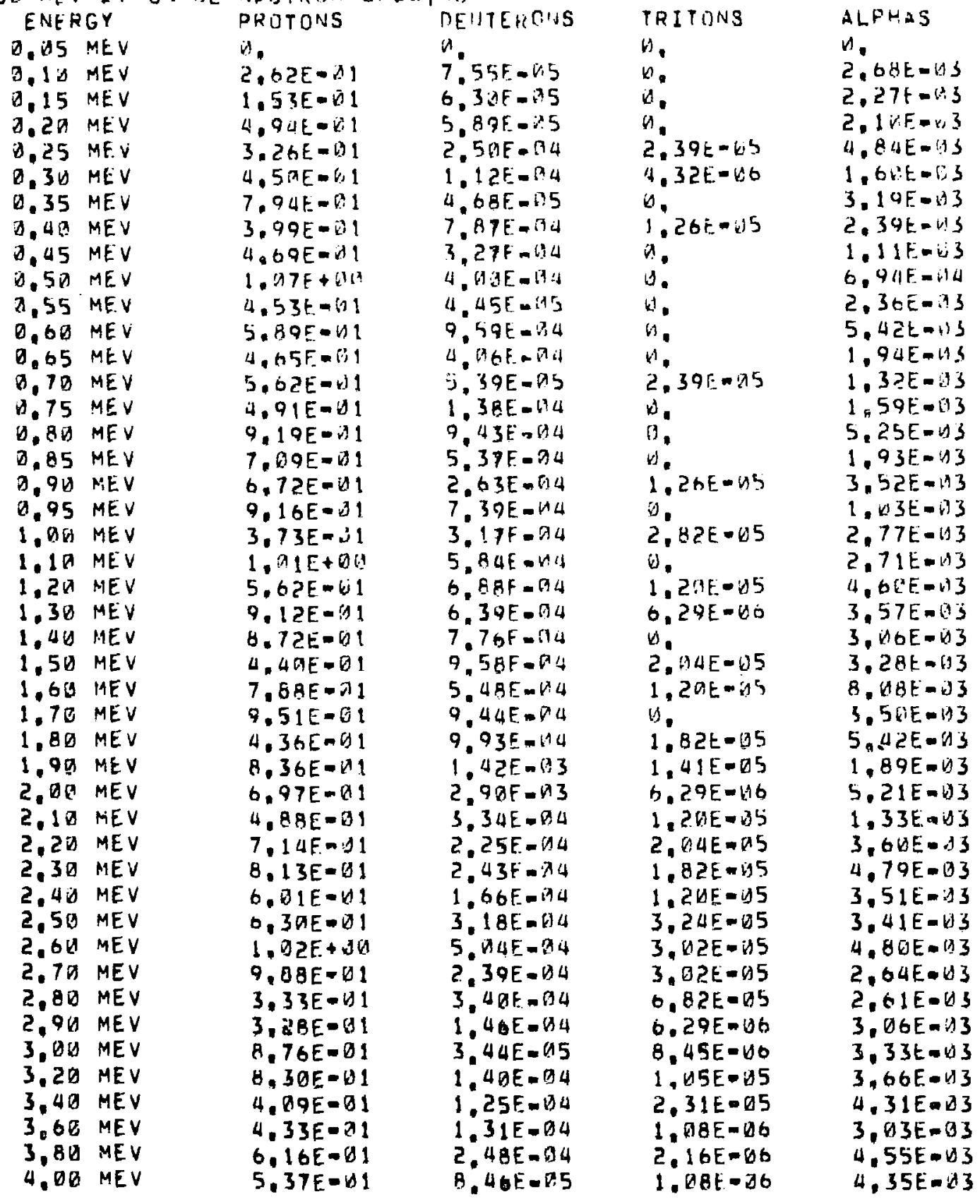


ISLOWING DOWN SPECTRUM AT M.20 CM. FOR ICRIJ MUSCLE TISSUE SO MEV D+ ON BE NFIJTROH SPECTRIJM

\section{ENERGY}

4.20 MEV

4.40 MEV

4.60 MEV

$4.80 \mathrm{MEV}$

5.06 MEV

$5.50 \mathrm{MEV}$

6. OU MEV

6.50 ME.

7. DU MEV

7.5ऽ MEV

8. HO MEV

8.5 3 MEV

9. AU MEV

$9.5 \%$ MEV

12. BA MEV

11. DO MEV

12.00 MEV

13.00 MEV

14. DO MEV

15. DO MEV

16. 16 MEV

17. DO MEV

18. GU MEV

$19.00 \mathrm{MEV}$

$20.00 \mathrm{MEV}$

$21.00 \mathrm{MEV}$

22. VI MEV

$23.00 \mathrm{MEV}$

$24.0 L M E V$

$25.00 \mathrm{MEV}$

26.00 MEV

$27.00 \mathrm{MEV}$

28. DU MEV

29. 10 MEV

$30.00 \mathrm{MEV}$

32,00 MEV

34.00 MEV

$36.00 \mathrm{MEV}$

$38.00 \mathrm{MEV}$

$40.00 \mathrm{MEV}$

42. DO MEV

प $.0 D$ MEV

46, DO MEV

$48 . D 0$ MEV

52. 00 MEV

ASVE, E(MEV)

$$
\text { FROTONS }
$$

$3,93 \mathrm{E}-\Delta 1$

7. $4 D E-01$

5. $38 E-B 1$

$5.3 Y E-D I$

$5.4 \lambda E-U 1$

$6.62 E-11$

$7.24 E=01$

5. $95 t-11$

$0.41 E-n 1$

b. $22 E-111$

$5,34 E-41$

$5.57 F=1$

$7.13 E-U 1$

$5,18 E-d !$

4. $B 3 E-D 1$

7. $. \Delta E-D I$

$6.56 E-01$

5. $13 E-01$

1. $B 6 E-11$

$7.12 E-192$

$6.73 \mathrm{E}-\mathrm{M2}$

$3.74 E=3$ ?

$4.45 E-32$

$5.12 E=12$

$1.69 E=82$

$1.23 E-D ?$ ?

1. $41 E-02$

3. $28 E-42$

औ.

0.

$1.79 E-52$

1. $D 7 E-O 1$

i.

D.

n.

1. ब1E- Q ?

1. $43 \mathrm{E}=32$

0.

$b$.

a.

0.

$2.15 E-142$

a.

b.

0.

7.62
DEUTERCINS

1. $8 D E=94$

8. 2.6E-15

1. $47 \mathrm{E}-1.4$

5.57E-.A5

$1.78 \mathrm{~F}=\mathrm{AL}$

9.8IE-35

1.7 SE $=04$

$7.85 E=1.55$

2. ดด $=14$

a

i

1

a

a

4

a

6

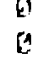

?

$\theta$

$$
a
$$$$
\text { r. }
$$

3

a.

b.

a.

().

3

a.

a.

b.

a.

a.

a.

0

0.

$\theta$

ด.

0

2.44
TRITUNS

$3.24 E-06$

2. $16 E-116$

$7,55 E-36$

iv.

i.

1

$\theta$

i.).

i),

4

a.

i)

$a_{1}$

U.

in

i)

0

0

a.

i)

4

b

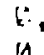

6.

a.

$\theta$

it.

is.

?.

i.

0

$b$

$B$.

is.

0

is.

0

0.

$\theta$

D.

3.

0.

2,37
ALPHAS

7. BUE-US

3. $25 E=0 j$

$5,26 E-13$

$1,5] E=3$

2. $26 E-133$

1. $35 E-03$

$2.53 E-43$

1. $41 t=13$

$2.49 F=15$

$6,7: 4 E-64$

$1,35 E-113$

$4.22 t=n 4$

$7.81 E=14$

2. $20 E-U A$

5. 2UE $=$ i1 4

1. $68 E-194$

$4.46 E-194$

$4.53 E-1.55$

$B .73 E-15$

$9.63 E=46$

2. $57 \mathrm{E}-\mathrm{dS}$

ด.

B.

U.

is.

u.

D.

11.

U.

G.

B.

0

i.

0.

ด.

U.

o.

$\theta$.

H.

i.

b.

a.

0

a.

o.

3.94 
ISLOWING DOHN SPECTRUH AT H. HA CM. FOR ICRL HUSCLE TISSIFE 53 MEV D+ ON HE NEUTRON SRE.CTRUM

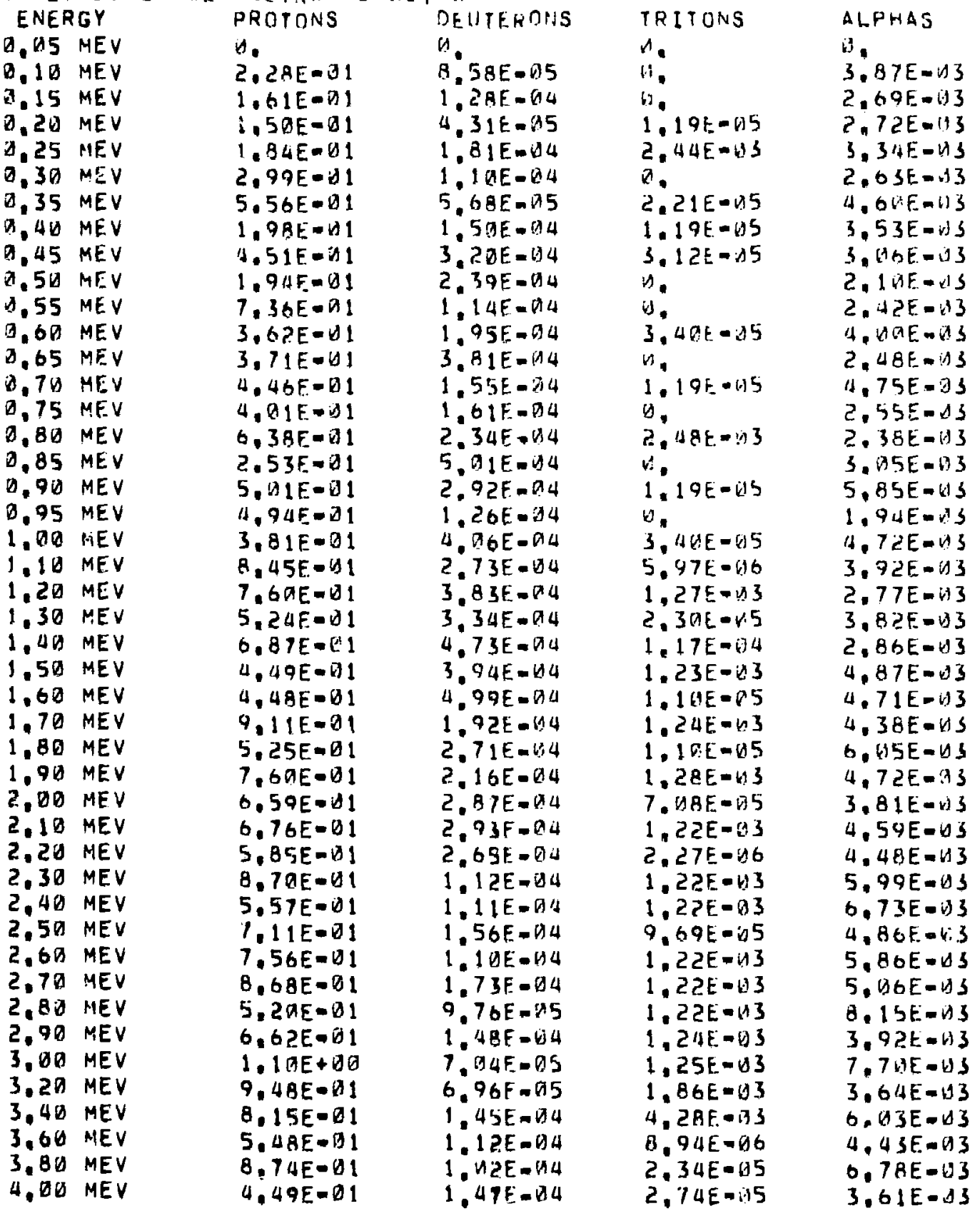


1SLOWING DOWN SPECTRLIM AT G.4M CH. FOR ICRU MUSCIE TISSUE 5. MEV D+ ON BE. NEUTRON SPECYRIJH

\section{ENERGY}

4. 2W MEV

4. 4O MEV

$4.60 \mathrm{MEV}$

4. 8 B $M E V$

5. DO NIEV

$5.5 \mathrm{~W} \mathrm{MEV}$

6.90 MEV

$6.50 \mathrm{MEV}$

7. 6 W MEV

7.513 MEV

8.UD MEV

8. 50 MEV

7. UA HEV

$9.50 \mathrm{MEV}$

10. $D O$ MEV

$11.00 \mathrm{MEV}$

12. OO MEV

$13.00 \mathrm{MEV}$

14.110 MEV

$15.40 \mathrm{MEY}$

$16.00 \mathrm{MEV}$

$17.00 \mathrm{MEV}$

18.0 NEV

19. औU MEV

20.DU MEV

21.60 MEV

22. OV MEV

23. DO MEV

$20.00 \mathrm{MEY}$

$25.00 \mathrm{MEV}$

26.0 0 MEV

$27.00 \mathrm{MEV}$

$28.00 \mathrm{MEV}$

29.00 MEV

30. OU MEV

32.00 MEV

34. $B D$ MEV

36. $10 \mathrm{MEV}$

38. $\triangle O$ MEV

40. HO MEV

42.DO MEV

44. $00 \mathrm{MEV}$

46. DO MEV

48.00 MEV

50, GO MEV

DAVE. E(MEV)

\section{PROTONS}

5. $77 E-101$

4. 2 DE- 11

$6.46 E-1$

5. $33 E-U 1$

7. $21 E=91$

$5.47 E-B !$

$7.5 \mathrm{AE}=\mathrm{A1}$

b. $2 B E-1$

6. BRF-U1

7. 34E-11

$7.76 E=01$

$5.55 E-D 1$

7. $74 E-V 1$

$6.45 E-41$

$6 . \mid 2 E-x) \mid$

5. $44 E=01$

$4,93 E-Z 1$

$4,87 E-41$

2. 'SRE- 1

$7.53 E-12$

2. 7BE-b?

7. $14 E=\square 2$

$2.99 E-142$

$2.61 E-62$

$3.65 E-1 A 2$

$2.96 E-D 2$

1. $17 E=1$

1. 5 IJE $=$ DI

$7.99 E-133$

2.9 $2 E-\triangle 2$

4. 7 SE- 13

1. $51 E-U 2$

$1,36 E=02$

0.

$3.46 E-42$

,

0.

$\theta$

a.

2.57E-U2

b.

a.

a.

$\theta$.

b.

7.65
DEUTEQONS

$1,11 E=134$

1. 3.JE-A4

1. $\triangle 2 E-A_{4}$

Q.37F-И5

1.75E-U 4

$9.76 \mathrm{t}-1.5$

$1.67 \mathrm{~F}-144$

7. 4 HE- iTS

$1.55 E-\triangle 4$

1. $1 E-1 E 4$

2. $14 \mathrm{E}=\mathrm{in} 4$

2. $A 1 F-B S$

4. G $3 E-15$

3. $52 E-115$

$7.70 E-145$

2. $52 \mathrm{~F}=45$

$6,93 E-75$

n.

.7.

a.

b

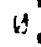

a)

a

a

u

$\theta$

i)

$$
\text { a }
$$

a

0

0

0

it

0.

a.

a.

0

a.

0

4.32

\section{TRITONS}

2. $45 E-45$

$5.07 E-175$

2. $45 E=65$

2. $45 E-\cos$

$5.64 E-115$

+. $.3 E-U 5$

$9.84 E-15$

2. 1OE-25

2. 10E-65

3.15E- 5

$1.34 E-W 5$

a.

1.

id

va

ij.

b.

a.

4.

$\because$.

r.

w.

b.

v.

3

v.

u.

v.

3

0

a.

$\theta$

V.

0

1

G.

3

b.

i.

a

0

D.

b.

2.69
ALPMAS

$3.72 E=13$

$3,2 U E-U S$

4. $12 E-13$

$2.45 E-43$

$3,39 E=U 3$

$2.49 E-V 3$

$4.75 E-b 3$

2. $18 \mathrm{BE}-13$

$3.94 E-113$

$1.04 E-13$

$1,63 E=03$

6. ИQE-U4

$1.41 E=133$

$7.13 E=44$

$1.85 E-U S$

?. $21 E=134$

$5.55 E-04$

$7.77 E .175$

$1.62 E-144$

$0.72 E=136$

$1.81 E-45$

1

1).

$\theta$.

y.

v.

a.

a.

i)

औ.

b.

v.

a.

G.

a.

D.

0

v.

b.

B.

b.

b.

o.

a.

B.

4.25 
1SLOWJNG DOWN SPECTRIIM AT A.6O CM. FOR ICRII MIJSCLE TISSUIE 50 MEV D+ ON HE NEUTRON SFECTRIM

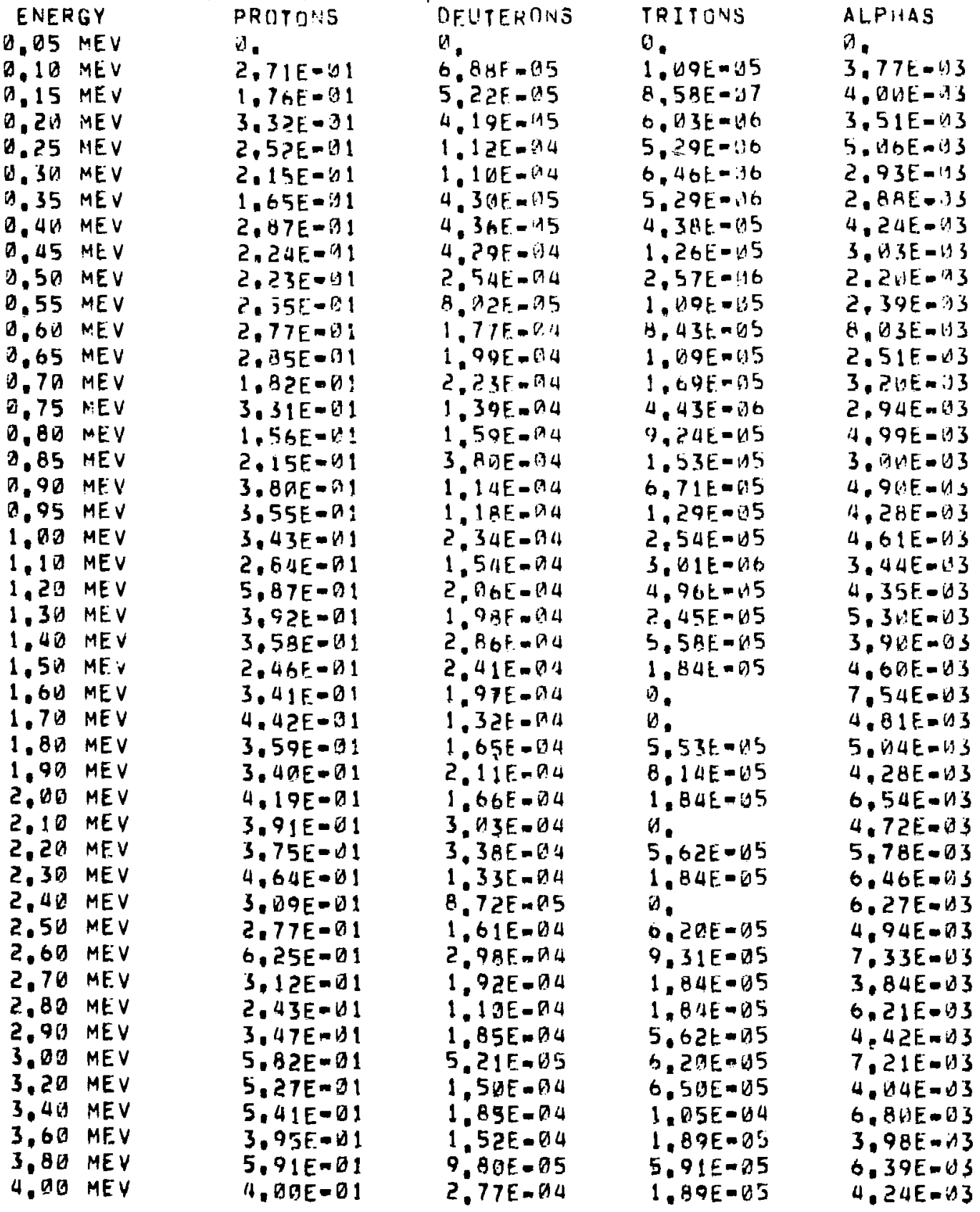


ISLOWING DOWN SPECTRUM AT D.OA CH. FOW ICRU MUSCLE TISSUE 5U MEV D+ ON HE NEUTRON SFETTRUM

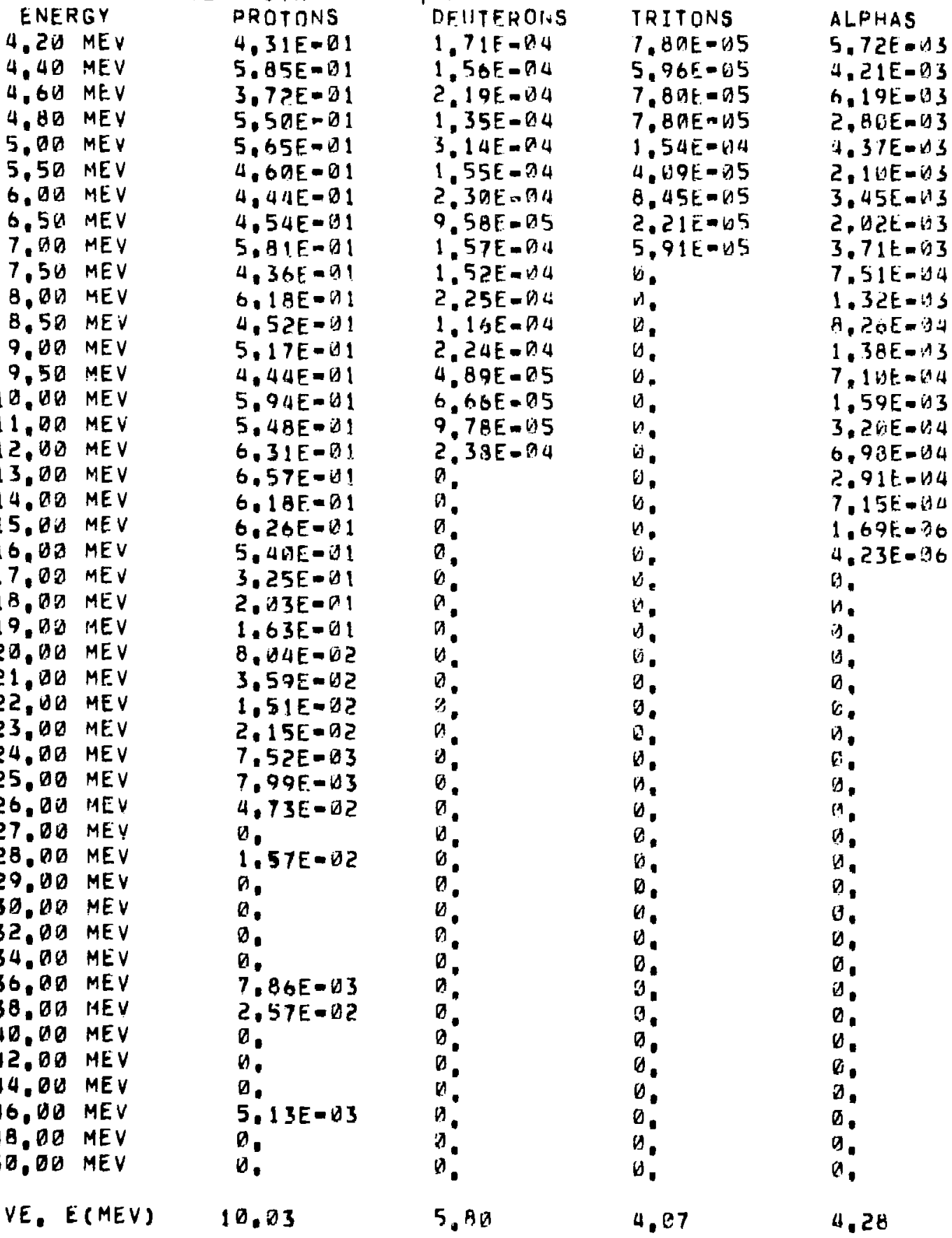


ISLOHING DOWN SPECTRUM AT 1, E C:A. FOR ICRU MISCLL TISSUE 5D MEV D+ ON BE NE.UTRON SPECTRUUIA

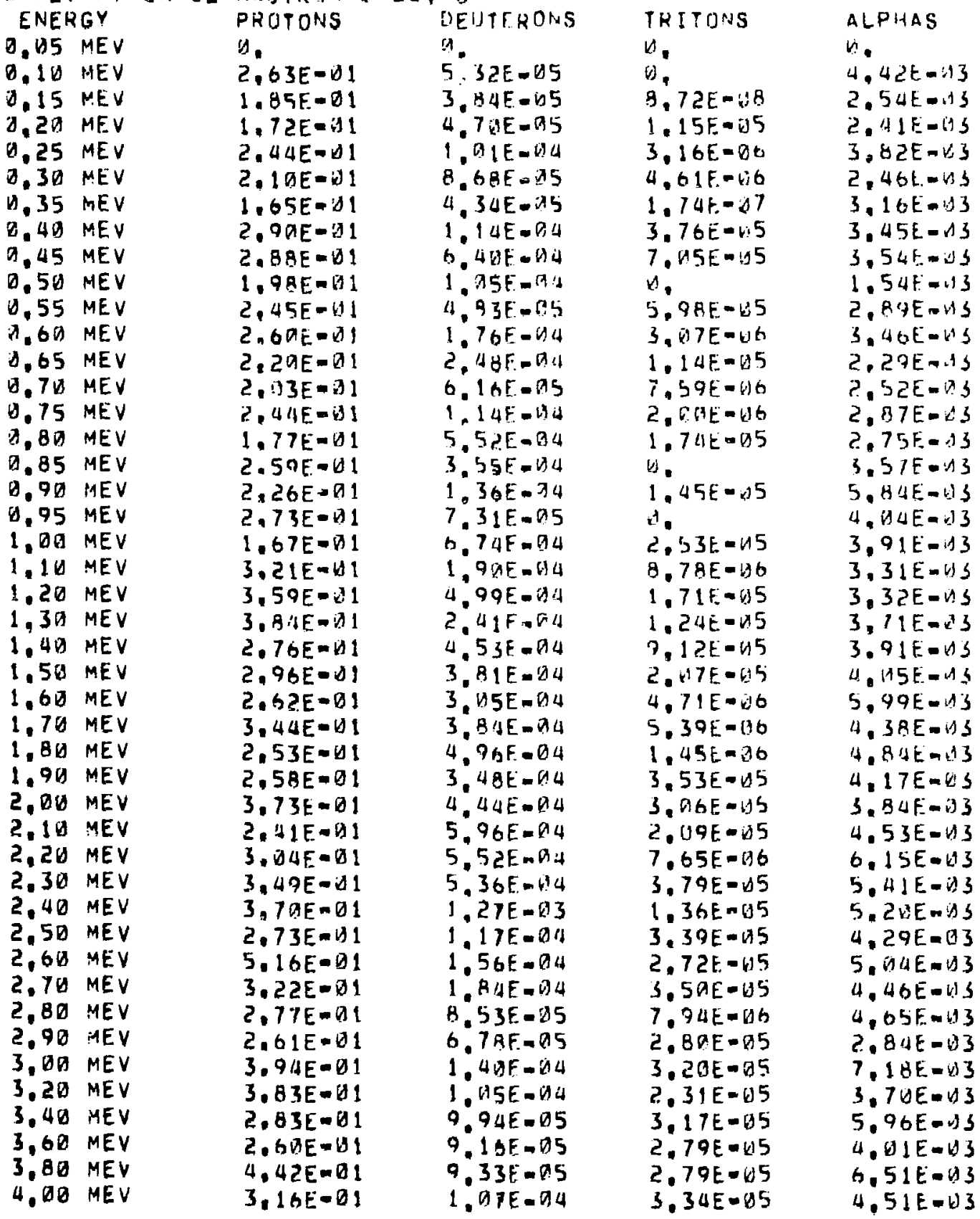


ISLOHING DOAN SPECTRIIFA AT 1, GA CM. FOR ICRU MUSCLE TISSUE 5D MEV D+ DN HE VEUTROIN SPECTRUM

\section{ENERGY}

4.20 MEV

$4,4 D$ MEV

$4.60 \mathrm{MEV}$

$4,8 \mathrm{MEV}$

5. DU MEV

$5,5 \%$ MEV

6. OU MEV

6.5 M MEV

7. 10 MEV

$7.5 \lambda \mathrm{MEV}$

8. BG MEV

$9.50 \mathrm{MEV}$

$9, \triangle 0$ MEV

$9,50 \mathrm{MEV}$

$13, B S$ MEV

$11, U$ NIEV

12. $1 \mathrm{MEV}$

$13,00 \mathrm{MEV}$

14. Bi MEV

15, DU MEV

16. OU MEV

$17.69 \mathrm{MEV}$

1., HA MEV

$19.01 \mathrm{MEV}$

2). HU MEV

21.61 YEV

22.00 MEV

$23, D \triangle M E V$

$24.00 \mathrm{MEV}$

$25.00 \mathrm{MEV}$

26. AD MEV

$27.00 \mathrm{MEV}$

28.00 MEV

$29.00 \mathrm{MEV}$

$30.00 \mathrm{MEV}$

32.00 MEV

34.DO MEV

$36.00 \mathrm{MEV}$

38,00 MEV

$4 \pi, D V$ HEV

42.00 MEV

44.00 MEV

46. OO MEV

48,00 MEV

$50,0 D$ MEV

GAVE. E(MEV)
PROTONS

3. $25 E_{-}=1$

4. $15 E-151$

$2.63 E-01$

$3.76 E-31$

3. $70 E-M 1$

$3.71 E-B 1$

4. t) $3 E-21$

$3.99 E-81$

4. $5 R E-B 1$

$3.86 E-1$

$4.67 E-B 1$

4. 37E- 11

$5.16 E-11$

3. $52 E-11$

$4.43 E-W 1$

$5.13 E-1$

$4.911 E-11$

4. $24 E-11$

$4,32 E-W 1$

$3,75 E-61$

$3,69 E-41$

$3,79 E-U 1$

3. $613 E-61$

2. 4 TE- औ 1

2. $43 E-01$

2. $\$ B E-A 1$

2. $21 E-\Delta 1$

1. $53 E-01$

$1.23 E-\square 1$

1. $\triangle B E-B 1$

1. CSE-D 1

$8,86 E-02$

1. $3 A E-U 1$

7. $33 E=D 2$

$5,6,7 E=U 2$

$1.79 E-92$

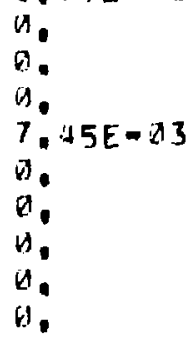

12.23
DE. UTFBDIUS

1.20F-Bid

$1.15 F-D 4$

1.6 BE - WA

2. $42 E-155$

6. $8 \cap F=05$

5.99F-05

8. $21 E=05$

9.3 $3 F=05$

1. $91 \mathrm{E}=1 \mathrm{M}$

3. SBE- $A 5$

$5.9 M F-105$

3. MBE-OS

$5.72 E-115$

2. BQE- $\$ 5$

$5.78 F=05$

a.

$a$

iv.

a.

$a$

a

a

$n$

a

a

.

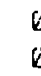

a.

$a$

a

$a$

a.

a.

a.

a.

3.28
TRITONS

2. $79 E=65$

4. $28 t-a 5$

$4.63 E-05$

$5.7 B E-05$

9. BIE- 15

2. B1E-U5

$5.91 E-65$

$1.48 E-05$

$7.38 E=4 S$

1. $48 E-65$

$1.89 E-195$

2. $63 E-05$

S. $5 B E-05$

i. .

i)

4

b.

a.

i.j.

b.

6

v.

$\theta$

$?$

b.

i.

b.

i.

v.

is.

b.

iv.

6

4.

v.

v.

b.

b.

(1)

U.

a.

$v$.

$\theta$

4.88
ALPHAS

$4.79 E-1.3$

$3.9 B \mathrm{E}-\mathrm{M}$

$5.11 \mathrm{E}=\mathrm{S}$

$3,51 \mathrm{~F} \cdot \mathrm{s}$

$4, D S E=133$

$3.46 E-B) 3$

7. UUE- V 3

$1.73 E-1) 3$

2. $52 E-D 3$

$1.18 \mathrm{t}-13$

$2,23 t-w 3$

1. $21 \mathrm{Em} 3$

1. A7E-US

$5,46 E-\triangle 4$

7. B $3 E-\triangle 4$

$5.97 \mathrm{E}=\mathrm{B}^{4} \mathrm{~L}$

$1,226=.43$

$4,16 E=$ तi $^{4}$

1. U2t-US

6. ACHE-Wo

1. $52 \mathrm{E}=\mathrm{V}, 5$

1).

is.

1.

4.

a.

9.

$\theta$.

n.

is.

$b$

i)

b)

0.

a.

a.

b.

n.

H.

b.

a

b.

b.

A.

U.

4.79 
ISLOWING DOWN SPFCTRHM AT Z, MG CM. FOR ICR!J MUSCLF. IISSUE 5H MEV D+ ON BE NEUTRON SPECTRUH

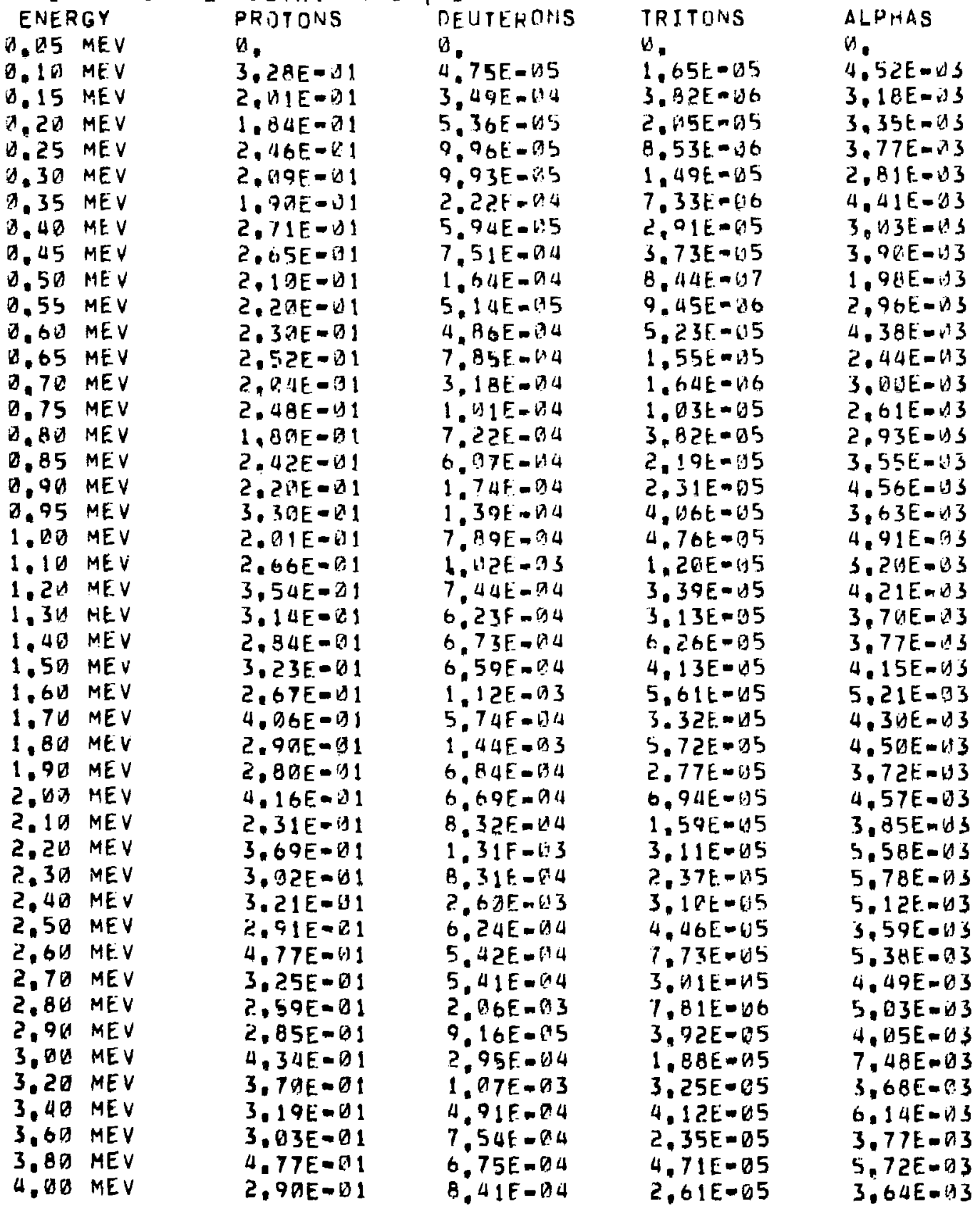


ISLOWING DOWN SPECTRUM AT 2. HA CM, FOR ICRIJ MUSCLE TISSUE 5O MEV D+ ON BE NEUTRON SPECTRUM

\section{ENERGY}

4.20 MEV

4.40 MEV

4.6月 MEV

4.8 MEV

5. 0 A MEV

5.53 MEV

$6 . D U$ MEV

6.50 MEV

7. DU MEV

7.50 MEV

8.DO MEV

8.50 MEV

9. MU MEV

9.50 MEV

10. OQ MEV

$11,00 \mathrm{MEV}$

12, $A D$ MEV

13. 月D MEV

14,00 MEV

15.00 MEV

16.0U MEV

$17.00 \mathrm{MEV}$

$18.00 \mathrm{MEV}$

19.DO MEV

2月, $\triangle 0$ MEV

21 . 10 MEV

22.DD MEV

23.00 MEV

24.00 MEV

25. OU MEV

26,00 MEV

$27 . M O M E V$

28. DO MEV

29.0ด MEV

$30.00 \mathrm{MEV}$

32. QD MEV

34. ME MEV

$36.00 \mathrm{MEV}$

38.00 MEV

40.00 MEV

42.00 MEV

44.00 MEV

46.00 MEV

48. PIG MEV

$50.00 \mathrm{MEV}$
PROTONS

3. 8DE-AI

$4.97 E-D I$

3. $25 E-\triangle 1$

4. $15 E-01$

$4.74 F=01$

$4.12 E=01$

$3.98 E-\angle 1$

4. $1 B E-1 B 1$

$4,98 E-W 1$

4. 15E- $1 !$

$4.26 E-11$

4. 3HE $=1$

$4.63 E-\Delta !$

$3,6 ? E-131$

$4.28 E-01$

5. O9E - 1

$5,16 E-V i 1$

$4.36 E-D 1$

4. $\triangle 9 E=\square 1$

$4.06 E-H 1$

$3,14 E=01$

$3.77 E-b 1$

3. $99 E-01$

3. $01 E-11$

$2.33 E=01$

2. 1 IIE-OI

$2.43 E-61$

$1.73 E=01$

$1.27 E-41$

$1: 44 E-\overline{1} 1$

1. $20 E-01$

$9,40 E=U 2$

$5.89 E-122$

$4.57 \mathrm{E}=U 2$

$4,96 E=02$

$3.35 E=62$

$2.62 E=0 ?$

$2.79 E=133$

$4.64 E=83$

$9.2 B E=03$

0.

D.

U.

0
DEUTERTNS

1. 39E-113

$9.24 E-A 4$

$8.6 \mathrm{HF}=\mathrm{BH}$

8.62E-3i

$1.30 F=X^{1} 3$

$6.15 E=114$

B. $11 F=4$

$7.44 \mathrm{E}-\mathrm{BA}$

1. R2F-A3

1.83E- .94

$2.47 E-144$

2. $6.5 E=1^{*} / 4$

1. $45 E-7.4$

1. $35 E-114$

$2.71 E-114$

$2.56 \mathrm{~F}-04$

$7.03 F-04$

$a$

a.

a.

n.

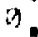

(i)

a.

a.

a.

b.

a.

a.

a.

9

a.

$a$

$a$

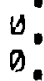

0

a.

$a$

0

9

5.34
TRITDNS

2, 2AE-WS

3. $136 F-05$

$6.36 E-115$

1,3 ? E $=015$

$1.88 E-65$

$1.85 t-65$

3. $\{5 E-155$

1. $94 E-145$

$3.57 t=05$

$0.72 E-5 h$

1. DIE- 15

$1,34 E-45$

$2.69 E-05$

i.

b.

i.

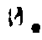

A.

b.

4.

is.

n.

i)

E!

(1).

U.

v.

$b$

i).

iv.

$b$.

a.

$\theta$

i.

$v$.

v.

n.

b.

0.

i.

的。

b.

b.

b.

4.04
ALPHAS

$4.52 E=33$

$3,03 E=U 3$

4.14E-.13

$3.21 E-4) 3$

$4,43 E=43$

2. $37 E-W 3$

4. $65 E-1) 3$

1. $761 E-13$

2. $87 E=43$

1. $2 W E-U 3$

2. $12 E=13$

$7.54 k=04$

i. $18 E-D_{3}$

$5.44 E=E^{\prime} 4$

$8.54 E-D 4$

$5.36 E-114$

1. $13 E-63$

1. OBE- $\$ 4$

1. $77 E=: 34$

1. $14 \mathrm{E}=6 \mathrm{Q}$

2. 7 UE $=19$ it

5. 39E-W $\%$

1. $4 \Delta E-N O$

H.

b.

i.

田:

b.

4.

a.

i.

a.

0.

w.

n.

D.

b.

b.

b

0

0

(1)

$\theta$

D.

4.46 
1SLOWING DONN SPECTRIJM AT 4 , GO CH, FOR ICRU MUSCLL. TISSUE 5D MEV D+ ON BE NEUTRON SPFCTRUM

\section{ENERGY}

D. O5 MEV

0.13 MEV

0.15 MEV

0.20 MEV

0.25 MEV

0.30 MEV

0.35 MEV

D. 4 MEV

0.45 MEV

0.50 MEV

0.55 MEV

0.60 MEV

0.65 MEV

0.70 MEV

0.75 MEV

$0.80 \mathrm{BEV}$

$D, 85 \mathrm{MEV}$

0.90 MEV

0.95 MEV

1. DO MEV

$1.10 \mathrm{MEV}$

1.2U MEV

$1.30 \mathrm{MEV}$

1.49 MEV

1.50 MEV

1.6D MEV

1.70 MEV

$1.80 \mathrm{MEV}$

1.90 MEV

2.00 MEV

2.10 MEV

2,20 MEV

2.30 MEV

2.40 MEV

2,50 MEV

$2.60 \mathrm{MEV}$

2,70 MEV

2. 80 MEV

$2.90 \mathrm{MEV}$

3.DO MEV

3.20 ME.V

3.40 MEV

$3.60 \mathrm{MEV}$

3.86 MEV

$4,00 \mathrm{MEV}$
PROTONS

1).

$3.04 E-1) 1$

1.97E-U1

1. $78 E=B 1$

2. $7 A E-B !$

2. $97 E=01$

1. $73 F-01$

2.70E-U1

$2,90 E-101$

2. $11 E-01$

2. 2TE-O1

2. $60 E-01$

2. 28E-hI

2. I IE-OI

$2.53 E-D 1$

1. $99 E-\square 1$

$2.33 E-01$

2. 20E- 1

2. $50 E=01$

$1.69 F-B 1$

$2.74 \mathrm{E}=1$

$3.24 E-01$

$2.99 E-i d$

$2,76 E=B !$

3. $27 E-M I$

2,64E-01

3.66E-DI

3. $94 E-\square 1$

2. $B 3 E-B 1$

$3.23 E-01$

2.67E-1DI

3. D6E-01

3. $12 E=01$

$3,2 \triangle E=01$

2. $74 E-41$

4. $46 E-01$

3. 2. $8 E=01$

2. $47 E-01$

$2.82 E-81$

$3.92 E=01$

3. 50E-BI

3. 27E-D 1

$3.07 E-01$

$4,32 E=31$

$2,62 E=1$
DELTTERTNS

t.

$4.75 E-05$

$7.5 B E=0.5$

5.6EF- 15

1. П9E-ПИ

$2.55 E-54$

5. ด उE- -15

8. 36F-V 5

2. $21 E=144$

1.79E-b 4

5.18E-W5

?. 1 OE $\triangle 14$

4. $46 E=64$

8. $75 E-05$

1. DBE-D 4

1. $3 \mathrm{aE}-\mathrm{T}^{4}$

4. 87E-O 4

1. $54 E=04$

9.39E- 15

2. $19 F-04$

1.82E- 84

3. $52 E=44$

3.16F-O4

2. $87 \mathrm{E}-04$

3. $23 E-74$

$3,32 F-04$

1. $63 E=04$

3. $83 \mathrm{E}-\mathrm{H} 4$

1. $54 F-O A$

3. $24 E-U A$

1. GYERTA

3. $41 E-04$

1. $91 E-14$

1. $72 E-04$

3. $42 E-134$

2. $97 E-34$

2. $48 E-04$

2. $64 E=114$

2. $24 E-B 4$

2. $29 E-14$

?. $80 F-O 4$

$5.35 E-04$

1. 39E-1) 4

$1.94 E=04$

2. $27 E-04$
TRITONS

B.

7. ด9E-U6

7. $\angle 5 E-116$

$5.35 t-136$

1. 39E- 05

$1.93 E-04$

$9.78 E-U{ }^{\prime}$

$5.73 E-105$

2. $14 E-115$

$3,46 E=16$

2. $65 E-65$

2. $96 E-105$

1. $43 E-35$

1. 8 . $8 E-E 4$

3. $72 E-196$

$5.23 E-155$

4. $62 E-B T$

2. $59 E-15$

$1.90 E-34$

$3.94 E-05$

$7.68 E-06$

i. $365-01 \mathrm{~d}$

$1,65 i-b 5$

$1.01 E-04$

$4.10 E-\triangle 5$

$1.31 E=-44$

1. $14 E-04$

$1,2 Q E=114$

$3,82 E-015$

1. 32E-G 4

1. $29 E-104$

2.17E-RI

1. $26 E-V 4$

2. (1) $3 E-1) 4$

?. $53 E=04$

5. $7 E-64$

$3,64 E=65$

4. $58 E-155$

$1.84 E-45$

4.16E- 05

3. 8RE- US

$4.91 E-115$

1. $62 E-05$

$1.91 E=05$

$4.94 E-05$
ALPHAS

$d$.

4. V2E-VIS

$3.6 U E-03$

$3.35 E=b 3$

4. $14 E-$ in 3

$2,82 E=43$

4. (UUE-X) 3

$3,65 E-13$

$3.49 E-U 3$

$2.11 \mathrm{E}-1 \mathrm{~S}$

$3,84 E=193$

$5.3 W F-U 3$

$2.46 E-13$

2. $54 E=\omega 3$

2. $82 E=d 3$

$3.60 \mathrm{~F}-\mathrm{Al} \mathrm{S}$

2. $714 E=U j$

$5.97 E=13$

$3,05 E-13$

4. $76 E-U S$

3. $38 E-v S$

$4.13 E-43$

4.5 A -13

$4.34 E-03$

$3.93 E-b 3$

$5.76 E-A 3$

4. $8 B E-B 3$

$5.35 E-13$

$4.37 E-63$

$5.07 E-13$

$4.26 E=143$

5. BRE-U3

$7.16 E-03$

b. $28[-\Delta 3$

$4.29 E-143$

$5.77 E-03$

$\triangle .37 E-153$

$5.16 E=03$

$4.53 E-V S$

$6.93 E-\Delta 3$

$4.613 E-113$

7. 2 ZE -1.3

$4.66 E-113$

$7.31 E=B 3$

$4.16 E-13\}$ 
ISLOWING DOWN SPECTRIJM AT 4 DA CM. FOH ICRU MUSCLE YISSUE 50 MEV D+ ON BE NEUTRON SPECTRUIM

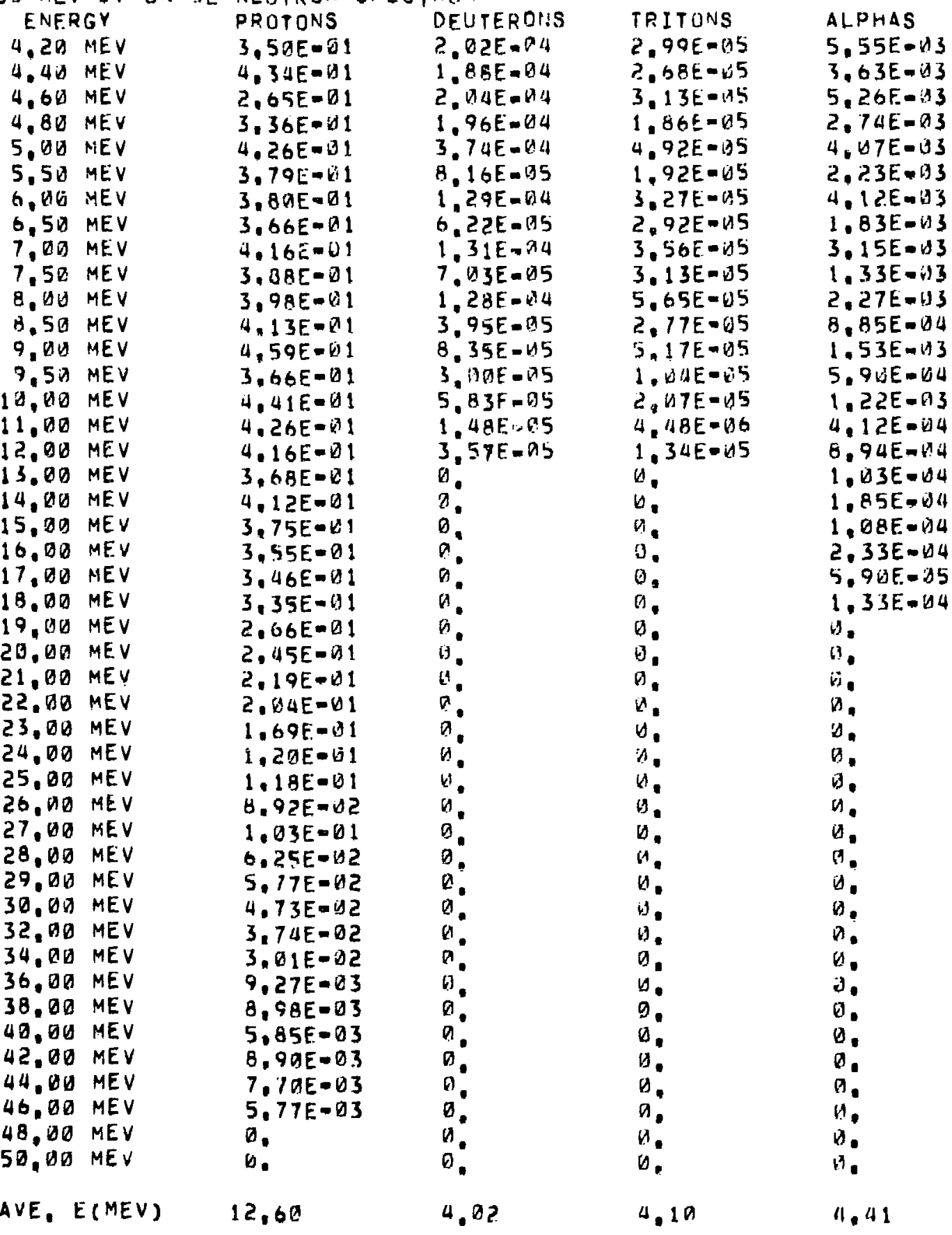


ISLOWING DOUN SPECTRUM AT 6. HU CM. FOR ICRU MUSCLE. TISSUE 50 MEV D+ ON GE NEUTRDIV SPFCTRUM

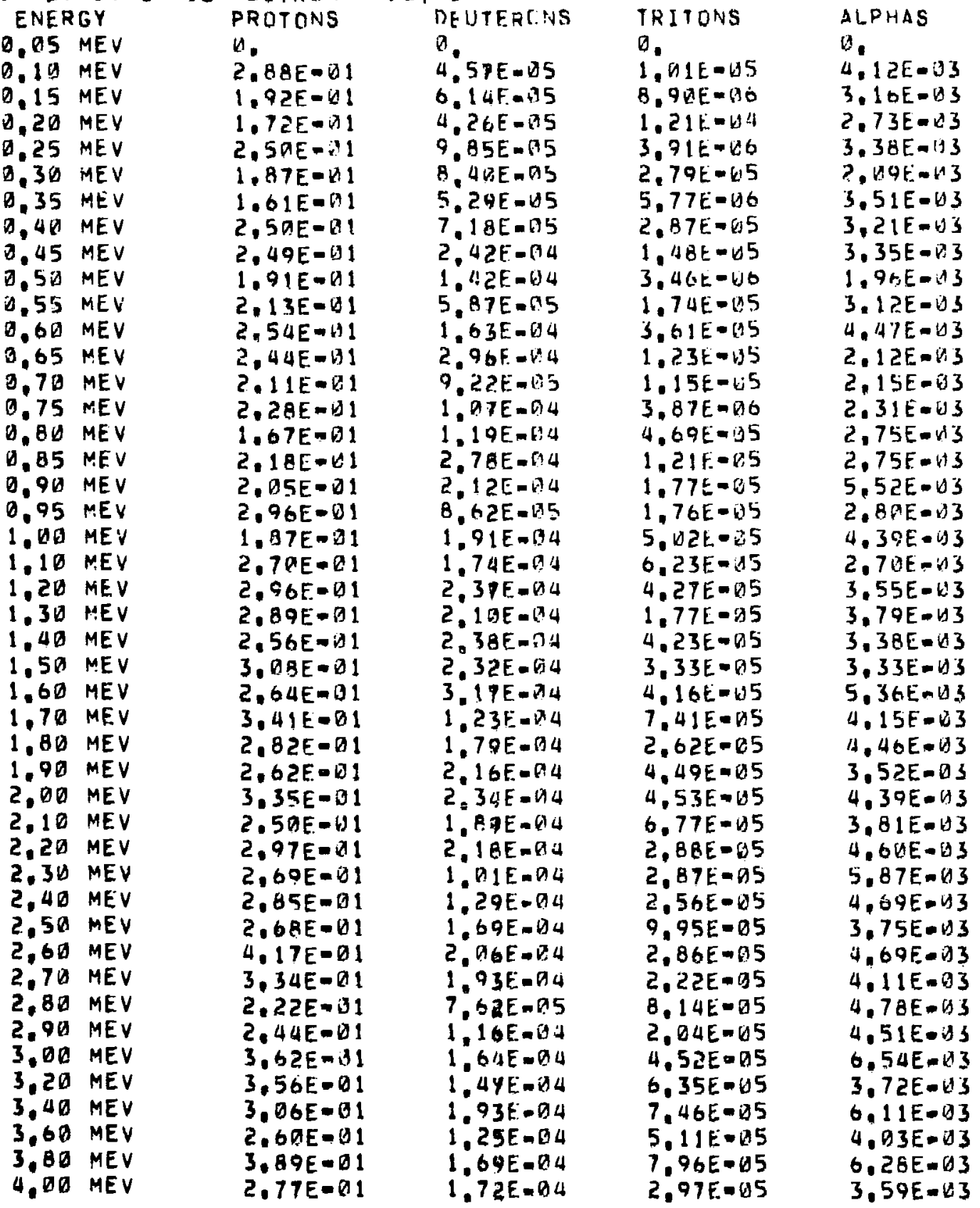




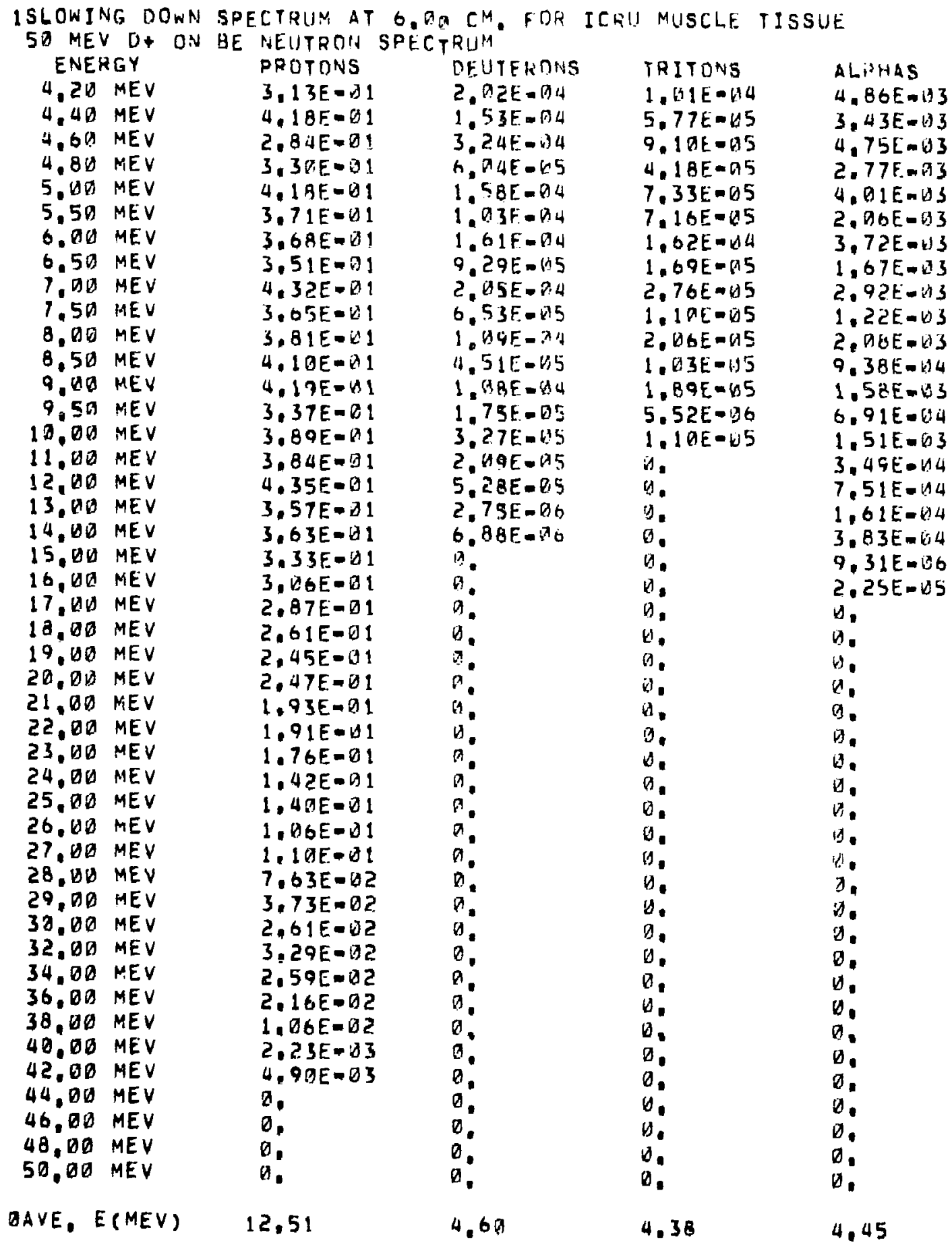


ISLOWING DONN SPECTRIIM AT 1:, C.M. FOR ICRU MUSCLE IISSUE 50 MEV D + ON RE NEUTRON SPECTRUM

\section{ENERGY}

D. DS MEV

D.10 MEV

0.15 MEV

0.26 MEV

0.25 MEV

0.3 MEV

0.35 NEV

D. $4 D$ MEV

0.45 MEV

0.50 MEV

0.55 MEV

0.60 MEV

0.65 MEV

0.70 MEV

0.75 MEV

0.8D MEV

$0.85 \mathrm{MEV}$

$0.90 \mathrm{MEV}$

0,95 MEV

1.DO MEV

1.10 MEV

1.20 MEV

$1.30 \mathrm{MEV}$

$1,40 \mathrm{MEV}$

$1.50 \mathrm{MEV}$

$1.60 \mathrm{MEV}$

$1.70 \mathrm{MEV}$

1.80 MEV

1.90 MEV

2, $D D$ MEV

2.1ऽ MEV

$2.20 \mathrm{MEV}$

2.30 MEV

2.40 MEV

2.50 MEV

2,60 MEV

$2.70 \mathrm{MEV}$

2.80 MEV

$2.90 \mathrm{MEV}$

3. DG MEV

3.20 MEV

3.40 MEV

3,60 MEV

3,80 MEV

4. DO MEV
PROTONS

$\because$.

2.77E-31

1.74E-i]

$1.52 E-U 1$

$2.30 F=121$

$1.77 E-01$

1. $54 E-01$

2. 32E- 21

2. 36E-01

$1.90 \mathrm{E}-31$

1.99E- 01

2. 35E-11

2. DÁE - OU1

1. $B 8 E-31$

$2.3 ? E-\square 1$

1. $5 B E=D 1$

2. $18 E-D 1$

$1.98 E-B 1$

c. $53 E-d]$

$1.71 E-B 1$

2.S1E-DI

2.84E-21

$2,14 E-D 1$

2, $51 E-31$

2. $48 E-01$

$2,42 E-01$

$3.41 E=01$

$2,36 E=U 1$

2. $51 E-D 1$

$3.22 E=\Delta 1$

$2,33 E=01$

$2.93 E-01$

2. $61 E-V 1$

2. $81 E-01$

2. $42 E-01$

$3.84 E=1$

3. $\triangle 3 E=0$ !

$2,15 E-01$

$2.41 E=01$

3. $29 E-01$

$3.39 E=U 1$

2. 7AE- $D 1$

2. $73 E-01$

$3,86 E-01$

$2.92 E=1$
DEUTERONS

.

$4.6 \% E-D 5$

3. $8 G E=05$

$7.96 \mathrm{~F}-6.5$

9. 36E-D!5

2. $42 E-A 4$

6. $18 E-E 5$

$9.34 \mathrm{E}-155$

2. $32 \mathrm{Z} E=0.4$

$2.63 E-174$

$8.58 E-15$

$1.62 E-34$

$2,3 G E-D 4$

2. 87E-O4

$1.14 E-14$

$1.47 E-011$

$1.1 S E-D 4$

$1, B O E-V^{2}$

$1.65 \mathrm{E}=1 \mathrm{~T}$

2. $77 E-Q 4$

3.

$4.25 E=04$

1. $8 I E=04$

3. A7E-A4

3. $96 \mathrm{E}-\mathrm{A} 4$

$7.93 E=04$

1.3OE-A 4

$3.44 E-94$

2.75E= 44

$3,12 E-14$

1. $26 E-1) 4$

$3.69 E-1) 4$

1. $47 \mathrm{E}-04$

3.27E-DI

1. $82 E=04$

$3.98 E=D 4$

1. R5E- $A 4$

$3.51 E-194$

1. $72 E-D 4$

$3.96 E-04$

$5.45 E-134$

5. $47 E-114$

$1.65 E=04$

2. $12 E-124$

3. QIE-OA
IRITUNS

is.

$5,58 E-\square 5$

$5,58 E-16$

2. 8 UE $=\square 5$

$\downarrow .85 E-06$

$3.39 E=5$

$5,37 E=05$

1. $213 E-15$

$4.43 E-65$

3. $16 E=115$

6. 40E-WS

1. $93 E-W 4$

6. $2 \Delta E-E S$

$1.33 E-B$ i

3. $26 E-06$

$2.37 E-1) 5$

$3.03 E-45$

$6.69 E-16$

3. 6UE- 05

1.87E-US

$1.67 E-15$

$3.96 E-05$

1. $5 E=114$

$3.69 E-25$

$3,0 D E-05$

$4,24 E-65$

5. $\triangle 4 E=115$

$1.72 E=154$

2. $10 E-105$

3. D2E-U5

$7.16 E-B 6$

$9.13 E=\$ 5$

1. $74 E=\square 5$

1. $44 E-135$

1. $24 E-64$

$2,45 E=95$

2. $* 9 E-05$

$9.73 E=05$

$1.79 E-115$

1. $64 E-04$

$2.03 E-05$

$6.23 E=05$

$5,39 E-65$

$6,69 E-115$

$\because 82 E-165$
ALPHAS

(1)

$3.45 F_{n}=03$

3. WHE $=W 3$

$2.56 E=B S$

3. ? $\{E-U\}$

2. $28 E-V 3$

$3.35 E-143$

2. $81 E-03$

$2.83 E-13$

$1.77 E-43$

2. $86 \mathrm{E}-\Delta 3$

$3.50 E-k) 3$

$2,49 E-143$

2. $28 E-n 3$

$2,27 E=113$

2. $49 E-43$

2. $42 E-113$

$4.43 E=13$

$2.85 E-13$

$3.8 B t=13$

2. $B 2 E=\square 5$

3. 2 AE- 03

$3.37 E-W$

$3,69 E=U$

$3.2, E-43$

$4.5 W E-D 3$

3. $73 E-4 s$

4. $1 \Delta E-U 3$

3. $14 E=13$

$3,85 \mathrm{E}-(1) .3$

$3.16 E=43$

$4.65 E-43$

4. $66 E-53$

$4,51 E-1) 3$

$3.29 E=\Delta 3$

4. 97E-U3

$3.49 E-\mathrm{HS}^{3}$

$4,21 E=13$

$3,8 G E-B 3$

$5,66 E-d 3$

$3,37 E=13$

$5,76 E-H S$

$3,36 E=03$

$5,49 E=193$

$3,09 E-13$ 
ISLOWING DOWN SPECTRUIA AT IW.A CM. FOR ICRU MUSCLE TISSUE $5 D$ MEV D+ ON BE NEUTRON SPECTHUM

\section{ENERGY}

4.20 MEV

4. 4D MEV

4.6D MEV

4.80 MEV

5. DO MEV

$5.50 \mathrm{MEV}$

6. DO MEV

$6.50 \mathrm{MEV}$

$7.00 \mathrm{MEV}$

$7.50 \mathrm{MEV}$

3. DU MEV

B. $50 \mathrm{MEV}$

9. Dด MEV

$9.5 \square$ MEV

$10.00 \mathrm{MEV}$

$11.00 \mathrm{MEV}$

$12,00 \mathrm{MEV}$

$13,00 \mathrm{MEV}$

14,05 MEV

15, OH MEV

16.00 MEV

17.00 MEV

18.0L MEV

19.00 MEV

$20.00 \mathrm{MEV}$

$21.00 \mathrm{MEV}$

22. $10 \mathrm{MEV}$

23,00 MEV

24.00 IHEV

25. OU MEV

26. OC MEV

27. OD MEV

28.00 MEV

29.0D MEV

30.00 MEV

32.00 MEV

$34.00 \mathrm{MEV}$

36.00 MEV

$38.0 D M E V$

40.00 MEV

$42.00 \mathrm{MEV}$

44.06 MEV

46. 10 MEV

48.00 MEV

50.09 MEV

DAVE, E(MEV)

\section{PROTONS}

3. 22E- 1

$4 . \triangle A E=D 1$

2. 69E $=01$

$3.03 E-11$

4. $D 8 E-A 1$

3.27E- 1

$3,53 E-41$

3. $46 E=131$

4. D $9 E=U 1$

3. $53 E-1$

$3.58 E-01$

$3.52 E-G 1$

$3.97 E-01$

3. $15 E-$ iो 1

$3.66 E-W 1$

$3,75 E-61$

3. $99 \mathrm{AD}-D 1$

$3.47 E-01$

$3.36 E-01$

$3,4 D E-11$

$2.62 E-131$

$2.66 E-01$

$2.41 E 001$

2. $14 E-Q 1$

2. $90 E-191$

$1.98 E-01$

$1.67 E-01$

$1,43 E-1] 1$

$1.3 B E=\square 1$

$1.23 E-01$

$7.91 E-D 2$

7.1 RE-A2

$6.24 E-02$

5. $21 E-12$

3. $12 E=02$

$2.32 E-02$

$2.5+E=102$

$1.66 E-02$

$1.15 E-02$

$1.27 E-02$

$5.97 E=13$

$2.63 E-1) 3$

0.

0.

B.

12.41

\section{DEUTERONS}

D. BGE $=4$

2. $34 \mathrm{E}=\mathrm{CA}_{4}$

4. $41 E-D 4$

1. $12 E-D 4$

1. $31 E=(14$

2. $27 \mathrm{E}-\mathrm{A} 4$

$3.95 E-b 4$

2. $19 E=$ GI 4

2. $91 E=0.4$

1. $51 E-134$

1. $86 E-144$

2. $30 E-1 A 4$

1.76E-WI

1. $24 E-D A$

?. $48 E=94$

?. $4 E=\square$

$5.58 E=14$

a.

$B$

$a$

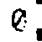

a.

0

Ei:

a

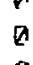

a

$\theta$

$\theta$

a

$a$

0

a.

b.

0

0

ด.

$a$

$a$

a.

a.

a.

$a$

6.21
TRITONS

$6.66 E-05$

$9.81 E-15$

1. $66 E-G 4$

$1.24 E-34$

2. $47 \mathrm{E}-\mathrm{i} \mathrm{i} / \mathrm{S}$

1. $29 E-45$

$2.54 E-015$

$1.12 E=55$

. $91 E-U 5$

. 46E- 16

$.69 E-05$

$3.87 E-156$

7. $C 1 E-D 6$

1. $48 E=96$

$2.95 E-106$

u.

U.

b.

n.

0.

4.

$\theta$

n.

v.

1

4

$n$

0

b.

$\Delta$

a

b.

1.

is

v.

.

b.

id.

i]

$\theta$

0

0

$\theta$

3.62
ALPHAS

4. $18 E-x 3$

$2,90 E-J 3$

$3.99 E=63$

2. $41 E=U 3$

3. $49 E=B 3$

$1.79 E-03$

$3.49 E=33$

$1.27 E-b 3$

2. 19E-13

$7,31 E=64$

$1.54 E-W 3$

$7.25 E-114$

$1.25 E-13$

4.71E-U4

9.92E-W4

2. $72 \mathrm{Em}$ is 4

5. 66E- I4

$9,32 E-U 5$

$1.82 E-04$

$4.26 E-15$

$9.78 E=65$

1. $32 E-\$ 7$

?.97E- 17

D.

$U_{1}$

0

D.

$a$

$D$

0

a.

10

$\theta$

D.

d.

$b$.

$D$

b.

a.

a.

v.

0.

0.

6

4,26 
ISLOWING DOWN SPECTRUM AT 14. C'9, FOE ICRU MUSCLE IISSJE 50 MEV D+ ON BE NEUTRON SPECTRUM

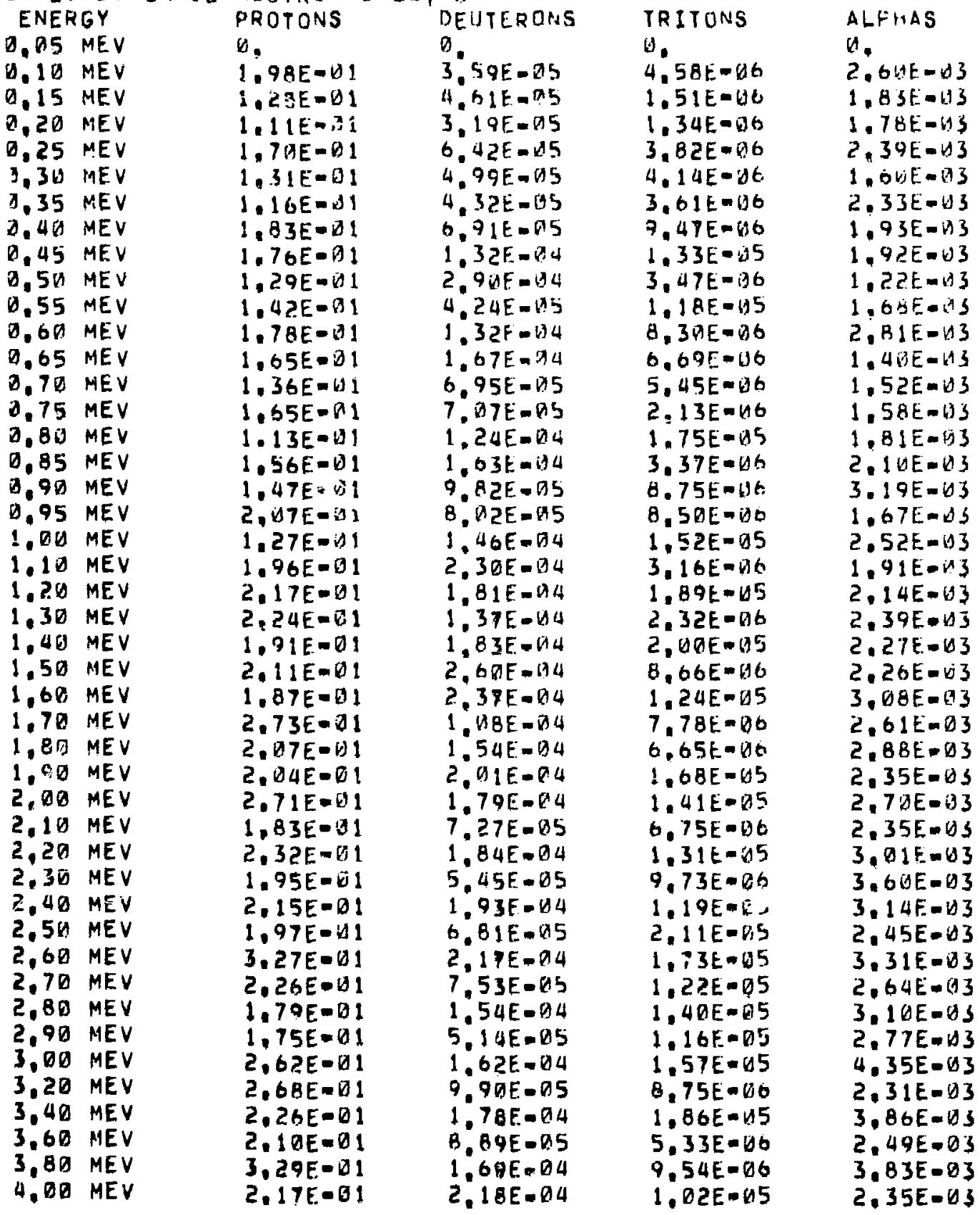


1SLOWING DOWN SPECTRUM AT 14.G CM. FOR ICRIJ MUSCLE TISSLE 5 D MEV D+ ON BE NEUTRON SPECTRIH

\section{ENERGY}

4.20 MEV

4.40 MEV

4.60 MEV

4.8D MEV

5. $D 0$ MEV

$5.50 \mathrm{MEV}$

6.09 MEV

$6.50 \mathrm{MEV}$

7. $\triangle$ ME MEV

7.50 MEV

8. QU MEV

8.50 MEV

9. DO MEV

9.5 B AEV

10.00 MEV

$11.00 \mathrm{MEV}$

12.00 MEV

$13.00 \mathrm{MEV}$

$14.00 \mathrm{MEV}$

15.00 MEV

$16.0 \mathrm{MEV}$

17.00 MEV

$18,00 \mathrm{MEV}$

$19.00 \mathrm{MEV}$

$20.00 \mathrm{MEV}$

$21.00 \mathrm{MEV}$

22,00 MEV

23.06 MEV

24.00 MEV

$25.00 \mathrm{MEV}$

26. OH MEV

27. $10 \mathrm{MEV}$

28.DV MEV

29.DO MEV

30. $00 \mathrm{MEV}$

$32.00 \mathrm{MEV}$

34.00 MEV

$36,00 \mathrm{MEV}$

$38,00 \mathrm{MEV}$

40. $10 \mathrm{MEV}$

$42.00 \mathrm{MEV}$

44. DO MEV

$46.00 \mathrm{MEV}$

48.06 MEV

50,00 MEV

DAVE. E(MEV)
PROTONS

2. $45 E-01$

$3,28 E=01$

2. $D 7 E-01$

$2.45 E-11$

3. $22 E-151$

2. $83 E=$ is 1

2.76E-01

2. $81 E-1$

3. $19 E=D 1$

2. $38 E-01$

$2.96 E-11$

3. $02 E=1$ !

3. $23 E-11$

$2,61 E=01$

3. $05 E-01$

3. $07 E-01$

$3.25 E-41$

3. QDF-DO 1

2. $93 E-61$

2.90E-O 1

2. $3 B E-\square 1$

2. $39 E-11$

2. ИBE-D1

1. $80 E-01$

1.67E- 11

1.59E-DI

$1.52 E-\triangle 1$

1. $14 E-D I$

$7.76 E=U 2$

7. D2E- $\$ 2$

$5.56 E-D C$

6. $99 E-12$

$3.79 E-02$

3. $29 E-W 2$

$2.69 \mathrm{E}-02$

$2.60 E-\square 2$

1. $42 E=02$

$9.9 B E-03$

$6.70 F-V 3$

$8.05 E-1 B 3$

$5.97 E=103$

$2.63 E-103$

$\theta$

$\Delta$

a.

12.29
DEUTEROHS

$4.21 E=04$

$4.93 E-05$

$6.49 E-95$

3. 2UEMDS

5. U1E-05

A. 3 BE- $A 5$

$6.64 E-D S$

$3.14 E-D .5$

$6.675-05$

3. I 2E- 05

5. $2 D E=15$

2. 17F- 15

4. $46 E=A 5$

1. $63 E-15$

2. $86 E=05$

1. D. $3 E-05$

2.19E- 15

$1.93 E=06$

3. BGE $A B$

a.

a

0

A.

is.

a.

ต.

a.

a)

$a$

0

b.

a

$\theta$

$\theta$

a.

0

0.

a.

$\theta$

a.

3.91
TRITONS

$1.30 E=05$

1. $2 B E-05$

1. 1 AE -65

$8.68 E=$ Wh

1.7 UE $-M 5$

$6.34 E-06$

1. 3PE-195

$2.91=006$

$4.72 E-66$

$3.5 \Delta E-\Delta b$

7. $41 E=05$

$1.37 \mathrm{Em}$

2. $73 \mathrm{E}-0 \mathrm{t}$ :

$2.77 E-G 6$

$6.23 E=66$

$1.34 E-00$

$3.67 E-06$

0

b.

b.

0.

B.

(A.

ด.

a.

a.

y.

9.

b.

t.

$\theta$

b.

9

3.

$\theta$

b.

n.

D.

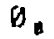

$\theta$.

0.

0

0.

b.

4.56
ALPHAS

2.96E-03

2. $155 E-13$

$2.73 E=13$

$1.72 E-13$

2. $50 E-0.3$

$1.43 E-1) 3$

$2,69 E-03$

1. $07 \mathrm{E}=03$

1. $85 E-113$

$7.63 E-44$

1.27E- .3

5. $58 E-104$

9. $\triangle B E-D A$

$4.07 E-144$

$7.36 E=D 4$

2. $8 B E=124$

$5.96 E-\square 4$

$7.63 E-65$

$1.45 E-64$

$3.35 E-155$

4. $45 E-05$

$4.18 E-115$

$7.93 E-15$

3. 84E-VS

8. $44 E=55$

$b$.

a.

0

U.

a

a.

$\theta_{\mathrm{s}}$

i.

6.

0.

$\theta$

B.

0.

0.

$\Delta$

3.

a.

$\theta$.

0

U.

4,61 
IINITIAL SPECTRUM AT 3. BOAV.BS CM. FOR SHONKA A-15G PLASTIC 5 I MEY O+ ON BE NEUTRON SPECTRIIM

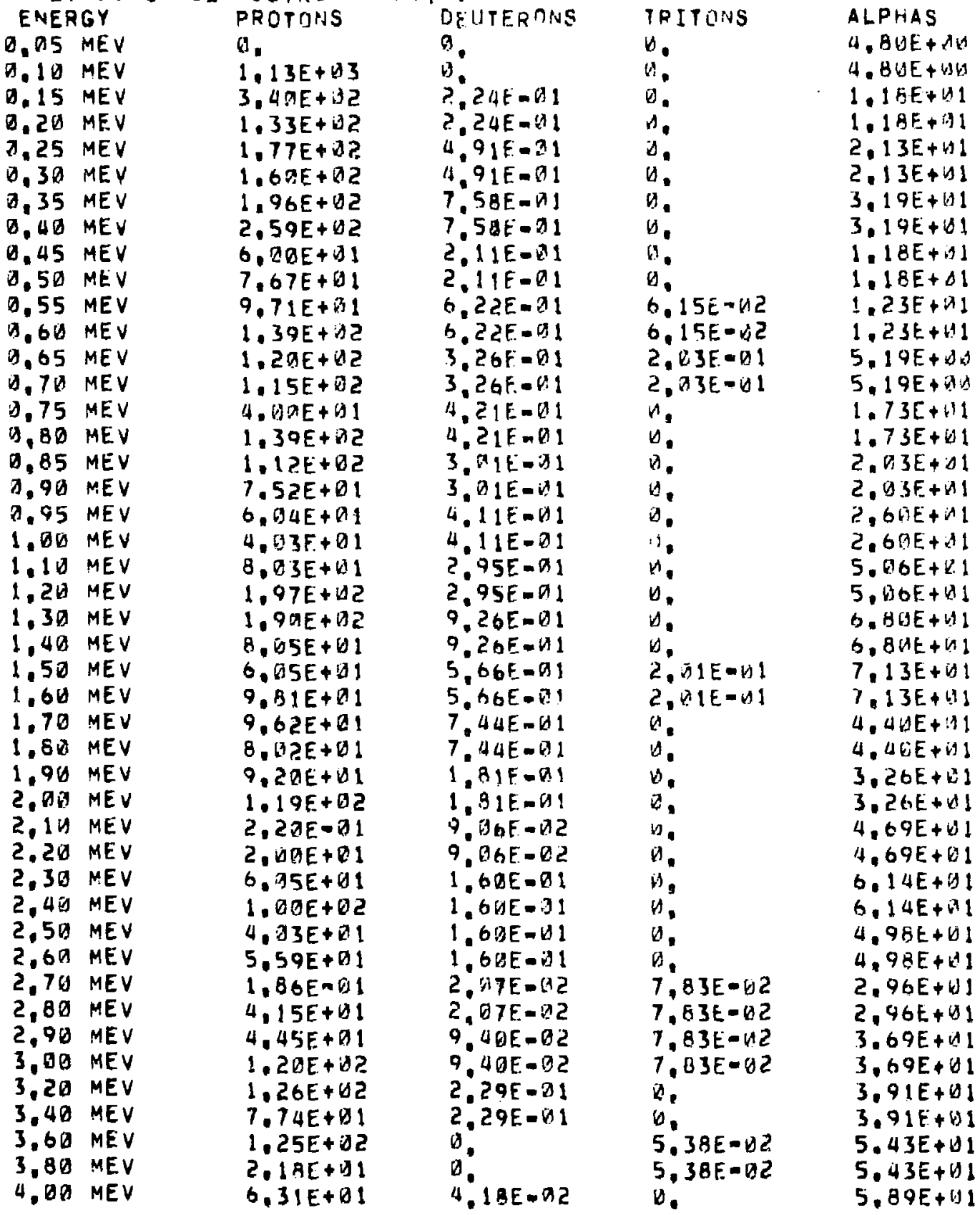


IINITIAL SPECTRUM AT O.OH-U,OS CH. FOR SHDNKA A-ISH PLASTIC 50 MEV D+ ON HE NEUTRON SPECTRUM

\section{ENER'GY}

4,20 MEV

$4,40 \mathrm{MEV}$

$4.60 \mathrm{MEV}$

4.BO MEV

5,01 MEV

$5,50 \mathrm{MEV}$

$6.0 O$ MEV

$6.50 \mathrm{MEV}$

7. OO MEV

7.50 MEV

ค. BU MEV

8.50 MEV

9. GD MEV

$9.50 \mathrm{MEV}$

19 OU MEV

11.00 MEV

$12,00 \mathrm{MEV}$

$13.02 \mathrm{MEV}$

14. $D 6$ MEV

15. OD MEV

16.0口 MEV

17. $00 \mathrm{MEV}$

18. OD MEV

$17.00 \mathrm{MEV}$

2D. DO HEV

$21.00 \mathrm{MEV}$

22.00 HEV

23.04 MEV

24.00 MEV

25, DO MEV

$26.00 \mathrm{MEV}$

$27.01 \mathrm{MEV}$

$28,00 \mathrm{MEV}$

$27.01 \mathrm{MEV}$

30. DU MEV

$32,00 \mathrm{MEV}$

34.00 MEV

36. DO MEV

38. OU MEV

4D, OU MEV

42.00 MEV

44.00 MEV

$46.00 \mathrm{MEV}$

48. OU MEV

50,00 MEV

HAVE, E(MEV)

$$
\text { PROTOIS }
$$

4. BUE+DI

3. $90 E+D 1$

1. $\triangle 2 E+12$

1. QDE +U

$2.26 E+B 1$

$0.25 E+D 1$

$1,8 U E+(1) 2$

$2.05 E+01$

$4,05 E+1$

$1.61 E+\square 2$

1. $619 E+02$

1. $2 Q E+B 2$

8. $D(G E+D I$

3. 5DE+ 01

E. OBE+U1

4. $14 E+11$

3. $) 3 E+(1)$

1. $19 E+\theta 2$

$5 . B 7 E+B 1$

$b$.

8. G(AE) + 1

2. $1 A E+B 1$

k.

A. ACE+Z1

4. MUE+ 1

a.

i).

6. :HQE+ 1

2. $U M E+D 1$

o.

b.

2., $\triangle B F+D 1$

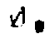

a.

6

,

a.

$\Delta$

:

$v$.

5.

a.

b.

i.

is.

9.82
DEUTERONS

4. $18 E-A 2$

a.

:.

(1.

D.

$1.12 E=-1) !$

$1.12 E=B !$

$1.35 E-B 1$

$1.35 E-1$

?. $15 \mathrm{E}-1) 1$

$2.15 E-O 1$

$1,62 E-D 1$

$1,62 E-131$

3. $42 E-0$ ?

$3,42 E-132$

म.

3.

is.

a.

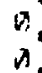

a.

is

is

is

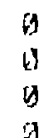

a

2

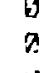

$$
\text { o }
$$$$
2
$$$$
2
$$

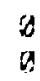

$a$

a.

a.

a.

a.

(1)

3.59
TRITONS

G.

u.

$\theta$

$\Delta$.

0.

a.

b.

if.

$4.46 E-W 2$

$4.46 E=92$

$4,42 E-b 2$

$4,42 E-02$

$9.56 E-192$

9.56E-DI2

4,51E-BDC

$4,51 E-52$

4.

v.

is:

4.

v.

L.

$\theta$

,

a.

0.

a.

0.

$a$

b.

a.

is.

$\theta$.

i.

is.

i.

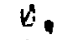

0.

$\theta$.

b.

a.

6.

6.

6.

7,24
ALPHAS

5.89E+11

$6.32 E+11$

$6,32 E+W 1$

3. $2 R E+U 1$

3. $2 \Delta F+\triangle 1$

$1.14 E+12$

$1.14 F+V 2$

3.7ИE+ 1

$3,7 U E+W 1$

2. $87 E+01$

$2.97 E+d 1$

3. $59 E+d 1$

$3.59 k+\Delta 1$

2. $32 E+V H$

2. $82 E+W v$

2. $1 W E+v v$

2. 1 AE + $A B$

$1.193 E+111$

$1, D 3 F+b 1$

1. $\Delta 2 E+\Delta 1$

1. $\triangle 2 E+\forall 1$

$\Delta$.

$\forall$.

$4.15 t-152$

$4.15 \mathrm{t}-\mathrm{UL} 2$

a.

$n$.

i.

a.

0 ,

n.

a.

0.

0.

8.

$n$

D.

6.

w.

a.

(1)

4.

a.

n.

$\theta$.

5,51 
1INITIAL SPECTRUM AT $9,0 J-6,10$ CM. FOR GHONHA A-15O PLASTIC 50 MEV D+ ON BE NEUTTRDIN SPECTRIIM

\begin{tabular}{|c|c|c|c|c|c|}
\hline 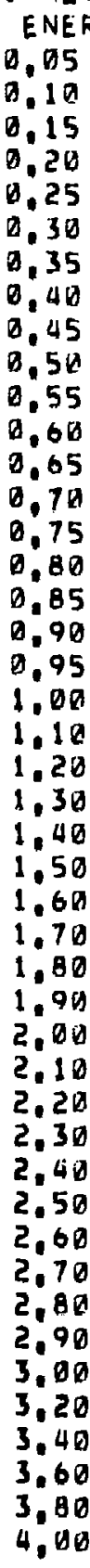 & 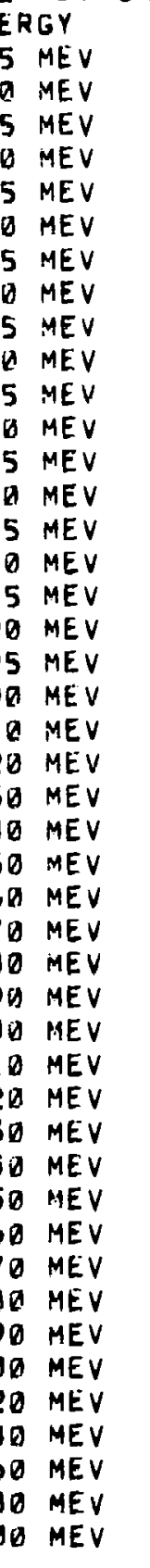 & 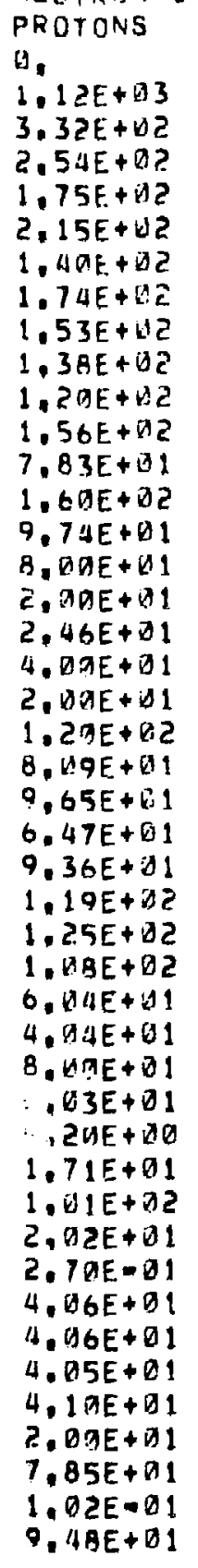 & 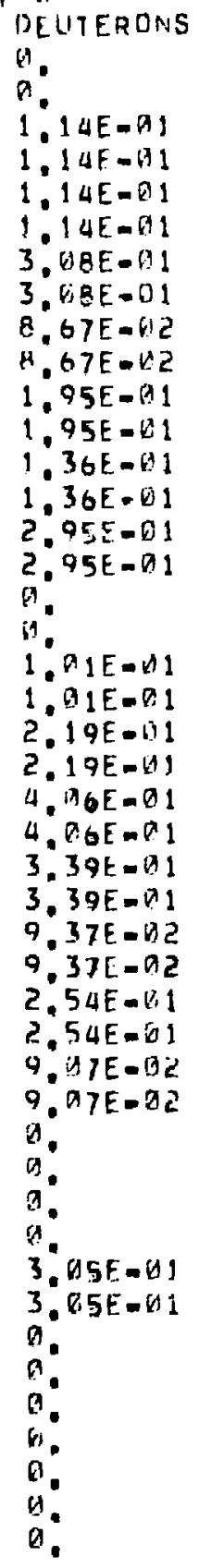 & 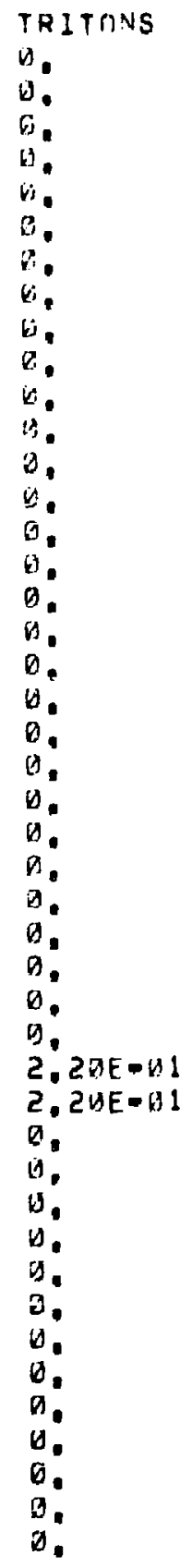 & 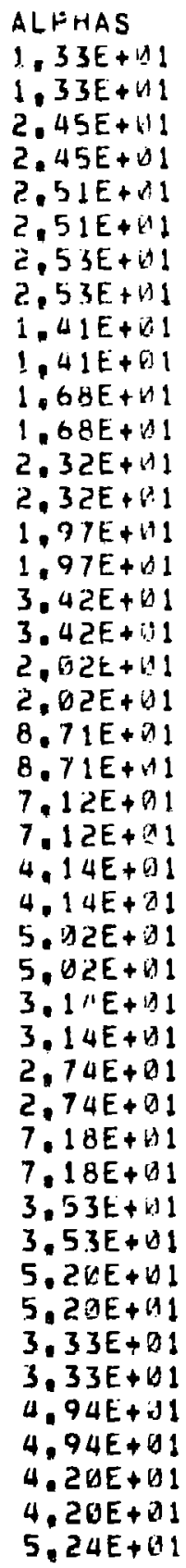 \\
\hline
\end{tabular}


1INITIAL SPECTRUM AT Q.65-V. IG CM. FQR SHONKA A-15E PLASIIC SA MEV D+ ON BE NEUTRON SPECTRUM

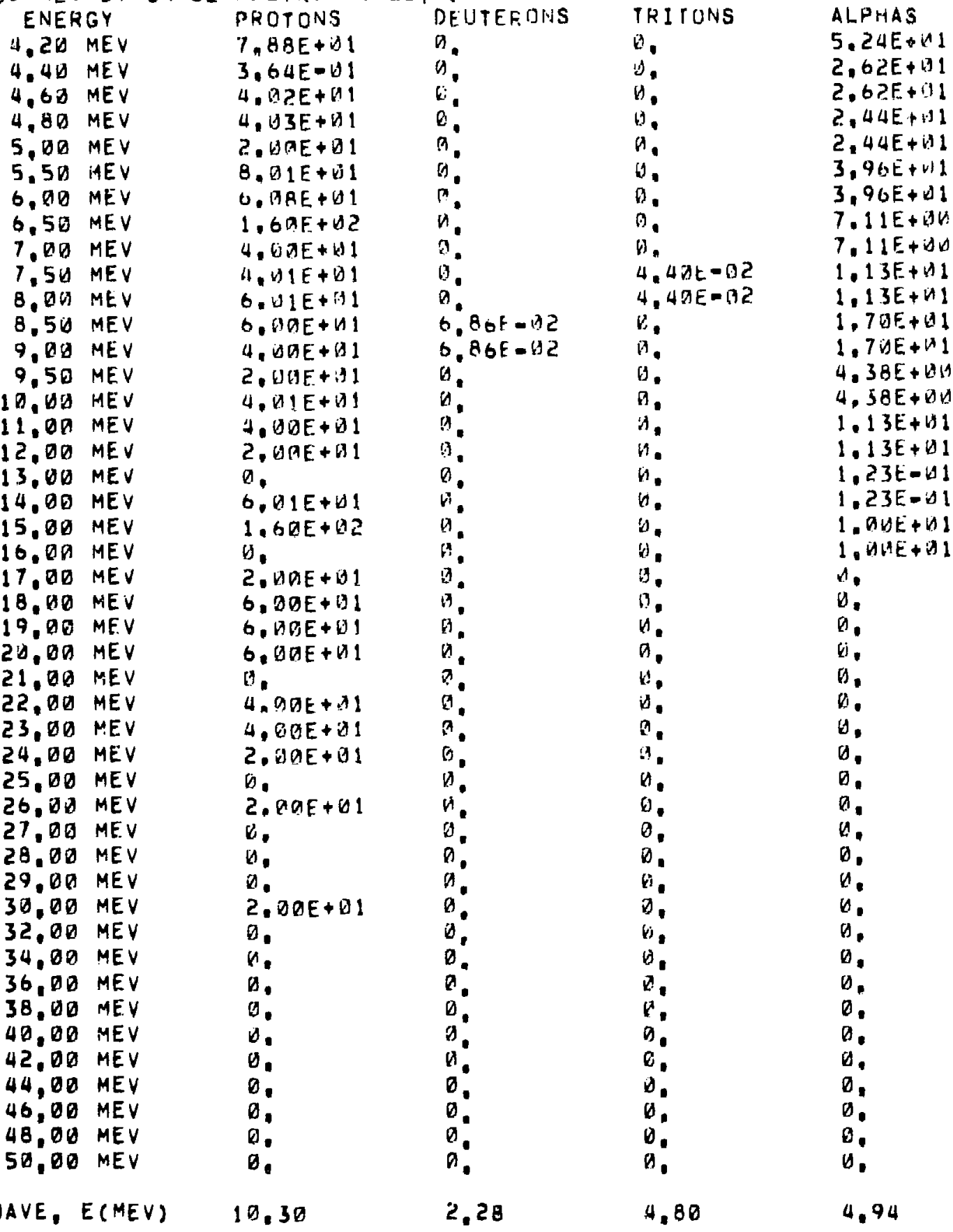


IINITIAL SPECTRUM AT M.1M-M.15 CM. FUR SHOHNA A-15O PLASTIC 50 MEV D+ DN HE HEUTRDN SHECPRUH

\section{ENERGY}

D. OS MEV

O. IV MEV

0.15 MEV

D.20 MEV

Q.25 MEV

D. 30 MEV

0.35 MEV

0.40 MEV

$9.45 \mathrm{MEV}$

$0.50 \mathrm{MEV}$

2.55 MEV

0,60 MEV

$0,65 \mathrm{MEV}$

0.70 MEV

0.75 MEV

O.86 MEV

D.85 MEV

$0.90 \mathrm{MEV}$

0.95 MEV

1, DD MEV

$1.10 \mathrm{MEV}$

$1,2 \square$ MEV

1.3 MEV

1,40 MEV

1.50 MEV

1,68 MEV

1,70 MEV

$1.9 \mathrm{~J}$ MEV

$1.91 \mathrm{MEV}$

$2,00 \mathrm{MEV}$

$2.10 \mathrm{MEV}$

2.20 MEV

2.39 MEV

2, 4S MEV

2,50 MEV

2,00 MEV

$2,70 \mathrm{MEV}$

2,80 MEV

$2.90 \mathrm{MEV}$

3. SO MEV

3,20 MEV

3.46 MEV

3,6U MEV

3, 80 MEV

4. DO MEV
PROTONS

i).

1. $115 E+43$

5. $11 j E+U ?$

3. $5 A E+D 2$

$3.55 E+42$

2. $69 E+10 ?$

$1.76 F+112$

$1.16 E+112$

$9.53 E+31$

$1.57 E+b 2$

$3.72 E+01$

$1.37 E+.12$

4. BGE+C)

3,7ME+d1

$3.73 E+n 1$

$2.33 E+1$

6. $14 E+31$

3. $B \cap E+ \pm 1$

1.79E+ D?

4. $: 22 E+41$

8. $k^{3} 3 E+31$

b. $2 A E+131$

$9.13 E+J 1$

7. B9F. d I

7. S9E+ U1

$4.15 E+61$

$1, G Q E+$ Q Q 2

8. $(13 E+1)$

6. $\triangle 2 E+D 1$

$9,78 E+U 1$

$1.89 E-11$

$1.18 E+12$

4.10E+ DI

4. $\angle B E-D 1$

$4.63 E-1]$

$2.35 E+00$

1. $42 F+B ?$

$3, B B E+D 1$

4. 25E+

4. $92 E+01$

$1.53 E+\theta 0$

$9.93 E+101$

$9 . B 7 E+21$

$4 . A 2 E+A 1$

4. $\theta Q E+D 1$
DEUTERIINS

r.

(i).

4. $34 E-B 1$

$4.34 F-w^{2} !$

$2,16 E-81$

2. $16 E-M 1$

4. $27 E-a 1$

4. $27 \mathrm{E}-\mathrm{HI}$

4. $6 \Delta F-r 1$

4. $6.1 E-n 1$

b. DUE $=$ A

6. $36 F=n 1$

4. $6 ! A=01$

11. $6 D F-A 1$

$2.1 \mathrm{BE}=\mathrm{Al}$

2. $13 F=01$

a.

a

$5.21 F=\| 1$

2. $21 F=01$

4. $91 \mathrm{E}-11$

$4.9 \mid E-11$

$9.73 E-C^{3} 1$

$9.73 E-H 1$

5. i $6 E-11$

5. $16 f-4: 1$

$9.15 \mathrm{~F}-12$

9.15F-te?

9. $2 z E-4 z$

9.22E-112

$1.46 F=1$

1.9bF-U1

9. $P 2 E=2$

$9.22 E-k 2$

i.

a.

it.

B.

b.

in.

13

$a$

in

10
TR」TUids

b.

b.

!)

W

6

6

Gi.

b.

is

in

i

b)

$\rightarrow$

it.

a.

r.

ii.

b)

4

b.

19

11.

*.

in

b.

$\therefore$

H.

k.

v.

bi:

月.

4.

$\forall$.

(1)

i.

".

$\Delta$.

$\theta$.

is.

a.

4

a.

$n$.

v.
AIPTAS

2. $57 F+N$

2. $57 E+19$

3. $38 \mathrm{E}=\mathrm{Wl}$

$3,5 \mathrm{Et}-\mathrm{J1}$

$2,71 E+16$

$2.71 E+w 6$

$2.43 E-41$

2. $43 E-11$

S. $46 E+19 t^{\circ}$

5. $4 u k+d k$

$4.32 E+11$

i), $32 E+11$

$1.3 \quad 3 E+11$

$1,3 i n+\cdots 1$

$1.76 E+11$

1.7hE+U1

2. $1 E+\cdots$

$2 \cdot 21 E+11$

$1.62 E+11$

$1, b 2 E+11$

1. $8 v E+11$

1. $8 n t+b 1$

$4.8(t+1)$

4. $81 t+191$

$3.55 E+11$

3. $55 E+11$

$3.33 \mathrm{E}+11$

3. V $5 E+1$

1.17E+D1

1. $17 E+1$

$3.6(\mathrm{UF}+\mathrm{Dl} 1$

$3.60 E+11$

2. 36E+ 11

2. $30 E+11$

4. $15 E+1$

4. $\mid 5 E+w 1$

3. $4 B E+6\}$

$3.48 E+11$

$1,62 E+b 1$

$1.62 E+111$

$4.23 E+? 1$

is, $23 E+1 !$

$3.18 E+11$

$3, \mid 8 E+b 1$

$5.57 E+11$. 


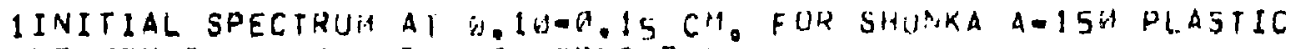
5D MEV D+ ON HE NE!ITEONA 3'RCTR!M

\section{ENERGY}

4.2. MEV

$4,40 \mathrm{MEV}$

$4.60 \mathrm{MEV}$

$4, B O \mathrm{MEV}$

5. HA MEV

$5.50 \mathrm{MEV}$

6. UV MEV

h. 5 A MEV

$7.99 \mathrm{AEV}$

$7.50 \quad M E V$

B. DU MEV

3.50 MEV

7. UW MEV

7. 5 M MEV

10.DO MEV

11,39 ME V

12. तN ME MEV

13. DD MEV

14, DU MEV

15. OU MEV

16. DU MEV

17. OU MEV

18.0D MEV

$19 ., D D M E V$

2\%. DY MEV

21. UW MEV

22. UU MEV

23. UD MEV

24. DU MEV

25. OA MEV

26. DO MEV

27. US MEV

29, DU MEV

29.00 MEV

$30,0 \pi \mathrm{MEV}$

$32.00 \mathrm{MEV}$

34. DU MEV

36.0 10 MEV

$38 ., D D$ MEV

40. WD MEV

42, DO MEV

$44.00 \mathrm{MEV}$

$46.00 \mathrm{MEV}$

$48,90 \mathrm{MEV}$

$50.90 \mathrm{MEV}$

GAVE, E(MEV)
PRUTIONS

$3.90 E+21$

$9.72 E+19$

$2.09 t+1$

$0.13 t+31$

$1.12 E+92$

5. $7 R E+D 1$

1.2AE+ 1 ?

$0.30 E+61$

4. WGE +UI

1. WGE+ II?

1. $2 A E+v 2$

B. HME+U1

4. $265+\ldots 11$

9. त7Fः:1!

6. ADE +DI

1. $\checkmark A E+U$ ?

1. $4 a E+b 2$

6. $A B E+31$

2, $D 1 E+11$

8. जीE + प1

6. $\triangle O E+1$

4. $3 a+n+b 1$

4. $9 a E+41$

$A, n a E+b 1$

B. $B \cap E+B 1$

4. $0: E+B 1$

i.

$v$.

2. $\triangle A E+B$

Z. $A E E+11$

n.

3.

2. $D A E+M 1$

i.

2. QUEE+ DI 1

a.

i.

i.

$n$.

Y.

$v$

i.

4.

.

i.

10.01
DEUTERINS

s.

(1)

13.

3.

$3.4 \triangle F-42$

$3,44 E-112$

a.

:

3.44t-31?

3. 4 UE - ? ?

3. $43 \mathrm{~F}-\mathrm{AC}$

$3.43 E-2$ ?

9 .

i.

$3.43 E-D 2$

$3.43 F-H_{2}$

(1)

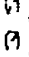

1

$a$

in

(1)

a

is

i)

is

9

a

$n$

B.

a

a

a

\section{a.}

\section{a.}

$$
\text { a }
$$

\section{a}

a

i.

2.43
TRITONS

A.

a.

$\therefore$

a.

i.

".

u.

4. $35 E-12$

$4.35 E-112$

i.

is.

i.

b.

i.

$v$.

$4.468-12$

4. $16 E-9$ ?

i.

b.

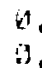

3

i.

il.

b.

$n$

A.

i.

i.

b.

is.

b.

i.

H.

is.

G.

$n$

19

$v$.

a.

$d$.

1.

$H$

i.

$y$

9.53
ALPHAS

$5.57 E+1]$

$1.77 E+91$

$1.77 E+11$

$5.48 E+11$

$5.4 a E+1$

$3,7 V E+11$

3. /11E+ +1 1

7. nut $E+(u) 1$

7. BAE + DI

$3, \theta|E+n|$

$3,87 E+31$

$2,52 E+11$

$2.59 k+11$

$1,64 E+4 t$

$1.64 E+10$

2. $19 E+i 1$

2. $19 E+21$

$4.0 D E-B C$

$4.6 B E-x \cdot 2$

$9.21 E=Q 2$

$9,21 E=x^{\prime} 2$

$4,18 E-12$

4. JHE-LC

it,

n.

is.

vi.

$v_{1}$

$d$.

vi.

19

B.

in.

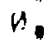

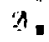

b.

is,

a.

a.

$n$

U.

$\forall$.

V.

$\Delta$.

0.

5.75 


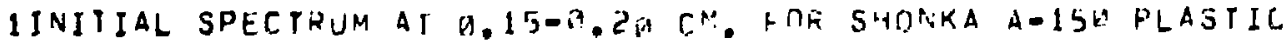
5D MEV D+ DN HE HEUTRON SPECTR!yP

\begin{tabular}{|c|c|c|c|c|c|}
\hline ENE & & 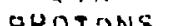 & DEUTFRDUS & TRITINS & AlPriAs \\
\hline 0.85 & HEV & u. & 3 & 6 & $7 . H \triangle E+1 ! k^{\prime}$ \\
\hline 10 & MEV & $1.01 E+63$ & H & $\because$ & $7 . \forall \angle E+\omega$ \\
\hline .15 & MEV & $0.37 c+112$ & $3.36 F-1$ & $\because$ & $1,7 \geq F_{2}+41$ \\
\hline 0.20 & MEV & $2 . B A E+\triangle 2$ & $D E-B 1$ & 6. & $1.72 E+1.1$ \\
\hline 0.25 & MEV & $2.390+12$ & $7 E=B I$ & $\overrightarrow{v i}$ & $9.5 A E+k 16$ \\
\hline 0.30 & MF V & $3.1 d E+k i$ & $3,37 \mathrm{E}-41$ & $v$. & $9.5: E+1: 6$ \\
\hline 0,35 & MEV & $1.9115+02$ & $3.7, E=A_{1}$ & $t^{1}$ & 4. $\left.K_{b} E+1\right\}$ \\
\hline 0.40 & MEV & $2.12 E+v_{i} ?$ & $3.7 \mid E-21$ & v. & 4. USE +1 \\
\hline 0.45 & MEV & 1. nine + n? & $2.22 E-B 1$ & 0. & $2.38 t+41$ \\
\hline 0.50 & $M E V$ & $1.1 \cap E+62$ & $2, P \geq F-1$ & $1 \%$ & $2.33 E+11$ \\
\hline 3.55 & IAE $V$ & 8. 49E +.19 & $? \cdot 1 \mathrm{E}-21$ & 4. & $2.018+61$ \\
\hline 0.61 & MEV & $7.77 E+\dot{B} 1$ & Z. $1 B F=01$ & $\therefore$, & $2.6|E+b|$ \\
\hline 0.65 & MEV & $1,21 E+32$ & $2.1 A E_{-}=11$ & $\because$ & $7.50 E+i b$ \\
\hline $0.7 \mathrm{u}$ & MEV & $3.85 t+41$ & $2,1 \dot{m} F=1$ & $\because$ & $7.58 E+66$ \\
\hline$\lambda .75$ & MEV & $2 . W B E+B 1$ & $3.11 F=1$ & 1 . & $3.22 t+1$ \\
\hline 3.84 & MEV & 4. $12 k+n 1$ & $11 F=1$ & a. & $3.22 E+111$ \\
\hline 0.85 & MEV & $6.0 .5 E+51$ & $1.7 \triangle E=b^{2} 1$ & b. & $1.39 E+[\pi 1$ \\
\hline 0.90 & $M E V$ & $5.87 t+61$ & $4.74 E-P 1$ & $\dot{v}$ & $1.39 E+61$ \\
\hline 0.95 & MEV & $8,0, E+61$ & $6.58 \mathrm{~F}-1.1$ & 1. & $1.25 F+n 1$ \\
\hline 1.01 & MEV & $7.91 \mathrm{E}+11$ & $38 F-0^{2} 1$ & 6. & $1.25 t+1 ! 1$ \\
\hline $1.1 \%$ & MEV & $1.79 E+12$ & $54 E-01$ & u. & $5.1 \angle E+1$ \\
\hline 1.215 & MEV & $5.91 E+61$ & $5 \triangle E=1$ & 0. & $5.14 E+1$ \\
\hline 1.35 & MEV & $6.5 P E+11$ & $7.1 \mathrm{~B} E-01$ & $E$ & $3.59 E+11$ \\
\hline 1.40 & ME V & 1. BOE + J2 & $i \cup F=A$ & 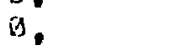 & $3,59 E+: 11$ \\
\hline 1.50 & MEV & $1.05 E+A ?$ & $7 E-01$ & H. & $3, \||E+1|$ \\
\hline 1.60 & $M E V$ & $7.93 F+Z 1$ & $37 t=k \cdot 1$ & is. & $3 . v 1 E+1$ \\
\hline 1.70 & MEV & $2 . M B F+01$ & 7.7 . & $2.17 E-W 1$ & 4. $82 E+1: 1$ \\
\hline 1.88 & MEV & $1.772-12$ & GEE $=P 1$ & 2. $19 \varepsilon-11$ & $4.82 E++1$ \\
\hline 1,919 & MEV & $9.58 F+11$ & $2 F-B I$ & 落. & $1.76 E+k 1$ \\
\hline 2,00 & MEV & $8,11 E+11$ & $92 f-11$ & n. & $1.70 f+n 1$ \\
\hline 2.10 & MEV & $2 \cdot \geqslant 3 E+1$ & $r$ & 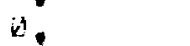 & $5.13 E+01$ \\
\hline 2.21 & MEV & $6.12 E+21$ & $\theta$ & $w$ & $5.12 t+11$ \\
\hline 2.30 & MEV & $4,66 E+\{1$ & $3 \Delta E-112$ & $d$ & $4,72 E+31$ \\
\hline 2.47 & MEV & $7.94 E+8.1$ & $9.31 \mathrm{E}=n 2$ & 6 & $4.72 E+11$ \\
\hline 2.50 & MEV & $2.13 E+01$ & $1 . D Q E-Q 1$ & $9,64 E-102$ & $2.95 E+61$ \\
\hline 2.69 & MEV & 1. & $1.6 M E-O 1$ & $9.6 \triangle E-\angle 2$ & $2.95 E+131$ \\
\hline 2,71 & MEV & $6.62 E+01$ & a. & b. & $75 E+d !$ \\
\hline 2.60 & MEV & $6.05 E+61$ & 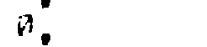 & G. & $2.75 E+11$ \\
\hline 2.90 & MEV & $2 . \triangle O E+b 1$ & $n$ & $9.64 E-022$ & 4. $12 E+11$ \\
\hline 3.00 & MEV & $2 . \triangle B E+61$ & $n$ & $9.64 E-62$ & $4.12 E+61$ \\
\hline $2 A$ & MEV & $3.91 E+D 1$ & $4.12 E-22$ & $5.29 E-W 2$ & $3.15 E+v i ?$ \\
\hline 3.40 & MEV & $2.25 E+01$ & $4.12 E-92$ & $5.29 E-62$ & $3.15 E+11\}$ \\
\hline 3,60 & MEV & $2.21 E+D 1$ & & b. & $1.99 E+01$ \\
\hline $\begin{array}{l}3.8 \text { is } \\
4.00\end{array}$ & $\begin{array}{l}\text { MEV } \\
\text { MEV }\end{array}$ & $\begin{array}{l}5.90 E+111 \\
4.01 E+01\end{array}$ & $\begin{array}{l}4 \\
2\end{array}$ & b. & $\begin{array}{l}1.99 E+11 \\
3.57 E+11\end{array}$ \\
\hline & & & & & \\
\hline
\end{tabular}


IIVITIAL SPECTRUM AT A.15-6.2W CM. FOR SHOHYA A-15G PLASTIC SA MEV D+ ON AE NFUTLTA SFECTRUM

EN!ERGY

4.26 MEV

4. 4. MEV

4.60 MEV

4.81 MEV

5. OU MEV

$5,5 U$ MEV

$6, H O M E V$

$6.50 \mathrm{MEV}$

7. HE MEV

$7.5 \square$ MEV

9. 4 . MEV

8.50 MEV

$9 . E D$ INEV

$9.5 \%$ MEV

$1 \%, D N M E V$

11. DQ MEV

12. OD MEV

13,00 MEV

14. SIS MFV

$15 . \triangle S$ MEV

16. BD MEV

17. OO MEV

18. OOQ MEV

19. DO MEV

$2 A, O D$ MEV

21 , OU MEV

22. BA MEV

23. 19 MEV

24. DU MEV

25, OA MEV

26. DU MEV

27.00 MEV

28, BD MEV

29, 0 M MEV

$37.01 \mathrm{MEV}$

32.00 MEV

34. OU ME:

36, OD MEV

38 , UU MEV

$4 D, D B$ MEV

$42,00 \mathrm{MEV}$

44. OD MEV

46.00 MEV

48. DO MEV

50.00 MEV

DAVE, E(MEV)

$$
\text { PROTOMS }
$$

1.85E- 1

$3.5 R E+11$

$6.86 E-111$

2. $45 E+1$

9.5 2 H +1

4. 19E + ${ }^{A} 1$

1. $20 E+192$

6. UUE $U+1$

?. WRF + 1

3. $52 F+41$

C. AटE+ 1

8. $11 E+U 1$

b. KI $R E+E I$

b.

2. MQE + I I

4. D7E + DI

1. $4[E+02$

2. $(1)+E$ ?

6. $06 E+D 1$

2. HSE + U1

1. $2 a E+12$

2. $M A E+\mid T 1$

$\theta$

t.

4. BAE + 11

v.

C. $\mathrm{B}^{2} \mathrm{AE}+\mathrm{x}^{2} 1$

a.

4. वUEE+BI

2. WUE + W1

2. VIAE + W1

?. WOE + (I)

2. $) A E+b I$

a.

2. DAE + DI

$\theta$.

$\Delta$.

2. ARE E E 1

$\Delta$.

$\theta$.

U.

D.

0.

a.

Q.
DIFUIFRUAS

?. $99 \mathrm{~F}-\mathrm{DI}$

7. 2 HE $=A 2$

7. PLE - A '

$1 .\{4 \mathrm{~F}=1$

$1,1+F=1$

3. $+3 F=n 2$

3.4 SE -82

6. F5E-l? 2

$0.65 f-k 2$

3. $34=-1$

3. $94 E-U 1$

$6.67 \mathrm{E}-\mathrm{HZ}$

$6.87 F-92$

1. $A 3 E-6 i 1$

1. $\triangle 3 E-Q !$

1. MTE-V:1

1. $A F-D 1$

in.

b.

ค.

a.

1.

A

a.

i).

a.

a.

a.

$n$

a

a.

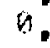

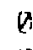

v

$\nabla$

$a$

b

a.

$a$

$a$

a.

$B$

0

4.66
TRITONS

ii)

1. $3 A E-H 1$

1. $3 A F-$ is

is.

4

B. $814-12$

$5.81 \mathrm{t}-\mathrm{t}^{2} \mathrm{C}$

if

i)

i.

ti.

i.

b.

$8.92 E-192$

$8.92 t=42$

i).

i.

$4.51 E-412$

is $51 t=12$

w.

0

as

in.

in

v.

b.

a.

w.

v.

b.

b.

b.

v.

เ)

b.

b.

E.

$\theta$.

b.

o.

a.

a.

b.

4.

0.

7.05
ALHHAS

3. $57 \mathrm{~F}+19$

1. $58 t+11$

1. $58 \mathrm{BE}+111$

2. 7 BE + E 1

2. $70 \mathrm{E}+\mathrm{HI}$

$2.63 t+31$

2. $63 E+1$

$3.65 t+11$

$3.65 E+131$

?. BDE +. 11

$2,6(1) E+M 1$

4. $56 E+11 \%$

4. $5 E E+144$

3. $10 E+\Omega^{2} 0$

3. $40 E+10 x^{2}$

2. $27 E+11$

$2.27 E+x^{\prime} 1$

1. B $2 E+10$

1. $42 E+46$

$1.66 E=1$

1.66E-111

$4.15 F-142$.

$4.15 \mathrm{E}-\mathrm{L}^{4} 2$

$3,94 E-.12$

$3.99 E-W 2$

a.

b).

H.

in.

D.

G.

11.

is.

$n$

n.

O.

a.

$v$.

b.

V.

0

0

i.

a.

b.

5.34 
1INITIAL SFECTFUM AT $9.2 U-4.4 C C^{M}$, FOR SHOHAA A-1SD PLASTIC 5B MEV D+ ON RE NEUTQTN SPECTRUM

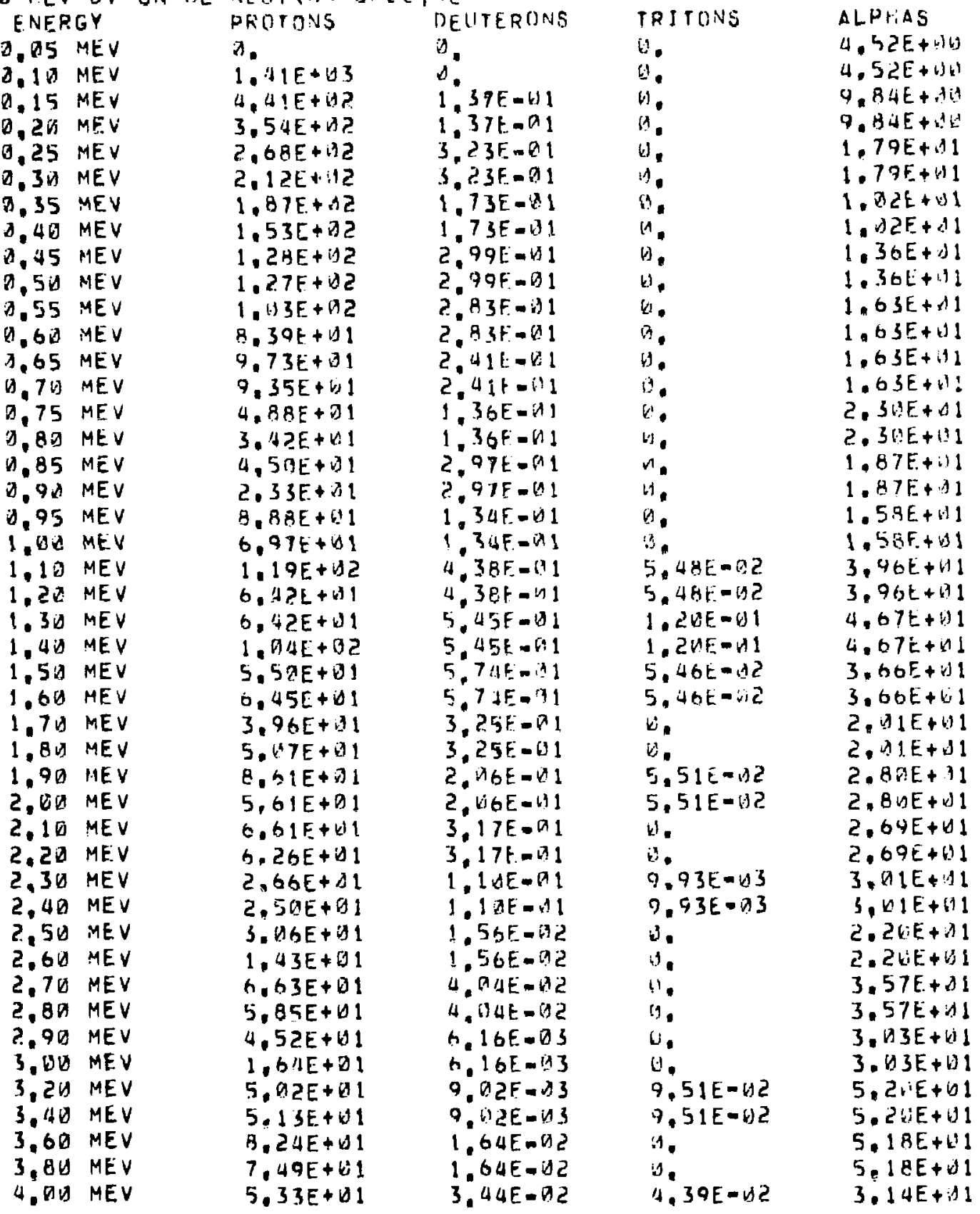


1INITIAL SPECIRUM AT M. 2H-B, UI CH. FOR SHUNKA A-15: PLASTIC 5D MEV D+ ON BE NEUTRMN SPECTRUM

\section{ENERGY}

4. 20 MEV

4. 4. MEV

4. Si MEV

4.80 MEV

5. 7.3 MEV

$5.5 H$ MEV

6. .91 HEV

6.50 MEV

7. UV MEV

7.5 MEV

8. IU MEV

8. 5 Q MEV

9. BU MEV

$9.5 \%$ MEV

1D. BO NEV

11. HO MEV

12. BO MFV

13. UO MEV

14. 13 MEV

15. DO MEV

16. OD MEV

17. US MEV

1B. VIS MEV

14.0O MEV

2). DU MEV

21. UU MEV

22. AN MEV

23. AD MEV

24. DU MEV

25, OU MEV

26. VU MEV

27. DO MEV

23.DO MEV

29. VO MEV

$30,0 D M E V$

32, OU MEV

34.06 MEV

$36,00 \mathrm{MEV}$

38. BU MEV

40.QD MEV

U2, DO MEV

44,00 MEV

46.DO MEV

4月, OU MEV

$5 \partial .00 \mathrm{MEV}$

DAVE. E(MEV)
PFITTHAS

3. $A 7 E+61$

7. $12 F+11$

3. उAE+HI

S. $5 \mid E+i 11$

4. AME + WI

1. $A 6 E+02$

8. $4\{E+A 1$

$5.39 E+B 1$

$6.9 R E+U 1$

4. $34 t+61$

$5.74 E+131$

5. Ma $+x 1$

1. w $44 E+B)$

G. VOCE + H1

E. $91 E+D 1$

1. $A 5 E+13$ ?

R. U। $1 E+4 !$

5. $93 E+11$

5. $46 E+k 1$

7. WOE + $\because 1$

5..) $A E+11$

3. $5 \cap F+L I$

3. 기 $E+$ a 1

5. $98 E+11$

4. $50 E+A 1$

2. $57 E+\lambda$

4. $B A E+b 1$

1. औ

1. $5 \pi E+41$

1. $50 \mathrm{~F}+\mathrm{k}^{3}$ :

1. WAE + J1

i.

n.

a.

a.

A.

(i)

$v$.

5. HAE + DH

a.

b.

b.

4.

v.

9.75

\section{DFUTEROI:S}

3. $4 a t=1 c^{2}$

$1.90 \mathrm{AH}$

$1,9 M E-02$

$7.85 E-U C$

$7.85 E-0.2$

2. $99 \mathrm{E}-\mathrm{AC}$

2. $995=$ H?

1. $1 F-n 2$

$1.81 \mathrm{~F}-\mathrm{BI}$

$4.39 F=112$

$494-112$

$6.94 E-02$

6. ता E - I2

$1.71 E-\because ! 2$

$1.71 E-A^{2}$

1.71E- I?

$1.71 E=02$

$i$

i)

$\theta$

n.

$r$.

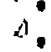

b.

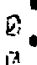

.

$a$

n.

$r$

is.

i

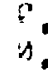

$A$

P)

$n$

a.

a.

a.

b.

i)

2.95

\section{TPITONS}

4. 39E- U2

is.

n.

n.

$\because$

$2.93 \mathrm{~L}-42$

$2.93 E-\Delta Z^{2}$

i.

b.

$1.11 E-12$

$1.11 \mathrm{E}=\ln 2$

a.

19

$1,12 E-12$

$1.12 E-42$

$1.11 E-12$

1. $11 E-\| \bar{E}$

प!

k.

d)

i).

at

i.

in

b.

b.

o.

4

i.

in.

w.

a.

i).

0

il.

t'.

1.

$v$.

(i).

(1).

0.

i.

$i$

v.

4.03
ALF"RAS

$3,14 E+111$

$3.55 E+11$

$3.55 E+11$

3. $63 E+d 1$

$3.835+61$

$4.5 N E+: 11$

$4,5, E+41$

4. $26 E+111$

$4.2 h t+11$

2. $35 F+41$

$2,35 E+11$

1. $45 E+.1$

1. $45 E+1$

$7.34 t+164$

$7.34 E+19.9$

1.7 viE. +1

1. $7 E E+H 1$

1.5HE + WG

7. $5 B E+B^{3} V$

6. $M A E-W 2$

6. $P B E-112$

2. $50 \mathrm{HE}+\mathrm{HA}$

?. $5 v^{0} E+J n$

0.

b.

a.

is

.

().

in.

v.

A.

V.

$\because$

n.

a.

v.

i.

n.

v.

i.).

b.

H.

0.

0

5,78 
IINITIAL SPECTRUM AT Q.4HEG, HA CH. FGF SHOINKA A-15A PLASTIC 5V MEV D+ ON BE NEUTRON SPLCTRIIO,

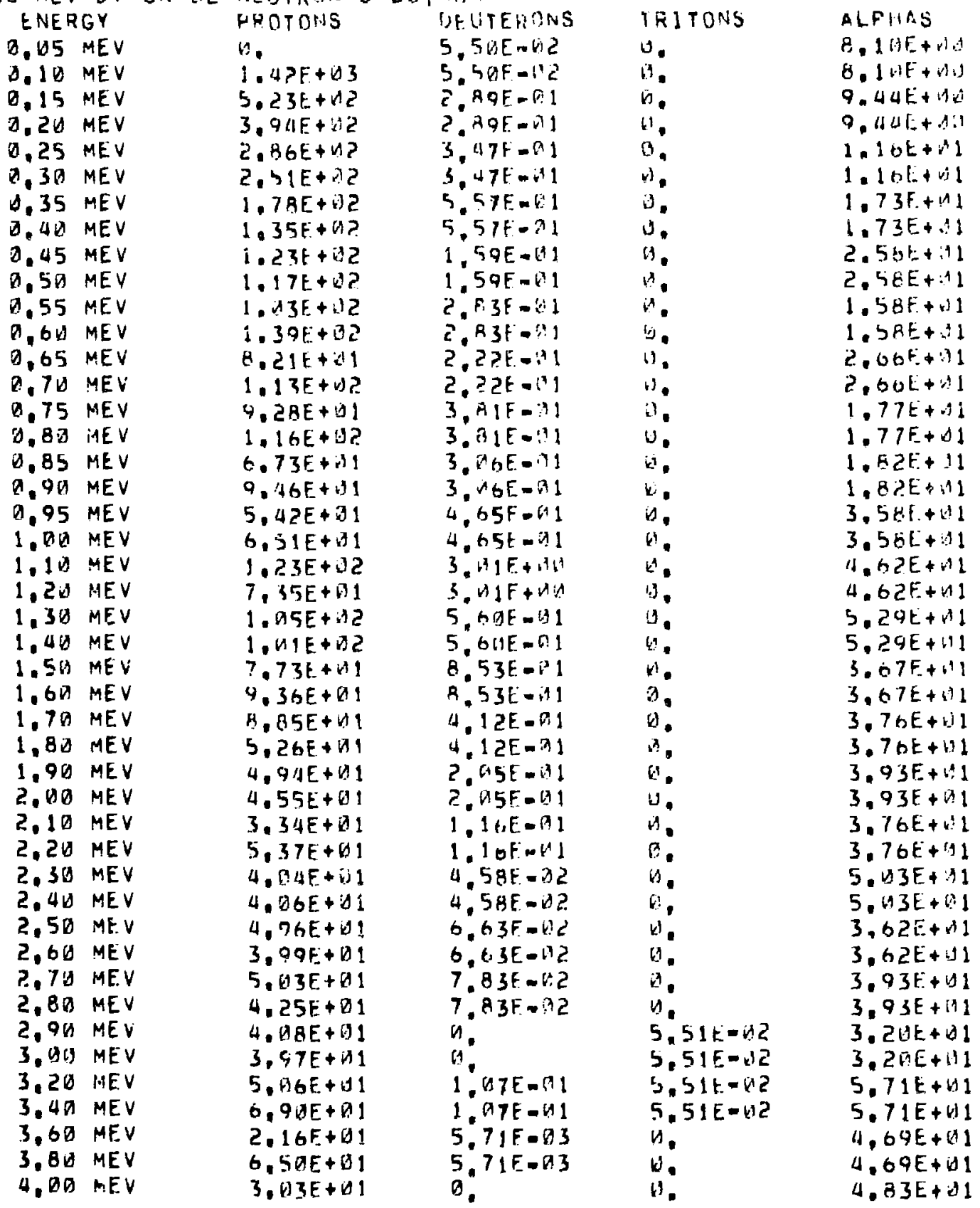


IINITIAL SPECTRUIA AT $9.46-0,6$ GN. FIIN SHOSKA A-15G PLASTIC 53 MEV D+ ON BE WEUTRON SPECTRIII

\begin{tabular}{|c|c|c|c|c|c|}
\hline ENER & GY & PROTGISS & DF I TFFONS & TFITONS & $A L P H A S$ \\
\hline 4.20 & MEV & $5,09 E+11$ & $\pi$ & $n$ & 4. $83 t+71$ \\
\hline 4.40 & MEV & $4.69 E+\Delta 1$ & $n$ & $\Delta$ & $4.07 E+v 1$ \\
\hline 4.60 & MEV & $7.40 \bar{E}+11$ & $a$ & $m$ & $4.67 E+b 1$ \\
\hline 4.87 & MEV & $3.87 E+61$ & U & $2.27 t=n ?$ & $3.02 E+111$ \\
\hline 5.01 & MEV & $5.91 E+61$ & ta & $2.27 E-192$ & $3,62 E+11$ \\
\hline 5,50 & MEV & $7.7 B E+D 1$ & 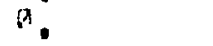 & $2.27 \mathrm{~L}=\mathrm{U} 2$ & $5,91 \bar{E}+w 1$ \\
\hline 6.90 & MEV & $7.97 F+01$ & $v_{1}$ & $? .27 E-G 2$ & $5.91 E+\Delta 1$ \\
\hline 6.50 & MEV & $6.3 \cap E+1$ & a. & a & $4.15 E+111$ \\
\hline 7.00 & MEV & $6.71 E+61$ & $A$ & $i$ & $4.15 E+11$ \\
\hline 7.56 & MEV & $6.95 E+.11$ & $n$ & 14 & $2.71 E+11$ \\
\hline a.nus & MEV & d. $8 \geq E+11$ & $i A$ & $\because$ & $2,71 E+31$ \\
\hline 8.56 & ME V & $7 . H E+W 1$ & $5.53 E-12$ & $\mathrm{~N}$ & $1.8 \Delta E+v 1$ \\
\hline 9.60 & MEV & $5.67 E+11$ & $5.53 E=142$ & $!$ & 1. BSE + H1 \\
\hline $9.5 n$ & MEV & $9.32 E+1: 1$ & $2.76 F=112$ & $\because 4$ & $7.49[+61$ \\
\hline $0 ., n$ & MEV & $5,83 F+\angle 1$ & $2.76 F-A ?$ & $\omega$ & $7.49 t+x^{2}$ \\
\hline .00 & MEV & $5.49 E+01$ & $2.76 E-142$ & $H$ & $1.52 E+101$ \\
\hline - bin & MEV & $7.98 E+D 1$ & $2.76 F=02$ & in & $1.52 E+41$ \\
\hline .00 & MEV & $B .73 E+b 1$ & 0 & $\therefore$ & $2.6 B E+.6$ \\
\hline .60 & MEV & $4.50 E+111$ & $n$ & $\Delta$. & $2.68 E+110$ \\
\hline OO & MEV & $3.50 E+: 1$ & 9 & $\therefore$ & $4.41 E-02$ \\
\hline .00 & MEV & 7.UEE + 1 & 4 & v. & $4 . b 1 t=.2$ \\
\hline .00 & MEV & $4.40 E+01$ & $\ddot{n}$ & $r$. & 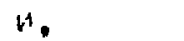 \\
\hline 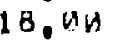 & MEV & 3, W & is. & bit, & 19 \\
\hline$\Delta B$ & $M E V$ & 4. $A E E+4$ & A. & th. & 14. \\
\hline 40 & MEV & 4.5 AE $+E^{2} 1$ & H. & $1 \%$ & Q). \\
\hline.$\Delta B$ & MEV & $5.6 E+11$ & $n$ & w. & $b$. \\
\hline $.0 n$ & MEV & $2.50 F+11$ & $\theta$ & 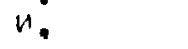 & !. \\
\hline 00 & MEV & $2 . W A E+B 1$ & 6 & it & M. \\
\hline$B D$ & MEV & . & 8 & $A$ & $n$. \\
\hline 00 & ME.V & 5.QAE+A17 & $i$ & 4 & 4 \\
\hline 10 & MEV & 1. DAE +K1 & 4 & $b$. & 0. \\
\hline$\theta 0$ & MFV & $1.5 A E+1$ & 8 & H. & $n$ \\
\hline$O B$ & ME V & 0. & B. & $n$ & W. \\
\hline$\theta 0$ & MEV & $b$. & $\theta$ & $a$. & $\theta$ \\
\hline$\pi 0$ & MEV & $v_{0}$ & $H$ & b. & u. \\
\hline$\theta 0$ & MEV & $5 . \ln B E+w(1)$ & n. & u. & $n$ \\
\hline$O V$ & MEV & 5, SUE + UA & $n$ & 0. & $H$ \\
\hline U日 & MEV & $5 . U A E+\square G$ & 5 & a. & 6. \\
\hline 00 & MEV & in. & ה & 9. & 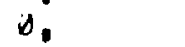 \\
\hline 10 & MEV & i. & i). & $n_{0}$ & $n$ \\
\hline$D B$ & MEV & A. & 3 & v. & $\Delta$. \\
\hline 40 & MEV & 5.BOE+ DO & 0. & 月. & 1 \\
\hline 00 & MEV & 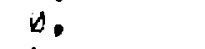 & 6. & 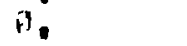 & in. \\
\hline & MEV & $n$. & . & a. & D. \\
\hline$\Delta 0$ & MEV & $n$ & o. & it. & is. \\
\hline$E$ & $E(M E V)$ & 10.14 & 1.98 & 4,18 & 5.13 \\
\hline
\end{tabular}


IINITIAL SPECTRUM AT M.OAH1. OQ CH. FIR SHOHKA A-I5H PLASTIC 5D MEV D+ ON BE NFUITRDN SPECTR!!M

ENERGY

B. 05 MEV

$0.16 \mathrm{MEV}$

0.15 MEV

$0.20 \mathrm{MEV}$

0.25 MEV

0.31 MEV

$0.35 \mathrm{MEV}$

1. 40 MEV

$0.45 \mathrm{MEV}$

0.50 MEV

0.55 MEV

$0.60 \mathrm{MEV}$

0.65 MEV

0.70 MEV

0.75 MEV

$0.80 \mathrm{MEV}$

0.85 HEV

0.90 HEV

0.95 MEV

1. OO MEV

$1.13 \mathrm{MEV}$

1.20 MEV

1.315 MEV

1. 4V MEV

1.5. 0 MEV

1.GU MEV

1.70 MEV

$1.80 \mathrm{MEV}$

1.90 MEV

2. $00 \mathrm{MEV}$

2.10 MEV

2.20 MEV

2.30 MEV

2,40 MEV

2. 50 MEV

2,60 MEV

2.70 MEV

2,80 MEV

$2.90 \mathrm{MEV}$

3. DU MEV

3.20 MEV

3.40 MEV

3.60 MEV

3.8I MEV

4. $\triangle D$ MEV
PROTONS

i.

$1.43 E+\$ 3$

$5.24 E+42$

$4.06 E+102$

3. $25 E+42$

3. $\cap 1 E+12$

2. $23 E+0 ?$

$1.98 E+22$

$1.74 E+112$

1. 7 BE + U2

$1.8\lfloor E+82$

1. $33 E+12$

1. $29 E+1 D 2$

1. $99 E+$ मी

8. $47 E+\square 1$

$9.82 E+b 1$

$7.4(E+c) 1$

$7 . D 3 E+A 1$

$7.18 E+11$

7. $59 E+\Delta 1$

1. $11 E+42$

1. $3 b E+i] z$

1. $33 E+132$

1.17E+ 12

1. $13 E+12$

7. $11 E+B 1$

$6,63 E+D:$

$0.43 E+01$

$5.92 E+1$

4. $94 E+B 1$

$5.79 E+1$

$3.53 E+01$

4. $83 E+111$

$5.516+11$

3. AQE $\$ 1$

$4.74 E+D 1$

3. $17 E+B 1$

3. $51 E+B 1$

3. $45 E+11$

3. $4 Q E+U 1$

$9.33 E+01$

5. $16 E+B 1$

$6.62 E+01$

5. $34 E+i 1$

4. BOE + II
MEUTERDNS

1. $4 G F-\triangle 2$

1. $16 E-U 2$

$1,11 E-A 1$

$1.11 E=\| 1$

$1.73 E-O 1$

$1.73 E-a 1$

2. A5E-AI

?. $55 F=1$

1. $96 E-01$

$1.96 E=01$

2. $215=91$

2. $21 E-91$

1. $45 E-91$

1. $+5 F-31$

2. UGE-A!

2. $\because 6 E-O 1$

? 3 IF $A I$

2. $31 F-n 1$

3. 39F- $=1$

3. 39E-D 1

$5.53 E-B 1$

$5.53 E=A 1$

4. M9E- 11

4. $) \cap E-A 1$

$4.52 F-41$

$4,52 F-U 1$

2. $31 E-61$

?. $31 E-31$

$1.95 E-91$

$1.95 E-8.1$

$5.43 E-42$

5. $\triangle 3 E-V I 2$

2. $77 \mathrm{~F}-\mathrm{HL}$

$2.77 \mathrm{E}-1.2$

2. $87 \mathrm{E}-112$

2. $87 \mathrm{E}-42$

4. $57 E-62$

$4.57 E=02$

U.

a.

5. $5 F-62$

5. D5F- II

$1.22 E-61$

1. $22 E=01$

$4.17 E-02$
TRITINS

n.

v.

13.

b.

i)

4

5. $\triangle \triangle E D-\triangle 3$

$5, D \triangle E-W 3$

A.

$\pi$

d.

B.

in.

a.

b).

b.

b.

b.

is.

H.

$2.48 E=32$

$2,48 E=11$ ?

2. $43 E=42$

$2.43 t-02$.

$5.17 E-42$

$5.17 t-k 2$

9.

a.

$2.59 E-102$

2. $59 E-152$

!!

4.

n.

b.

3. $74 \mathrm{E}-18$ ?

3.74E- $\lambda 2$

?. $64 E-02$

$2.64 E-12$

1. 5 hE $-\not 2$

1. $56 E-H 2$

2. $43 E-\Delta 2$

$2.43 E-62$

$4,8 \geq E-\triangle 2$

$4.82 E-112$

4.
ALPHAS

$7.6(E+: 1 W$

$7.61 E+d k$

1. $2 A E+\square 1$

1. $20 E+11$

$1,55 t+111$

1. $55 E+.11$

1. $27 E+11$

1. $27 E+11$

1. $27 E+d 1$

1. $27 E+111$

1. $19 E+11$

1. $19 E+1$

$1.89 t+11$

$1.89 \mathrm{~F}+11$

$1.62 F+1$

1. $62 E+.11$

2. $14 E+B \mid$

2. $14 E+31$

1. $5: J E+11$

1. $50 t+41$

3. $75 E+41$

S. $75 E+01$

3. $B 2 E+H$

$3.82 E+41$

2. $35 E+1$

2. $35 t+61$

2. $88 E+11$

2.88E+11

3,2 Q $Q E+A 1$

3. 24E+U!

3. $U 3 E+11$

3. $13 E+11$

$2.37 E+41$

2. $37 E+11$

3. तो $1 E+11$

3. $01 E+01$

2. $|4 E+4| 1$

2. $14 E+n)$

2. $03 E+d$

2. $\{3 E+11$

4. $B 4 E+D I$

4. BUE:U1

$3.76 E+D I$

$3.76 F+U 1$

3. 4 VIE 41 
IINITIAL SPECTRUM AT D.6A-1, QD CM. FOF SHUNKA A-15G PLASTIC SO MEV D+ ON HE NEUTRON SPECTRUM

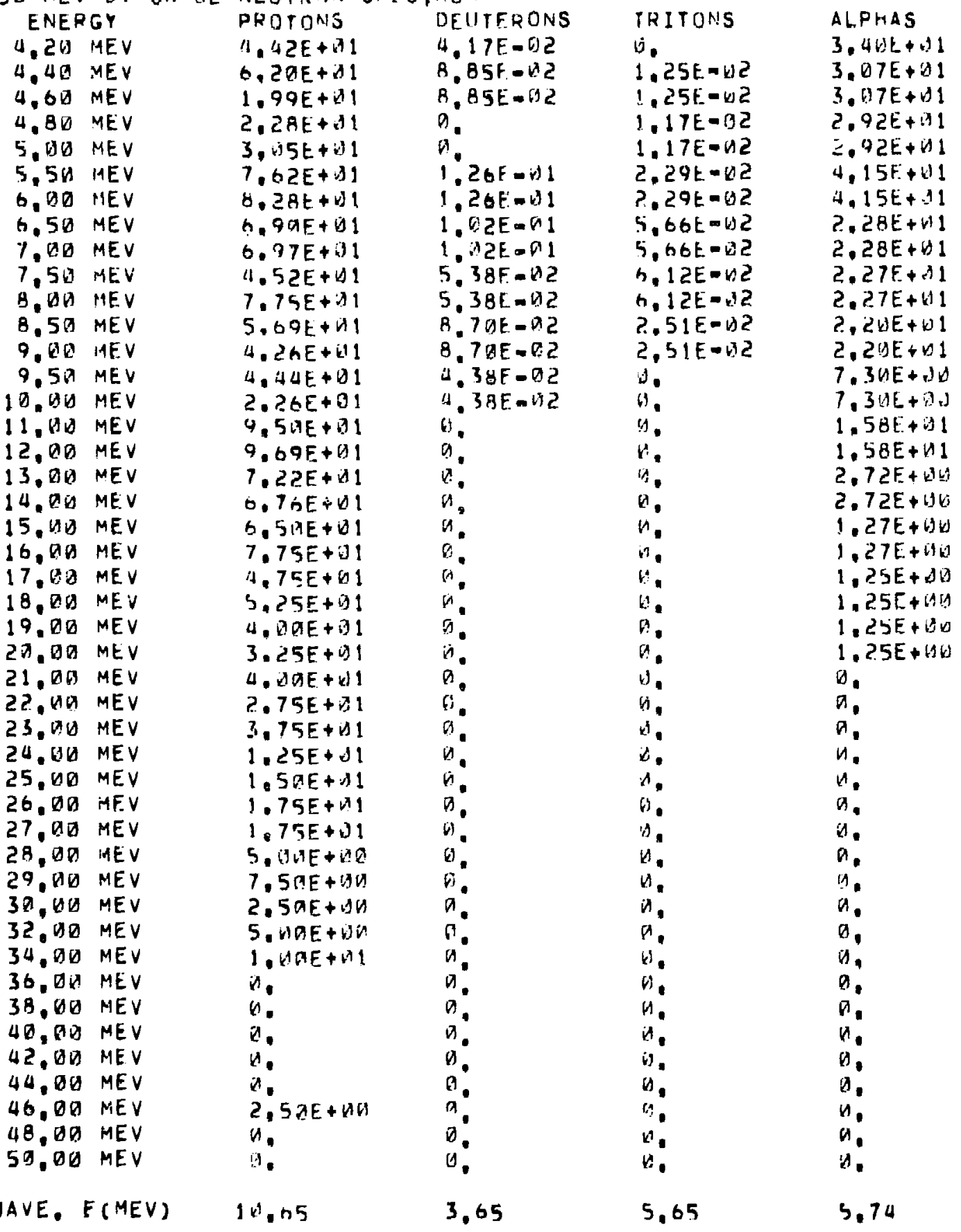


IINITIAL SPECTRUM AT 1, HV-2.AB CH. FOR SHONRA A-15U PLASTIC 50 MEV D+ ON HE NEUTRON SPECTRUM

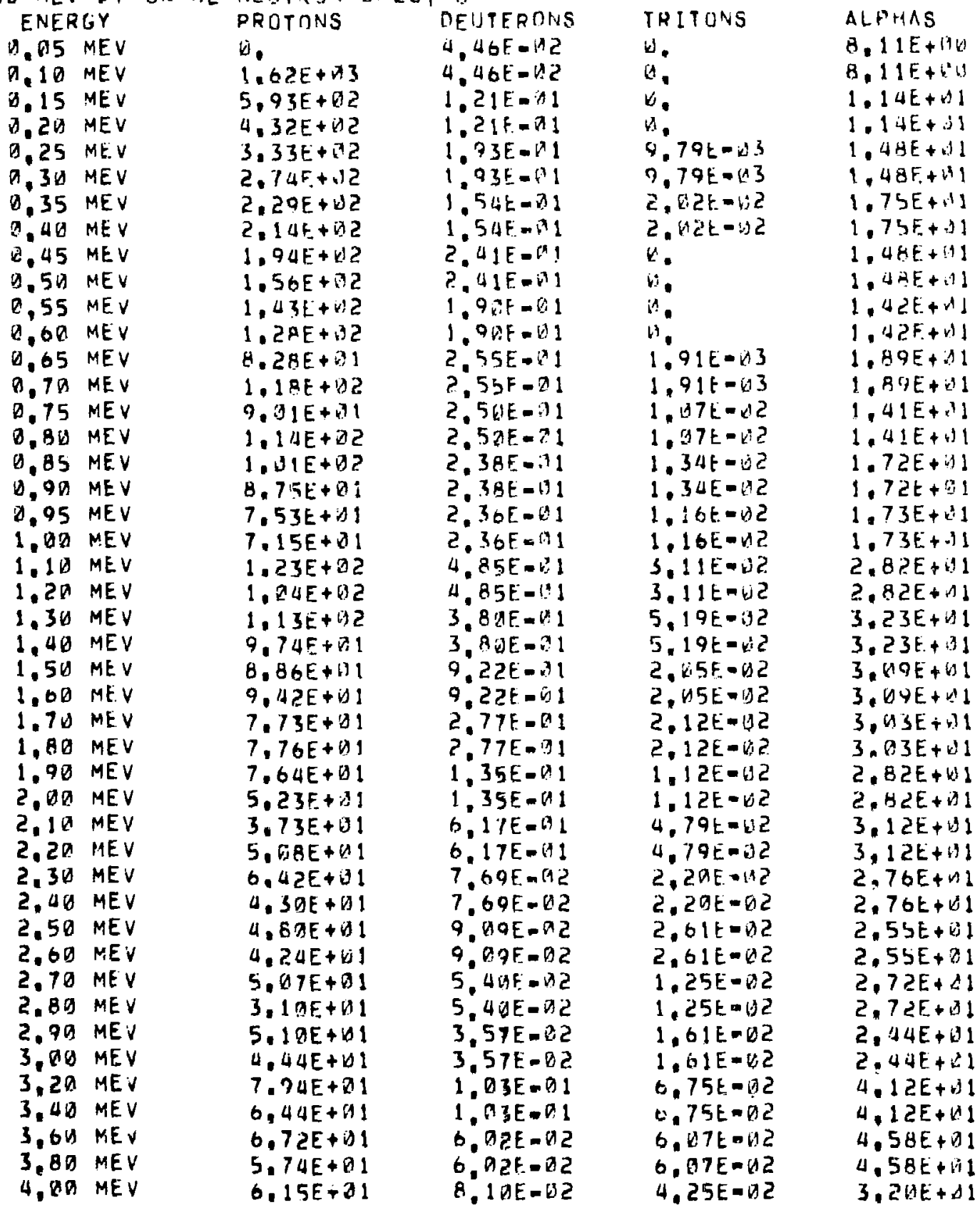


IINITIAL SPECTRUM AT 1, MH-2, 9G CM. FOR SHOMKA A-15H PLASTIC 5D MEV D+ ON BE NEUTRON SPECTRUIM

\section{ENERGY}

4. 2 D MEV

4. 40 MEV

4.60 MEV

4.BO MEV

5. $\angle O$ MEV

5.50 MEV

$6.2 U$ MEV

6.50 MAV

7. $10 \mathrm{MEV}$

7.50 MEV

8.00 MEV

$8.50 \mathrm{MEV}$

$9.00 \mathrm{MEV}$

9.50 MEV

10.00 MEV

11. RQ MEV

12. $O D$ ME V

$13.60 \mathrm{MEV}$

$14.00 \mathrm{MEV}$

$15.00 \mathrm{MEV}$

16. 10D MEV

17. DO MEV

18.0O MEV

$19.00 \mathrm{MEV}$

$20.0 \mathrm{HEV}$

$21.00 \mathrm{MEV}$

22. B MEV

$23.00 \mathrm{MEV}$

$24.00 \mathrm{MEV}$

25. OD MEV

$26.00 \mathrm{MEV}$

27.02 MEV

$28.00 \mathrm{MEV}$

29. 90 MEV

$30.10 \mathrm{MEV}$

$32.00 \mathrm{MEV}$

$34.00 \mathrm{MEV}$

$36.60 \mathrm{MEV}$

$36.00 \mathrm{MEV}$

$40.00 \mathrm{MEV}$

$42.00 \mathrm{MEV}$

44. 00 MEV

46. OO MEV

$48,00 \mathrm{MEV}$

50, UO MEV

DAVE, E (MEV)

\section{PROTCNS}

4. $65 E+B 1$

$5.43 E+131$

4.12E+ 121

4.8AE+M1

3. $4 A E+31$

9. $55 E+31$

7. 1 RE + SI

8. $46[+x] 1$

6. $.3 E+91$

$7.3 h E+: 11$

$7.17 E+111$

8. $26 E+v 1$

5. $94 E+\Delta .1$

$5.45 E+21$

$4.98 E+31$

1. $\triangle 4 E+\pi 2$

$9.41 E+01$

7.96E+ 91

5. $8 B E+1$

5. $87 E+\square 1$

$4.50 E+D 1$

$5.86 E+b 1$

3. $79 E+b 1$

2. 7 AE + 11

3. $40 E+B 1$

2.8 $8 E+\Delta 1$

1. $62 . E+B 1$

2. $2 A E+1$

1. $30 E+01$

8. $\angle B E+U V$

9. DgE + ba

6. IGE + Wh

6. JUE + DB

3. $.0 Q E+D C$

b.

6. DOE+DD

B, UAE + $\triangle A$

3. $\triangle O E+O E$

2. DOE + OQ

b.

1. $\triangle O E+D$

2. AOE + BG

i.

$\Delta$

0.

9.77

\section{DEUTEROAS}

8. 1 AF.

$6.05 E-9 ?$

6. Q5E-kं

$4.28 \mathrm{~F}=32$

$4.28 E-f$ ?

$1.24 \mathrm{E}=!^{A} 1$

1. $24 E-01$

6. $0.9 E-1$

6. OQ - E I

1.16E-1

1. $16 E=M 1$

$6.59 F-0 ?$

$6.59 F-V^{2} 2$

$7.22 t-C^{\prime} c$

$7.22 E-A 2$.

2. $B \zeta E-A ?$

ट. BउE- $A 2$

1. $7 E-A 2$

$1 \div(17 E-1) 2$

1.71E $=13$

1.71E-A3

1

i.

a.

$\theta$

D.

a.

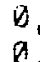

a.

a.

D.

i.

$\theta$

a

0

is

0

0

$a$

$a$

4.65
TRITONS

$4.25 E-02$

$8.01 E-13$

B. QIE- $1 E 3$

2. 9 GE - ज2

2. 3 A $=\triangle$ ?

5. $28 E-112$

$5,28 E-k .2$

$5.35 E-\mathrm{U} 2$

$5.65 E-112$

3. $\triangle 6 E-14$ ?

3. $26 t=12$

$6.91 k-k^{3}$

$6.91 E-03$

2. $611 E-b 2$

$2.64 E-12$

$6.73 E-65$

$6.73 E=33$

$6.78 E-G 3$

6, 7PE- 3

in

0.

v.

1).

a.

$b$

!

b.

0.

4

0

b.

is

io.

a.

0

0

0

$b$.

b.

i.

a.

D.

b.

$b$.

b.

5,65
ALPHAS

3. $2 . U E+01$

$2,65 E+71$

$2,65 E+61$

2. $21 E+21$

2. $21 E+i 1$

4. $83 E+11$

1., $83 E+c, 1$

$3.12 E+d 1$

$3 . \mid 2 E+11$

2. $17 E+1$

2, $17 E+11$

$1,27 E+\ln \mid$

1. $27 E+.1$

1. $15 E+1$

1. $15 E+1$

$7.85 E+06$

$7.85 t+17 n$

$4.73 E+3 i 4$

$4.73 E+b u$

2. $M R E+$ W

2. ABE + HA

1. $B U E-D 2$

1. $64 \mathrm{E}-112$

$3,63 E-133$

$3.83 E=03$

$A$.

b.

a.

is.

$a$.

o.

a.

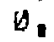

$v_{n}$

$b$.

$a$

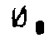

$\theta$

b.

ne

a.

$\theta$

$\theta$

a.

a.

5.43 
1IN]TIAL SPECTRUM AT 2,BG-4. TA CM. FOH SMUNKA A-1SG PLASTIC 5D MEV D+ ON BE AEUTRON SPECTKUM

\section{ENEAGY}

1. 55 MEV

0.10 MEV

0.15 MEV

Q.20 MEV

D. 25 MEV

$0.30 \mathrm{MEV}$

0.35 MEV

$0.43 \mathrm{MEV}$

0,45 MEV

0,50 MEV

0.55 MEV

0.60 MEV

3.65 MEV

U.7U MEV

1.75 MEV

$\triangle .8 \% \mathrm{MEV}$

0.85 MEV

0.912 MEV

0.95 MEV

1. DO MEV

$1.10 \mathrm{MEV}$

$1.20 \mathrm{MEV}$

1.30 MEV

$1.40 \mathrm{MEV}$

$1.50 \mathrm{MEV}$

$1.60 \mathrm{MEV}$

$1.70 \mathrm{MEV}$

$1.80 \mathrm{MEV}$

1.90 MEV

2,DU MEV

2.10 MEV

2.?B MEV

2. $3 U$ MEV

2. 40 MEV

2.5D MEV

2.611 MEV

$2.70 \mathrm{MEV}$

2.80 MEV

2.90 MEV

3. DU MEV

3.20 MEV

3.40 MEV

3.6D MEV

3.84 MEV

4.DV MEV
PROTONS

ง.

$1.71 E+63$

$0.75 E+0 ?$

$4.91 E+122$

$3.82 \mathrm{E}+42$.

$2.89 E+02$

2. STE + th2

2. $\triangle 8 B E+\triangle 2$

$1,93 E+17 z$

1. $80 E+82$

1. $48 E+172$

$1.26 E+112$

1. $20 E+D 2$

1. $12 E+12$

1. $12 E+M 2$

1. $U 1 E+\Delta 2$

$9.89 E+111$

8.: $9 E+01$

$7.91 E+21$

$7, Q 2 E+B 1$

$1.32 E+M 2$

$1.17 E+102$

1. $19 E+D 2$

3. $45 E+101$

9.83E+A1

7. $35 E+U 1$

8. $65 E+01$

$7.69 E+01$

$7.49 E+61$

$5.44 E+11$

$5,87 E+B 1$

$6.64 E+D 1$

6. $\{4 E+\Delta\}$

$4,43 E+B I$

$5,08 E+11$

$4.19 E+11$

$4,02 E+\square I$

$4.89 E+y 1$

$4.35 E+01$

$4.25 E+01$

$5,98 E+61$

6. $75 E+11$

$6.67 E+01$

5. $26 E+01$

$6.13 E+U 1$
VEUTEROHS

$1.66 E=112$

$1.66 E-02$

8. $52 E-A 2$

$9.52 E-A 2$

$1.52 E-11$

$1.02 F-U 1$

$1.84 F=01$

$1,945=01$

2.11E-O1

?. 11F-ज1

2. $2 F=1$

2. $A 2 E-A 1$

?. ALE-VI

2. $94 E-P ?$

2. $20 E-U 1$

?. $26 E-G 1$

$2,11 E-11$

2. 1 IE-U 1

2. $20 E-1$

?. 2 SE $=1$

4. $17 E-A 1$

$4.17 E-91$

$3.63 F-01$

$3.63 \mathrm{~F}-111$

3. BGE-91

$3.86 F-V 1$

1.96E-O1

$1.96 \mathrm{~F}-01$

$1.33 F=n !$

4. $33 E-\pi !$

9. $25 \mathrm{E}-1 \mathrm{C2}$

$8.25 E-k i 2$

$3.47 \mathrm{E}-02$

3. $\triangle 7 E=112$

$3,35 t-01$

$3.35 E-A 1$

$3.72 \mathrm{E}-\mathrm{AC}$

$3.72 E-142$

2. 7 GE- 11

2. $7 \mathrm{bE}-A 1$

?. 5ZE-112

2. $52 \mathrm{~F}-92$

$4.45 E-02$

4. $45 E-6: 2$

?. $41 F=92$
TR:TUNS

b

is.

ive

is.

bi.

b.

(1).

1. $22 E=42$

1. $22 E=17$ ?

is.

3 .

ij.

A.

$4.55 E-6: 3$

$+.55 t-13$

2. $D 8 E-\backsim 3$

2. BRE- 43

4. $85 E-43$

$4,85 E=43$

2,,$O E=01$

2. GUE- 11

5. $19 E-W 3$

5. WOE-W 3

$5,67 E-U 3$

$5.67 t-13$

1. $56 E-B 2$

$1.56 E-W 2$

$1.11 L=62$

$1,11 E=42$

2. 38t-112

2. 38E- 12

1. $52 E-42$

$1.52 E-62$

1. 7 RE-112

$1.78 E=\square 2$

1. $43 E-n 2$

1. $\triangle 3 E=112$

$9,87 E=43$

$9.87 t=63$

$4.6 \Delta t-112$

4. $6 U E-42$

$1.76 E-B)$

$1.76 E-122$

2. $13 E-h i 2$
$A L F+A S$

9. $\left.33 E+H_{k}\right)$

6. $33 E+13$

1. $26 E+11$

1. $26 E+61$

1. $29 E+11$

1. $29 E+11$

$1.53 E+k i 1$

$1.53 E+\cdots 1$

i. $02 F+i$

$1.62 E+11$

$1.61 E+U 1$

1.61E+ 11

1. $72 E+11$

1. $72 F+11$

$1.79 E+41$

$1.79 E+41$

1. $83 E+11$

1.B3E+ $B 1$

1. $7 k^{\prime} E+k^{4} 1$

$1,7 b E+W 1$

3. $55 E+61$

$3.55 F+1$

$3.65 E+11$

$3.65 E+.11$

$3.41 t+31$

$3.41 E+71$

$2.97 t+11]$

$2.97 E+61$

$3.14 E+11$

$3,14 E+11$

3. $19 E+1$

3. $19 E+11$

3. 12E+ 11

$3,12 E+41$

3. $2 B E+M I$

3. $28 t+11$

$2.73 t+11$

2. $73 E+41$

2. $B 5 E+11$

$2.85 F+U 1$

$4.06 E+11$

4. hot + U1

$3.95 E+x 1$

$3.95 E+11$

$3.44 E+01$ 


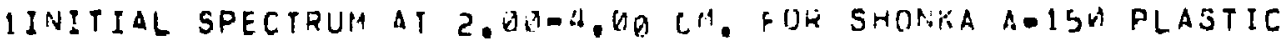
5A MEV D+ ON BE IFITRDA SPECTH(I"

\section{ENERGY}

4. $2 D$ MEV

4.40 MEV

$11.60 M ! . V$

4. BI MEV

5.DV MEV

5.50 MEV

$6.0 D M E V$

$6.5 D$ MEV

T. O. MEV

$7.50 \mathrm{MEV}$

8. $A O M F V$

8.51 MEV

$9.7 D M E V$

$9.50 \mathrm{MEV}$

13.OV MEV

11. OQ MEV

12. DD MEV

13. BU MEV

14. A MEV

15.0D MEV

16.0U MEV

$17 . A G$ MEV

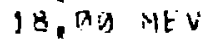

14. DU MEV

20. OO MEV

2!. OA PIEV

22. UO MEV

23. UD MEV

$24.00 \mathrm{MEV}$

25. UD MEV

$26,0 \Omega$ HEV

27,0 MEV

23.OO HEV

29. QO MEV

3ब. MO IAEV

32. OD MEV

34. DO MEV

36. DO MEV

38.00 MEV

4D, HO MEV

42. OH MEV

44. OO MEV

46.00 MEV

48.0O HEV

$50.00 \mathrm{MEV}$
PROTOAS

4. 3? 201

4. $56 E+d 1$

$3.5 ! 1 E+11$

4. 5 AE+U1

$3.83 E+1$

8. $54 E+11$

$9.55 E+01$

$7.53 E+b 1$

1. $42 E+b 1$

$6.7+1 E+1$

$D, 2 ? E+b 1$

D. $M 9 E+W 1$

6. $24 E+D 1$

4. $99 E+1$

5. $04 E+21$

Bं. $39 \mathrm{~F}+$ + 1

$7.61 E+01$

h. 2 HE + U 1

5. $57 E+11$

5.64E+ +1

3. $89 E+61$

4. $08 E+1$

3. 5 HIE +31

2. $35 t+1$

2. $50 E+\lambda 1$

$2.65 E+111$

3. $19 E+01$

$1.85 E+11$

1. $45 E+01$

1.,$a E E+1$

$1, n a E+D 1$

1. $I \pi E+b 1$

1. $05 E+41$

4. 6) $3 E+A B$

1. DAE + WE

5. 5 HE + b.

1. $50 E+6.6$

2. $190+A G$

I. IVF+ $O Q$

b.

5. BUE-OI

1. MAE+ OU

3.

4.

U.
DEUTEROAS

2. $41 E-42$

$3.13 E-1$

3.13F-O1

2. $71 E-112$

2. $71 E-92$

1. $12 E-41$

1. $12 \mathrm{~F}-1$

5.77E- 22

$5.77 \mathrm{E}=\Delta 2$

4. 39E-U2

4. 39F- 12

3.62E- 112

3. $62 \mathrm{~F}-\mathrm{n} ?$

?. 5 AE-A?

?. 5 UE $=42$

$6.17 E-6$

$6.17 E-M 5$

$0.25 E-G$

ค. ?5E- 5

B. $55 E-134$

8. $55 E=$ W

b.

is.

b.

it.

i.

4

i

a.

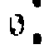

1.

a.

b.

i.

D.

(1)

4.

4

3.

a.

9

0.

3.40
TRITOHS

2. $13 E-\Delta 2$

$1.56 E-6 j 2$

$1.56 \mathrm{t}-02$

$1.76 E-32$

1. 7GE M Uू

$2.25 E-\Delta 2$

ट. $25 \mathrm{E}-\mathrm{W} 2$

$P .42 E=\| ?$

$2.42 E-n 2$

is. $35 E-13$

8. 55E- 3

i. $22 E-112$

$1.22 E-13$

2. $27=-13$

‥ $27 f-5$

?. $24 t=23$

?. $24 E-63$

1. $13 E-613$

$1.13 E-r^{4} 3$

).

i)

a.

V.

Vi.

4.

b.

D.

b.

4.

b.

v.

iv

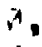

1.

U.

U.

1.

(A.

W.

v.

H.

1

0

1

U.

4.16
ALPHAS

$3.44 E+11$

3. $14 E+41$

3.11E+O1

2. $5 B E+\square 1$

2. $53 t+11$

$4.6 n E+\square 1$

4. $60 E+U 1$

$2,95 E+d 1$

2. $98 E+11$

2. $24 E+11$

2. $2.4 E+d 1$

$1.58 E+111$

1. $5 H E+H 1$

I. $U \backslash E+b \mid$

1. $H] E+W 1$

1. $|b E+v|$

1. $16 E+V 1$ !

5. 36E+1U

5. 3oE + $1 k^{4}$

2. $03 E+14$

2. $U 3 E+6 U$

$7.53 E-\Delta 1$

$1.53 E=1$

2. $54 E-V 1$

2. $54 E-41$

0.

a.

4.

a.

b)

vis

b.

a.

U.

\&

v.

G.

A.

i.

b.

0.

4.

d.

4.

9.42

5.55

OAVE. E(MEV) 


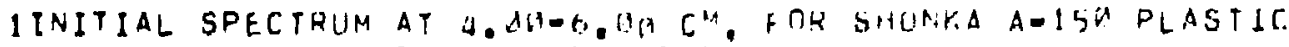
S) MEV D+ DN BE NFUTR II! SPECTR\|PA

\section{ENERGY}

1. OS MEV

1. 1D MEV

D. 15 MEV

$0.20 \mathrm{MEV}$

$0.25 \mathrm{MEV}$

2.3 MEV

0.35 MEV

$0.4 W$ HEV

$0.45 \mathrm{MLV}$

0.50 MFV

0.55 MEV

0.60 MEV

$0.65 \mathrm{MEV}$

$0.70 \mathrm{MKV}$

3.75 MEV

3.80 MEV

0.85 MEV

0.96 MEV

0.95 MEV

1, Q6 MEV

$1,10 \mathrm{MEV}$

$1,20 \mathrm{MEV}$

1,3 MEV

$1.40 \mathrm{MEV}$

1.5U MEV

1,60 MEV

1.70 MFV

1. BH MEV

$1.90 \mathrm{MEV}$

2. OU MEV

$2,10 \mathrm{MEV}$

2.20 MEV

2.3 M MEV

2.40 MEV

2,50 MEV

$2.60 \mathrm{MEV}$

$2.70 \mathrm{MEV}$

2.8D MEV

2.90 MEV

3.90 MEV

3.20 MEV

3.40 MEV

3.613 MEV

$3.80 \mathrm{MEV}$

$4 . \triangle 9$ MEV

\section{PRUTONS}

W.

$1.71 E+43$

$0.69 E+42$

14. 9 वरE+ त

$3.42 E+32$

$2,74 E+J 2$

2. $3 A E+D 2$

1. $94 \mathrm{E}+12$

$1.815+d ?$

1. 5 AE + U?

$1.47 E+142$

1. $29 t+02$

1. $45 E+63$ ?

1. $13 E+62$

1.1 1 1 + V2

9. MAE + DI

$8.5 B E+H 1$

B. 32. E + HI

B.91E+U1

$7.58 E+1$

1. $43 E+112$

$1.23 E+0 ?$

$1,18 E+192$

1. Got + is?

9. $29 E+b 1$

1. $5 E+2 ?$ ?

$7.97 E+B 1$

$7.87 E+d 1$

B. $12 E+v 1$

$6.46 E+D 1$

5. $54 E+U 1$

5. $72 E+\triangle 1$

$5.85 E+\Delta 1$

$5.60 F+U 1$

4. $41 E+41$

$4.17 E+b$ !

$4.49 E+131$

4. $53 E+(1) 1$

3. $23 i+01$

$3.925+b 1$

$0.87 E+11$

6. 9 ME + DI

$6.12 F+11$

5.17E+01

$5.79 E+71$
DFUTERORS

2. 5GE-U2

$2.50 E=112$

1. $95 F=01$

1. HSF- 1

1. $\triangle Q F-D I$

1. $\triangle 9 E-M 1$

$1 . \& T E-A 1$

1. $87 E=A 1$

1. $65 E-11$

1. hjF-nI

4. $33 F=0$ !

$4.33 E-D 1$

?. $67=99$

2. I:7E-OI!

1.6QE - .1

$1.605-01$

$1.53 F-D_{1}$

$1.53 F=V 1$

$1.72 F=61$

$1.72 E-O 1$

4. $21 E-91$

4. $2 \backslash E-A 1$

3. 7 BE - 1

3.7RE-BI

$3.53 F=1$

$3.53 t-31$

5. $f A F-A 1$

5. ARE-H1

3.7 $\triangle \mathrm{DE}-\mathrm{B}$

3.7 पE $=01$

$6.93 E-92$

6. $93 E-1 \mathrm{C}$

5.7 7 形 - A?

5.7.1E-

4. औIF- И?

$4 . Q 1 E-W ?$

$4.8 A E=62$

4. $8 \mathrm{at}-0 \mathrm{Z}$

2. $32 E=02$

$2.32 E-102$

$6.52 E-02$

6. $52 E-02$

2. $74 \mathrm{E}=01$

2. $74 E-D ! 1$

4. $16 E-02$
TRITUNS

$\therefore$

N.

b.

F.

a

$v$

v.

A.

b.

4.

$6.91 t-135$

$6.31 t-03$

1. in $3 E-142$

1. $\triangle 3 E=\| d 2$

Q. $04 E-64$

a. BUE-W4

1. HZE-UC

1. $\triangle 2 E-U 2$

1. $76 E=63$

1.76E-A3 3

1. $6 A E-62$

$1.60 E-B 2$

$1.77 E-U 2$

$1.77 \mathrm{E}-1 \mathrm{Bz}$

2. $57 \mathrm{t}-32$

2. $57 \mathrm{k}-2 \mathrm{C}$

1. $U 1 E-42$

1. UIE $1 \mathrm{BL}$

1. 17E-U?

$1.17 E-U 2$

1. $56 E-42$

1. $56 E-13$ ?

1. $35 E-12$

1. $35 E-v 2$

1. $.6 \mathrm{GE}-\mathrm{H}^{3}$

1. $D 6 E-103$

1. $64 t-42$

$1.64 E-52$

$9 . \Delta 3 E-B 3$

$9.43 E=03$

3. प9E-सेट

$3,096-192$

2. $21 E-42$

2. $21 E-42$

1. $22 E-12$
ALPHAS

$9.65 t+. A A$

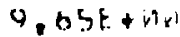

1. $28 E+41$

1. $24 F+i j L$

$1.36 E+111$

1. $30 E+.11$

$1.67 E+.11$

$1.67 E+11$

$1.54 t+11$

$1.54 t+111$

1. $26 E+11$

1. $26 E+11$

$1.37 E+11$

$1.37 t+41$

$1.49 E+11$

1. $40 t+31$

$1.53 E+\infty 1$

$1.53 E+11$

$1.7\lfloor E+D\rfloor$

$1.71 E+11$

S. $16 E+B 1$

$3.18 t+11$

$2.94 E+y !$

$2,94 E+t)$

$2.77 E+41$

$2.77 E+11$

$2.98 E+11$

2. $98 E+11$

$3.11 E+11$

3.11E+19

2. $56 E+W 1$

2. $56 E+41$

2. $42 E+1$

2. $42 E+41$

2. $25 E+11$

2. $25 E+D 1$

$2.52 E+01$

2. $52 E+11$

2. $22 E+k]$

2. $22 E+11$

4. $35 E+01$

$4.35 E+v 1$

3. $89 E+\cdots 1$

$3.89 E+U 1$

$3.19 E+(1)$ 


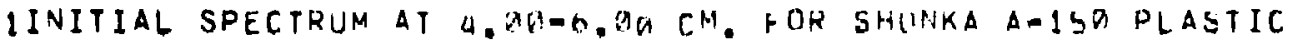

50 MEV D+ ON BE NEUTRON SPECTRUM

\section{ENERGY}

4.2D MEV

4. 4 U MEV

4.61 MEV

4.8 MEV

5. 10 MEV

$5.50 \mathrm{MEV}$

6,00 MEV

6.50 MEV

7.DO MEV

7.50 MEV

8.0O MEV

8.50 MEV

9.01 MEV

$9.50 \mathrm{MEV}$

$10.00 \mathrm{MEV}$

11.0ด MEV

12.00 MEV

13.00 MEV

14.DO MEV

$15.00 \mathrm{MEV}$

16. 10 MEV

17. AD HEV

18.0 MEV

$19 . \mathrm{UV} M E V$

$20.00 \mathrm{MEV}$

$21.00 \mathrm{MEV}$

22. DO MEV

23, OD MEV

24,00 MEV

25. OD MEV

$26.00 \mathrm{MEV}$

27. OD MEV

28.0V MEV

$29.00 \mathrm{MEV}$

$30.00 \mathrm{MEV}$

32.0O MEV

$34.00 \mathrm{MEV}$

$36.90 \mathrm{MEV}$

$38.00 \mathrm{MEV}$

40.00 MEV

42. DO MEV

44,00 MEV

$46,00 \mathrm{MEV}$

48.00 MEV

50. HO MEV

\section{PROTONS}

4.71E+b1

$4.44 E+D 1$

$5.13 E+01$

5. $D 1 E+D 1$

$4.49 E+61$

$9.29 E+31$

$8.76 E+111$

$7.56 E+11$

$6.71 E+61$

$6.41 E+41$

A. $8 \lambda E+M 1$

$6.58 E+11$

$5.09 E+D 1$

$4,68 E+11$

$4.15 F+09$

$7.05 E+Q 1$

$6.34 E+01$

$4.95 E+141$

$5.68, E+01$

$5.23 E+D I$

4. $42 E+B 1$

$4.39 E+[) 1$

3. $45 E+01$

$2.84 E+11$

2. ORE + BI

$2.20 E+D ?$

$1.85 E+\angle 1$

1. $19 E+01$

1.95E+ 01

9. $90 E+00$

1. $05 E+b)$

1. $B U F+O 1$

B. (1) $A E+D$

5. $5 A E+69$

5. OAE + RIP

5. $\triangle Q E+\square D$

4. $B D E+D E$

3. $D O E+B a$

1. $5 U E+0$ ?

0.

a.

1. $O A E+D O$

0

6

A.
DEUTCROINS

4. $16 \mathrm{~F}$-192

2. $33 E-3$ ?

$2.33 E-1.2$

3. $26 E-D C$

3. $26 E$. FI

9. EIE-A2

9.0IE-AZ

1. $\triangle O E=D !$

1. $\triangle 6 F=A 1$

5. $\triangle B E-\angle 2$

5. BE-AZ

b. $18 E-M 2$

6. $18 E-n_{2}$

1. $74 F-02$

1.74E- त?

$1.91 E-92$

$1.91 E-132$

4. $3 E E-Q 3$

$4.38 E-03$

$a$

$a$

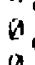

v.

ii

i)

$a$

a

$Q$

8

$a$

a

0

a.

a.

0

0

a.

0

0

0

3.62
TRITUAS

1. $P .2 E-112$

2. $42 E-U$ ?

$2.42 \mathrm{~F}=\mathrm{K2}$

2. $13 E-42$

$2.03 t-112$

$3.75 t-132$

$3,75 E-1.2$

$1.96 E-92$

1. $96 E-12$

$1.51 E=0$ ?

$1.51 \mathrm{E}-1.2$

$1.36 E-O 2$

$1.36 E-12$

1. $28 E-D 2$

1. $28 E-n ?$

$4.90 E-03$

$4.96 E-63$

$3.38 E-113$

$3,3 B E=113$

i.

U.

v.

w.

i.

v.

4.

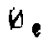

b.

3

i.

i.

0

0

0

,

$\forall$.

a.

U.

a.

b.

b.

$n$

$\theta$

0

,

5.84
ALPHAS

$3,19 E+11$

2. $42 E+11$

$2,42 E+\Delta 1$

2. $H 4 F+31$

2. (3) $4 E+11$

4. $14 E+d 1$

a. $14 E+b 1$

$2.69 E+11$

2. $69 E+61$

$2, U 3 E+11$

2. $03 E+01$

$1.33 E+01$

1. $33 E+11$

$5.57 E+b)$

$5.57 E+60$

1. $|U E+| U \mid$

1. $1 W E+1$

2. $16 E+96$

2. $16 E+b k$

$1 . \forall 3 F+44$

1. HSE+ HU

$7.59 E-V 1$

$7.59 \mathrm{~L}-\mathrm{A} 1$

2. $56 t-111$

? SRE-I

D.

n.

a.

b.

b.

a.

b.

b)

4

$\theta$

v.

G.

a.

a.

a.

a.

$\theta$

$\theta$

a.

b.

5.35 
IINITIAL SPECTRUM AT G.MA-11. 50 MEV D+ ON BE NEUTRON SPECTRUM

\section{ENERGY}

2. $M S$ MEV

0. II MF.V

D. 15 MEV

0.20 MEV

$0.25 \mathrm{MEV}$

0.3ด AEV

$3.35 \mathrm{MEV}$

Q. $\triangle$ M MEV

$0.45 \mathrm{MEV}$

d. SD MEV

$0.55 \mathrm{MEV}$

0.60 MEV

3.65 MEV

0.70 MEV

0,75 MEV

D. $80 \mathrm{MEV}$

0.85 MEV

0.90 MEV

0.95 HEV

1.DO MEV

1. 12 MEV

1.2W MEV

1,36 MEV

$1.4 U$ MEV

1.50 MEV

1.65 NEV

$1.70 \mathrm{MEV}$

1.80 MEV

$1.90 \mathrm{MEV}$

2. UD MEV

$2.10 \mathrm{MEV}$

2.20 MEV

2. 3U MEV

2. $40 \mathrm{MEV}$

2.50 MEV

$2.60 \mathrm{MEV}$

$2.70 \mathrm{MEV}$

$2,80 \mathrm{MEV}$

2.90 MEV

3. OD MEV

3.2W MEV

3, 40 MEV

3.60 MEV

3. BU MEV

4.00 MEV
PRCTONS

B.

1. $58 E+63$

5. $95 E+b ?$

4. $22 E+4 ?$ ?

$3.26 E+1) 2$

$2,84 E_{-}+142$

2. $199 E+62$ ?

1. $94 \mathrm{E}+12 \mathrm{Z}$

1.89E+ 12

1. $5 ? \mathrm{E}+\mathrm{A2}$

1. $45 E+\Delta 2$

1. $12 E+H 2$

4. $B \geq E+\square 1$

1. $) R E+G 2$

9.19E+ 1

$9.235+11$

8. $17 E+01$

$7.90 E+D 1$

$8.6 ! 5 E+U 1$

$6.96 F+61$

$1.27 E+12$

1. $12 E+152$

1. $3 B F+\square 2$

6. $4 B E+D 1$

B. $81 E+\Delta 1$

B. $53 E+01$

$7.44 E+1$

$7.69 E+B 1$

6. $55 E+51$

$6.66 E+101$

$5.77 E+\Delta 1$

$5.37 E+\$ 1$

5. $14 E+11$

$5,64 E+01$

4. $43 E+01$

$4.71 E+B I$

$4.50 E+B 1$

$4.29 E+D I$

3. $82 E+01$

4. $13 E+D 1$

$6.64 E+101$

5. 39E + BI

$5.78 E+d 1$

$5.92 E+\mathrm{d} 1$

$5, \mid 5 E+b 1$
['EUTERUNS

1. $62 E-W 2$

$1.62 E-12$

1. GSE- DI

1. $M: B E=G 1$

$1.13 E-D 1$

1. $|R E-D|$

1. $69 E-D 1$

$1.69 E=1$

1. $72 E-91$

1. $72 F-n 1$

$1.73 F-61$

$1.73 E-61$

$i .65 E-W 1$

$1.65 F-01$

1.5 RE $=1$

$1.58 \mathrm{~F}-01$

$1,6 \mid E-n !$

1. $h 1 E=01$

3. $12 E-A 1$

3.12F-m

3.6QE-HI

$3, b B E-A 1$

3. $55 E-i) !$

3. $55 E-D 1$

3. आ $1 E-41$

3. $\mid E-U 1$

1.76E- 11

1.76E-HI

1.? $\{E-V 1$

1. 2 IF-A

5,3 IF-

$5.37 E-142$

1. $85 E-51$

$1.85 E-91$

4. $\triangle 1 E-B 2$

4. $1 E-52$

1. $5 B F-91$

1. $58 E-11$

$2.66 E-132$

$2.66 E-92$

1.78E- 1

1. $78 E=\square$

1.47E- B)

1. $47 E-31$

2. $5 \mathrm{BD}=0 \mathrm{C}$
TPIT TNS

A.

1.

is.

U.

2. $42 E-03$

2. $42 E-43$

$4.05 E-03$

4. $65 E-V 3$

1. $\forall B E-b 2$

1. $68 t-12$

a.

(1).

b. SOSE $=63$

ค. NGE $=3$

6. 9OE - 13

5. 119k-113

$3.69 E-13$

$3.69 F-13$

1. $28 \mathrm{E}-62$

1. $28 E-42$

1. $12 E-02$

1. $12 E-42$

$7,93 \mathrm{k}-1.3$

$7.73 E-b 3$

1. $54 E=42$

$1.54 t-42$

1. $34 E=\$ 2$

$1.34 E-d ?$

$3.11 E-D 3$

$3.11 E-H 3$

1. $79 E-12$

1. 7 AE-BC

9. 1 ULE-K3

9. 1 DE $=03$

1. $13 E-12$

1. $13 E-6$ ?

$4.35 E-3$

$4.35 E-W 3$

$4.52 E-1) 3$

$4.52 \mathrm{E} M \mathrm{~S}$

2. $84 \mathrm{E}=\mathrm{G} 2$

2. BUE-DL

1.70E-VZ

$1.7 A E-\triangle 2$

2, $82 E-b 2$
ALPHAS

$7,7 W E+11 U$

$7.70 E+H V$

1. $1 W E+11$

1. $1 U E+1$

1. $32 E+11$

$1,32 E+1$

$1.24 E+11$

1. $24 E+B 1$

1. $32 E+1$

$1.32 E+U 1$

$1.32 E+1$

$1.32 E+1$

$1.5 B F+11$

1. $5 B E+11$

$1.37 E+11$

$1,37 E+1$

1. $5 P E+11$

1. $58 E+141$

1. $51 \dot{E}+b 1$

?. $51 E+b 1$

$2,91 E+W 1$

2. $91 E+41$

2. $54 E+b 1$

$2.54 E+11$

$2.03 E+11$

$2.63 E+d 1$

2. $58 E+11$

$2.58 E+d 1$

$2.52 E+1$

2. $52 E+11$

$2.52 E+11$

2. $52 E+1$

2. $43 E+11$

2. $43 E+1$

?. $30 E+U 1$

2. $30 E+D 1$

$2.3 \Delta E+H 1$

$2,3:+E+b 1$

2. $199 E+61$

2. $196+11$

3. $72 E+111$

3.72E+ 1

3.11E+U1

3.11E+DI

2.69E+HI 


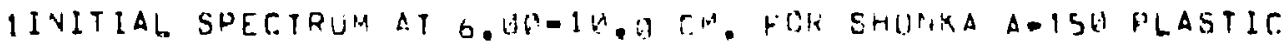

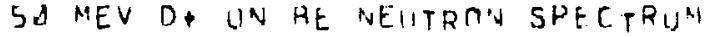

\section{ENERGY}

4. 20 MEV

1. $43 \mathrm{MEV}$

4.6H MEV

4.83 I:EV

5. BOM MEV

\$. SA MEV

6. HA IIEV

6.53 MEV

7. HS MEV

7.5:1 MEV

H. B.1 MEV

$8.5 t^{A} M E V$

9. DO MEV

$9.5 \%$ MEV

$17.0 H M E V$

11. HO MFV

12. UA MEV

13. DU MEV

$14.00 \mathrm{MEV}$

15,00 MEV

$16,90 \mathrm{MEV}$

17. DH MEV

18,00 MEV

19, OD MEV

20, DO MEV

$21, D O M E V$

22, DD MEV

$23 . \Delta B N E V$

24.00 MEV

25, IO ME M

26. OD MEV

$27.01 \mathrm{MEV}$

$28,00 \mathrm{MEV}$

$29.01 \mathrm{MEV}$

30. AU MEV

$32.00 \mathrm{MEV}$

$34.00 \mathrm{MEV}$

36. DO MEV

38. DO MEV

4D. DU MEV

42, DU MEV

44, QO MEV

46. OD MEV

$48.00 \mathrm{MEV}$

50.00 MEV

$$
\text { PRUTUAS }
$$

4. $27 E+v 1$

1. $55 F+1$

1. $14 E+U 1$

3. $36 E+1$

$3.06 E+D 1$

B. $52 E+1$

A. $3 I E+b 1$

7 , $4 E+D$ !

$0.14 t+61$

5. S3E + II

4. $I I E+d 1$

$4,67 E+4 ! 1$

$4.0 .1 E+41$

4. $13 E+11$

$4, \mid 7 E+01$

$7.63 E+41$

$6.45 E+01$

6. $14 E+(1) 1$

$5.55 E+61$

$4.62[.+11$

4. $22 E+D 1$

3. $17 E+01$

$2.41 E+61$

2. $62 E+D 1$

2. $4 M F+D I$

2. $23 E+J 1$

1.78E + 91

1. $5(A E+01$

1. $17 E+11$

1. $.25 E+01$

$7.75[+0.7$

$8.75 F+13$

$4.75 E+11 B$

4. $25 E+O U$

2. $5 A E+\Delta A$

4. $25 E+6 \lambda$

$3.25 E+60$

1.5 $9 E+60$

5. $D() E-(d) 1$

$1.25 E+19$

7. 50E-BI

$1.25 E+\pi$

i.

is

9.

9.15
DEllाEर्याis

2. $50 F-02$

2. $82 E-\lambda ट$

2. $82 F-\lambda 2$

2. $99 E-U 2$

$2.795-132$

1. $94 \mathrm{~F}=1$

$1.94 E-1$

8. $56 \mathrm{~F}-42$

B. $56 E=12$

$4 . M 7 E=A^{2}$

A. $.77 \mathrm{E}-\mathrm{H}$ ?

3. वसE $-?$ ?

$3.94 E-d z$

1. $56 t-n 1$

1. 5 SE- b' 1

3. $21 \mathrm{E}-\mathrm{XI}$

3. $215=132$

7. त1 . A 3

7. $1 \mathrm{~F}-\mathrm{B} 3$

ค. $55 E-44$

B. $55 \mathrm{E}-\mathrm{a} / \mathrm{A}$

a.

it.

i.

w.

b.

is.

C.

a

9

i)

$\theta$

D$$
\text { in }
$$

i).

i.

A.

.

b.

4

in.

is.

4.63
I RITCH:

2. $42 \mathrm{E}-\mathrm{E} 2$

$2.47 \mathrm{E}-\mathrm{BC}$

?. $47 \mathrm{E}-12$

1. $20 E-192$

$1.26 t-12$

2. 75t-.12

ว. $75 E=.12$

3. $37 \mathrm{E}-112$

$3.37 E=\square 2$

$1.79 E-12$

$1.77 \mathrm{~F}=11$ ?

$5.57 E-43$

$5.57 t-113$

$5.97 t=03$

5. $77 E-63$

1. $\triangle 2 E-\lambda 2$

1. $M 2 L-W 2$

$1.67 E-315$

$1.67 E-33$

(b)

it.

b.

4.

t.

(i).

a.

4.

$\theta$

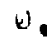

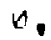

i).

v.

b.

a.

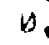

A

i.

1.

a.

is.

i).

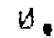

0.

in

b.

5.95
ALPIAS

2. $09 E+91$

2. $97 E+11$

2. $b 7 E+61$

$1.84 t+11$

$1.84 E+41$

5. $8 w E+W 1$

3. 8 HE+W!

2. $47 E+41$

$2.47 E+11$

1.52E+ 11

$1.52 t+11$

$1, M b E+\mid 11$

1. $.9 b E+11$

1.5BE+ 113

$7.5 B E+d v$

A. $6 n E+x^{2} b$

B. $60 E+10 \mathrm{~A}$

5. $2.4 E+1.4$

5. $2.4 E+4 v$

$1.53 E+D W$

1. $53 E+V$

1. $\triangle 2 E-11$

1. $42 E-31$

3. 7BE- 11

3. 7 BE- -11

a.

B.

$+1$

1.

3.

b.

is.

$b$

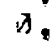

b.

1.

$a$

u.

a.

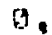

(1)

万.

id.

b.

b.

5.52 


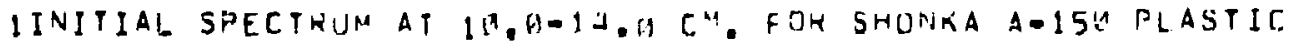
5A MEV D+ ON HE NEUTROS SFECTRULA

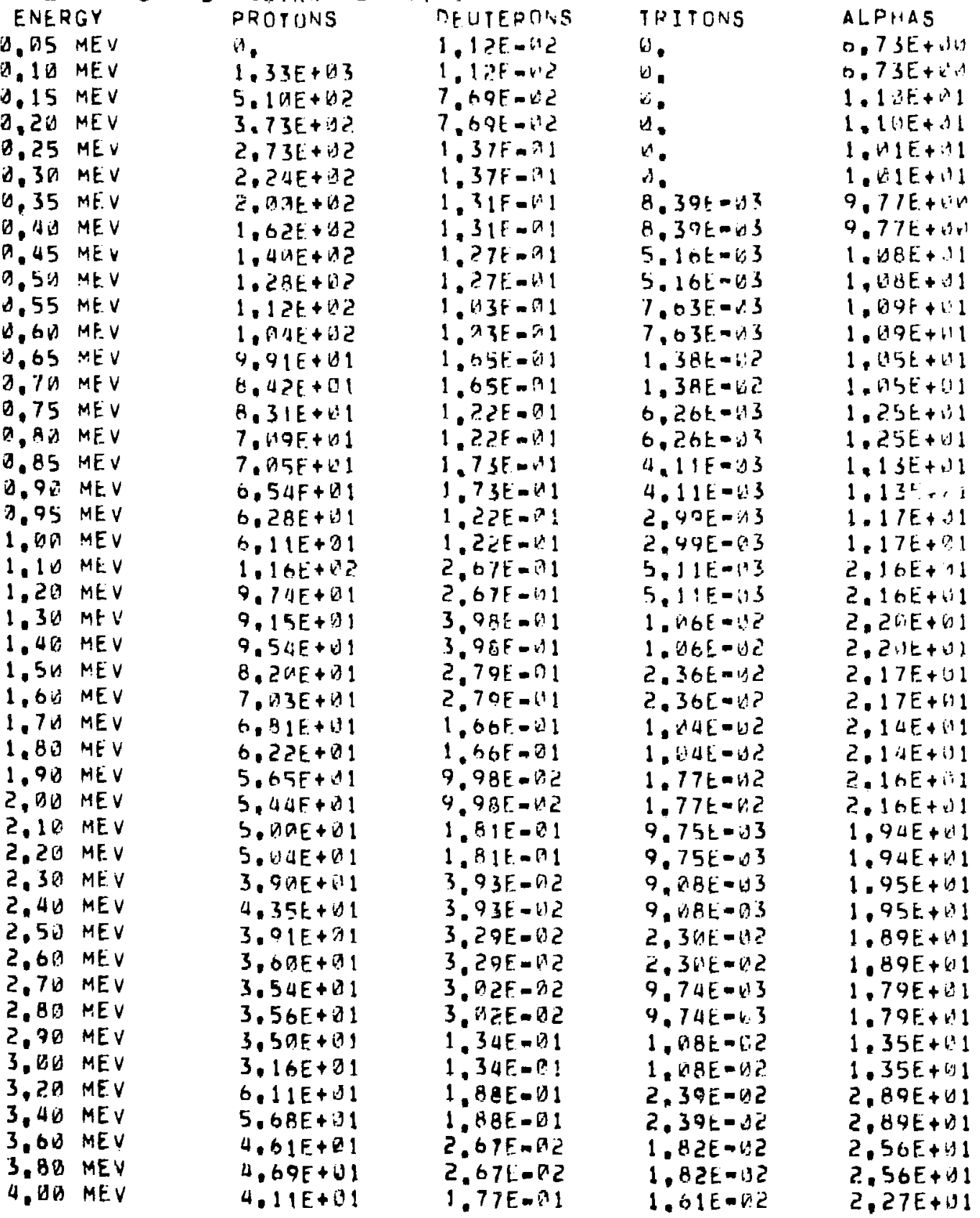


IINITIAL SPECTRUM AT 1R.MO14.A CF, HOR SHOHRA A-15A PLASTIC 50 MEV D+ ON HE HEUTROH SPFCTRUM

\section{ENERGY}

4.2 21 MEV

4.4\% MEV

4.67 MEV

4.8 M MEV

5. 11 MEV

$5,5, M E V$

6,00 MEV

6.50 MEV

7. DID MEV

7.5. MEV

8. HD MEV

8.50 MEV

9. 14 MEV

9.5D MEV

10. WJ MEV

11. MI MFV

$12.01 M E V$

13. DD MEV

14. 月. MEV

15. 00 MEV

16. $10 \mathrm{MFV}$

$17.01 \mathrm{MEV}$

18. BV MEV

19. HI MEV

2ग. OD MEV

21. OO MEV

22. DD MEV

23. TR MEV

24. DV MEV

25. OA MEV

26. DS MEV

$27.0 D \mathrm{MEV}$

28. DA MEV

29. HQ MEV

3D. DO MEV

$32.00 \mathrm{MEV}$

$34 . D O M E V$

36. OV MEV

$38.0 \square$ MEV

42. ED MEV

44.DV MEV

46. 96 MEV

48. OU MEV

50.0 M MEV
40. OD MEV
PEOT ONS

3.7 $2 E+B 1$

4. DU $4 E+B 1$

3. $85 E+11$

3. 8 Q $+1 ? 1$

3. $P 41 F+.11$

$7.91 E+.91$

$6.5 a E+.1$

5. $2 A E+t^{n} 1$

5. $7 a E+11$

5. $12 E+H^{2} 1$

$4.47 E+11$

4. $25 E+W 1$

$3.95 F+11$

3. $2 R E+B 1$

$3.16 E+A 1$

$0.77 E+111$

$5,44 E+11$

4. $8 B E+11$

4. $94 E+11$

$3.73 E+k^{3} 1$

3. $61 E+.11$

2. $35 E+.91$

$2.67 E+11$

2. $\Delta \Delta_{E}+\Delta 1$

2. $\triangle B F+d 1$

2. $15 E+d 1$

1. $6 U E+111$

1. $25 E+1$

$9.75 E+\lambda \lambda$

9. DAE + UD

7. WRE + DA

6. $D Q \square E+2 B$

4. $75 E+B: 4$

$2.25 E+10$

3. $9 A E+B D$

$3.75 E+10$

4. $D O E+4 A$

2. $\triangle A E+B D$

1. $53 E+10$

1. $25 E+1)$

5. ADE $=31$

2. SUE-!]1

0.

$2.5 \cap E-01$

A.

9.28
DH UTFETIS

1.77F-1

1. $98 E-12$

1. $98 t=02$

3. ARE-Oz

3. agt - in

2. त $\mathrm{F}-\mathrm{A}$

?. H8F-A1

$6.50 E-A \mathrm{C}$

0.

a.

a

a

a

$a$

$a$

A

$\rightarrow$

$$
\text { a }
$$

$$
\text { a }
$$

$$
\text { a }
$$

a

a.

a.

$a$

a.

b.

a.

0

i.

DAVE, E (MEV)
$1.96 F=F^{2} 1$

$1.96[\mathrm{mi1}]$

$6.66 \mathrm{~F}-\mathrm{AC}_{\mathrm{C}}$

1. $47 E-.1$

$1,47 E-\cap 1$

?. 2 B $E-x^{2} c$

5.20 की 2

2. $25 E-102$

5. $25 F-125$

$3.975-13$

$3.97 E=M$

4

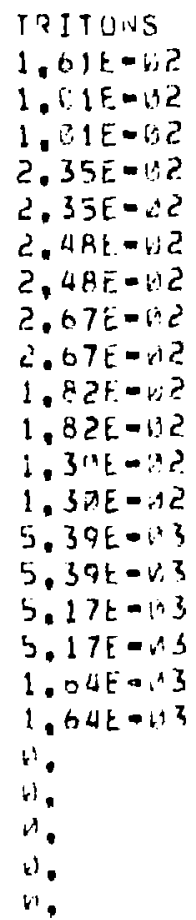

it.

a.

$i$

is.

$\therefore$

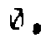

4

1.

b.

U.

i.

,

U.

(1).

b.

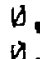

b.

.

$\theta$

0.

a.

1).

4.76
ALPHAS

$2.27 E+A 1$

$1.64 E+b$ ।

$1.64 E+11$

$1.47 t+01$

1. $47 E+1$

2. 9 BE + 1

$2.98 E+7.1$

$1.73 E+11$

$1.73 E+11$

1. $4 R E+11$

1. $4 B t+D I$

A. $A$ AE +1 in

8. $84 E+M 1$

$7.36 E+14:$

$7.36 t+1161$

$6.6 A L+14$

$6,68 t+14$

$2.511 E+: 16$

$2.52 E+96$

1. $1 E+4$

1. $B \perp E+A M$

3. $82 F_{-}-101$

$3.82 t-d 1$

2. $2 R E=113$

$2.2 B E=: 13$

$n$.

$n$

n.

ט.

i.

b.

a.

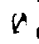

$\theta$

a)

b

n.

b.

0.

n.

b.

a.

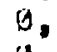

B.

5.39 


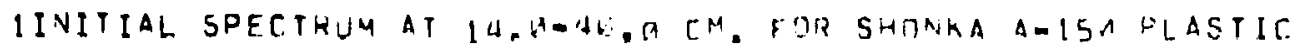
5A MEV D+ ON HE NEUTTRMN SPECTRUM

\section{ENERGY}

D. U5 MEV

D. II MEV

D.1S MEV

त.2. MEV

0.25 MEV

3,30 MEV

D. 35 MEV

D. UD MEV

0,45 MEV

7. 50 MEV

$\lambda .55$ MEV

0.60 MEV

$\triangle .65$ MEV

0.10 MEV

0.75 MEV

D. 9G MEV

D.8S MEV

$0.90 \mathrm{MEV}$

0.05 MEV

I. BS MEV

$1.1 \mathrm{M} M \mathrm{MEV}$

1.20 MEV

1.3 $15 \mathrm{MEV}$

$1.40 \mathrm{MEV}$

1.50 MEV

$1,60 \mathrm{MEV}$

$1.70 \mathrm{MEV}$

?. BO MEV

$1.96 \mathrm{MEV}$

2. DO MEV

2. IV MEV

2.20 MFV

$2.30 \mathrm{MEV}$

2,4 , MEV

2.5У MEV

2.60 MEV

$2,7 \pi$ MEE V

2.8 8 MEV

$2,94 \mathrm{MEV}$

$3.00 \mathrm{MEV}$

$3.20 \mathrm{MEV}$

3,40 MEV

$3.60 \mathrm{MEV}$

$3, B D$ MEV

4. $\triangle O$ MEV
PROTINS

a.

$0.72 E+12$

?. $53 E+\Delta z$

$1,82 E+42$

$1.3 A E+D E$

$1.14 E+32$

$9.79 E+U 1$

$8.43 E+49$

$7.27 F_{-}+U 1$

$6.8 B E+11$

$5.72 E+11$

$5.47 E+.11$

4. IAE+MI

$4.45 t+11$

$4.15 E+41$

4, U1E+iII

$3.76 E+11$

3. $47 E+11$

3. $22 E+x^{2} 1$

3. $2 I 1 E+U 1$

$5.35 E+D 1$

5. $15 E+11$

$4.53 E+11$

4. $\cdots: E+11$

3. is $3 E+31$

$3.47 E+11$

3. 3 ME + 11

3. $27 E+11$

2. $B(E+B)$

2. $74 E+b 1$

$2.69 E+V I$

$2.49 E+b !$

$2.37 E+11$

2. $24 E+11$

$2.15 E+O 1$

1. $94 E+V 1$

$1,93 E+11$

$1.75 E+W 1$

$1.73 \mathrm{E}+\mathrm{B} 1$

$1.71 E+D I$

3. $2 \nabla E+\Delta 1$

$2,945+01$

$2.02 E+i j 1$

$2.59 F+11$

$2,28 E+\pi 1$
DEUTFRDNS

b. 7מE- 13

6. $76 \mathrm{E}=13$

4. 1 HF -192

4. $18 F-1 \lambda 2$

4. $2+1 E-a ?$

4. $z^{n}[-2 \zeta$

$5.67 E=112$

5.67F-V2

$5.60 E-W 2$

5.66F-W2

5. $97 \mathrm{E}-\mathrm{WL}$

5.97E. .

5. $77 \mathrm{~F}=112$

$5.77 \mathrm{E}-\mathrm{A}$

6. $A B E-112$

6. "BE- ${ }^{14}$ ?

5. BSE- $\bar{c}$

5. $n 3 F-12$

B. $07 F-12$

B.67E-UC

1. $4 S E-11$

$1.46 F=01$

$1.16 \mathrm{~F}=1$

$1.16 F=11$

1. ISF-Li

:18E-J1

$1.12 E=11$

$1.12 F_{=}=11$

6. $74 \mathrm{~F}=42$

6. itaf = I?

$4.16 E-W 2$

1. $16 E=W_{2}$

3. EIF $=D 2$

$3.81 F-6 ?$

1. $4 b F=Q 2$

$1.4 \mathrm{OE}-4 ?$

3. $\triangle U E=" 2$

$3 . A G=2$

B. $65 E=63$

8.65E-113

$3.61 \mathrm{E}=\mathrm{W}$

$3,61 F=4 ?$

$3.48 E=W ?$

3. $48 E=112$

3. $5 \mathrm{GF}=\pi 2$
TRITOAS

$\rightarrow$.

H.

$+1$

4.

7. $2 \Gamma \mathrm{A}=\mathrm{A \mu}$

7. COE

$7.54 t-14$

$7 . j+t=4 a$

$1.85 t-g 3$

$1.85 \mathrm{~L}-\mathrm{B} .4$

$1.35 E-13$

$1.45 t-w 3$

C. $27 \mathrm{E}-1 \mathrm{C}$

2. $27 \mathrm{E}=\mathrm{W}$

4. $37 E-13$

$4.37 E-: 43$

1. $64 t-113$

$1.00 t=3$

z, c.lt-4t?

$2.21 t-42$

$4, .11-113$

$5.81 t-3) 3$

$6.46 E-33$

5. HGE $=113$

$0.09 E-13$

$6.69 t=63$

$7,111<-13$

$7,+11 t=n^{2} 3$

7. $S 3 E=\square 3$

$1.53 E-13$

$0.7 i^{4} t=z^{2} 3$

8.7at-dis

$6.29 t-i) 3$

6. $119 E-0^{4} 3$

$2.51 E-112$

2.51E-11?

$4.14 E-H^{2}$

4.14E-bi2

$6,0 B E-W 3$

$6.68 E-143$

9. $\triangle A B E=H 3$

3. $W(B E-U) 3$

$1,02 E-B 2$

1. $112 E-132$

$7.81 E-v^{3}$
ALEHAS

2.73t?

2.73E+ +1

4. $4 x E+v^{\prime} t$

$4.4 x \cdot t_{-}+1 \cdot 1$

S. $23 E+.11$

$5.2 S E+19 k$

5. $2 B E+1.1$

5. $28 E+41$

$5.52 E+r i$

$5.52 E+1$.

5. $42 E+1 n$

5. $42 E+1.1$

5. $67 E+11$

$5.47 E+14$

ᄃ. $8 / F+1: i$

S. $87 E+A v$

G, $15 E+1, A$

b. $15 \mathrm{E}+(1)$

h. 3:t $+: 1+1$

b. $S 1: E+d A$

1. $E V^{\prime} E+d$

$1 . ? \cdot E_{0}+11$

1.?2E+ +11

1.22E+11

$1, \mid 3 F+1$

$1.13 E+11$

1. $i c^{2} E+x \mid$

$1.12 F+11$

1. $\{2 E+d\}$

$1 \cdot 1<E+11$

$1.11 E+11$

$1 .|G E+1|$

1. . $\triangle E E+1$

$1.014 E+11$

9.8JE+

9. $B \perp E+v Q$

9.7 $\mathrm{KE}+11 \mathrm{t}$

9.7WE+U

$9.25 E+1)$

9.25E+.1.

$1.59 E+N 1$

$1.59 E+1 D !$

$1.39 E+1 i 1$

$1.39 E+111$

$1,|+E+d|$ 


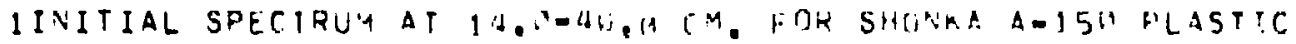
S3 MEV D. ON JE NEITBOA SHECTHIM

ENERGY

4. 2 MEV

4. 48 iat. $V$

4.66 MF.

4.8. $M E V$

5. OS MEV

5,50 MEV

6.0 ME. $V$

3.5W MEV

7 . WI MEV

7.52 MEV

8. UN MEV

8.5. HEV

9. $\triangle 1 . M$ MEV

7.5 0 HEV

10, $D U$ ME.

11. तU MEV

12. OU MEV

13. 9 Q MEV

$14.0 \Omega$ MFV

15. DO MEV

16. LU NIV

17. DO MEV

18. VIV RIEV

19. $C$ MEV

20. $00 \mathrm{MEV}$

21. $O S$ MEV

22. DQ MEV

23. WH MEV

24. DU MEV

25. $3 \mathrm{MEV}$

$26.00 \mathrm{MEV}$

$27 . \angle Q$ MEV

$28 . \angle O M E V$

29. $\triangle 10$ MEV

3W, UD MEV

32. OL MEV

34. OO MEV

$36.01 \mathrm{MEV}$

38.00 MEV

$40.00 \mathrm{MEV}$

$42 . \triangle D M E V$

44. 14 MEV

46.0. MEV

48, DO MEV

$50.0 \mathrm{MEV}$
FरणTO?S

2. $211 E+d 1$

$1.72 E+41$

$1.97 E+11$

$1.71 E+11$

$1.78 L+01$

4. $34 E+\Delta 1$

$3.565+.13$

3. $211++n 1$

2. $7.1 E+.11$

2. $58 E+v 1$

$2.44 E+11$

2. $22 E+11$

2. $.7 F+61$

1. $92 E+11$

$1.96 E+111$

$3.23 E+61$

2. $02 E+61$

2. $55 E+W 1$

2. $\triangle 4 F+1$

$1.98 E+11$

$1.64 E+H 1$

1.,, $1 E+61$

$1.33 E+.11$

1. $18 E+d 1$

9. $02 E+1 A$

H. $92 E+W$

8. $23 E+16$

B. AOE + JA

b. 35E + 115

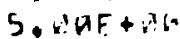

$5.5 \mathrm{JE}+\mathrm{A} .1$

$3.15 E+B^{3}(3)$

2. $42 E+b i$

2. $27 E+v H$

2. $12 E+w 11$

2. $73 \mathrm{~F}+\mathrm{wH}$

2. $50 E+\ln$

1. $B E+d(t)$

$7.31 \mathrm{E}-\mathrm{b} 1$

4.62E-U1

$3.85 E-n 1$

$2,31 E-111$

7. $69 E-11$ ?

$1.15 E-U 1$

$3,85 E-42$

9.52

\section{[CEITR RTIXS}

3. $5,1,5=112$

$1,11 E-12$

$1.11\left[-12^{2}\right.$

$1.33 E=k i 2$

$1.33 E-192$

7. तIL

7. MIE-?

4. 1 Q

$4.18 f-c^{2}$

6. $5 E=42$

b. $75 F-A^{2}$

$1.86[-112$

1. HoF - H2

$9.72 E=13$

$0.72 F_{-}=1+3$

11.7 If $=1.3$

4.7 If -6 ?

$1.915-b\}$

$1.91 F^{-3}=3$

6. $581-15$

6. $585-45$

9.

1.

B.

a.

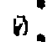

$?$

(i)

0

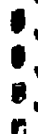

$n$

in

H.

is

a.

5.30
TRIT行

$7 . b 1[-1]]$

$5.16 E-.13$

5. 16E- 113

5. 4 GE - 3

1. $\triangle A E-13$

$3, V 1 E-H C$

$3.61[-12$

8. H6E-U3

B. ERE-W3

h. $23 t-t+3$

$0,23 E-13$

2. $39 L-12$

c. $39 E-12$

2. $58 \mathrm{E}-63$

$2,58 \mathrm{R}-13$

3. $67 \mathrm{E}=13$

2.07E-1.3

4. $v 6 t-d u$

id fitt-bid

8. 7 GE-T'5

3.7 VIE- 65

b.

i.

b.

t).

.

$\checkmark$

6.

in.

d.

d.

a.

b.

U.

is.

4.

$t$

i.

0

a.

is.

$v$

b.

6

n.

5,59
ALPHAS

$1.14 E+1$

1. $(12 t+w 1$

1. $H 2 E+d 1$

$8,72 t+b i$

a. $72 E+1: 1$

$1.63 E+11$

$1.53 E+21$

1. $J A E+41$

1. $\triangle B E+111$

$7.27 E+19 W$

7. $27 E+1) .1$

5.d $d t+d b$

5. $11 E+4$

$3.57 E+1.1$

$3.57 E+d U$

$4,57 E+A E$

$4.57 E+64$

1. $74 E+v v$

1. $74 E+d W$

6. 65E-U1

$0.65 t . .11$

3. $M 9 E-H 1$

3. $89 E-H 1$

$2,51[-11]$

$2.51 E-n !$

4.

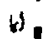

4

1.

it.

i.

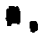

0

3

U.

$v$

a.

a.

i.

4.

a.

a.

o.

a.

4.

5,66 


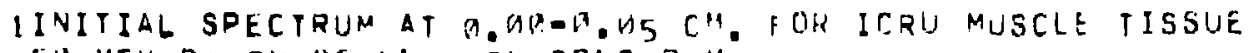
5A MEV D+ ON BE NEUTRDA SPECTRIYA

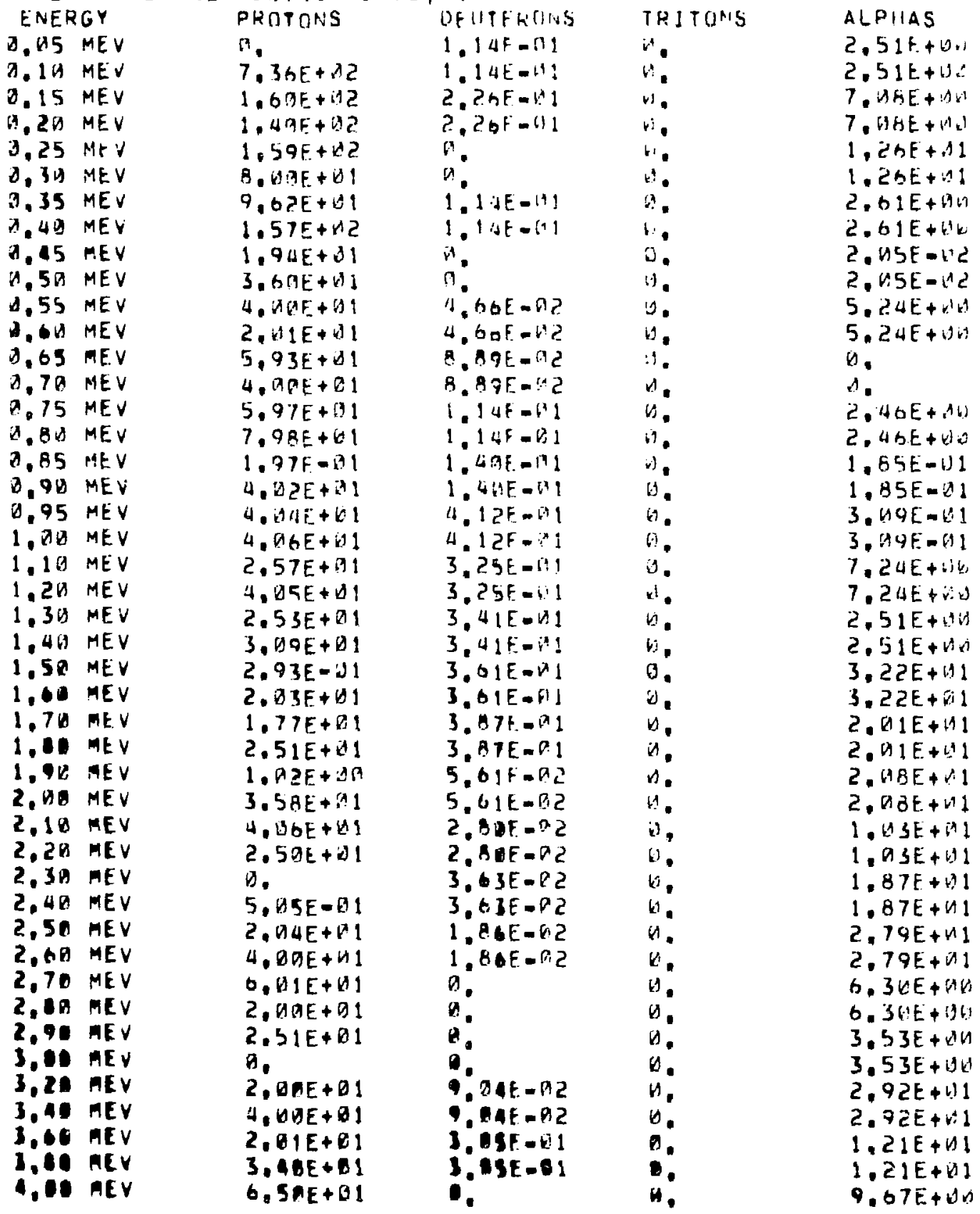




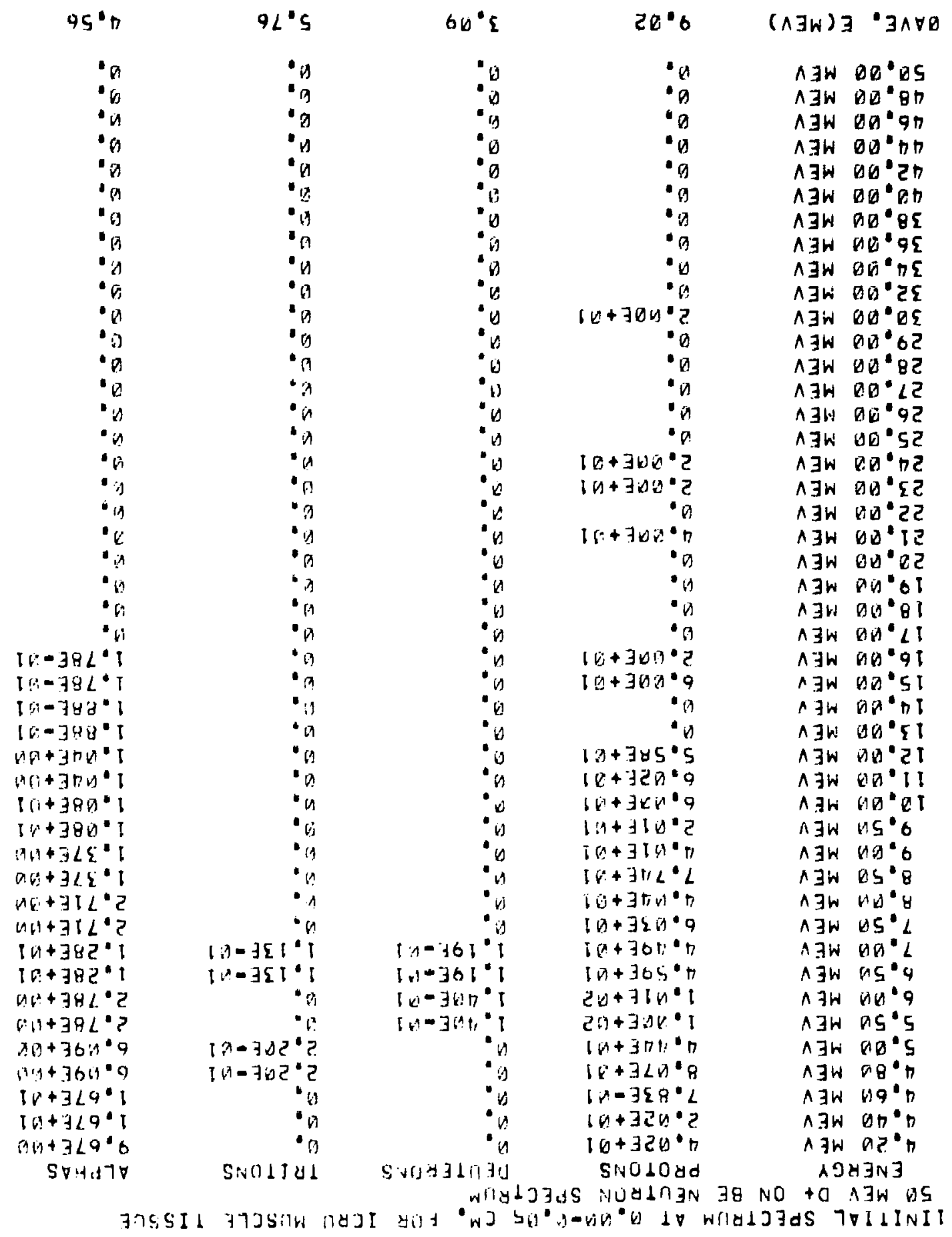


1INITIAL SPECTRUM AT M.05-0.1A CM. FOR ICRIJ MUSCLE IISSIJE 52 MEV D+ ON BE NEUTRON SPECTRUUM

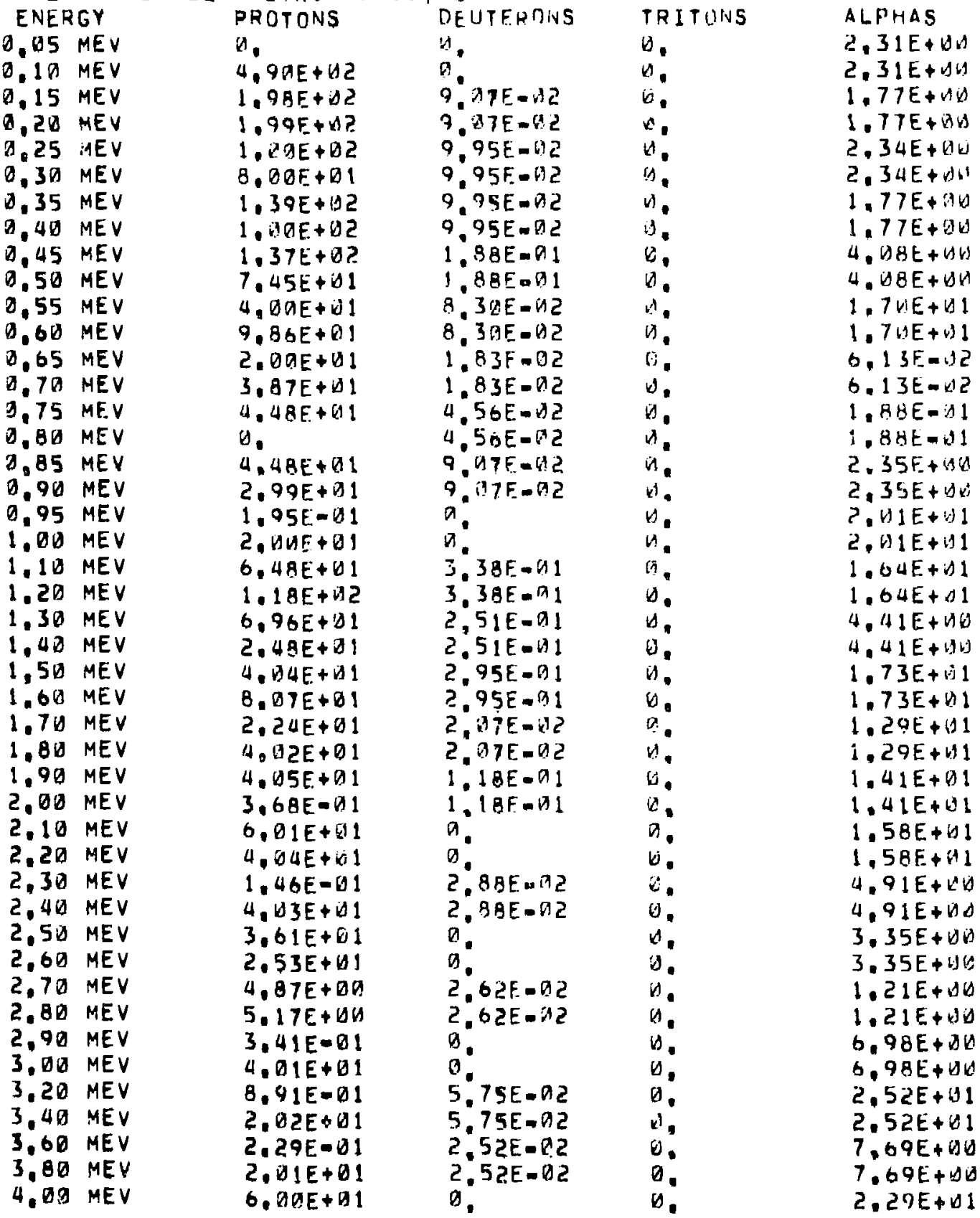


IINITIAL SPECTRUM AT O, OS-, 1 G CM, FOR ICEII MUSCLE TISSUE SII MEV D+ ON HE NEIITROIV SPE.CTRLIM

\section{ENERGY}

4. 2D MEV

4. 40 MEV

4.60 MEV

$4.80 \mathrm{MEV}$

5.8 MEV

5.50 HEV

$6 . M B$ MEV

6.50 MEV

7. DQ MEV

$7.5 U$ MEV

8,00 MEV

8.50 MEV

9. DO MEV

$9.50 \mathrm{MEV}$

10.00 MEV

$11.00 \mathrm{MEV}$

12. DO MEV

13.011 MEV

14.00 MEV

15,00 MEV

$16,00 \mathrm{MEV}$

17.DO MEV

18. UA MEV

19.0 MEV

2D. DG MEV

21,00 MEV

22.00 MEV

23. 00 MEV

$24.00 \mathrm{MEV}$

25. OA MEV

$26.00 \mathrm{MEV}$

27.00 MEVV

28.09 MEV

29,00 MEV

30.00 MEV

32, OO MEV

34.00 MEV

36, DO MEV

$38.00 \mathrm{MEV}$

40.00 MEV

42.00 MEV

44, DO MEV

46,00 MEV

48,00 MEV

50,00 MEV

DAVE, E(MEV)
PHOTONS

1. BhE +01

v.

1. $B 9 E+W 1$

$7.87 E+B I$

2.BSE+ HI

$7.72 E+01$

$7.7 D E+D 1$

$5.48 E+31$

6. $\triangle B E+D 1$

$6.03 E+01$

2. $1 E+B 1$

2. DQEE+ 1

4. AQE + UI

2. DIE+ II

$\theta$.

1. $20 E+42$

6. $V A E+11$

2. BAE + II

4. (1AE +D)

8. UaE +1

b.

$6.9 A E+01$

$\theta$.

4. DQTE+D1

$\theta$.

$2,09 E+01$

a.

U.

v.

v.

0

a.

i.

2. OGE + OI

$b$.

,

b.

0.

B.

0.

D.

b.

b.

0.

b.

9.45

\section{DEUTERCA.S}

n

a

a

a)

(1).

(3)

in

$n$.

a

a

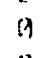

a

$a$

a

$a$

4

a

$\theta$

$a$

a

a

$$
a
$$

$$
a
$$

a.

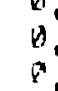

$\theta$

0

a.

$a$

$a$

b.

a.

$\theta$

1.41
TRITOAS

is.

b.

$a$

b)

is

3.

w.

i)

v.

i.

W.

(3)

$v$

b.

(1)

bis

i.

D.

a.

H.

i.

a.

v.

a.

$\theta$.

B.

a.

i)

b.

$\Delta$.

n.

b.

0

3.

$v$.

$\theta$

$\theta$.

u.

0.

0.

$D$.

0 .

5,76
ALPHAS

2. $29 E+41$

$5.64 E+0 v$

$5.64 E+60$

1. $27 E+10$

$1,27 E+W U$

1. $93 E+11$

1. $33 E+t^{2} 1$

$1.90 E+\Delta B$

$1,9 v E+d u$

7. 25E-6? 1

$7,25 E-W 1$

$1.63 E+00$

1. $A 3 E+116$

$1 .|| E+|1|$

1. $1 I E+D 1$

7. $\triangle 4 \mathrm{E}=\mathrm{W})$

7. $\triangle 4 E-W 1$

1. $\triangle 2 E+B 1$

1. AT.E + B1

$\theta$.

A.

G.

b.

H.

w.

o.

a.

$D$.

$\theta$

U.

$\theta$

b.

a.

0

n.

$\theta$.

$\Delta$.

B.

a.

0.

a.

0.

4.

D.

0.

6.15 
IINITIAL SPECTRIJM AT 0.1B-0.15 CM. FOR ICRU MUSCLE TISSUE 50 MEV D+ DN BE NEUTRON SPECTRUM

\begin{tabular}{|c|c|c|c|c|}
\hline $\begin{array}{l}\text { ENERGV } \\
0.05 \mathrm{MEV} \\
0.10 \mathrm{MEV} \\
0.15 \mathrm{MEV} \\
0.20 \mathrm{MEV} \\
0.25 \mathrm{MEV} \\
0.30 \mathrm{MEV} \\
0.35 \mathrm{MEV} \\
0.40 \mathrm{MEV} \\
0.45 \mathrm{MEV} \\
0.50 \mathrm{MEV} \\
0.55 \mathrm{MEV} \\
0.60 \mathrm{MEV} \\
0.65 \mathrm{MEV} \\
0.70 \mathrm{MEV} \\
0.75 \mathrm{MEV} \\
0.80 \mathrm{MEV} \\
0.85 \mathrm{MEV} \\
0.90 \mathrm{MEV} \\
0.95 \mathrm{MEV} \\
1.00 \mathrm{MEV} \\
1.10 \mathrm{MEV} \\
1.20 \mathrm{MEV} \\
1.30 \mathrm{MEV} \\
1.40 \mathrm{MEV} \\
1.50 \mathrm{MEV} \\
1.60 \mathrm{MEV} \\
1.70 \mathrm{MEV} \\
1.80 \mathrm{MEV} \\
1.90 \mathrm{MEV} \\
2.00 \mathrm{MEV} \\
2.10 \mathrm{MEV} \\
2.20 \mathrm{MEV} \\
2.30 \mathrm{MEV} \\
2.40 \mathrm{MEV} \\
2.50 \mathrm{MEV} \\
2.60 \mathrm{MEV} \\
2.70 \mathrm{MEV} \\
2.80 \mathrm{MEV} \\
2.90 \mathrm{MEV} \\
3.00 \mathrm{MEV} \\
3.20 \mathrm{MEV} \\
3.40 \mathrm{MEV} \\
3.60 \mathrm{MEV} \\
3.80 \mathrm{MEV} \\
4.00 \mathrm{MEV}\end{array}$ & 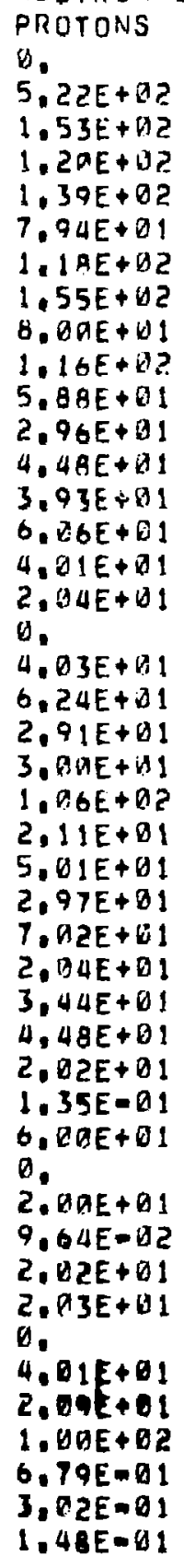 & 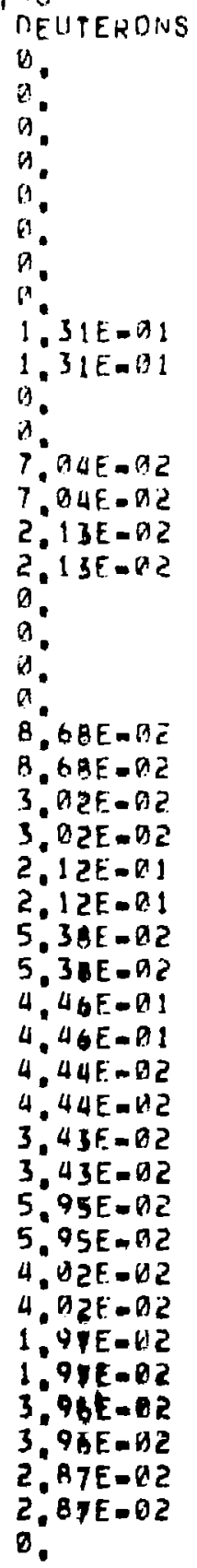 & 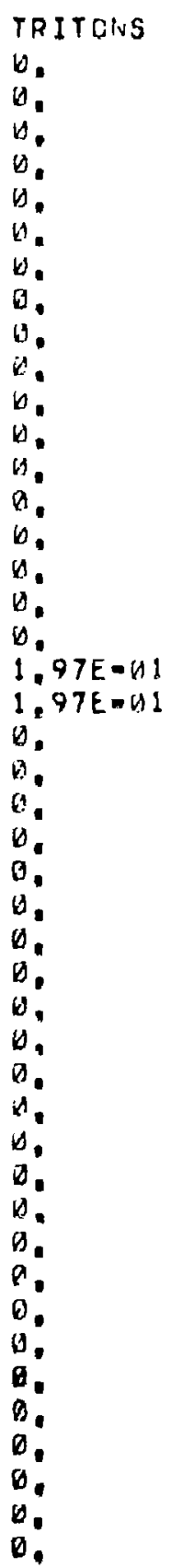 & 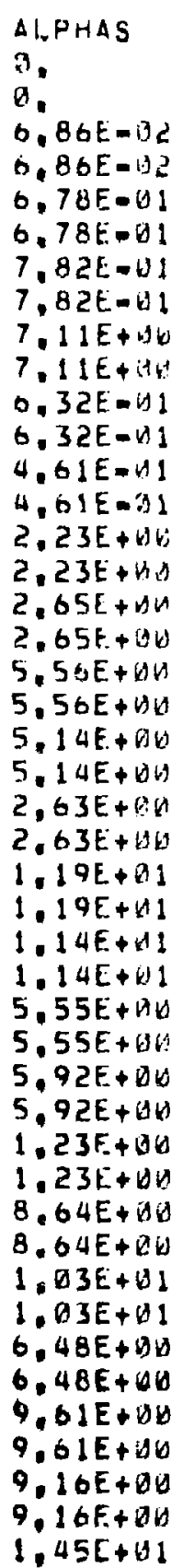 \\
\hline
\end{tabular}


IINITIAL SPECTRUM AT O.,1H-M.15 [H. FOR ICRU MUSCLE TISSUE 50 MEV D+ ON RE NEUTRON SPECTR!IM

\begin{tabular}{|c|c|c|c|c|c|}
\hline ENER & GY & D0075 & DFUTFRDNS & TRITONS & ALPHAB \\
\hline 4.20 & MEV & 4. $08 E+01$ & a. & 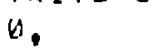 & $1.45 F_{-}+01$ \\
\hline 4.40 & MEV & $2.05 E+01$ & H: & 0. & $3.15 E+1$ \\
\hline 4.60 & MEV & $2.01 E+01$ & Q. & b. & $3.15 E+K 1$ \\
\hline 4.80 & MEV & $2.97 \mathrm{E}-91$ & $2.36 E-112$ & 6. & $1.24 E+64$ \\
\hline 5.00 & MEV & $2.02 E+1$ & $2,36 \mathrm{~F}=12$ & 0. & $1.24 E+b 1$ \\
\hline 5.50 & MEV & $4,09 E+101$ & in. & 4 & $1.82 E+191$ \\
\hline 6.00 & NEV & 4. HGE + OI & a. & $\theta$ & $1.82 E+191$ \\
\hline 0.56 & MEV & $3,87 E+13 !$ & $\theta$ & 19 & $6,69 E+(76)$ \\
\hline 7.00 & MEV & $4.13 E+01$ & H. & n. & $b, 69 E+b k$ \\
\hline 7.50 & MEV & $6.03 E+11$ & 4 & ki. & $2.4[1 E+\infty 1$ \\
\hline 8.00 & MEV & 2. BUE + 1 & a. & is & $2.4 E E+B 1$ \\
\hline 8,56 & MEV & $2.03 E-01$ & 0 & $\theta$ & $6.86 E=111$ \\
\hline 9.00 & MEV & $2.60 E+11$ & $\theta$ & 0 & $6 . B O E-U 1$ \\
\hline 9.50 & MEV & $4 . D 1 E+D 1$ & 4 & $n_{1}$ & $4.4 G E-U I$ \\
\hline 10.00 & MEV & $b$. & 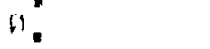 & a. & $4.4 G E=K 1$ \\
\hline 11.00 & MEV & $D . \Delta A E+\cup 1$ & $A$ & $\Delta$ & $9.86 E=01$ \\
\hline 12.00 & MEV & $\triangle D O F+1$ & $a$ & 0 & $9.86 E=1$ \\
\hline 13.00 & MEV & $v_{1}$ & $n$ & 0 & $2,89 E=101$ \\
\hline 14.00 & MEV & $6 . d a E+01$ & $a$. & 6 & $2.89 E=11$ \\
\hline 15.00 & MEV & $0.00 E+01$ & 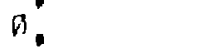 & n. & $4.90 E-02$ \\
\hline 16.00 & MEV & $2.0 B E+01$ & 9 & $\vec{n}$ & $4.9 W E-D 2$ \\
\hline $17.0 \mathrm{G}$ & MEV & $4.00 E+01$ & $a$. & 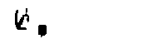 & is. \\
\hline 18.00 & MEV & $2.00 E+01$ & u. & b. & 0. \\
\hline 19.09 & MEV & 6. $00 E+01$ & $a$ & ?ִ & 0 \\
\hline 20.00 & MEV & $2.60 E+01$ & 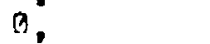 & b. & 0 \\
\hline 21.00 & MEV & 0. & 0. & 6 & B. \\
\hline 22.00 & MEV & 0 & 0 & 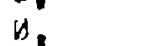 & Q \\
\hline 23.00 & MEV & $2.00 E+01$ & $a$ & $n_{1}$ & B. \\
\hline 24.00 & MEV & $2.09 E+01$ & E & $v_{1}$ & d. \\
\hline 25.00 & MEV & 0. & $n$ & D, & 9. \\
\hline 26.00 & MEV & $D$. & a. & 0 & 0. \\
\hline 27.00 & MEV & $2 . D D E+01$ & 0 & 0 & n. \\
\hline 28.00 & MEV & D. & $\theta$ & 0 & 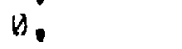 \\
\hline 29.100 & MEV & a. & $\mathrm{H}$. & 8 & (1. \\
\hline 30.00 & MEV & 0. & 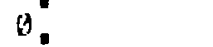 & 0. & 0. \\
\hline 32.00 & MEV & $2.00 E+01$ & a. & $\theta$. & 0. \\
\hline 34.00 & MEV & $B$. & 0. & 0. & 0. \\
\hline$\theta 0$ & MEV & b. & D. & 0. & $a_{1}$ \\
\hline 38,00 & MEV & 0. & 9. & 0. & 0. \\
\hline 40,00 & MEV & 0. & 0 & 0. & 0. \\
\hline 42.00 & MEV & 0. & e. & 0 & a. \\
\hline 44.00 & MEV & $2.00 E+O 1$ & 0. & 0. & $\theta$ \\
\hline 46,00 & MEV & & 0 & 0. & u. \\
\hline 00 & MEV & a. & 9. & 0. & a. \\
\hline 20 & MEV & 0. & 0 & W. & C \\
\hline A & $E(M E V)$ & 13.04 & 1.99 & 0.95 & 5.27 \\
\hline
\end{tabular}


IINITIAL SPECTRUM AT B.15-A.CA CM. FTR ICRU MUSCLE IISSUE 50 MEV D+ ON BE NEUTRON SPECTRUM

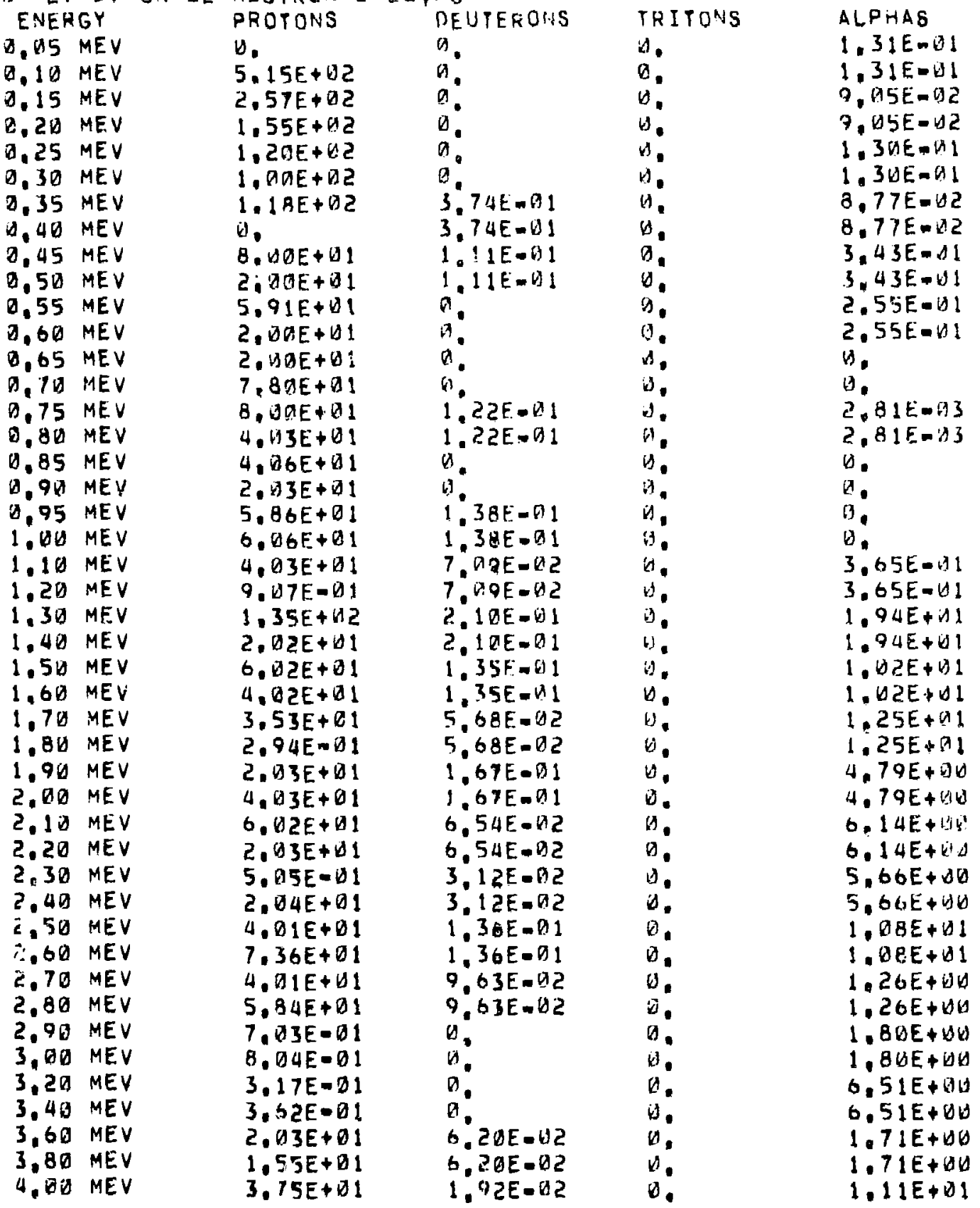


IINITIAL SPEETRUM AT D.15-0.2A CM. FOR ICRLI MUSCLE IISSUE 50 MEV D+ ON HE NEUTRON SPECTRUIA

\section{ENERGY}

4.20 MEV

4.40 MEV

4.60 MEV

$4,84 \mathrm{MEV}$

5. 00 MEV

5, 50 MEV

6,0 O MEV

$6,50 \mathrm{MEV}$

7.00 MEV

$7,50 \mathrm{MEV}$

$8,00 \mathrm{MEV}$

8,50 MEV

$9.00 \mathrm{MEV}$

$9,50 \mathrm{MEV}$

10. QD MEV

$11,00 \mathrm{MEV}$

12,00 MEV

13,00 MEV

14,00 MEV

15,00 MEV

$16,00 \mathrm{MEV}$

$17,00 \mathrm{MEV}$

$18.00 \mathrm{MEV}$

19,00 HEV

20,00 MEV

21, DO MEV

22,90 MEV

23,00 MEV

24, UB MEV

25, OD MEV

$26,00 \mathrm{MEV}$

$27.00 \mathrm{MEV}$

28.00 MEV

29.0D MEV

30.00 MEV

32. DO MEV

34.00 MEV

36,00 MEV

38,00 MEV

$40.00 \mathrm{MEV}$

42.80 MEV

44.00 MEV

46. 10 MEV

48, DO MEV

50, R० MEV

GAVE, E(MEV)
PROTONS

2. $144 E+1$

2. $A 2 E+01$

4. $\triangle 3 E+J 1$

$2.95 E=01$

$6,13 E=01$

6. $05 E+D 1$

$7.83 E+81$

$7.94 \mathrm{E}+01$

4. $02 E$ क D 1

$6.03 E+61$

$4.01 E+D 1$

$4 . D 1 E+\square I$

4. OAE $+D 1$

2. $R A E+Q 9$

$\Delta$.

B. DAE + 1

4. $8 Q E+D$.

1. $A B E+D 2$

$\theta$.

4. DGE+ DI

4. $B(B E+B 1$

0.

$2,00 E+\square 1$

4. $A D E+B 1$

$\theta$,

2.00E\$01

0.

2. $00 E+D 2$

,

0.

$\theta$.

$2, B Q E+Q 1$

$\theta$.

$\theta$.

0.

$\forall$.

$2,00 E+01$

$\theta$.

$\Delta$.

$\theta$.

0.

i.

0.

$\theta$

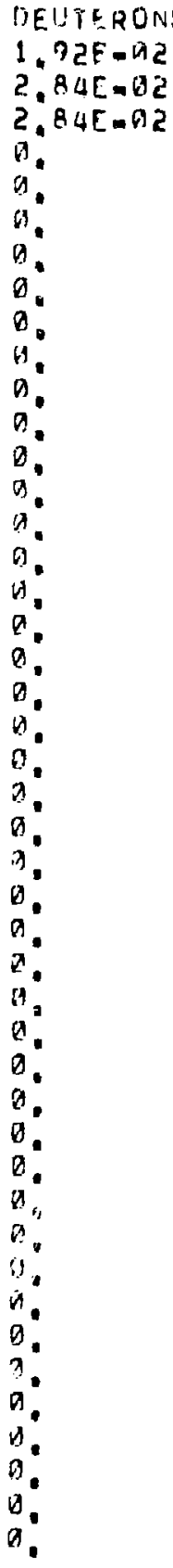

DEUTLRONS

TRITONS

O.

is:

$\because$

ti:

$\theta$.

a.

b.

i.

n.

$v_{8}$

1.

i.

$n$

4

u.

,

i:

a.

$\theta$

$\partial$

$\theta$

$\theta$.

is.

9

$\Delta$.

$\theta$

b.

?..

E.

0

is.

$B$

.

$\theta$

$\theta$.

$\theta$.

0

$\theta$.

n.

$\theta$.

0.

9.

$\theta$

$\theta$,

0.

0.95
ALPHAS

$1.11 E+01$

$1,2 U E+B 1$

$1.2 b E+61$

$1.60 E+\Delta V$

$1.64 E+36$

$1,39 E+11$

$1.39 E+01$

2. $94 E+120$

$2.90 E+B A$

$2,91 E+410$

$2.91 E+B H$

$1.53 E+\theta 0$

$1,53 E+100$

6. 1 DE-U!

6. $\mid \triangle E=D !$

$8.47 E-11$

$8,47 E-\Delta 1$

$1.12 E$ DU 1

$1 . \mid 2 E-B 1$

2. 57E-62

$2,57 E-1 / 2$

b.

0.

a.

$D$.

(1.

$\theta$.

$\Delta$.

a.

is:

n.

n.

0.

0.

0.

v.

0.

B.

a.

$\theta$

,

4

0

B.

a.

4.62 
IINITIAL SPECTRUM AT Q.2H-Q. 4 G CH. FOR ICRU MUSCLE TISSLIE 50 MEV D+ ON BE NEUTRON SFECYRUM

\section{ENERGY}

D. 05 MEV

Q. 10 MEV

$0.15 \mathrm{MEV}$

$0.20 \mathrm{MEV}$

0.25 MEV

$0.30 \mathrm{MEV}$

ด. 35 MEV

3. 4U MEV

0.45 MEV

2.50 MEV

$0.55 \mathrm{MEV}$

$0.60 \mathrm{MEV}$

0.65 MEV

$0.70 \mathrm{MEV}$

0.75 MEV

$0.80 \mathrm{MEV}$

0.85 MEV

0.90 MEV

0.95 MEV

1. DO MEV

$1.18 \mathrm{MEV}$

1,20 MEV

1,30 MEV

1.40 MEV

$1.50 \mathrm{MEV}$

$1.60 \mathrm{MEV}$

$1.70 \mathrm{MEV}$

$1,80 \mathrm{MEV}$

1.90 MEV

2.08 MEV

$2.10 \mathrm{MEV}$

2,20 MEV

$2.30 \mathrm{MEV}$

$2.40 \mathrm{MEV}$

2.50 MEV

2,60 MEV

2,78 MEV

2.80 MEV

2.90 MEV

2.00 MEV

3,20 MEV

3.40 MEV

3.68 MEV

3.60 MEV

4.00 ME.V
PROTONS

D.

7.19E+192

$2.95 E+1) 2$

$2.3 \square E+D 2$

$1.3 A E+Q 2$

1. Q B $A E+D E$

$6,25 E+11$

$6.8 Q E+11$

$9.25 E+i$ !

$7.25 E+D 1$

$7.75 E+01$

$4.83 E+0$ ?

4. $49 E+D 1$

$3, \triangle A E+Q 1$

4. DOE + I

$4.64 E+\theta 1$

$1.14 E+11$

$2.99 E+(11$

$5.12 E+11$

$2.5 D E+B D$

$1.01 E+02$

$3.53 E+01$

$6,30 E+1) 1$

$3.01 E+D !$

$5.93 E+01$

$3.6 B E+01$

$2.22 E+01$

$3.15 E+81$

$2.53 E+B 1$

$4.78 E+\square 1$

$3 . \forall 3 E+D 1$

2. $47 E+01$

2. BSE + OI

3. UGE+ +DI

$1.84 E+01$

$1.4 B E+B 1$

1.99E+B!

$1.04 E+11$

1. OYE + BI

$2.53 E+0:$

$2.56 E+D 1$

$4.76 E+01$

$2.5 B E+01$

$2.53 E+101$

3. $03 E+01$
DEUTERONS

$2.73 E-02$

2. $93 E-02$

$5.38 E-102$

$5,38 E-02$

ด.

:

7. GDE-AL

7. DDF-A

3. $9 A[-32$

3.9 $\triangle E-112$

1.2 $2 \mathrm{EE}-\mathrm{BI}$

$1.2 z E-91$

5. $19 E-02$

5. $99 E-02$

$5.75 E-02$

$5.75 E-92$

$1.64 E-01$

$1.64 \mathrm{E}-01$

$9.39 E-02$

$9.39 E-02$

2. 36E- 11

$2,36 E-E 1$

2.12E-DI

$2.125-11$

2. $23 E \mathrm{EO} 1$

2. $25 E-A_{1}$

$9.90 E-H 2$

$9.99 E-102$

2. $56 E+Q 65$

2. $56 E+06$

1.19E-GL

1.19E- 11

$5.10 E-02$

$5,1 B E-02$

$1.35 \mathrm{E}-02$

$1.35 E-92$

5. 3RE $=02$

$5.32 E-D 2$

3. $36 E-D 2$

3. $36 E-02$

$2.65 E-102$

$2,65 E-02$

2.47E- И2

2. $47 E-02$

2.71Eन0?
TRITUNS

D.

b.

b.

b.

a.

$b$.

b.

is.

i.

E.

$B$

4

(1)

$\forall$.

$\theta$.

$\theta$.

0.

E.

(3)

0.

B.

$\theta$

$5.13 E-102$

$5,13 E-C ?$

a.

a.

0.

D.

D.

$\theta$

$\theta$

$\forall$.

0.

0.

$\nabla$

0.

$3.92 E-52$

$3.92 E-02$

B.

1.

$2.54 E+D B$

$2.54 E+00$

0.

0.

2.
ALPHAS

$1.29 E+D B$

$1.29 E+1) 0$

$7,52 E-01$

$7.52 E-61$

$2.05 E+B 1$

2. DSE + A

$4,43 E+00$

4. $43 E+4 B$

2. $94 E-01$

$2.94 E-11$

2. 94E+

$2.04 E+B 1$

$2,52 E+11$

$2.52 E+A y$

$9.53 E-31$

$9,53 E-D 1$

$1.69 E+20$

$1.69 E+46$

$3,29 E+60$

$3.29 E+416$

$5 . \Delta 7 E+Q 13$

$5.07 E+B Q$

$3,84 E+Q 15$

3. $B 4 E+D Q D$

$5,37 E+1)$

$5.37 E+\triangle D$

$7.20 E+\theta 0$

$7.20 E+\theta 0$

$6.87 E+\forall Q$

$6.87 E+\triangle D$

$5,92 E+0 D$

$5.92 E+06$

$5.63 E+B D$

$5.63 E+D E$

$1.41 E+Q 1$

$1.41 E+D I$

$1,20 E+\theta 1$

1. $2 \Delta E+B 1$

$4,62 E+\forall D$

$4,62 E+\forall B$

$1.20 E+131$

$1,20 E+01$

1. $45 E+01$

$1,45 E+91$

I. OOE+ + ? 
IINITIAL SPECTRUM AT O.2A-O,4g CM. FOR ICRU MUSCLE TISSUE 5O MEV D+ ON BE NEUTRON SPECTRUM

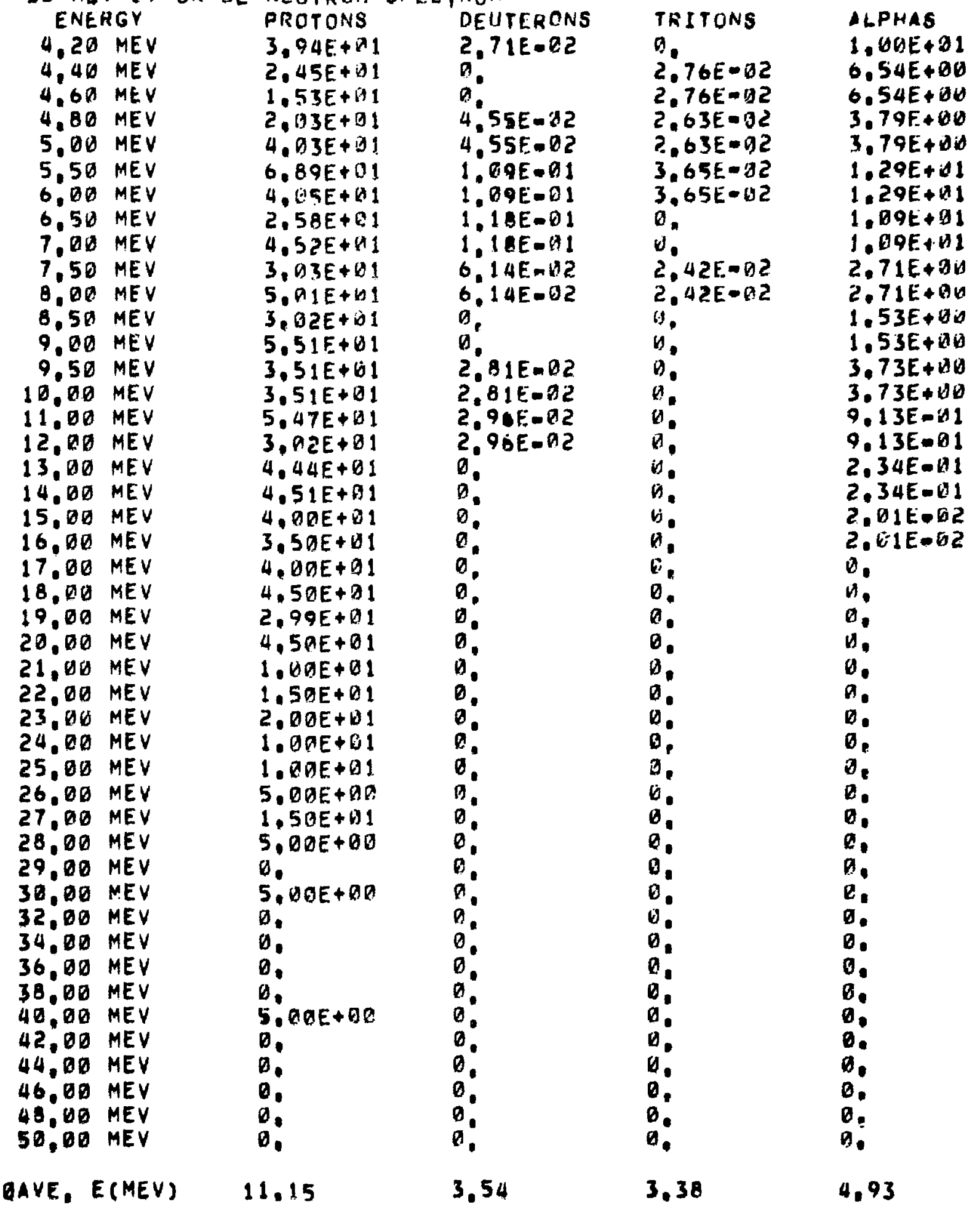


IINITIAL SPECTIUM AT O. $40-0.60$ CM. FOR ICRU MUSCLE 1TSSUE. 50 MEV D+ ON BE IFUTRON SPECTRU'

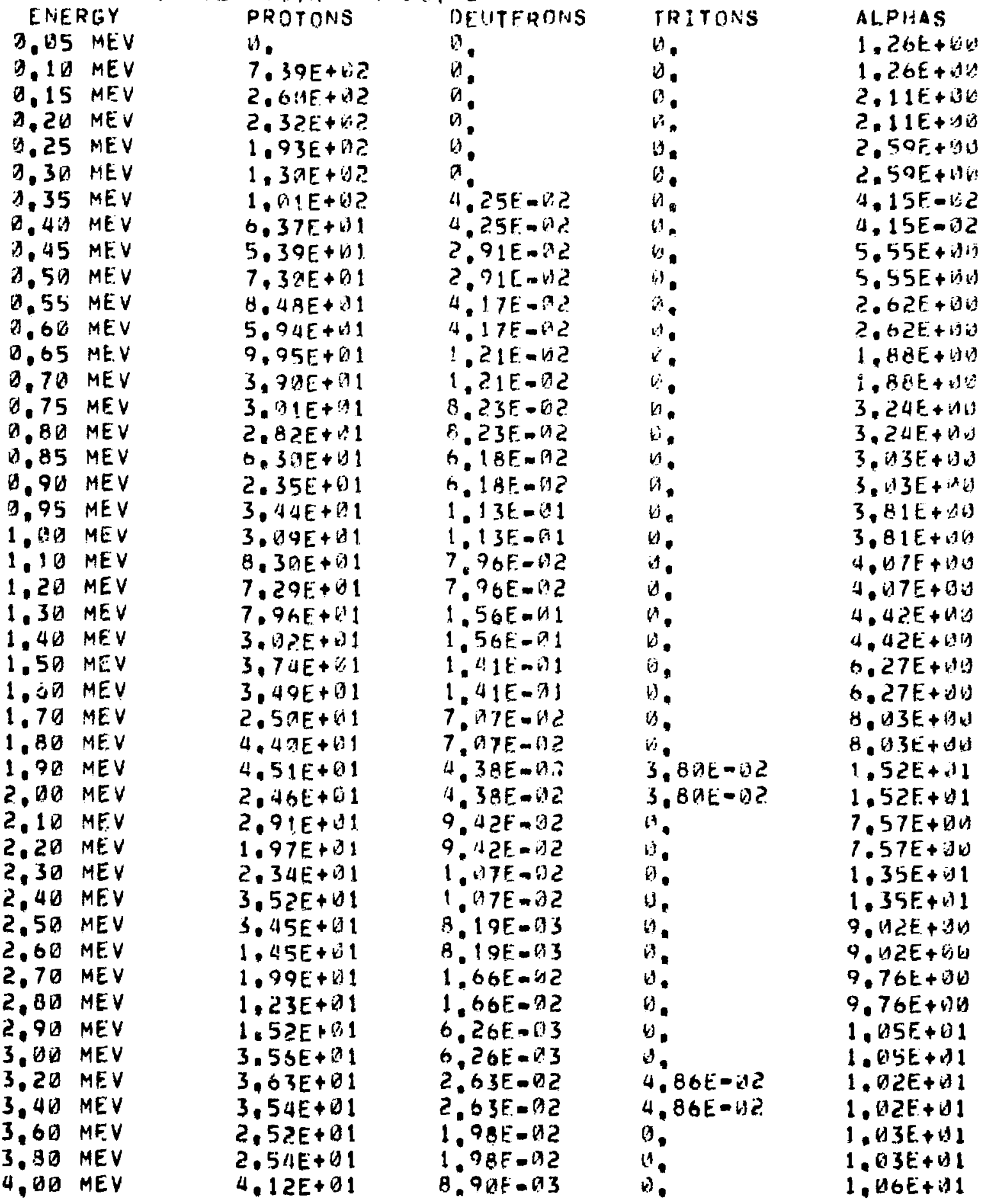


IINITIAL SPECTRUM AT $0.40-1.60$ CM, FOR ICRU MUSCLE TISSUE 5D MEV O+ ON RE HEUTRUN SPFCTRUMM

\begin{tabular}{|c|c|c|}
\hline & & \\
\hline 20 & MEV & $\therefore+b 1$ \\
\hline 40 & MEV & $95 E+d 1$ \\
\hline 60 & MEV & $64 E+11$ \\
\hline 80 & MEV & $33 E+11$ \\
\hline 5.00 & MEV & $2 E+\Delta 1$ \\
\hline 5.50 & MEV & $G E+D 1$ \\
\hline 6.00 & MEV & $15 E+1$ \\
\hline 6.50 & MEV & $3 E+131$ \\
\hline 100 & MEV & $A P E+11$ \\
\hline 7.50 & MEV & $\triangle 2 E+101$ \\
\hline B. 10 & MEV & $1 E+D 1$ \\
\hline 3.50 & MF:V & $2 E+01$ \\
\hline 100 & MEV & $96 E+H I$ \\
\hline 1.50 & MEV & VHE $B 1$ \\
\hline 1.00 & MEV & $52 E+01$ \\
\hline 1.00 & ME.V & $17 E+11$ \\
\hline 2.60 & MEV & $7 E+G 1$ \\
\hline .00 & MEV & $; X E+01$ \\
\hline .00 & MEV & $1 A E+01$ \\
\hline dat & MEV & $5 O E+U 1$ \\
\hline 00 & MEY & $30 \ddot{E}+b 1$ \\
\hline$D O$ & MEV & $5 \triangle E+B 1$ \\
\hline 100 & MEV & $5 G E+D 1$ \\
\hline 10 & MEV & $\Delta E+B 1$ \\
\hline (1) & MEV & $A \Delta E+D 1$ \\
\hline 00 & MEV & $5 . M B E+W G$ \\
\hline$\Delta \theta$ & MEV & $\theta$ \\
\hline 20 & MEV & $30 E+\Delta 1$ \\
\hline 00 & MEV & 1. $\triangle 0 E+11$ \\
\hline 00 & AIEV & $1 . D E+D 1$ \\
\hline & MEY & $1.5 m[+1] 1$ \\
\hline 0 & MEV V & 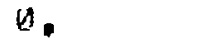 \\
\hline 00 & MEV & 6 \\
\hline 00 & MEV & $a$ \\
\hline 00 & MEV & $\theta$ \\
\hline$\theta 0$ & MEV & 0 \\
\hline$\Delta \theta$ & MEV & $\varphi$ \\
\hline 00 & MEV & $1 . \square E+D 1$ \\
\hline va & MËV & $\Delta$ \\
\hline$G Q$ & MEV & b. \\
\hline$O B$ & MEV & n \\
\hline & MEV & p \\
\hline & MEV & S. IUUE \\
\hline & $\begin{array}{l}\text { MEV } \\
\text { MEV }\end{array}$ & $\begin{array}{l}0 \\
0\end{array}$ \\
\hline & Mic & \\
\hline
\end{tabular}

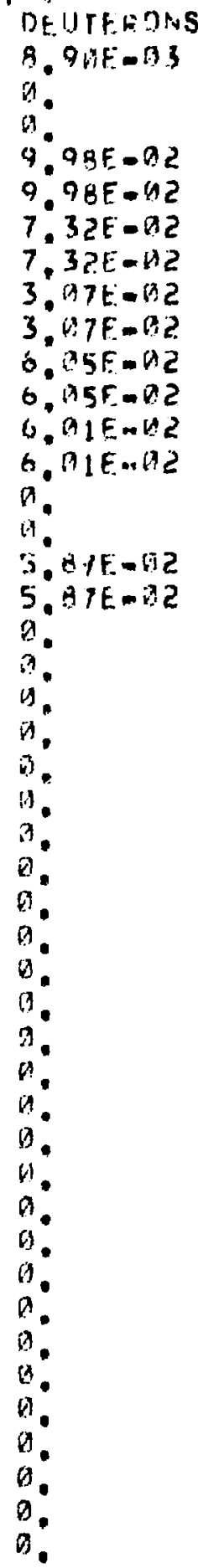

TRITINS

ALPHAS

1)

1. MOE+ BI

1. $19+\div+1$

1. $D Q E+I 1$

प.

7. $84 E+196$

4.97E- 112

$7.846+148$

$1.35 E+31$

b. $79 E-62$

$1.35 t+b 1$

$6.7 D E-U 2$.

1. $025 .+11$

$2.66 \mathrm{~F}=62$

1. $(125+1) 1$

$1.73 E+B K$

$1.73 E+i N n$

1. $58 E+110$

1. $58 E+A M$

$3.33 E+116$

$3.33 E+10)$

8. $31 E-Q 1$

R. 31t-A1

2. $O B E+H)$

$2.63 E+130$

6. $1 k E-U 3$

$6.10 E-03$

1.

b.

in

i).

b.

i.

b.

i.

A.

d.

v.

u.

b.

i.

3

13.

v)

(1)

u.

A.

4.

w.

a.

i'.

H.

B.

b.

Q.

i.

.

i.

औ!

is,

D.

0.

0

b.

n.

b.

n.

(3.

(i).

(i).

.

$\theta$.

i.

GAVE. E(MEV)

10.77

5.92

5.08

5.22 
1INITIAL SPECTRUM AT D.6\%-1. DO CM. FOH ICRU PUSCLE TISSUE

5D MEV D+ ON BE NEUTRON SPECTRUN"

\section{ENERGY}

2, D5 MEV

0.10 MEV

0.15 MEV

0. 21) MEV

$0.25 \mathrm{MEV}$

Q.3 3. MEV

$4.35 \mathrm{MEV}$

$0.40 \mathrm{MEV}$

0.45 MEV

$0.50 \mathrm{MEV}$

0.55 MEV

$0.60 \mathrm{MEV}$

$0.65 \mathrm{MEV}$

0.70 MEV

0.75 MEV

0.80 MEV

0.85 MEV

0.90 MEV

0.95 MEV

1. DO MEV

$1,10 \mathrm{MEV}$

$1.20 \mathrm{MEV}$

$1.3 \square \mathrm{MEV}$

1.40 MEV

1.50 MEV

1.60 MEV

1.70 MEV

$1.80 \mathrm{MEV}$

$1.90 \mathrm{MEV}$

2.00 MEV

2.10 MEV

2.20 MEV

2.30 MEV

2.40 MEV

2.50 MEV

$2,60 \mathrm{MEV}$

2.70 MEV

2.80 MEV

2.90 MEV

3.DD MEV

3,20 MEV

3.40 MEV

$3.60 \mathrm{MEV}$

3.80 MEV

4,DQ MEV
PROTOIS

D.

7.9.9E+

$3.42 E+32$

$2.13 E+12$

$1.27 E+02$

$1.4 B E+D ?$

$1,03 E+02$

$1 . G A E+12$

9. $B D E+B 1$

$7,62 E+11$

$7.29 E+$ पू

$7,3 \cap E+11$

$6.8 B E+B 1$

$5,1 O E+B 1$

4. $17 E+31$

$6.26 E+21$

$4.54 E+\Delta 1$

$4.31 E+11$

2. $2 B E+D 1$

$4.11 E+D 1$

$6.7 G E+B 1$

$4 . B A E+b 1$

$5.71 E+H 1$

$7.10 E+d 1$

$4.30 E+D 1$

$3.92 . E+B 1$

6.17E+D1

$4.04 E+D 1$

3. $A 6 E+\Delta !$

$3.12 E+6 !$

$3.7 ! E+01$

3. 2hE+ DI

2. $4 A E+D 1$

$2.43 E+B 1$

$1.99 E+B 1$

$2.95 E+111$

2. $B A E+01$

$1.66 E+01$

$2.72 E+11$

2. $50 E+01$

$5.21 E+D 1$

$3,31 E+111$

3. $26 E+01$

$3.18 E+1$

$2,75 E+01$
DEUTEROIS

a.

$3.35 E-A C$

$3.25 F-b 2$

$4.93 E-7 \bar{C}$

$4.93 E$ a. त?

$6.98 E-O ?$

$6.93 E-B 2$

E. MGE- U?

$8,0 G E=02$

$3.44 E-6 ?$

$3,44 E-6+2$

$3,695-A 2$

$3.69 E-02$

5. $4 \Delta E-A$ ?

$5.44 E-A 2$

$1,35[-D]$

$1.35 E-11$

$9.21 E-W 2$

$9.21 E-02$

G. B?E-F.?

$6: \therefore-112$

1. $29 E-B 1$

1. 2TE- 11

$5.53 E-42$

$5.53 E-1 A 2$

3. $47 E$ NAC

3. $47 E-22$

$3.33 E-112$

$3.33 E-D 2$

2.BIE-D?

2. B1E-Q2

$4.4 A E=$ C

$4.4 U E-Q 2$

$2.94 E-02$

$2,94 \mathrm{E}$. 02

$1,55 E-V Z$

$1.55 E-\cap ? 2$

$2.16 E-02$

$2,16 E-32$

$2,73 E=12$
TEITONS

D.

b.

$\theta$.

$b$.

i.

.

in

v.

$2.42 E-02$

$2.42 E=132$

6.

b.

D.

$\forall$.

$\because:$

9

$2.75 E-112$

$2.75 \mathrm{~F} \cdot \mathrm{na}$

$2.73 t-19 ?$

$2.73 E-V 2$

6.

V.

$5.39 t-112$

5. DQE- B?

$\forall$.

a.

B.

$\theta$

0.

B.

$\theta$.

b.

is.

0.

$2.45 E-3 ?$

$2,45 t-62$

(1).

0.

0.

vi.

is.

10.

(1)

.

$B$.
ALFIIAS

$2,13 E+96$

$2.13 E+B E$

2. $17 E+20$

$2 . \forall 7 E+110$

$2.06 E+49$

2. $136 E+192$

$9.34 E-41$

$9.34 E-n 1$

$1.50 E+115$

$1.56 E+119$

h. URE-II

6. $1 B E-V 1$

?. $3 \ddot{O F}+3 \mathrm{k}$

$2.38 k+3 b$

?.97E+ 19

$2.97 E+10$

$7.99 F-132$

$7.99 t-51$

$3.66 E+611$

$3.66 E+14$

$3,43 E+13 n$

$3.43 E+16$

$7.50 E+100$

$7.5 A E+40$

$1,35 t+31$

$1.35 E+41$

$4.71 E+.16$

$4.71 E+4.4$

6. $(A) E+4)$ id

$0.03 E+\ln B$

7. B6E+ (1)

$7,86 E+46$

$3.54 E+B .3$

$5.54 E+3$

9, $15 E+21$

$9.15 E+\angle G$

$7.25 E+140$

$7.25 E+10$

4. $98 E+19$

$4.98 E+a b$

$1.24 E+01$

1.2 $24 E+U 1$

$9.0 B E+D B$

9. DRE + Q U

$5,73 E+B D$ 
1INITIAL SPECTRUM AT O.6Q-1. AT CH. FOF ICRII MUSCLE IISSUE 50 MEV D+ ON BE NEUTRON SPECTRUA

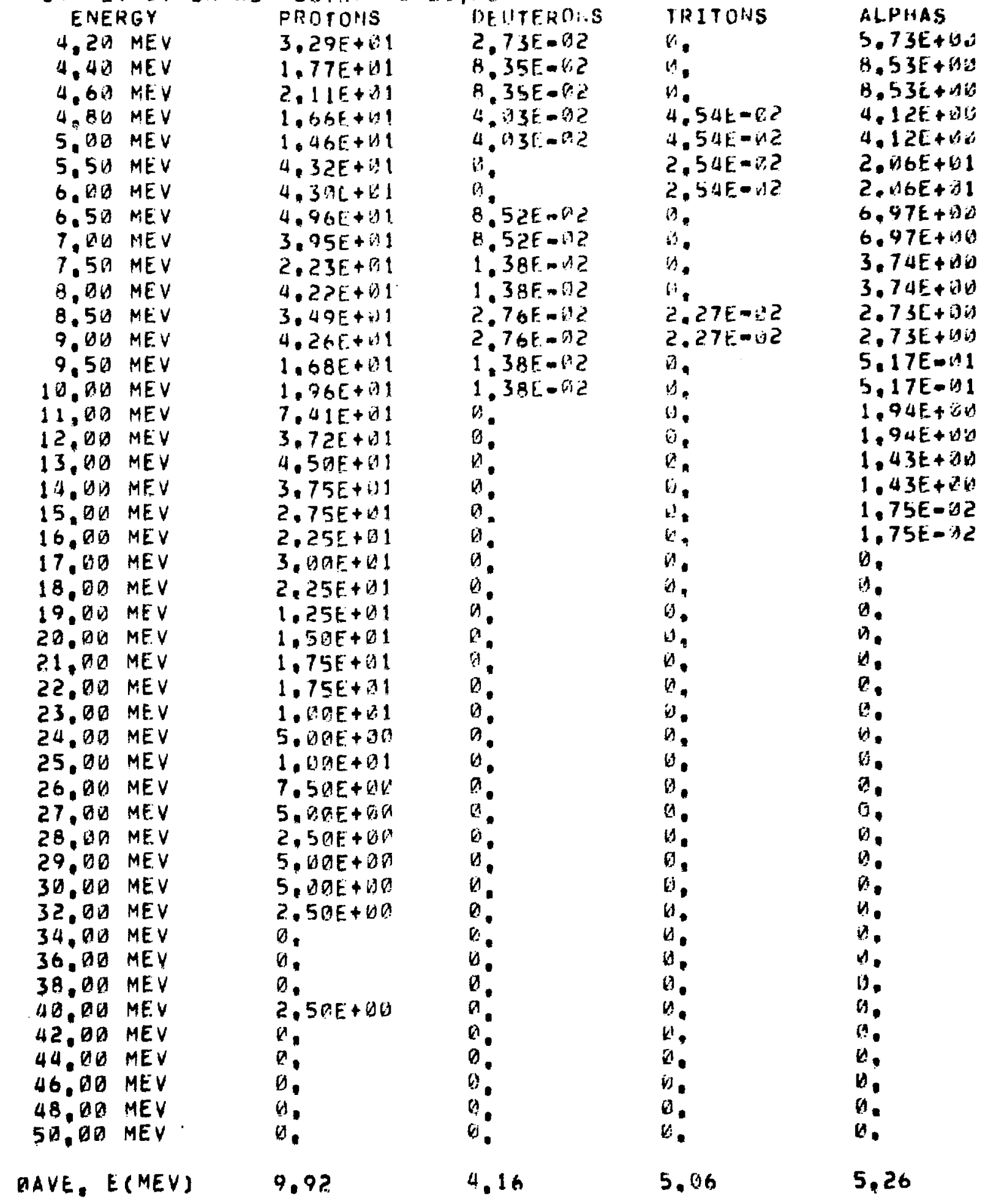


IINITIAL SPECTRUM AT 1.OQ=2,9D CM, FOR ICRU MUSCLE IISSUE 50 MEV D+ ON BE NEUTRON SPECTRUM

\section{ENERGY}

D.DS MEV

$0.10 \mathrm{MEY}$

0.15 MEV

$0.20 \mathrm{MEV}$

$0.25 \mathrm{MEV}$

$0.30 \mathrm{MEV}$

$0.35 \mathrm{MEV}$

Q. 14 MEV

$0.45 \mathrm{MEV}$

$0,50 \mathrm{MEV}$

$0,55 \mathrm{MEV}$

$0.60 \mathrm{MEV}$

$0.65 \mathrm{MEV}$

$0.70 \mathrm{MEV}$

0.75 MEV

$0.80 \mathrm{MEV}$

$0.85 \mathrm{MEV}$

0,90 MEV

0.95 MEV

1. $Q D$ MEV

$1.10 \mathrm{MEV}$

$1.20 \mathrm{MEV}$

$1.30 \mathrm{MEV}$

$1.40 \mathrm{MEV}$

1,50 MEV

$1.60 \mathrm{MEV}$

$1.70 \mathrm{MEV}$

$1.80 \mathrm{MEV}$

$1.90 \mathrm{MEV}$

2,00 MEV

$2,10 \mathrm{MEV}$

2.20 MEV

2.30 MEV

$2.40 \mathrm{MEV}$

2,50 MEV

2.60 MEV

2.70 MEV

2.80 MEV

$2,90 \mathrm{MEV}$

3.OD MEV

3,20 MEV

3.40 MEV

3,60 MEV

3,80 MEV

4.00 MEV
PROTONS

a.

$8.50 E+02$

3. $30 E+02$

$2,52 E+D 2$

$1.78 E+02$

$1.55 E+192$

$1.11 E+02$

$1 . \triangle 3 E+D 2$

$6,76 E+01$

8. D5E + 01

$7.40 E+91$

$7.79 E+11$

$5.45 E+01$

$5.89 E+01$

$5.62 E+111$

$4.35 E+C 1$

3. $54 E+11$

4. 6) $4 E+01$

4. $48 E+01$

$3,79 E+11$

$6.54 E+01$

$5.32 E+61$

5. $80 E+01$

$5.23 E+01$

$5.34 E+d 1$

$5.75 E+01$

$4.65 E+01$

$4.13 E+0^{\circ}$

$2.99 E+101$

$3,31 E+01$

$3.31 E+01$

$2.65 E+01$

$2,62 i+01$

2. $49 E+D 1$

$2, D 9 E+\nabla 1$

2. $80 E+D 1$

$1.57 E+11$

2. $59 E+D 1$

$2.51 E+01$

2. $D G E+B 1$

$4.99 E+01$

$4.24 E+01$

$3.54 E+01$

2. $34 E+01$

$2.17 E+01$
DEUTERONS

9.

6

$5.38 E=03$

$5,38 E-D 3$

$1.69 E-02$

$1.69 E-72$

$1.56 E-02$

$1.56 E-D 2$

2.92E-02

2. 92E-G2

$7.25 E-M 2$

$7.25 E-B 2$

$2.89 E=02$

2.89E-O2

5. $80 E-12$

$5,80 E-02$

$6.019-02$

6. $1, E=02$

$3.75 E-02$

$3.75 \mathrm{E}-\mathrm{AZ}$

$1.545-01$

$1.54 E-B 1$

$9.4 B E-B 2$

9.485 .02

$1.99 F-91$

1. OPF-01

$7.72 f-112$

$7.72 E-102$

$6.90 E=192$

$6,70 E-112$

$6.12 E-92$

$6.10 E-\theta 2$

1. $B 3 E+90$

$1.03 E+110$

$4.6 B E-12$

$4.6 B E-02$

$5,31 F-01$

5. $31 E-01$

2. $20 E-62$

2. $20 E-1.2$

4. 22E 02

$4.22 E-02$

$2.99 E-12$

2,99E-DL

$2,1 B E-D 2$
IRITONS

$\theta$.

a

a.

a.

$8,41 E=03$

$8,41 E$ - 33

$3.7 \Delta E-$ is 3

3.74E- $\{3$

0.

b.

0

b)

$b$

i.

b.

D.

$b_{0}$

id.

a.

d.

1. [9E-i]2

1, $\triangle 9 E-A C$

2. BSE- $D C$

?. $(45 E-b) 2$

$9.93 E-03$

$9.93 E-33$

2.1GE-G2

$2.16 E-62$

$1 . ด 9 E-V 2$

1. $\triangle 9 E-\triangle$ ?

$1 ., 18 E-D 2$

1. $98 E-D 2$

$8.48 E-U 3$

B. $48 E-73$

$2.13 E-02$

$2.13 E-02$

$7.93 E-Q 3$

$7,93 E-H 3$

a.

6.

$5.63 E-63$

$5.63 E=03$

1. $) B E-D 2$

1. GEE- 02

v.
ALPHAS

6. 6 ite $=1$

6. $6 U E-Q 1$

$2.89 E+W Q$

2. 89E+UD

$2,4 U E+\Delta D$

2. $40 E+: 10$

$8.96 E-U 1$

B.96E-A1

$1.34 E+\Delta U$

$\therefore 34 E+D E$

$1,84 E+B C$

$1,84 E+B 6$

$2.43 E+96$

$2.43 E+00$

1. $79 E+410$

$1.79 E+110$

$2.59 E+16$

$2.59 E+D D$

$3,32 E+\Delta B$

3. $32 E+V 0$

$6,17 E+100$

$6.17 E+09$

$4.43 E+136$

$4.43 E+B H$

$4.79 E+00$

$4.79 E+60$

$9.13 E+150$

$9.13 E+116$

$4.37 E+B C$

$4.37 \mathrm{E}+916$

$8.54 E+150$

$8,54 E+140$

$9.92 E+100$

$9.92 E+00$

$6.94 E+D y$

$0.94 E+C 13$

$8.14 \mathrm{E}, 16$

$8,14 E+01$

$8.73 E+01$

B.7 SE+DE

$1.40 E+W 1$

$1,40 E+01$

$1.26 E+01$

$1.26 E+01$

$8,55 E+00$ 
IINITIAL SPECIRUM AT 1.OHAZ, HU CM. COR ICPU MUSCLE TISSUE 50 MEV D+ ON BF NEUTRON SPECTRUM

\section{ENERGY}

$4.20 \mathrm{MEV}$

4.40 MEV

4.62 MEV

4.80 MEV

5.00 MEV

5,50 MEV

$6 . \triangle B \mathrm{MEV}$

$6.50 \mathrm{MEV}$

7,00 MEV

7.50 MEV

6. DO MEV

8,50 MEV

9,00 MEV

$9.50 \mathrm{MEV}$

$10.00 \mathrm{MEV}$

$11.90 \mathrm{MEV}$

$12.00 \mathrm{MEV}$

13,00 MEV

14. HD MEV

$15.00 \mathrm{MEV}$

16. TO MEV

17. DO MEV

$18,00 \mathrm{MEV}$

$19 . D D$ MEV

$20.00 \mathrm{MEV}$

$21.00 \mathrm{MEV}$

2?.DO MEV

$23.0 V M E V$

$24.00 \mathrm{MEV}$

25, UO MEV

26,00 MEV

$27.00 \mathrm{MEV}$

$28.00 \mathrm{MEV}$

$29,00 \mathrm{MEV}$

$30.00 \mathrm{MEV}$

32.00 MEV

34. DO MEV

36.00 MEV

38,0 MEV

40.00 MEV

42,80 MEV

44,DD MEV

46,00 MEV

48,00 MEV

$50.00 \mathrm{MEV}$
PROTONS

$3.69 E+01$

$3.08 E+01$

3. 3AE+AS

$2.51 E+21$

2. $28 E+21$

$6.30 E+21$

$3.63 E+11$

4. $91 E+01$

5. $\triangle 4 E+B 1$

$4,16 E+01$

3. $26 E+B 1$

$3.64 \mathrm{~F}+01$

$2.98 E+B 1$

2. $95 E+11$

1. $78 E+01$

$5.35 E+\Delta 1$

$5,39 E+D 1$

4. $49 E+01$

3. $57 E+01$

$2.90 E+101$

2. $7 D E+01$

3. $\triangle O E+O 1$

2. $10 E+01$

2, $3 \Delta E+B 1$

1. $40 E+1$

1 ? $Q E+D$ ?

$1.90 E+01$

1. $20 E+\theta 1$

5. $0 A E+B O$

9.DOE + DQ

$9 . \triangle A E+60$

6. DDE+ OD

3. $00 E+D G$

$2, B O E+B D$

3. $\triangle Q E+Q D$

3.DQE + OD

3. $R$ CIE+OE

O.

$\theta$.

$1.0 D E+O A$

0 .

$\theta$.

0.

0.

0.

9.86
DEUTERONS

2. $18 E-02$

$5.17 E-91$

$5,17 E-D 1$

$5.27 E=01$

$5.27 E-a_{1}$

1. $52 E-02$

$1,52 E-12$

1. U1E+ AU

1. O1E+ Dú

$4.4 B E-112$

$4.4 B E-D 2$

0

a.

$1.15 \mathrm{E}-02$

$1,1 \mathrm{SE}=02$

5. $\triangle D E-D 1$

5. $0 \mathrm{ED}=1$

5 .

0.

9.

(1.)

.

:

a.

9

,

ด.

9.

0.

ต.

0.

9.

0.

0.

9.

$a$

D.

ต.

$B$

a.

a.

0

9.

7.03
TRITONS

U.

1. $D Z E=A 2$

1. उ2E M $\triangle 2$

,

$\theta$

$1.08 E-02$

1. $\$ 8 E-62$

$1.116 E-02$

1. BGE-GL

v.

is.

1. D2E-O2

1. $12 E=02$

a.

b.

b.

b.

b.

V.

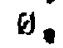

0.

$\theta$

g.

0.

$\theta$

.

i.

0

0

$\theta$

$\theta$

0.

a.

0

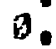

$\theta$

$\theta$

0

a.

0

$\theta$.

0

0.

4.37
ALPHAS

$8.55 E+915$

$5.91 E+U V$

5. $91 E+\Delta K$

$7.35 E+B 6$

$7.35 E+190$

$1,23 E+\Delta 1$

$1.23 E+91$

7. $44 E+100$

$7.44 E+100$

$4.67 E+D D$

$4.67 E+00$

$1.63 E+100$

$1.63 E+06$

$1.17 E+\theta 0$

$1.17 E+\theta 0$

2. $\triangle B E+O B$

2. $98 E+B D$

$1,29 E-1$

1. 29E-DI 1

5. $12 E=1$

5. $12 E-D 1$

$1,21 E=\square 3$

$1.21 E-03$

$\theta$.

a.

0.

0.

v.

b.

0.

0.

ด.

a.

a.

a.

$\theta$.

a.

0.

$\theta$

3.

0

0

$\theta$.

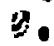

4

5.06 
1INITIAL SPECTRUM AT $2,0 D=4,09$ CM, FOR ICRU MUSCLE TISSUE 50 MEV D+ ON BE NEUTRON SPECTRUM

\section{ENERGY}

D. B5 MEV

0.10 MEV

$0.15 \mathrm{MEV}$

$0,20 \mathrm{MEV}$

$0.25 \mathrm{MEV}$

Q.30 MEV

0.35 MEV

0.40 MEV

0.45 MEV

0,50 MEV

0.55 MEV

$0.60 \mathrm{MEV}$

0,65 MEV

0.70 MEV

0.75 MEV

$0.80 \mathrm{MEV}$

0.85 MEV

0.90 MEV

$0.95 \mathrm{MEV}$

1.00 MEV

1.20 MEV

1,20 MEV

$1,30 \mathrm{MEV}$

1. UE MEV

1.50 MEV

$1,60 \mathrm{MEV}$

$1,70 \mathrm{MEV}$

1.8 M MEV

$1,90 \mathrm{MEV}$

2.DO MEV

2,10 MEV

2.20 MEV

$2,30 \mathrm{MEV}$

2.40 MEV

$2.50 \mathrm{MEV}$

$2.60 \mathrm{MEV}$

2,70 MEV

2.80 MEV

2.90 MEV

3.00 MEV

3.20 MEV

3.40 MEV

3.60 MEV

3.80 MEV

4.DO MEV

\section{PROTONS}

D.

$9.23 E+02$

$3.65 E+02$

$2.39 E+02$

$2.17 E+D 2$ ?

$1.63 F+42 ?$

$1.26 E+D ?$ ?

$1.07 E+02$

1. $01 E+0$ ?

9.17E+DI

B. OIE +01

7. $\triangle B E+D 1$

$7.26 E+01$

$5,61 E+01$

$5.63 E+21$

$5.26 E+11$

5. $25 E+01$

$4.26 E+011$

$4.89 E+101$

3. $75 E+01$

$5,86 E+11$

$7.78 E+01$

$5.98 E+01$

4. $84 E+D 1$

$5.21 E+01$

$5.82 E+01$

$4.52 E+81$

$4.54 E+61$

$3.76 E+01$

$4.64 E+01$

$3.60 E+11$

$2.86 \mathrm{E}+11$

2. $32 E+01$

$3.84 E+61$

$2.60 E+21$

$2.70 E+01$

$3.11 E+81$

$2,14 E+B 1$

2. $49 E+B 1$

$2.24 E+(0)$

$3,06 E+01$

4. $14 E+01$

$3.72 E+01$

$3,53 E+01$

3. $26 E+D 1$
DEUTERONS

D.

0.

1.5SE-D?

$1.55 E-112$

$1,60 \mathrm{E}=0$ ?

1. GBF-g2

2. $32 E-1$ ?

2.32E-ด2

$4.14 E .02$

$4.14 E-192$

$4.75 E-A 2$

$4.75 E=0 ?$

$4.56 E-B 2$

$4.56 E-02$

$7.29 E-112$

$7.29 E-112$

$3.92 \mathrm{~F}-02$

$3,92 E-B 2$

$5,47 E-A 2$

5.47F-A2

1. $25 F-01$

1. 25E-31

$3.82 E-01$

3. $B 9 E-D 1$

1. $1 \forall E=\theta 1$

1. $|B E-B|$

$9.43 E-B 2$

$9.43 E=B 2$

$6.97 E-02$

$6.97 E-02$

4.39E-02

$4.39 E=02$

2. 81E-01

2. $81 \mathrm{E}=\mathrm{B1}$

2.8YE-OZ

2.87E-02

2. 70E-OI

2.7EE-O1

$3.52 E-02$

$3,5 \mathcal{R} E-82$

$2.21 E-02$

2. $21 E-02$

2.86E-01

2. B6E-01

$5,17 E-02$
TRITONS

$D$.

a.

B.

$\theta$

$1.5 A E=D 2$

$1.50 E=B 2$

5. DOEE-DS

5. DOE-U3

$5,89 E=B S$

5.89E-B 3

$1.15 E-G 3$

$1.15 E-03$

$\theta$.

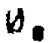

$\theta$.

a.

6.

$\forall$

.

$\theta$.

1. $16 E-$ is 2

$1,16 E-B 2$

0.

$y$.

$1.48 E-62$

1.4AE-D?

0 .

0.

$1.42 E-02$

$1.42 E-02$

$1.0 B E-D 2$

1. $\triangle B E=Q 2$

b.

b.

b.

$\theta$.

2.19E-02

$2.19 E-02$

$9, B B E=Q 3$

$9,8 B E=93$

2.07E-Q2

2.07E-D2

3. $49 E-13$

3. $49 E-23$

$1.27 E-62$
ALPR,AS

$6,73 E-01$

$6.73 E-n 1$

$1.71 E+611$

$1.71 E+O L$

$1.74 E+09$

$1.74 E+14$

$2.67 E+B E$

$2.67 E+0 n$

$2.28 E+Q 1 n$

2. $28 E+116$

$2.60 E+b 11$

$2.60 E+110$

$2,45 E+150$

$2.45 E+60$

$3.23 E+B D$

$3.23 E+6 U$

$2,80 E+B D$

2. $80 E+\theta U$

$2,96 E+90$

$2.96 E+\forall \theta$

$5, B D E+\forall B$

$5,8 B E+D O$

$6.91 E+Q 0$

$6.91 E+0 D$

$7.76 E+00$

$7.76 E+60$

$6.52 E+105$

$6.52 E+D B$

$9.05 E+00$

$9.05 E+01$

$9.91 E+100$

$9.91 E+00$

1. $17 E+11$

1. $17 E+01$

$9.5 B E+D B$

$9.58 E+00$

$8.95 E+106$

$8.95 E+00$

$5,46 E+0 B$

$5.46 E+D B$

$1.68 E+01$

$1.68 E+61$

$1.33 E+01$

1. $33 E+d 1$

1. $10 E+01$ 
IINITIAL SPECTRUM AT $2.00-4.0 Q$ CM. FOR ICEU MUSCLE TISSUE 50 MEV D+ ON BE NEUTRDN SPECTRUM

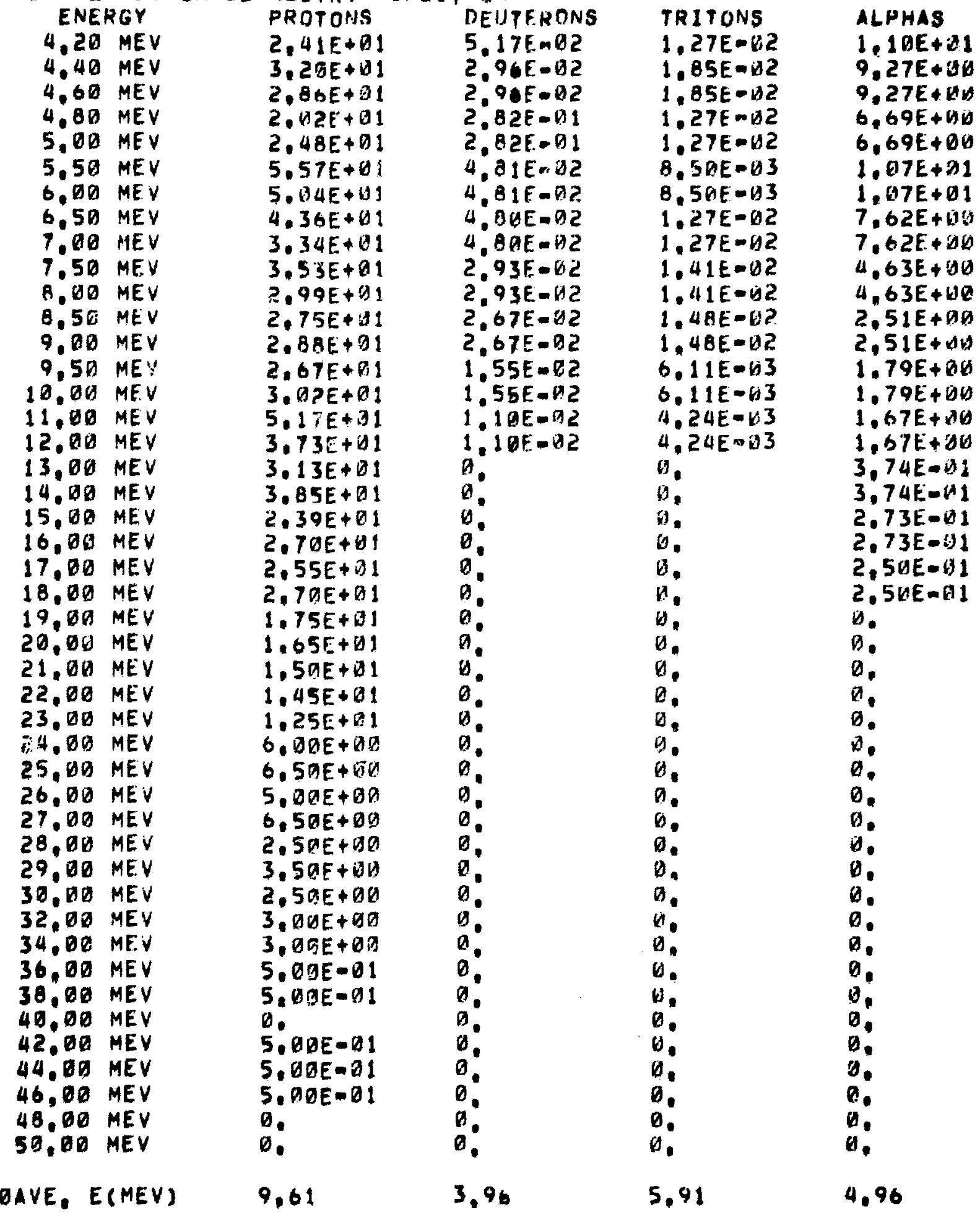


1INITIAL SPECTRUM AT 4.DO-6.9A CN. FOH ICRU MUSCLE IISSUE. SO MEV D+ ON BE. NEUTRON SPECJRUH

\section{ENERGY}

D. 05 MEV

D. 10 MEV

$\checkmark .15$ MEV

$0,20 \mathrm{MEV}$

Q.25 MEV

3.30 MEV

$0,35 \mathrm{MEV}$

Q. 4 M MEV

0.45 HEY

$0.50 \mathrm{MEY}$

$0.55 \mathrm{MEV}$

$0,60 \mathrm{MEV}$

$0.65 \mathrm{MEV}$

0.79 MEV

0.75 MEV

$0.8 \mathrm{MEV}$

$0.85 \mathrm{MEV}$

$0.90 \mathrm{MEV}$

0.95 MEV

1. . O MEV

1.10 MEV

1.2D MEV

$1,30 M E V$

$1.40 \mathrm{MEV}$

1.50 MEV

$1.60 \mathrm{MEV}$

$1.79 \mathrm{MEV}$

$1.80 \mathrm{MEV}$

1.90 MEV

2. DO MEV

2.10 MEV

2.20 MEV

2,30 MEV

2. 40 MEV

2.50 MEV

2.60 MEV

2.70 MEV

$2,8 \mathrm{MEV}$

2.90 MEV

3. OQ MEV

3.20 MEV

3.40 MEV

3.6日 MEV

3.80 MEV

4. $\triangle O$ MEV

\section{PROTCNS}

D.

9. 6 BE+ 12

$3.47 E+b 2$

$2.57 E+W 2$

$1.87 E+132$

1. $45 E+172$

$1.286+12$

B. $74 E+B I$

$9.36 F+01$

$7.93 E+D I$

$6.92 E+B 1$

$6.58 E+1$

5. $4 A E+11$

5. $8 ? E+11$

$5,33 E+01$

$5,61 E+01$

$4.12 E+01$

$3.7|E+D|$

4. $11 E+61$

$3.3 B E+D 1$

$7.74 E+B 1$

$7.34 E+101$

6. $17 E+\square 1$

6. $33 E+01$

$6.62 E+D 1$

5. $48 E+01$

5. $121 E+b 1$

4. $25 E+1$

4. $7 h E+U 1$

3. $4 \Delta E+O 1$

3. $7 B E+D 1$

3. $5 U E+01$

2. $82 E+01$

2. $19 E+01$

3. $96 E+01$

3. $U 2 F+1) 1$

2. $90 \mathrm{Cg}+01$

Z. $13 E+01$

2. $43 E+01$

2. $29 E+01$

$4.20 E+101$

$4 .|1 E+D|$

3. $01 E+01$

$3.41 E+01$

2.97E+01

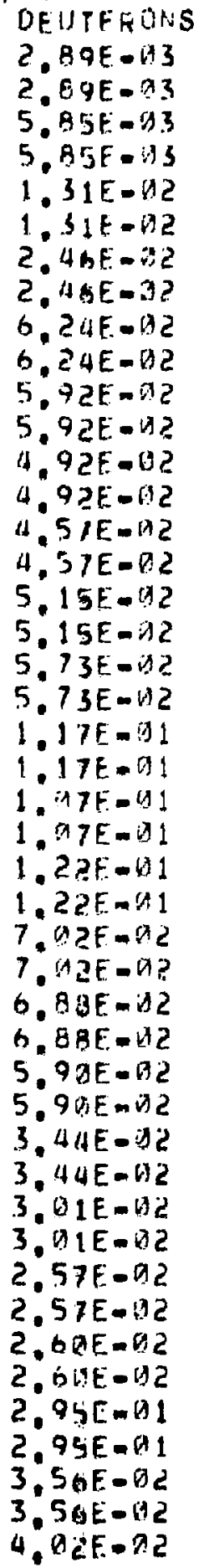

DEUTFEUNS

IPITONS

A1.PHAS

7. $99 E-11$

$7.99 E-11$

1. $13 E+\angle D$

$1.13 E+10 V$

1. $82 E+1 K$

$1.82 E+4 w$

$1.96 t+\Delta V$

1.96E+AR.

2. $12 E+A B$

2. $\triangle 2 E+B d$

2. $09 E+30$

$2.69 E+00$

2. $1 E+(E)$

2. BIE+WU

2. 4 $5 E+b l$

2. $45 E+3 i)$

2. $02 E+B u$

2. $\Delta 2 E+\forall \Delta$

$2.64 E+W 1$

$2,64 E+A N$

5. $24 E+U D$

5. $24 E+06$

$5.96 E+26$

$5.96 E+d i$

$5.53 E+40$

$5,53 E+00$

$5.47 E+B A$

$5.47 E+00$

$6.3 B E+1 B$

$0.8 \mathrm{k} E+14$

7. $42 E+i v w$

$7.42 E+1$

$7.29 E+\Delta W$

$7.29 E+14$

$6.60 E+B W$

6.6 W $E$ +D

$6.65 E+14$

$6.65 E+v v$

$7.1 A E+W$

7. $1 U E+1 U$

1. $23 E+11$

$1.23 E+11$

1. $29 E+d 1$

$1,29 E+41$

$B, 2 B E+M$ 
IINITIAL SPECTRUM AT GOERAR, FG C:A. FUR ICRU MUSCLE TISSUE 50 MEV D+ DN BE NEUTRON SPECTRIJA

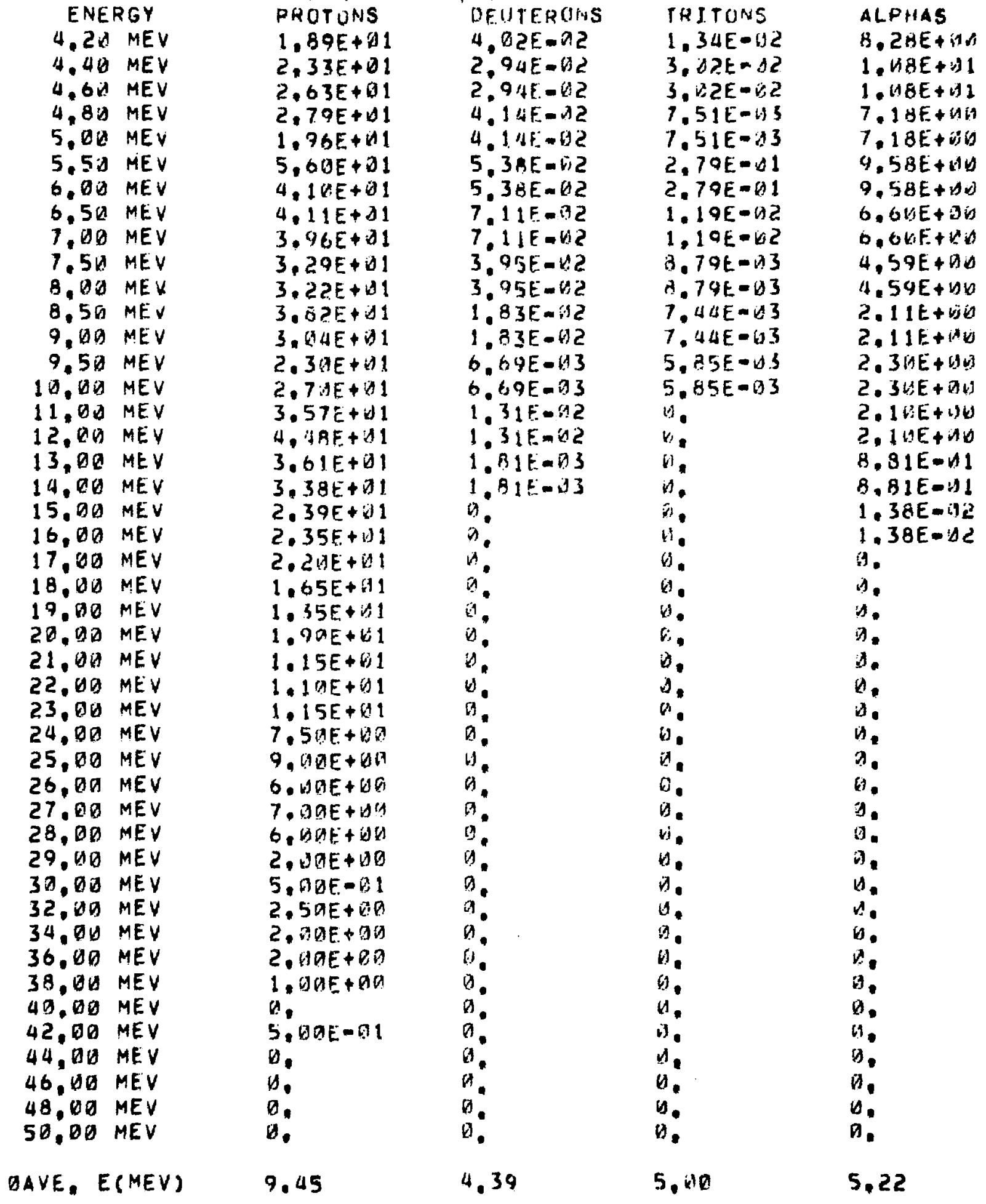


1INITIAL SPECTRUM AT G.DAOIKOA CM. FOR ICRII MUSCLE TISSUE 50 MEV D+ ON BE NELTTKON SPECTRUM

\section{ENERGY}

D. DS MEV

0.10 MEV

$0.15 \mathrm{MEV}$

$0.20 \mathrm{MEV}$

D. 25 MEV

$0.30 \mathrm{MEV}$

$D .35 \mathrm{MEV}$

$0.40 \mathrm{MEV}$

0.45 MEV

$0.50 \mathrm{MEV}$

$0,55 \mathrm{MEV}$

0.64 MEV

0.65 MEV

$0.70 \mathrm{MEV}$

0.75 MEV

$0.80 \mathrm{MEV}$

$0.85 \mathrm{MEV}$

$0.90 \mathrm{MEV}$

0.95 MEV

1.01 MEV

$1.10 \mathrm{MEV}$

1.20 MEV

$1.30 \mathrm{MEV}$

$1.40 \mathrm{MEV}$

$1.50 \mathrm{MEV}$

$1.60 \mathrm{MEV}$

1.70 MEV

$1 . B O$ MEV

$1.90 \mathrm{MEV}$

2, DO MEV

2.10 MEV

$2,20 \mathrm{MEV}$

2.30 MEV

$2,40 \mathrm{MEV}$

$2.50 \mathrm{MEV}$

2.60 MEV

2.70 MEV

2.80 MEV

2.90 MEV

3. DD MEV

$3.20 \mathrm{MEV}$

$3,40 \mathrm{MEV}$

3,60 MEV

3,80 MEV

4.BD MEV
PROTONS

$\theta$.

8. $45 E+92$

3. $69 E+142$

$2.29 E_{-}+22$

1. $79 E+B$ ?

$1.37 E+1) 2$

1. $1 A E+6$ ?

$9.84 E+B 1$

$8,53 E+11$

$9.66 E+91$

B. 1RE+U1

$6.7 D E+191$

$6.68 E+11$

$5.73 E+01$

$4.87 E+01$

$4.89 E+B$ I

4. $\mid 8 E+11$

$4.71 E+81$

$3.96 E+111$

$3, B 2 E+11$

$7.38 E+61$

$6.61 E+1$

$5.74 E+\& 1$

$5.72 E+61$

$5.01 E+1)$

$4.35 E+01$

$4.74 E+B 1$

$3.96 E+\$ 1$

3.7 ZE +11

$3.30 E+61$

$4.39 E+01$

$3.36 E+D 1$

$3,25 E+C 1$

$3.05 E+01$

$2.69 E+161$

$2.55 E+101$

$2.34 E+91$

$2.53 E+01$

$2.34 E+01$

$1.85 E+B 1$

3. $3 B E+D 1$

$3.43 E+61$

$3.38 E+01$

$3.36 E+U 1$

$2.99 E+81$
CFUTEODINS

4. DOE -03

$4.06 E-03$

2. $82 E-1) S$

2. B.2F- 83

B. $18 E-A 3$

B. $18 F-.13$

$2.62 E-112$

$2,62 \mathrm{~F}-112$

$3.75 E-1.2$

$3.75 E-112$

3. 3 UF $-A$ ?

$3,3 Q E-A^{A} 2$

$4.03 E-12$

$4.03 E-0$ ?

$3.97 E-A 2$

$3.978-52$

$3.96 \mathrm{E}-92$

$3.96 E-82$

$4.63 E-02$

$4.63 E-\pi^{2} ?$

$1,|3 E-A|$

$1.93 E-11$

1. IXE-UI

1.1 BE - B !

$9.79 E-12$

$9.79 E-82$

$8.54 E-02$

$8.52 \mathrm{~L}-0.2$

$6.22 E-42$

$6.22 E-42$

$3.9 B E-D 2$

$3.98 E-D 2$

$2.98 E-02$

2.98E- И2

$4.25 E-12$

$4.25 E-02$

2. $22 \mathrm{E}-12$

$2.22 \mathrm{E}-92$

$1.84 E-02$

$1.84 E-D 2$

1.65E-Bi 1

$1.65 \mathrm{~F}-01$

2. BIE-02

2. DIE-DL

$2,62 E-D 2$
TRITONS

B.

n.

6

8.

$2.34 E-63$

$2,04 t-n 3$

b.

b.

$2.55 E-1.3$

$2.55 E-1) S$

B. JAE - is 3

$8.19 E-$ i 3

$4.75 E-13$

$4,75 E=(1) 3$

$5.11 E-13$

$5,11 E=\operatorname{B} 3$

$5.34 E=-3.3$

$5.34 E-A 3$

6.

n.

$7,11 E-03$

$7.11 E=03$

3. 3 AE $=$ त3

$3.3 a E=63$

$7.66 E-03$

$7.66 E-43$

$1.35 E-01$

$1.35 E-1$

$1.03 E-\angle 2$

1. $\square 3 E-\square 2$

$5.03 E-13$

$5.03 E-D 3$

$7.62 E=W 3$

$7.62 E-U 3$

$2.37 E-33$

$2.3 T E-V 3$

$8.43 E-D 3$

$8.43 E-1.3$

$6.95 E-13$

$6.95 E-1) 3$

$7,29 E-\triangle 3$

$7.29 E-D 3$

$1.10 E=02$

$1.12 E-02$

$1.09 E-02$
ALPHAS

1. $19 E+D U$

1.19E+1001

2. $25 E+4: 3$

2. $2 S F+U B$

$2.11 E+111$

2. $11 E+W E$

2. $14 E+10$

2. $14 E+A k i$

2. $2 B E+A$.

$2.28 E+b v$

$1.64 E+110$

$1.64 E+9.1$

2. $\triangle 6 E E+13:$

2. $B G E+U A$

2. $\Delta G E+W D$

2. $\triangle B E+3 W$

$2.18 E+114$

2. $1 B E+13 A$

2. $31 t+b v$

$2.31 E+U v$

$5,71 k+10$

3.71E+vin

$5.11 E+B V$

5. $11 E+b 1$

$5.21 E+U$.

5. $21 E+D W$

$6.44 E+(06$

6. $4 \Delta E+D n$

$5.80 E+0 V$

$5,8 D E+D U$

$6.26 E+\Delta 6$

$6.26 E+101$

6.17E+dB

$6.17 E+v i v$

$7.14 E+\vec{B} D$

$7,14 E+U D$

$6.66 E+194$

$6.66 E+\Delta h$

$6.53 E+d D$

$6.53 E+\Delta \theta$

$1.25 E+111$

1. $25 E+91$

1. $15 E+11$

$1.15 E+11$

8.97E+ JU 
IINITIAL SPECTRUM AT 19,O-14. CM, FOR ICRU MUSCLE TISSIJE 50 MEV D+ ON BE NEUTPONN SPECTRUM

ENERGY

D.D5 MEV

$0.1 D \mathrm{MEV}$

$0.15 \mathrm{MEV}$

$0.20 \mathrm{MEV}$

0.25 MEV

0.30 MEV

0.35 MEV

0.40 MEV

$0.45 \mathrm{MEV}$

$0.50 \mathrm{MEV}$

$0.55 \mathrm{MEV}$

0.6 MEV

0.65 MEV

$0.70 \mathrm{MEV}$

$0.75 \mathrm{MEV}$

0.80 MEV

0.85 MEV

$0.90 \mathrm{MEV}$

$0.95 \mathrm{MEV}$

1. OD MEV

$1.10 \mathrm{MEV}$

1.20 MEV

$1.30 \mathrm{MEV}$

1. 40 MEV

$1.50 \mathrm{MEV}$

$1.60 \mathrm{MEV}$

$1.70 \mathrm{MEV}$

$1.80 \mathrm{MEV}$

$1.90 \mathrm{MEV}$

2. $\triangle O$ MEV

2. 10 MEV

$2.20 \mathrm{MEV}$

$2,30 \mathrm{MEV}$

2. 40 MEV

2.50 MEV

$2.60 \mathrm{MEV}$

2.70 MEV

$2.80 \mathrm{MEV}$

$2,90 \mathrm{MEV}$

3.00 MEV

3.20 MEV

$3.40 \mathrm{MEV}$

3,60 MEV

$3.80 \mathrm{MEV}$

4.DO MEV
PRIITONS

Q.

$7.34 E+D ?$

2. $93 E+Q 2$

$2.15 E+0$ ?

1. $49 E+12$

$1.31 E+112$

$1.03 E+0$ ?

$9.52 E+01$

$7.96 E+01$

$7.84 E+01$

$6.44 E+01$

5.27E+41

$4,77 E+111$

$4.82 E+11$

$3.97 E+D !$

$5.99 E+11$

3. $71 E+01$

$3.23 E+01$

3. $41 E+i 1$

$3.11 E+01$

$5.96 E+01$

$5,34 E+1$

$5.41 E+B 1$

$4.63 E+91$

4. $25 E+1$

$3,70 E+B 1$

$4,17 E+11$

3. $45 E+01$

$3,56 E+01$

$3.53 E+11$

$3.31 E+01$

$2.52 E+B I$

$2.45 E+81$

2. $44 E+01$

2. $48 E+01$

$2.57 E+1$

2. $04 E+B 1$

2. 8 AE +01

$1.85 E+01$

$2.03 E+B 1$

2. $80 E+B 1$

3. $05 E+01$

$2,67 E+91$

$3.07 E+11$

$2.33 E+01$
DEUTERUIIS

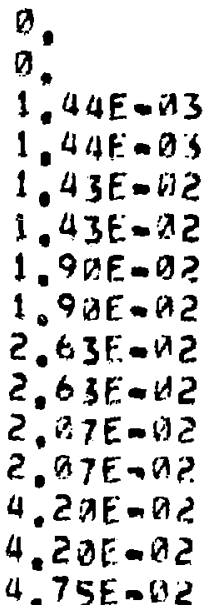

$4.75 E-112$

$4.75 E-a_{2}$

3. 36F-A2

$3,36 E-112$

1. $56 \mathrm{E}-\mathrm{D} 1$

$1,56 E-61$

8. 35E-A?

$8.35 E-02$

$2.19 F=14$

2.19E-G1

$9.795-D 2$

$9.7 \% E-112$

$4.84 F-92$

$4.34 E-32$

$1.73 E-1$

$1.73 E-131$

$1.62 E-21$

$1.62 E-91$

2. MUE-QL2

2. $14 E-D 2$

$1.5 A F_{-}-172$

$1.58 E-B 2$

$2.48 E-O Q 2$

2. IBE-A?

$1.41 E=02$

1. $41 E-D 2$

2. $586-02$

2. $58 E-D 2$

$1.74 E-D 2$

$1.74 F-02$

2.67E-AI
TRITONS

1.

A.

$\Delta$.

(7).

a.

b.

2. $14 E-1) 3$

2. $14 E-63$

$7,84 \mathrm{E}-0.3$

1. $B 4 E=13$

$5,13 E-\sin$

$5.13 E-13.5$

1. $2.5 E-b 1$

1. $25 E-G 1$

$6.56 E-4: 3$

$0.56 E-\triangle 3$

$3.61 E-a^{3}$

$3,61 E-3$

H. 19E- id

$8.19 E-33$

$2.74 E-V 3$

2. $74 E-63$

1.8ME- 3

$1.80 E-D 3$

$7.45 E-U 3$

$7.45 \mathrm{E}-\mathrm{b} 3$

D.

i.

$\theta$

0.

7. 39E $=\mathrm{ij}$

$1.39 t-w 3$

$2,32 E-03$

$2.32 E-B 3$

$5,18 E-03$

$5,1 B E-D 3$

1,, $7 E-10$ ?

1. $17 E-W 2$

$1.13 t-02$

$1.13 E-132$

7. 92E-3S

$7,92 E-03$

i. $71 E-03$

$1.71 E-B 3$

$6.55 E-143$
ALPHAS

7. 2SE-OL

7. 23E- 11

1. $11 E+v j j$

$1.11 E+10$

1. $47 E+6$

1. $47 E+b i t$

$1.83 E+4 u$

1. $B 3 E+d k$

$1.66 E+00$

1. $60 \mathrm{E}+\mathrm{HI}$

1. $45 E+4.3$

1. $45 E+() !$

1. $85 E+4 s$

1. BSE+Wis

1. $34 \ddot{E}+2 !)$

1. $34 E++1 i$

1. $73 E+419$

1. $73 E+i 3$

1. $O U E+W 11$

$1.6(1 E+b)$

4. $31 E+(1)$

4. $31 E+1] \psi$

4. $44 E+66$

$4.44 E+b 0$

4. $74 E+d d$

$4.74 E+0.6$

$5.87 E+U$

$5.87 E+i j 6$

$4.96 E+1.1$

$4.96 E+(B)$

$4.78 E+10$

$4.78 E+6 n$

$5.35 E+v 6$

$5,35 E+i H$

$5.94 E+\angle B$

$5.94 E+W$

5. $28 E+09$

$5.28 E+U G$

$5.1 A E+b B$

5. $14 E+D V$

$9,46 E+00$

$4.46 E+41$

B. $98 E+4 d$

B. $9 A E+1 B$

$5.61 E+136$ 
IINITIAL SPECTRUM AT 19, AO14. A CA. FOR ICRU MUSCLE TISSUE.

5B MEV D+ ON HE NEUTRDH SPECTRUM

\section{ENERGY}

$4.26 \mathrm{MEV}$

$4.40 \mathrm{MEV}$

$4,60 \mathrm{MEV}$

4,80 MEV

5. 00 MEV

5,50 MEV

6,00 MEV

$6,50 \mathrm{MEV}$

7. 01 MEV

$7.50 \mathrm{MEV}$

$8,00 \mathrm{MEV}$

$8,50 \mathrm{MEV}$

9,0 , MEV

$9.50 \mathrm{MEV}$

$10,00 \mathrm{MEV}$

$11.00 \mathrm{MEV}$

12.00 MEV

13. DB MEV

$14,00 \mathrm{MEV}$

15,00 MEV

$16,00 \mathrm{MEV}$

$17.00 \mathrm{MEV}$

$18.00 \mathrm{MEV}$

19,00 MEV

$20,00 \mathrm{MEV}$

21,00 MEV

2?.OB MEV

$23,00 \mathrm{MEV}$

24.00 MEV

25.04 MEV

26. 10 MEV

27.00 MEV

$28.00 \mathrm{MEV}$

29.00 MEV

$30.00 \mathrm{MEV}$

32.00 MEV

34.00 MEV

$36.00 \mathrm{MEV}$

$38.00 \mathrm{MEV}$

$40.00 \mathrm{MEV}$

42.00 MEV

44.DO MEV

46. DO MEV

48, DD MEV

50.00 MEV

\section{PKOTONS}

$2.57 E+01$

$2.47 E+11$

$2.18 E+11$

$1.91 E+b 1$

$\ln 8 Z E+11$

4. 43E+ WI

$3.6 R E+D 1$

$3,25 E+01$

$2.81 E+01$

$2.89 E+01$

$2, b 2 E+B 1$

$2.65 E+01$

2. $O G E+D 1$

$1.9 A E+B 1$

$1,94 E+B$ !

$3,2\{E+\Delta)$

$2.97 E+\Delta 1$

3. $16 E+B 1$

2. $74 E+41$

$2.37 E+111$

1. $79 E+11$

1.92E+C1

$1,57 E+01$

1. $1 G E+\nabla 1$

1. $B A E+B 1$

1. $15 E+Q 1$

$\ln 23 E+01$

B. $75 E+B D$

4. 5 BE +0.3

$4 . \triangle Q E+\Delta G$

3. $O A E+B O$

$3,75 E+10$

$1.75 E+B E$

$1.75 E+00$

$1,25 E+40$

$2,50 E+00$

1. $00 E+D A$

$7.5 D E-01$

$2.50 E=01$

5.DतE-DI

5.20E-DI

$2.5 D E-D 1$

$\theta$.

0.

$\Delta$

9.19
DELITERONS

2. 69E-DI

$1.17 E-82$

$1.17 E-92$

$1.15 E-112$

$1,15 E-\theta_{2}$

3.11E-O2

$3.11 F-02$

2. $32 E-92$

$2.32 t-12$

$1.81 E=n$ ?

$1.31 E-A 2$

$1.25 E-02$

$1.25 \mathrm{E}=62$

$7.20 \mathrm{E}-1 \mathrm{~B}$

$7.2 \mathrm{gF}-\mathrm{a} 3$

$5.18 E-83$

$5,08 E-D S$

9 . A6F-DOA

$9.9 G E-B A$

$\theta$.

9.

i.

a.

a.

a.

:

a.

9

D.

$\theta$

:

क.

0

0

0 :

$\theta$

0.

0

0

0

0.

0

3.63
IRITOUS

b. 5SE- 3

$4.61 t-b 3$

$4.61 E=43$

$3.97 E-U 3$

$8,97 E=03$

$1.23 E=\square$ ?

$1,23 E-D 2$

$2,41 E-03$

$2.41 E-B 3$

$2,67 E-() 3$

$2.67 E-A 3$

$\theta$.

$\theta$.

$\ln 14 E-63$

1. $14 E-\{3$

$1.18 E-i 33$

$1.18 E-153$

is.

a.

b.

$\theta$

a.

(n)

,

$B$.

$n$

$\theta$.

a.

$n$

$\theta$.

v.

0.

b.

D.

.

(1).

4.

$\theta$

0.

$\theta$

0

:.

().

$\theta$.

3.79
ALPHAS

$5.61 E+d v$

$4.97 E+d U$

$4.97 E+U E$

$4.27 E+U B$

$4.27 E+B i$

$7.66 E+106$

$7.66 E+\Delta 0$

4. $3 \Delta E+D D$

4. 3tE+ $B t$

$2.15 E+4 t$

$2.75 E+D 0$

$1.42 E+v i s$

$1.42 E+31$

$9.67 \mathrm{E}-\mathrm{A} 1$

$9.67 E-b 1$

1.17E+dis

$1.17 E+213$

$1.94 E=01$

$1.94 E-31$

$1.25 E-U 2$

$1.25 E-\Delta 2$

$1.25 E-111$

$1.25 E-21$

$1.25 E-11$

$1.25 E-\triangle 1$

$\theta$

).

n.

0.

a.

b.

$\theta$.

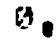

D.

a.

$\theta$.

3

a.

0.

9

0.

0.

0

$\theta$

0.

4.96 
IINITIAL SPECTRUM AT $14, P M 40^{\circ}$, CH. FOF ICEL MUSCLE TISSUE 50 MEV D+ ON RE NEUTRDN SPECTRUM

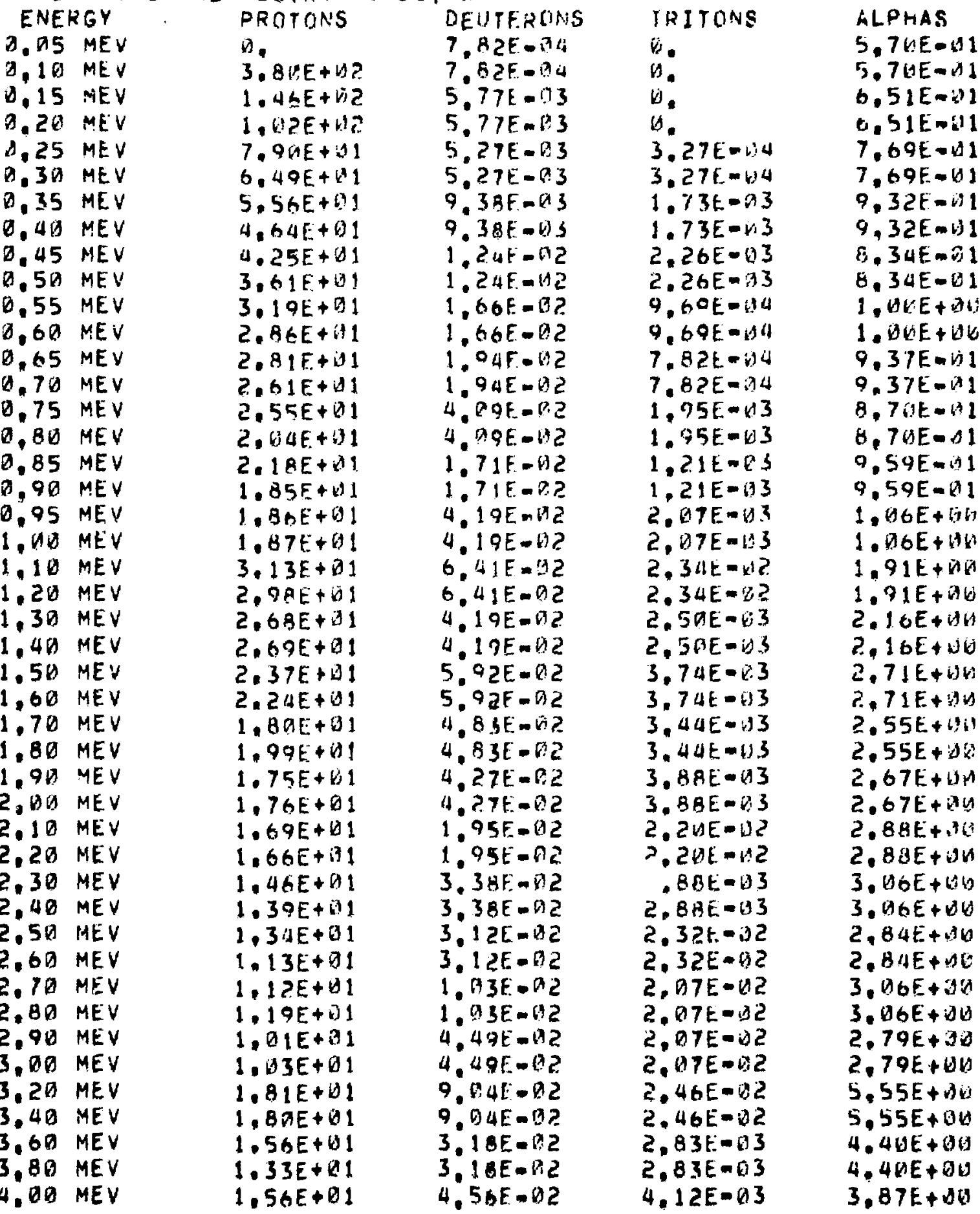




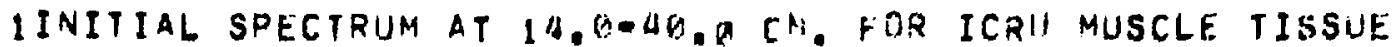
5O MEV D. ON BE NEUTROA SPECTRUM

\section{ENERGY}

4.2O MEV

4. 40 MEV

4,66 MEV

4.80 MEV

5. DO MEV

$5.50 \mathrm{MEV}$

6.00 MEV

6.5口 MEV

7. OQ MEV

$7.50 \mathrm{MEV}$

8. 00 MEV

8.50 MEV

9. DO MEV

$9.50 \mathrm{MEV}$

10.01 MEV

$11.00 \mathrm{MEV}$

$12.00 \mathrm{MEV}$

13.DO MEV

$14.09 \mathrm{MEV}$

$15.00 \mathrm{MEV}$

16.00 MEV

17. 10 MEV

18. OO MEV

$19.00 \mathrm{MEV}$

20.01 MEV

21.2O MEV

$22.00 \mathrm{MEV}$

$23.00 \mathrm{MEV}$

24.00 MEV

$25,00 \mathrm{MEV}$

26. $00 \mathrm{MEV}$

$27.01 M E V$

28. $\triangle O M E V$

$29.00 \mathrm{MEV}$

$30.00 \mathrm{MEV}$

$32.00 \mathrm{MEV}$

34.00 MEV

36. $10 \mathrm{MEV}$

38.08 MEV

40. DO MEV

42. DO MEV

44.00 MEV

46. $00 \mathrm{MEV}$

48,00 MEV

50.00 MEV
PROTONS

$1.31 E+D 1$

1. $29 E+191$

1. $18 E+A 1$

1. $A T E+Q 1$

9.8CE+ DO

$2.26 E+01$

$2.22 E+11$

$1.62 E+\square 1$

1. $62 E+21$

1. $45 E+11$

1. $S B E+01$

1. $45 E+01$

1. $26 E+01$

1. $\triangle 6 E+\Delta 1$

9.58E+ 0 .

1. $82 E+01$

1. $55 E+11$

$1.50 E+D 1$

$1.28 E+B 1$

$1,28 E+01$

$1,03 E+41$

$9.56 E+00$

7. 8 BE + $Q 4$

$0.6 B E+00$

$7.3\{E+D 0$

$5.65 E+00$

5. $69 E+00$

$3.65 E+b$ ?

3.5DE+DO

3. $42 E+D V$

2. $46 E+00$

1. $96 E+09$

$1.65 F+B n$

$1.46 E+09$

1. $\triangle B E+\triangle D A$

$1.81 \mathrm{E}+0$

1. $O A E+Q B$

$9.62 E-D 1$

$5.77 \mathrm{E}=01$

$3.46 E=01$

$7.69 E-02$

$2.69 E-01$

0

$\theta$

0

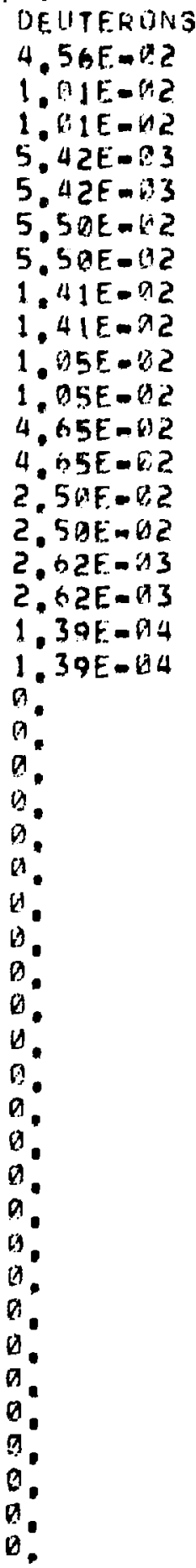

TRITUNS

4. $12 E-B 3$

$4.01 t-b 3$

4. $01 E-13$

2. $16 E-6) 3$

i. $16 E-13$

$7.31 f=-3$

$7.31 E-133$

$5.16 E-143$

$5,16 E-63$

1. BLE-B3

1. BAE - O 3

1. $15 E-15$

1. $15 \mathrm{E}-\mathrm{B}$

8. $91 E-104$

$8.91 E-04$

1. $\triangle O E-Q 3$

1. $\triangle 6 E=$ in 3

$1.53 t-B$

$1.53 E-64$

b.

(1).

b.

9

a.

0.

b.

10

17.

a.

0

$D$

0

A.

0.

0.

b.

0.

0

$d$

a.

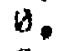

0

$\theta$

i).

4.02
ALPRIAS

3.87E+Dh

3. $32 E+U G$

3. $32 E+D V$

2. $47 E+: B V$

2. $47 E+2 b$

$4.2 B E+4 G$

4. $2 B E+D V$

$2,34 E+6 B$

$2,34 E+Q 0$

$1.61 E+C B$

$1.61 E+B n$

$9.58 E-V 1$

$9.58 E-H A$

$6,195-11$

6. $17 E-111$

7.96E-DI

$7.9 E E-11$

$3,86 E-11$

3. B6E-A1

$2.49 E-42$

2. 49E-U2

$1.93 E-192$

1. $93 E-\Delta 2$

3. B5E-U2

$3.85 E-02$

a.

0

$\square$.

0.

a.

D.

0.

0.

a.

$\Delta$.

a.

0.

$\theta$

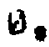

a.

4

D.

$\theta$

a.

5.24 
1SLOWING DONN SPECTRIJM AT H, GOCM, FOR SHONKA A-1SR PLASTIC:

42 MEV P+ ON HE NEUTRON SPECTRI!H

ENERGY

D. US MEV

$0.10 \mathrm{MEV}$

D.15 MEV

D. 20 MEV

0.25 MEV

0.34 MEV

$0.35 \mathrm{MEV}$

0. 40 MEV

2.45 MEV

0.50 MEV

0.55 MEV

D. OU MEV

0.65 MEV

0.7 MEV

$0.75 \mathrm{MEV}$

0.80 MEV

0.85 MEV

$0.90 \mathrm{MEV}$

0.95 MEV

1. DQ MEV

1.1 14 MEV

1.20 NEV

1.30 MEV

1,40 MEV

$1,50 \mathrm{MEV}$

1.6U MEV

$1.70 \mathrm{MEV}$

$1.80 \mathrm{MEV}$

1.90 MEV

2. OU MEV

2.1D MEV

2.20 MEV

$2,30 \mathrm{MEV}$

2.40 MEV

2. $50 \mathrm{MEV}$

$2.60 \mathrm{MEV}$

2.70 MEV

2.80 MEV

$2.90 \mathrm{MEV}$

3.0D MEV

$3.20 \mathrm{MEV}$

3. 40 MEV

3.60 MEV

3.80 MEV

$4.00 \mathrm{MEV}$
PROTONS

U.

$1.74 E+0 B$

1. $32 E+B H$

$2.15 E-62$

6. 4 $4 E-42$

$9,52 E-11$

$2.76 \mathrm{~F}-\mathrm{k}) 1$

$2,91 E+14 B$

$5.58 E-01$

1. $.6 E+36$

8.9AE-D1

2. B1E+3a

1. $41 E+4 B$

$1.52 E-D 1$

2. $25 E+196$

1. $11 E-31$

1. $7 A E+D O$

$2.79 E-01$

4. $38 E-31$

4. DDE-DI

2. $33 E+01$

8. 36E-O1

$7.72 E=B 1$

$0.3 B E-\triangle !$

1. $18 E+60$

$9.015-1) 2$

1. $47 E+100$

$1.07 E+D G$

I. $6 ! E+B Q$

$9.49 E=1$

$6.93 E-i J 1$

4. $76 E-611$

9. 3AE- DII

$4.76 E-D 1$

7. $26 E-D !$

$5.37 E-02$

$1.31 E-04$

$1.58 E+10$

$9.44 E=D 5$

Z. ÓZE-1B2

$4,66 E=02$

$1.32 E-02$

$5,61 E-B 2$.

$4.25 E-02$

7. $24 E-A 2$
DEUTERONS

D.

9

in

4.

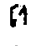

A.ME-TS

1

a.

a.

6. $83 f=-115$

a.

4. $74 F-35$

a)

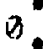

4. DE-VS

2. $43 E-106$

4. M $4 F-V 5$

4. तUE-OS

$\theta$

4. $4 E=95$

$5.43 F-15$

1. AIE-D4

1. 2 ? $E=16$

$1.40 E-05$

0

1. $22 E-10 h$

1. AUE-US

1. $22 E-4 n$

$1.22 E=26$

$1.52 E-U 5$

2. $43 E-116$

1. $76 E-A 5$

0

1. $2 B E-45$

1. $2 B E-05$

a.

1.2BF. 95

1. 2BE-0. 5

1. 2RE-HS

1. $20 E-05$

1.2.8E- -5

3.83E-OS

is.

$a$

9.
TRITUNS

is.

H.

in.

,

i.

i.

v.

a.

W.

4

11

in.

i.

$\theta$

b)

i.

v.

i.

in.

a.

i.

冋.

0

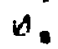

i.

6.

v.

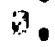

a.

a.

0

E.

a.

is

a.

b.

$v$

a.

7

$\theta$

D.

is.

a.
ALPHAS

D.

8.47E-34

$2, B \cap E=U 4$

1. $46 E=15$

3. 2TE-J4

1. $) 3 E-x 3$

$0.45 E-4 \angle$

1. $\triangle B E-B 3$

9.99E- 34

$2.13 E=.14$

$\triangle, 82 E-114$

5,7 BE- IS

1. $\triangle 7 E-13$

1. $58 E-134$

$7.92 E-134$

$3.60^{\mathrm{N}} \mathrm{E}-\mathrm{D4}$

1. $19 E-V 3$

1. $11 E-U 3$

4. 9BE- $\triangle 4$

1. $29 E=43$

$5.07 \mathrm{E}-(1) 14$

$5.26 E-134$

$5.51 E-44$

$5.52 E-11$

$3.32 E=U 3$

4. 39E-L4

8. IOE -34

1. $23 E-v 3$

3. $A 2 E-14$

4. DUE-HA

2. $37 E-13$

$3.35 E-144$

$3.72 E-114$

$3.91 \mathrm{E}=\mathrm{L}(\mathrm{t})$

2. $89 E-03$

S. (11 $1 E-04$

1. $43 E-144$

$4.27 E-N 4$

2. 8 (6) $\mathrm{E}=\mathrm{H} 3$

$2.14 \mathrm{E}-\mathrm{UL}$

1.50E- 03

$3.83 E-04$

$1.53 E-d 3$

$1.52 E-03$

$4.23 E-63$ 
1SLOHJNG DOWN SPECTRUM AT A. BA CM. FCR SHONAA A-ISK PLASTIC 42 MEV P+ DN BE NEUTRON SPECTRUM

\section{ENEHGY}

4.2O MEV

4,40 MEV

$4.60 \mathrm{MEV}$

4.8 M MEV

5. OU MEV

5.50 MEV

$6.00 \mathrm{MEV}$

$6.50 \mathrm{MEV}$

7. OD MEV

$7.50 \mathrm{MEV}$

8. DO MEV

$8.50 \mathrm{MEV}$

3. 10 MEV

9.50 MEV

10. OU MEV

11. DO MEV

$12,013 \mathrm{MEV}$

13. DO MEV

$14.0 \%$ MEV

$15.00 \mathrm{MEV}$

$16,00 \mathrm{MEV}$

17.OS MEV

18, OU MEV

19. HU MEV

20.00 MEV

$21.01 \mathrm{MEV}$

22. OD MEV

23. DO MEV

$24.00 \mathrm{MEV}$

25.00 MEV

26. AO MEV

$27.90 \mathrm{MEV}$

$28.00 \mathrm{MEV}$

29. OO MEV

3月.DO MEV

32. DO MEV

34.00 MEV

36.0 MEV

38.00 MEV

$40.00 \mathrm{MEV}$

42,00 MEV

$44,00 \mathrm{MEV}$

$46.00 \mathrm{MEV}$

$48.00 \mathrm{MEV}$

50. OO MEV

GAVE. E(MEV)
PROTONS

5. 106E-102

$1.63 \mathrm{E}-\mathrm{JC}$

$3.36 E-1]$ ?

$3.34 E-02$

$4.96 E-02$

$6.59 E=02$

$4.82 E-\lambda 2$

2.1 ? $F-114$

$2.2 A F \cdot D 4$

4. 3ME 205

ट. $79 E-195$

a.

a.

is.

(1)

i.

.

G.

n.

i.

$\theta$.

3

a.

$\theta$

ते

B.

b.

0.

b.

b.

$\theta$

1.

a.

a

$b$

8.

0.

i.

a.

D.

0.

b.

$\theta$.

0.

1.46
DEUTERONS

$b$

13

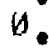

i.

4.

9

7

?.

A.

19

a

3

in.

(9)

a.

0

9

a

$\pi$

$A$

$n$

3

a.

i.

a.

9.

$\theta$

a.

a.

5.

0

a.

a.

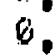

0.

a.

1.67
TRITONS

?.

b.

a.

4

t).

ㄴ.

de

b.

is.

(t).

b.

0.

3

b.

4

it.

w.

is.

$b$.

a.

0.

3

1.

3.

i.].

得.

19.

a.

0

0

*.

U.

(1)

$v$.

1

U.

i.

0

a.

0

2.

.

0

1.

1.

0
ALPHAS

4.15E-US

8.83E-DS

$9.32 E-B S$

$1.095-104$

1. $52 E-14$

$7.13 E-A S$

7. $42 E-B 4$

2. $16 E-15$

3. BHE- 15

$5.79 E-14$

2. B3E-15

2. $28 E-36$

$5.73 E=\square 4$

2. $15 E-[16$

$5.75 E-44$

2. $33 E-14$

$5.6 n E-D 4$

$5 . O B E=214$

1. $42 E-D 3$

is.

$B$

9.

$\Delta$

i)

i.

id.

0

0

$\theta$

a.

0.

i.

is.

i.

i).

เ.

i)

iv

i)

$\theta$.

$\Delta$.

a.

u.

a.

0.

6.16 
ISLOWING DOWN SPECTRUM AT TIDS CM. FOR 6HONKA A-15G PLASTIC 42 MEV P+ ON BE NEUTRON SPECTRUN!

\section{ENERGY}

7. Q5 MEV

Q. II MEV

Q.15 MEV

$0.20 \mathrm{MEV}$

0.25 MEV

$0.31 \mathrm{MEV}$

0.35 MEE

0. 40 MEV

0.45 MEV

$0.50 \mathrm{MEV}$

0.55 MEV

0.60 MEV

0.65 MEV

0.70 MEV

0.75 MEV

$0.80 \mathrm{MEV}$

0.85 MEV

$0.9 \square$ MEV

0.95 MEV

1.OO MEV

1.10 MEV

$1.20 \mathrm{MIEV}$

$1.30 \mathrm{MEV}$

$1.40 \mathrm{MEV}$

$1.50 \mathrm{MEV}$

$1.60 \mathrm{MEV}$

$1.70 \mathrm{MEV}$

$1,80 \mathrm{MEV}$

1.90 MEV

2,00 MEV

2,10 MEV

2,20 MEV

$2.30 \mathrm{MEV}$

2. 40 MEV

$2.50 \mathrm{MEV}$

$2.60 \mathrm{MEV}$

2.70 MEV

2.80 MEV

2.90 MEV

3.DD MEV

3,20 MEV

$3,40 \mathrm{MEV}$

3,60 MEV

$3,80 \mathrm{MEV}$

4, BB MEV
PRUTONS

$D$.

$2.56 E+64$

1. $27 E-11$

3. $94 E-11$

$1.28 E+20$

$1,78 E+\angle C$

$2,37 E+B C$

$2.26 F+9 G$

$3,45 E-131$

1.27E+ BO

$0.61 E-01$

$2.24 E+00$

$5,83 E=(x)$

$4.99 E=D 1$

$2,54 E-R 1$

6. QAE-OI

$3,26 E+D D$

5. $92 E-61$

$4.11 E-1)$

3. $15 E+109$

$3.45 E+130$

$1.69 E+10 E$

3. $39 E-D 1$

$1.49 E+\theta H$

$6.830-20 !$

$1,2 Q E+0)$

$2.62 E+60$

$2.41 E+80$

$4.35 E-61$

1. $55 E+170$

$4,39 E-B 1$

$1.17 E+100$

$9,66 E-01$

$2.05 E+01$

$1.16 E+0 B$

$9.76 E-01$

4. $17 E-D 1$

$1.55 E+80$

$2.3 D E-B 1$

$1.87 E+B A$

$7.51 E-01$

$6.53 E-B 1$

5.1BE- 11

1.11E+QG

4. $1 A E-B 1$
DEUTERONS

A.

$7.98 E-06$

$7.98+-05$

3.07E-A5

2.3 $3 E-75$

$2,62 E-05$

$1.41 \mathrm{E}-125$

3. $27 \mathrm{E}-1 \mathrm{AS}$

1. DPF-D 4

$7.895-145$

$2.46 F .05$

$6.31 E-174$

2. $\triangle 4 \mathrm{E}=64$

4.98E- 15

$9.65 E-14 h$

$7.98 E-96$

$8.93 E-195$

$9.65 E-00$

4. $\triangle 7 E=05$

$9.64 \mathrm{~F}$. B 5

5. Bott-as

$4.408-45$

$4.78 E=0.5$

$3.51 F=04$

$B .76 E-A 5$

$1.81 E-04$

2.59E-AS

$2.12 E=0.5$

$4.25 E-05$

$3.41 E-24$

1. 2 a $=05$

$2.15 \mathrm{~F}=0 \mathrm{~T}$

$1.24 E-115$

$3.36 t-04$

$3.38 E=05$

$8.31 E-D 5$

$3.11 F-04$

9 . FIE- D6

9 , OQF-OH

$9.09 E-B 6$

$1.77 E-B 4$

$1,78 E-64$

$1.51 E-(A)$

$1.68 E-D 4$

$1,51 E-04$

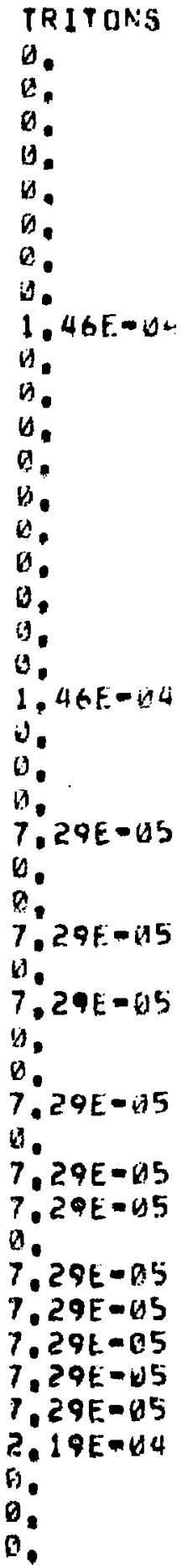

ALPHAS

O).

6. $14 E=14$

2. BBE $=13$

$1, D 5 E-42$

$2.59 E-133$

4. 28E-13 3

5. $59 E-0.3$

$1,56 E-13$

b. $42 \mathrm{CE}=3$

1. $41 \mathrm{Ea}-13$

2. $51 E-14$

$2.87 \mathrm{E}=42$

$1.32 \mathrm{E}-\mathrm{A} 3$

$1.71 E=.53$

$3.94 \mathrm{E}-2 \mathrm{~S}$

$2,93 E-42$

7. ARE-113

5.MSE-AO 3

$3.19 E-133$

$1.51 t-12$

$5.89 \mathrm{E}=\mathrm{d}$

3. GDE.mu3

$2.45 E-13$

6. 7TE-DS 3

$1,52 E-D 2$

2. $3: E-E^{2} 3$

$2.94 E-13$

7.31E-W 3

$5,95 t-63$

$3, B 8 E-13$

2. IUE-D2

$1.28 E-W C$

2, 2UE-03

Q.1BE-GI

1.37E-G?

4.2 (B) -1$) 3$

$3,35 E-23$

2. $10 E-42$

$1.68 E-132$

5, BUE-DS

$9.46 E-03$

8.45E-G 3

$1.22 \mathrm{EM} 2$

$7.01 E-D 3$

$1.74 E-112$ 
1SLOWING DOWN SPECTRUM AT G. 5 CM. FOR SMONKA A-ISH PLASTIC 42 MEV P + ON BE NELTRON SPECTRUM

\section{ENERGY}

4.2D MEV

4. 4D MEV

4.60 MEV

4. 80 MEV

5. OO MEV

5.50 MEV

6. OU MEV

$6.50 \mathrm{MEV}$

$7.00 \mathrm{MEV}$

$7,50 \mathrm{MEV}$

8.DO MEV

8.50 MEV

$9.00 \mathrm{MEV}$

$9.50 \mathrm{MEV}$

10. OU MEV

11.OD MEV

$12,00 \mathrm{MEV}$

13.00 MEV

$14, \square D$ MEV

15, MEV ME

16.00 MEV

$17.00 \mathrm{MEV}$

$18.00 \mathrm{MEV}$

19. OV MEV

20. OD MEV

$21.00 \mathrm{MEV}$

$22.00 \mathrm{MEV}$

23,DD MEV

24.DO MEV

25.DO MEV

26.00 MEV

$27.00 \mathrm{MEV}$

$28,90 \mathrm{MFV}$

29. 00 MEV

$30.00 \mathrm{MEV}$

$32,00 \mathrm{MEV}$

34.00 MEV

36. MO MEV

38.0 MEV

$40.90 \mathrm{MEV}$

42.00 MEV

44. OO MEV

46.00 MEV

$48.00 \mathrm{MEV}$

$50.00 \mathrm{MEV}$
PROTONS

1.67E+OG

1. $A 7 E+B A$

3. $49 E-A 1$

9. $2 A E-D 1$

$3.44 E-111$

$5,34 E-B 1$

4. THGE-DI

B. $68 E-B$ ?

1. $75 E-61$

6. 3ПE - BZ

$6.20 E-B 2$

1. $29 E-h 1$

c.

1. $58 E=31$

(1.

1. $48 E-01$

¿. BतE- 12

1. $11 \mathrm{E}-\mathrm{O} 1$

$1.49 F-61$

$1.65 E-0$ ?

$7.29 \mathrm{E}-\Delta \mathrm{C}$

$\Delta$.

$1.4 A E-D 1$

4. $16 E-02$

1. $3 E-W 1$

0.

$5.95 \mathrm{E}-02$

0.

$0.77 E-02$

7.1 ?E-口2

1. 5?E-B1

$\theta$

4

a.

a.

$5.21 E=b 2$

ib.

a.

0

$b$.

a.

u.

0.

0.

b.
DEUTEROWS

1.68F-0. 4

3. D2E -104

1. $68 E-34$

$4.53 F=H A$

$7.72 E-12$

1. 3ذE-05

1. 35E-05

2. D2E- 15

5. $39 E-195$

a.

i.

a.

0

9

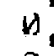

D.

$b$

3

a.

a

0

d

9

a

$D$

a

$a$

$a$

$a$

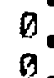

a.

$a$

0

$a$

a.

3.53
TEITONS

$\theta$.

1.

H.

$b$.

0

0

a.

n.

B.

2 .

11

$k$.

i.

is.

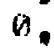

is

G)

is

$\theta$

(a)

U.

i.

is.

$\theta$

a.

U.

6

v.

b.

g.

b.

$\theta$

0.

a.

n.

a.

$\theta$

$\theta$

H.

0

b.

0

b.

2.52
ALPHAS

3. И3E-U2

8. $15 E-E .3$

1.23E-D2

2. TDE $=5$

1. $72 E-12$

$2,64 E=04$

$4.55 E=113$

4. ZAE-DS

5.57E-A 3

$5.33 E-V 3$

$7.94 E-A 3$

$5,39 E=3$

1. ATE-A2

8.83E- 15

1. $B 1 E-D 4$

1. $21 E-94$

$3.29 E=14$

$3.52 E-A b$

$7.03 E$. IS

a.

n.

Q.

9.

ต.

b.

U.

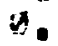

a.

a.

U.

b.

D.

n.

D.

a.

U.

a.

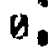

B.

d.

$4:$

A.

a.

(6.

is.

4.43 
ISLOWING DOWN SPECTRUM AT T.1A CM. FOR SHUNKA A-15A PLASTIC 42 MEV P+ ON BE NEUTRON SPFCTRUM

\section{ENERGY}

D. $\triangle 5$ MEV

0.10 MEV

Q.15 MEV

$\pi, 20$ MEV

$0.25 \mathrm{MEV}$

0.30 MEV

0.35 MEV

$0.49 M E V$

0.45 MEV

0.50 MEV

$0.55 \mathrm{MEV}$

$0.60 \mathrm{MEV}$

$0.65 \mathrm{MEV}$

$0,70 \mathrm{MES}$

0.75 MEV

$0,8 \triangle \mathrm{MEV}$

0.85 MEV

0.90 MEV

$0.95 \mathrm{MEV}$

1,00 MEV

$1.10 \mathrm{MEV}$

$1,20 \mathrm{MEV}$

$1,30 \mathrm{MEV}$

$1.40 \mathrm{MEV}$

$1,50 \mathrm{MEV}$

$1.60 \mathrm{MEV}$

1.70 MEV

$1.80 \mathrm{MEV}$

$1.90 \mathrm{MEV}$

2.00 MEV

2.10 MEV

2,20 MEV

2,30 MEV

2,40 MEV

$2,50 \mathrm{MEV}$

$2.60 \mathrm{MEV}$

$2,71 \mathrm{MEV}$

$2,86 \mathrm{MEV}$

2,90 MEV

$3,00 \mathrm{MEV}$

3,20 MEV

$3.40 \mathrm{MEV}$

3.60 MEV

3,80 MEV

4,00 MEV
PRUT CAS

W.

$1.11 E-01$

$1.92 E+100$

7. $46 E=01$

$5.54 E+D A$

$7,6 \triangle E-D 1$

1. $23 E+10$

1. $91 E+B$ a

$1.55 E+B A$

$1.18 E+D B$

1. $20 E+19$

$1.53 E+605$

$6.16 E-D 1$

$2,39 E-61$

$1.73 E+100$

$2,72 E-D 1$

3.11E+ 19

$4.17 E-D ?$

$4,7 A E=\square 1$

$5.28[-11$

$1.21 E+6 i]$

$1.54 E+\Delta E$

$1.33 E+60$

$1.77 E+10$

$1.15 E+B A$

$6.49 E-B 1$

$1.59 E+B D$

$6.05 E-01$

$6,37 E=B 1$

$1.14 E+100$

$1.13 E+B D$

$1.95 E+1 !$

7. $\triangle O E=01$

$1.77 E+U A$

1. $10 E+\Delta B$

1.DQE+DO

$4,37 E-01$

$1,0 I E+D Q$

$7.91 E-91$

$5.44 E-01$

$1.36 E+0(4$

$1.05 E+00$

$4.26 E-9 !$

$8,55 E-11$

Q. 36E-Q1
DEUTERONS

ด.

3.74E-US

i.

2. $49 E-05$

$1.32 \mathrm{~F}-\mathrm{O}$

7. $\triangle B E-115$

2. $\triangle 9 E-05$

$4.41 E=05$

$4.97 E-05$

$1.68 E-6)$

1.

$1.56 E=0.4$

$4.14 E-155$

$3.74 E-115$

$7,09 E-U 5$

$2.22 E$ ans

$1.75 E=B 4$

9. 3AE -OS

0.

$9.3 M E-Q 5$

$1.53 E-114$

$2.40 E-D \triangle$

$3.18 E-45$

$1.26 E=17$

$5,21 E-65$

$6,: 7 E-95$

A. $14 t-a 5$

$1.23 E-34$

1. $34 t-04$

1. BAE- 14

$\theta$

I. A TEADS

1.BTE-BS

a.

1:87E-AS

1. BT.E-AS

1.BTE-DS

$3.74 E-05$

$3.74 E-05$

$7.49 E=15$

$B$.

$a$

0.

a.
TRITONS

D.

0 ,

v.

D.

b.

a.

$\theta$.

is.

0.

0.

$\theta$

b.

n.

$\theta$

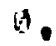

is

$\because$

$a$

b)

B.

$\forall$.

$\theta$

0.

$\theta$

$v$

$\Delta$

u.

0.

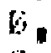

a.

v.

n.

:

0.

$v$

0

v.

$n$

a.

b.

i).

$b$.

ti.

b.

ii.
ALPHAS

$v$

$1.12 E-1) 3$

7.51E-D4

व. $39 F=(1) 3$

$5.6 \mathrm{SE}-1 \mathrm{H} 4$

$4.89 E-134$

2. $12 E-1) 3$

9. 32E-44

9. $39 E-114$

B. $15 E-34$

$5.62 E-D 4$

2. $38 E-13$

$3.73 E-v 3$

$7.95 E-\Delta 4$

$6.395=13$

$7.73 E-V 3$

$7,31 E-64$

$9.76 E-N 4$

$2,62 E=+3$

1. DQE-A3

$1.59 E-33$

1 . GI5E- 3

$3.54 E-H 3$

$1,21 E-12$

$2.98 E-03$

$7.65 E=03$

$1.34 E-133$

$2,66 E-143$

$0.97 E-13$

5. $33 E-A 3$

2. $53 E-D 3$

B, 6IE-33

$1,71 E-43$

$1,96 E-d S$

$5, h 2 E-$ in 3

2.7BE-AS

$1.18 E-132$

$1.83 E-V 3$

$6.27 E-D 3$

$2,37 E-63$

$4.46 E-(D) 3$

$3.64 t-3 s$

$6,795-33$

2. 29Enkis

2. $97 \mathrm{E}=3$ 
1SLONING DOWN SPECTRUM AP O.10 CM. FOR SHORKA A-1SD PLASTIC 42 MEV P + ON BE NEUTRON SPECTRIJM

\section{ENERGY}

4.20 MEV

4.40 MEV

$4.69 \mathrm{MEV}$

$4, B$ B MEV

5,O0 MEV

5,50 MEV

$6,00 \mathrm{MEY}$

6,50 MEV

$7,90 \mathrm{MEV}$

7.50 MEV

8. $O U$ MEV

B. SD MEV

$9.00 \mathrm{MEV}$

9.50 MEV

$10.00 \mathrm{MEV}$

11,00 MEV

12.00 MEV

13. OS MEV

14,06 MEV

15, DO MEV

16. R0 MEV

17,00 MEV

19.0 MEV

$19.00 \mathrm{MEV}$

$20.00 \mathrm{MEV}$

$21.00 \mathrm{MEV}$

22.00 MEV

23.00 MEV

24. OU MEV

25. DO MEV

$26.00 \mathrm{MEV}$

27.DO MEV

28.UD MEV

29. $D 0$ MEV

$32.00 \mathrm{MEV}$

$32.00 \mathrm{MEV}$

$34.00 \mathrm{MEV}$

36. OO MEV

$38.00 \mathrm{MEV}$

$40.00 \mathrm{MEV}$

$4 ? .00 \mathrm{MEV}$

44.00 MEV

46.00 MEV

48. 00 MEV

$59.00 \mathrm{MEV}$

DAVE, E(MEV)
PROTONS

$1.47 E+\Delta B$

1. $\triangle 9 E+D Q$

$9,87 E-1) 1$

4. $5 B E-B 1$

$1.45 E+10$

$8,66 E-31$

$9.6 \square E-61$

$3.67 E-D 1$

$4.72 E-D 1$

$3.64 E=61$

$1.15 E=1$

$2.27 E-k 1$

$1,02 E-B 1$

$6.37 E-91$

$6.13 E-62$

$1 . \forall 2 E-B !$

$2,71 E-01$

$1,33 E-B 1$

$4.30 E-92$

$3,30 E-11$

$1.41 E-U 1$

$1.44 E-D 1$

$1.57 E-01$

1. $51 E-01$

S. 15E- 12

$5.95 E-02$

$5,95 E-B ?$

$1,31 E-01$

7. $19 E-02$

1. $52 E-31$

$\theta$.

$\forall$.

5.

8, 94E-U?

9 ,

$5,21 E-02$

$n$,

*

9

i.

i.

.

n.

$\theta$

6.79
DEUTERDNS

$n$

$a$

$a$

a.

D.

a.

is.

0.

$n$

$n$

a.

s.

bis.

$\theta$

a.

a.

a.

0

1

$a$

0

$a$

n.

a

9

a.

n.

a.

0.

a.

a.

a.

a.

a.

:

1.35
TRITONS

a.

,

i.

i.

b.

a.

0.

is.

$b$.

9.

0.

9.

$n$

B.

(9.

vi.

1.

0.

$\dot{6}$

:

,

v.

o.

v.

b.

b.

$b$.

vi.

is:

ค.

$\pi$

ที.

b.

a.

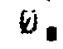

$\theta$.

B.

0.

b.

H.

$\theta$

n.

$\omega$

2.52
ALPHAS

2.23E-DS

$4.38 E-.33$

2. $58 E=-3$

$5,31 E=\operatorname{s} 3$

$5.69 E-14$

2. $A 5 E=13$

$6.18 \mathrm{E}-\mathrm{B} 3$

3. $3 \mathrm{GE}=\mathrm{AD} 3$

6. $35 E-.13$

3. 7 BE-D4

6. ASE-DIG

3. B5E- 14

$6.74 E-i) 4$

$9.33 E=115$

$2,64 \mathrm{E}=174$

6. $28 E-45$

$1,8 B E=114$

a.

is.

H.

O.

$\theta$

a

a.

$\theta$

a.

B.

D.

b.

a.

9.

$\Delta$.

G.

(3.

a.

,

n.

.

D.

6.

u.

$\forall$.

a.

a.

$\Delta$.

4.44 
1SLONING DOWN SPECTRUM AT 0.15 CM. FOH SHONKA A-1SU PLASTIC 42 MEV P+ ON GE NEUTROAN SPECTRUM

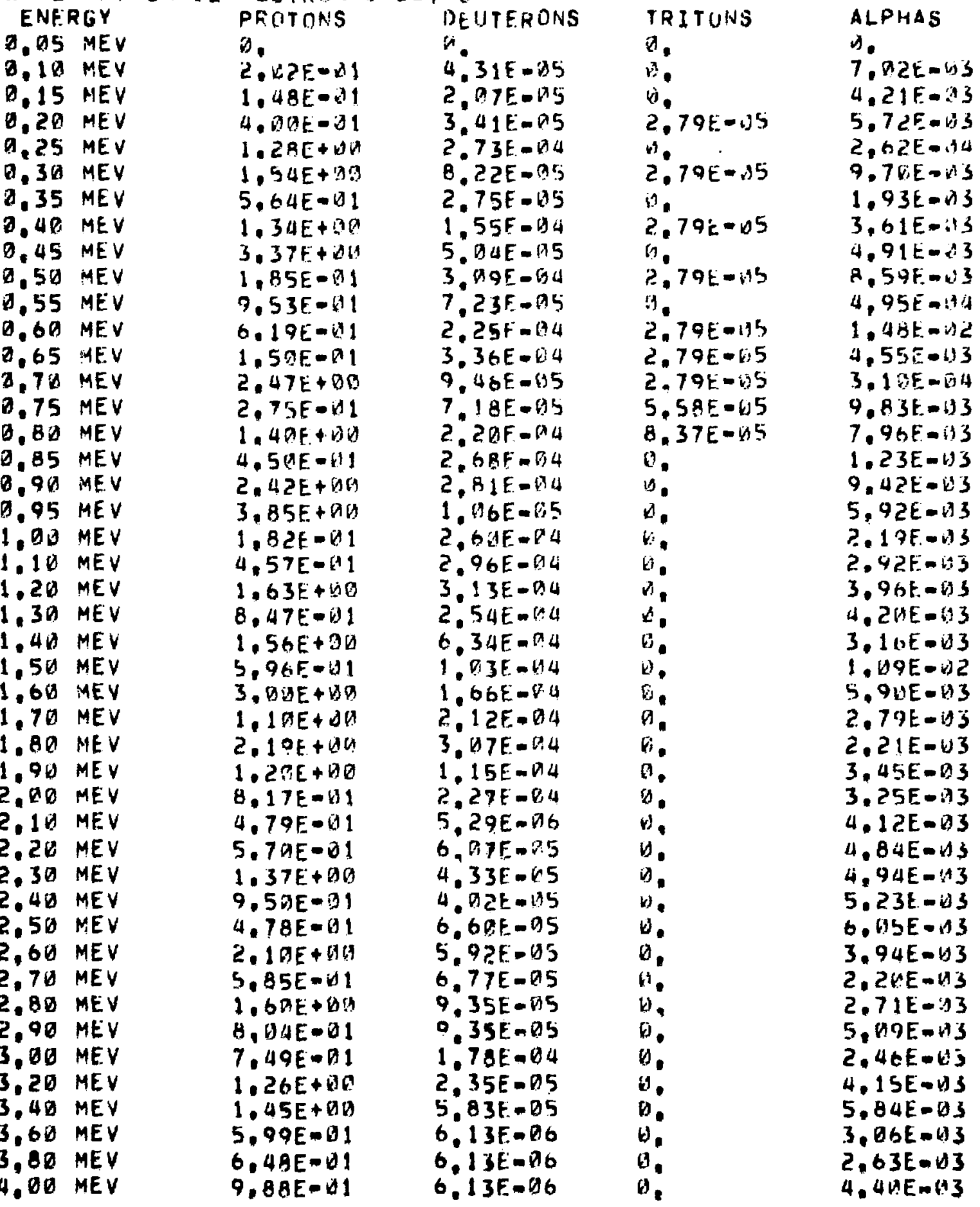


ISLDWING DOWN SPECTRUM AT D. 15 CM. FOR SHONKA A-15V PLASTIC 42 MEV P+ ON BE NEUTRON SPECTRUM

\section{ENERGY}

4.20 MEV

4.4D MEV

4.60 MEV

4.80 MEV

5. DO MEV

5.50 MEV

6.00 MEV

6.5O MEV

7. $O Q$ MEV

7.5B MEV

8. OA MEV

8.50 MEV

$9.00 \mathrm{MEV}$

$9.50 \mathrm{MEV}$

10,00 MEV

$11.00 \mathrm{MEV}$

$12.00 \mathrm{MEV}$

13. QV MEV

14. $D$ MEV

$15.00 \mathrm{MEV}$

$16.00 \mathrm{MEV}$

$17.90 \mathrm{MEV}$

$18.20 \mathrm{MEV}$

$19.00 \mathrm{MEV}$

$20.00 \mathrm{MEV}$

21.DQ MEV

$22,00 \mathrm{MEV}$

23. BW MEV

$24.00 \mathrm{MEV}$

$25.00 \mathrm{MEV}$

26.06 MEV

$27 . \triangle B$ MEV

$28.00 \mathrm{MEV}$

29.00 MEV

33.00 MEV

32. OU MEV

$34.00 \mathrm{MEV}$

36.60 MEV

$38.00 \mathrm{MEV}$

4D. DD MEV

42. DO MEV

44. OQ MEV

46. OD MEV

48. DO MEV

50,00 MEV
PROTOIS

1. $24 E+10$

$7.97 \mathrm{E}-\mathrm{DI}$

$9.23 E-01$

$7.54 E-O 1$

5.86E-U1

$3.46 E-B 1$

$9.25 E-61$

$6,18 E=01$

2. 62E-11

2. $45 E=01$

2. $895-02$

3. $22 E=121$

4. $19 E-101$

$2.96 E-11$

0.

$2.57 E-01$

$1,43 E-D 1$

$2, B \triangle E-B !$

$1.3 \triangle E-01$

$8.03 E-D 2$

1. $\triangle 8 E-\square 1$

$3.13 E-1$

9.14E-W

$1.1\lfloor E-G !$

$5,15 E=0 \mathrm{C}$

5. 95E-U2.

$1.31 E-1$

7.19E-192

1. $52 . E-11$

7. 1OE-U2

a.

if.

$6.94 E-02$

a.

1. $V A E-B 1$

$a$.

0.

D.

b.

a.

D.

a.

D.

a.

6.84
DFUTERDISS

1. A AE-Bis

1.66f-. 5

$2.33 t-0.5$

5. $4 B F=46$

$9.14 E .16$

a.

0

i.

a

a

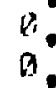

a

a.

4

a

a

$a$

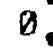

0

.

a.

$a$

0

$\theta$

b

a

$a$

6

0

0

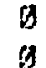

$a$

a.

a.

$a$

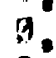

0.

0

1.53
TRITONS

1.

1

v.

$\theta$

$n$.

$b$

0.

D.

a.

to

$B$

in.

13

b.

i).

b.

4.

y.

a.

6

s.

3.

i.

a.

U.

a.

1.

4

H.

a.

y.

6.

D.

$B$

0.

a.

0.

a.

$\theta$

n.

6

Q.

n.

n.

i.

C. 58
ALPHAS

5.72E-B3

$9.31 E-114$

$1.15 E-1) 3$

B. $75 E-\triangle 4$

1. $14 E=\square 3$

$8.74 E-114$

2. D9E-H3

3. $7 D E-W L$

$5 . B 1 E-Q 4$

$3.17[-(1)$ is

$4.99 E-44$

2. $42 E-64$

5. $19 E=04$

$1,15 E-B 4$

$2,82 E=04$

$8.62 E-05$

$2.12 E-14$

4. 7UE- DS

9.4 KE- 05

0.

D.

a.

b.

a.

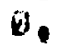

0

a.

$D$

a.

a.

a.

n.

0.

a.

i.

$\Delta$

$\theta$

0

0

a.

0

0

0

is.

0.

2.90 
1SLOWING DOWN SPECTRIIM AT G.2U CM. FIR SHOAKA A-15O PLASTIC 42 MEV P+ ON BE INEUTRON SPCCTRUMM

\section{ENERGY}

$0.05 \mathrm{MEV}$

$0,19 \mathrm{MEV}$

3.15 MEV

$0.20 \mathrm{MEV}$

0.25 MEV

$D .30$ MEV

0.35 MEV

$0.40 \mathrm{MEV}$

0.45 MEV

$0.50 \mathrm{MEV}$

$0,55 \mathrm{MEV}$

$0,60 \mathrm{MEV}$

0.65 MEV

$0,7 \mathrm{U}$ MEV

0.75 MEV

$0.80 \mathrm{MEV}$

0.85 MEV

Q.90 MEV

0.95 MEV

$1.00 \mathrm{MEV}$

$1.10 \mathrm{MEV}$

$1.20 \mathrm{MEV}$

1,30 MEV

1. 40 MEV

1.51) MEV

$1.60 \mathrm{MEV}$

$1.70 \mathrm{MEV}$

1.89 MEV

1.90 MEV

2.0B MEV

2.10 MEV

$2,20 \mathrm{MEV}$

$2.30 \mathrm{MEV}$

$2.40 \mathrm{MEV}$

$2.50 \mathrm{MEV}$

$2.60 \mathrm{MEV}$

$2.70 \mathrm{MEV}$

2.8U MEV

2,90 MEV

3, $\triangle 0$ MEV

$3,20 \mathrm{MEV}$

$3.40 \mathrm{MEV}$

$3,60 \mathrm{MEV}$

$3,80 \mathrm{MEV}$

4. DO MEV
PROTONS

$D$.

1. $12 E+E$

1. $59 E-11$

1. TSE WD 1

2. $42 E-01$

$1,51 \mathrm{E}-31$

1. $85 E-01$

$1.65 E-31$

$2, B 2 E+\Delta \theta$

$4.74 E+60$

$3,25 E-D 1$

$1.64 E+D 0$

$1.94 E-11$

$1.61 E+B A$

$1.48 E-61$

1. $55 E-\lambda t$

$2.29 E+00$

$5.75 E-111$

$1.51 E+1) 1$

$2.35 E-111$

$6,66 E-D 1$

$1.70 E+D C$

$2.35 E-11$

$1.62 E+D O$

B. $22 E-01$

7. $B \geq E-\triangle 1$

8. $\triangle B E-[1$

$1.14 E+A Q$

$7.36 E-01$

$2.48 E-41$

$1.37 E+60$

$3.10 E-01$

$1 . \triangle 5 E+0 A$

O. DQE W U1

$5.27 E-01$

$9.02 E-U 1$

$3.65 E-91$

$3,54 E-01$

7. $D 1 E-D 1$

1. $\triangle A E-B 1$

$6.56 E-B 1$

$7,74 E-D I$

$5,2 B E-D 1$

$4.52 E-01$

$5.49 E-D 1$
UF UTEROIS

7 .

$4.55 E-35$

$1.81 E=B 4$

$111 t-3$

$2.78 E-15$

$7.94 E-.95$

$1.75 F-64$

4.?. $1 E-$ is 5

$11.56 F=04$

$1.79 \mathrm{E}=\mathrm{vid}$

$1.186=04$

1. $\triangle b E=\lambda a$

4. $39 E-13$

$1.49 E-74$

$1.9: E=0.4$

$2.66 F-G 4$

$4.56 E-b^{4}$

$4.3 \mathrm{GE}=3$

$1.10 F-T_{4}$

?.92F- 14

$2.15 E-13$

$1.79 F-P A$

?. $17 E-123$

2. $21 E-15$

2. $2 b E-W 3$

b. $+3 \mathrm{~F}=\mathrm{H} 3$

$2.13 F=4$

4. 46E- 115

$1.22 E-04$

2. $17 E-114$

$1.73 E-04$

$1.23 E-\bar{x} .4$

$1.47 E-194$

$1.74 E-H A$

1.7 VE-114

2. $166 \mathrm{E}-114$

2. $42 E={ }^{4} 4$

3. $86 E-04$

A. TIE-AS

$? .20 F-05$

$7.38 E-0 S$

$1.31 E-D 4$

$5,181-$ ins

1. $42 E-1) 4$

2.66E-A'
TRITONS

i.

$7.58 E-.35$

2.

$1.36 E-$ is

i.

1. $36 E-1 j 5$

i).

$9.04 E-65$

$\because$.

$1,36 t=115$

i.

$1,36 t-w 5$

$\because$ OE-O5

$1,365-15$

B. $71 E-65$

$1.18 \mathrm{t}=$ his

b.

6.

$7.68 E=65$

i.

$3.84 \mathrm{E}-95$

3. $34 E-G 5$

$3.84 E-15$

$\% .68 E-45$

$1,68 E-135$

$1.54 t=h 4$

it.

b)

is

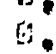

$n$

b.

$n$

is.

i.

i.

i.

,

9

औ.

$a$

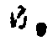

W.

Q.
ALPHAS

$d$.

4,1 CE $=$ W 3

3. $18 \mathrm{~F}-113$

$3.28 E=$ is 3

$4.68 E=V B$

$1,54 E-x i 3$

2.29E-33

2. OSE-:3 3

$3.43 E-23$

1. DVIEAR 3

2. BUE WS

$9.93 E-53$

h. $69 E=h 4$

$4.26 E-V) 3$

$2.68 E-4 C$

1. $U$ 3E- 12

$4.5 D E-133$

4. 3t:E-IS 5

4. $2 E E-13$

D. BIEABS

$4.98 E-43$

$3, T E E=13$

$5.43 E-13$

$5,72 E-13$

7.19tans

3. $29 E-B 3$

$4.71 E-i 3$

4. $60 E-13$

$7.36 E=43$

$1.47 E-112$

2.7UE-US

$9,57 E-d 3$

$4.42 E=-13$

B. BEE-T3

$8,43 t=13$

b. $27 E-13$

h. 25E-A3

1.51E-112

1,91E-Uद

$2,24 E-b 3$

$5,76 E-13$

9. Bóf.-J3 3

1.07E-G?

3.85 tul 3

$9,58 t-i 13$ 
ISLOWING DOWN SHECTRUM AT H.2A CM. FOR SHUNKA A-15O PLASTIR. 4? MEV P+ ON BE NEUTTRON SPECTRUM

\section{ENERGY}

4.20 MEV

4. 40 MEV

4.60 MEV

4.80 MEV

S.DO MEV

$5.50 \mathrm{MEV}$

6.00 MEV

$6.50 \mathrm{MEV}$

$7.0 Q$ MEV

1.50 MEV

B. DO MEV

$8.50 \mathrm{MEV}$

Q. DO MFV

$9.50 \mathrm{MEV}$

10. DO MEV

11. UA MEV

$12.00 \mathrm{MEV}$

$13,00 \mathrm{MEV}$

$14.01 \mathrm{MEV}$

15.00 MEV

$16,00 \mathrm{MEV}$

17.00 MEV

18.00 MEV

$19.0 \mathrm{MEV}$

$20,00 \mathrm{MEV}$

$21.00 \mathrm{MEV}$

2.2.00 MEV

23.00 MEV

$24.00 \mathrm{MEV}$

25.0 MEV

$26.00 \mathrm{MEV}$

27. DO MEV

28, DO MEV

29.00 MEV

30. AD MEV

32.00 MEV

$34.00 \mathrm{MEV}$

$36.00 \mathrm{MEV}$

38. UD MEV

40,00 MEV

42 DO MF.V

44.00 MEV

46.00 MEV

48.00 MEV

50.OD MEV

AAVE, E (MEV)

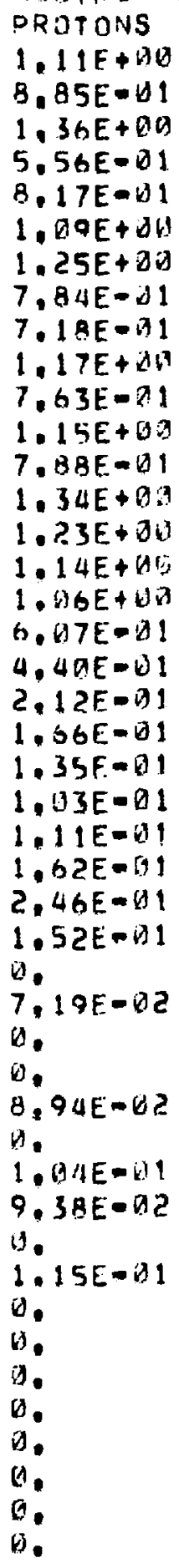

8.94

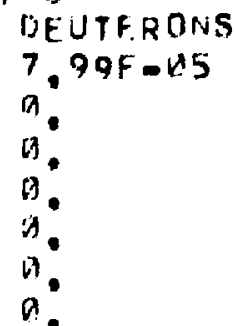

19

b)

(4)

1)

a

4

1.

9

14

$b$

$a$

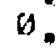

$a$.

a.

$b$

0

$\theta$

0

$\theta$

in.

$\theta$

6

u

a

9

i)

$a$

a.

a.

0.

9

1.32
TRITCNS

3.

9

B.

$\Delta$

H.

b.

9.

is.

vi.

(1)

+j.

i).

n.

in.

is

is.

$\Delta$

6

a.

0

3.

$\theta$

is.

B.

$\theta$

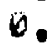

i.

is.

n.

0

b.

6.

n.

0.

0.

6

$\theta$

1

a.

(1)

$\theta$.

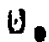

(1).

0

1.06
ALFHAS

3. $47 \mathrm{E}-\mathrm{WS}$

4. $D, E, E-I 3$

1. $29 E-1) 2$

1. NHE 1 ज2

2.1 Git-t3 3

$5.53 t-1) 3$

1. $3 E E=(B) 2$

$1, D 3 E$ A 3

$3.96 E-64$

1. $56 t-1 / 3$

$3.48 E-44$

1. $47 E-3$

$1.62 E-63$

9. $43 E=115$

$1.5 \zeta E=6 ! 3$

2. 1UE-A3

3. 57E-1;3

5.16E-1.15

1. M ZE -14

s.

$b$.

$n$.

is.

0.

0.

a.

$\theta$.

a.

a.

4.

i.

b.

$\Delta$

$\theta$

in.

$b$

0.

a.

a.

a.

0.

0.

a.

a.

0.

4.56 
ISLOWING DOWN SETCTRIIM AT 4,4 G CM. FOR SHDHKA A-15A PLASTIC 42 MEV P ON HE INEUTRON SPECTPUM

\section{ENERGY}

0.05 MEV

$0.10 \mathrm{MEV}$

0.15 MEV

A. 20 MEV

0.25 MEV

Q.38 MEV

0.35 MEV

0.40 MEV

0.45 MEV

$0.50 \mathrm{MEV}$

$0.55 \mathrm{MEV}$

$0.60 \mathrm{MEV}$

0.65 MEV

0.70 MF.V

0.75 MEV

0.8 MEV

$0.85 \mathrm{MEV}$

$0.90 \mathrm{MEV}$

0.95 MEV

1.00 MEV

$1.10 \mathrm{MEV}$

$1.20 \mathrm{MEV}$

1.30 MEV

1.40 MEV

$1.50 \mathrm{MEV}$

$1.61 \mathrm{MEV}$

$1.70 \mathrm{MEV}$

1. Bด MEV

$1.90 \mathrm{MEV}$

2. DO MEV

$2.10 \mathrm{MEV}$

$2.20 \mathrm{MEV}$

$2.30 \mathrm{MEV}$

2.40 MEY

$2.50 \mathrm{MEV}$

2.60 MEV

$2.70 \mathrm{MEV}$

$2,80 \mathrm{MEV}$

$2,90 \mathrm{MEV}$

$3 . D 0 \mathrm{MEV}$

$3,20 \mathrm{MEV}$

3,40 MEV

3.61 MEV

$3,80 \mathrm{MEV}$

4,00 MEV

\section{PFOT ONS}

औ.

$2.8 \pi E+3 \pi$

1. 3 $3 E+$ a is

$1.77 E-D 1$

2. 2 A -01

$1.73 E-B i$

$1.78 E-131$

$2,79 E-O 1$

$0,17 F-11$

1. $41 \mathrm{E}+\mathrm{BCH}$

2. $86 E-01$

$1.18 \mathrm{t}-\bar{D} 1$

$1.67 \mathrm{~F}=1$

$5.0 P E-01$

Q. TAE-DI

1. $19 E-B 1$

1. $54 E+60$

3. 3तE-DI

$1.65 E+00$

3. $43 E+79$

4. $41 E-D 1$

$7.61)=11$

$1.68 E+B H$

1. $95 E+64$

$1.30 E+D O$

6. $9 B E-D 1$

$1, B 4 E+D G$

8.78E-G1

$1.11 E+50$

$5.43 E-61$

7. $95 E-B D$

B. $13 E-B 1$

$4,9 B E-B D 1$

$9.47 E-B 1$

$2.71 E+00$

$1.93 E+03$

$1.48 \mathrm{E}+3 \mathrm{OC}$

$9,51 E-B 1$

$5,12 E=41$

$1.22 E+00$

$1.44 E+00$

$1.25 E+\theta H$

$6.58 E-B 1$

$1.95 E+06$

$1.64 E+0 V$
DEUTFRONS

a.

$1.16 f-65$

$7.56 E=05$

7. 31 IF-MO

$0.43 E-515$

$5,33 E-25$

$9.131-05$

9 . 3BE-AS

$1.34 E-D^{4}$

$9,33 E-A 15$

$2.95 F-05$

$1.74 F-A S$

$1.36 E-$ A A

$8.64 E-0 S$

7. BTE-AS

1. DIE-CA

1. $O A E-D A$

$2.57 E-D 4$

3.21E-US

?. $85 E-04$

$1.26 E-014$

$1.14 E-D 4$

$9.90,-V 5$

$2.32 t=04$

$1.73 \mathrm{E}-0.44$

2. $32 E-94$

$1.95 E=04$

1. UIE-O 4

1. DIE-DL

$2.04 t-64$

$9.15 E-05$

$1.42 E-P / 4$

$1.70 E-04$

$1.93 E-B 4$

$1.53 E-04$

3, $22 F-B 4$

$2.64 \mathrm{~F}=04$

2. DOF-OA

$2.10 E-04$

?. $23 E-B 4$

$1.2 B E-A 4$

1. 2OE-G

$1.17 E-04$

2.59E-04

$1.79 E=134$
TRITONS

(A.

$14.0 \geq E-06$

b.

2.B $B E-O 5$

1. $41 E-O S$

0.

c.

4. $\triangle 3 E=$ मI6

3. $48 E-135$

$n$.

$b$.

$1.41 E=05$

2. $47 E-05$

$1.41 E=05$

.

3.8HE-DS

i.

$3,48 E-05$

$4.63 E-B O$

$3,48 E-615$

2.65E- 15

$4.77 E-B 5$

2. 27E-DS

9.0LE-DS

4. G3E-US

B. BSE- 16

$\checkmark$.

$\Delta$

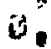

$\theta$.

0.

a.

(1.

0.

$3.47 E-105$

3.

E.

:

6

D.

$1.74 E-65$

a.

3.

0.

$1.74 E-05$
ALPHAS

i.

1.55E-1) 3

3. $4 \mathrm{E} E-6) 3$

$6.43 E-53$

1. USE-A?

$3,11 E=(13)$

$5.84 E=63$

$3.82 E-43$

$4.43 E-13$

1. 3GE $=113$

$1.96 E-1) 3$

$1,(A 1 E-D) 2$

1.68E- I 3

$2.47 \mathrm{t}=\mathrm{x}^{2} \cdot 3$

$1.0: 50-02$

b.v3t-us

$6.41 E-A 3$

$5.49 c=43$

3. $82 E-n 3$

5. $15 E-13$

$9.49 E-33$

$4.42 E-D 3$

$4.58 t-n 3$

$7.91 \mathrm{E} D 3$

$7,27 \mathrm{E}=\mathrm{B} 3$

3. $27 \mathrm{E}=0.3$

$7,48 \mathrm{~F}=\mathrm{BS}$

$6.03 E-03$

5.57E-D 3

$8.43 E-15$

$7.27 t-13$

1. $\triangle B E-I J 2$

$4.23 E-A 3$

7. 52E-A 3

$5.38 E-(13$

$7.59 E=U 3$

$5,41 E-03$

$7.57 E-43$

$1.15 E=A 2$

$4.72 E-63$

3. OBE- $\triangle 3$

$7.85 E-143$

$1.12 E=02$

B.7BE-B3

$7.51 E-B 3$ 
1SLOWING DOWN SPECTRIIM AT A, AG CM, FOP SHONKA A-15B PLASTIC 42 MEV P+ ON HE TNEUTRON SPECTRUA

\section{ENERGY}

4.2G MEV

4.40 MEV

$4,60 \mathrm{MEV}$

4.8 ME.V

5. $A B$ MEV

$5.5 \mathrm{ASEV}$

$6.00 \mathrm{MEV}$

6.50 MEV

7. $\triangle A$ MEV

$7.50 \mathrm{MEV}$

8. OD MEV

3.50 MEV

$9.00 \mathrm{MEV}$

$9.50 \mathrm{MEV}$

$10.00 \mathrm{MEV}$

11.ON MEV

12.01 MEV

13.DG MEV

14. DU MEV

15.DQ MEV

16. OU MEV

17. OU MEV

$18,00 \mathrm{MEV}$

$19.00 \mathrm{MEV}$

20.DO MEV

21. DO MEV

$22.0 D \mathrm{MEV}$

23. UO MEV

$24.00 \mathrm{MEV}$

25.0D MEV

$26.00 \mathrm{MEV}$

$27.00 \mathrm{MEV}$

$28.00 \mathrm{MEV}$

29.00 MEV

$30.00 \mathrm{MEV}$

$32,00 \mathrm{MEV}$

$34.00 \mathrm{MEV}$

$36.00 \mathrm{MEV}$

$38.00 \mathrm{MEV}$

$40,00 \mathrm{MEV}$

42.00 MEV

44.00 MEV

46. OD MEV

$48.90 \mathrm{MEV}$

$50.00 \mathrm{MEV}$

DAVE, E(MEV)

\section{PROTONS}

7.8Af-i] 1

$6.5 A E-A 1$

1. $25 E+B$

1. $39 E+G A$

8.27E-DI

$4.32 E-U 1$

$8.57 E-B 1$

$7.13 \mathrm{~F}-\mathrm{W}$ ?

9. $19 E-111$

8. $2\{E-41$

$7.33 E-B 1$

1. $D 1 E+B E$

$8.67 E-81$

6. $197 E-11$

1. $14 E+Q O$

$7,71 E=\square 1$

8.7TE-U1

$7.17 E-k 1$

$3,89 E-01$

2. $50 E-B 1$

$3.91 E=1$

$3.52 E-B 2$

$1.05 \mathrm{~F}-1$

$8.18 E-U 2$

$1.35 E-D 1$

4. 16E-D?

$1.34 E-11$

0.

1. $\triangle 4 E-61$

$3,43 E-132$

$9.38 E-B 2$

2. $\triangle 1 F-A 2$

$6.37 E=02$

$4.47 E-D 2$

$2.53 \mathrm{E}-\mathrm{B} 1$

$5,21 E=02$

$2,87 \mathrm{E}-0 ?$

0.

$1.71 F-02$

$v$

b.

0.

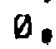

0.

8.47
DFUTERONS

9. $2 D E-1 D 5$

$1.39 E-A 4$

?. $3 D F=A 4$

3. $62 F-D S$

4.

$5.32 E-05$

$5.32 F=135$

1. $45 F-$ is 5

$5,32 E-65$

5. 32E-AS

b. $77 E=.5$

5. 32E-ES

6. $77 E-D S$

B. $22 E-15$

1. $40 E-A 4$

$7.74 E-B S$

1. $74 \mathrm{E}-114$

0

b.

$b$

0

a.

D.

6.

a.

b.

$\theta$

b.

0.

a.

0

0

0

a.

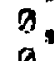

0

3

a.

0

0.

1

0

0

5.60
IR IT TONS

k.

$1.74 E=05$

A.

a.

$1.74 E-05$

$6.95 E-16$

$1.39 t-155$

$1,39 E-B 5$

$1.39 E-35$

2. $08 E-05$

$4.865-45$

n.

$s$

a.

0

0.

$n$

0

$n$

0

6

a.

$a$

0

0

0

(i).

0.

$b_{a}$

b.

a.

$d$

a.

,

b.

9.

a.

0

$a$

a.

4

$\theta$

$\theta$

i)

4.70
ALPHAS

6.7BF. 13

$3.6 \cup E-133$

7.61E-DS

5.6UE-i'3

$5,64 \mathrm{~F}=\mathrm{L}^{13} 3$

2. $89 E-13$

7. $B E=13$

$2,55 E-1) 3$

4. $41 E-133$

2. $11 E-\Delta 3$

4. BUE-L 3

3. B6E- I 4

7. SUE-SL

1. $\triangle B E-114$

4. 35E- 14

2. $11 E-44$

$3.93 E-114$

$1,61 E=95$

$3,22 E-15$

$\theta$.

.

D.

$\theta$

0

$\theta$

$\emptyset$

a.

0.

$a_{9}$

0

$\theta$

3

A.

0

0

0

a.

a.

0.

$\theta$

0

a.

3.84 
ISLOWING DOWN SPECTRUM AT O. GO CM. FOH SHONKA A-ISA PLASTIC 42 MEV P+ ON BE NEUTRON SPFCTRUM

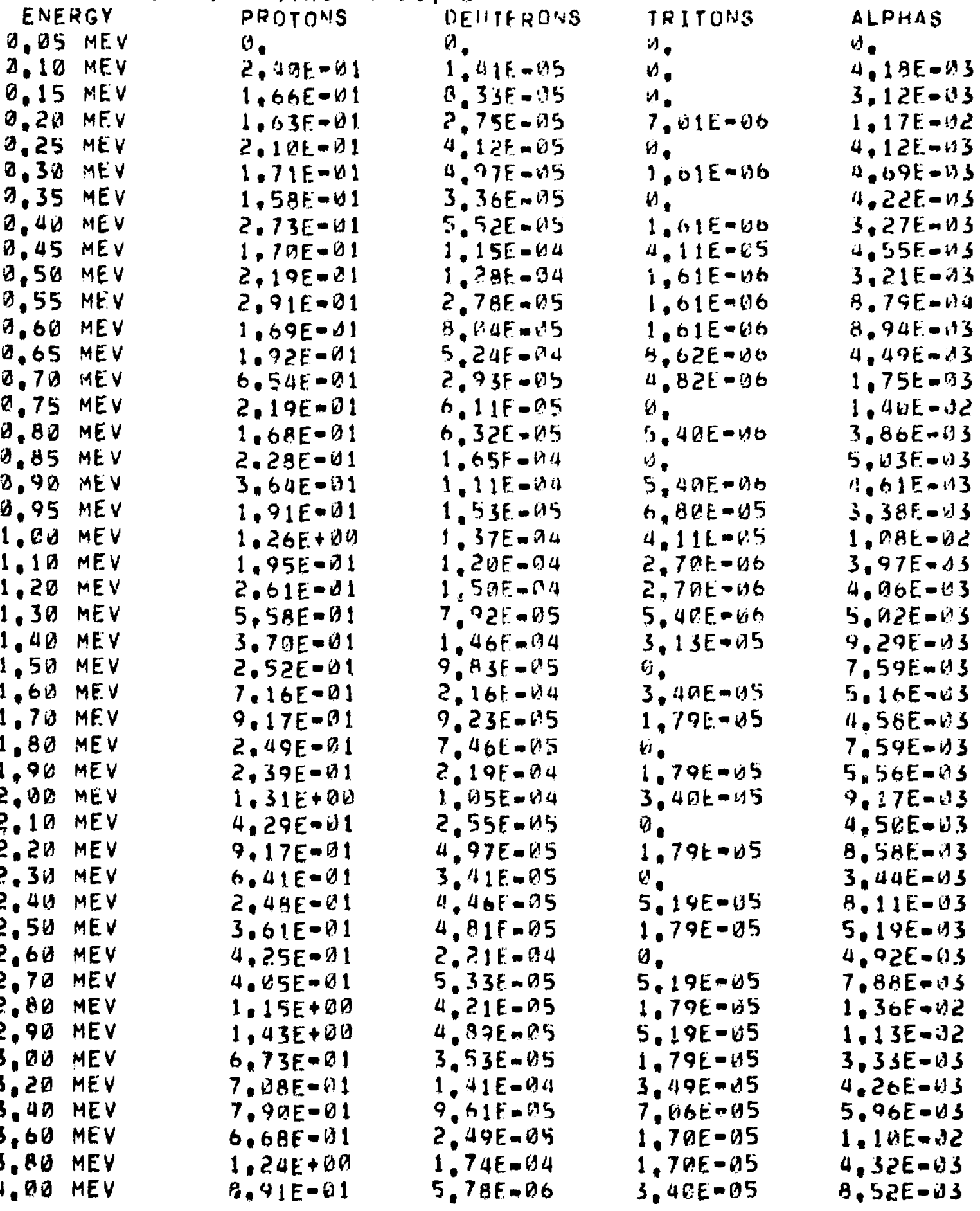


1SLOWING DOWN SPECTHUM AT D, GQ CM. FUR SHONKA A-15G PLASTIC 42 MEY P+ ON BE NEUTRON SPECTRUM

$$
\text { ENERGY }
$$

$4.20 \mathrm{MEV}$

4.40 MEV

4,60 MEV

4.80 MEV

5.00 MEV

5.50 MEV

6. AD MEV

$6.50 \mathrm{MEV}$

$7.00 \mathrm{MEV}$

$7,50 \mathrm{MEV}$

$8,00 \mathrm{MEV}$

$8.50 \mathrm{MEV}$

$7.00 \mathrm{MEV}$

$9,50 \mathrm{MEV}$

$10.00 \mathrm{MEV}$

$11.00 \mathrm{MEV}$

12,00 MEV

13. DO MEV

14.00 MEV

15,01 MEV

$16.00 \mathrm{MEV}$

17. OO MEV

$18.00 \mathrm{MEV}$

$19.00 \mathrm{MEV}$

$29.00 \mathrm{MEV}$

$21.00 \mathrm{MEV}$

$22.00 \mathrm{MEV}$

23. DO MEV

24.00 MEV

$25.00 \mathrm{MEV}$

26.00 MEV

27.0 MEV

28. OQ MEV

$29,00 \mathrm{MEV}$

30. $\triangle O M E V$

$32.00 \mathrm{MEV}$

$34,00 \mathrm{MEV}$

36,00 MEV

$38.00 \mathrm{MEV}$

$40.00 \mathrm{MEV}$

$42.00 \mathrm{MEV}$

44,00 MEV

46.01 MEV

48. DD MEV

50,DE MEV
PRUTONS

1. $53 E+6 D$

1. $14 E+0.3$

1. $14 E+813$

$1.3 A E+B D$

5. $12 E-61$

$1.11 E+Q 1]$

$1.16 t+20$

B. 93E- 1

$6.47 E-(1)$

$9.94 E-D 1$

$6.96 E-B 1$

7. $19 E-61$

$7.28 E-01$

$1.10 E+D A$

$7.29 E-91$

$7.45 E-\theta 1$

$8,51 E-91$

$9.64 E-01$

$6.18 E=91$

$6,43 E-D 1$

$5.27 E-61$

3. $B G E-01$

$0.06 E-01$

$3.86 E-01$

$2.5 B E-D 1$

$1.66 E-91$

$7.96 E=02$ ?

$1.63 E-01$

$3.39 E-02$

3. $19 E-B 1$

$8.06 E-\square 2$

$2.01 E=0$ ?

$1.36 E-111$

$2.23 E-022$

B. $08 E=02$

$2.69 E-02$

0.

$3,28 E-02$

$\theta$

i.

$\theta$.

$\theta$.

g.

G.

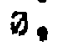

DAVE, E(MEV)

DEUTERDNS

1. 1 OE-D 4

$6.82 E-96$

1. ज2EनTA

$6.82 E-116$

$1 . \triangle 2 F-B 4$

Q. $43 E-95$

$9.79 E=D 5$

$1.94 E-D 4$

3. DUE-DA

O.

D.

a.

$\theta$

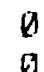

(1)

:

a

$a$

$a$

a

0

a.

9

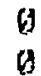

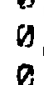

0

0

0

0

$a$

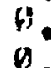

a.

0

0.

a.

0.

9

3.94
TRITONS

1. TVE-WS

S. 1AE- 45

E. SAE-GS

$\theta$.

i.

B.

F.

a.

B.

$n$

$\theta$.

k.

0.

a.

i.

6.

9.

9.

9.

$\theta$.

$n$

bi.

b.

a.

0.

if.

4.

s.

a.

b.

a.

0.

a.

$\Delta$.

i.

$\theta$

B.

$\theta$

U.

b.

a.

a.

0.

0.

a.

3.67
ALPMAS

D. $R 4 E-133$

$6.68 E-J 3$

1. $12 E-D 2$

$9,68 E-D 3$

4. $85 E-M 3$

$3.81 E=13$

$6.72 E-03$

$3,52 E-03$

$4,7 \angle E-D 3$

1. $44 E-133$

$6,5 \mathrm{GE}-14$

1. $35 E-13$

$1.77 E-93$

$2,59 E-34$

$1.91 E-03$

$1.65 E-03$

2. 8 QUE -3

$1.61 \mathrm{E}=1.55$

$3.22 E-145$

i.

.

b.

ต.

a.

9.

9.

b.

a.

0.

is.

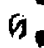

0.

0

a.

$\theta$

$\theta$.

$B$.

$\theta$

0.

a.

$\Delta$

0.

$\theta$.

0.

4.53 
1SLOWING DOWN SPECTKLIM AT 1, DG CM. FOR SHONKA A-15H PLASTIC 42 MEV P+ ON BE. NEGITRTN SPECTRUIM

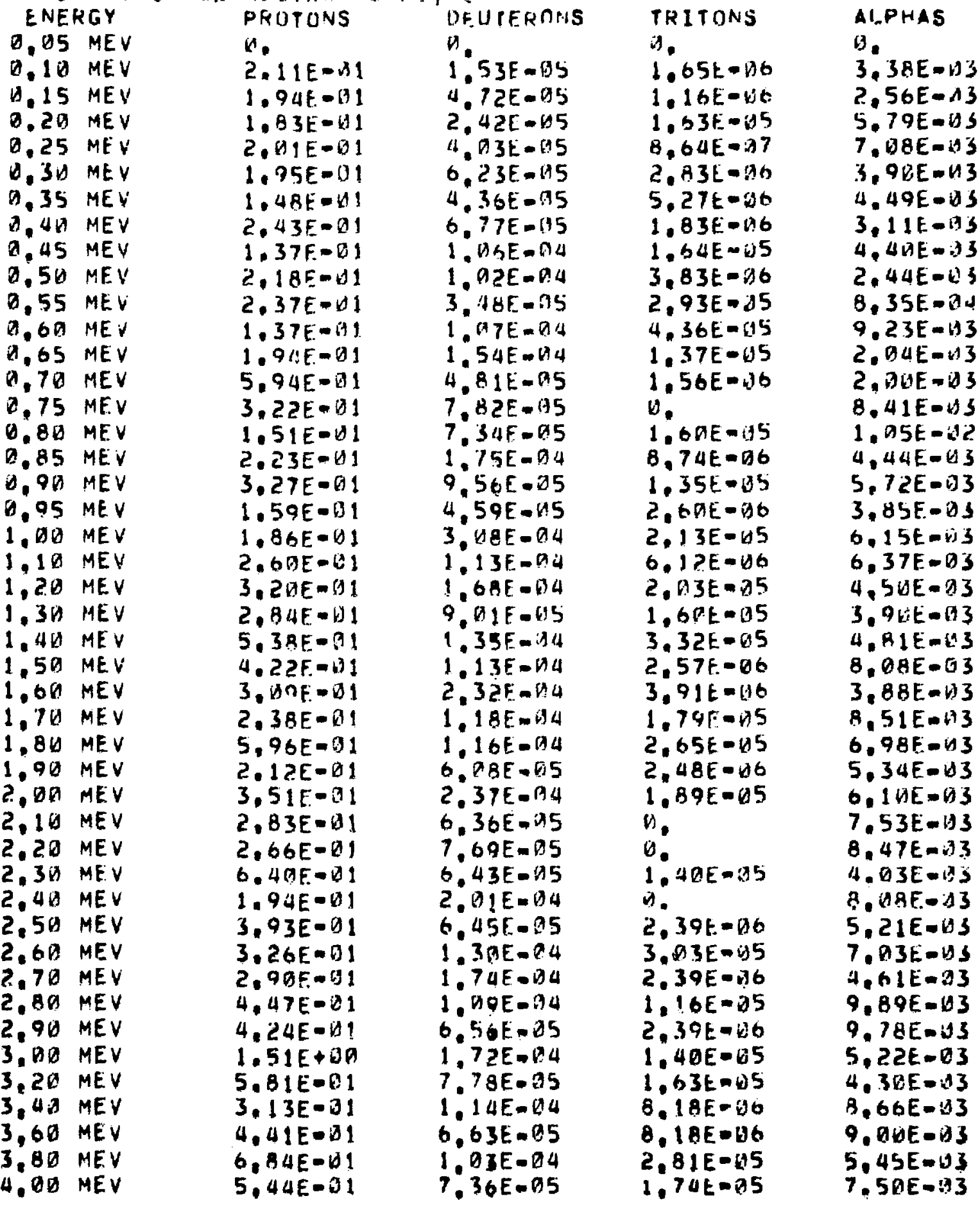


ISLOWING DOWN SPECTRIJM AT 1 , CM. FOR SHONKA AOISU PLASTIC 42 MEV F+ ON HE NEUTRON SPECTRUM

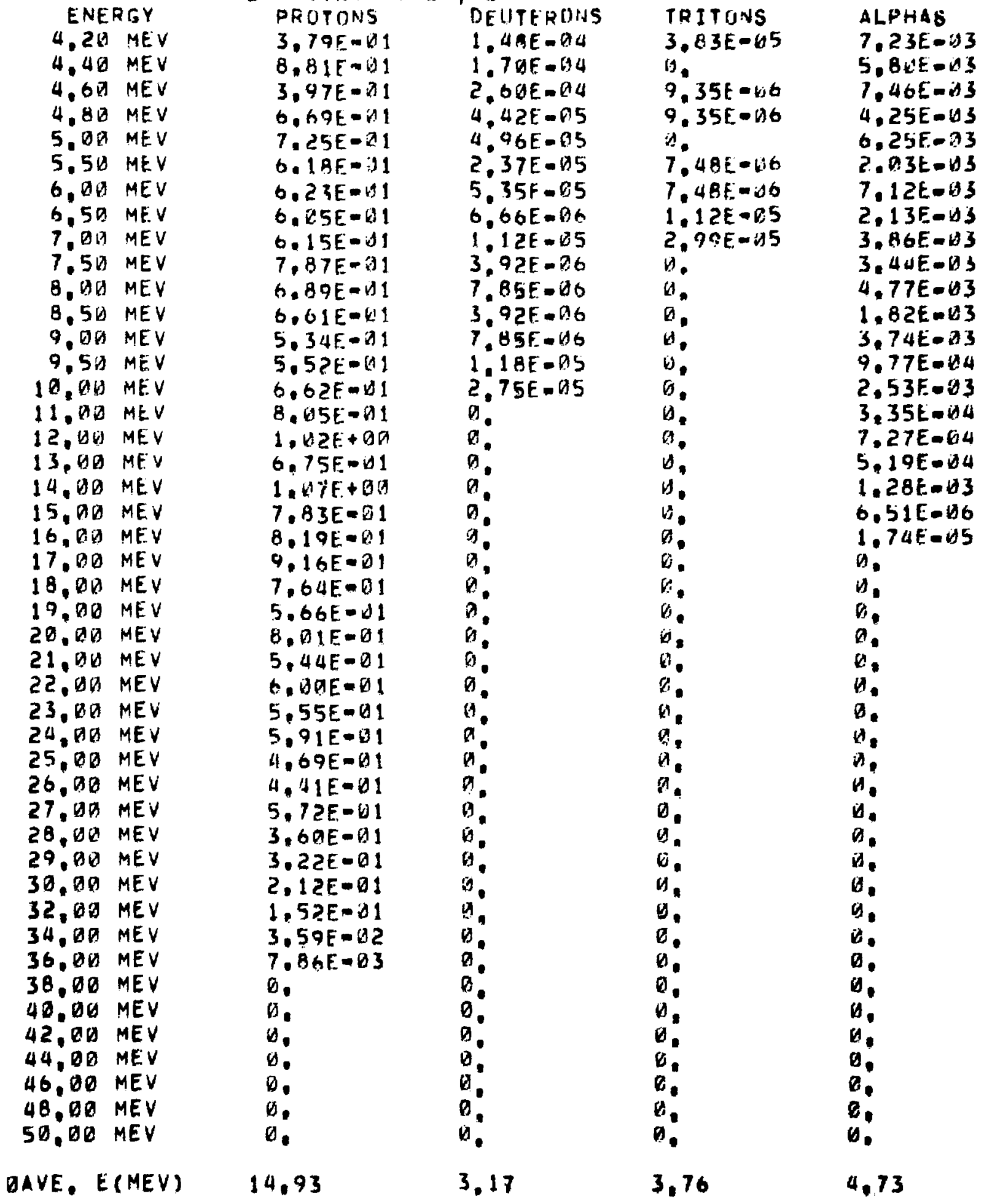


ISLOWING DOHN SPECTRUM AT 2, GA CM. FOR SHUNKA A-15D PLASIIC 42 MEV P+ ON BE MEUTRON SPECTRUM

\section{ENERGY}

J. OS MEV

Q.10 MEV

A. 15 MEV

8.20 MEV

0.25 MEV

Q.3 3 MEV

$0.35 \mathrm{MEV}$

0,40 MEV

0.45 MEV

$0.50 \mathrm{MEV}$

$0,55 \mathrm{MEV}$

$0.60 \mathrm{MEV}$

0.65 MEV

$0.70 \mathrm{MEV}$

0.75 MEV

$0.80 \mathrm{MEV}$

0.85 MEV

0.90 MEV

0,95 MEV

1.90 MEV

$1.10 \mathrm{MEV}$

1,20 MEV

$1,30 \mathrm{MEV}$

$1.40 \mathrm{MEV}$

$1.50 \mathrm{MEV}$

$1.60 \mathrm{MEV}$

$1.70 \mathrm{MEV}$

$1,80 \mathrm{MEV}$

$1,90 \mathrm{MEV}$

$2.00 \mathrm{MEV}$

2.10 MEV

2.2U MEV

2.3V MEV

2, 40 MEV

$2,51 \mathrm{MEV}$

2,66 MEV

2,70 MEV

2,Bด MEV

2.90 MEV

3.00 MEV

3.20 MEV

$3.40 \mathrm{MEV}$

3.60 MEV

3,80 MEV

4. OD MEV
PROTONS

4.

E. $49 E-01$

$1.98 E-61$

1.9BE- 11

$2,36 E-B 1$

2. $16 E-01$

$1.82 E-01$

C. $33 E-D 1$

$1.83 E-61$

$2.05 E-01$

3. $B \triangle E=D 1$

$1,81 E-B 1$

2. $16 \mathrm{EF}-\mathrm{B} 1$

6. 85E-ด1

$2,63 \mathrm{E}-\mathrm{Bl}$

$1.59 E-B 1$

$2,73 E=D !$

$3,81 E-Q 1$

2. $12 E-B 1$

$1.8 M E-B 1$

$2.72 E-\Delta 1$

2.9 2E-B1

$2.75 E-B 1$

$5.45 E-1$

4. $42 E=\square 1$

$3.56 E-\triangle 1$

?. 93E-D!

H. 6 ?.E $=1$

$2.65 E-01$

$3.45 E-61$

$3.9 B E-D 1$

$2.86 \mathrm{E}-01$

6. $21 E-31$

2. $14 E-\Delta 1$

$4.25 E-01$

3.75E- 11

2. $89 E-B 1$

$5.15 E-01$

$5.06 E-101$

1. $05 E+00$

4. 19E-O1

$2,67 E-11$

$4.20 F-01$

$5.96 E-B 1$

$5,69 E-111$
DEUTEROINS

a.

1.17E-A4

Q. 21E-US

8. 39F. -94

$6.42 E-95$

1. $56 E=0.14$

$5.94 E-635$

6. $56 E-185$

$1.95 E-04$

$2.31 E-154$

$3.18 E=0.5$

$2.35 E .04$

$1.26 E-C) 3$

$1.44 \mathrm{~F}=\mathrm{CA}$

$1.90 E-64$

$2.7 E E-B 4$

2. $165-B 4$

$9.26 E-204$

$2.55 E-65$

3. 4 XIE $=14$

$1.17 F-D 3$

$1.64 F-1 \mathrm{~S}$

$2.22 E-113$

$5,75 E-A 4$

$6.43 E-34$

2. 9BE-IS

$1.87 E-34$

1 QVEE-VA

2. HTE- 14

1. $85 E-153$

$1.69 E-193$

$1.07-04$

6. 8OE-24

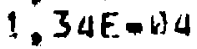

$5.84 E-V 5$

$2,43 E-D 3$

$9.19 E-05$

$7.06 E-04$

9. D2E-35

2. $33 \mathrm{E}-\mathrm{U} 3$

$1.12 E-93$

2. $13 E-(1) 3$

3. $38 E-A 5$

$1.33 E-03$

B. BQf-OA
TRITOPS

E.

$8,7 D E=\Delta E$

1 . $A B E-A B$

4. $42 E-V D$

$1,86 E-106$

$3.01 E-06$

3. U3E- BV

$6.29 E-05$

$1.24 E-D 5$

$6.94 E-46$

1. $06 E-05$

$7.93 E-115$

1. $\triangle B E-G 5$

3,72E-UG

2. $6 A C-66$

1. $29 E-155$

3. $46 E=116$

2. $42 E-B 6$

$7.45 E-06$

$B, 1 \theta, E=A G$,

$4,87 E-\Delta 6$

1. $47 E=05$

$6.27 E=0.6$

$2.55 E-05$

$2.20 E-05$

1. $49 E-45$

A. $19 E=11 t_{3}$

$3,02 E-155$

8. $86 E-116$

$4.95 t-196$

$2.60 E-d 5$

$3.2 \Delta E-B h$

$4.95 E-46$

$3.24 E-06$

$2,2 \Delta E-\triangle 5$

$3,96 E-B S$

$4.72 E-\Delta 6$

6. 7 OE- BS

$1.93 E-15$

1. $38 E-\square 5$

4. TRE-WS

$1.57 E-65$

$3.21 E-06$

2. 8 BE-G5

$3.15 E-H S$
ALPWAS

a.

1. $H \in E-B 3$

1.81E-US

7. WUE-CI

$6.238-43$

2. $92 E-B 3$

$3,32 E=03$

2.62E-A3

3. $2 S E-$ IS 3

$2.01 E-D 3$

$9.36 E-14$

8. $45 E-H 3$

3. 2UE-U3

1.79E-U3

5.7 VE- 13

1. HIE-WL

$3.21 \mathrm{E}-\mathrm{in} 3$

$3,84 E=\Delta 3$

2. $71 E-43$

$5.58 E-6.3$

$5.55 E-03$

$3.27 E=A 3$

4. DIE-A 3

$7.52 E-H 3$

$6.87 E-M S$

$3, \gamma U E-B 3$

$5.91 E=(13$

$5.79 E-13$

$4.63 E-133$

5. U9E-W 3

b. GBE-HS

9. $14 E-$ is 3

3 , उ $3 E-63$

S.56E-US

$5.22 E$ and 3

5,37E-DS

$5.51 E-03$

B. $33 E=13$

$9.11 E=[13$

$3.79 E-B 3$

$5,15 E-\triangle 3$

S. $31 E-B\}$

A. $79 E-113$

$5.8 W E-03$

h. OBE-US 
1SLOWING DOWN SPECTRUM AT 2, DA CM, FCK SHONKA A-15B PLASIIC 42 MEV P+ ON BE HEUTRON SPECTRIIM

\section{ENERGY}

4.20 MEV

4. 4 M MEV

$4,60 \mathrm{MEV}$

4.80 MEV

5. 00 MEV

5,50 MEV

$6.00 \mathrm{MEV}$

$6,50 \mathrm{MEV}$

7,00 MEV

7.5D MEV

$8.00 \mathrm{MEV}$

$8.50 \mathrm{MEV}$

$9 . D O M E V$

9.50 MEV

10.00 MEV

$11.00 \mathrm{MEV}$

12, OS MEV

$13,00 \mathrm{MEV}$

14. 00 MEV

$15,00 \mathrm{MEV}$

$16.00 \mathrm{MEV}$

$17,00 \mathrm{MEV}$

$18,00 \mathrm{MEV}$

$19.00 \mathrm{MEV}$

$20,00 \mathrm{MEV}$

$21.00 \mathrm{MEV}$

22.00 MEV

23.09 MEV

24.00 MEV

25.0Q MEV

26. Q0 MEV

$27.0 B \mathrm{MEV}$

$28.00 \mathrm{MEV}$

$29.00 \mathrm{MEV}$

$30.00 \mathrm{MEV}$

$32,30 \mathrm{MEV}$

$34.00 \mathrm{MEV}$

$36.00 \mathrm{MEV}$

38. AB MEV

$40.00 \mathrm{MEV}$

42,00 MEV

44.00 MEY

46, DO MEV

48,00 MEV

SD,DO MEV

DAVE, E(MEV)
FUOTONS

4. 2XE-T1

$5.72 E-B 1$

5.97E- G1

$3.94 E-D 1$

$7.32 E-91$

5. $05 E-B 1$

$6,13 E-61$

$5.55 E-01$

$6.07 E-D 1$

$6,2 A E-11$

$11.42 E-\theta 1$

$6.88 E-i) 1$

$5.85 E-01$

5. 4AE - 1

5. $96 E-O 1$

$6,59 E-01$

$6.78 E-01$

$6.19 E-11$

$6.88 E-\triangle 1$

$5.55 E-\| 1$

$5.73 E-B 1$

$4.91 E-D 1$

$5,6 U E=D 1$

4. $46 E-01$

6. 25E-D1

$4.38 E-D 1$

$5.3 Q E-B 1$

4. $1 \mathrm{BE}-\mathrm{B} 1$

$3.77 E-A 1$

4. 32E-D1

$2.33 E-D 1$

$2.85 E-1$

2.53E-D1

$2.45 E-d 1$

3. DOE-तI

$1.45 E-D !$

$9.57 E-02$

$8.54 F-92$

$1.71 E-H 2$

a.

$\theta$

a.

B.

a.

n.

14.79
DFUTEROAS

9. 32E-A 4

?. $41 E-B 3$

$2.57 E-A 3$

$4.29 E-115$

$4.93 E=h 4$

3. $43 E-B 4$

$5,39 E=D 4$

$5.95 \mathrm{~F}-\mathrm{BS}$

5. B $3 E-B A$

7. $B$ BE- 94

$4.93 E \mathrm{mO4}$

7.7:3F-

$1.37 \mathrm{E}-03$

$3.59 E-(14$

$7.22 E-B 4$

$6.73 E-06$

$1.52 E-95$

9.

9.

:

:

:

a.

a.

$\theta$

$\theta$

0.

in:

,

$\theta$

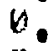

G.

$\theta$.

$a$.

a.

0

0.

.

.

D.

4.

0 :

4.90
TRITUNS

$2.96 t .-35$

$8.86 E-166$

$4,12 E-415$

$2,69 E-155$

$8, B 6 E-116$

$2.84 E-25$

$3.64 k-05$

$3.99 E-65$

7. UAE- 65

2. $\triangle G E-65$

$3,32 t-105$

$1.85 E-45$

$4.22 E-U 5$

$8.73 E-36$

2. $K 4 E-15$

6.

i.

6.

$n$.

$\theta$

a.

0.

$\theta$

a.

is.

a.

0.

n.

a.

,

0.

0.

$\therefore$

$\Delta$.

0.

i.

0

.

is.

a.

$\theta$

.

u.

,

0.

5.64
ALPHAS

7. 53E-A3

$4.50 E-B) 3$

$6,11 E-13$

$5,79 E-$ id 3

$5.27 E-B]$

$1.91 \mathrm{E}-\mathrm{AS}$

$5,19 E-B 3$

2,6 AE- I 3

$4,32 E-B 3$

$1.82 E-113$

2. 95E-D. 3

$\because 63 E=93$

$2,96 E-13$

5,3 BE- -44

$1.75 E-13$

$3.22 \mathrm{E}-14$

$B, 39 E=4$

2. $57 \mathrm{E}-\overline{\mathrm{B} S}$

$2,67 E-6)$

$2,21 E-B A$

2. $27 E-W \Delta$

$2,17 E-B 4$

4. 34E- II 4

$4.34 E=$ B 11

O, BQE-H 4

is.

a,

b.

o.

0.

U.

b.

0

ต.

B.

D.

$n_{1}$

$\theta$.

9

$g$

a.

0.

b.

$\theta$.

a.

5.10 
1SLOWING DOWN SPECTRUM AT H.HG CH. FOR SHONKA A-15V PLASTIC 42 MEV P+ ON BE NEUTRON SRECTRUM

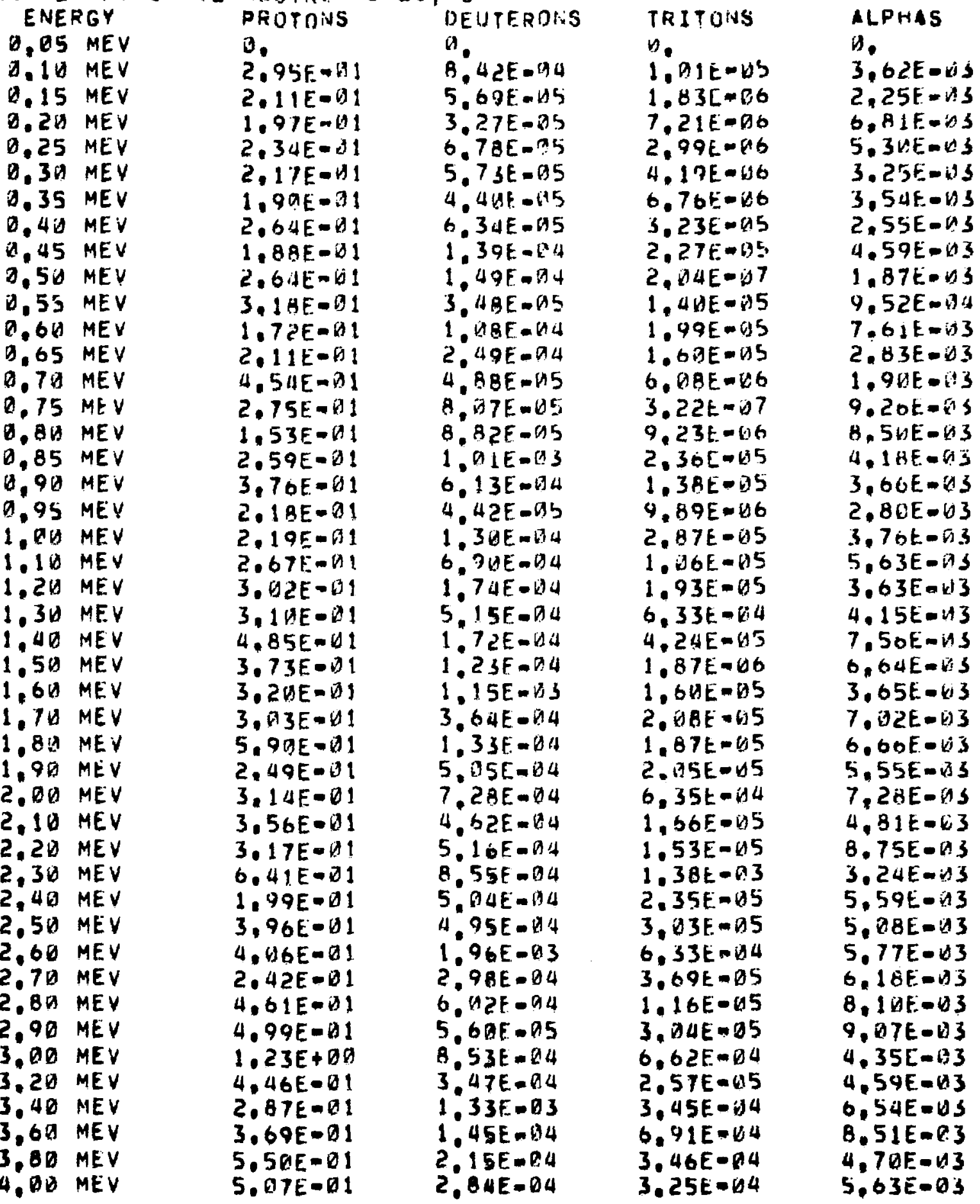


1SLOWING DOWN SPECTRUM AT 4, GO CM. FOR SHONKA A-15H PLASTIC WL MEV P+ ON BE NEUTRONA SPTCCTRUMA

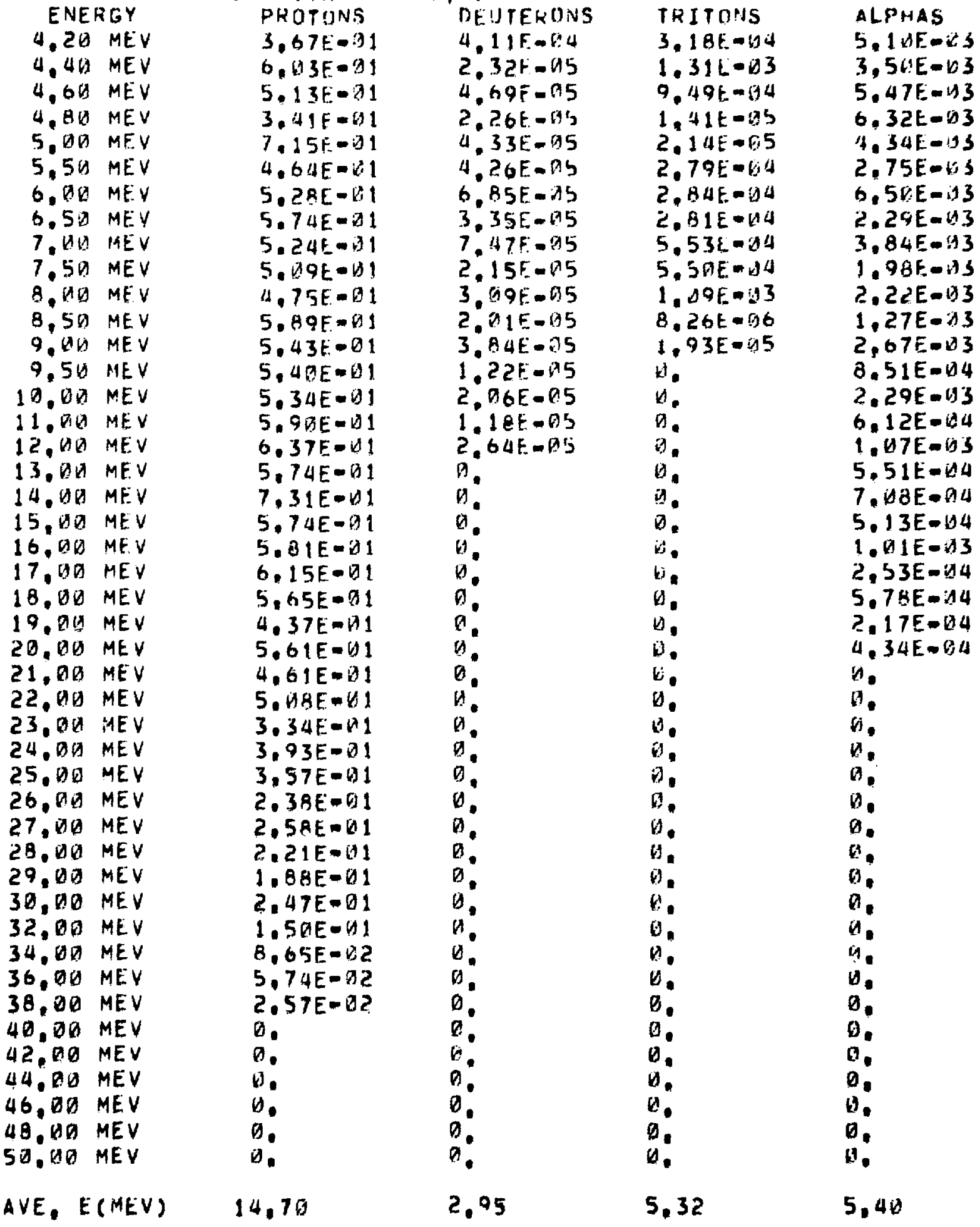


ISLOWING DONN SPECTRUIA AT 6, MA CH. FOH SHONKA A-I5H FLASTIC 42. MEV P+ ON BE NE:ITROH SPECTRUM

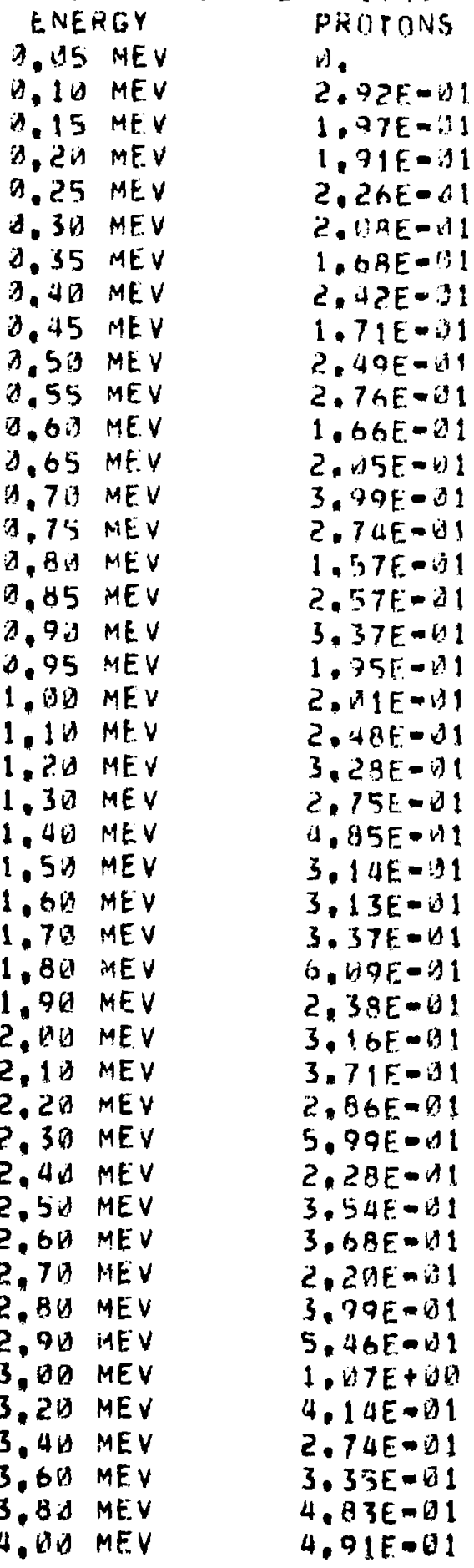

UEUTERTIIS

i.

8. $96 F-\pi i$

$1.79 t=-14$

1.99E- 95

6. BIF-li's

$1.5116-24$

$3.01 \mathrm{~F}-\mathrm{N}$ SS

1. $63 E=(34$

$2.29 t=64$

$3.03 E-94$

2. $98 E-D S$

1. $37 E-A 4$

2. ? IF $=4$

4. $84 E=.95$

6. $1 B E-15$

R. $87 E-05$

$1.73 f-84$

1. $1 \mathrm{f}-14$

$2.35 E-145$

1. $70 E=94$

1. $4 U E-34$

1. $53 E-194$

1. $10 E-6.1$

2. $25 F-14$

1. $42 F-6 a$

2. $14 \mathrm{~F}-\mathrm{n} 4$

$1.14 \mathrm{~F}-34$

1. $97 \mathrm{~F}-\mathrm{O}_{4}$

7.6? $5=115$

1. $75 E-44$

5.13E-OS

9.31E-U5

$5.36 E-1: 5$

1. $27 E-t^{2} \cdot 1$

7. $22 E-05$

1.27E-OA

$1,1 \mathrm{LE}-\mathrm{D}$

9 . IDE $=$ DS

7. 2AF-115

9.98F-05

7. A2F. IDS

$1.12 E-14$

3. $51 E-15$

$7.97 E-1 A S$

$5.74 F=95$
TRITUNS

W.

$7.73 E-16$

2.1 $14 E-$ Nio

Y. $48 E-36$

$2,64 t=196$

7. $41 E=146$

$5.22 E-116$

2. $57 \mathrm{E}-45$

1. $32 E-d 5$

4. SBE-Wh

S. HGE-US

2. RAE- 15

1. $31 E-45$

5. $2 A E=136$

5. $32.3 E-16$

2. 5RE -15

1. $26 E-115$

1. $69 t=65$

$7.21 E-06$

$1.35 E-35$

1. $25 \mathrm{f}-\mathrm{U} 4$

$3.37 E-.15$

1. $14 E-15$

4. $\{3 E-15$

1. 5 UE- 15

$1.35 E=195$

2. $13 E=45$

$1.67 t .05$

2. $36 E-45$

2. ?9t-n5

1. $25 E-15$

1. 15E-4!4

$3.55 E-05$

1. $11 \mathrm{~L}-05$

2. $35 E-115$

$5,50 E-65$

2. $25 E-05$

$2.57 E-1.15$

1. $2.2 t-x$

J. $35 E-\checkmark 4$

1. $711 E-05$

$2.31 E-U 5$

$6.01 E-135$

$3.53 E-15$

$3.48 E-N^{2}$
ALPHAS

$\forall$

2. A9E-U3

2. $39 E-4$

$6.33 t=.13$

$7,11 E-3$

S. प1E-OS

$3.37 E=13$

?. $97 k-43$

$4.97 E-13$

2. $27 E-n 3$

1. $195-33$

A. $24 E=d 3$

$2.09 E-13$

1. $64 E-W 3$

$7.89 E-13$

1. $A 3 E-12$

A. 6 GE- US

$4.3 E-3$

2.07t-As

3. 4 IE-3S

6. $12 E-103$

$3.18 E-\gg 3$

$4.1 B E$ - A 5

7. $\mathrm{XHE}=\mathrm{H}$

$0.75 E=3$

$3.75 E-13$

6. $49 E-n 3$

5.7AE-US

6.4 BE- 3

$6.49 E-13$

5. BSE $=3$

9. OIE-U!S

3. 27E-US

4. $85 E-13$

6. $13 E-3$

6.37E-AS

$7.15 E-13$

Q GHE-JS

$1 ., D S-12$

$4.29 E-43$

$5.18 E-33$

A. MAE- D 3

Q.11E-A3

4. $84 E=143$

$5.51 E-! 3$ 
1SLOWING DOWN SPECTRUM AT G. GG CM. FOR SHUNKA A-15H PLASTIC 42 MEV F+ UN BE REUTKON SPECTRUMT

$$
\text { ENERGY }
$$

4. 2W MEV

4.4\$ MEV

4. OM MEV

4. BI MEV

5. AQ MEV

5,50 MEV

6, QU MEV

$6.50 \mathrm{MEV}$

7. U. MEV

$7.5 \mathrm{~B}$ IIEV

8, DU MEV

9.5A MFV

9. 10 MEV $V$

9,50 MEV

1D, DO MEV

$11, D O M E V$

12. $100 \mathrm{MEV}$

$13.00 \mathrm{MEV}$

$14.00 \mathrm{MEV}$

$15.00 \mathrm{MEV}$

$16.00 \mathrm{MEV}$

17. BD MEV

18. DU MEV

19, OU MEV

26, BO MEV

21. OH MEV

22, MEV MEV

23. $\square$ MEV

24.01 MEV

25, DA MEV

26, OH HEV

27. BU MEV

$28, H$ MEV

29, OH HEV

$30.0 \%$ MEV

32. DQ MEV

34. $\triangle O M E V$

36. OO MEV

3B. DO MEV

AD. OU MEV

42. UU MEV

$44.0 \mathrm{MEV}$

46. $\triangle D$ MEV

48. QU MEV

$5 B .00$ MEV

gAVE, E(MEV)
PROTONS

$3.25 E-31$

$4.96 E-01$

4. $11 E-B 1$

3. $11 E-U 1$

6. $2 \Delta E-H 1$

4. $47 E-B) 1$

$5.43 E-61$

$4,65 E-01$

5. $40 .-11$

5. $22 \mathrm{~F}-\mathrm{BI}$

4.6 $3 E-41$

5. $21 E-A 1$

$5,39 E-A 1$

$4.54 E-U 1$

5. (ACE-b)

$5.73 E-D 1$

5. $25 E-\triangle 1$

$4.84 E-11$

$6.22 E-\triangle 1$

5. $44 E-01$

5. $\{6 E-31$

$4.895-11$

$4,93 E-61$

$3,69 F-91$

4.7 OE-01

4. $\triangle U F=R 1$

$4.17 E-11$

$3.79 E-31$

$3.79 E-A 1$

$3.33 E-1$

$2,22[-\pi 1$

2. $97 E-U$ !

2. $38 E-11$

$1,62 E-\square 1$

$1.345-11$

9. BSE-Q?

$9.7 \square E-B C$

$3.66 F-02$

$4.65 E-112$

9.2AE-43 3

a.

a

$\theta$

i)

$D$.

14.69
DFUTFRONS

$7.59 E-15$

$7.42 E-U 5$

$8.58 E-14$

5.9AE-US

1. $4 E=D^{2} 4$

$3.41 F-145$

5. G1E-OS

?. $6.6 E-V 5$

b. 7 UE-AT

$1.94 E=.45$

$3,8,3 E-155$

$8.44 E-1.6$

1. $42 F=D F$

1. 2BE- II5

2. 5 SE $=5$

b. $63 E=15$

1. $49 E-65$

a.

a.

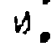

a.

a.

4

it:

it.

6

V.

a

$a$

G.

$a$

a

is

is

0

i

i).

a.

0

3.57
TPITONS

9. ofte-ns

1. $01 E-65$

6. B1t-ks

$2,44 t-$ ins

3. $41 E-45$

$4.83 t-15$

$1.13 E-114$

a. $35 t-116$

$1,32 E-135$

5. $P: 9 E-P I 6$

$8.13 E-196$

5. $14 E-6:$

1. $15 E-5$

$4.4 R E-66$

1. $15 t-15$

n.

b.

$n$

i.

4 .

k.

1.

d.

is.

$n$

is

4

i.

6.

V.

b.

it

$\Delta$

t.

0

G.

ti.

bi.

i)

$b$.

6.

b.

i).

9

if.

4.29
ALPRAS

5. 3HE-MS

II. $66 E$ on 3

6.) B E=33$

4. $99 t-113$

$4.25 E-43$

2. $24 E-$ W 3

$5,97 t-143$

$2.43 E-43$

$3.91 \mathrm{E}-\mathrm{H} 3$

1. $81 \mathrm{~K}-\mathrm{Ni}$

$2,615-13$

1. $12 \mathrm{E}=\mathrm{AB}$

2. $13 E-13$

4. $3 \mathrm{EE}-\mathrm{AAt}$

$1.2 B E-B$

$4.69 E-14$

8. 5SE-11:

1.73E-B⿵

$2.73 E-14$

1. $44 E-0.4$

2. 26E-A4

$1.43 E-14$

$3,396-i, 4$

$7.24 E-135$

1. $45 E-1) 4$

d.

1).

(1)

i.

a.

U.

a.

0.

a.

(1)

(i)

a.

Q.

is.

to.

a.

$n$.

6

v.

4.52 
1SLOWING DONN SPECTRIIM AT IK.A CM. FOK SHONKA A-15:A PLASTIC 42 MEV P + ON BE NEUTRON SPECTRUH"

\section{ENERGY}

D. 15 MEV

0.10 MEV

$0.15 \mathrm{MEV}$

0.213 MEV

B. 25 MEV

Q. 3И MEV

$0.35 \mathrm{MEV}$

0. 4 M MEV

D. 45 MF.V

0.50 MEV

$0.55 \mathrm{MEV}$

0.60 MEV

0,65 MEV

0.70 HEV

0.75 MEV

0.80 MEV

0.85 MEV

P. 90 MEV

Q.95 MEV

1. OU MEV

1.1A MEV

$1.20 \mathrm{MEV}$

$1.30 \mathrm{MEV}$

1. 4 D MEV

$1,51)$ BAEV

1,60 MEV

1.78 MEV

1 , BS MEV

1.95 MEV

2. OU MEV

2.10 MKV

2.2 MEV MEV

2. 3U MEV

2.46 MEV

2.5D MEV

2.64 MEV

$2.70 \mathrm{MEV}$

$2.80 \mathrm{MEV}$

2,90 MEV

3. NO MEV

$3.20 \mathrm{MEV}$

3.40 MEV

3,60 MEV

3,80 MEV

4.00 MEV

\section{PROTONS}

i.

2. $19 E=(1)$

1. $35 E-31$

$1,7 G E=+1$

$1.99 E-D 1$

$1.8 \cap E-i I 1$

1. SGE -13

2. $15 E-91$

$1.54 E-x^{\prime} 1$

$2.23 E-01$

2. $45 E-11$

1.47K-01

$1.94 \mathrm{E}-01$

$4.43 E-81$

2. $24 E-D 1$

$1.45 E-D 1$

2. $06 E-11$

$3.15 E-11$

1.7 $7 \mathrm{E}=\mathrm{D} 1$

1. $3 E E-111$

2. 36E-(1)

$2.34 E-1$

2. 5 ITE-DI 1

$4.05 E-b 3$

$3,24 t-v 1$

2. $57 E-01$

$2.88 E-11$

$5.35 E-111$

2. $17 E-B 1$

$2.55 E-21$

3. $12 E-61$

$2,72 E-C 1$

$5,42 E-1)$ !

$1.98 E-101$

3. $3 \cap E-13$

$3,51 E-111$

2. DIE-U1

$3,51 E-D 1$

4. $35 E-01$

$7.49 E=61$

3.68E- -1$]$

$2.56 E-D 1$

$3,26 E-61$

$4.7 A E-A 1$

S. B6E-O1
DEUTERONS

$\theta$.

1. Dot-as

$1.264=94$

$1.795=195$

$1,27 \mathrm{E}-\mathrm{W} 14$

1, iaf $-x$ क

$2.62 F-115$

1. $25 E-1) 4$

$9.12 E-04$

1. $29 E-n 3$

8 . $B 8 E-15$

$1.22 F-14$

$3,80 E-14$

$3.45 E-D S$

$1.13 E-114$

$3 . S T E-174$

$1,285=114$

$5.61 F=14$

$2,35 \mathrm{~F}-\mathrm{WS}$

3. RI [- त 4

9.76\%-ri4

2. 2 OF- 04

3. 45 E-64

$6.7 \mathrm{AF}=134$

5 , SDE=:14

6.4 IVE- 14

$5.535-24$

9. 5 LERVA

ค. 93E- 4

8. $45 E-14$

2.15t-nu

8.19E- 15

$1.33 E-63$

$4.13 E-? .4$

$6.82 F=115$

$7, B O E-614$

$1,21 E-(1) 3$

B.79E- 44

$6.73 E-65$

$1,13 E-93$

$4.75 E-174$

$1.19 E-1,3$

$6.75 \mathrm{~F}-0.4$

$3.25 E-04$

2. $55 E-04$
TRITONS

औ.

5. $2 B E-36$

$1.63 E-B 6$

r. 13 tant

$1.44 \mathrm{E}=10$

$4.95 t-616$

3. H?t -06

1. $35 t=n 4$

$5.73 E-16$

$4.6 .91-616$

$1.60 t=65$

$1.85 t-195$

$8.37 t-16$

S. S9E-vit

$2.32 t-116$

1. id $T E=$ D 5

A. 51E-

3. 5TE- 16

8.87t-176

$1.74 E-114$

$3.75 E-16$

2.22t-ids

$6.01 E-60$

2. जU $4 E-05$

$0.47 t=65$

$3,99 E-115$

1.2 2 A E- 25

7.5 AE-W'

$1.82 t=n s$

$1, \nabla, 3 t=-1.4$

1. 1 RE $=35$

$0.36 E-35$

$4.25 E-115$

$7.21+-35$

2. $) 7 k-05$

$7.09 E-95$

$1,31 E-34$

$1.50 E-114$

$1.27 E-15$

$4.90 t-d 5$

$3.65 t-135$

$4.32 E-v 5$

8. $\Delta ? E-116$

$2.40 E-155$

$1.56 E-135$
ALPHAS

$\checkmark$.

2. $411=-13$

$1.83 E-.63$

3. $35 E-03$

3. $92 t=n 3$

2. ant-as

P. $S O E-T 3$

2. 47E- 113

$3.94 E=33$

$1.79 \mathrm{E}$ - B 3

$6.70 E-14$

$0.48 E-1) 3$

$2.23 E-13$

$1.6 \mathrm{OE}-\mathrm{H} 3$

6. $92 E-103$

6. $54 E-D 3$

$3.38 E-03$

$3.15 E-A 3$

1.78E-A3

5. BUE-A3

$4.75 E-113$

$2.43 E-13$

$2,92 k-i 13$

$5.43 E=0.3$

$5.45 E-13$

$2.71 E=03$

$5.45 E=63$

D. $\triangle 4 E-B S$

4. 3UE-O3

$5,92 E-1.3$

$5.37 E=135$

$6.45 E-1.3$

$2,34 E=193$

$5,89 E-13$

$3.89 E-13.3$

$4.93 E=.13$

$4.7 G E-13$

$7.43 E=-13$

8. पIUE-J3

$3.73 E-43$

$3.27 E=63$

$5.20 E-V j$

7. 22E-13

$3.74 E=03$

$4.75 E-13$ 


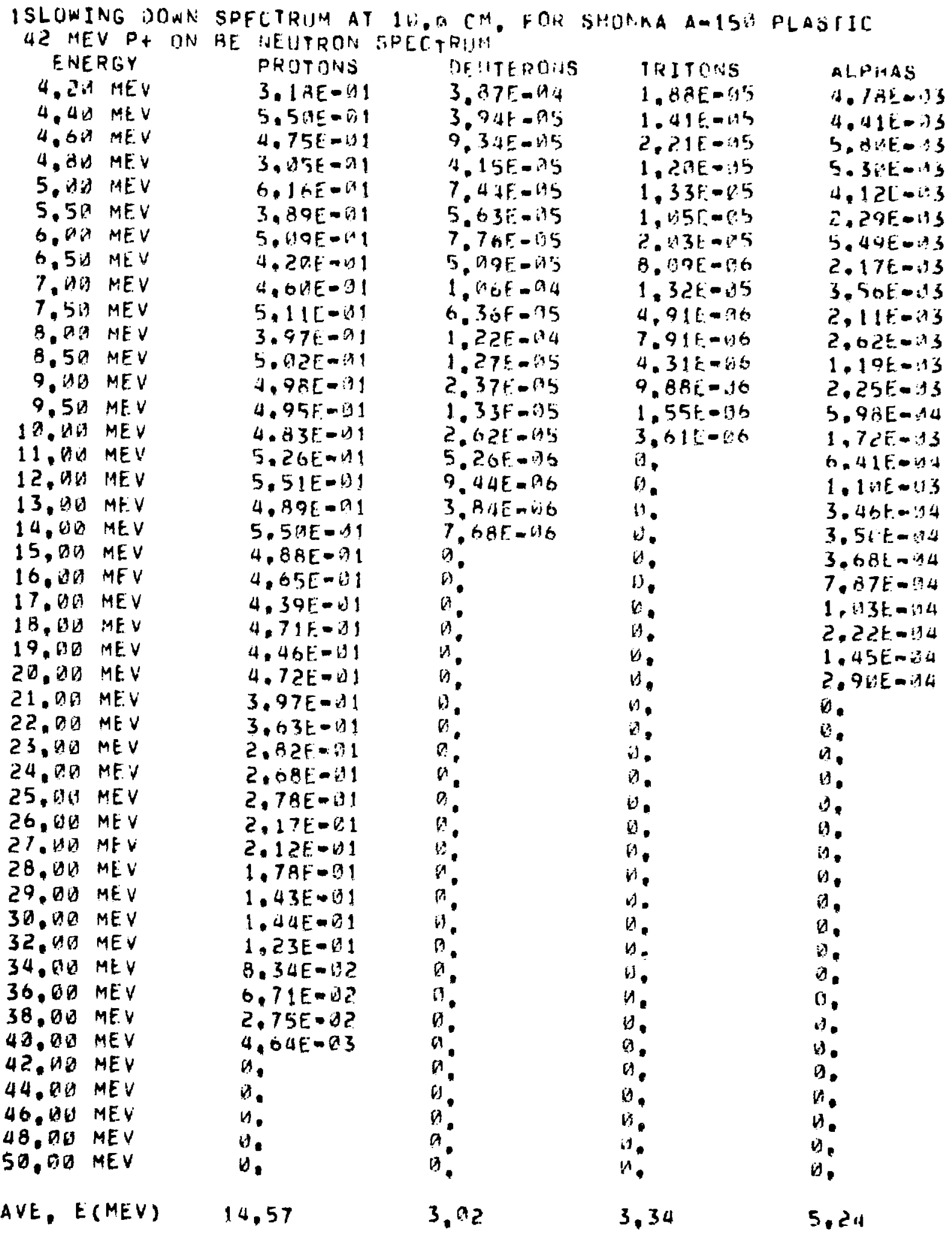


ISLONING DOWN SPECTRUM AT 14, C Ri. FOR SHLINKA A-1SU PLASTIC 42 MEV P+ ON HE NEUTRON SPECTRUM

\section{ENERG:}

0.05 MEV

D. 10 MEV

D. IS MEV

D.20 MEV

0.25 MEV

0.36 MEV

$0.35 \mathrm{MEV}$

0. 40 MEV

$0.45 \mathrm{MEV}$

$0.50 M E V$

0.55 MEV

$0.6 \mathrm{M}$ MEV

0.65 MEV

$0.7 D$ MEV

$0.75 \mathrm{MEV}$

D. BD MEV

0.85 MEV

Q.90 MEV

0.95 MEV

$1.0 Q$ MEV

1.10 MEV

1.20 MEV

1.30 MEV

1. $4 \triangle M E V$

1.5U MEV

1.68 MEV

$1.7 \$ \mathrm{MEV}$

1.8E MEV

1.9月 MEV

2. HQ MEV

2.10 MEV

2.20 MEV

2. $30 \mathrm{MEV}$

2. $40 \mathrm{MEV}$

2.50 MEV

2.6 $6 \mathrm{MEV}$

$2.70 \mathrm{MEV}$

2.81 MEV

$2.90 \mathrm{MEV}$

3. 00 MEV

3.20 MEV

3. 40 MEV

$3.60 \mathrm{MEV}$

3.80 MEV

4,DO MEV
PROTONS

$A$.

1.5BE-DI

$1.37 E-01$

1. $32 E-i 1$

$1.5 B E=B 1$

$1.49 E-D\}$

1. $26 E=1$

1. $\triangle R E-O 1$

1. $19 E-131$

$1.64 E-61$

$1.94 E-01$

1. $15 \mathrm{E}-1) 1$

1. $42 E-31$

4. $D B E-D 1$

$1.84 E-11$

$1.15 E-11$

1. 7 AE-A1

$2,32 E-41$

1. $42 E=31$

$1.44 E-\lambda 1$

$1.81 E-1$

2. $23 E-01$

1. 96E - D 1

3. $39 E-U 1$

2. $78 E-d 1$

2. $3 \Delta E-1$

2. $2 Q E-v 1$

4. $32 E-1$

$1.66 E-D 1$

1.96E-U1

2. $67 E-D !$

2. 13E-U1

4. $43 E-B 1$

$1.54 E-D 1$

2.

2.87F-v1

$1.61 E=01$

$2.98 E-d 1$

$3.60 E-01$

$0.98 E-1$

$3.12 E=U 1$

1.96E-DI

$2.54 E-D 1$

$3.97 E=01$

$3.33 E-01$
DEUTEFOWS

0.

$6.34 E=110$

3. $31 E$ abs

4. 5, F F- 15

$7.77 E-05$

$2,64 E-\$ 5$

1. $, 1 E-1,4$

4. $2 D F-135$

9.44E-A5

$1.90 \mathrm{GE}-\mathrm{D} 4$

4. $82 E-\triangle 5$

1. $72 E-B 4$

$1.96 E=04$

1. $05 E=1 A$

$9.68 E-175$

6. $75 E-105$

$1.56 E-64$

$7.49 E-A 4$

1. BOE- 35

1. $27 E-B A$

2. $36 E-04$

2. $26 E=\{4$

1. $25 E-b a$

$2.22 E=\lambda 4$

1. A6t-n't

2. $2 \times E-134$

$4,59 \mathrm{E}-\mathrm{A}$

$1,11 F=114$

1. 2hE-DL

2. $T \triangle E-\cap 4$

$8.46 E-15$

1. $64 F-09$

5. $2 J E=1+4$

$1.62 E-134$

b. $5 ! E-5$

$2.31 \mathrm{E}-\mathrm{M} 4$

3. $94 \mathrm{E}-\mathrm{B} 4$

1. $43 E-B 4$

$5.99 E-05$

5. AIE-A

1. यIF-AI

4. ISE-AH

2. $12 E-B$

2. 7 BE -04

$3.67 \mathrm{E}$ - BU
TRITONS

4.

$6 . \triangle B E=40$

$5.73 E-1.7$

$3.61 t-$. 35

$1.68 t-\mathrm{J} 6$

3. $24 E-66$

4. $49 E-116$

$9.75 E=k 6$

4. $45 E-106$

7. $\triangle 6 E-67$

1. $11 \mathrm{E}-\mathrm{U} 5$

$5.14 E-45$

$7.96 E-116$

3. $27 E-46$

B. $58 E=17$

3. $91 E-116$

$9.43 E=115$

4. $46 t-3 i b$

$9.65 E-b 0$

1. $26 E-115$

$2, W 4 E-15$

1. $24 E-15$

2. 4 IE- 15

3. $4 B E-\square 5$

2. $61 \mathrm{E}-\mathrm{JS}$

$6.33 E-05$

$3.51 E-45$

$1.96 E-45$

1. ARE -15

1. $67 E-35$

$4.39 E-Q B$

$2.73 E=05$

1. $\triangle A E=5$

7. $29 E-1 A 6$

$1.53 E-15$

$9.91 k-016$

$3.97 E-155$

4. 116E-U6

1. $71 \mathrm{EM}$ B

$4.12 E-65$

1.2AE-BS

2. $27 E-45$

1. $95 t-6 S$

$3.37 E-E^{5}$

3. $59 E-1 \mathrm{~S}$
ALPHAS

S.

1. $02 E-i 3$

1. $\triangle 1 F-13$

3. $h 1 E=13$

$3.65 E=-13$

1. $B 5 E=\square 3$

2. $58 E-14$

1. $48 E-193$

3. 1 UE-11 3

1. 1 SE $=3$

$5.08 E-114$

4. $D E-63$

1. $S 8 E=13$

$1.25 E-13$

$5.95 E-23$

$5.83 F=43$

2. 1 ot $=x^{3}$

$2,26 E=\omega 3$

$1,61 E-13$

4. $23 E-13$

3.40E-W 3

$1.93 E-d j$

2. 2.6t- 93

$4.34 t-13$

3.69E- 3

2. $2 E E-H 3$

$4.23 E=13$

$4.75 E-13$

$3,54 E-U 3$

4. $47 E-43$

$3.85 E-33$

$5.13 \mathrm{EIN} 3$

1. RAE- 43

4. $25 E-03$

2. 99E-1 3

$3,33 E-03$

4. VAE- 43

5. RUE- 13

$6.74 E-143$

$2.7 \nabla E-1.3$

$2.75 E-13$

$4.62 E-13$

$5.83 E-13$

$2.77 E-45$

$4.26 E=1.3$ 
ISLOWING DOWN SPECTRUM AT 14, G CM. FOK SHOSKA 1 -ISH PLASTIC 42 MEV P + ON BE NEUTRON SPECTRUM

\section{ENERGY}

4.2D MEV

4. 4 U. MEV

4.69 MEV

4. BO MEV

5. $\triangle$ MEV

$5.5 A$ MEV

6.00 MEV

$6.50 \mathrm{MEV}$

$7.00 \mathrm{MEV}$

$7.50 \mathrm{MEV}$

Q. AV MEV

$8.5 \square M E V$

$9.0 B M E V$

$9,5 D$ MEV

10,00 MEV

$11.00 \mathrm{MEV}$

$12,00 \mathrm{MEV}$

13,00 MEV

$14,00 \mathrm{MEV}$

15. 00 MEV

$16.00 \mathrm{MEV}$

$17.00 \mathrm{MEV}$

$18.00 \mathrm{MEV}$

$19,00 \mathrm{MEV}$

$20.00 \mathrm{MEV}$

$21.00 \mathrm{MEV}$

22. DU MEV

23. MO MEV

$24.0 D$ MEV

25. OD MEV

26. UD MEV

$27,0 B$ MEV

28, OB MEV

$29.00 \mathrm{MEV}$

$30.00 \mathrm{MEV}$

$32.00 \mathrm{MEV}$

34.00 MEV

36. OU MEV

38.40 MEV

$40 . B D O$ MEV

42. OU MEV

44. OV MEV

46. 00 MEV

48.00 MEV

$59.00 \mathrm{MEV}$
PROTONS

2.71E-H1

4.67E-UI

$3.67 E-U 1$

$2.63 E-41$

5. 4OE-kม 1

3. $12 E-W 1$

4. $\triangle T E-O 1$

$3.59 E-111$

4. $36 E-11$

4. $35 E-D I$

3. $35 E-11$

4. 2RE- 31

$4.35 E-01$

4. $113 E-, 11$

4. I5E-U1

4. $74 E-O 1$

$4.6 \Delta E-1) 1$

4. $24 E-D 1$

4. 66E - 11

4. $3 B E=B 1$

4. (A)E-DI

$4.2 B E=01$

4. $24 \mathrm{E}-\mathrm{B} 1$

3. $92 E-21$

$3.97 E-A 1$

$3,51 E-11$

3.27E-U1

$2.55 E-01$

2. $5 U E-U 1$

2. $78 E-A 1$

2.07E- 1

2. DSE- 61

$1.87 F=01$

$1.62 E-D 1$

$1.5 \Delta E-U 1$

1. $12 E-D 1$

6. TRE-B?

$4.42 .5-D 2$

1. $66 E-D 2$

$9.28 E-03$

a.

a.

a.

$v$

a.
DFUIEROPSS

$5.47 E-b^{2} 4$

3. GUE-AS

B. DTE-DS

2. ATE-115

5. POE MUS

$3.97 E-95$

5.74E- 15

2. $51 E-05$

7. I 3E-OS

$4.33 E-15$

5. DUE -15

3. 27E-AS

6. $49 E$ M D 5

$4.31 E-16$

6. A7E-116

$5.97 E=196$

1. 34E-115

a.

$a$

$a$

a

9

a

$a$

$a$

0

a.

0

0

0

0

$\theta$

i)

2

a.

a.

0

0

$b$

3.51
1P i TOHS

4. $45 E=135$

$1.2: E-65$

1.79E- 15

$8.73 E-16$

$1.13 \mathrm{t}=1 \mathrm{~d}$

$7,50 E-W 6$

1. $2 A E-35$

R. $44 E=66$

1. 3BE-?S

(1. $D 1 E-A 6$

1. $19 E-W 5$

3. $12 E-116$

$0.75 E-06$

2. $78 E-196$

$0.48 E-16$

b.

?

(?)

a.

a.

4

d.

u.

a.

u.

D.

G.

$B$

0

v.

0

a.

0

$n$

in

i)

w.

B.

iv

b.

a.

a.

4

(1)

3.89
ALP!HAS

$3.57 E-103$

3. GCE-US

4. THE-US

$4.21 E-13$

$2,90 E=$ तI 3

$1.84 E-A 3$

$4.67 E-.33$

$1.81 E-3$

3. BLEEDA 3

2. HUEDAS

2. VOE $=33$

1. UAE-A3

2. WBF- 3

6. $45 E-14$

1. $84 E-35$

6. $78 E-34$

1. $14 E-33$

$4.50 E-134$

$6.55 E-14$

2. $26 E=14$

5. 6IE- UA

a.

i.

a.

i)

4

0

a.

D.

$\theta$

4.

$\theta$

1.

$\theta$

$\theta$.

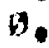

0

Q

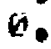

i).

a.

a.

(9)

0.

5. 31 
1SLOWING OOWN SPECTRUIA AT 9,PA CH, FOR ICRU MUSCLE. IISSUE 42 MEV P+ ON BE NEUTROA SPECTRUM

\section{ENEKGY}

0.05 MEV

D. IO MEV

$0.15 \mathrm{MEV}$

$0.20 \mathrm{MEV}$

0.25 MEV

$0,30 \mathrm{MEV}$

0.35 MEV

0,40 MEV

$0.45 \mathrm{MEV}$

0.5O MEV

$0.55 \mathrm{MEV}$

$0.6 \mathrm{MEV}$

$0.65 \mathrm{MEV}$

0,70 MEV

0.75 MEV

0.80 MEV

0.85 MEV

$0.9 D$ MEV

$0.95 \mathrm{MEV}$

1. DU MEV

1.10 MEV

1.20 MEV

1,30 MEV

1.40 MEV

1.50 MEV

$1.60 \mathrm{MEV}$

$1,70 \mathrm{MEV}$

$1.80 \mathrm{MEV}$

1.90 MEV

2. 00 MEV

2.10 MEV

2. $2 \triangle \mathrm{MEV}$

2.30 MEV

2. 40 MEV

2,50 MEV

2,60 MEV

2,70 MEV

$2,80 \mathrm{MEV}$

2,90 MEV

3. 30 MEV

3.29 MEV

3.40 MEV

3,60 MEV

3,80 MEV

4,00 MEV

\section{PROTONS}

a.

$1.99 E+D 0$

S. $S A E-6$ ?

3. SUE-61

$2.71 E+C H$

1.7 $A E-A 2$

$1.40 E+.70$

$1.87 E=31$

$1.61 E+36$

$1,34 E+Q .3$

4.7 IAE-DI

$3.55 E+06$

$2.20 E+13$

$2.61 E-\lambda 1$

$9.53 E-\lambda 1$

$4.43 E-A 3$

$5.35 E-L 1$

$5.20[-0 ?$

$7,61 F=21$

4.97E- 1

$1,35 E+A C$

6. $24 E-B) 1$

$2.25 E-U$ ?

$1,19 E+b$ ?

5. 3ZE- W 1

1. $56 E-B 1$

$1,47 E-1) 2$

4.83E-9?

$2,51 E-31$

1.3OE-D2

2. $21 E-11$

$1.03 E-03$

$5,39 E-62$

$7.46 E-B) 4$

$2,8 A E-\triangle 12$

$3,31 E-92$

$4.81 E-42$

$2,5 X E-B 2$

$9.71 E-(3) 3$

$3,34 E-1) 2$

6. A1E-DZ

$7.38 E-1) 2$

$5.27 E-22$

$6.23 E-03$

$3.83 E=32$
DE JTEKOPS

a.

$1.37 E-515$

$2.74 E-155$

$4.11 E-i 15$

$2.371-0.15$

a.

a.

$? 13 E-135$

$3.36 E-1: 4$

9.

0

$2.37 E-6.5$

2. $13 \mathrm{E}-195$

0.

a

B.

$4.54 t=95$

$3.36 \mathrm{E}-134$

a.

9.

$2.25 t=0.5$

2. $25 E-85$

1.27E-RS

$2.25 E-175$

$1.14 E-114$

$3.32 E-155$

$3.321=-115$

$6.64 E-05$

2. $37 E-45$

$4.74 E-175$

0.

9.

(1)

$\theta$

6.

$9.165 . .95$

0.

9.

9.

v.

a.

$4.53 E-15$

0 .

0.

$4.585=05$
TRITONS

i.

4.

$6.19 E=05$

b.

$1.17 E-x^{2}$

$\theta$.

$0.19 t-145$

$1.32 E=-4$

$1.79 E-B 4$

$1.86 E-34$

0 .

$5.53 E-15$

a.

$5.53 t=25$

6.

$5.53 E-2,5$

0.

$5,53 E=65$

0.

$5.53 E-115$

$5,53 E-6 i 5$

$1,38 E-B 4$

0.

B.

0.

,

$\theta$

$\theta$

$\theta$

$b$.

0.

$3.51 E-65$

a.

0

$\theta$

$\theta$.

$\theta$

it.

$\Delta$

ט.

$1.75 E-105$

Q.

$\theta$

$\theta$.

$1.75 E-.55$
ALPHAS

$\theta$.

$1.64 E-.13$

2. 3SE-A3

$4.91 E-\lambda 3$

1. UIE-A 3

$2.98 t-23$

$1,8 Z E-63$

$3 . U 4 E-33$

1. 7VEA:I3

$2.66 F-03$

$1.33 E-43$

3.7 bE $-1 \mathrm{AB}$

$1.67 E_{-}-\triangle 3$

$1.05 E-1 ! 3$

$1.67 E-193$

$4.18 E-33$

$1.72 E-$ it 3

$2,78 E-1 \times 3$

1. $\triangle 4 E-13$

$2,63 E=\sqrt{3}$

$2,48 E=\square 3$

2. $D 4 E=13$

$1.02 E-D 3$

B. $31 \mathrm{E}=114$

$2.16 E-D 3$

$1.54 c-1.3$

$7.58 E-04$

$1.03 E-33$

$3.02 E-03$

$1,05 E-13$

5. $13 E-34$

2.79E-B 3

$8.43 E-B 4$

$3.93 E-33$

$1.81 E-D 4$

$2.48 E-\Delta 3$

$1 . D B E-33$

$1.42 E-03$

$1,23 E-03$

2. $46 E-D 3$

$1.66 E-83$

$3.86 E-93$

$1.98 E=03$

$3.27 E-93$

$1.96 E-514$ 


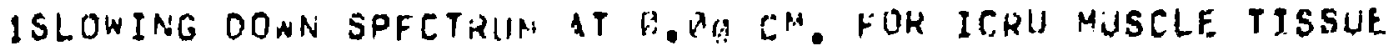
42 MEV P+ ON BE REUTTRON SPECTPLIA

\section{ENERGY}

4.21 MEV

4. 4 M MEV

4,61 MEV

4.8 M MEV

5. WO MEV

$5,50 \mathrm{MEV}$

$6.919 \mathrm{MEV}$

6,50 MEV

7, QO MEV

7.50 MEV

8.

$8.58 \mathrm{MEV}$

$9, E$ MEV

$9.5 \% \mathrm{MEV}$

10.01 MEV

11 . DU MEV

12, DA MEV

13, U.S MEV

$14.09 \mathrm{MEV}$

15. NO MEV

$16.00 \mathrm{MEV}$

17.00 MEV

18.AS MEV

19.019 MEV

$20.00 \mathrm{MEV}$

21.0D MEV

22.00 MEV

$23.00 \mathrm{MEV}$

24, DD MEV

25. OD MEV

$26.01 \mathrm{MEV}$

$27.0 \mathrm{MEV}$

28.03 MEV

29, DO MEV

$30.00 \mathrm{MEV}$

32.00 MEV

$34.00 \mathrm{MEV}$

36,00 MEV

$38.00 \mathrm{MEV}$

40.0D MEV

42.DQ MEV

44. BD MEV

46.00 MEV

48. TO MEV

$50.00 \mathrm{MEV}$
DR门TONS

2. $195-12$

$1,26 E-32$

3. 4 AE- IT?

2. $42 E-B$ ?

3. 4 GE- $\rightarrow$ ?

$5.32 E-: 12$ ?

$3.88 \mathrm{H}=32$

$4.45 E-144$

$3,22 E-B / 4$

$5,03 E-134$

$2,63 E-1) 4$

3. S6E $=14$

3.56E- I 4

5. $27 E-O A$

$3.56 E-04$

$6.2 A E-B A$

$1.41 E-$ is 3

1.39E-A4

$3,72 E-B 4$

$\theta$.

is.

a.

0.

0

a.

$\because$.

$\theta$.

B.

$n$.

u.

0.

n.

D.

n.

$\theta$

b.

a.

4.

$\theta$

0.

0.

1,08
DEUTFEOHS

H

$4:$

$4.5 B E=-15$

.

$4.58 t-015$

1. B $3[-05$

3.67E-0.5

1. $33 E-2.5$

5. TeF-AS

$5.54 E-05$

1.28E- -94

b.

$a$

0

$a$

$a$

n.

6

9

a

a

$a$

a

D

a

9

a

0

0

0

0

0.

:

0.

a.

:

4.75
TR ITOI:S

G.

19.

1. $75 t-45$

b.

.

7. 1) $1 E-? h$

$7.81 t-1.6$

1. $4 C E-125$

7. WIE-WB

$1.4 B E-115$

1. $4 B^{4} E-615$

2.10E-AS

$4.91 E-U 5$

a.

a.

n.

is,

b.

n.

.

$\theta$

a.

U.

,

D.

D.

9.

0.

(3)

$\theta$.

a.

is.

$\Delta$.

9

$\Delta$

0

$\Delta$.

*.

n.

b.

(3)

U.

0.

G.

b.

4.02
ALPHÁS

1. BgE-x4

$1.56 E-\triangle 4$

$1.71[-.14$

1. $2 J E-64$

$1.34 E-64$

$1.1 S E=A 4$

2.77F-d U

$4,37 E-15$

$6.15 E-15$

$1,28 \mathrm{E}=1.45$

2., (17) - -15

b.

$6.63 E-1 h$

$6.83 E-T 6$

$6.83 E-136$

$6.83 E=1 \%$

6. $83 E=16$

1. $37 E-A 5$

$2.73 E-45$

th.

a.

a.

a.

a.

$u$.

$a$

a.

a.

a.

b.

n.

$\theta$

0.

D.

$\theta$

0.

ค.

$\theta$

u.

$\emptyset$.

0.

a.

D.

$b_{\text {. }}$

a!

2.23 
ISLOWING DOWN SPECTRUM AT D. CON. FOA ICRU MUSCLE TISSUE AZ MEV P+ ON BE NEITRON SPERTRUHA

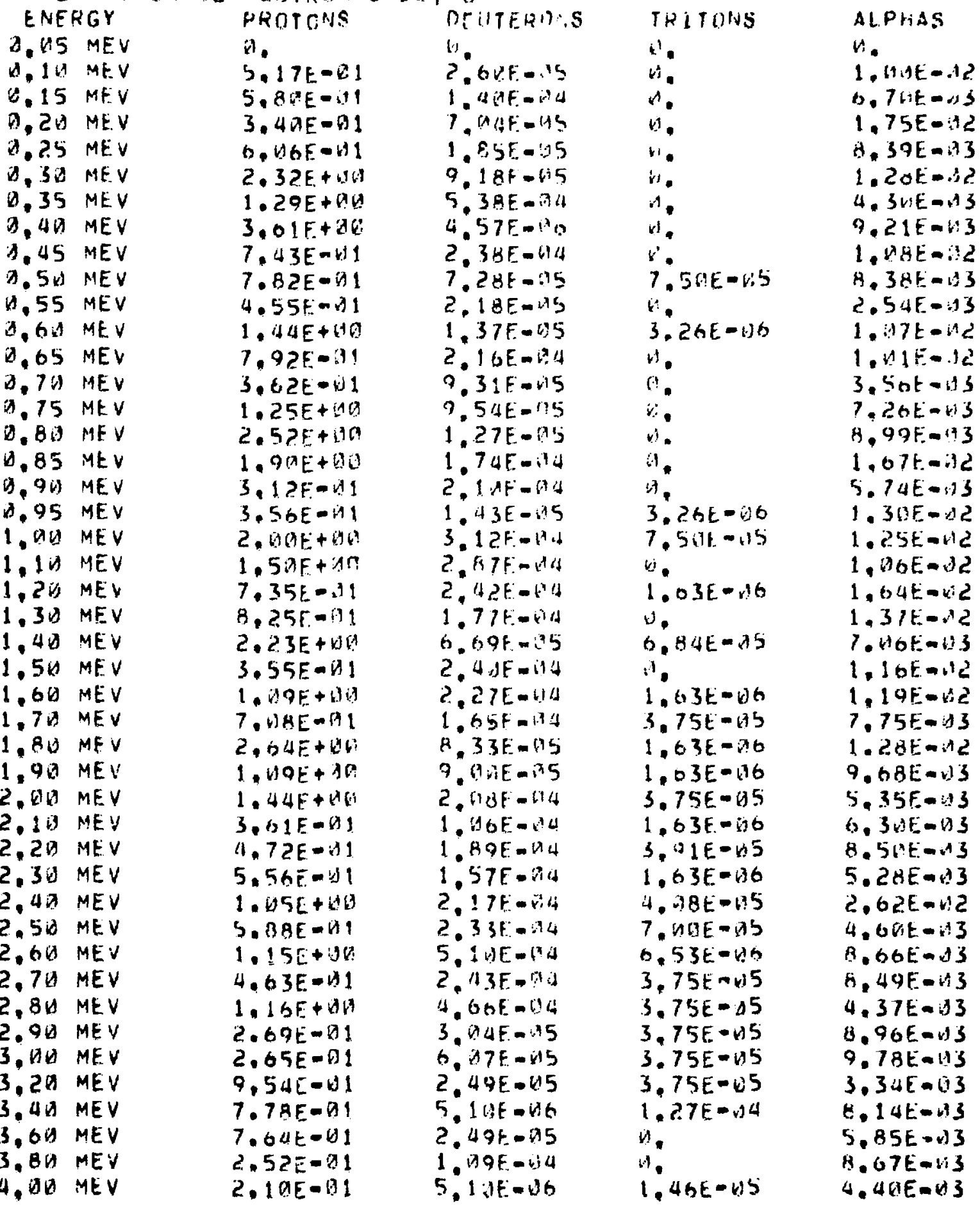


ISLOHING DOWN SPECTRIIA AT H, US CM. FOR ICRLI MUSCLE IISSUE 42 MEV P+ ON BE NEUITRDIN SPECIRUM

\section{E.NERGY}

4.29 MEV

4. 4 MEV MEV

$4,00 \mathrm{MEV}$

4.86) MEV

5.01) MEV

5,50 MEV

6,60 MEV

6.5 5 MEV

7. $\triangle 6$ MEV MEV

7.5A MEV

8. OO MEV

8.5 5 MEV

9. $\triangle O$ MEV

$9,5 \pi$ MEV

18. QS PEV

$11.00 \mathrm{MEV}$

12. (HD MEV

13.0U MEV

14.00 MEV

$15.00 \mathrm{MEV}$

16. DO MEV

17. $U$ U MEV

18. प0 MEV

19,00 MEV

20.01 MEV

$21.00 \mathrm{MEV}$

$22.90 \mathrm{MEV}$

$23.00 \mathrm{MEV}$

24. $015 \mathrm{MEV}$

$25.00 \mathrm{MEV}$

$26.00 \mathrm{MEV}$

$27.00 \mathrm{MEV}$

28. $\triangle \triangle ~ M E V$

$29.00 \mathrm{MEV}$

30. NO MEV

$32.9 G \mathrm{MEV}$

34. DO MEV

$36.00 \mathrm{MEV}$

$36, D D$ MEV

40. DO MEV

42.DQ MEV

44, DO MEV

46, DO MEV

48,00 MEV

$50.00 \mathrm{MEV}$

DAVE, E(MEV)
PRIITONS

$7.33 E-B)$

3. $[: 3 E-1]$

$7.23 E-11$

$2.39 E-A 1$

$6.47 E-(1)$

3. $96 E-11$

$3.06 E-11$

$3,12 E-B 1$

$2.7 D E-W 1$

$9.65 E-32$

1. 86E- 91

8. $\triangle U E-\triangle 5$

$1.79 E-\Delta D 2$

1. $48 E-112$

v.

$5.17 E-B 2$

1. $99 E-\triangle 2$

$4.5 \cap E-12$

$2,75 E-\Delta 2$

$9.1 B E-\triangle 2$

$\theta$.

3. $12 E-62$

$1.16 E-B 2$

t.

$6.54 E-D 2$

a.

n.

$n$.

n.

$\theta$

$2.02 E-J Z$

0.

$3.95 E-02$

$\theta$.

$\theta$.

,

6.

a.

9.

u.

b.

$\Delta$

n.

a.

4.

3.89
DEUTERONS

7. 2GE-WIS

2. $49 E-195$

1. IUE-JU

2. $49 E=.15$

3. ARE-US

8. $44 E-15$

$1.36 \mathrm{~F}-\mathrm{i} 14$

3. $57 \mathrm{E}-15$

$9.23 E-1.5$

$9.23 E-05$

1. 34E-n4

$1.89 E=.15$

1. RQE- 35

1. RQE-DT

$1.89 E-195$

$3,77 E-$ त 5

8. $49 E-05$

0.

$1.68 E-45$

0

i.

a)

0

a

a.

a.

9

a.

a.

a.

1.

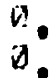

:

:

a.

$a$

i.

is.

9.

$\theta$.

4.74
TRITONS

11

$1.46 E-Q S$

is.

kं.

$1.46 E-15$

$5 . B 4 L-36$

$1.17 E-45$

$1.17 E-$ * 5

$1.17 t=55$

$1.75 E-195$

$4.09 E-65$

.

H.

0.

t.

!!

$n$

$\forall$.

0 .

$v$

n.

a,

i.

$n$

b)

a.

$\because$.

b.

a.

.

$v$.

4.

in.

$v$.

a.

i.

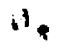

$\therefore$

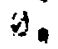

$\theta$

6.

ii:

$H$

0.

4,14
ALPHAS

2. II E E 3

1. 32E- 22

$0.29 E-1) 3$

$4.3 E-(13$

6. $52 E-D 3$

$4.94 E-113$

1. 3? E-ML

$4.47 E_{-113}$

H. ST.E-US

$3.93 E-A 3$

8. 25t-L's

$0.46 E-n 3$

$1.51 E-12$

$3.465=33$

?. $38 E-I 3$

3.07E-U 3

7. $40 t-k] 3$

$3.52 E-143$

B. $22 t-L^{3} 3$

2. $17 E=N 5$

$3.25 E-.45$

2. 28E- 15

$6,158 E-15$

o.

i.

a.

a.

n.

$\theta$.

a.

4

n.

n.

i.

m.

$\theta$

$a$

9

$\Delta$.

$\because$

0.

in.

is:

a.

$b, 37$ 
ISLOWING DOWN SPECTPIIM AT H. In CP. FUF ICRU MUSCLE TISSUE 42 HEV P+ ON BF NEUTRON SPECTRIIP

\section{ENERGY}

D. 15 MEV

Q. 11 MEV

D. 15 MEV

D.2W MEV

0.25 MEV

H. 32 MEV

0.35 MEV

i). 40 MEV

0.45 ME.V

0.50 MEV

0.55 MEV

$0.6 B$ MEV

0.05 MEV

$0.7 \triangle \mathrm{MEV}$

0.75 MEV

0. 8П MEV

B. 85 MEV

0.90 MEV

0.95 MEV

1.DD MEV

1.10 MEV

1.2U MEV

$1.36 \mathrm{MEV}$

$1 . \triangle 0 \mathrm{MEV}$

1.50 MEV

1.6U MEV

1.70 MEV

1. BU MEV

$1.90 \mathrm{MEV}$

?. $\triangle O$ MEV

2.1U MEV

2.2』MEV

$2.30 \mathrm{MEV}$

2. $40 \mathrm{MEV}$

$2.50 \mathrm{MEV}$

2.60 MEV

$2.70 \mathrm{MEV}$

$2.80 \mathrm{MEV}$

2.90 MEV

3.OU MEV

3.20 MEV

3. 40 MEV

3,60 MEV

3.80 MEV

4.60 MEV
PRGTONS

4.

5. $39 t-191$

1. $4 E+H A$

B. $85 \mathrm{E}=\mathrm{x}^{3}$ ?

4. $4(E-))$

5. $A M E-1$

$6.51 E-$ तो

7. $24 E-101$

4. $11 E+w i$

5. A3E-II

7. 1 at-d 1

5.94[-21

$8.32 t-v 1$

1. 2 UTE + 16

9. BOE-WI

$3.79 E+19$

$1.35 E+C A$

$1.976+2)$

1. $335+36$

8. BOF-DI

1. $25 E+2 E$

$8,33 E=01$

7. B8E- औI

4. $17 E-1$

1. ITE +4

$5.33 F-\omega 1$

2. $14 E-01$

$6.77 E-191$

1. $31 \mathrm{E}+\mathrm{BW}$

6.911E-111

1. $? \mathrm{BF}+\mathrm{BU}$

$3.03 E-19 i$

$6, B 2 E-D 1$

4. उSE-:II

$1.73 E+18 \mathrm{G}$

1. $4 ? E+B ?$

8.34E-U!

$1.61 E+4 A$

6. 16GE-J1

Q.3 $9 E-d 1$

$4.83 E-81$

$1.16 E+B O$

$3,43 E-\partial I$

$0.54 E-0 I$

2. $91 E-D 1$
DEUTEROISS

b.

क. $221-65$

2. मOE $A 4$

1. $57 \mathrm{E}-:=4$

4. 29E-Hid

$1.27 \mathrm{E}-\mathrm{V}$ i

4. $31 E-1^{2} 5$

$7.36 F=115$

2. ?At-1AG

$1.9 B E-34$

1. $83 \mathrm{BH}-\mathrm{HA}$

3.61E-14

4. $12 E=$ is 4

2. RQE

9.63E- 15

2. T1E-GI

4.3QE-A4

1. RGE- MA

$7.93 E=05$

5. 2 ZE-OA

2. $995-04$

?. $73 E-i^{7} 4$

2.7 $8 E=A 4$

5. प? $E-r^{\prime} 4$

3. $31 \mathrm{E}-\mathrm{A} 4$

4. BTE-W4

2. $13 E-A 4$

3. $16 E=D 4$

2. $56 E-04$

5. $07 E-34$

$5.99 E$ MAS

3. $565-45$

5. .9E- 65

1. $45 E-A 4$

1. 2 . $D-14$

2. $05 \mathrm{~F}-\mathrm{MA} \mathrm{A}$

2.57E-R5

2. $57 E-115$

2. $157 \mathrm{E}-05$

2. $57 \mathrm{~F}=0 \mathrm{US}$

2. $57 E-115$

$7.72 E-15$

.

0

i.
TRITOHS

iv.

(1)

1. $21 E-n s$

4.

1. $21 E-45$

b.

1. $21 E-75$

3. $39 E-114$

2. प? E-115

3. $03 E-W S$

.

V.

$3.27 E-.14$

i.

i.

6

$\theta$

14.

$9.16 E-05$

A.

B.

bi.

a.

b)

$n$

$4.5 B E-.15$

0.

0.

a.

$4.5 B E-M 5$

b.

t.

U.

$4,58 E-05$

k.

4.

4. 5RE $=175$

$b$.

b.

4.58E- 55

2. 2OE-E 5

2. $29 E-05$

2. $29 E-185$

2. 29E-k5

2. $20 \mathrm{E}-415$
ALPHAS

:3.

$9.51 E-i 13$

9. $3 U E-t 1 S$

$1.64 t-142$

$4.88 E-L^{7} 3$

1. $29 E-42$

8. $01 \mathrm{Em} 3$

1. 19E-112

1. $59 E-12$

Q. 09E-US

4. $55 E-k 3$

1. $70 E-1 ; 2$

1. $01 E-B i$

3. $17 \mathrm{E}=\therefore 3$

$5.32 E-15$

$9.57 \mathrm{E}-3$

$1.27 E-112$

$5.24 E-63$

1. WRE-12

8, 62E-O 3

$1.34 E=112$

2. $10 E-12$

7. $.9 E-U 3$

3. $48 E=: 13$

1. $29 E=V^{3}{ }^{2}$

B. $13 \mathrm{E}=13$

$2.35 E-12$

1. $+3 E-82$

$1.22 E-12$

5. $76 E=U 3$

$3.37 E-.13$

$9.73 E-\bar{Z} 3$

4. $48 E=, 13$

1. $15 E-+12$

5. $71 E-33$

$1.37 E=: 2$

8. $90 E-H 3$

B. $31 E-\cap 5$

1. $11 E-12$

1. $54 E-62$

3. $55 E=k 3$

5.7 OE - l? $^{3}$

1. $16 E-112$

1. $11 E-i s 2$

$4.38 E-\& 3$ 
1SLOKING DONN SPECTRIIM AT R.19 CM. FOH 1CHU MUSCLE 11SSUE 42 MEV P+ ON BE ISU UIROU SPECTRUIA

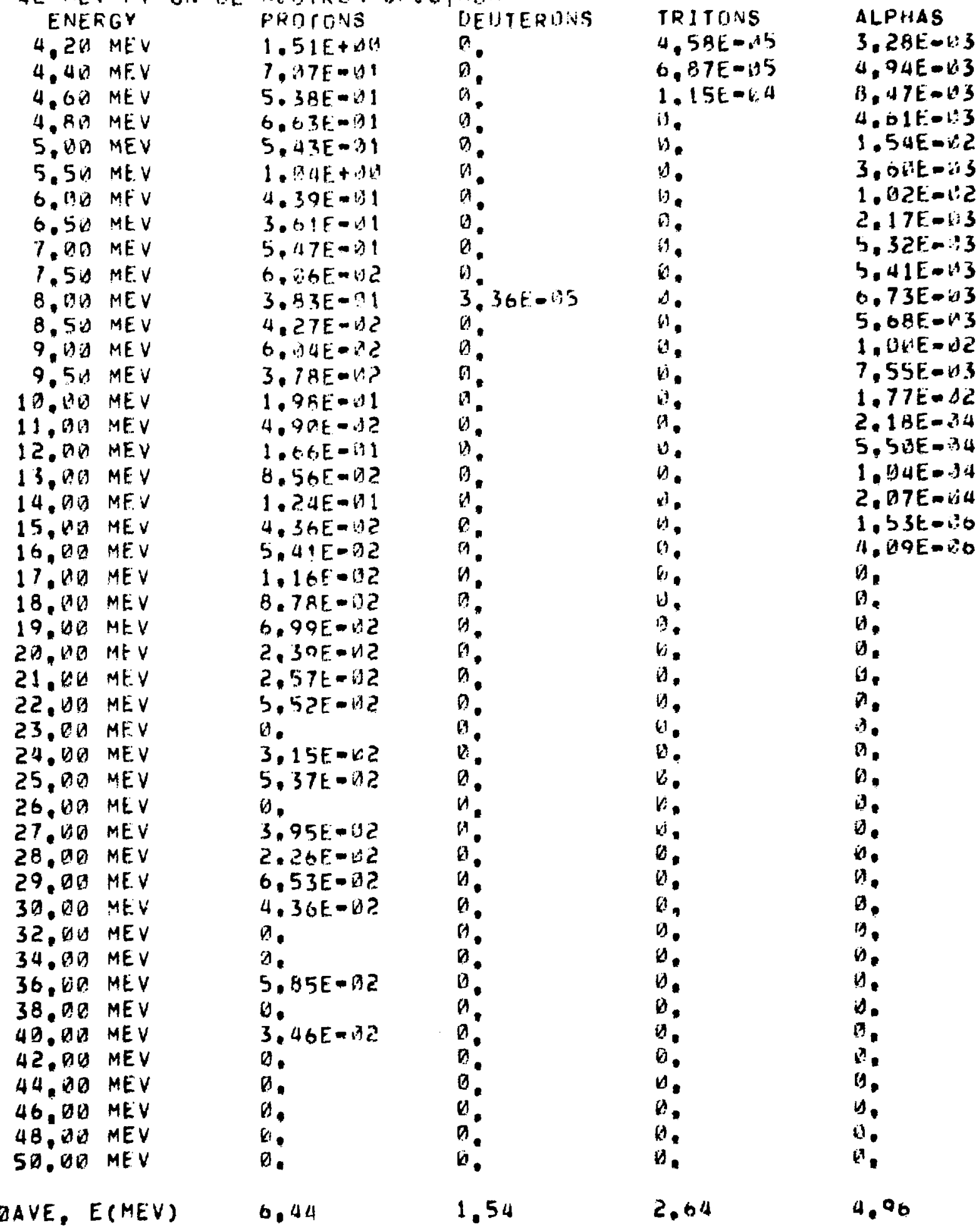


1SLONING DOWN SPECTRIIM AT W. IS C CA. FOH ICRLI MUSCLE IISSUF 42 MEV P+ ON HE NEIITRON SPECTRUMA

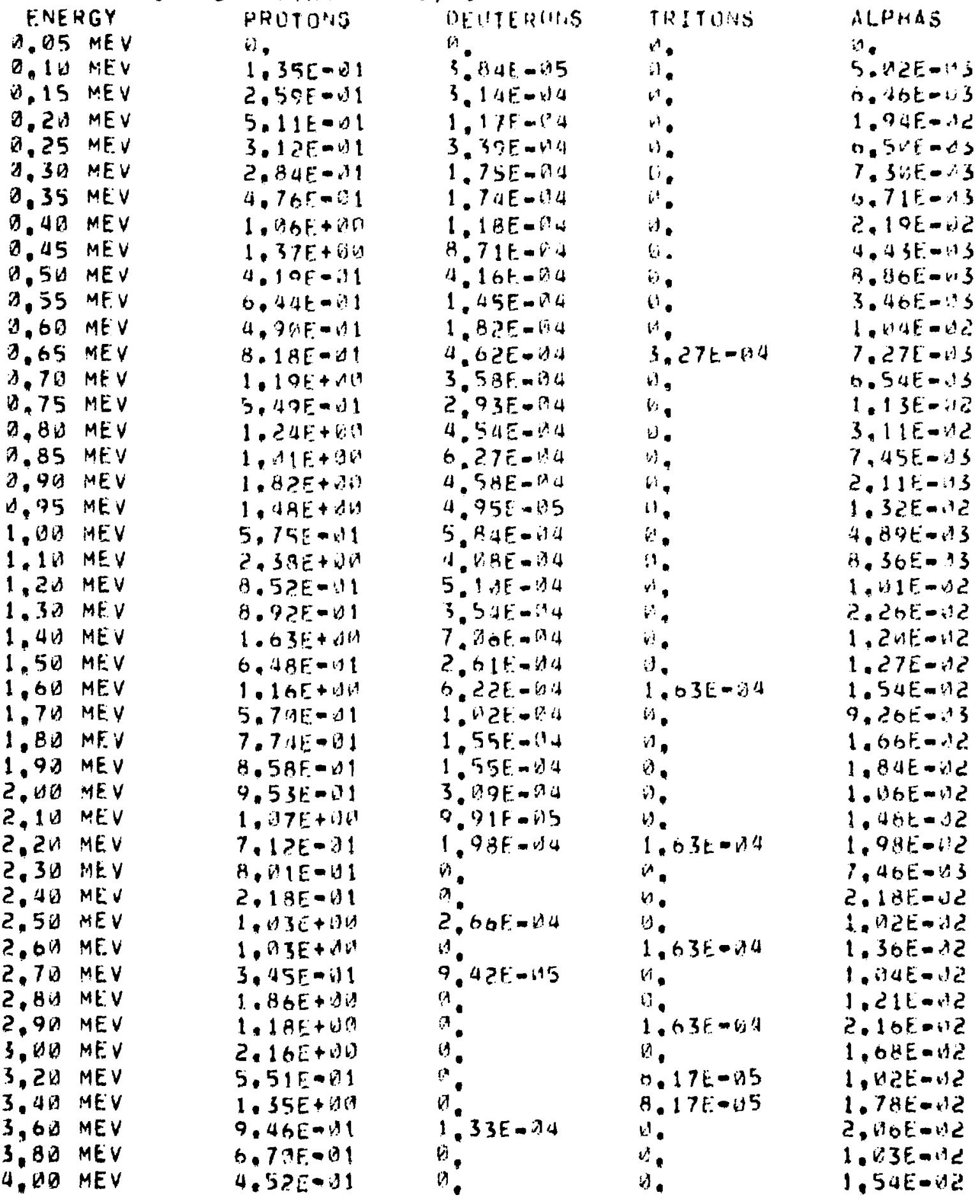


ISLOWING DOWN SPECTRUM AT 1.15 CM. FOR ICRU MUSCLE IISSUE 42 MEV P+ ON BE NEUTRON SPECTRIJM

\section{ENERGY}

4.20 MEV

4. 40 MEV

$4,60 \mathrm{MEV}$

4,8. MEV

$5,00 \mathrm{MEV}$

5,5B MEV

$6 . B 0 \mathrm{MEV}$

$6.50 \mathrm{MEV}$

7.Oด MEV

$7,50 \mathrm{MEV}$

B.DQ MEV

$8.5 \mathrm{MEV}$

$9.01 \mathrm{MEV}$

9,5 MEV

1D. $D$ MEV

11.00 MEY

$12.00 \mathrm{MEV}$

$13,00 \mathrm{MEV}$

$14.00 \mathrm{MEV}$

$15,0 \mathrm{O} M E V$

16, OQ MEV

17,01 MEV

18, Q0 MEV

$19.08 \mathrm{MEV}$

20. HO MEV

21.DO MEV

$22,00 \mathrm{MEV}$

23,00 MEV

24. OS MEV

25, OU MEV

$26.00 \mathrm{MEV}$

$27.00 \mathrm{MEV}$

28, DO MEV

29,00 MEV

$30.00 \mathrm{MEV}$

32. ON MEV

34. DD MEV

$36.00 \mathrm{MEV}$

38, OO MEV

$40 . U D$ N.E.V

42. DQ MEV

44,00 MEV

46.010 MEV

48. 00 MEV

50. HO MEV
PROTONS

$1.19 E+B D$

$5.83 E-J 1$

$1.39 E+B C$

$1.26 E+17$

$2.51 E-41$

$4.73 E-D 1$

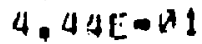

$4, D 3 E-D 1$

4. DSE - DI

$1.71 E-B !$

$1.8 \cup E=D 1$

I. $\angle G E-A 1$

$1.19 F=1$

2. 6 AE- DI

9. $\triangle 19 E-B 2$

$3.56 E-B 1$

1, GBE- 02

$1,11 E-\Delta 1$

3. $8 ? E-D ?$

$5.69 E-d ?$

$8.78 E-42$

$5,01 E-0 ?$

$4,89 F-172$

$4.00=-82$

$2.57 E-112$

8.10E-D?

a.

3. 15E- 122

$5,37 E-\| 2$

9.

$3,95 E-U 2$

$2,26 E=4$ ?

6. $53 E-B$ ?

4. 36E- 11 ?

a.

$\theta$

$5.34 E=0 ?$

1. $17 E-D 1$

$\Delta$,

3. $46 E=12$ ?

9.

0.

$\theta$.

0.

$\theta$.

7.28
DEUTEROIS

$4.71 E-155$

$1.3 .3 E=04$

0.

0

$1.33 E-B 4$

$7.21 E-95$

$7.21 E=0.5$

$1.9 D E-6: 4$

7. 21E-15

$1.8 H E-25$

$1.88 E-65$

$1.8 B E-(1) 5$

1. 9AE-AL

1. $8 B E-145$

$1.88 F-05$

$3.77 E-05$

$8.48 E-115$

2.06E-05

a.

ए.

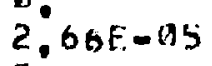

$a$

?.

a.

a.

9

0

3

a.

b.

6

a.

$\theta$.

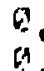

is

a.

:

$\therefore$

a.

a.

3.911
TRIT ONS

$\Delta$.

$n$

i.

$\therefore$

$n$

$\therefore$

$n$

n.

$n$

i)

i.

3

$n$

is.

in.

$H$

$1.63 E-45$

i.

i).

$\mathrm{Hi}^{\circ}$

i.

vi.

is.

$\theta$

$\theta$

$\theta$

$a$

$n$.

v.

.

.

G.

0

u.

U.

.

a.

a.

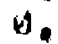

a.

.

n.

$\because$

a.

3.45
ALPTIAS

6.75E-:33

$1.43 E-112$

$1.5 \Delta E-H$ ?

$1.0 T E-32$

$9,23 E-v 3$

$1, \pi 2 E-32$

$1,94 \mathrm{H}-4,2$

$1.12 E-n 2$

$1.26 E-i 22$

$5.31 t-43$

$7,66 E-V 3$

$5.00 E-3$

$1.37 \mathrm{E}-\mathrm{WI}$

2. $25 E-133$

2. $91 \mathrm{E}-\mathrm{W} 3$

$2.15 E-63$

2.7 $4 E-193$

$3,75 E-k 13$

$7.49 E-143$

$1.53 E-V 0$

4. (B) $9 E-1) 6$

a.

a.

b.

v.

a.

$b$.

i.

a.

i.

1.

1).

i.

n.

19.

$\therefore$

is.

i.

D.

i.

n.

is.

i.

a.

i)

5.46 
ISLUWING DOWN SPECTRIIA AT U. 2R CM. FOR ICRU MUSCLE TISSUE 42 MEV P+ ON B3E PEUTROAN SPERTR JH

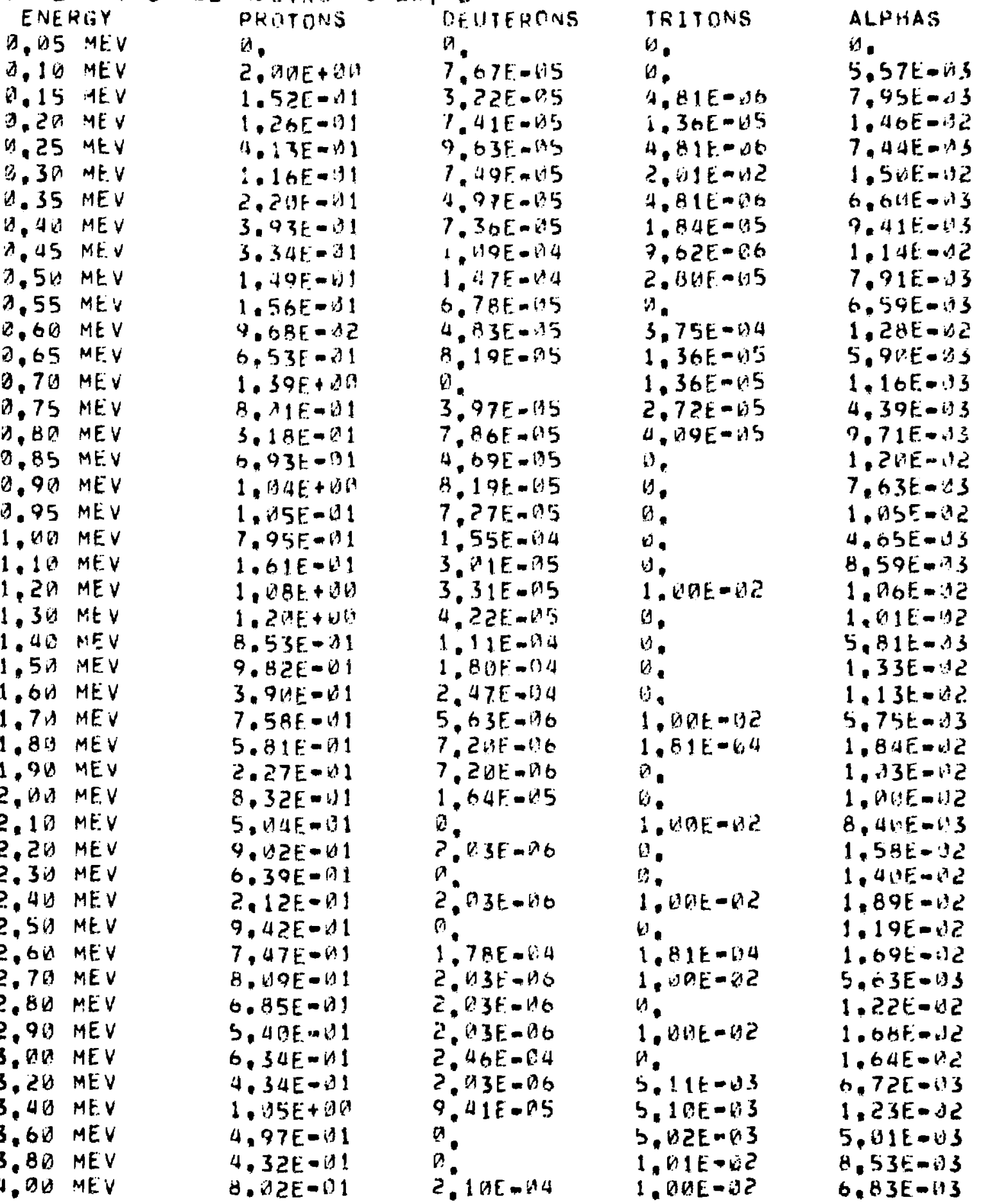


1SLOWING DOWN SPECTRIIM AT G. ZU CM. FOR ICQU MUSCLE IISSUE 42 MEV P+ ON BE NEUTRON SPEC.TFLI!

$$
\text { ENERGY }
$$

4,2B MEV

4. 40 MEV

4.6D MEV

4.8G MEV

5. 0.3 MEV

$5,5, M E V$

6. 0 Q MEV

6. 5 M MEV

7. 10 MEV

$7.50 \mathrm{MEV}$

8. $D$. NEV

8.5O MEV

9. MU MEV

9.56 MEV

10. AO MEV

11. DO MEV

12. $\triangle B$ MEV

13. OQ MEV

$14.00 \mathrm{MEV}$

15. DO MEV

$16, D G M E V$

17. OU MEV

18. OO MEV

19.0D MEV

$20,00 M E V$

21. DU MEV

2.2. OO MEV

23, MEV

24. $\triangle D$ MEV

25, 00 MEV

$26.00 M E V$

27. $O U$ MEV

28. OU MEV

29.DO MEV

37. (7) MEV

32.00 MEV

$34,00 M E V$

36,10 MEV

$38.00 \mathrm{MEV}$

40. $90 \mathrm{MEV}$

42.00 MEV

$44.00 \mathrm{MEV}$

$46,00 \mathrm{MEV}$

48.00 MEV

$50.00 \mathrm{MEV}$

DAVE, E(MEV)
PROTONS

$7.94 E-U 1$

$6.12 E-11$

$5.13 E-11$

$1.19 E+190$

3. $99 E-i 1$

$9.75 E-11$

5.7 ? E -1

7. $42 E-31$

$6,19 E-61$

$9.75 E-31$

$9.735-131$

7. $49 E-01$

9. $25 E-11$

$7.13 E-111$

8. 18E-1 1

$9.36 t-01$

8. $196 \mathrm{~F}-01$

$7.17 \mathrm{~F}-11$

4.89E- 1

$2.37 E-B 1$

$3.90 E-32$

$7.52 E=02$

$4.78 E-18$

$5.52 E=4 ?$

$2.57 E-12$

$3.15 E-02$

2. DZE-D2

$3.34 E-12$

3. $95 E-x^{3} 2$

1.

$9.34 E-42$

$1.13 E-D 1$

4. 36E-U2

d.

o.

$\theta$

1. $12 E-01$

1. $46 E-2.1$

D.

3.46E-102

i).

D.

0

i.

i.

9.59
DEUTEROLS

a

17

$8.81 \mathrm{f}-45$

1. 22E-V 4

B. 8if-ns

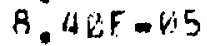

1. $19 E-114$

8. ACE- IS

2. DBF- II

$1.54 \mathrm{~F}-11 \mathrm{a}$

3. 4 AE -14

4.87F-bS

$9.75 E-195$

1. $46 \mathrm{~F}=64$

$3.41 F-134$

a.

Q

$2.66 P-35$

4

0

0

b.

a.

0

8

0

a.

$a$

0

0

a.

$b$

$b$

D

a.

B.

6

$n$

6.55
TRITDNS

2. 52E- 12 ?

$\because$

9. $35-195$

$\therefore$

9. $3 t-15$

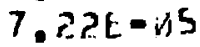

$7.22 \mathrm{~F}-\mathrm{BS}$

1. DBE - ति

$2,89 E-U 4$

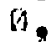

is.

W.

is.

b.

b.

n.

$\because$

i.

B.

is.

.

a.

i).

i.

t.

v.

$\theta$.

$B$.

$B$,

$B$.

$B$

$\theta$

$\theta$.

$\theta$

0.

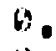

i.

b.

vi.

i.

0

b.

i.

0.

3.13
ALPHAS

7.2AEDIJ 3

6. $26 t=1.3$

1. $U 7 E-i)$ ?

2. BVE- 3

$3.84 E-15$

$2,67 E-33$

$0.44 F-13$

9. 1 WE $=$ W

$1,33 E-43$

1. DUE- 13

$1.56 E=13$

1. $\{R E-x) 3$

2. $44 E-B 3$

$3,57 F=k 14$

1. $B 2 E-03$

1. $99 E-14$

$5,6 U E-044$

$2.96 E-i 5$

$5.64 E-35$

$8,51 E-36$

2. 27E- 65

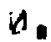

a.

a.

A.

0

i)

b.

1.

A.

i.

i.

$\Delta$.

$\theta$.

is.

9.

a.

$b$

b.

a.

i.

$\theta$

y.

0.

$v$.

3.25 
ISLOWING DOWN SPECTRUM AT G.4G CM. FOR ITRU MUSCLE IISSH. 42 MEV P+ DN HE NEUTRON SPECTRUM

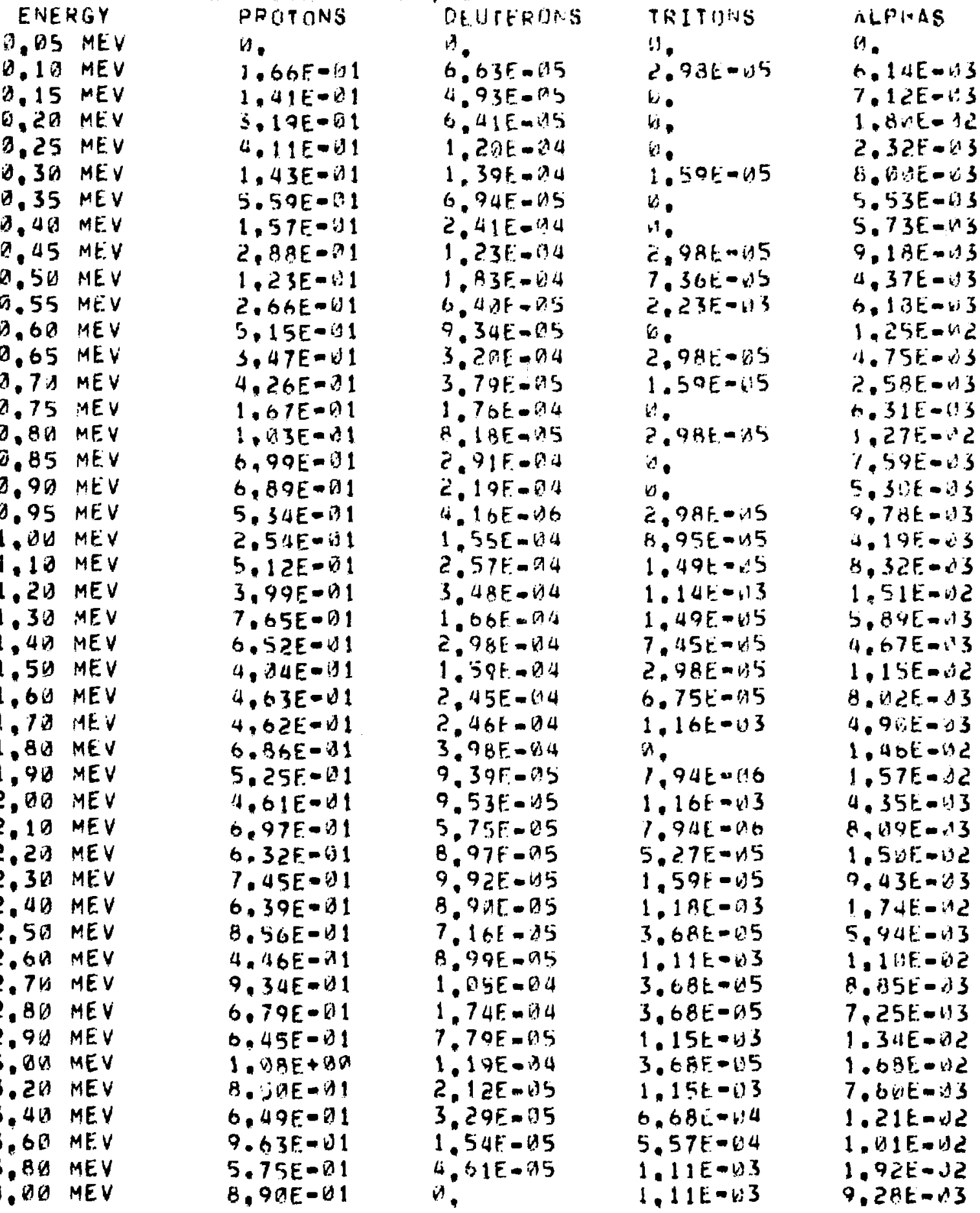


ISLOWING DONN SPECTRUM AT H.AR CM. FOR IERU MIJSCLE IISSUE प2 MEV P \& ON HE NEUTRON SPECTRIIH

\section{ENERGY}

4.20 MEV

4. 4 MEV

4.6U MEV

4.80 MEV

5. 00 MEV

$5,50 \mathrm{MEV}$

6.00 MEV

6.50 MEV

$7.00 \mathrm{MEV}$

7.50 MEV

8. (A) MEV

8.50 MEV

9. 00 MEV

$9.50 \mathrm{MEV}$

$10.00 \mathrm{MEV}$

$11,00 \mathrm{MEV}$

12,00 MEV

13. UO MEV

14.00 MEV

$15.00 \mathrm{MEV}$

$16.00 \mathrm{MEV}$

$17.00 \mathrm{MEV}$

18.00 MEV

19.010 MEV

$20.90 \mathrm{MEV}$

21. 10 MEV

22,00 MEV

$23.0 \checkmark M E V$

24. OD MEV

25.00 MEV

$26,00 \mathrm{MEV}$

27.00 MEV

$28, \triangle 8$ MEV

$29.00 \mathrm{MEV}$

32.00 MEV

32. $O G$ MEV

$34, O D M E V$

$36 . D O M E V$

38.01 MEV

40.00 HEV

$42.00 \mathrm{MEV}$

44.0\% MEV

46. OU MEV

4Q, HO MEV

50.00 MEV
PROTONS

6. 92E-1 1

$9.27 E-01$

$5.5 E-B D$

$5.29 E-91$

1. तA9E+ UIG

$6.75 E-\Delta 1$

7. $27 E-(B) 1$

$7.46 E-01$

$7.86 E-W 1$

S. $18 E-11$

$6,695-11$

6. SUE-O1

$4.75 t-b 1$

$5.15 E-11$

4. $68 E-D 1$

$5.42 E-41$

5. $99 E-A 1$

$3.17 E-01$

5. I AE - U I

$3.32 E=\emptyset 1$

1. $49 E-01$

Q. $04 E-62$

1.1 1 E-U1

2. $11 E-y 2$

5. $71 E=02$

1.6BE-B1

1. $27 E-U 1$

4. $43 E-142$

3. $93 E-U$ ?

$1.79 E-112$

3. 16E-A2

1.87E- 02

$9.87 E-13$

1. $13 E-D 1$

1. $28 \mathrm{E}-1$

1. $T 1 E-\Delta 1$

$3.46 E-02$

i.

vis.

G.

Q.

U.

a.

i.

0.

9.64
DE IITERUNS

1).

a

a

$a$

$a$

a

$B$

0

a

a

(1)

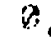

$Q$

8

a

a

a

c.

0

6

0

$b$

a.

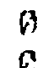

6

6

a

b.

$\theta$

a

0

9

4

a.

$\theta$

0

9

1.54
TRITOHS

$3.34 E-A S$

6

1.

i.

d.

v.

it.

4

iv.

i.

i.

0.

ii.

is.

tip

in

t).

in:

E.

6.

b.

ti.

i.

v.

k.

i)

vi.

ki.

G.

bi.

b.

b.

v.

ivi.

in

b.

k.

b.

bi.

bi.

vis

it.

8.

b.

$\theta$

3. 119
ALPHAS

Q. 3UE-T3

7. $42 E-11 S$

1. $M 2 E=12$.

$6.02 E=13$

$7,64 E-113$

$0.49 E-\triangle 3$

$1.23 E-n 2$

5. $\triangle 2 E=143$

Q. $73 E-03$

$9 . W 9 E-13$

$9.78 E-.13$

$6.71 E-33$

1. $3 I E-12$

3. UALE-B 3

$6.8 / E-.33$

$3.93 E-\Delta 5$

B. $78 F-03$

2. 0 V $E=-13$

$3.69 t-53$

1. $97 \mathrm{E}-13$

$5.08 E-1.3$

in.

b.

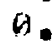

Q.

6

in.

0

औิ.

i.

b.

b.

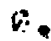

s.

i)

vis

b.

6.

i.

6.

b.

$\because$.

0

6.

b)

6.64 
ISLOMING DOWN SPECTRUM AT H.6H CM. FUR ICRU MUSCLE TISSUF 42 MEV P + ON BE NEUTROA SPECTRUM

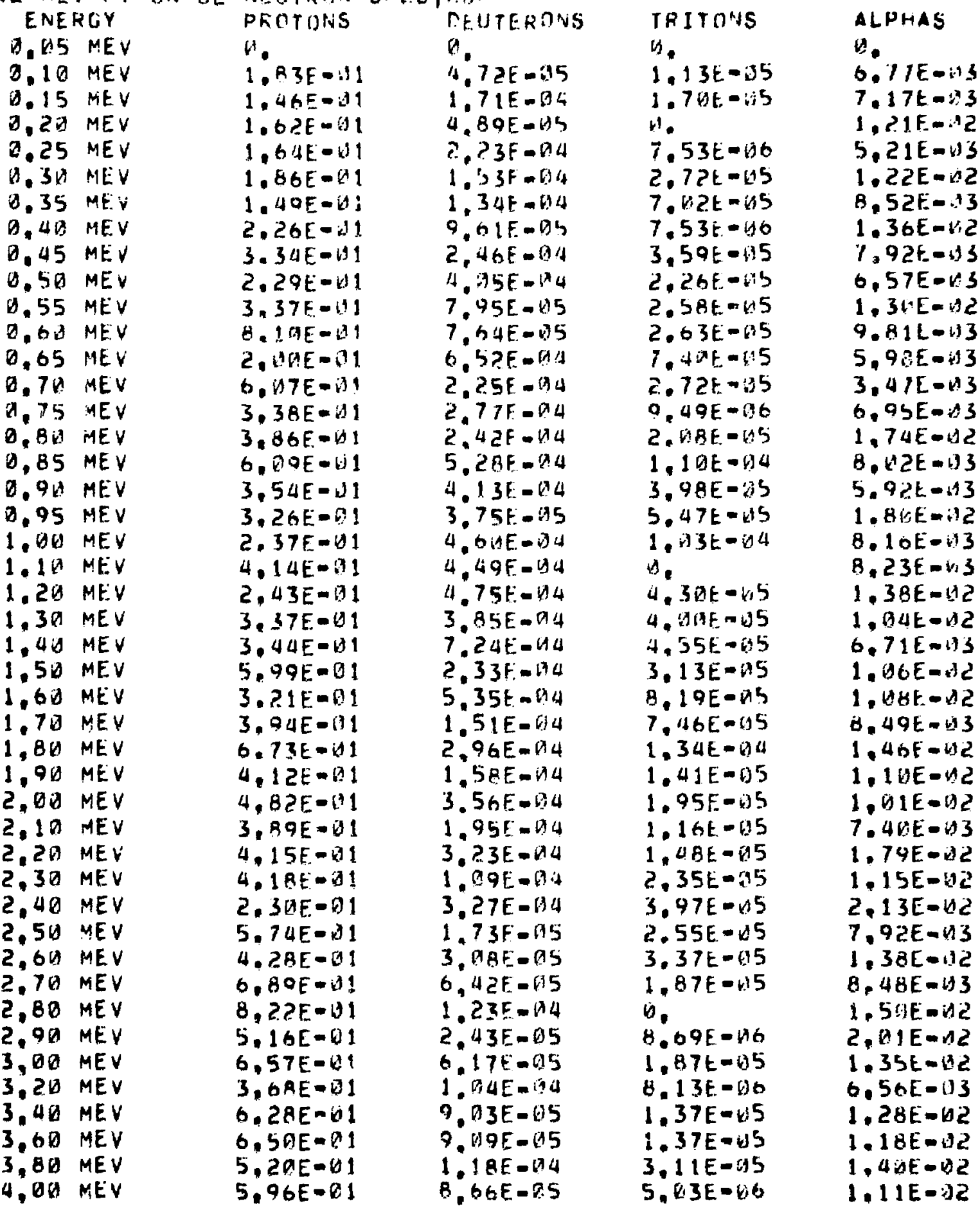




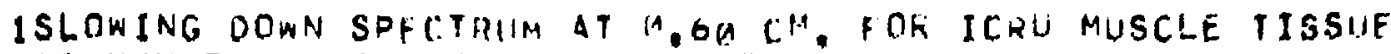
42 MEV P+ ON HE NEUTRUN SPECTRUH

ENERGY

4.20 MIEV

$4.40 \mathrm{MEV}$

4. 60 MEV

4,8 MEV

5. $\triangle 0$ MEV

5.50 MEV

6. DQ MEV

6.5V MEV

7. OQ MEV

$7.50 \mathrm{MEV}$

8. DQ MEV

$8,50 \mathrm{MEV}$

$9.00 \mathrm{MEV}$

$9.50 \mathrm{MEV}$

10.00 MEV

11. 00 MEV

$12.00 \mathrm{MEV}$

13.00 MEV

14.00 MEV

15. $91 \mathrm{MEV}$

16. DO MEV

17. $\mathrm{OH}$ MEV

18, DO MEV

$19,01 \mathrm{MEV}$

$20.00 \mathrm{MEV}$

$21,00 \mathrm{MEV}$

22. $00 \mathrm{MEV}$

$23.00 \mathrm{MEV}$

24.0D MEV

25, UQ MEV

$26.00 \mathrm{MEV}$

27.09 MEV

28. DO MEV

$29,00 \mathrm{MEV}$

30, QU MEV

32,00 MEV

34. UD MEV

36. UD MEV

38.UD MEV

$40.00 \mathrm{MEV}$

42. DO MEV

$44 . D Q$ MEV

46.00 MEV

$48,00 \mathrm{MEV}$

$50,00 \mathrm{MEV}$

gAVE. E(MEV)

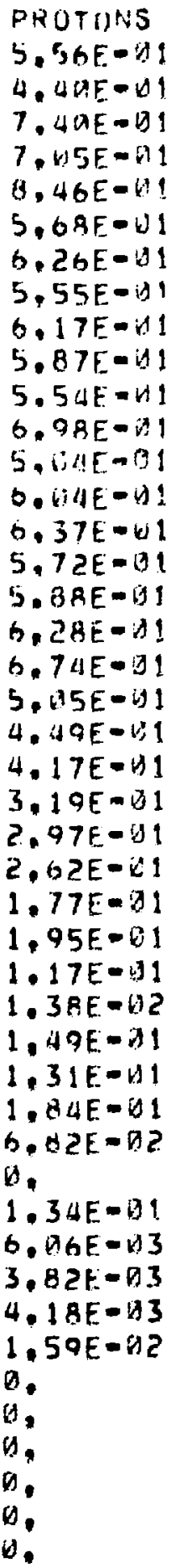

PROTIINS

S. SGE-DI

$4.4 Q E-D 1$

7. $\triangle A E-D 1$

7.VISE-DI

Q. $46 E-4 !$ !

$5.08 E-W 1$

$6.2 B E-11$

$5,55 E-1 d 1$

$0.17 E-11$

$5.87 E-191$

$5.5 \Delta E=11$

$6.98 E-1)$

$5.611 E-01$

0. GIUE-y!

$6,37 E-61$

$5,72 E=B 1$

5. $38 E-B 1$

$6,28 E-A !$

6.74E- 01

S. $15 E-V 1$

4. WE-VI

4. $17 E-111$

$3.19 E-B 1$

$2.97 E-11$

$2.62 E-i 1$

$1.77 E-81$

$1.95 E-B 1$

$1.17 E-11$

$1.3 R E-62$

1 प $A E=D 1$

$1.31 E-U 1$

$1.84 E=01$

$3.02 E=02$.

b.

$1.34 E-D 1$

6. QGE -13

$3.82 t-0.3$

$4.18 E-133$

$1.50 E-112$

0.

$\theta$

s,

0

11.19
DE IITEROUNS

1. OCE-AA

1.6 or - th 4

1. $51 \mathrm{E}-\mathrm{BI}$

$1.86 \mathrm{E}-\mathrm{BT} 4$

2. $9 U E-114$

2. $31 E-\lambda 5$

$4.32 E-.75$

$1.96 E-115$

$2.95 E-155$

$1.33 \mathrm{~F}-\mathrm{AS}$

1. $69 E-1.5$

$1.59 E-0.5$

$3.71 E-05$

a.

a

$a$.

9

$a$

$\theta$.

D.

:

is

b

0

6.

0

0

:

a.

a.

a.

a.

.

a.

6

a:

n.

$\theta$

0.

2,56
TRITONS

8.81E-VO

1. 38E-05

2. $42 E-35$

$3.74 E-B 6$

$0.23 E-116$

H.

U

w.

b.

0.

U.

$i$

e.

b.

b.

b.

a.

vi.

6.

a.

w.

b.

b.

$n$

is

0

b.

a.

D.

0.

$\theta$.

$\Delta$

0.

b.

n.

i.

a.

A.

,

$\theta$,

is.

0

6.

1.92
ALPHAS

1. $29 E-02$

$1.43 E-1) 2$

$1.37 E-12$

1. $12 E-12$

$1.5 v E-w^{3} z$

B. $4 D E-B 3$

$1.76 E-A 2$

8. VDEE-113

$1.12 E=02$

$5.75 E-113$

Q. $48 E-13$

$6.3: E-13$

1. $12 E-112$

$1.71 E-13$

$6.06 E-13$

$3,53 E=13$

$7,53 \mathrm{E}-\mathrm{B} 3$

$5.45 E-24$

$1.09 E-13$

$7.13 E-1) 4$

$1,05 t-03$

$1,57 E-13$

$3,85 E=03$

D.

V.

U.

D.

0.

a

(5)

b.

b.

$n$

$\forall$.

n.

$\theta$.

a.

D.

in,

o.

D.

0.

n.

n.

is.

6,01 
1SLDKING DOWN SPFCTRUM AT 1. DO CM. FUK ICRU MUSCLE IISSUE 42 MEV P+ ON BE NEUTRON SPECTRIMM

\section{FNERGY}

D. DS MEV

3.1 M MEV

0.15 MEV

D. 20 MEV

3.25 MEV

$0.30 \mathrm{MEV}$

$0.35 \mathrm{MEV}$

0.40 MEV

D. 45 MEV

1. SO MEV

0.55 MEV

$0.60 \mathrm{MEV}$

0.65 MEV

D. 7D MEV

$0.75 \mathrm{MEV}$

D. BU MEV

D. $85 \mathrm{MFV}$

0.96 MEV

0.95 MEV

1.00 MEV

1.10 MEV

$1.20 \mathrm{MEV}$

$1.30 \mathrm{MEV}$

$1.40 \mathrm{MEV}$

$1.50 \mathrm{MEV}$

1.60 MEV

1.70 NEV

1. BD MEV

1.90 MEV

2. DU MEV

2.10 MEV

2.2 $\triangle M E V$

2.30 MEV

2. $40 \mathrm{MEV}$

$2.50 \mathrm{MEV}$

$2,60 \mathrm{MEV}$

2.7B MEV

2.80 MEV

2.90 MEV

3. BD MEV

3. 2月 MEV

3.40 MEV

3.60 MEV

3.8 $\triangle \mathrm{MEV}$

4. $19 \mathrm{MEV}$
WROTOHS

b.

2. 37E-O1

$1, B 3 F-11$

1. $8 \mathrm{NE}-W !$

1. $03 E-11$

2. $03 F-01$

1. $3 O E=31$

2. 2 AE-U1

$2,34 \mathrm{E}-\mathrm{BS} 1$

1. $98[-w]$

$2.92 E-11$

$1.77 \mathrm{k}-11$

$2.27[-11$

$4,15 E-11$

2. $4 R E-\triangle 1$

$1.64 E-11$

2. $45 E-1$

2. $76 E=11$

2. $30 E-A 1$

2. $54 E-11$

$2.44 E=1$

2. $/ A E-D 1$

3. $22 E-b 1$

$2.33 E-01$

$3.99 E-W 1$

3. $2 O E-1$

3. G) $\triangle E-\triangle 1$

$4.76 E-01$

$2.37 \mathrm{~F}-61$

S. $3 E-1$

$3.2 A F-1$

4. WhE -01

3. $12 E-1$

$3,76 E-11$

$3,24 F-21$

$2,87 E=1$

$2.37 E-01$

4. 1 ? E- 01

$2,8 b[-01$

$6.23 E-11$

3. 4 RE-OI

$3.47 E-61$

$3.73 E-H 1$

$3.87 E-01$

$3.53 E-01$
OFUTFROAS

it.

1. A $\triangle E=D S$

i. $18 \mathrm{BE}=\mathrm{W}$

5. AUE-OTS

1. $\left.H_{6} 6-1\right) 4$

$9.655-65$

3. 50 o $=195$

1. $41 t=-174$

3.1 I. . -1.4

1. $3 \mathrm{PF}-\mathrm{B} / 4$

5.651 .05

1. $28 F=194$

2. $47 E-34$

$4.55 F-\pi 4$

$7.92 E-W$

$7.65 E-113$

3. $22 E=D A$

1. $22 E=.14$

?. $43 E-15$

2.16f-ila

1. $91:-194$

2. 3UF- 14

3. $96 \mathrm{GE}=03$

2. $296-i 14$

$2.79 E=14$

$3.63 E=114$

$1.63 \mathrm{~F}-\mathrm{A} 4$

2. $71 \mathrm{~F}=\mathrm{AL}$

2. $18 \mathrm{Bt}=4$

J. $88 E-74$

$3.78 F-N^{3}$

1. $45 E-v^{4}$

1. 9AE-IS

5. 24 a -45

$1.93 E-15$

3. 9 ?t-D3

1. $36 E-H 4$

9. 2. 3E- 10

?. $40 E-05$

3. $73 t=143$

9. A9E-OS

2. $92 E-43$

4. $77 E-145$

2. $U 6=-13$

1. 9 BE- 13
TRITONS

$\because$.

$1.26 E-155$

3.

i. $33 E=$ in

i.

$2.69 E=10$

i.

1. $33 E-635$

1. $2 t E-.25$

1. $17 E-65$

$3.37 E-13$

$0.54 E-155$

1. 4 UIE $=15$

2. 69E =1त

$2.07 E=x^{2} t$

1 1. $O G E=05$

2. 3 ht $=$ is

i.

2. $52 E=45$

$1.17 \mathrm{~F}=.5$

t. 32E- 116

$4.16 \mathrm{k}=\mathrm{k} .5$

1. B $1 E-15$

$5.67 t-65$

$1.08 t-15$

3. $27 E-\triangle S$

2. जटE-W5

3. 2UE-US

$1.714 .-15$

5. $85 E-115$

b. $18 E-197$

b. $51 E-36$

1. $32 \mathrm{C}-15$

$3.35 E=05$

5. $24-35$

S. $46 E-A^{5} 5$

4. $\triangle D E-1 S$

$5.16 E-36$

$2.58 E-15$

7. $2 Q E-45$

3. 2 RE- -35

$5.68 E-145$

2. $46 E-45$

$8.15 E-45$

1. $79 E-v 5$
ALPHAS

i.

6. 27E-13

5. HUE-WS

1. $22 \mathrm{E}-\mathrm{d} 2$

3. 3OE-A3

H. $19 E-H$

10. $96 \mathrm{E}=-\mathrm{s}$

$7.34 E-43$

7. USE $=.13$

'5. $41 E=.33$

1. W $2 E-v 2$

$7.72 E-13$

$5.72 E-v 3$

$3.48 \mathrm{E}=13$

1. $82 E-13$

$1.54 E=W$

$7.8 B E-13$

$3.57 \mathrm{~F}$ - ws

9.72E-.1S

$3,61 F=23$

$\forall, \rightarrow 3 E-i 3$

1. $13 E-42$

$8.83 E-43$

b. $75 E-43$

B. $71 E-43$

i. 1 V $E-x 2$

$9.97 E-13$

1. VI2E-112

1. $n 3 E-1 C$

8., $5 E-U S$

7. 3WE-WS

$1,16 E-12$

?. $2 E=-13$

1. $42 t-.32$

h. $27 E-.3$

$1.19 E=32$

B. $26 E=+13$

$8.81 E-13$

1. 38E- -2 ?

$1.17 E-1 C$

$5.46 E-1.5$

$9.76 E=113$

9.81E-LS

1. 32E-U2

8. $40 E-33$ 
ISLOWING DOWN SPECTRUM AT I. R'A CM. FOR ICRII MUSCLE TISSUF: 42. MEV P O ON BE NEUTRON SPECTRUM

\begin{tabular}{|c|c|c|c|c|c|}
\hline \multicolumn{2}{|c|}{ ENERGY } & PROTONS & DEUTEROWS & TRITITAS & ALPHAS \\
\hline $4,2.0$ & MEV & $3.30 E-61$ & $2.3 S E=A^{\wedge} 3$ & 5. $44 E-115$ & $18.97 E-k, s$ \\
\hline 4.40 & MF V & $4.58 E-01$ & $3,7 b E=133$ & $4.29 E-95$ & $7.36 F-33$ \\
\hline 4.60 & MFV & $9.44 E-6 !$ & $5.75 F-113$ & $5,30[-05$ & $9.27 E-143$ \\
\hline 4.80 & MEV & $4.22 E=.11$ & $B, \because 9 E-A 5$ & $4,42 E-.15$ & $7.53 t-13$ \\
\hline 5,00 & MEV & $3.94 E-181$ & $1.7 \% E-114$ & $7.46 t-015$ & $1.15 \mathrm{E}=\mathrm{t}^{2} \mathrm{Z}$ \\
\hline 5.50 & MEV & $4.65 E-1.1$ & $1.41 E-n 4$ & $3.35 t=65$ & $5.41 E-13$ \\
\hline 6.00 & MEV & 3. $43 E-D 1$ & $2.77-84$ & $6.58 E-135$ & 1.2. $1 E-42$ \\
\hline 6.50 & MEV & $4.55 \mathrm{E}-\triangle 1$ & $1.51 \mathrm{~F}-94$ & $2.10 E-65$ & 5.6 WE $=13$ \\
\hline 7.60 & MEV & 4. $16 E-51$ & $3.34 E-04$ & $5.31 E-15$ & 7. $31 \mathrm{E}=\mathrm{W}$ \\
\hline 7.50 & MEV & $4.89 \mathrm{E}-01$ & $7.84 F=65$ & $2,11 E=106$ & $6.74 F=3$ \\
\hline 8.00 & MEV & $4.21 E-b 1$ & $1.83 F-144$ & $4.52 E-66$ & $6.4 E=13$ \\
\hline 8.50 & MEV & $7 . B \backslash E=01$ & $5.22 E-195$ & $1.2 \Delta E-06$ & $5.42 E=03$ \\
\hline 9.60 & MEV & $4.45 E-31$ & 1. $22 E-64$ & $2.48 E-116$ & $9.61 \mathrm{E}-\mathrm{bs}$ \\
\hline 9.50 & MEV & $3 . B A E=A 1$ & $1.27 E-36$ & $3.72 E-\left[x_{0}\right.$ & $4.33 E=43$ \\
\hline 10.60 & MEV & $3.93 E-n i$ & $2.96 E-1.6$ & $B .67 E=36$ & 1. $66 E-12$ \\
\hline 11.00 & MEV & $4.7 D E-M 1$ & 4 & n. & $3.83 E-13$ \\
\hline 12.00 & MEV & $5,27 E-11$ & V & $u_{0}$ & $6,4 \partial E=n 3$ \\
\hline 13.00 & MEV & $5.19 F=1$ & 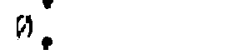 & b. & $3 . \angle B E=W 3$ \\
\hline 14.00 & MEV & $0.77 E-11$ & 7 & $n$ & $5,93 \mathrm{E}-13$ \\
\hline 15,00 & MEV & $4.58[-21$ & 4 & 0. & $1, B 4 E=43$ \\
\hline 16.00 & MEV & $4.35 E-41$ & $n$ & 6. & $3.22 E-\Delta S$ \\
\hline 17.00 & MEV & $4.16 E-01$ & 0 & 0 & $1.44 E=03$ \\
\hline 18.00 & MEV & $4.93 E-01$ & $\theta$ & 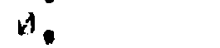 & $3.16 E-13$ \\
\hline 19.00 & MEV & 3.RAE-a1 & $B$ & 0 & B, HVE- 14 \\
\hline 20.00 & MEV & $3.25 E-\lambda 1$ & $n$. & $w$ & $1.4 \cap E-U S$ \\
\hline 21.00 & $M E V$ & $3.58 E-11$ & $n$ & 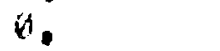 & A. \\
\hline $22.0 x$ & MEV & $3.41 E-01$ & (1). & $n$ & D. \\
\hline 23.90 & MEV & $2.995-61$ & $n$. & 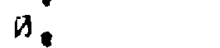 & a. \\
\hline 24.00 & MEV & $2.9 ? E-W 1$ & 4. & 0 & $\dot{v}$ \\
\hline 25.00 & MEV & $2, D B E=01$ & 0 & $n$ & $n$ \\
\hline 26.00 & MEV & $2.72 E-01$ & i) & ij. & n. \\
\hline 27.00 & MEV & $2.50 E-101$ & n & $v$ & 4 \\
\hline 28.96 & MEV & $1.54 E-61$ & 10 & 3 & i. \\
\hline 29.00 & MEV & $1,44 \mathrm{E}=01$ & a. & 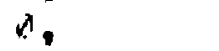 & $n$ \\
\hline 33.00 & MEV & $1.91 E-01$ & $a$ & $v$ & $\theta$ \\
\hline 32.00 & MEV & $9.73 E-92$ & a & $n$ & 4. \\
\hline 34.00 & MEV & $4.13 E-12$ & is. & in & D. \\
\hline 36.64 & MEV & $3.665-112$ & $\dot{v}$ & $n$ & b. \\
\hline 38.00 & MEV & $1.3 E-62$ & n. & $N$ & $v$. \\
\hline 40.00 & MEV & 0. & $a$ & b. & i. \\
\hline 42,50 & MEV & $u_{0}$ & 0 & 1 & a. \\
\hline $44.0 \mathrm{Hi}$ & MEV & $\theta$ & 4. & $b_{0}$ & a. \\
\hline 46.00 & MEV & 0. & $a$ & !. & 0 \\
\hline 48.00 & MEV & $v$ & $a$ & 10 & $a$ \\
\hline $53.0 v$ & $M E V$ & 0. & $\theta$ & 0 & $n$ \\
\hline AVE & (MEV) & 14.19 & 3.74 & 4.34 & 7.33 \\
\hline
\end{tabular}


ISLOWING DOWN SPECTRUM AT 2. AO CM. FOR ICRL MISELE IISSUE 42. MEV P+ ON BE NE!JTRON SPECTRIIM

\section{ENERGY}

D. D5 MEV

D. 10 MEV

D. 15 MEV

0,21 ME.V

0,25 MEV

0.3 MEV

0.35 MEV

d. 4 MEV

0.45 MEV

$0.50 \mathrm{MEV}$

$0.55 \mathrm{MEV}$

b.61) MEV

$\triangle .65 \mathrm{MEV}$

$0.70 \mathrm{MEV}$

0.75 MEV

0.84 MEV

9.85 MEV

D. 90 MEV

0.95 MEV

1. MD MEV

1.1D MEV

1.20 MEV

1.36 MEV

1. 40 MEV

1.50 MEV

$1.60 \mathrm{MEV}$

1.70 MEV

$1.80 \mathrm{MEV}$

1.91 MEV

2. $\triangle \triangle M E V$

2.1 $\triangle M E V$

$2.20 \mathrm{MEV}$

2.3H MEV

2. 4 D MEV

2.51 MEV

2.60 MEV

$2.70 \mathrm{MEV}$

2. BW MEV

2.9! MEV

3. DD MEV

3.2U MEV

3. 40 MEV

3.60 MEV

$3.80 \mathrm{MEV}$

4. O MEV

\section{PROTINS}

i.

$2.43 E-91$

$1.97 E-101$

$1.66[-11$

2. $D, 1[-0]$

2. $U$ ? $E-k 1$

$1.57 E-31$

2. $15 E-11$

2. $13 E-61$

1. $85 E-61$

$3.14 E-D 1$

1. $62 E=01$

2. 25E-U1

$4.75 E-01$

$2.95 E-\| 1$

1.7 AE-DI

$1,77 E-91$

$2.34 E-B)$ ?

3. $14 E-B 1$

2. ПAE-D1

2. $44 E-51$

$2.5 D E-21$

$3.05 E-01$

2. $29 E-01$

$5 . \Delta 1 E-D 1$

3. $36 E-D 1$

2. $71 E-01$

$3.15 E-01$

2. $7 \Delta E-U 1$

2. $92 E-U 1$

4. $27 E-101$

$3.60 E-101$

$4.37 E-B 1$

$2.91 E-B 1$

$2.71 E-D 1$

$3.33 E-B 1$

2. $44 E-01$

3. $35 \mathrm{E}-11$

2. 9RE-01

7. $32 E-U 1$

$3.91 E-31$

$3.4 Q E=D 1$

$3.86 E-11$

4. $11 E=1$

4. ?IE-OI
DEUTEROIS

a.

5.56E-OS

1. $35 E-W 4$

5. DRE-DIS

1. $47 F=124$

1. $25 E-114$

5. $31 E-35$

1. $35 E-24$

$3.91 F-n L$

?. $33 F-04$

$9.16 E-A S$

$9.71 f=65$

$3.52 E-14$

$9.68 E-W 4$

1. $65 E-0.04$

1.50E-OU

8. BUE-OA

4. ? BE-M 4

3. $33 E=05$

3. $50 F-04$

2. $93 E-14$

$4.47 E-D 4$

2. $72 E-C 4$

4. D RE - 44

3. $59 F-614$

6. SBE-CA

2. $75 E-34$

?. $68 \mathrm{E}=\mathrm{B} 4$

$5.76 E-04$

2. $57 E=04$

3. 3 AE WI IA

$2,35 \mathrm{~F}=\mathrm{AA}$

1. $42 E-04$

1. $79 E-04$

3. B6E-OA

$3.72 F-94$

6. $42 E=14$

$6.69 E=134$

5.61E-0.5

$9.56 E=05$

$1.73 E-04$

$4.23 E-04$

1. $49 k-04$

3.67E-HA

$1.9 T E-B 4$
IFITUNS

a.

5.4\%t-nts

2. $86 \mathrm{~F}-47$

h. $51 t=06$

$1.44 E-1) 6$

$1.63 E-45$

2. $86 E-47$

1. $\triangle A E=B 6$

4.8 it $-\dot{k} \mathrm{j}$

$1.93 E-6 j 5$

1. $23 E-V 5$

1. 1At $=05$

4. $27 E=16$

3.19E-US

$9.14 E=67$

$5.75 t-06$

1. $35 E-05$

4. $26 E-b 6$

?. $14 \mathrm{E}-05$

$3.73 E-45$

1. $19 \mathrm{E}-\mathrm{N} 5$

$4.20 E-115$

6. $42 E-16$

$3,54 E-15$

1. $298-116$

2, SRE-U5

2. $74 E-.15$

1.29E-1D5

1. $24 E-$ W'

7. $2 \in E-\triangle 5$

$8.30 E-D 6$

$3,16 E-15$

$2,81 E=05$

$5.69 E-65$

$4.63 E-105$

$1.52 E-115$

4. EUE-DS

$1.63 E-05$

3. 20E- 65

$7.15 E-195$

2. 23E-05

5. $2 U E-15$

1. $35 t-05$

$3.96 E-95$

$1.07 t-05$
ALPRIAS

C.

4. BOE-A3

5. $8 U E-113$

1. $\$ 5 E-\square 2$

$3.51 \mathrm{E}-\mathrm{US}$

$9.24 E-B 3$

9. vet-us

$9.53 \mathrm{E}-\mathrm{is}$

$6.25 E-13$

5. $75 E-x 3$

6 . VUE-WS

$1,01 E-1.2$

8. $44 E-13$

2. $67 E-W 5$

6. $13 E-43$

1. $48 E-62$

$3.56 E-13$

$4.55 E-0 S$

1. D9E- 1.2

$7.4 B E=13$

8.1 1 EE-A3

1. $25 E-12$

7. ?.YE-03

$6,9, E-.33$

1. $15 E-W 2$

T. 2UE-OS

$7.83 E-\square 3$

1. $U 7 E-42$

1. $25 E-D 2$

6. 3BE-it

9. $48 E-153$

$1.55 E-B 2$

6. $17 \mathrm{E}-\mathrm{B} 3$

$1.63 E-52$

$6,28 E-n 3$

$1.22 E-02$

$5.29 E-1: 3$

$7.76 E-A S$

$1.64 E-\forall i 2$

1. $V 7 E-A_{K}$

$6.24 E-13$

$1.23 E-112$

1. $17 E-92$

1. 3RE- 12

$9.58 E=3$ 
ISLOWING DOWN SPECTRLIM AT 2. MG CM. FOR ICRU MUSCLE TISSUE. 4? MEV P+ ON BE NEUTRON SFECFFUIA

\section{ENEKGY}

4. 2 ME $V$

4.40 MEV

$4.60 \mathrm{MEV}$

4. 8 M MEV

5.00 MEV

5.5 A MEV

6. DO MEV

6.50 MEV

7. DO MEV

1.50 MEV

8. HO MEV

$8.5 B$ MEV

9. DO MEV

9.50 NEV

12,00 MEV

1), $\triangle Q$ MEV

$12, B 0$ MEV

13, 60 MEV

14, DU ME.V

$15,00 \mathrm{MEV}$

$16,00 \mathrm{MEV}$

$17.00 \mathrm{MEV}$

$18.00 \mathrm{MEV}$

$19.0 Q$ MEV

$20 . B D M E V$

21.00 MEV

$22,00 \mathrm{MEV}$

23.09 MEV

$24.00 \mathrm{MEV}$

25.DU MEV

26. OU MEV

$27 . O B M E V$

$28.00 \mathrm{MEV}$

$29.00 \mathrm{MEV}$

$30.00 \mathrm{MEV}$

$32.00 \mathrm{MEV}$

34. MO MEV

$36,00 \mathrm{MEV}$

$38.00 \mathrm{MEV}$

40.0D MEV

42. OD MEV

$44.00 \mathrm{MEV}$

46. DO MEV

48. DO MEV

$50,00 \mathrm{MEV}$

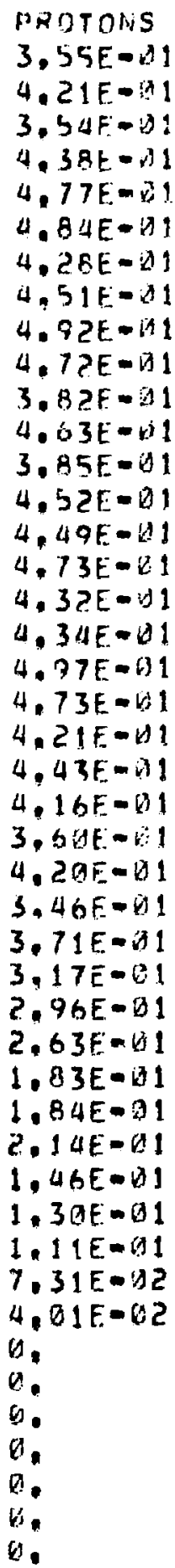

14.28
DEUTERDINS

5. MIAF-M 4

$2.65 E=144$

$6.816-194$

3.97E-ins

2. $62 \mathrm{E}-1.4$

2. $965-114$

2. $49 E=04$

2. $75 \mathrm{~F}-94$

7. 1 UF $-E 4$

$5.94 F-95$

1. $25 E-174$

1. $82 E-75$

4. DAE - V 5

1. $\triangle B E-05$

3. $42 F=05$

2. 4 A F -67

2. $40 E-n ?$

$4.8 A E-A 7$

9. 6 G $\mathrm{E}=\mathrm{CA} 7$

a.

a.

a

b.

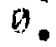

$a$

a.

0

a.

0

a.

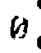

D.

o.

0

$\theta$

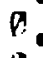

$n$

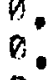

c.

a.

0

$\$ .02$
TRITUNS

5. $73 E-.95$

2.97E-GS

4. $) 4 E-155$

1. $46 E-05$

1. 82E-115

1. $56 E-135$

$3.95 \mathrm{f}-\mathrm{k} .5$

2. $06 E-106$

5. $03 E-16$

4. 31E-A?

1. $1 E-k b$

i)

ti.

is

$i$.

t:

B.

6.

in.

$\theta$

3.

v).

3.

b.

if

a.

$1)$

(1)

$\theta$

in

b.

a.

a.

i)

b.

i.

i.

A.

0

0

0

.

.

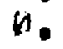

0

3.42
ALPHAS

$3.51 \mathrm{E} \rightarrow \mathrm{S}$

1. $20 E-B 2$

1. $10 t-02$

7. $42 E-B 3$

9. ORE-B⿵ 3

6. ALE - fis

1. $25 E-192$

5.4IE-US

8.2JE-A3

$4,76 E-V 3$

$7.61 E-03$

3. $36 E=-13$

6. $59 E-V 3$

2. $26 E-B S$

$6.17 E-i s 3$

1. $\triangle 4 E-S S$

?. $37 \mathrm{E}-173$

8.96E-34

2. $16 E-4 S$

6. D2E-DG

$1.37 E-45$

$1.56 \mathrm{~F}-1 \mathrm{BO}$

3. $44 E-40$

$9.25 E-D I$

$2,16 E-116$

$a$.

$\Delta$.

0

0.

b.

D.

a.

$\theta$.

$n$

(1).

$\theta$

0.

$\theta$

D.

B.

0.

D.

i)

a.

a.

5. 14 
1SLONING DOUN SPECTPUM AT 4 , GU CM, FOR ICRI! MUSCIF IJSSIJE 42 MEV P+ ON BE NEUTRON SFECTFIJ

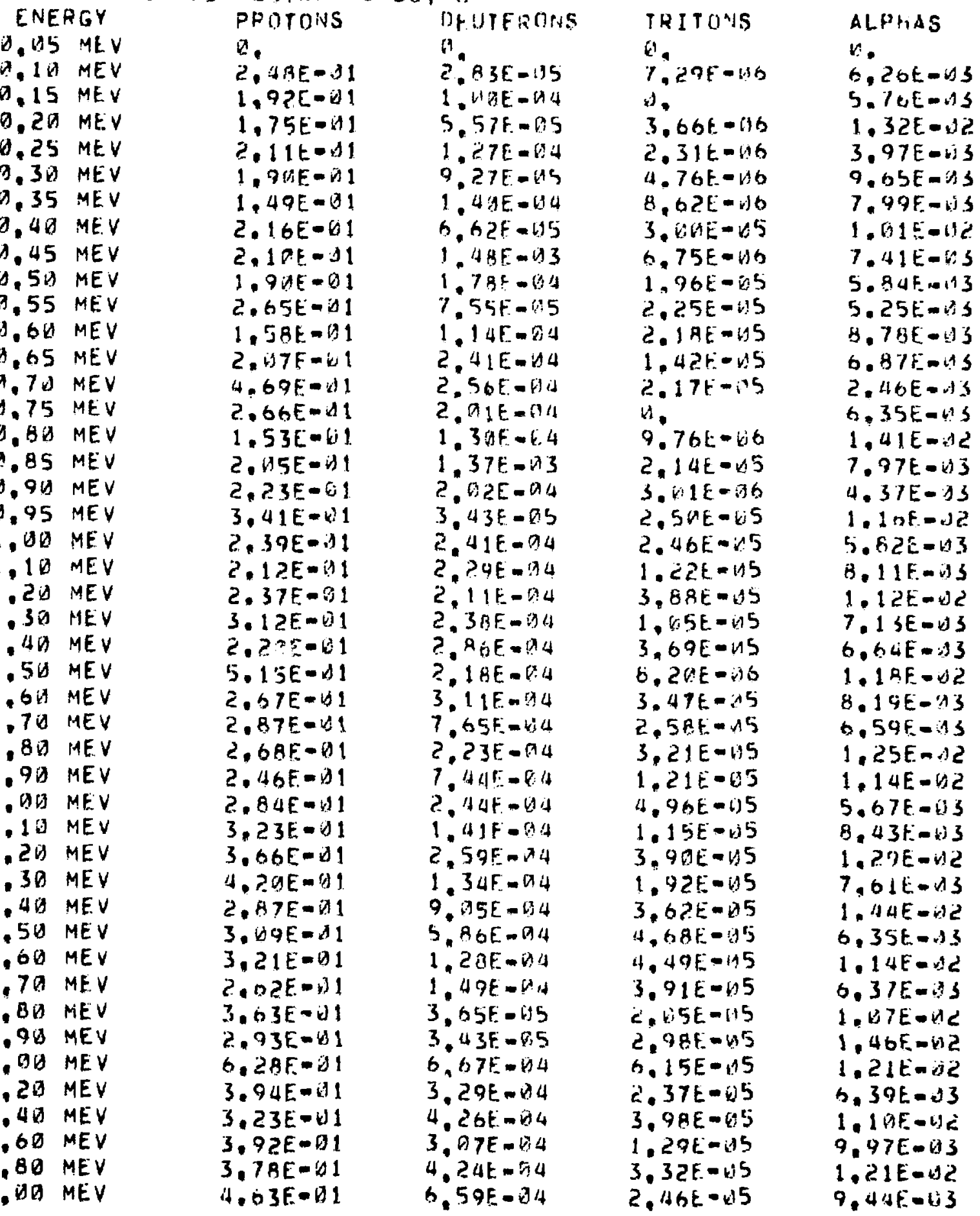


ISLOWING DOWN SPECTRUM AT 42 MEV P DN HE HEUTRON SPECTRUM

\section{ENEAGY}

4. 20 MEV

4., 40 MEV

4.60 ME.V

4.80 MEV

5. $D O$ MEV

5.50 MEV

$6.1 \%$ MEV

6,50 MEV

7.8D MEV

7.50 MEV

8. $\triangle 0$ MEV

$8.50 \mathrm{MEV}$

$9.00 \mathrm{MEV}$

$9.50 \mathrm{MEV}$

10. OU MEV

$11.00 \mathrm{MEV}$

12. $O O M E V$

13.09 MEV

14.0O MEV

$15,0 \mathrm{MEV}$

$16,00 \mathrm{MEV}$

17.0 MEV

18.00 MEV

$19.00 \mathrm{MEV}$

2D. OO MEV

$21.0 B$ MEV

22. DO MËV

23.60 MEV

24.DO MEV

25,00 MEV

$26,00 \mathrm{MEV}$

27. OO MEV

$28, D V M E V$

29, UD MEV

30. $\triangle O$ MEV

32,00 MEV

34. DO MEV

36. UO MEV

3 3. OU MEV

40. GU MEV

4?.OV MEV

44.00 MEV

46,DO MEV

48.60 MEV

$50.00 \mathrm{MEV}$

$$
\text { PROTONS }
$$

3. उBE-GI

4. 3AE-DI

3.76E-U1

4. 59 $9 E-01$

$4.39 E-01$

$4.50 E-01$

$4.43 E-31$

$3.7 M E-D 1$

4. 26t-01

5. $19 E-U 1$

4. $1 \mathrm{GE}-61$

$4.32 E-61$

4. 35E-D1

$4.83 E-41$

$4.725-41$

4. $\triangle 6 E-B I$

$4.74 E-01$

$4.67 E-61$

4. $\triangle A E-D 1$

4. 4 पE $U 1$

4. $25 E-B 1$

$3.63 E-! 1$

3. QUE $=1$

$3.36 E-31$

$3.37 E-B 1$

3. $24 E-D 1$

3. $\triangle B E=\square 1$

$2.61 E-U 1$

3. $17 E-B 1$

$2.94 E-01$

$2,17 E-01$

$1.97 E-61$

1. 7 2 E $-B 1$

$1.29 E-D 1$

$1.47 E-01$

$1,17 E-61$

$7,65 E-12$

$5.16 E-02$

2. $\triangle 7 E-B 2$

$6,05 E=193$

$B$

b.

0

0

0
DEUTERONS

$3.83 E-D 4$

3. पतE-त 4

$6.67 E=0,4$

9.61E-0i4

1. 32E-A3

3. $94 \mathrm{E}-134$

$8.59 E-A A$

6. BIE-AS

1. 5BE-AL

5. OUE-OS

8.99E-VS

2. $98 E-1) 5$

5.9 $4 E-05$

1.6 6 IF -15

2. $85 E-95$

1. 37F. $\square 5$

3. DQE-D5

$\theta$

6

a.

a.

$a$

$a$

0.

$a$

$b$

a

0

in.

17

is.

a.

is.

a.

0

0

a.

$a$

0

$n$

$a$

$a$

4.19
TRITUIS

3. $0.5 E-05$

2. $34 E-95$

$3.38 E=$ is 5

1. $52 E-45$

2.78E- 95

$1.2 B E=05$

2. $90 E-45$

$7.85 E=k 6$

1. $V 5 E-05$

9. 25E- 66

$1.18 E-D 5$

1. DIE- 15

2. ज) $1 E-65$

3. 6 . $E E=136$

5. $26 E-$ IS 6

4. 9 सE - IA 6

1. 1DE- 15

u.

$\theta$

it.

17

b.

a.

6.

a.

a.

a.

1.

i.

id.

i).

ij.

D.

i.

b).

vi.

G.

U.

H.

b.

b.

0.

0

4.

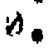

4.72
ALPrAS

8. HCE $=3$

9. $32 E-V S$

1. $V 5 E-D 2$

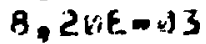

B, DUEAOS

5. G1E-US

1. $20 E-B 2$

$5.78 E-G 3$

7.78E-OS

$5,8 U E-\triangle 3$

6. 9UE $=$ U 3

4. $36 E-13$

$7.95 E-13$

$2.23 E=03$

6. 2WE-IS

$? .73 E-O S$

$5,72 E=A S$

1. $47 E-63$

2. $33 E-03$

$9.93 E-14$

$1.77 \mathrm{E}-03$

$5.18 E-14$

$9,21 E-1) 4$

$4.20 E-G 4$

B. BUE $=14$

0.

$\Delta$.

b.

$\theta$

$\theta$

0

$\Delta$.

0.

i)

is

i.

n.

a.

4

i)

a.

the

D.

0.17 
1SLOWING DOWN SPECTRIIM AT 6, BG CM. FOR ICRU MUSCIE IISSUE. 42 MEV P + ON HE NEUTKONN SPECTRUM

\section{ENERGY}

D. OS MEY

D. 1 B MEV

D. 15 ME.

0.2 MEV

0.25 MEV

$0.30 \mathrm{MEV}$

0.35 MEV

0.40 MEV

0.45 MEV

$0.50 \mathrm{MEV}$

$0.55 \mathrm{MEV}$

$0.61 M E V$

0.65 MEV

0.79 MEV

$0.75 \mathrm{MEV}$

2.86 MEV

B. BS MEV

0.94 MEV

$0.95 \mathrm{HEV}$

1. $\triangle 0$ MEV

1.10 MEV

1.26 MEV

1.30 MEV

1.40 MEV

1.50 MEY

1.60 MEV

$1.70 \mathrm{MEV}$

$1.80 \mathrm{MEV}$

$1.90 \mathrm{MEV}$

2. DO MEV

2.10 MEV

2. 23 HEV

2.3U MEV

2. $40 \mathrm{MEV}$

$2.50 \mathrm{MEV}$

$2.60 \mathrm{MEV}$

2.70 MEV

$2.80 \mathrm{MEV}$

$2.90 \mathrm{MEV}$

3. UO MEV

3.20 MEV

3.40 MEV

3.60 MEV

$3.80 \mathrm{MEV}$

4.OU MEV

\section{PROTONS}

0.

$2.35 t-41$

1.89E- 11

$1.77 \mathrm{~F}-11$

2. $\triangle 6 E=1$

2.01E-01

1.5 $) E-61$

2. $14 E=01$

2. $U A E-U 1$

$1, B+E-11$

2. $57 E-131$

$1.62 E-91$

2. $04 E-1$

4. $19 E-B 1$

2. $51 E-111$

1. $5 \mathrm{HE}-31$

$1.92 E-\nabla .1$

2. 2 तE-OI

$2.87 E=01$

2. $29 \mathrm{E}-01$

2.16F-O1

2. $26 E-1$

2. $89 E-31$

$2.11 E-11$

$4.56 E-11$

$2.53 E-U 1$

$2.59 E-B !$

$2.61 E=01$

$2,21 E=k 1$

$2.61 E=01$

3. $41 E=B 1$

$3.74 E-D 1$

3. $60 E-B 1$

3. $09 E-\triangle 1$

2. 5OE-AI

$2.64 E-B 1$

2. 3OE-O1

3.13E-D1

$2.87 E-01$

$5.58 E-91$

$3.23 E=$ औI 1

$2.63 E-11$

3. $11 \mathrm{E}-1) 1$

3. $14 E-01$

4. O2E-BI
DEUTERUNIS

a.

3. 28E-95

1. $11 E-04$

$5.37 F-05$

$9.985-05$

$8.77 E-35$

$4.46 E=05$

$9.98 E=05$

$1.55 \mathrm{E}=\mathrm{AA}$

1. $4 U E-114$

9.18E. 145

8. 66F- 45

2. 37E-OA

$1.98 E-144$

1. $145=14$

1. 12E- 124

2. $48 F=0.4$

1. $70 E-04$

$2,48 \mathrm{~F}-1.5$

2. $95 E-104$

1. 82F-64

2. $03 \mathrm{E}=4$

1. $75 E-94$

2. GUE-T4

1. $45 E-94$

2. $91 E-B 4$

1. $18 E-54$

1.7 AE -14

$7.75 F-145$

1. 1 $4 E-Q \mathrm{AJ}$

$4.62 E-95$

$7.92 E=05$

$2.59 E=05$

$0.31 E-15$

1. $88 E-25$

$8.63 E=05$

1. $15 E=A 4$

$4.0 Q E-O 5$

4.69E- 15

$7.21 E=05$

5.14E- 15

9.27f-. 5

3. DIE-O5

$6.33 E-U S$

$4.85 E-A S$
TRITUNS

4.

$7.31 E=E B$

$6.14 \mathrm{E}=\mathrm{B} 7$

$4.30 \mathrm{E}-196$

$9.69 E-07$

$7.15 \mathrm{t}-196$

$2.82 E=106$

$6.67 F=24$

6. $80 \mathrm{E}=16$

1. DIF. -15

$1.55 E-46$

$3.94 E=05$

$1.14 E-05$

$9.53 E-110$

2. $04 \mathrm{~F}=06$

1. $12 E-05$

1. $A \geq E-45$

$4.18 F=66$

$3.71 E-E 5$

2. $91 E=015$

h. BAE-V6

1.96E-D5

$0.36 E-16$

$2.95 E-65$

1. $D O E=5$

3. $41 E-45$

1. $23 E=5$

$2,61 E-05$

1. $04 \mathrm{~F}-05$

2.,$A B E-25$

$8.36 E-66$

$3.48 E-34$

1. 2?t -05

$3.49 E-05$

$2.65 E-05$

$9.51 t-04$

1. $6 \mathrm{BE}-\$ 5$

6. $12 . E-46$

8. VISE-US

2. $U 1 E-05$

1.8 ?E- 04

$2.3 B E=\$ 5$

$0.06 E-110$

$1.60 E-65$

$6.33 E=04$
ALFHAS

b.

$5.23 E-63$

$5.45 E=35$

$1.11 E-U 2$

4. $62 E-13$

$9.72 E-93$

$6.86 E-13$

8. 47E-US

$6.2 b E-W 3$

5. DOE-AS

$4.57 E-03$

$9.19 E-B S$

6. ASE- A3

2.83E-V3

$5.62 E-0 S$

1. $58 E-1$ ?

7. 8 GE-AS

$4.35 E-U \vdots$

$1.06 E-12$

$4.94 E-B 3$

7.98E-

1. DGE -12

$7 . \triangle 4 E-13$

$6,15 E-13$

I. DUE-OZ

$7.42 F-13$

$7.74 E-b 3$

1. $01 E-\Delta C$

1. BAE-VZ

$5,47 \mathrm{E}-W 3$

$6.98 E-\pi 3$

1. $33 E-12$ ?

$7 . V 35=03$

1. $31 \varepsilon=02$

D. 2SE $=13$

1. $15 E=12$

$0.44 E-\triangle 3$

$1.32 E-102$

$1.44 E-6 z$

$1.08 E-02$

$6.31 E=13$

1. KBE- 12

$9.51 E-13$

$1,23 E=112$

$8.70 E=03$ 
1SLONING DOWN SPECTRUM AT 6, OH CM. FTH ICZU MUSCLE IISSUE. 42 MEV P + ON HE NËUTROH SPECTRIMM"

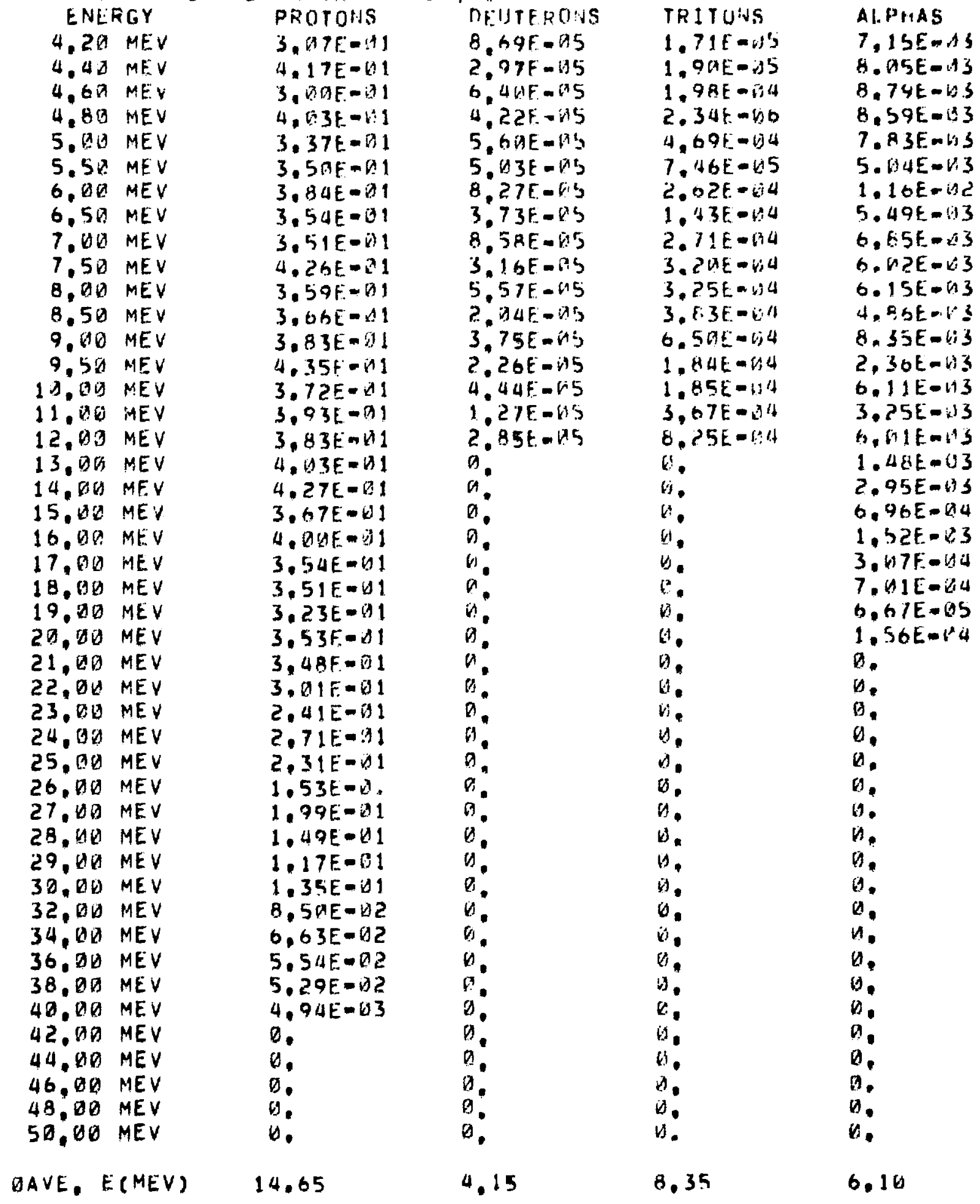


1SLOHING DOWN SPECTRUM AT 1W. CM. FOA ICRU MUSCLE TISSUE L2 MEV P+ DN BE NEUTRON SPECTRUM

\section{ENERGY}

$D, 05$ MEV

D. 1 i) MEV

0.15 MEV

0.20 MEV

D. 25 MEV

U. 31 MEV

D. 35 MEV

DU MEV

$0.45 \mathrm{MEV}$

7. 5 MEV

$D, 55 \mathrm{MEV}$

0.60 MEV

0,65 MEV

1.70 MEV

D.75 MEV

$0.80 \mathrm{MFV}$

0.85 MEV

$0.90 \mathrm{MEV}$

$0.95 \mathrm{MEV}$

1.OU MEV

1.1 MEV

1.2Q MEV

1.30 MEV

1.40 MEV

1.50 MEV

$1.60 \mathrm{MEV}$

$1.70 \mathrm{MEV}$

1.BD MEV

$1.70 \mathrm{MEV}$

2. HO MEV

2.11 MEV

2.2丁 MEV

$2.30 \mathrm{MEV}$

2. 4R MEV

2.50 MEV

$2.60 \mathrm{MEV}$

$2.70 \mathrm{MEV}$

$2.86 \mathrm{MEV}$

$2.90 \mathrm{MEV}$

$3.00 \mathrm{MEV}$

$3.20 \mathrm{MEV}$

$3.40 \mathrm{MEV}$

$3.60 \mathrm{MEV}$

$3.80 \mathrm{MEV}$

4.0O MEV
PROTONS

2.

2. DISE-DI

$1.65 E-11$

1. $50 E-D 1$

1. $\theta O E-W I$

$1.74 E-11$

1. $29 E-11$

$1.67 E-01$

1.6BE- 11

$1.55 E-$ i 1

2. $10 E-U 1$

1. 3BE-U1

$1.71 E-U 1$

3. $47 E-D !$

2. $27 E-11$

1. $33 \mathrm{~F}=61$

1. 56E-D1

1. $79 E-D 1$

$3.02 E-B 1$

2. $26 t-01$

$1.77 E-M 1$

1.98E-W1

2. $45 E-$ id 1

$1.74 E-B 1$

$3.93 E-61$

2. $2,6 E=U 1$

2. $2|E-B|$

2. $52 E-11$

2. $\triangle P E-U 1$

2. 1 I $A E-01$

2. $94 E-1$

3. $11 \mathrm{E}-01$

3. $21 \tau-b 1$

?. $39 E=01$

2. 3AE-O1

2. $54 E-B !$

2. $24 E-B 1$

$2.77 E-11$

2. $53 E-01$

$5,18 E=0 !$

3. $05 E-D 1$

2. $6 \triangle E-B 1$

2.81E-01

$2.74 E-101$

$3.22 E-01$

\section{DEUTEROI+S}

2.52F m ils

8. MAE-AT

$3.15 E-15$

$9.23 E-45$

$6.765-05$

$5.37 E-05$

$7.3 B F=115$

1. $59 E=114$

$1.15 E-194$

5. SQE-O5

$9.73 E=195$

2. ATF $=04$

1. $86 \mathrm{E}=14$

1. $12 E-\bar{R} 4$

$9.70 F-05$

2. $34 \mathrm{E}-614$

$1.815-04$

2. กิวt-45

$1.87 F-34$

1. $A, j E-1) 4$

$1.035-14$

3. $4(4 E-6) 1$

2. $5 b E-14$

1. $36 E-64$

$2.63 E-94$

1. $21 F=G 4$

1.71F-AI

1. $B 14 E-B 4$

$3.1 U E-O A$

$1.92 E-1.5$

9. $85 E-15$

4. $1 E-15$

2. D:AE -14

3. 21 $E-795$

2. $4 \mathrm{BF}-\mathrm{Ai}$

1. $15 \mathrm{~F}=0 \mathrm{~A}$

4.915 .05

$4.37 \mathrm{~F}=15$

$6.65 E-0.5$

1.? $2 E=64$

$7.57 F-05$

$1.65 E-04$

$1.59 E-14$

$5.47 E-145$
TRITONS

13

$4.93 \mathrm{E}-\mathrm{Db}$

2. $49 E=196$

$4,29 E-W 6$

2. $20 E-10$

3. $2 D E-B b$

$3.93 E-d h$

1. $87 E-45$

8.5 DE-66

$7.6 t-66$

1.6UE- 45

$1.72 E-45$

$6.92 E=146$

$7.67 E-U S$

1. $27 E-6 \mathrm{C}$

7. ROE- 146

$1.68 E-65$

B. 1 t t $=06$

$\therefore, 29 E-B A$

1. 37F-WS

$0,19 t-06$

S.EQE-DS

9.28t. -116

2. $29 E-155$

$5,67 E-10$

1. $231-34$

1. $53 E-65$

1. $42 K-415$

$4.95 E-36$

1. $48 E-U A$

$1,44 E-06$

1. $35 E-45$

d. $\$ 19 E-116$

$1,26 E-14$

3. $23 t-05$

1. $5 n t-0.5$

1. 20E -114

$7.10 E-D 6$

$1.67 E=155$

1. $48 E-04$

7. 32E-G5

8.47E-US

$6,52 E-115$

6. B6E- 35

7. OAF.U 5
ALPHAS

(1).

$1.23 E-13$

$4,81 E-13$

$9.79 E-W 3$

$3.71 \mathrm{E}-1 \mathrm{~B}$

A. $58 E-13$

5. 4 IE -13

7. त $4 E-13$

$5.27 E=115$

4. $3 D E-113$

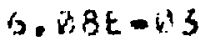

7. $41 E-13$

$5.47 \mathrm{t}-23$

2. GAOE- $=3$

S. UQE- 13

$1,13 E-n i 2$

$6.53 E=43$

3,6 WE-1:3

?. IQE-US

4. $21 E-143$

6. $32 E-$ it

$9,89 E-433$

$5.98 E-\operatorname{in} 3$

$5.57 \mathrm{~L}-13$

9. U1E- is

$6.93 E=43$

$5.01 t-63$

$9.89 t=-13$

$3.71 E-w s$

$4.67 E-43$

6. $74 E=13$

1. $23 E=U 2$

$0.53 E=1 S$

1. $15 E-22$

$5.44 E-13$

$9.49 E-A S$

$5,48 F=M S$

$\theta$. ULE-

$1.13 t-02$

$9.38 E-33$

S. $07 E-D 3$

$9.85 t-13$

$B, D L E-D S$

1. $11 E-42$

6. $86 E=13$ 
1SLOWING DOWN SPECTRUHA AT 16.G CM. FOR ICRU MUSCLE TISSUE 42 MEV P+ ON BE NEUTRON SPLETRUM

\section{ENERGY}

4. 20 MEV

4. 4A MEV

4.60 MEV

$4.86 \mathrm{MEV}$

5. (1) MEV

$5.50 \mathrm{MEV}$

6. Q1) MEV

$6.50 \mathrm{MEV}$

$7.06 \mathrm{MEV}$

$7.50 \mathrm{MEV}$

8. QO MEV

8.5月 MEV

9. DO MEV

9,50 MEV

10. OO MEV

$11.00 \mathrm{MEV}$

12, OU MEV

13.DQ MEVV

14.DQ MEV

$15,00 \mathrm{MEV}$

16. $10 \mathrm{MEV}$

17, OU MEV

18. $\triangle O M E V$

$19.00 \mathrm{MEV}$

20.OA MEV

21. OD MEV

$22.00 \mathrm{MFV}$

23,00 MEV

$2400 \mathrm{MEV}$

25. MO MEV

26. OQ MEV

$27 . D D$ MEV

$28.00 \mathrm{MEV}$

$29,00 \mathrm{MEV}$

$30.40 \mathrm{MEV}$

$32.0 U$ MEV

34.00 MEV

36.00 MEV

$38.00 \mathrm{MEV}$

$40.40 \mathrm{MEV}$

42, DD MEV

$44.00 \mathrm{MEV}$

46.DD MEV

48. OU MEV

50,OO MEV
PKOTONS

2.7AE-W1

3. $52 E-11$

$2.66 \mathrm{E}-101$

$3,5 \mid E-D 1$

3. $17 E-01$

$3.44[-1] 1$

$3.65 E-11$

$3.43 E-41$

$3.64 E-111$

$3.93 E-01$

3. $48 E=31$

3. $46 E=A 1$

3.5 A $=$ H 1

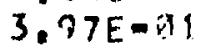

$3,57 E-E !$

3. $85 E-1) 1$

$3.54 E_{-1}-1$

$3.92 E-1$

3. $82 . \mathrm{D}=1$

3. $A \triangle E-B 1$

3. $42 E-B 1$

3. $25 E=11$

$3.37 E-W 1$

3. WAE-O 1

3. $\{B E-W 1$

2. $90 \mathrm{E}-\mathrm{B} 1$

2. $66 \mathrm{~F}-\mathrm{U1}$

2.11F=日1

2.53E-

2. i) $6 E-$ i 1

1. B1E- 11

$1.76 E-U 1$

$1.60 E-01$

$1.23 E-01$

1. $25 E-01$

?. $84 F-U$ ?

$7.13 E-12$

$4.74 E-02$

$3.51 E-\triangle Z$

4. $32 E-13$

b.

a.

a.

a.

14.73
DEUTEKDIS

$1.86 E-04$

1. 15E-04

1. $73 E-64$

1.24E-1) 4

1. 38E-A4

$1.56 E=014$

3. $\triangle 2 E=\square 4$

5. $40 E=95$

1.13E-H4

5.8OE-V5

1. $12 E=04$

$1.5 n E-115$

3.1 1 UE -195

1. $75 E-0.5$

$3.76 E-115$

$4.56 E-116$

$9.66 E-116$

7. GUE-VI

1. $32 E-110$

(3)

D.

i.

0

$a$

0.

a.

.

a.

a.

a.

a.

$a$

$\theta$

0.

a.

$\theta$

a.

0

$a$

a.

0

4.26
THITUNS

$1.39 t-6 j 4$

$1.84 E=114$

3. $4 M E-b 4$

1. $41 E-45$

2. $27 E-15$

$1.55 \mathrm{E}-45$

3. 3 Q -115

B. $79 E-176$

$1.41 E-65$

$h, 13 E-96$

9.75E- 16

5. 12E $=06$

1. 1 $4 E-115$

1. $26 E-B 6$

2. $45 E-116$

$7.27 E-V 7$

$1.64 E-106$

(i.

U.

a.

a.

A.

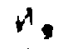

u.

i).

$\theta$.

v.

b.

b)

b.

4

it.

$\theta$

a.

0

i).

6

b.

b.

b)

4

is

$\theta$

3.88
ALPF:AS

5.7 of $-A\}$

7. 3UE- US

$7.76 E-13$

$6.32 E-43$

$6,47 E-D 3$

4. $56 E-D^{3} 3$

9. T9E-US

4. $39 E-\triangle 3$

ค. $52 E-73$

4. 1 UE- 13

S. $03 \mathrm{E}-\mathrm{S} 3$

2. $47 E-03$

$5, D E=13$

$1.02 t-A s$

a. vut - is 3

$1.75 t-n s$

$3,21 E-13$

$1,13 E-G S$

?. HSE-QS

6. 32E- $=14$

1. $22 E=\square 3$

$3.71 E-b 4$

$9,5 \mathrm{H} E-134$

$3.42 E=1.35$

$7,99 E-105$

a.

1).

b.

b.

14

1).

a.

i.

a.

1.

H.

v.

u.

a.

G.

b.

0.

$\theta$

D.

a.

5.78 
1SLOWING DOWN SPECTRUM AT 14.A CM. FOR ICRU MUSCLE IISSUE 42 MEV P+ ON BE NEUTRON SPECTRUA

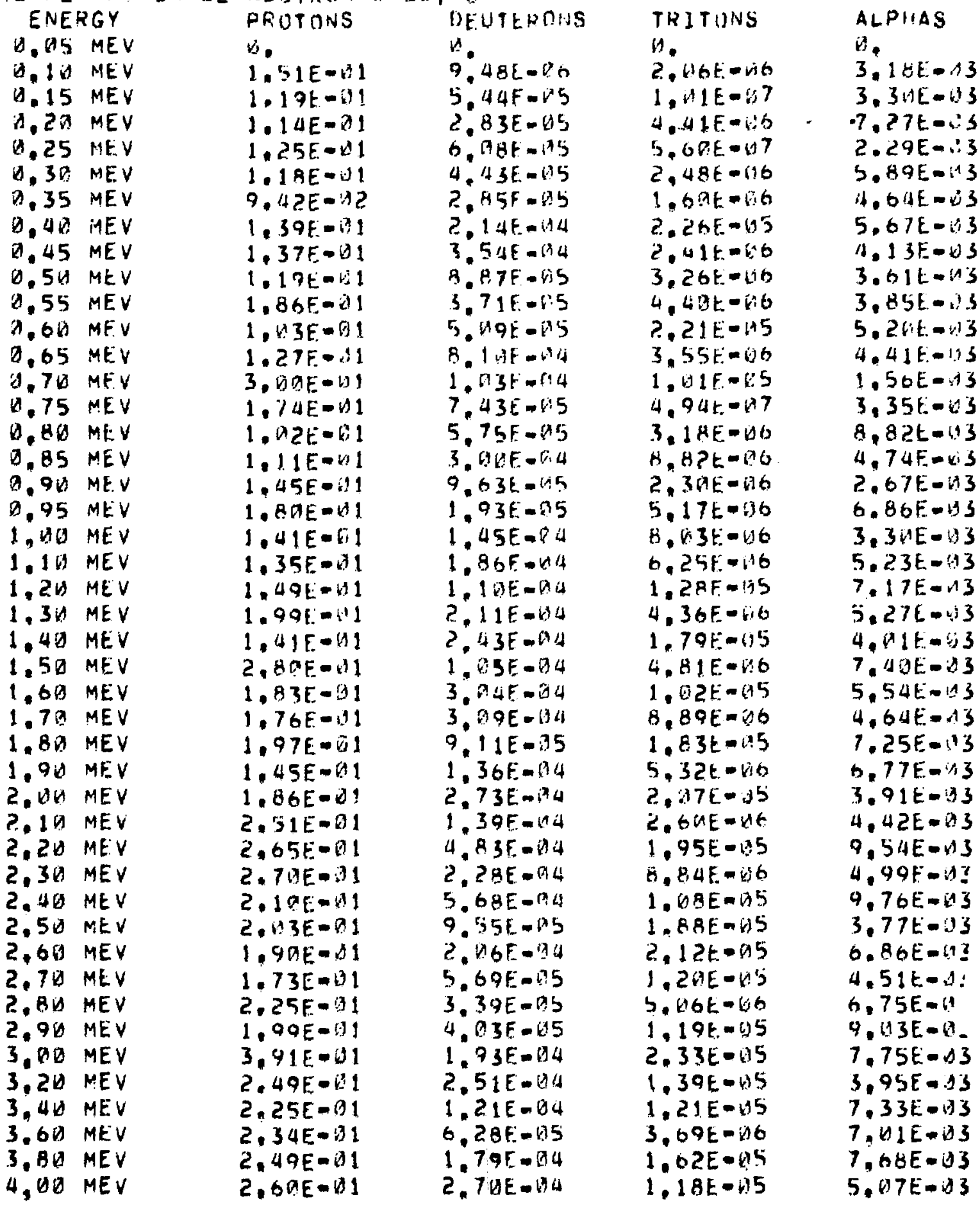


ISLOWING DONN SPECTRIIM AT IL, CM. FON ICPU MUSCLE IISSUE Q2 MEV P+ DN BE NEUTEON SPECTRUM

ENERGY

4.26 MEV

4,40 iUE

$4.60 \mathrm{MEV}$

4.8D MEV

5. $A D$ MEV

$5.5 \%$ MEV

6. OL MEV

6. 50 MEV

7. $\triangle U$ MEY

7.5A MEV

8. UV MEV

8.5 $\triangle$ MEV

9.00 MEV

$9.50 \mathrm{MEV}$

1F. $O D$ MEV

$11 . H B$ MEV

12. M MEV

$13 . D D$ MEV

14. $A V$ MEV

15. $A O M E V$

16. OA MEV

$17.0 \mathrm{MEV}$

18, BO MEV

19.0O MEV

2Q. OO MEV

$21.0 D M E V$

$22.00 \mathrm{MEV}$

23.00 MEV

24, UA HEV

25,00 MEV

26. OD MEV

$27.00 \mathrm{MEV}$

28. BO MEV

$29.0 \mathrm{~V} M E V$

30.00 MEV

$32.00 \mathrm{MEV}$

34.00 MEV

36. OU MEV

$38.00 \mathrm{MEV}$

$40.00 \mathrm{MEV}$

42. BD MEV

$44, D O M E V$

46,00 MEV

$48.00 \mathrm{MEV}$

$50.6 U$ MEV

RAVE. E(MEV)
PRUTINS

2. 5 hE -1

2. $1 F=1$

2. $4 h E=31$

2. $7 \cap E-31$

2. $81 E-11$

3. $15 E=61$

2.81F.-31

2. $855-1$

2. $86 E-A 1$

3. $25 \mathrm{E}-\mathrm{Cl}$

2. $67 \mathrm{E}-01$

$2.83 E-01$

2. $.99=1$

3. $49 E-61$

3. $35 E-01$

$3.11 E-1$

2. $\triangle T E-D 1$

3. $24 E-61$

$2.92 E-111$

$2.81 \mathrm{E}=31$

2. $11 E-M !$

$2,64 E-11$

$2.64 t-11$

$2.53 t-k 1$

2. $44 E-111$

2. $25 E-31$

2. $24 E-k i 1$

$1.66 E-11$

1. BAE-A1

$1.71 E-W 1$

1. $5 A E-\lambda 1$

$1.24 E-01$

1. $27 E-D 1$

$8.78 E=.32$

$9.77 E-W 2$

$8.64 E-22$

$5.77 E-02$

2. $14 E-112$

1.5 2 (1) - 62

$0.95 E-93$

0 .

0

b.

$\theta$

b.
DEUTEKONS

$1.17 E-n_{A}$

1. $27 F=174$

3.27F.

2. MGF-Ma

a. $77 F=14$

1. $59 E-B 4$

2. $53 E-14$

1.78F-D.4

2. 36F-A 4

2. 1 IE- D

11. $205-B 4$

1. $3 E-05$

1. $95 E-175$

1. $43 E-95$

?. $76 \mathrm{~F}-1 \mathrm{~S}$

$6.28 E-176$

1. 13E- 15

$4.50 E-176$

8. $99 E-116$

$n$

$\theta$

4

3

ti

a

a.

$n$

a

a

$a$

$a$

0

$b$

$a$

a

$a$

0

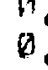

0

0.

4.71
TRITONS

i. $x^{3} 3 t=.15$

$6.6 B E-116$

i. $47 \mathrm{E}-0 \mathrm{~S}$

2. $8 U E-.16$

1. $10 E-.15$

$9.50 E-40$

i. $51 t-15$

1. $15 E-1.5$

1. $72 E-15$

B. $37 E-116$

1. $21 E-115$

$7.26 E=06$

$1.66 E-115$

$1.15 E=06$

$1.15 \mathrm{c}=06$

$1.79 E=t)$

$3.32 E-16$

1. $V 3 F-U 6$

2. $5 F-126$

i.

i.

b)

w.

0

i.

4.

1

u.

b)

त.

i)

U.

iv.

b.

(1)

t.

a.

b)

b.

i.

ti.

b.

b.

5.40
ALPHAS

5. 7 TE $\triangle 3$

6. HUE-OIS

$5.91 E-$ in 3

S. $3 G E-A S$

$6.19 E-A 3$

$3.57 E-3$

$7.56 E-1.3$

$3.98 E-B 3$

$6.935-\pi 3$

$3.51 F-.13$

4. $02 E-43$

2. $61 E-N S$

$4.67 E-193$

1. 3YE $=.33$

$3.2 U E=113$

1. $85 F_{-}=3$

$3.67 \mathrm{E}=\mathrm{A3}$

$9.22 E-H 4$

$1.65 E=.33$

$4.56 E-34$

$7.0 \Delta E-t^{2} 4$

4. 4 GE - $\mathrm{HI}_{4}$

$9.93 E-144$

1. $30 \mathrm{E}-14$

2. $92 E-14$

b.

b.

in.

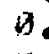

4

i.

id.

e.

0

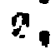

1

0

1.

$D$

3.

a.

n.

b.

a.

6.13 
IINITIAL SPECTRUM AT A.BB-W. HS CM. FOR SIHOHKA A-1SU PLASTIC 42 MEV P+ ON BE NEUTPOIV SPECTRUM

\section{ENERGY}

D. 05 MEV

7.10 MEV

0.15 MEV

0.20 MEV

$0.25 \mathrm{MEV}$

$0.31 \mathrm{MEV}$

$0.35 \mathrm{MEV}$

0.4 MEV

0.45 MEV

$0.5 \square \mathrm{MEV}$

$0.55 \mathrm{MEV}$

$0.60 \mathrm{MEV}$

$0.65 \mathrm{MEV}$

$0.70 \mathrm{MEV}$

0.75 MEV

D. 8 G MEV

D.85 MEV

$D, 9$ MEV

0.95 MEY

1.90 MEV

$1.10 \mathrm{MEV}$

$1.20 \mathrm{MEV}$

$1,30 \mathrm{MEV}$

$1.40 \mathrm{MEV}$

1.50 MEV

$1.60 \mathrm{MEV}$

$1,70 \mathrm{MEV}$

1.8 \& MEV

$1.90 \mathrm{MEV}$

2.00 MEV

2.10 MEV

2.20 MEV

2.30 MEV

$2.40 \mathrm{MEV}$

$2,50 \mathrm{MEV}$

2,60 MEV

$2,7 @$ MEV

2,80 MEV

$2,90 \mathrm{MEV}$

3. DO MEV

3.20 MEV

3,40 MEV

3.60 MEV

$3,80 \mathrm{MEV}$

4.DO MEV
PROTONS

i.

4.5 $53 E+92$

$1.60 E+112$

$6.00 E+01$

$1.31 E+42$

$1.94 E+01$

3. $91 E+\theta 1$

9. $B 1 E+01$

4. BดE +DI

$3.79 E+D 1$

$1.16 E+D 2$

2. $U D E+D 1$

$\theta$.

$\theta$.

6. BQAE+ is 1

2.1 $13 E+61$

$9.51 E=\Delta 2$

$6.43 E+B 1$

2.5UE-DI

6.

$1.59 E+02$

4. $02 E+91$

$3.82 E+101$

$6.92 E+01$

$5,79 E+B 1$

$2.04 E+D 1$

$5.87 E+11$

8. DOE DI

4. ODE + B 1

$5,78 E+11$

2. DटE+ 1

$3.81 E+51$

4. UDE+UI

2. ज1E+O1

2. $\triangle a E+b 1$

2. $\Delta U E+B 1$

$6 . B O E+B 1$

$2.03 E+D 1$

2. DIE+ 1

a.

4. OPE+!) 1

$5.23 E+01$

$9.9 B E+D 1$

$6, D I E+D 1$

7. DQE $+D 1$

\section{DFUTEROIS}

0.
$0:$
$0:$
$0:$
$0:$
$0:$
$0:$
$0:$
$0:$

$1.41 E-A^{2} 1$

$1.41 E-B ! 1$

6.

$n$.

$7: 23 E-6) ?$

7. $23 \mathrm{E}-92$

:

:

a.

1:.

$1.93 \mathrm{E}-02$

1 . $93 E-12$

:

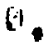

2. $43 E-112$

2. $4 \Delta E-A 2$

i.

a:

:

5.

$5,64 E-25$

$5.64 E-U 2$

a.

म.ं
TRITONS

D.

$\lambda$

r.

b.

is.

is.

is,

n.

$b$.

in.

$n$.

$n$.

b.

i.

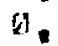

$n$.

is.

$n$

a.

H.

,

is

$\theta$

$D$

4

$n$

$\theta$

$\pi$

$n$.

b.

$b$.

is.

b.

n.

$n$.

u.

b.

o.

n.

4.

$1.92 E-11$

$1.92 \mathrm{E}-\mathrm{B} 1$

4.

b.

a.
ALPHAS

1. $\triangle 2 E-D 1$

1. $\triangle 2 E-D 1$

$1.31 E=1$

$1,31 E=1$

$3.29 E-41$

$3.29 E-01$

$2.52 E+3.1$

2. $52 E+b \theta$

$2.32 E+04$

$2.32 E+a: i$

$3,41 E-11$

$3.41 E-1$

$n$.

4.

$4, B 2 E-D 1$

$4.82 E-61$

2. $52 E+4 \Delta$

2. $52 E+5(5)$

$1.71 E+B 1$

$1.71 E+B 1$

$1.53 E+D 1$

$1.53 E+D 1$

2. $4 B E+A B$

?. $4 A E+A B$

$1.50 E+46$

$1.50 E+\Delta 0$

$2.76 E+10 j$

$2.76 E+100$

$6.97 E+d U$

$6.67 E+40$

$1,55 E+11$

$1.55 E+111$

4. $B 1 E+B L^{2}$

4. $D 1 E+i^{A} U$

$1.62 E+C 1$

$1.62 E+11$

$3.56 E+1060$

3. 5 VIE+ DU

$1,27 E+D 1$

1. $27 E+11$

$1,33 E+d 1$

$1.33 E+111$

3. $54 E+46$

3.54E+DD

$4.86 E+11$ 
IINITIAL SPECTRUM AT D, BA-O, H5 CM. FOR SHONKA A-15O PLASTIC 42 MEV P + ON HE NEUTROH SPECTRUM

\section{ENERGY}

4. 20 MEV

4.40 MEV

4.60 MEV

4.80 MEV

5. $\triangle 0$ MEV

5.50 MEV

6. 00 MEV

6.5 A MEV

7. $O 2$ MEV

7.5 MEV

8.00 MEV

8.5口 MEV

9. DQ MEV

9.50 MEV

$10,00 \mathrm{MEV}$

11. 10 MEV

$12.80 \mathrm{MEV}$

13. DO MEV

14.00 MEV

$15.0 \% \mathrm{MEV}$

$16.00 \mathrm{MEV}$

$17,09 \mathrm{MEV}$

$18,00 \mathrm{MEV}$

19. $\triangle D M E V$

$20.00 \mathrm{MEV}$

$21.00 \mathrm{MEV}$

$22.00 \mathrm{MEV}$

$23.00 \mathrm{MEV}$

24.00 MEV

25.00 MEV

$26.00 \mathrm{MEV}$

$27.00 \mathrm{MEV}$

$28.00 \mathrm{MEV}$

$29.0 M E V$

30.100 MEV

32. UO MEV

34. OU MEV

36,00 MEV

38.OU MEV

40.00 MEV

42.00 MEV

$44.00 \mathrm{MEV}$

46. DD MEV

4B. SO MEV

50.BD MEV

\section{PROTONS}

5.69E+ 11

4. $U 1 E+Q 1$

8. $1 E+91$

2. $01 E+D 1$

6. DIE+01

$7.86 E+11$

$3.73 E+01$

$2,86 E=01$

4. $.9 E+01$

1. UAE $=$ त I

4. D1E+U1

2. $19 E+B 1$

औ.

2. $31 E+11$

U.

3. 그믄 1

2. $b . M F+1$

S.

2. DOE $+D 1$

2. $90 E+01$

4. DOE + 31

a.

2. $\partial G E+B 1$

2. $B A E+B 1$

4. JดE + DI

a.

2. $B O E+O 1$

U.

2. DAE+D1

2. $D O E+21$

4. BOE+ 11

b.

0.

6

a.

2. $\triangle O E+01$

$\therefore$

0

0

n.

$a$

$\Delta$

$\theta$.

$\theta$

0.
DEUTEROAS

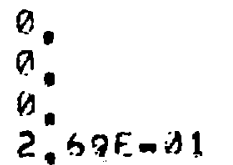

$2.69 E-31$

a.

n.

1.81t-U2

1. BIF-A2

$\Delta$

in.

$v$

$n$

a.

a

(1)

()

$a$

$a$

a.

a.

$\Delta$

o.

b.

$a$

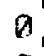

0

0

$n$

b.

$\theta$

a.

a.

0

in.

a

$a$

$a$

0

3.90
TP I TONS

0.

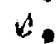

is.

i.

iv.

(i)

b.

a.

i.

i.

0.

v.

$v$

0 .

b.

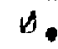

1.

$v$

is

i.

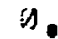

b.

in.

v.

0.

$v$

$\checkmark$

.

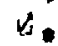

$D$

D)

D.

U.

$u$.

b.

w.

is.

$\dot{\theta}$

o.

n.

is.

i.

$b_{0}$

$\theta$

0.

3.20
ALPHAS

4. $B \rightarrow E+41$

$1.25 t+41$

1. $25 E+11$

1. $A 9 E+11$

1. $D 9 E+D 1$

$1.64 E+b i j$

$1.64 E+V 0$

1. $24 E+B D$

1. $24 E+10$

1. $\triangle S E+11$

1. $n 6 E+11$

1. $\Delta 2 E+\Delta 1$

1. $(4-2 E+4) 1$

$8.60 F-42$

B. $6 V E-V Z$

$5,3 B E-B]$

5. $3 B E=\square 1$

1. $) 1 E+B 1$

1. $) 1 E+U 1$

i.

$\theta$

n.

n.

a.

o.

$\theta$

8.

b.

$\theta$

9.

$n$

$\theta$

$\theta$

0.

v.

6.

b.

n.

v.

0.

a.

u.

0.

0.

6.42 
IINITIAL SPECTRUM AT O.J5-O.1A CH, FOR SHOHKA A-ISU FLASTIC 42 MEV P + ON HE NEUTRON SPECTRIJM

\section{ENERGY}

D. DS MEV

3.10 MEV

Q.15 MEV

0.20 MEV

0.25 MEV

$0.30 \mathrm{MEV}$

$0.35 \mathrm{MEV}$

$0.4 D$ MEV

$0.45 \mathrm{MEV}$

0.50 MEV

0,55 MEV

0.60 MEV

0.65 MEV

0.70 MEV

0.75 MEV

$0.81 \mathrm{MEV}$

0.85 MEV

$0.90 \mathrm{MEV}$

0.95 MEV

1. OU MEV

1.10 MEV

1.20 MEV

$1.30 \mathrm{MEV}$

1. $40 \mathrm{MEV}$

1.50 MEV

1.60 MEV

1.70 MEV

1.8D MEV

1.90 MEV

2. OO MEV

2.10 HEV - -

2.20 MEV

2.30 MEV

2. 40 MEV

2.50 MEV

2.60 MEV

$2.70 \mathrm{MEV}$

2.80 MEV

2.90 MEV

3. OD MEV

3,20 MEV

$3.40 \mathrm{MEV}$

3.60 MEV

3.80 MEV

$4.00 \mathrm{MEV}$
PRDTONS

4.

5. $25 E+11 ?$

2.7PE IS?

$9.79 E+191$

$3.95 E+31$

7. $54 E+b 1$

A. OOE + OI

B. $\triangle A E+B 1$

3.76E+01

8. DOE + 11

1. $O Q E+42$

$5,86 E+11$

9.

2. $6 O E+k^{2} 1$

4. $2 E+b 1$

1. $43 E-61$

2. $4 \mathrm{HE}+01$

$6.27 E-02$ ?

2. $02 E+101$

$3.84 E+61$

6.U3E+ 11

9. $8 B E+61$

$6.48 E+4 !$

$6.03 E+41$

$2.03 E+D 1$

5. $85 E+D 1$

6. $43 E+01$

5. $38 E+191$

$9.59 E+D O$

2. $D A E+B 1$

की $02 E+61$

$5,76 E+D 1$

2. $91 E+61$

2. $144 E+13$

6. $D 2 E+61$

1. $20 \mathrm{E}=\mathrm{V} 1$

2. 54E- 21

$2.05 E+01$

L.

4. DAE+ OI

2. DQE + 1

4. DAE +DI

$5.71 E+B 1$

1. $09 E-U 1$

7. $+1 E+B 1$
DEUTEKONS

a.

a.

a.

B.

17

i.

6.995 .112

6. 99E ma2

a.

6.

$0.98 E-0.2$

$6.98 E-12$

14

0

B.

i)

$4.64 E-1.2$

$4.64 E-142$

$1.57 E-01$

$1.57 E-101$

0.

a.

3. $85 F-D 2$

3. $85 E-02$

$2.90 E-112$

$2.90 E-12$

1. $34 E-D 1$

$1,34 \mathrm{~F}=01$.

a.

a.

a.

$b$

o.

a.

?.

2. $47 E-172$

2. $47 E-02$

$?$

a.

0

is.

a.

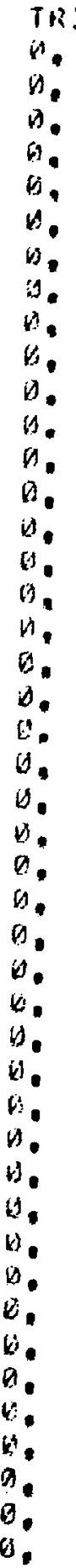

ALPiAs

A.

$\theta$

1. $49 E-b^{3} 1$

1. $49 E-B 1$

A. $17 E-B 1$

4. $17 E-101$

$1.95 E-\Delta 2$

$1.95 E-112$

3. $24 E-A 1$

3. 2AE-A1

1. $35 E-11$

1. 3SE- 11

1. $\triangle E E-B 1$

1. $46 E-B 1$

$1.67 E-(1) 2$

1.67ENO?

$1.25 E-G 1$

$1.25 E-B 1$

3. $05 E-1$

3. $\triangle 5 E-161$

1. $25 E-01$

$1.25 E-01$

1. $\triangle B E+D 1$

$1, G A E+\square 1$

1. $\triangle B E E+D 1$

1. $66 E+191$

1.20E+ 20

1. $26 E+B O B$

$3.85 E+B D$

$3.85 E+0$.

1. $22 E+61$

1. $22 E+i 1$

1. $2 \Delta E+B V$

1. $2 G E+D V$

1. $13 E+61$

1. $13 E+D 1$

3. $47 E+4 V$

3. $47 E+B B$

1.73E+ $9 B$

1. $73 E+Q V$

2. $27 E+131$

2. $27 E+\Delta 1$

1. $2 M E+D C$

1. $2 B E+B G$

4. $3 B E+\triangle B$ 
IINITIAL SPECTRUM AT $9.05-6.1 G$ CH. FOK SHONKA A-ISU FLASTIC 42 MEV P+ ON BE NEUTRON SPECTPUM

\section{ENERGY}

4.2U MEV

4.40 MEV

4.60 MEV

4. 80 MEV

$5.00 \mathrm{MEV}$

$5.50 \mathrm{MEV}$

6. $O D$ MEV

$6.50 \mathrm{MEV}$

7. DO MEV

$7.50 \mathrm{MEV}$

8. UA MEV

$8.5 B$ MEV

$9 . \triangle V M E V$

$9.50 \mathrm{MEV}$

16. OB MEV

11. OO MEV

12.00 MEV

13, OO MEV

14. OQ MEV

15.0V MEV

16. DO MEV

17.0Q MEV

$18 . \triangle O$ MEV

$19.00 \mathrm{MEV}$

$20.00 M E V$

$21.00 \mathrm{MEV}$

22. OO MEV

23.00 MEV

24.00 NE.V

25. DD MEV

26.00 MEV

$27.00 \mathrm{MEV}$

28.0 MEV

29.00 MEV

$30.00 \mathrm{MEV}$

32. OA MEV

34.01 MEV

36.00 MEV

$38.00 \mathrm{MEV}$

40.00 MEV

42.00 MEV

44.00 MEV

46.00 MEV

$48.60 \mathrm{MEV}$

$50.00 \mathrm{MEV}$

GAVE, E(MEV)

\section{OROTONS}

4. $\triangle G E+U 1$

2. $\Delta P . F+\Delta 1$

6. $\triangle 4 E+\square 1$

2. $33 E-\Delta 1$

4. $1 E+D 1$

4. GQEE+D1

7. $87 E+$ BI

6. $12 E+d 1$

1. $19 E+\square 2$

2. $b 3 E+11$

6. DOE +OI

6. $A R E+D$

2. $\triangle A E+B I$

2. $10 E+\Delta 1$

6. $19 E+1$

2. $A M E+D 1$

1. $\Delta A F+\Delta z$

2. $\triangle A E+\triangle 1$

2. OAE +Q1

4. $\triangle A E+B 1$

2. GAE + DI

4. BaE +1

b. VOE + D 1

2. GaE+ 1

2. ODE +O1

ह.

2. OGE + 1

2. UAE+D 1

0.

$\theta$

a.

o.

a.

2. DดE+01

$\theta$

0.

$\theta$

$\theta$

0

0.

a.

iv.

a.

b.

$a$

9.93
DFUTFROHS

V.

a.

a.

n.

r.

a.

$\theta$

a.

a.

i)

?.

v.

a.

$a$

a.

b.

b.

$\theta$

i.

a.

a.

i.

$\theta$

$a$

a.

a.

$a$

a.

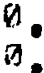

a.

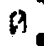

A.

H.

a.

$a$

a.

0

$a$

$a$

1.40
TR I T ONS

$\%$

6

a.

b.

$\therefore$

a.

$\because$

1.

i.

t.

E.

a.

A.

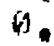

vi.

i.

a.

b.

b.

v.

a.

i.

in.

6.

$b$

b.

b.

i.

b.

b.

a.

0.

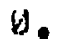

i.

$D$.

9.

b.

D.

0.

0.

a.

0.

3.20
ALPHAS

4. $3 B E+13$

2.B7E+ $B D$

$2.87 E+i d\}$

1. $A \in E+(A)$

1. $\triangle C E+\triangle B$

$5.42 E-H 1$

5. $42 E-B 1$

$1.12 E+B 1$

1. $12 E+n 1$

7. B TE-V1

7. $\triangle 7 E-11$

$9.48 E-1$

$9.48 E-11$

$3.41 E-D 1$

$3.41 E-11$

$4.15 E-A 1$

$4,15 E-01$

to.

$b_{2}$

b.

G.

b.

ด.

a.

a.

$\theta$.

$\theta$.

a.

a.

b)

a.

6

a.

a.

$\theta$.

a.

$\theta$.

a.

.

$U$

b.

$\theta$

$b_{0}$

a.

b.

4.33 


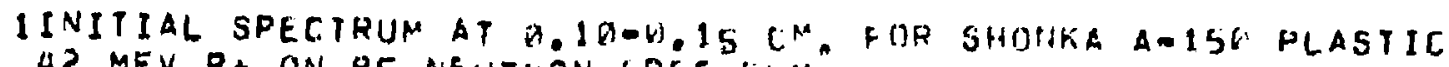
42 MEV P+ ON BE NEUTHON SPECTKLIP

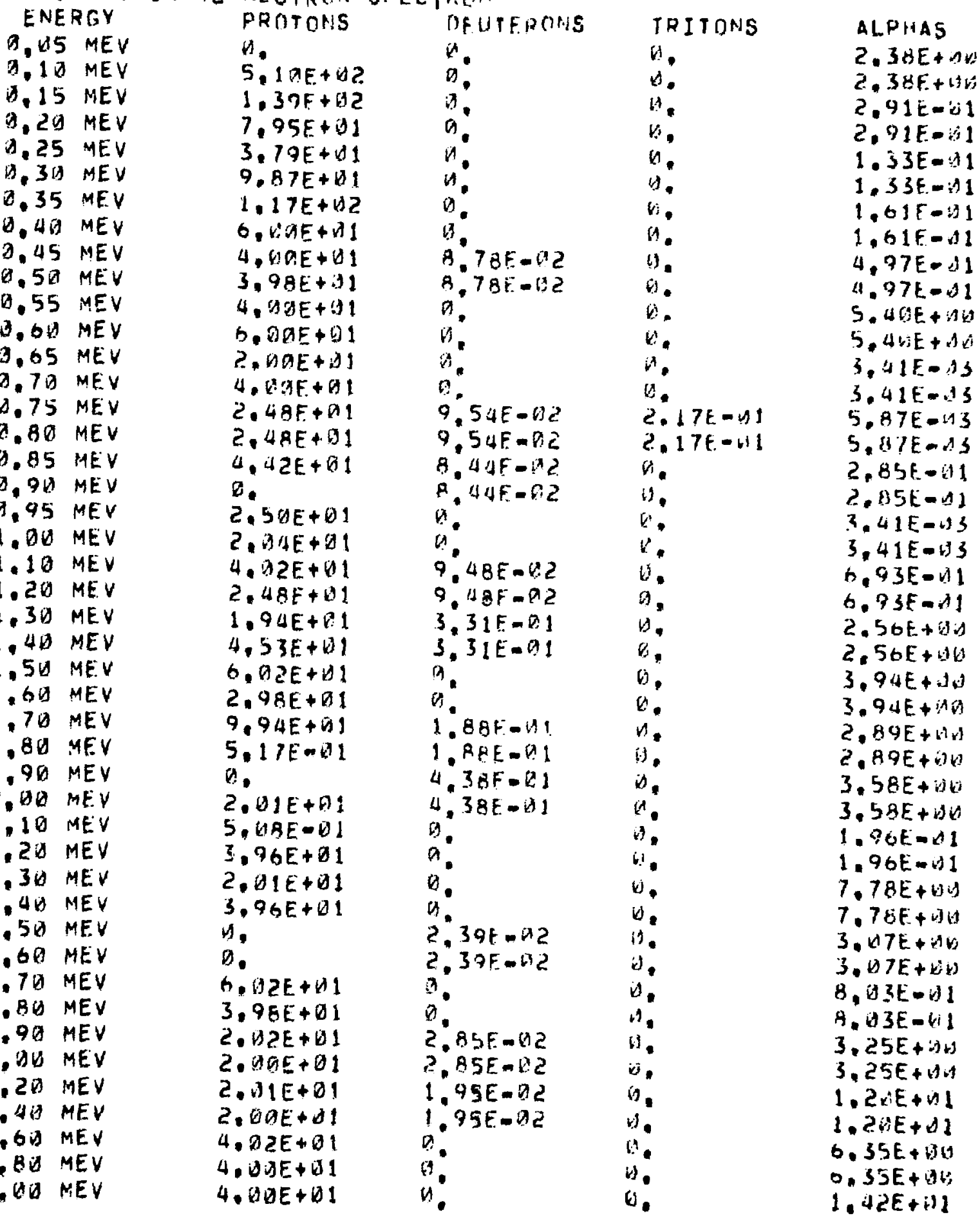


1INITIAL SPECTRUM AT 0.1H-10.15 CH, FCR SHONKA A-15U PLASTIC 42 MEV P+ ON BE NEUTRON SPECTR!II

\section{ENERGY}

4. $20 \mathrm{MEV}$

4. $40 \mathrm{MEV}$

4.60 MEV

4.80 MEV

5. DO MEV

$5,5 \square M E V$

6. $\triangle 0$ MEV

$6.50 \mathrm{MEV}$

7. OD MEV

$7.50 \mathrm{MEV}$

8. DU MEV

$8.5 B$ MEV

3. DO MEV

$9.50 \mathrm{MEV}$

$19.00 \mathrm{MEV}$

$11.00 \mathrm{MEV}$

12. DO MEV

13.DO MEV

14. $20 \mathrm{MEV}$

$15.00 \mathrm{MEV}$

16.00 MEV

$17.00 \mathrm{MEV}$

$18.00 \mathrm{MEV}$

$19.00 \mathrm{MEV}$

$20.00 \mathrm{MEV}$

21.00 MEV

$22, \partial D M E V$

$23.00 \mathrm{MEV}$

$24.01 \mathrm{MEV}$

25.00 MEV

26. OD MEV

$27.01 M E V$

28.20 MEY

29.00 MEV

$30.00 \mathrm{MEV}$

32.00 MEV

$34, D V$ MEV

$36.00 \mathrm{MEV}$

$38.00 \mathrm{MEV}$

40. UG MEV

42. UV MEV

44. BW MEV

46. OD MEV

$48.00 \mathrm{MEV}$

50.00 MEV

GAVE, E(MEV)
PROTONS

2. $\triangle 6 E+D 1$

1. $51 \mathrm{E}-01$

2. $02 E+B 1$

8. $D 2 E+11$

2. $U A F_{-}+1$

2. USE+Q1

1. $91 E+\square 2$

6. D $3 E+b 1$

4. OUE + O I

8. $02 F+01$

4. OAE + A1

4. DIE+ II

$\theta$.

$2, B D E+01$

$\theta$.

4. DOE +DI

2. $D A E+D 1$

2. $\triangle A E+B 1$

2. $d a F+3 !$

B.

D.

$6.00 E+D I$

2. $v a E+b 1$

b.

2. AGE + DI

B.

6

a.

$\Delta$.

2. $\triangle A E+D 1$

b.

b.

b.

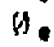

$\theta$

i)

0

$\theta$

b.

b.

0.

0.

0.

b.

0.

$8,4 ?$
DEUTERDIS

$1: 98 E-92$

1. 9 \&E $=$ 的

0

0

a.

H.

0

$n$

0

0

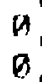

$\theta$

0

9.

6

$a$

0

$B$

0

6

$a$

0

0

0

a.

a.

a.

a.

$B$

0

0

ด.

0

0

D.

B.

1.50
TRITUNS

$\Delta$.

a)

n.

a.

v.

$\Delta$.

$\theta$

i.

b.

0

n.

i.

b.

0.

if.

w.

a.

U.

b.

n.

D.

is.

n.

b.

*

(1).

G.

b.

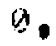

i.

i.

b.

(1).

4.

1

$\dot{v}$

6.

a.

i1.

b.

9.

2 .

0

o.

9.75
ALPHAS

1. $42 E+\Delta 1$

7.1 GE-YI

7. IGE-DI

1. $14 E+\Delta \Delta$

$1.14 E+4$

$1.39 E+41$

$1,39 E+11$

I. $2.2 E+1 A v$

1. $22 E+\Delta v$

1. $\triangle 4 E+B i$

1. $A 4 E+B n$

9. $13 E-B 1$

$9.13 E-B 1$

3. 39E-DI 1

$3,59 E-$ id 1

4. $5 U E-U 1$

4. $5 W E-U 1$

1. $32 E-V 1$

$1.32 E-W 1$

i.

a.

U.

n.

$\Delta$

$\Delta$

$\theta$

a.

$\Delta$

b.

$b$.

a.

0.

ib.

g,

S.

.

b.

9.

6.

,

a.

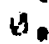

$a$

0.

i).

4.60 
1INITIAL SPECTRUM AT O,15-9,2日 CM. FOR SHONKA A-15\% PLASTIC 42 MEV P+ ON BE NEUTRON SPECTRUM

\section{ENERGY}

D. 15 MEV

$0.10 \mathrm{MEV}$

D.15 MEV

$0.20 \mathrm{MEV}$

0.25 MEV

0.3 B MEV

$0.35 \mathrm{MEV}$

0.40 MEV

$0.45 \mathrm{MEV}$

$0.50 \mathrm{MEV}$

$0.55 \mathrm{MEV}$

0.60 MEV

$0.65 \mathrm{MEV}$

0.7 U MEV

0.75 MEV

0.8 D MEV

D.BS MEV

$0,90 \mathrm{MEV}$

Q.95 MEV

1. $\triangle D$ MEV

1.19 MEV

$1.20 \mathrm{MEV}$

$1,30 \mathrm{MEV}$

$1.40 \mathrm{MEV}$

$1.50 \mathrm{MEV}$

$1.60 \mathrm{MEV}$

$1.70 \mathrm{MEV}$

$1.80 \mathrm{MEV}$

1.9 MEV

$2.00 \mathrm{MEV}$

2.10 MEV

2.20 MEV

2,30 MEV

2.40 MEV

2.50 MEV

2,60 MEV

2,70 MEV

2,80 MEV

2.90 MEV

3. DO MEV

3.20 MEV

3.40 MEV

3,60 MEV

$3,80 \mathrm{MEV}$

$4,00 \mathrm{MEV}$

\begin{abstract}
PROTONS
\end{abstract}
D.

$4,33 E+D 2$

$1.76 F+1 ?$

2. $11 E+\dot{102}$

1. AQE + D2

1. $4 B E+112$

1.94E+9?

4. ODE + B I

1. $16 E+\square 2$

4. $\partial A E+01$

$6 . B D E+W 1$

$5.09 E+01$

$7.57 E+191$

6. () $2 E+31$

6. DPE+DI

$7.32 E+D !$

7. BQF- $=$ BD

$2,16 E+1$

4. DIE DI

B.

2. $45 E+1$

$2.99 E+61$

$2.49[+61$

2. $A Q E+Q 1$

$5.41 E+00$

4. $51 E+D 1$

$1.57 E+81$

$1.95 t+11$

$7.91 E+01$

$2.04 E+61$

4. B2E+BI

4. $\triangle A E+D$ I

1. $9(9 E+B)$

$1.52 E-01$

4.03E+ 01

$4.51 E+D !$

2. DIE+ 1

$\Delta$.

2. OAE +01

1. $80 E+\angle 1$

Q. QAE + DI

$2.03 E+01$

2. 26F- 01

$1.55 E-\theta 1$

$4.01 E+101$
DEUTEROHS

a.

a.

9

:

:

0

$1.74 E-n 2$

$1.74 E-62$

a:

D.

$9.48 E-62$

$9.48 E-192$

n.

.

$1.86 t-01$

$4.86 E-01$

$6.27 E-92$

6. 2.7E-VIC

?. $\triangle A E-D 2$

$9,4 B E-D 2$

$1.61 E-31$

$1.61 \mathrm{E}-\mathrm{OI}$

$1.98 E-n z$

$1.98[-102$

2. $22 E-B 1$

2. 2?E-A1

.

a.

Q.5UE-192

$8,50 E-102$

6. $54 \mathrm{~F}-12$

$6.54 E-22$

$1.9 \mathrm{UE}-\mathrm{OZ}_{2}$

$1.7 U E-\forall 2$

$4,53 E-02$

$4.53 E-92$

$1.61 \mathrm{E}-01$

$1.61 \mathrm{E}-01$

2. $8 U E-D 2$

2. $H A E-D 2$

2. $47 \mathrm{E}-D 2$

$2,47 E-112$

$5.53 E-D 2$

$5.53 \mathrm{E}-\mathrm{BC}$
TRITONS

B.

.

0.

n.

.

0.

0.

v.

a.

$\theta$

$\theta$

i.

n.

B.

$\theta$.

$v$.

$n$.

$n$.

$\theta$

a.

U.

6.

6

b.

$2,68 E-\theta 1$

$2,08 E-G 1$

0 .

b.

0.

$b$,

is

$\theta$.

$\theta$.

$B$.

$b$.

$\theta$

$D$

$D$.

n.

E.

is.

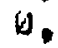

$\theta$

v.

$\theta$
ALPHAS

$4.12 E-\triangle 1$

4. $12 E=D 1$

7.21E-DI

$7.21 E-B 1$

b.

b.

6.9 GE-GL

$6.90 f-b 2$

$6.94 E-$ BI

$6,94 E-i) 1$

1. $\triangle 5 E+61$

$1.05 E+b 1$

1. $199 E+11$

1. GGE + II 1

4. $12 E-V) 1$

A. $12 E-61$

1. $156 E+b 1$

1. 106E+ 1

2. $44 E+140$

$2.44 E+40$

1. $135 E^{\circ}+1$

1. $B 3 E+W 1$

$7.6 B E+W B$

$7.68 E+1)$

2. $36 E+131$

2. $36 E+1$

1. $22 E+\Delta 0$

1. $22 E+B D$

$6.77 E-(1)$

$6.77 E-1$

$3.73 E+\Delta \theta$

$3,73 E+A v$

$7.26 E+4 D$

$7.26 E+96$

$2,36 E+111$

2. $36 E+111$

$5,64 E+U V$

$5.64 E+B D$

7.94E-D1

$7.94 E-01$

$2.24 E+121$

$2.24 E+111$

1. $B 2 E+B 1$

1. R2E+01

?. $91 E-D 1$ 
IINITIAL SPECIRUM AT D.15-1.2G CM. FOR SHONKA A-15O PLAETIC 42 MEV P+ ON BE NEUTRUN SPECTRUM

$$
\text { ENERGY }
$$

4.2W MEV

$4.40 \mathrm{MEV}$

4.6D MEV

4.81 MEV

$5,00 \mathrm{MEV}$

$5,50 \mathrm{MEV}$

6.00 MEV

$6,50 \mathrm{MEV}$

7. DIO MEV

$7.50 \mathrm{MEV}$

8,00 MEV

8,50 MEV

9 9 $\triangle$ MEV

$9.50 \mathrm{MEV}$

10. QOU MEV

11. UO MEV

$12,00 \mathrm{MEV}$

13,00 MEV

14. DO MEV

15.00 MEV

16.09 MEV

17. 00 MEV

18.01 MEV

19, OD MEV

20. DO MEV

21. DO MEV

$22.00 \mathrm{MEV}$

23.DO MEV

$24.06 \mathrm{MEV}$

$25, O D$ MEV

$26,00 \mathrm{MEV}$

27.0 MEV

2B. DS MEV

29. OB MEV

39.OD MEV

$32,00 \mathrm{MEV}$

34.00 MEV

36.00 MEV

38, OO MEV

4Q,DO MEV

$42,0 D$ MEV

44.00 MEV

46. UD MEV

4B. OO MEV

5D.00 MEV

GAVE. E(MEV)

\section{PROTONS}

$1.73 E-11$

$7,85 E-02$

2. $\triangle \triangle E E+D 1$

$5.60 \mathrm{E}-\mathrm{k}^{3} 1$

$2,01 E+01$

$6.05 E+01$

$6,07 E+01$

$6, \forall 3 E+\theta 1$

B. $1 B E+B 1$

$2.95 E+31$

$2,11 E-\lambda !$

4.D1E+ 01

$\Delta$.

4. बIE+ 11

2. WOE+ HI

1. $a b E+b z$

b. $\triangle A E+B 1$

$D, D G E+D !$

D.

D.

2. BBE + DI

2. $D A E+D 1$

2. $D D E+D 1$

$\theta$.

$D$.

4. DDE +D1

$\forall$.

D.

$y$.

0

o.

$\theta$

a.

G.

R. טOF+口1

$\theta$.

4. $B D E+21$

$\theta$.

0.

$\theta$

$a$

0.

D.

1.

$B$.

11.43

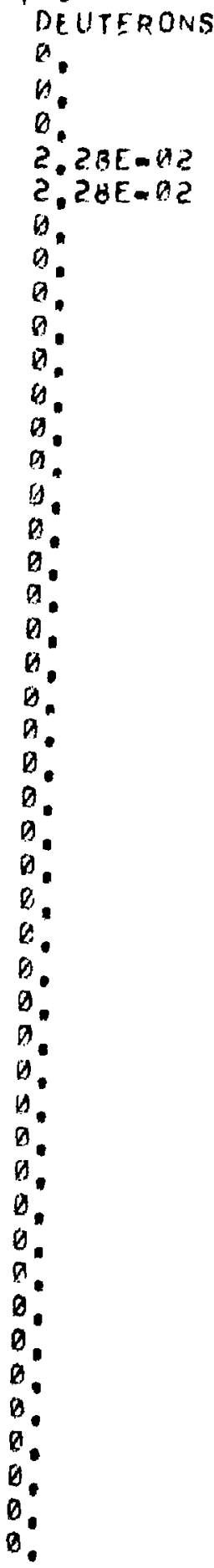

1.98

\section{TRITONS}

Q.

a.

Q.

9

(4)

$\Delta$

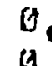

.

0

$\theta$

a.

0.

.

$\theta$

i.

is.

b.

b.

$\theta$

0

$B$.

b.

6.

$\theta$

b.

$\theta$.

i.

$\theta$

D.

0.

0

v.

0.

0.

1.50
ALFHAS

7.91E-AI

$5 . B 8 E+D \square$

5. $08 E+74$

1. $D 8 E+61$

1. BBE 61

$2.68 E+11$

$2.68 E+11$

1. $18 E+x y$

$1.18 E+U D$

$9.97 E-61$

$9.97 E . \Delta 1$

$7.44 E-11$

7. $\triangle A E-O 1$

$3.2 B E-B 1$

$3.28 E-01$

$1.64 E+111$

$1.04 E+D 1$

$1.61 E-11$

$1,61 E-d 1$

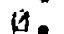

0.
0.
0.
0.
0.
0.
0.
0.
0.
0.
0.
0.
0.
0.
0.
0.

5.62 
IINITIAL SPECTPUM AT H.2O-D.4A CM, HOR SHOINKA AOI5O PLASTIC 42 MEV P + ON BE NEUTRON SPECTRUM

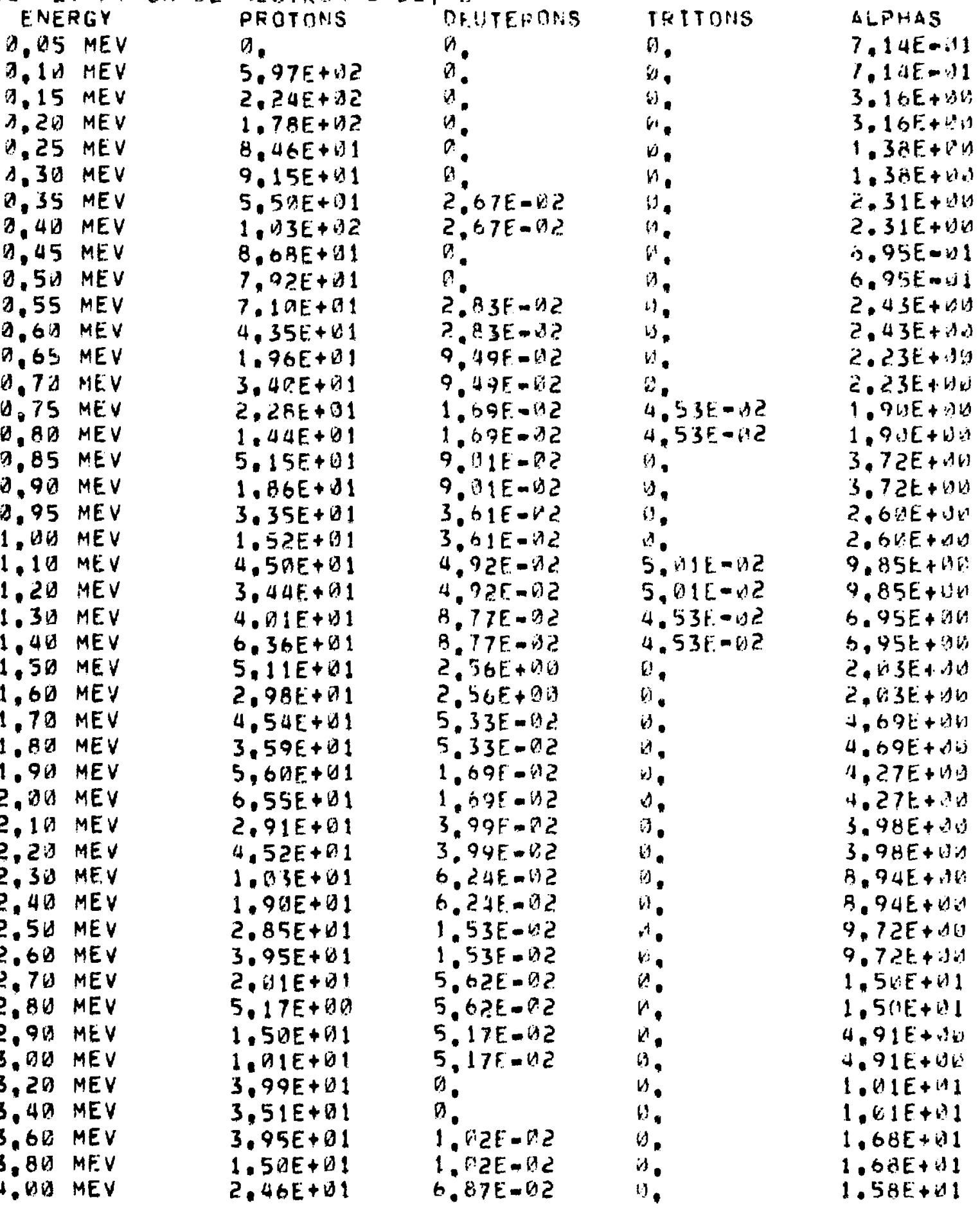


IINITIAL SPECTRUM AT $0.20-0.40$ CM. FGR SHQNKA A-15P PLASTIC 42 MEV P+ ON BE NEUTRON SPECTRUM

\section{ENERGY}

4.20 MEV

4.40 MEV

4.61 MEV

$4.80 \mathrm{MEV}$

5. 10 MEV

$5.50 \mathrm{MEV}$

6.00 MEV

$6.50 \mathrm{MEV}$

$7.01 \mathrm{MEV}$

7.50 MEV

$8.00 \mathrm{MEV}$

$8.58 \mathrm{MEV}$

Q.8Q MEV

$9.50 \mathrm{MEV}$

10.00 MEV

$11.0 E \mathrm{MEV}$

$12.00 \mathrm{MEV}$

13,09 MEV

14.0O MEV

$15.00 \mathrm{MEV}$

16.00 MEY

$17.00 \mathrm{MEV}$

18.01 MEV

19. OQ MEV

20.00 MEY

$21.00 \mathrm{MEV}$

22.00 MEV

$23.00 \mathrm{MEV}$

$24.00 \mathrm{MEV}$

25.0B MEV

$26.00 \mathrm{MEV}$

27.00 MEV

28.00 MEV

29.00 MEV

30, BO MEV

32.00 MEV

34.00 MEV

36.20 MEV

38.00 MEV

$40.00 \mathrm{MEV}$

$42.00 \mathrm{MEV}$

$\triangle 4.09$ MEV

46. DO MEV

$48.00 \mathrm{MEV}$

50.04 MEV

$$
\text { PRUTONS }
$$

$3.97 E+21$

2. 36E+ 1

$1.52 E+11$

$1.52 E+11$

$2.91 F+B)$

$3.96 E+01$

$3.53 E+01$

$3.03 E+21$

$3.52 E+D 1$

1. $51 E+11$

2. $5 \mid E+\lambda I$

4. $92 E+61$

3. $33 E+b$ !

$1.50 E+11$

2. $S A E+121$

4. $42 E+1) 1$

4. $5 U E+B 1$

4. $4 \Delta E+k 1$

2. $x^{1} a E+B 1$

3.5AE+ 1

3. 5 AE + 1

2. UGE + 11

2. 5 AF +01

2. UAE + DI

3. DINE+ 1

1. 5QE + D. 1

1. $5 \mathrm{ME}+1 \mathrm{I}$

b.

b.

i.

a.

5. DUE+ DO

1. 5OE + I

1. $\triangle B E+U 1$

5. QUE+UQ

2. $O B E+01$

1. $Q 0 E+B 1$

.

5. UaE+ 60

$B$.

$\theta$

0

4

i.

12. 12
DEIITERONS

$6.87 E-02$

1. $\triangle 2 E-D 2$

1. G?E-D?

i.

3.

a.

a.

1

a.

a

0.

a.

1 .

1. Bot - A ?

1. BDE-OZ

$3.6 B E-A 2$

$3.63 F-02$

0

i.

0

D.

A.

4

a.

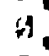

$a$

9

a

a.

a

$a$

a.

0

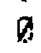

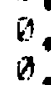

a.

b.

0

a.

0

a.

2. 74
TFITONS

$\Delta$.

D.

is.

$\theta$

$\because$

N.

i.

H.

i.

¿. $07 E-02$

¿., $17 E-02$

D.

a

b.

in

a.

a.

b.

4

9.

().

a.

b.

a.

11

a.

b.

0

0.

$D$.

u.

$n$

$\theta$

(1)

i.

D.

i.

W.

b.

a.

0.

$B$.

L.

a.

$d$

4.69
ALPHAS

1. $58 E+11$

1. $28 E+i 11$

1. $2 B E+B 1$

$4.3 B E+B A$

$4.38 E+D G$

6. $51 E+1 v$

8. $51 E+v^{2} v$

7. $80 \mathrm{E}+\mathrm{W}$

7. Bu:E+

5. $49 E+111$

$5.49 E+6 B$

9. $33 E-111$

$9.33 E-A 1$

3.7OE-MI

$3.70 \mathrm{END}$

2. $7 v E+i k$

$2.7 .1 E+B$

$7,3+E=$ H 2

$7.34 \mathrm{E}$ (1)

D.

i.

0.

Q.

a

a.

$a$

$a$

$B$.

D.

b.

Q.

$\theta$.

0.

0.

$n$

U.

D.

i)

0

a.

19.

$\theta$.

.

v.

0.

4.88 
IINITIAL SPECTRUM AT $A .4 D-0.60$ CH, FOR SHONRA A-15H PLASTIC 42 MEV P+ ON HE: NEUTRDN SPECTRUM

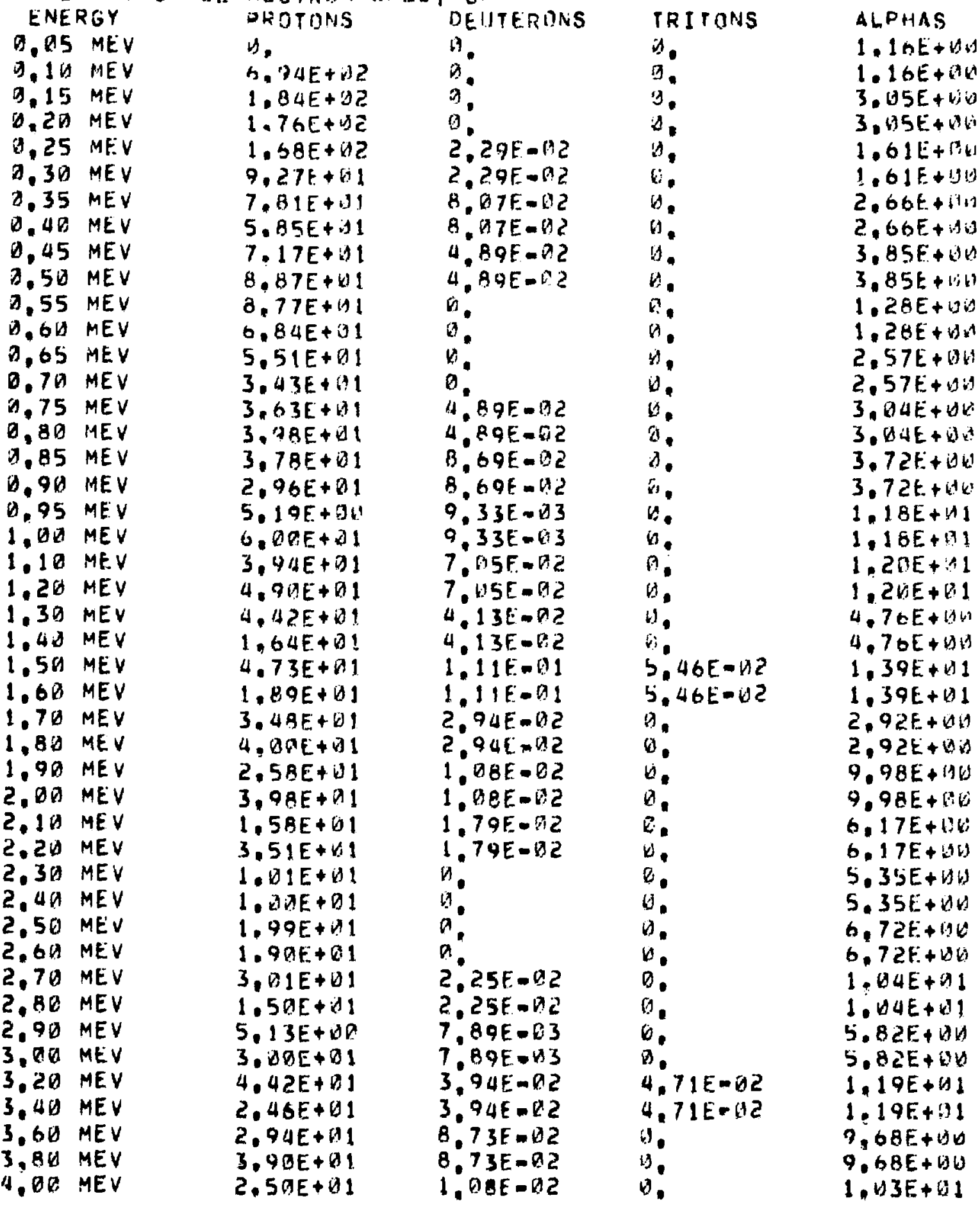


1INITIAL SPECTRUM AT U. 4B-H.6H CM. FOR SHONKA A-15H PLASTIC प2 MEV P+ ON BE NEUTRON SPECTPIIP.

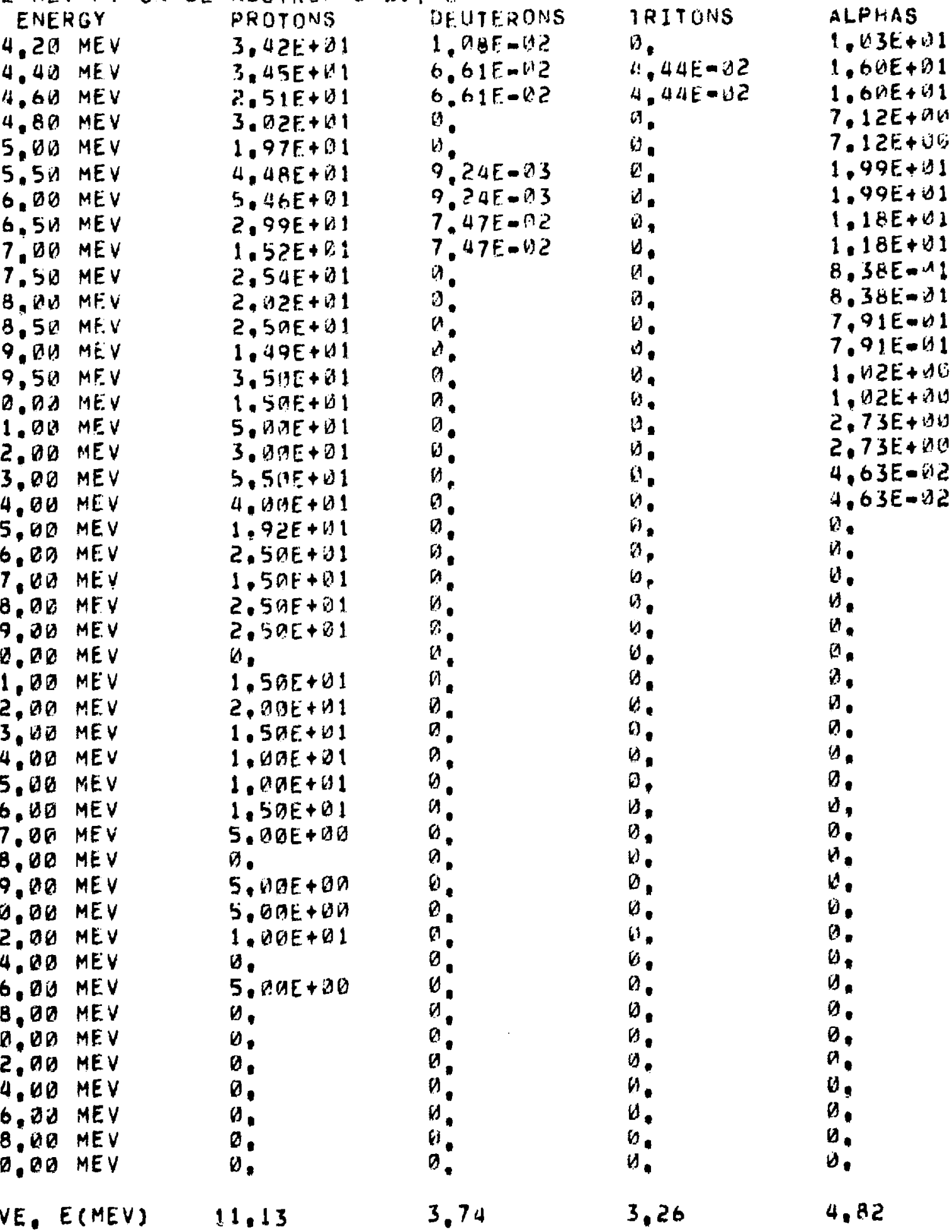


IINITIAL SPECTHUM AT B.OH-1,HA CM. FOR SHOINAA A-15D HLASTIC 42 MEV P+ ON BE NEUTRON SFECTRIIM

\section{ENERGY}

D. 25 MEV

0.10 MEV

0.15 MEV

$0.26 \mathrm{MEV}$

D. 25 MEV

0.30 MEV

7.35 MEV

Q. 4O MEV

D. 45 MEV

$0.56 \mathrm{MEV}$

$0.55 \mathrm{MEV}$

0.60 MEV

$0.65 \mathrm{MEV}$

0.70 MEV

0.75 MEV

Q.80 MEV

D.BS MEV

Q.9U MEV

0.95 MEV

1. DO MEV

1.10 MEV

$1.20 \mathrm{MEV}$

$1.30 \mathrm{MEV}$

1. 4 LE MEV

1.50 MEV

$1.60 \mathrm{MEV}$

$1.7 \mathrm{MEV}$

$1 . B O M E V$

$1.90 \mathrm{MFV}$

$2.00 \mathrm{MEV}$

2.1D MEV

2.20 MEV

2. 30 MEV

2. $40 \mathrm{MEV}$

2,50 MEV

$2.63 \mathrm{MEV}$

$2.70 \mathrm{MEV}$

2. aU MEV

2.9 $9 E \mathrm{MEV}$

3. OU MEV

$3.20 \mathrm{MEV}$

3. 4 D MEV

3.60 MEV

3.80 MEV

4. OU MEV

$$
\text { PROTONS }
$$
U.

7. $\triangle D E E+\triangle 2$

$2.62 E+W 2$

2. $D 2 E+112$

1. $18 E+.12$

1. $2 A E+w 2$

1. IUE. U2

6. $30 E+01$

5.96E+ 11

$7.69 E+11$

$4.47 E+11$

4. $26 E+111$

$4.45 E+d 1$

$3.07 E+91$

$5.25 E+41$

3. $41 E+D 1$

7. $81 E+01$

4. $29 E+\lambda 1$

2. $95 E+t^{3} 1$

5. BSE + D 1

$5.47 E+D 1$

$6.71 E+d 1$

S. $16 E+x 11$

$6.36 F+01$

$3.33 E+1$

2. $98 E+01$

4. $11 E+\vdots 1$

$2.54 E+d 1$

4. $6 R E+1$

5. 3 CE + ) 1

3. DeE+DI

1. $79 E+01$

4. $07 E+1$

1. $8 R E+U 1$

2. $25 i E+1$

2. $23 E+B I$

1. $156 E+131$

1. $97 E+1$

$2.21 E+01$

$2.79 E+01$

2. $76 E+01$

$3.11 E+91$

2. $93 E+01$

1.77E+ 11

3. $7 \Delta E+\forall 1$

\section{DEUTFROUS}

a.

$1.3 ? E-112$

1. $3 ? F-b$ ?

$1)$

0.

$1.53 E-A ?$

1. $53 E-D 2$

2. BAF-I. 2

2. $90 \mathrm{E}-142$

$3,51 E-12$

3.51E-92

1. $45 F-112$

1. $45 E-172$

$2.94 E-B I 2$

2. $94 \mathrm{E}-\mathrm{bl}$

a.

$\Delta$.

$7.97 E-N 2$

$7.77 E-12$

5.77E-12

$5.77 E-W 2$

5. $36 F=a^{x} 2$

5. הGE-WZ

4. $1 B E-112$

4. $16 E-1: 2$

6. $.7 E-12$

6. ATE-1C

5. A2E- $=2$

5. A2E-T.C

$3.36 E=W 2$

$3.36 E-x^{x} 2$

$3.26 E-12$

3.26f-d?

$1.514-142$

$1.515-122$

2. $98 E-11 c^{3}$

$2.98 E-12$

$1.54 E=12$

$1.54 E-112$

$1.61 \mathrm{E}-\mathrm{bl}$

$1.61 E-62$

1. UTE-02

1.07E-V?

3. $21 E=13$

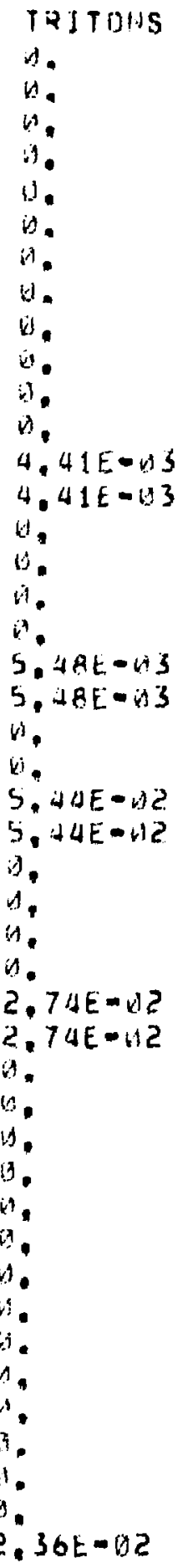

AI.PITAS

1. UIE+ $D: 1$

1. M1 $1 E+d a$

$1, v 1 \bar{E}+b u$

$1.81 E+C ?$

1. $85 E+1.2$

$1.85 t+40$

2. $13 E+3 A$

2 . $3 E+14$

2. $14 E+14 b$

2. $14 E+\| r$

2. $12 E+i 16$

$2,12 E+d x$

$3.33 E+4 d$

3. $33 E+14$

$1.31 E+6 t$

1. $31 F+61+1$

2. $01 E+b v$

2. $61 E+U 4$

Q.19F-DI

$9.19 E-W 1$

S. $93 E+A j$

$5,93 E+i^{t} i$

S. $26 F+416$

$3.20 E+3$

$7,94 E+B \alpha$

$7.74 t+10$

$7.35 E+\cdots$

7. $38 E+d t$

$5.97 E+A k$

$3.97 E+t)$

4. $58 E+60$

4. $58 E+D U$

3. $12 E+A n$

3. $12 E+j u$

7. $20 E+00$

7. $26 E+A$

9. $35 E+13 n$

$0.35 E+.4$

$6.915+111$

$0.91 E+.1\}$

1. $19 E+M 1$

1. $1 Y E+(51$

1. औ) $8 E+1\}$

1. $\triangle B E+1$

1. $14 E+41$ 
1INITIAL SPECTRUM AT B. 6O-1.0G CH, FOR SHOMKA A-IGLARLASTIC 42 MEV P+ ON BE NFI!TRON SPECTRIJM

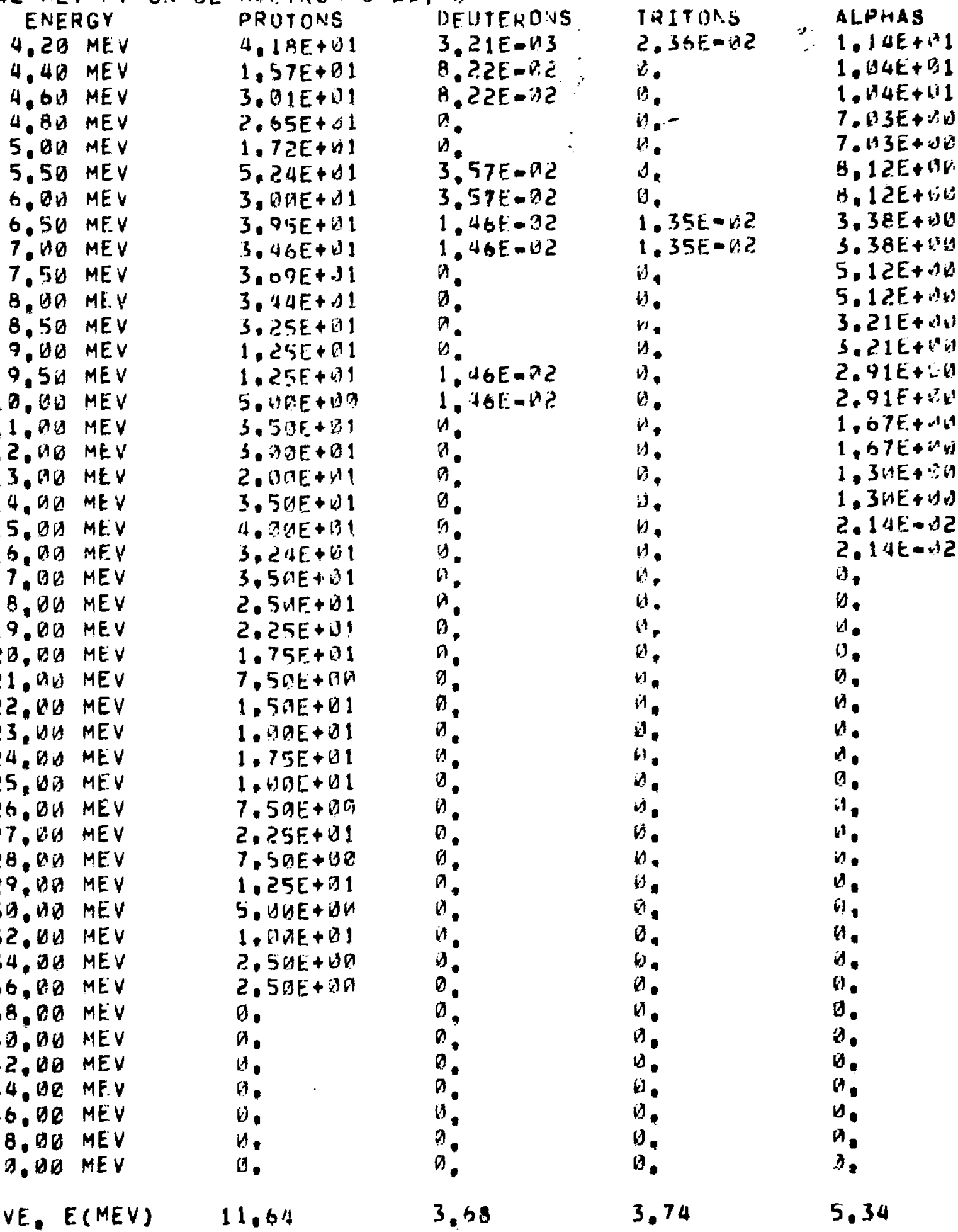




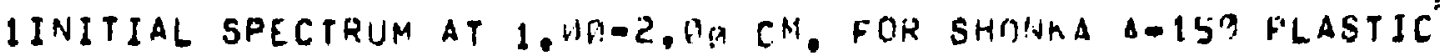
42 MEV P+ ON HE NEUTRON SHEC.TRIJM

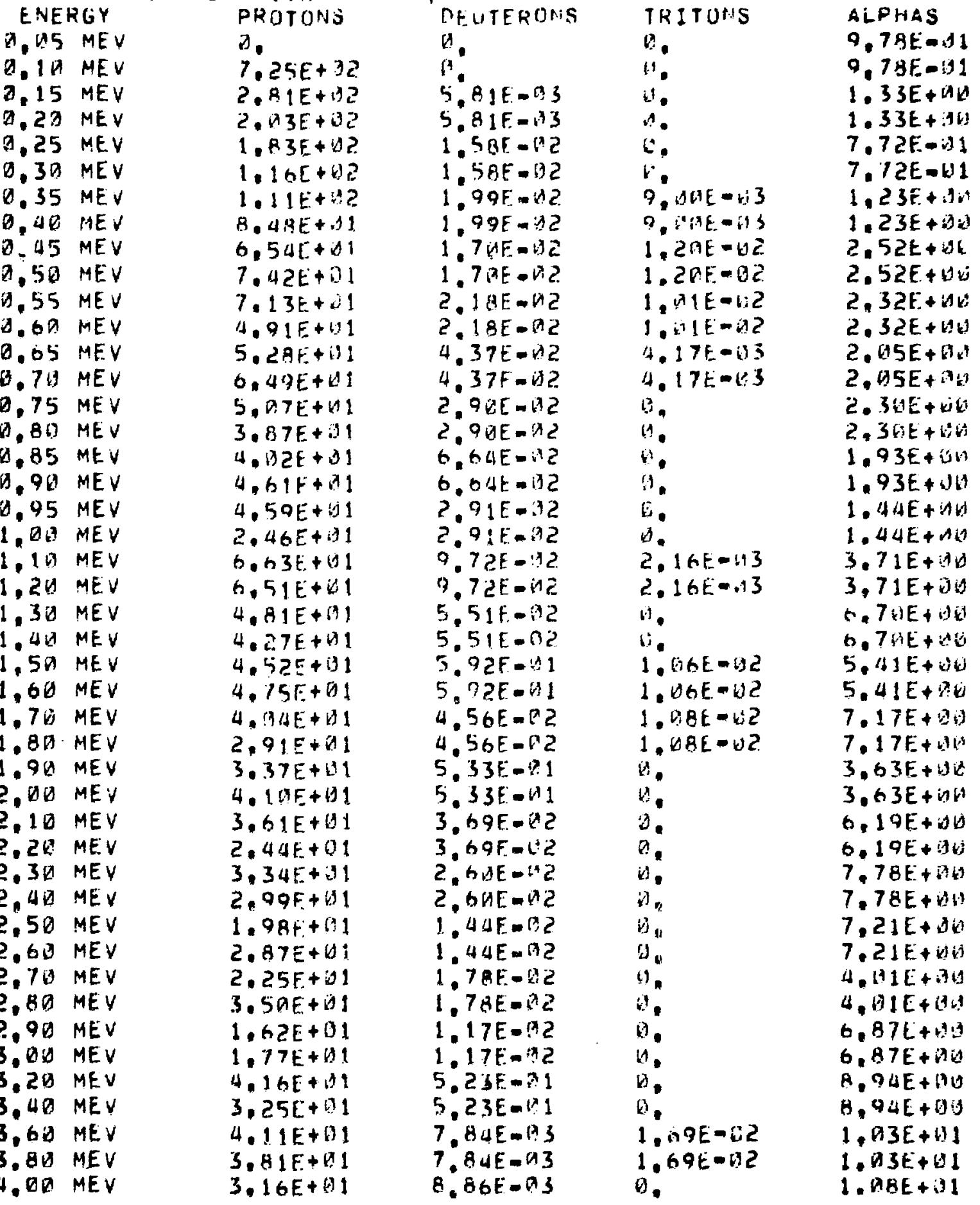


IINITIAL SPECTRUM AT I.MB-?. PR CM. FGIR SHOHKA A-15. PLASIIC 42 MEV P+ ON HE NFUTRON SPLCTPIJM

$$
\text { ENERGY }
$$

4.2U MEY

4. 4 ME

4.60 MEV

4,80 MEV

5, WU MEV

5.5D MEV

6. DQ MEV

6.5 I MEV

7. MG MEV

7.50 MEV

9. HU MEV

$B .50 \mathrm{MEV}$

9 . $\triangle$ MEV

$9.5 \square \mathrm{MEV}$

1.,DO MEV

$11.00 \mathrm{MEV}$

12, OB MEV

13. OQ MEV

14.AO MEV

15. JQ MEV

16. AQ MEV

$11.010 \mathrm{MEV}$

18. DU MEV

$19.00 \mathrm{MEV}$

2D. OD MEV

21.00 MEV

22, DO MEV

23, OU MEV

24. DI MEV

25. DO MEV

26. QU MEV

$27.119 \mathrm{MEV}$

28. HO MEV

29. DL MEV

$30, D V M E V$

$32.00 \mathrm{MEV}$

34. DS MEV

36. 10 MEV

$3 B .00 \mathrm{MEV}$

40.OD MEV

42. 19 ME

$44.00 \mathrm{MEV}$

$46,00 \mathrm{MEV}$

$48.00 \mathrm{MEV}$

$50.00 \mathrm{MEV}$

DAVE, E(MEV)

\section{PROTUNS}

1. EAE+B1

$2,555+i 11$

2. $17 E+11$

$2.86 t+11$

2. $\{2 F+11$

$5,33 E+b)$

4. $95 E+1 ! 1$

4. $85 E+31$

4. $4 M F+V I$

3. $\triangle R E+1$

$2.41 E+D 1$

$2.58 E+J 1$

$1.87 E+11$

$1.96 t+11$

2. $69 F+1$

$4.15 E+11$

$3.87 E+101$

3. $69 E+B 1$

3. $7 A E+B 1$

2. $49 E+111$

1.99E+ 1

$1.90 E+W 1$

1. $3 A E+B D 1$

1. $A Q D E+B 1$

1. $7 a E+b 1$

$1.19 E+191$

$1,19 E+191$

$1.6 \pi E+d 1$

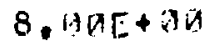

1.3 $30 E+D 1$

6.) \lambda E+4) ?$

5. MAE+ 30

6. $b 9 E+B D$

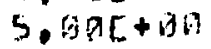

1. $\triangle A E+1$

6. $906+06$

4. $D D E+D U$

5. $\angle B E+B Q$

1. $20 E+B$

0.

10.53
DELTERDIUS

B. BGF-OB

5. $24 F-11$

$5,2+E-31$

1. 3 OE-B?

1. 3थF -12 .

2. 5\%F $-2 ?$

$2.59 \mathrm{E}-\mathrm{U} 2$

$4.95 E-3$ ?

4. $95 E-82$ ?

1.17F. - 2

1. $17 E-D 2$

$5.1: E-01$

$5.11 E=A$

1. $1 \square E-b 2$

1. J NE-D?

$5.64 \mathrm{E}-03$

$5.64 E-D 3$

17

0.

a.

a.

$\theta$

a.

0

v

$a$

D.

0

$a$

1

$a$

a

a

$a$

a.

$a$

0

3

0

i)

a.

5.34
TRITUNS

i.

b.

b.

b.

17.

V.

in

$1.91 t-x^{3} 2$

1. $01 E-02$

$4.85 E-43$

4. $45 t=43$

1. d $5 E=v 2$

$1.85 t-72$

4. YAE $=3$

4. $90 E-\lambda 3$

b.

is

i.

$n$

is.

$v$.

i.

b.

1

is.

in

a.

0

0

i.

0.

,

b.

b.

b.

b.

a.

a.

v.

$\dot{b}$

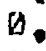

D.

.

B.

D.

6.38
ALPRAS

1. $148 E+11$

6. $26 E+\Delta \theta$

$6.26 E+\Delta V$

$4.34 E+94$

$4,34 E+40$

$8.90 E+A B$

$B, 96 F+D W$

5. $(10 E+A)$

5. AGE + HA

$3.76 E+i 3)$

$3.7 \cap E+A H$

2.87E+B

?. $87 \mathrm{E}+\mathrm{WH}$

9. 7 UE-U1

$9.7 R E=U 1$

8.93E-?I

$8.93 E-111$

$6.94 E-102$

$6.94 E-62$

$9.44 E-53$

$9.44 E-E 3$

i.

n.

S. $\left.6 Q^{4} E-1\right] 1$

5. HUE-U1

0.

a.

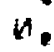

o.

a.

.

,

$\therefore$

(t)

a.

$\theta$.

3

n.

$\theta$.

a.

a.

vis.

a.

o.

5.92 


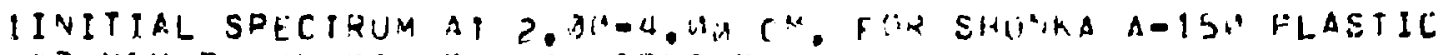
$42 M E V P+$ UN LE NUUTRON SPECTPUM

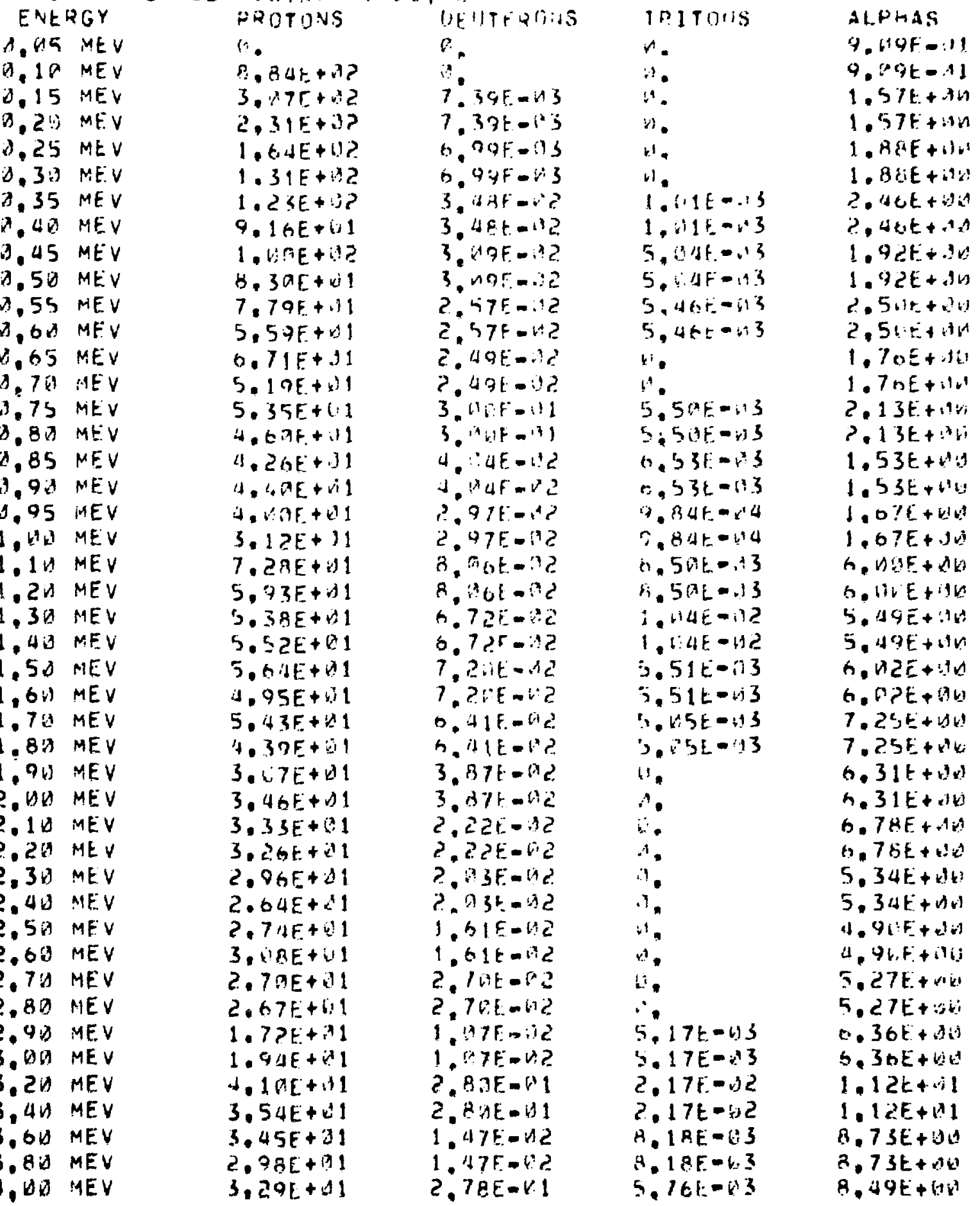




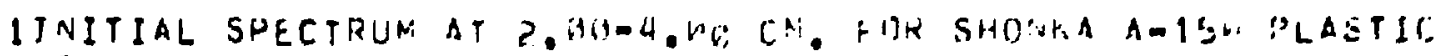
42 MEV $P+$ ON HE NEITROA SPECTRUI:

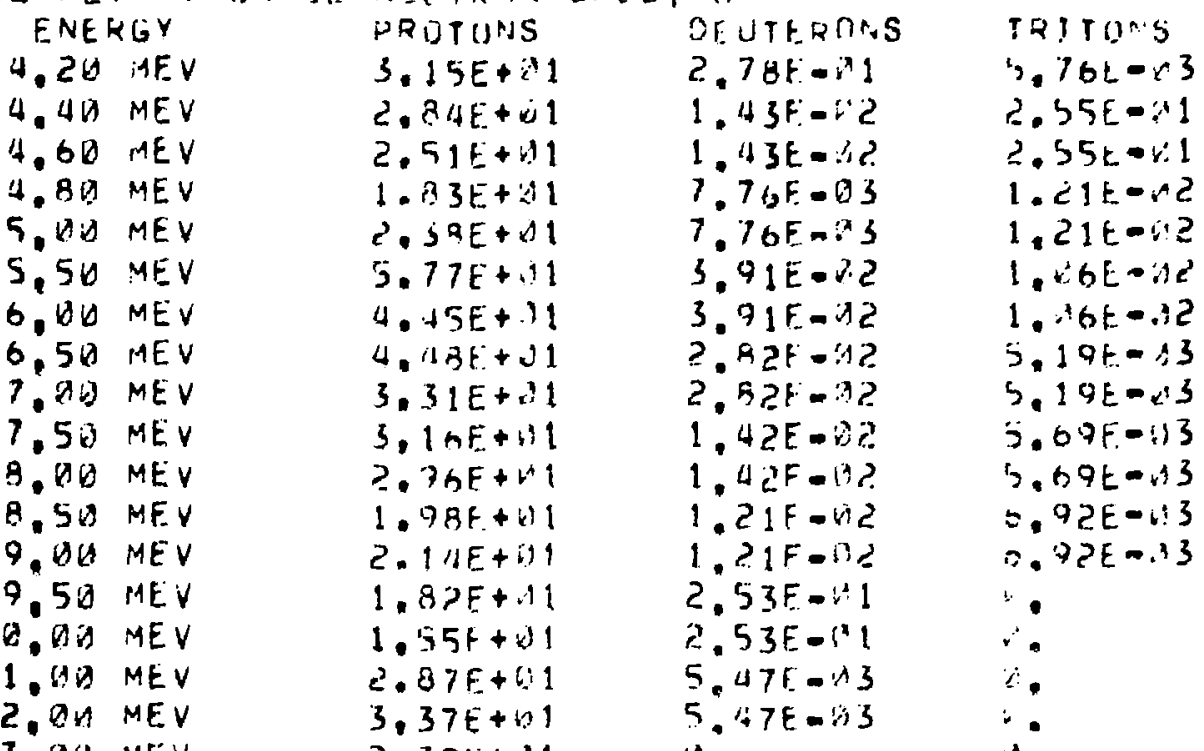

13. 10 MEV

$2.39 \mathrm{~F}+111$

14, BIS MEV

$3,: 9 F+6 !$

15. 50 MEV

16. $Q 0$ MEV

2. $\rightarrow A E+B 1$

E. $2 A E+B I$

$17.0 \mathrm{MEV}$

2. $83 E+B 1$

1B. QU MEV

19.01 MEV

$1.75 E+121$

1. B.iF+A1

20. DU MEV

l. $25 F+d 1$

1. $2 A E+41$

$1.65 E+11$

22. QU MEV

Q. $5(A F+D W$

23. DO MEV

1. $2: 1 E+111$

$24, O B$ MEV

25. UD MEV

9. ME+ IP

26. HO MEV

27. HO MEV

28.06 MEV

$29.40 \mathrm{MEV}$

30. DH MEV

32. OS MEV

34. OH MFV

36. OU MEV

$38.0 Q M E V$

40. OU MEV

$42.02 \mathrm{MEV}$

44. VD MEV

46, DV MEV

48. $\triangle 0$ MEV

$50.00 \mathrm{MEV}$

$7.5 \mathrm{OF}+106$

$5.50 E+\Delta A$

5. $0 A E+116$

4. $43 E+19 v$

$0.5 O E+0 k$

7. $D C E+A U$

4. COAE + IS

3. $D A E+b e$

1. $5 A E+10$

a.

it.

is

4

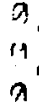

9

$a$

b.

i)

in.

1.

$\therefore$

is

is.

if.

if

$\because 1$

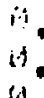

a.

in.

is:

QAVE, E(MEV)

16.21

5.12

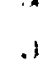

i.

1.

!

1

6

.

(9)

G.

,

4

4

1

i.

it

i.

$\therefore$

$\because$

$i$.

11.

b.

in.

3

$\pi$

19

is

0.

ALPHAS

H. $49 E+4: 3$

$5.65 E+A$

$3.05 t+\lambda$

$5.93 E+2 B$

$5.93 F_{-}+60^{3}$

9. $1 B E+\sin$

$9.15 t+k^{2} t$

$7 . \mathrm{xH}_{+}+\mathrm{BH}$

$7.6: 2 E+1:$ ?

2. $41 E+A$

2. $41 E+B .1$

2. $21 E+4 i]$

2. $218+1,3$

$1.92 E+\cdots$

$1.32 t+14$

Q.61E-11

F. $61 E-11$

3. 1 HE- 1

3. $1: E-51$

$7.585-31$

7. $58 E-i 1$

2. $5 \mathrm{is}^{2} \mathrm{t}-1$

2. $54 F-131$

?. SIF -.41

$2.5 \mathrm{BE}-3 \mathrm{l}$

is.

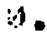

1.

n.

?.

a.

is.

in.

i.

to

1.

1.

1.9.

1.

b.

is.

1.

i.

s.

4.58

5,32 


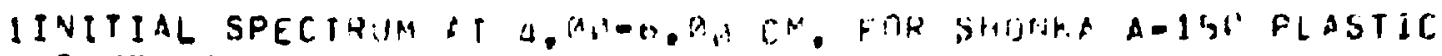
4? MEV P+ ON BE NEUTROIY SPLCTPUP.

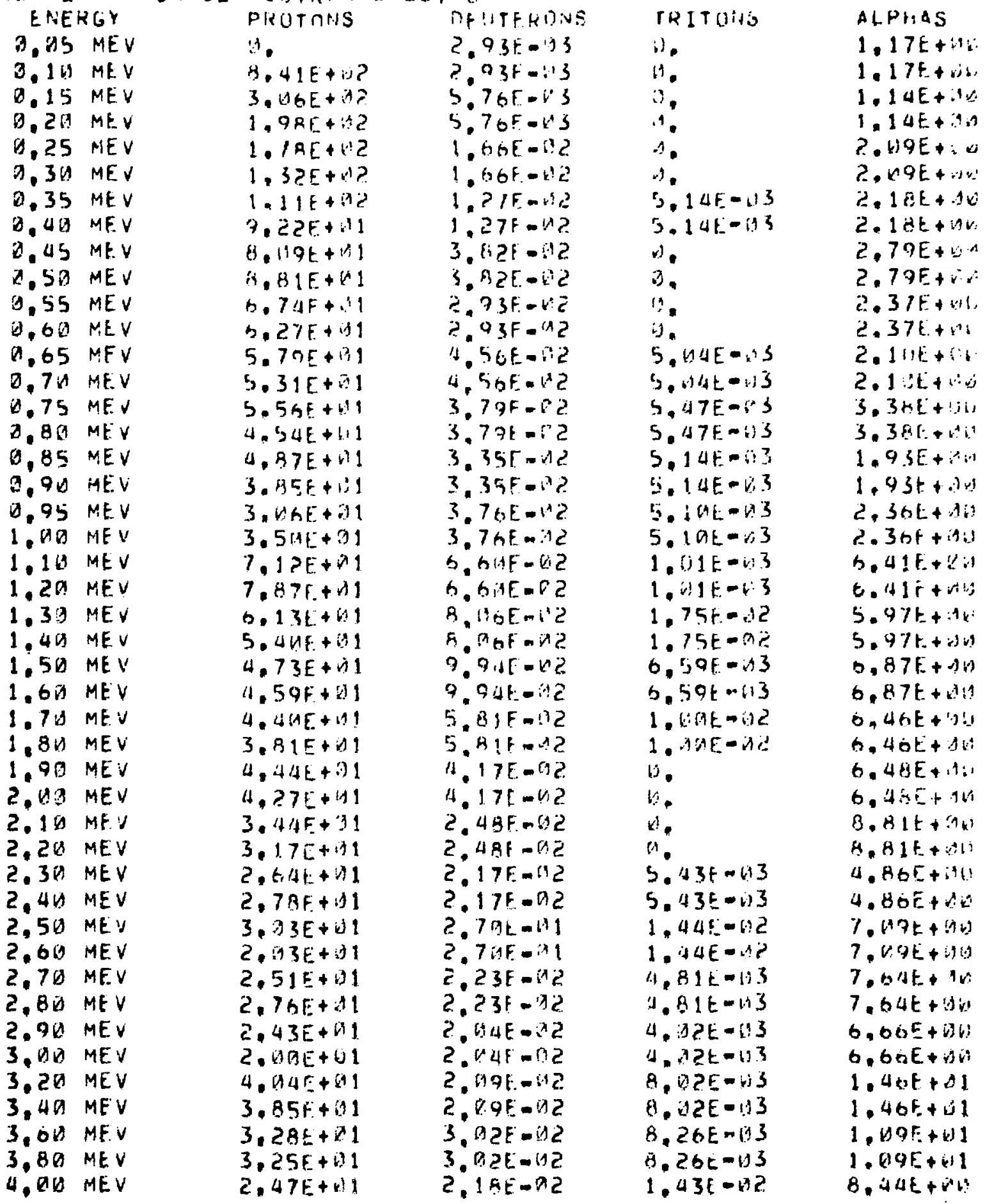




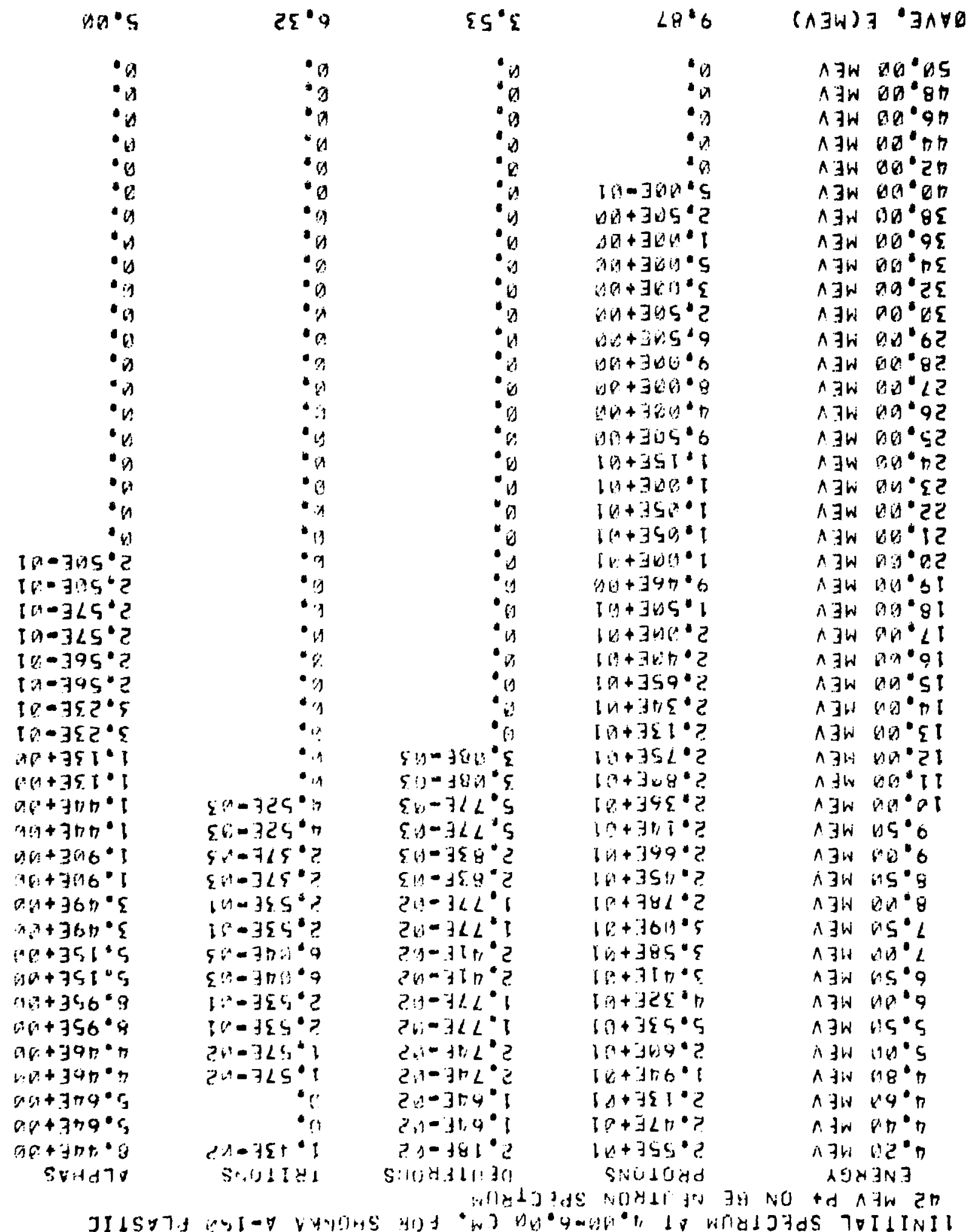

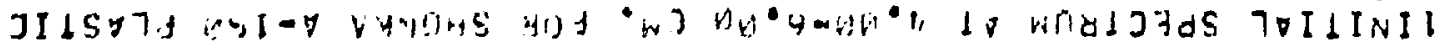


IINITIAL SPECIHUM AT 6.OH-IHER CH. FCR SIIINKA A=ISL NLASTIC 42 MEV P + ON BE NEUTRON SHECTRUN

\section{ENERGY}

0.05 MEV

7. 10 MEV

0.15 MEV

$0.20 M E V$

0.25 MEV

0.3 MEV

0.35 MEV

0.4B MEV

0. 45 MEV

B. 50 MEV

0.55 MEV

$0.61 \mathrm{MEV}$

0.65 MEV

$0.70 \mathrm{MEV}$

0.75 MEV

$0.80 \mathrm{MEV}$

$0,85 \mathrm{MEV}$

$0.90 \mathrm{MEV}$

$0.95 \mathrm{MEV}$

1. OO MEV

1,10 MEV

$1.20 \mathrm{MEV}$

1.30 MEV

1.40 MEV

1.50 MEV

1,60 MEV

$1.70 \mathrm{MEV}$

1.80 MEV

1.90 MEV

2. DU MEV

$2.10 \mathrm{MEV}$

2. 2D MEV

2.30 MEV

2.40 MEV

2.50 MEV

$2.60 \mathrm{MEV}$

2,70 MEV

2. BV MEV

$2.90 \mathrm{MEV}$

3.00 MEV

$3.2 A M E V$

3. $40 \mathrm{MEV}$

3.61 MEV

3. BD MEV

4,DO MEV

\section{PEOTONS}

U.

$7.43 E+a 2$

$2.95 E+102$

2. $34 E+12$

1. $51 E+42$

1. 3 id +4 ?

1. $13 E+12$

$8.835+(1)$

$7.92 k+61$

$7.32 E+01$

$6.54 E+01$

$5.85 E_{-}+1$

$5.39 E+11$

$5.54 E+11$

$4.45 E+1$

4. $55 E+71$

3. BUE +DI

3. $B 4 E+W 1$

3. $88 \mathrm{~F}+81$

$3.13 E+01$

$6.53 E+01$

5. $59 E+\Delta 1$

5. $21 E+D 1$

5. $12 E+1$

$4.55 E+11$

$4.65 F+D 1$

4.13E+D1

3. $87 E+\Delta 1$

3. $5 B E+11$

$3.81 E+01$

3. $23 E+1$

3. $17 E+01$

3. $05 E+01$

$2,68 E+\lambda 1$

2.59E+DI

2. $34 E+11$

$2,10 E+01$

1. $9 \square E+01$

$2,21 E+01$

$2.06 E+21$

3. $26 E+11$

$3.65 E+01$

3. $109 E+01$

$2,87 E+91$

2. $79 E+41$
DEUTFRUNS

D.

i.

5. $24 F-03$

5. $24 \mathrm{~F}=\mathrm{B}$

2. 1 IE-BS

2. $11 E-[13$

1. 3yE-12

$1.36 E-0 ?$

1. $42 E-111$

$1.12 F-B 1$

2.18E-D?

$2.1 B F-A 2$

1. $99 E-B C$

1. $99 \mathrm{~F}-\mathrm{AP}$

2. $10 F-A C$

2. $1 D E-C 2$

3. 57.E- D2

3.571 .172

$2.55 E-112$

2. $55 E-1)$.

1. $7|E-d|$

$1.71 E-A 1$

$7.365-92$

$7.365=112$

$4.68 E=11 ?$

$4.68 t-102$

$3.835-62$

$3.83 F-02$

$1.61 E-1$

$1.61 E-M 1$

2. $44 F-02$

2. $4 \Delta E-\Delta 2$

2. NUE $O$ OC

2. DUE $=92$

$1.41 E-22$

$1.4 I F-02$

1. $47 E-D 1$

$1.47 E-01$

1.74E-H2

$1.7 \Delta E-H Z$

2. $84 E=01$

?. $84 \mathrm{E}-191$

2. $69 E-01$

2. $69 E-01$

1. $47 E-01$
TRITONS

$\theta$

,

.

4

2. 5 AE $=3$

2. SAE-N3

1.

1.

2. 35E-V 3

2. $35 E-\Delta 3$

$7.74 E-13$

$7.74 E-\cap 3$

$2.95 E-43$

2. $35 E-V 3$

3. $37 F-M 3$

3. $37 E-33$

2. $73 E-x 3$

$2.73 E-13$

5. $14 t-54$

$5,14 t-04$

$3.42 E-0.3$

$3,42 E-B 3$

4.,$A E-V . S$

$4,60 E-x^{3} 3$

$4,7 \geq E-93$

$4.72 t=13$

2. $35 E-43$

2. $35 E-03$

$7.37 \mathrm{E}-1.3$

$7.37 \mathrm{E}-D 3$

2. $44 E-63$

2. $44 E-\backsim 3$

$\theta$.

b.

$7.37 E-U 3$

$7.37 E-13$

1. $3 \square E-B 1$

$1.39 E-B 1$

$5.29 E-03$

$5.29 E-U 3$

$3.38 E-13$

$3.38 E-W 3$

1. $74 E-02$

1. $? a F_{.}=\Delta 2$

$1.30 E-02$
ALPRAS

B. $79[-1]$

B. $74 \mathrm{E}-\mathrm{Al}$

$1.32 E+16$

1. $32 t+4$.

1. $.98 E+0) v$

1. $M B E+B$

1. $42 E+3 A$

1. $42 \mathrm{E}+1 \mathrm{~W}$

1. $36 E+113$

1. $38 E+3 U$

2. $100 E+A$ i

2. $4 h E+v k:$

2. $14 E+d v$

2. $\triangle A E+W \%$

$1.45 E+4$.

1. $45 E+14$

2. $38 t+1 v$

?. WBE+ +4

$2.15 t+4 k$

2. $15 E+U E$

4. $B O E+K 1 \vee$

$4.83 E+610$

4. B $1 E+4$ W

$4 . \Delta 1 E+\Delta v$

$5.51 E+10$

$5.51 E+U A$

5. $13 E+4 U$

$5.13 E+11\}$

6. 1 WE +1 (1)

6. $10 E+i t y$

$4.87 E+x i v$

$4.87 E+H E$

$5.49 E+U V$

$5.49 E+d W$

$5.75 E+d d$

$5.15 E+11$.

$4.39 E+4 D$

$4.39 E+00$

6. $D 1 E+06$

6. IIE+C

$7.90 E+10$

$7.90 E+64$

8. $05 E+6 v$

B. $15 E+D V$

$6.96 E+14$ 
IINITIAL SOECTRUM AT G,BG-IDGG CM. FOR SHONKA A-ISH PLASTIC 42 MEV P+ ON BE NEUTRON SPECTRUM

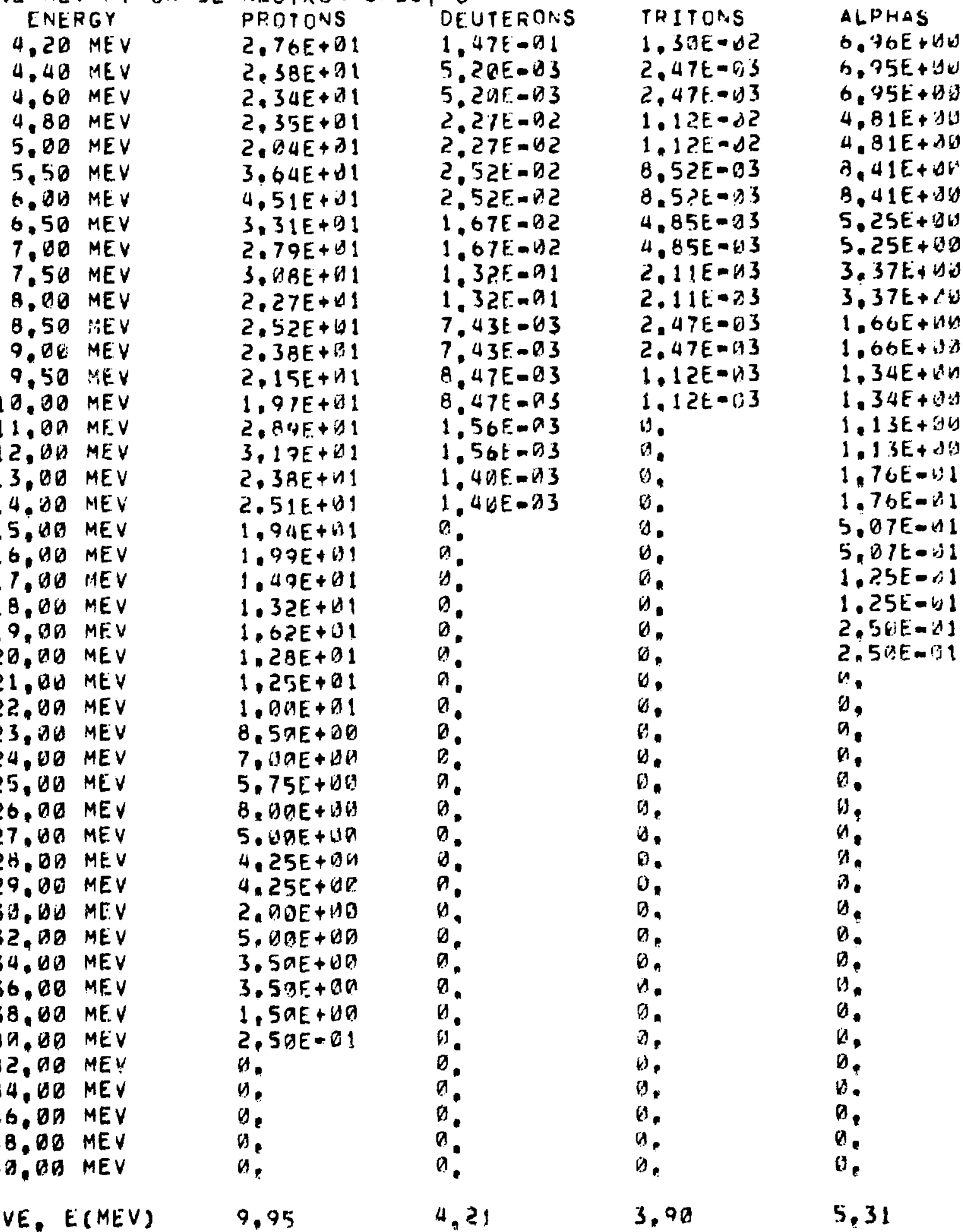


IINITIAL SPECTRUM AT 19.19-14.A CM, FOR SHONKA A-15O PLASTIC U? MEV P+ ON BE NEUITR? SPECYRUM

ENERGY

D. 5 MEV

$0.10 \mathrm{MEV}$

0.15 MEV

$0.26 \mathrm{MEV}$

0.25 ME.V

0.30 MEV

0.35 MEV

0.40 MEV

$0.45 \mathrm{MEV}$

0.50 MEV

$0.55 \mathrm{MEV}$

$0.60 \mathrm{MEV}$

0.65 MEV

0.70 MEV

0.75 MEV

0.BO MEV

$0.85 \mathrm{MEV}$

0.96 MEV

$0.95 \mathrm{MEV}$

$1.00 M E V$

$1.10 \mathrm{MEV}$

$1.20 \mathrm{MEV}$

1.30 MEV

1.40 MEV

1.5W MEV

1.60 MEV

1.70 MEY

1. .8 MEV

$1.90 \mathrm{MEV}$

2. $U 1$ MEV

2.1B MEV

2. 2W MEV

2.3 MEV

$2,40 \mathrm{MEV}$

$2.5 n \mathrm{MEV}$

$2.60 \mathrm{MEV}$

$2.70 \mathrm{MEV}$

2.80 MEV

2.90 MEV

3.00 MEV

3.2U MEV

3.40 MEV

3.60 MEV

3,80 MEV

4.DD MEV
PROTONS

b.

$6.72 E+12$

$2.6 P E+\Delta ?$

1. $77 \mathrm{E}+\mathrm{D}$ ?

1. 4 PE $+B 2$

1. $04 \mathrm{~B}+\mathrm{QL}$

9. $82 E+11$

8. $1 E+a_{1}$

$7.52 E+B !$

$6.51 E+01$

5.59E+ 11

4.78E+ BI

$4,77 E+0 !$

$4.67 E+01$

4. $20 E+D 1$

3. $91 E+01$

3. $7 \mathrm{AE}+01$

3. $1 a f+b 1$

3. (OBE + 1)

$3.34 E+111$

$5.63 E+01$

$5.75 E+01$

5. $40[+61$

3. $98 E+B_{1}$

$3.91 E+D 1$

$3,36 E+01$

3. $\triangle 2 E+Q_{1}$

3. 3HE + B1

3. $25 E+D 1$

2. $65 E+D 1$

2. $D E+B 1$

2.87E+ 11

2. $8 B C+91$

2. $\triangle 2 E+11$

$2.65 E+01$

$2.23 E+31$

$1.83 E+B 1$

1. $4 \mid E+A 1$

1. BAE+ O1

1. $4 A E+B 1$

2.8QE + 01

3. $3 F+b 1$

$2.59 E+31$

2. $07 E+11$

ट. $12 E+\square 1$
DEUTERIINS

1. $\triangle Q E=B$

$1,4 Q E-O B$

7. $14 F-B 3$

7. $14 E-\cap 3$

5. $1 ! E-D 3$

$5.11 E-05$

1. $15 f-42$

$1.15 F-12$

1. 4टE-VI

$1.42 E-01$

2. A.4E-

2. $D+F-B 2$

2. $45 E-02$

2. $45 E-22$

2. $43 F-P 2$

2. $43 E-02$

2. $45 \mathrm{~F}-\mathrm{PC}_{2}$

2. $45 F-\Delta 2$

2. $68 E-B 2$

2. $68 E-12$

$5.35 E-12$

$5.35 \mathrm{~F}-112$

6. 39F- 32

$6.39 \mathrm{~F}-1 \mathrm{ZZ}$

4. 66E-V2

4. . $6 E-02$

$1.71 E-91$

$1.71 \mathrm{E}-01$

1. $46 E-B 1$

1. $46 E_{-1}-1$

$2.15 F-B ?$

2. $15 \mathrm{E}-42$

$1.36 F-92$

1.36E-O?

$1.135-62$

$1.13 E-n 2$

1. $28 E-02$

1. $2 B E-9 Z$

1. $1 A E-A 2$

1. $10 E-D 2$

$1.64 E-02$

$1.64 E-B 2$

1. $\triangle Q E-O J$

1. $4 Q E-B 1$

1. $38 E-O 1$
TRIIUNS

$\theta$,

$\theta$.

$\theta$

0

$b$

$a$

a.

a.

$A .37 E-63$

$4,37 E-03$

4.69E-k.4

$4.69 E-104$

$9.39 E=14$

$9.39 E-B 4$

2. $57 \mathrm{E}-3$

$2.57 t-03$

1. IOE- $\triangle 3$

1. 1UE-DS

3.17E-A3

$3,17 t-n 3$

2. $49 E-A 3$

2. $49 E-43$

$2.55 E-03$

$2.55 E-03$

$7.63 E-113$

$7.63 E-43$

$7.21 E-3\}$

7. $21 E-B 3$

$7.46 E-B 3$

7. $46 E-B S$

$2.79 E-03$

2.7AE-U3

$4.96 E-m 3$

$4.96 E-.13$

6.

$\theta$

$6.90-193$

6. $19 E=U 3$

4. 9DE- 113

4. $90 E-D 3$

1. $25 E-b 1$

1. $25 E-01$

$0.75 E-D 3$

$0.75 E-03$

1.67E-B 3
ALPIAS

3. 47E-AI

3. $47 E-B 1$

$7.43 E=01$

$7.43 E-i) 1$

1. $06 E+10$

$1.06 E+00$

1. $07 E+V B$

1.07E+ 14

2. $07 E+\Delta A$

2. $D T E+D E$

$9.7 B E-B 1$

$9.7 \cup E-U 1$

1. $29 E+B G$

1. $29 E+O B$

1. $86 E+116$

$1.85 E+0 n$

$1.69 E+13 k$

$1.69 E+V 6$

$1.83 E+00$

$1.83 E+1911$

4. DEE+ 10

4. $B B E+D E$

$3.25 E+00$

$3.25 E+B$

$3,81 E+\omega 0$

3. B1E+BU

3. $15 E+100$

3. $15 E+0 B$

4. $42 E+B$ ?

4. $42 E+D n$

$4.79 E+10 \omega$

$4.79 E+0 D$

4. $52 E+\Delta, 3$

$4.52 E+B D$

$3.51 E+2 k$

3. $51 E+\Delta 0$

4. $36 E+B U$

$4.36 E+16 Q$

$4.62 E+36$

$4.62 E+46$

$7.86 E+B 6$

$7.66 E+16$

$5.42 E+B U$

$5.42 E+00$

$4.91 E+46$ 
IINITIAL SPECTRUM AT 10.KH-1A. CM. FDR SHDHKA A-15L PLASTIC 42 MEV P + ON BE NFUTRON SPECTRUM

ENERGY

4.20 MEV

4. 40 MEV

4.60 ME $V$

4.8B MEV

5. HO MEV

$5,50 M E V$

6. .9 MEV

$6.5 \square$ MEV

7. DV MEV

7.50 MEV

8. 3.3 MEV

8,50 MEV

7. 00 MEV

9.50 MEV

$12.0 D$ MEV

11. UG MEV

$12,00 M E V$

13,00 MEV

14.DV MEV

15. OU MEV

16.UA MEV

$17,00 \mathrm{MEV}$

18. 18 MEV

$19.00 \mathrm{MEV}$

20,0U MEV

21. BO MEV

2?,DD MEV

$23.00 \mathrm{MEV}$

$24.01 \mathrm{MEV}$

25,00 MEV

26. 19 MEV

27 D $D E V$

28.DO MEV

29. DO MEV

30. DO MEV

$32 ., D D M E V$

34. OD MEV

$36.0 B$ MEY

38. OO MEV

4D. OD MEV

$42,0 \square \mathrm{MEV}$

44.00 MEV

$46.00 \mathrm{MEV}$

$48.00 \mathrm{MEV}$

50,00 MEV
PROTONS

?. $27 E+131$

2. $28 C+61$

2. $21 E+b 1$

2. JIE $+D 1$

$1.69 E+01$

$3.84 E+101$

3. $16 E+111$

3. $13 E+Q 1$

2. $92 E+81$

2. $26 E+01$

2. $00 E+191$

1. $87 E+01$

1.8 $81 E+31$

2. $191 E+Q 1$

1. $49 E+01$

$3.35 E+61$

$2.83 E+D 1$

2. $17 E+01$

1.87E+Q1

1. $74 E+01$

$1.52 E+01$

1. $65 E+B 1$

$1.35 E+\square 1$

1. $52 E+D 1$

$8,00 E+D Q$

1. $15 E+01$

B. $50 E+4)$

7. GOE + $\triangle Q$

$5.75 E+100$

6. $90 E+00$

7. $25 E+100$

4. $5 ? E+B A$

4. $25 E+0$.

$5.25 E+B D$

3. DOE + DO

5. $110 E+\angle G$

3. $2.5 E+00$

$2.25 E+00$

$7.5 U E-D 1$

5., $90 E-O 1$

0.

0

0.

$b$

b.
DEUTEROINS

1. 38E-M1

$1.31 E-92$

1. $31 E-12$

1. 1 1 $19-92$

1. $10 E-D 2$

1. ? $3 E-H 2$

1. $23 E-42$

2. 39E-D2

2. $37 E-A 2$

1. BEE-L2

1. $98 \mathrm{BE}-12 \mathrm{Z}$

7. $2 / E-B 3$

$7.27 \mathrm{E}-1.5$

2. $26 E-03$

2. ?GE-33

2.7BE-A3

2.78E-A3

0

a.

D.

a.

$a$

$\theta$

9.

$\theta$

$a$

0

a.

$a$

$a$

0

0

(1)

0

$a$

0.

0

0.

3.57
TRITUNS

1.67E-313

5. $39 E-113$

5. $39 E-\square 3$

$1.95 E-43$

$1.95 t-13$

6. $1 R E_{-}=U 3$

6.1BE- 13

O. SRE- 13

$6.56 E-13$

$3.77 E-v 3$

$3.77 t-\dot{ } 3$

$1.35 E-03$

1. $35 E-b S$

1. $49 E-\checkmark 3$

1. $\triangle 9 E=13$

$\theta$.

i.

0

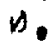

1.

U.

W.

13

0

0.

$n$

$\theta$.

D.

D.

i).

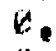

$\theta$

t)

0.

$\theta$

6.

$\theta$

U.

$\Delta$.

a.

0

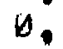

b.

0

3.93
ALPHAS

$4.91 E+10$

$5.43 E+16$

$5.43 E+10$

$3.35 E+3 i s$

3. $35 E+111$

5. $B B E+B: 1$

6. SHE + OD

4. BDE $+O B$

$4,86 E+010$

2. BSE+ U U

2. $B 5 E+60$

1.70E + D.

1.7UE + UO

1. $58 E+100$

1. $58 E+06$

1. $13 E+\Delta D$

$1.13 E+16$

$0.73 E-01$

$6.73 E-1$

5. $07 E-U 1$

$5,07 E-H 1$

$b$.

i).

b.

i.

B.

b.

0.

d.

0

U.

a.

a.

b.

B.

$\Delta$.

0.

D.

$\theta$

b.

0

b.

1

b.

b.

5.55 


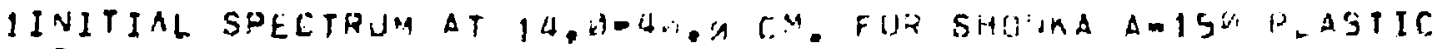

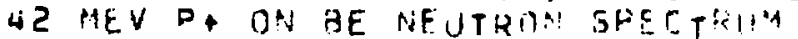

\section{ENERGY}

D. US NEV

Q. 1 G MEV

$0.15 \mathrm{MEV}$

$D, 2 \cap M E V$

$D .25 \mathrm{MEV}$

D.3 M MEV

0.35 MEV

T. $\$$ Q MEV

0.45 MEV

0.50 MEV

$D .55$ MEV

D. 6 II MEV

$\lambda .65$ MEV

0.7 MEV

0.75 MEV

D. BO MEV

0.83 MFV

$3.90 \mathrm{MEV}$

0.95 MEV

1. $\triangle B$ MEV

1.10 NiEV

1.2U MEV

$1.30 \mathrm{MEV}$

1. 4 H MFV

1.50 MEV

1. 6 O MEV

$1.70 \mathrm{MEV}$

1. BV MEV

$1.90 \mathrm{MEV}$

2. UU MEV

2. 11) MEV

2.RU MEV

2. $315 M E V$

2. $\triangle \mathrm{WJ} M E V$

2.51 MEV

2. $O Q$ MEV

2.7n MEV

2.80 HEV

2.90 MEV

3. WU MEV

3.20 MEV

3. 40 MEV

3, 6 D MEV

3. BQ MEV

4, WD MEV
PROTINS

$\omega$

3. गวE+ $\partial 2$

1. $S I E+32$

9.5.JF+b1

$7 .+3 E+01$

$5.8 \lambda E+111$

$4.79 F+61$

4. $22 E+W 1$

5. $35 E+01$

3. $47 E+1$

3. $30 E+$ WI 1

2. $99 E+111$

2. $197 E+\$ 1$

2. $24 E+11$

2. $)\{F+n\}$

1.97E+W1

1. $89 E+w 1$

$1.67 \mathrm{E}+\mathrm{Wl}$

1.69E+ +1

$1.63 F+11$

2. $38 E+11$

2. $76 E+191$

2. $29 k+x^{\prime \prime 1}$

2. COE +U1

?. $10 E+d 1$

1. $34 E+11$

1. $95 E+41$

1. $R O E+$ WI

1. SOEE+?

1. $64 E+11$

1. $51 E+(1)$

1. $37 E+61$

1. $172 E+1$

1. $32 E+41$

1. $18 E+1$

1. $\Delta 2 E+b 1$

1. $1 \lambda E+B]$

1. $\triangle R E+1$

1. DaE +

$9.74 E+G(1)$

$1.6 B E+01$

$1.60 E+01$

$1.43 E+41$

$1.40 E+61$

$1.23 E+1$
DF UTERUS

$\because$.

$3.171=\ln 3$

$3.17 i-1+3$

3. ?he $=13$

3. $20[-613$

7. $49+-63$

$1.47 t=43$

9. 3ise-ns

9. 3UF-AS

1. $13 \mathrm{~F}=62$

$1.13 F=19 ?$

5. $.7 \mathrm{E}-\mathrm{bic}$

$5.27 E-112$

$1.395-82$

1. 3पE - ते?

$5.5 .3 E-c^{2}$

$5.5 .9 E-12$

1. $47 E-V I C$

1. $47 E-9 ?$

a. $98 \mathrm{~F}=\mathrm{M}$ ?

$4.98 E-12$

7. 2 BE- $A 2$

7.? BE - II?

5. ACE-AZ

S. DAt - A 2

5. PRE $=x^{12}$

5. ABE- IT?

$3.56 E-W 2$

$3.56 \mathrm{~h}=\mathrm{AT} 2$

5. $95 \mathrm{E}-\mathrm{H} 2$

$6.95 E-62$

7. WSF - . 22

7 . 15F - M2

$9,43 E-13$

7. $48 F-43$

$2.65 F-12$

2. $65 E-02$

$2,615=02$

2. 6 if $=$ liz

1. $27 E-11$

$1.2 / F-01$

4. 9 O - IA $^{4}$

4. $89 F=02$

$6.5 \mathrm{DE}-\mathrm{H} 2$
Tip Tries

$\therefore$.

$+1$

1 .

it

1. 14t-ris

1. $14 E=-13$

$7.99 E-.35$

$1.49 E-.15$

is.

4).

$2.46 f-13$

2. 15t-15

1. 4 . $E=$ U 3

1. $46 E-63$

$3.45 E-i i 4$

5. $45 t .14$

2. 2 At -13

?. $28 \mathrm{t}-13$

1. $8 \mathrm{itc}-\mathrm{A}^{3}$

1. 4 1. E- - 3

$3.99 t-13$

S. Igt - it 3

1. DIAF -1 is

1. $5: 0=3$

2. $311 t=-112$

2. 3IE- A?

4. $58 \mathrm{~F}=03$

$4.58 E-33$

$5.33 E-.93$

$3.33 E-15$

2. 1 bt $=3$

2. 1 GE $=13$

2. $52 E-1] 3$

¿. T2E-AS

$2,11 E-H c^{2}$

ट. $11 t-v 2$

2. $1: 9 E-i j 3$

2. $\triangle 9 E-.35$

2. 1 HE-UZ

2. $1 \mathrm{~A} E-\mathrm{G} 2$

$3.59 E-U S$

$3.59 E-63$

2. $11 E=13$

2. $11 E=43$

2,1 GE $-14 ?$
ALPHAS

3.7GF-Ai

3. THE-A1

4, $3, T F-4:$

4. BSE- I1

$7.92 E-11$

$7.12 E-n 1$

$7.53 E-V 1$

$7.53 E-D 1$

7. IOE-VI

7. $16 F-1\}$

$0.9,1 F-11$

$6.9 W F-X 1$

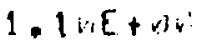

1. $1 \times E+1 \%$

6. $13 E-11$

\&. 1 3E- - I 1

B. SIE-U1

Q. $61 E-.11$

9. $2 k .5=11$

$9.2 B E-11$

1.81ftily

1. $8\{f-t i d$

2. $1 r^{2} f+w i t$

2. $12 E+H v$

2. $22 \mathrm{E}+19 \mathrm{~A}$

2. $22 E+11$

2. $35 E+W$

2. 35E+ $11:$

$2.57 E+16]$

2. $57 E+14$

2. $64 \mathrm{~F}+1 \mathrm{H}$

?. $\$ 4 E+d a$

$2.82 f+010$

2. $82 \mathrm{E}+\mathrm{W}$

$2.91 \mathrm{E}+\mathrm{HO}$

z. $91 E+36$

2. 6 OHE + DU

?. GUE + HW

$2.57 E+4 k$

$2.57 E+w)$

$4.72 E+46$

$4,7 \geq E+44$

4. $4 \mathrm{UE}+14 \mathrm{H}$

$4,4 V E_{-}+W 0$

$3.37 E+d G$ 


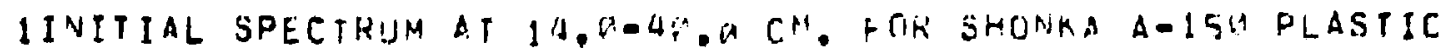
42 MEV P + ON BE NEUTRON SPECTRLII

\section{ENF.RGY}

4. 2U MEV

4.44 MEV

$4,6$. MEV

$1.80 \mathrm{MEV}$

5,13 MEV

5.50 MEV

6. OU MEV

6. SO MEV

7. HW MEV

7.5H MEV

B. LU MEV

B. 5И MEV

9. $\triangle V ~ M E V$

$9.5 \cup$ MEV

13. UV MEV

11. WU MEV

12. HI MEV

13. $10 \mathrm{MEV}$

14. OQ MEV

15. 100 MEV

$16.00 \mathrm{MEV}$

17. HO MEV

1B. $O O M E V$

19. ON MEV

29, WIO MEV

21. OU MEV

22, NO MEV

23. DV MEV

24,00 MEV

25. DU MEV

$26.90 \mathrm{MEV}$

27.0 IS MEV

28, $O M$ MEV

29,0 D MEV

30.0 MEV

32. MA MEV

34.01 MEV

36. UO MEV

38. DQ MEV

40. U1 MEV

$42.04 M E V$

44. DG MEV

$46.00 \mathrm{MEV}$

49.06 MEV

50. BU MEV

OAVE, E(MEV)

\section{PRATUIS}

$1,19 E+21$

$1.19 E+\Delta 1$

$1.16 F+31$

1. $: 4 \mathrm{E}+\mathrm{Q} 1$

$9.83 E+D H$

2. $1 \mathrm{AE}+\angle 1$

1. $96 E+191$

$1.63 F+b 1$

1. $46 E+31$

1. $28 E+d 1$

$1.2 M E+D 1$

7. $74 t+3 H$

1. $A B E+01$

$9.5 U E+d i)$

$8.9 B E+11 A$

1. $S 5 E+1$

1. $4 P E+11$

1. $2 P E+W 1$

1. $17 E+11$

1. $1 A F+31$

9.4RE + dV

$7,8 ? E+46$

$7.18 F+4 B$

6. $15 E+b$.

o. $31 E+k$.

$0.31 E+114$

$5.65 E+W(W$

$4.77 E+A U$

5. A AE + D

$4,31 E+019$

$3.05 E+130$

3. $5 \lambda E+A 1$

$2.73 E+34$

$2.23 E+30$

1.77E+B:

3. $46 E+60$

2. $46 E+3 i t$

1. $23 E+B A$

$7.3 S E-.11$

1. $5 \Delta F-d !$

Q.

in.

n.

$D$

is.

16.57
DF JTER?RS

h. $36 F-12$

6. $33 F-A^{A} 2$

6. 33E- 22

$4.63 E=1.3$

$4,6 \leq F-13$

$5.52 \mathrm{E}-2$

$5.52 F-92$

$2.96 E-k 2$

?. $9 E-0$ ?

2. $53 \mathrm{E}-\mathrm{n}$ ?

2. $53 \mathrm{~F} . \mathrm{MI}$

$2.37 E-N 2$

2. $37 \mathrm{~F}-7$ ?

$4.1 P E-122$

4.1 HE-h2

2. VIAR-D.2

2. $\rightarrow$ प्रF- 12

8. $23 E-M+1$

B. $23 E-1.4$

a.

.

in.

is.

.

w.

a.

a.

(i)

is.

it

a.

4

$n$

$a$

v)

a.

i)

is.

6

9

.

$5.3 i$
ThI TUivs

2. $16 t-v^{2} 2$

2., $715-43$

2. $71 E-x 3$

$9.25 E-24$

$9.25 E-64$

$3.7 \exists E-13$

3. $79 E-.3$

2. $21 E-A 2$

2, $21 E-V Z$

1. $\angle A E-B^{3} 3$

4. $A B E-23$

H. SAE- 14

Q SWE-W

$5.91 E-34$

$5.91 E-34$

$3.5 O E-P 4$

3. SPE-WI

$i$.

vis

i.

$r$

vi.

.

i.

vi.

W.

$\forall$

in.

G.

is.

$A$

1.

D.

(i).

c)

b.

$\omega$

b.

v.

S.

k.

bi.

U.

A.

6.

4.83
ALPRIAS

3. $37 E+64$

2. $82 E+b$.

$2.82 E+6 A^{4}$

2. $2 b E+i n$

2. 2hE+dx?

3. $B S E+d E$

3. $35 E+V I$

2. $2 b E+: A r$

2. $20 E+k i j$

1. $76 E+3$

$1,76 E+A 15$

9.7 of -41

$9,76 E-A 1$

$6.77 E-A 1$

$6.77 E-21$

ล. $25 \mathrm{E}-\Delta 1$

$8.25 F=-11$

$2.57 F-D 1$

2. 57 E - iI

1. $96 E-k 1$

$1.96 E-x 1$

$1.35 E-41$

$1.35 E-\square^{4}$

$7.69 E-1.2$

$7.69 E-1.22$

D.

B.

i.

A.

it.

a.

a.

4.

in.

1.

1.

1.

1.

H.

in.

vi.

H.

is.

is.

$\theta$

5,54 
IINITIAL SPECIRUM AT H.DOOH. RS CH, FOK ICRH MUSCLE IISSUE 42 MEV P + ON BE NEUTPRN SPECTRUM

\begin{tabular}{|c|c|c|c|c|}
\hline ENERGY & PROTOUS & V.F UTERDAS & TRITONS & ALPHAS \\
\hline$D . M S \quad M E V$ & a. & 4 & (1) & $2.33[+1 v)$ \\
\hline 0.10 MEY & $4.3 A E+3 ?$ & is & (i) & $2.33 t+4: 3$ \\
\hline J.15 MEV & $2.17 E+12$ & 1.178-1i1 & 6. & 4. $41 E+26$ \\
\hline 0.20 MEV & $7.56 E+2:$ & $1.17 E-1.1$ & 4. & $4.41 E+4$ \\
\hline$D .25$ MEV & $1.96 t+1.2$ & $B$ & 9 & 8. $A_{6} E+6 x^{\prime}$ \\
\hline $1,3 G$ MEV & S. BUE +1 I & 仿 & 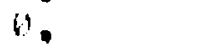 & $R \cdot B \cap E+D_{k}$ \\
\hline 35 ME $\checkmark$ & 8. vilif + 11 & $a$ & $\therefore$ & 1. $D(B E+11$ \\
\hline .40 MEV & v. & $\theta$ & u. & 1. $1 E+E+1$ \\
\hline .45 MEV & $9.56 E+11$ & $\theta$ & 2. $201-111$ & $1.1+E+31$ \\
\hline . $5 \mathrm{M} M \mathrm{MEV}$ & $2 . A A F+11$ & 6 & 2. $\left.2 N^{\prime} E-V^{\prime}\right)$ & $1.14 E+\| 1$ \\
\hline $55 \mathrm{MEV}$ & 6. D. & $1.175-111$ & $n$ & $6.74 E+6.6$ \\
\hline$O D$ MEV & $H$ & $1.17 \mathrm{E}-61$ & $\theta$. & $6.74 E+64$ \\
\hline 65 MEV & $6 . A M E+11$ & a. & $\theta$ & $7.33 E+14 E$ \\
\hline $7 \triangle M E V$ & n. & $\theta$ & is & $9.33 E+(91)$ \\
\hline 75 YEV & $2,3) \varepsilon+191$ & a & iil, & $7.34 E+30$ \\
\hline 30 MEV & $1.21 E-B 1$ & 6. & 6. & $7.34 E+46$ \\
\hline 5 MEV & $3.71 E+11$ & 19 & v. & $1.64 E+11$ \\
\hline$\triangle M E V$ & 2. $\triangle A E+B 1$ & 1 & $\theta$ & $1.64 E+11$ \\
\hline 95 MEV & $2 \cdot \operatorname{sos} 3 t+61$ & 4. & 3 & $2.39 E+11$ \\
\hline AA MEV & $3 . E R E+01$ & it. & it. & $2.39 E+11$ \\
\hline 10 ME V & $5.76 E+01$ & $1.17 \mathrm{E}-11$ & $2.21 E-1$ & $5.30 E+B 1$ \\
\hline$\triangle M E V$ & $6, W A E+B 1$ & $1.17 F-01$ & $2.21 E-.11$ & $5,3 b E+i 11$ \\
\hline $.30 \mathrm{MEV}$ & $0 . M A E+11$ & $n$ & a & $2.31 E+11$ \\
\hline $.4 V M E V$ & $4.40 E+11 !$ & H & *. & $2,311+n 1$ \\
\hline$S U$ ME. & 4. $\because A E+61$ & $1.17 t=u_{1}$ & H. & $2.34 E+1.1$ \\
\hline bE MEY & $\theta$. & $1.17 E-V 1$ & $H$ & $2,34 E+1$ \\
\hline$\triangle M E V$ & $2.43 E+01$ & $3.26 E-112$ & $a$ & $1.31 E+V 1$ \\
\hline OA MEV & & $E=02$ & a. & $1,31 E+Q 1$ \\
\hline QU MEV & $3,28 \mathrm{E}+11$ & $E=12$ & 0 & $1.48 E+91$ \\
\hline DE MEV & 2 OIPE + Oi & st. -12 & $H$ & $1.4 B E+v 1$ \\
\hline ID MEV & H. $4 R E+B I$ & a. & $w^{5}$ & 1. $43 t+11$ \\
\hline MEV & D. & $n$ & 1. & $1.43 E+1$ \\
\hline MEV & A. ABE+Q1 & 9 & $\theta$ & $1.97 E+1$ \\
\hline DL MEV & $4 . \forall A E+B 1$ & a. & $\mathrm{k}^{\prime}$ & $1.97 E+1$ \\
\hline ;0 MEV & d. & $3.68 E-H_{1}$ & $5,16 E-03$ & $1.03 E+61$ \\
\hline MEV & $2.00 E+B 1$ & $3.46 E-41$ & $3.16 E-12$ & 1. $13 E+11$ \\
\hline MEV & 1. $119 E+12$ & $3.11 F=w 1$ & $a$ & $S .41 E+D 6$ \\
\hline MEV & b. & $3.11 E=1$ & 0 & $5.41 E+10$ \\
\hline MEV & 0. & $6.616=02$ & 3 & 1. $1 E+\Delta 1$ \\
\hline$M E \ddot{V}$ & 6 & $6.61 E-02$ & B. & $1 \circlearrowleft 1 E+01$ \\
\hline MEV & 6. AAE + DI & $b$ & $1.82 E-11$ & $2.44 E+11$ \\
\hline MEV & $1.60 E+02$ & $\theta$ & $1.82 E-111$ & $2.44 E+v 1$ \\
\hline MEV & $7.82 E+01$ & , & $b_{1}$ & $1.56 E+B 1$ \\
\hline $\begin{array}{l}30 \text { MEV } \\
\text { MEV }\end{array}$ & b. & Q. & i. & $1.56 E+61$ \\
\hline MEV & $4.11 E+61$ & & v. & $3.98 E+611$ \\
\hline
\end{tabular}


IINITIAL SPECTHUM AT M. GHOB.B5 CM. FliK ICRIJ MUSCLE TISSUF. 42 MEV P ON BE NEUTRUN SPECTRUA

\section{ENERGY}

4.20 MEV

4.40 MEV

4.60 MEV

$4, B O M E V$

5. UИ MEV

$5.5 \square M E V$

6. DO MEV

$6.50 \mathrm{MEV}$

7. 10 MEV

$7.50 \mathrm{MEV}$

8. HO MEV

8.50 MEV

9. OO MEV

9.5D MEV

10. DO MEV

11. OG MEV

12.00 MEV

13. DU MEV

$14.00 \mathrm{MEV}$

15.DD MEV

16. DU MEV

17. GO MEV

18.DO MEV

$19.0 \%$ MEV

$20.0 D M E V$

$21.0 \triangle M E V$

22. UA MEV

23. OU MEV

24.00 MEV

25, DD MEV

26. OU MEV

$27 . A H ~ M E V$

28. 10 MEV

$29.00 \mathrm{MEV}$

30. BV MEV

32. OQ MEV

$34.00 \mathrm{MEV}$

$36.00 \mathrm{MEV}$

38.0D MEV

40. OD MEV

42. $\triangle O ~ M E V$

44. DO MEV

46. OQ MEV

48, DO MEV

50.00 MEV
PROTOUS

5. $82 E+\Delta 1$

$1.71 t+16$

1. OAf + DI 13

2. जIA $[+11$

2. $A C E+D !$

1. $1 \cap E+2 Z$

1. $3 \mathrm{BF}+\mathrm{BI}$

4. 17E+3!

2. $1 E+y 1$

2. $\lfloor\mid 1 E+U 1$

4. DOE + D

a.

4. $B A E+V 1$

2. $B A E+D 1$

is.

4. $) 3 E+131$

2. M5E

6. WAE + OI

2. $\triangle 3 E+D 1$

2. OAE + 1

a.

4. $H Q E+11$

2. GGE +1

a.

4. MBE+D1

$\Delta$.

is.

$D$.

i.

a.

2. MaE + 1

0.

2. AAE + O1

is.

1.

i.

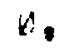

b.

v.

B.

B.

B.

U.

0.

a.

9.32
DEUTFROARS

a

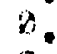

.

a.

2. $57 \mathrm{~F}-\mathrm{AI}$

?. $57 \mathrm{E}-1$

in.

(i)

$6.85 E-32$

$6.25 F-142$

a.

i.

$\because$

in,

$3.43 F-1.2$

$3.43 E-12$

$3.42 E-B 2$

$3.42 E-V 2$

D.

a.

o.

$n$

g.

$a$

a.

a.

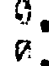

is

$n$

a.

a.

4

.

0

a.

0.

v.

5,90
TRITUNS

is.

is.

i.

in.

i.

i.

3.

4.

$4.43 E \cdot$.?

4. $43 L-b i 2$

$4,4 B E=A 2$

4. $\triangle R E-H 2$

1).

6.

i.

6

B.

D.

0.

$\theta$

i.

$\therefore$

$\theta_{1}$

is

b.

a.

is.

a.

in.

in.

H.

w.

U.

b.

v.

is.

is.

in.

$v$

is.

b.

Q.

4.

4.35
ALPHAS

3. OHE + AW

3. $98 E+116$

$3.98 E+A k$ ?

$1.63 E+11$

$1.03 E+1$

1. obit + in 1

1. OVE + B1

4. $42 E+04$

4. $42 \mathrm{t}+\mathrm{dA}$

1. $15 E+41$

1. $i S E+1$

2. $\mid 4 E+11$

$2.14 E+B 1$

1. $12 E+A B$

1. Oa $2 E+A n$

1. $\theta \Delta E+W 1$

1. $D 4 E+11$

1. $A 1 F+D 1$

1. $(1 F+1$

B. 37t-dC

8. 37E-D2

Q. $37 E-1) ?$

B. 37E-UZ

H.

1.

1.

a.

0.

19

,

is.

i.

0.

is.

4

1.

a.

$\theta$

b.

(i)

i.

b.

a.

a.

D.

6.27 
IINITIAL SPECTRUM AT D. A5-H.1A CM, FOM ICRLI MUSCLL IISSUF: 42 MEV P+ ON HE IEEUTRON SPFCFGUM

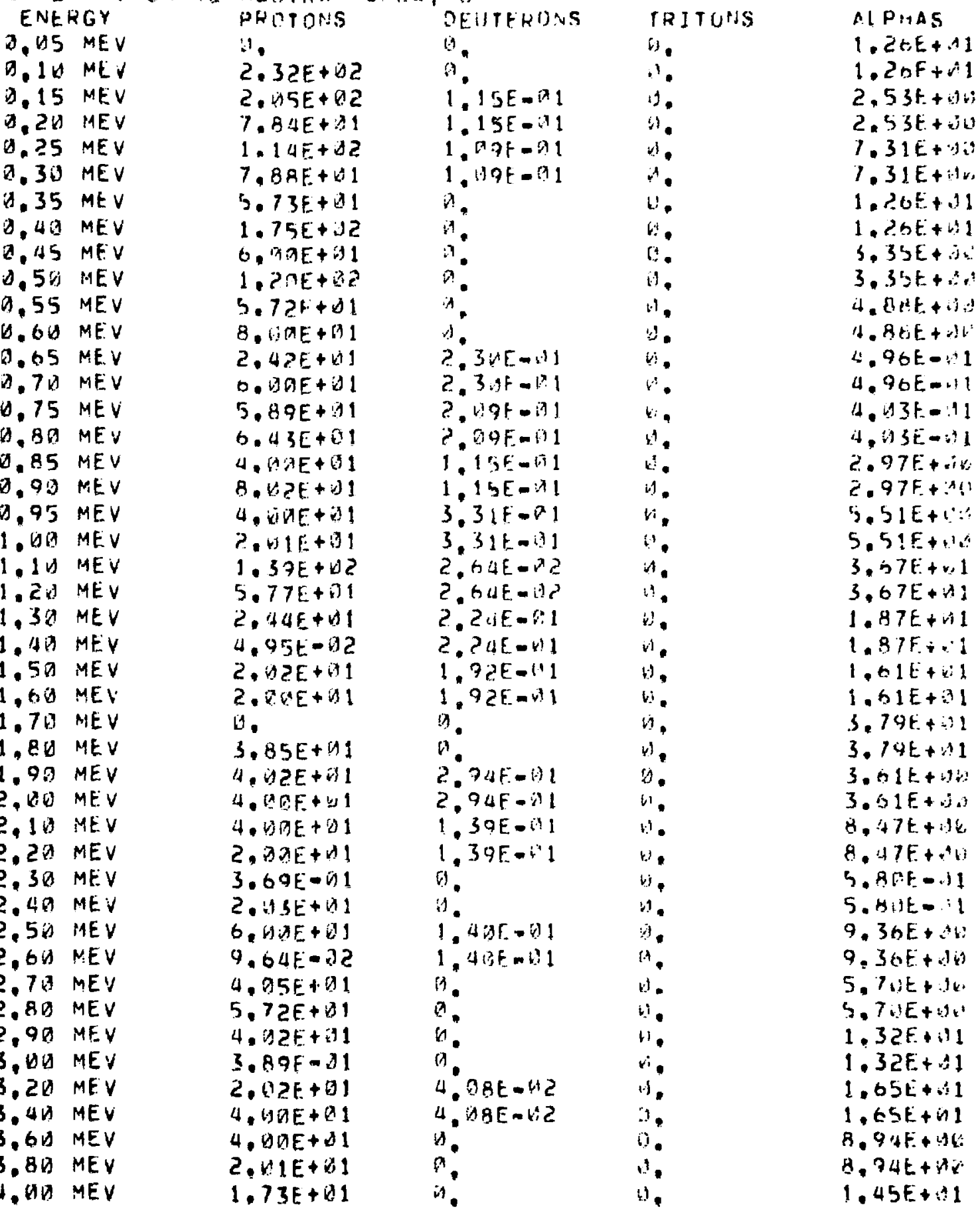


IINITIAL SPECTRUH AT O. U5-W.1G CM. FUK ICFL MUSCLE TISSUE 42 MEV P + ON BE NEUTRON SPLCTRUM

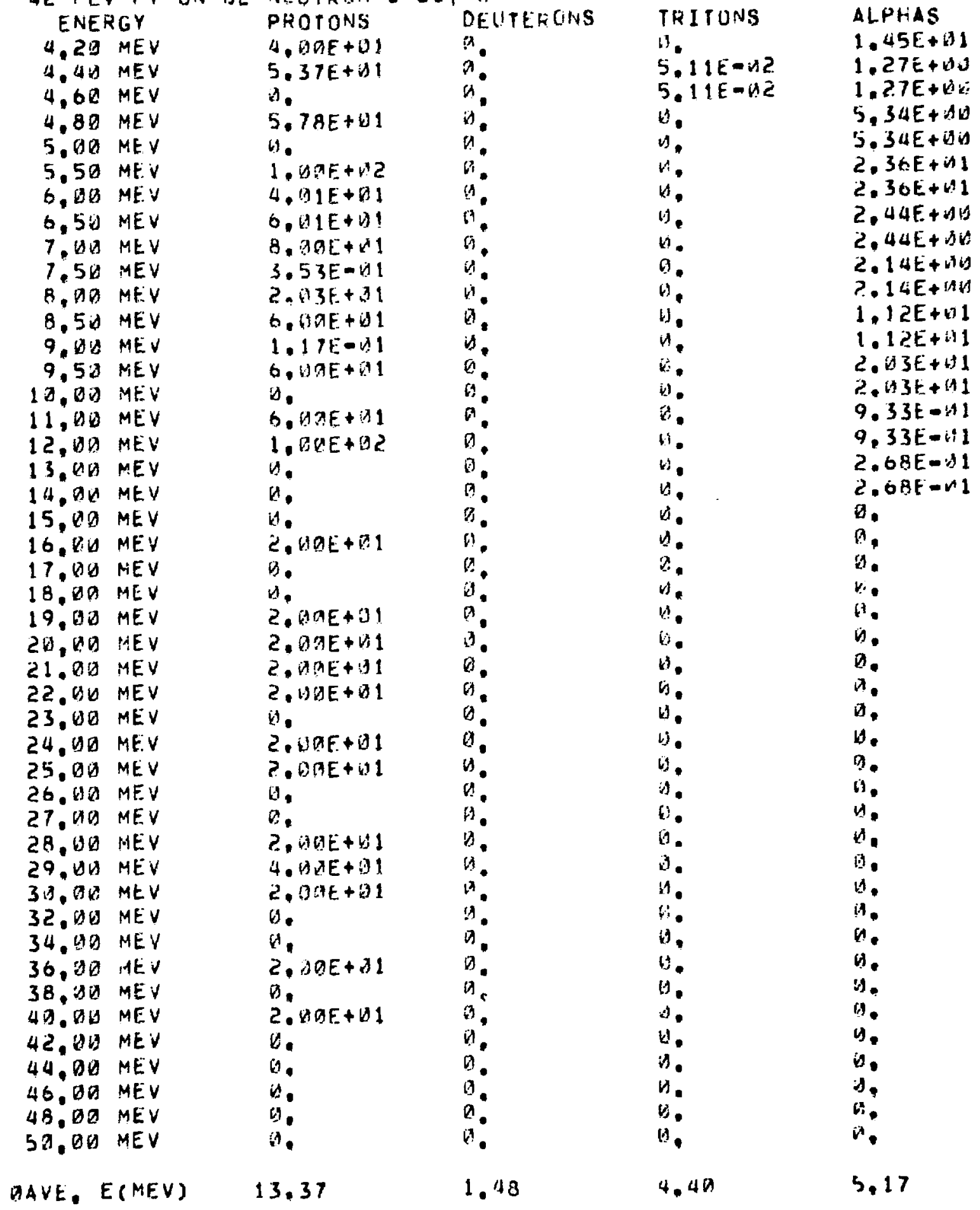


IINITIAL SPECIRUM AT U.19-1. $15 \mathrm{CM}^{\mathrm{M}}$. FOP ITRH MUSCLE IISSUE 42 MEV P+ ON BE NE!ITRON SPFCTRIIM

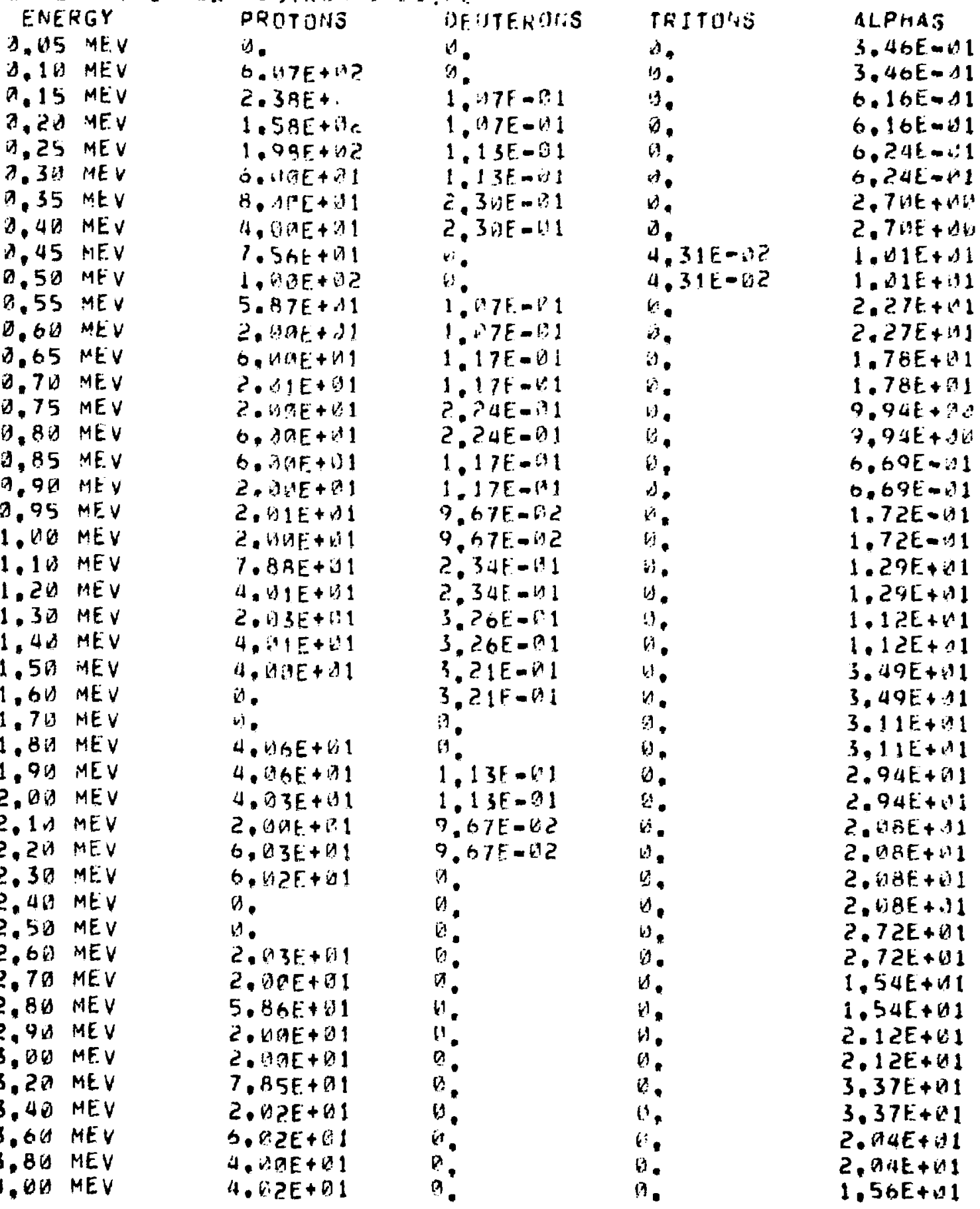




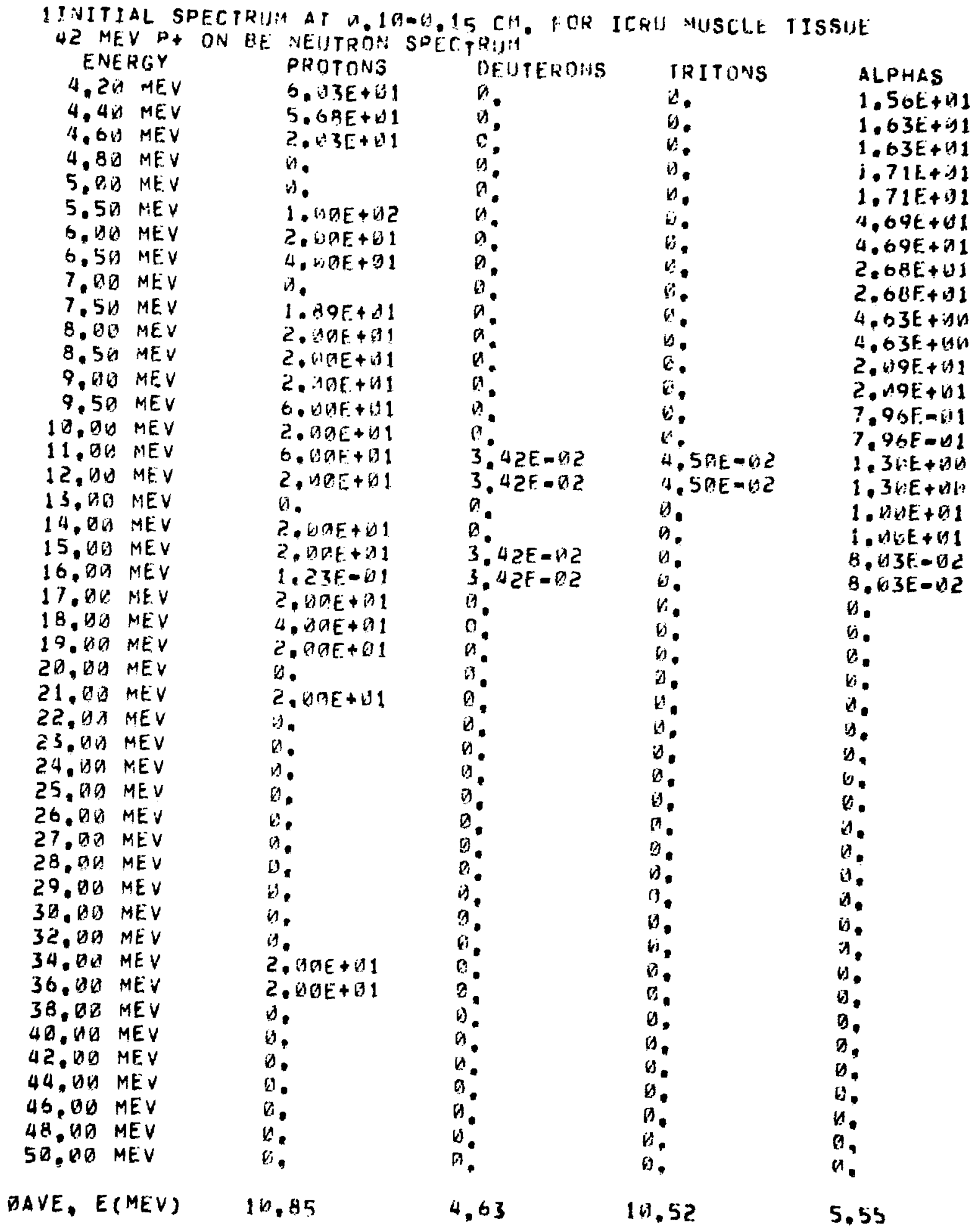


IINITIAL SPECTRUM AT H. 15-9,2A CM. FOR ICRU MUSCLE IISSHE 42 MEV P+ DN EE NEUTRON SPECTRUH

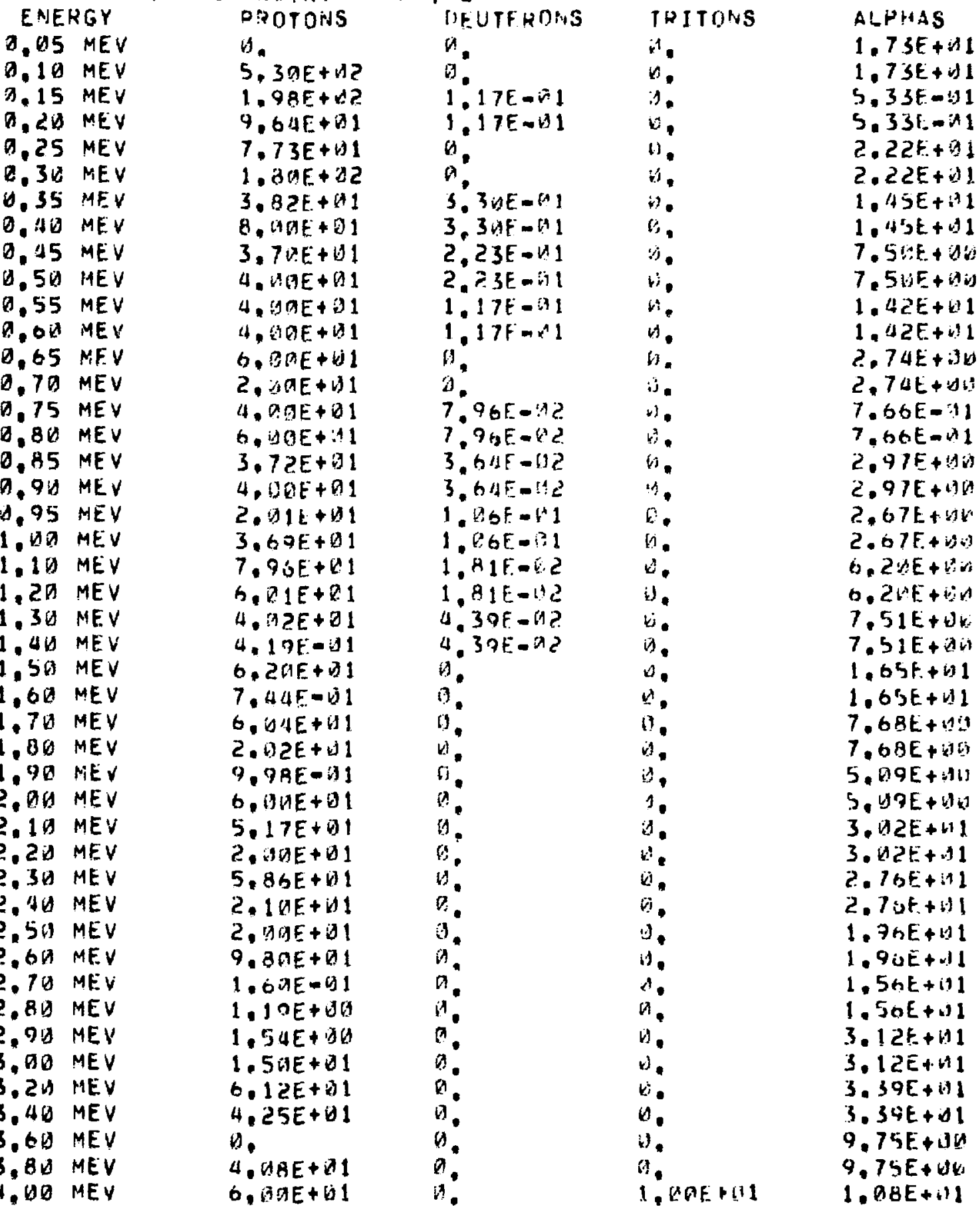




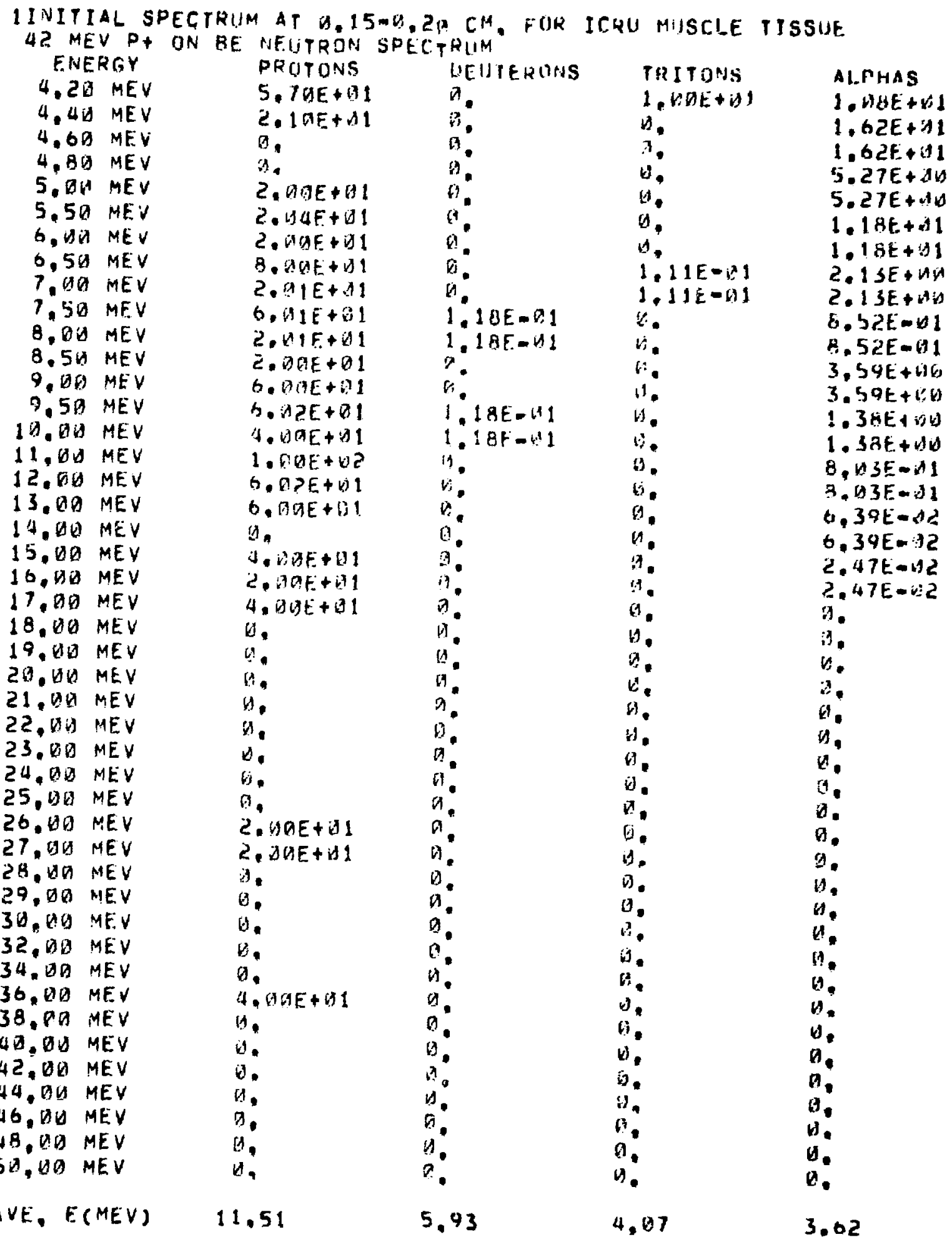


1INITIAL SPECTRUM AT A.20-A.4A CH. FOR ICRU MUSCLE IISSUE. 42 MEV P + ON BE NFLTROIV SPECTFIIIA

\section{ENERGY}

D. 15 MEV

0.10 MEV

9.15 MEV

3.20 MEV

0.25 MEV

Q.3D IEV

0.35 MEV

0. 4D MEV

0. 45 MEV

1.5\% MEV

R. 55 MEV

0.60 MEV

0.65 MEV

$0.70 \mathrm{MEV}$

0,75 MEV

$0.81 \mathrm{MEV}$

0.85 MEV

$0.90 \mathrm{MEV}$

0.95 MEV

1. $\triangle D M E V$

$1.19 \mathrm{MEV}$

1.20 MEV

1.30 MEV

1. 40 MEV

$1.50 \mathrm{MEV}$

$1.60 \mathrm{MEV}$

1.7V MEV

$1.80 \mathrm{MEV}$

1.9 MEV

2. OQ MEV

$2.10 \mathrm{MEV}$

$2.20 \mathrm{MEV}$

$2.30 \mathrm{MEV}$

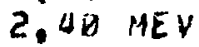

2. $50 \mathrm{MEV}$

2,60 MEV

$2.70 \mathrm{MEV}$

2. $8 \backsim$ MEV

$2.90 \mathrm{MEV}$

$3,0 V$ MEV

3.2V MEV

3. UR MEY

3.6D MEV

3.80 MEV

4. UD MEV
PIDTONS

i.

6. BRE + B

1. $79 t+2$

$2,14 E+02$

1. $54 E+132$

$6.42 E+21$

$5.50 E+1) 1$

9. $18 E+\cdots 1$

$3.83 E+A 1$

4. $33 F+1$

$5.8(7 E+A)$

3. $50 \mathrm{E}+01$

$6.7 \Delta E+b 1$

?. $47 E+51$

1. $11 E+D 1$

3. $55 E+01$

2. $46 E+31$

2. $13 E+11$

$3,45 E+111$

i. $35 E+1$

6. $51 E+B 1$

2. $96 E+11$

5. $47 E+B 1$

5. $00 E+\triangle 1$

$5.78 E+B 1$

1.97E+ 11

2. $59 E+01$

3. AIE 1

3. $59 E+D 1$

$1.99 E+11$

1.97E+ 1

$3.925+11$

2. $5.9 E+11$

1. $Q 7 E+61$

1. $92 E+41$

$1.41 E+111$

$3.57 E+01$

$2.39 E+131$

1. $54 E-11$

1. $84 E+01$

$3.05 E+x) 1$

$3.77 E+11$

$3.93 E+01$

$3.92 \mathrm{~F}+01$

3. $45 \mathrm{E}+1$
DFUTERTISS TRITONG

$5.85 t-42$

$5.85 E-142$

5. $5 B E-22$

$5,53 E-.72$

2. $635=12$

2. $6.5 E-192$

$5.14 E-12$

$5.14 E-n 2$

4. NDE $=A 2$

$4 . \forall B E-D ? 2$

$5,85 F-12$

$5,85 E-12$

it.

P.

1. D1E- 1

1. $11 \mathrm{~F}=\mathrm{A}$

4.

0.

$6.62 E=112$

$6.62 E=132$

1. $29 E=1$

$1.29 F-? 1$

3. 6 QEE- 1

3. $980 .-31$

2. 5 U 5 - 92

2. 5 Q $E-12$

2. $M T E-O 1$

2. $\triangle \triangle E-\triangle 1$

3. $55 E-1^{4} 2$

$3.55 F-72$

a.

a.

2. $4 B E-A 2$

2. $4 B E-02$

0.

n.

9

$\theta$

5. A2E- 12

5. $\triangle 2 E-n ?$

3. $1 B E-A 2$

3. 1 HE-OC

a.

a.
1.

.)

s.

D.

i.

4.

$n$

is.

$5.14 E-12 Z$

5. 1 UE-B2

a.

r.

v.

6.

5. 14E-32

5. 1 $4 E-92$

19.

i)

a.

n.

b.

$d$.

a.

a.

5. O1E-UZ?

5. DIE-H?

$\forall$.

b.

$b$

in

9

is.

$\theta$

0.

0.

b.

0

i.

U.

s.

5. 29t- 02

5. 29E- 82

w.

$n$

2. $50 E+06$
ALPHAS

1.27E+ 114

$1.27 E+(A)$

3. (ASt + WI:3

5. $\triangle 5 E+2 A$

2. BKE + D

2. BOEE + AS

$5.03 E+A L$

5, (1) $3 E+11 \%$

3.8UE+4t

3. BRE + DU

$6.46 t+0 k$

6. $46 E+3 v$

5. $199 E+A G$

5. $19 E+d v$

B. 5 ut +40

$B, 56 E+146$

$1.41 E+19 n$

1. $41 \mathrm{E}+\mathrm{H}_{\mathrm{k}}$ :

5. $23 E+00$

5. $2.3 E+46$

$1.8 \circ E+b 1$

$1,86 E+111$

B. $64 E+U$

B.64E+3:3

1. $11 E+11$

$1 .|1 E+\Delta|$

$1.63 E+111$

$1,63 E+11$

S. $54 E+01$

$B .54 E+d G$

1. $49 E+01$

1. $49 E+11$

1. $3 \Delta E+1$

1. $3: t E+11$

$B, 61 E+W$

8.61E+1)U

8. $80 E+4)$

8. $84 E+0 v$

1. $32 E+61$

$1.32 E+41$

3. $2 a E+d 1$

3. 2UE+U1

2. $23 E+41$

2. $2.3 E+M 1$

2. $\square I E+I !$ 
IINITIAL SPECTRUM AT O.2A-O. 4n CM. FOR ICRII MUSCL.L TISSIJF. 42 MEV P + ON BE NEIJTRON SPECTKU\%

ENERGY

4.2त MEV

4.40 MEV

4.6月 MEV

4.8 $8 \mathrm{MEV}$

5.06 MEV

5.5 TA TEV

6. DA MEV

$6.50 \mathrm{MEV}$

7. $O D M E V$

7.50 MEV

B, OD MEV

8.56 MEV

9. 90 ME $Y$

9.59 MEV

10.06 MEV

$1:, 00$ MEV

12. W. MEV

13. DO MEV

$14.00 \mathrm{MEV}$

15, DQ MEV

16. OA MEV

17. OU MEV

$18,00 \mathrm{MEV}$

19.0 $19 \mathrm{MEV}$

$20,00 \mathrm{MEV}$

21. OU MEV

$22 . U 0 \mathrm{MEV}$

$23.00 \mathrm{MEV}$

24.00 MEV

$25.00 \mathrm{MEV}$

26. DO MEV

$27.00 \mathrm{MEV}$

28. DO MEV

$29.00 \mathrm{MEV}$

$30.00 \mathrm{MEV}$

$32.00 \mathrm{MEV}$

34.00 MEV

36.00 MEV

3B. OO MEV

40.90 MEV

42,01 MEV

44.DD MEV

46. DO MEV

$48.02 M E V$

50.00 MEV

DAVE, E(MEV)
PROTONS

3. UUE+ $D 1$

3. $51 E+61$

4. $\triangle 3 E+\Delta 1$

1. $B 6 E+61$

$5,11+E+A D$

6. $1 k+1 k 1$

2. $52 E+B 1$

5. $49 E+B 1$

5. $9 A E+D 1$

2. $5 \lambda E+B]$

3. $22 E+1$

$3,57 E+1 D 1$

2. $5 \nabla E+D 1$

1. $51 E+01$

5. JUE + QU

3. 5 T $E+i 1$

3. AQE + D?

1. $5 A E+D 1$

3. UIE+ 11

2., $5 A E+A 1$

2. $9 B F+31$

4. $M E+D E+1$

2. $5 D E+D 1$

5. ADE + DU

2. $B \cap E+B 1$

n,

2. $M A E+Q 1$

2. MAE+A1

2. $\forall A E+B 1$

1. $5 \backsim E+D 1$

2. WAE + WI

1. $\triangle \cap E+b 1$

5. OOE + OA

5. DOE + UQ

5. GOE+ BO

1. $50 E+D 1$

i.

D.

$\Delta$.

0.

E.

b.

B.

a.

v.
DEUTERDINS

b.

2

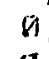

a.

4

a.

$\vec{n}$

i)

?.

a

a

0

$\theta$

9

9

19

11

9

B.

a

a.

a.

$a$

$a$

a.

a.

a.

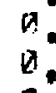

a.

a.

$a$

$a$

a.

1.42
TR 1 TOPS

2. $50 E+W H$

i.

V.

II.

(1)

3

$\theta$

D.

G.

is.

is.

$\Delta$.

$\Delta$.

$\theta$

(i).

i.

$n$

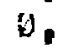

$\theta$

19.

v.

$n$

b.

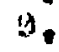

is.

b.

9.

$\because$

i.

0

a.

$\Delta$.

.

$\theta$

D.

E.

$\theta$

0

id.

0

$\theta$

$\theta$

a.

bs

0

3.93
ALPHAS

2. DIE + D1

$1.57 E+131$

$1.57 E+U 1$

4. $77 E+d M$

$4.77 E+46$

2.1 $1 \mathrm{E}+21$

$2.17 E+11$

$1.1, E+D 1$

1. $105+31$

1. $24 t+61$

1. $24 E+11$

$1.35 E+41$

1. $35 E+W 1$

3. $2 U E+D H$

3. $2 u E+d u$

1. $31 E+\Delta 1$

1. $31 E+\lambda 1$

2. $54 E+14$

2. $5 k E t+16$

5. $1 B E E+D A$

5. BWE + $A 19$

i.

0.

(3.

I.

a.

0.

D.

0.

0.

(1.

a.

$\theta$

b.

a.

0

id.

4

D.

a.

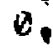

is.

i)

n.

D.

6.63 


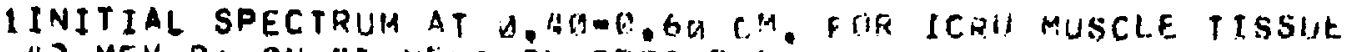

42 MEV $P+O N$ BE INEUTRON SPECFRII"

\section{ENERGY}

0.05 MEV

9. 1 G NEV

0.15 MEV

0.20 MEV

Q. 25 MEV

0.30 MEV

0.35 MEV

9. 4 M MEV

$0.45 \mathrm{MEV}$

1.. 54 MEV

0.55 MEV

$0.60 \mathrm{MEV}$

$0.65 \mathrm{MEV}$

D.7A MEV

0.75 MEV

J.BO MEV

Q. B5 MEV

$0.90 \mathrm{MEV}$

$0.95 \mathrm{MEV}$

1. OU MEV

1.1O MEV

1.20 MEV

1.30 MEV

1. UO MEV

1.50 MEV

$1.60 \mathrm{MEV}$

1.70 MEV

1.80 MEV

1.90 MEV

2. AD HEV

2.10 MEV

2. 20 MEV

2. 3U MEV

2. 40 MEV

$2,50 \mathrm{MEV}$

$2.60 \mathrm{MEV}$

2,70 MEV

2,80 MEV

2.90 MEV

3.0口 MEV

3.20 MEV

3,40 MEV

3,60 MEV

3, BO MEV

4.0 MEV

\section{PROTONS}

$\therefore$.

$0.58 E+11 ?$

1. $85 E+12$

$1.39 E+A ?$

$1,33 E+13$ ?

$9 .+3 E+01$

3. $B 9 E+11$

B. 5 . AE $+B 1$

9. $\triangle M E+D 1$

$9.83 F+\Delta 1$

$0.46 E+D 1$

4. $7 \mid E+91$

2. $69 E+19)$

$5,71 E+01$

$2.03 E+131$

$4.97 E+01$

3. $77 E+01$

1. $16 E+01$

3. $64 E+B 1$

$2.97 E+11$

$7.45 E+41$

$4.98 E+61$

$4.22 E+11$

$5.79 E+01$

1. $83 E+01$

3. $49 E+b 1$

3. उलE $+d 1$

4. $) / E+D 1$

$4.74 E+3 U$

$3.47 E+01$

3. जOE

3. $46 E+11$

1. $51 E+171$

1. $51 E+101$

3. $23 E+b 1$

3. $36 E+111$

3. $94 E+81$

$4.47 E+21$

2. $B D E+D 1$

2. $89 E+\triangle 1$

2. $56 E+01$

$2,97 F+1$

3. $15 E+D 1$

4. $27 E+B 1$

2. $515+61$

\section{DEUTF.ROAS}

1.

a.

?.355-1?2

2. $85 \mathrm{~s}=\mathrm{m} \cap \mathrm{A} 2$

1. $A 9 E-191$

1. $19 E-11 !$

$9.85 E-1.2$

$9,55 F-13$

$1.92 F-1$

1. $.20 F-D$

$7.29 F-142$

$9.29 F-B$

1. $58 E=M 1$

$1,5 B E-1$

1. $\triangle A E-A 1$

1. $D \Delta E-B 1$

i. $14 E-M 1$

1,4 At - i 1

$7,615-92$

$7.61 F-12$

$3.51 \mathrm{~F}-\mathrm{A} !$

$3.51 \mathrm{E}-1$

$3,22 f-1$

3. ?2E- ${ }^{1} 1$

2. $12 F-1$

2. $121=\because 1$

1. $24 E-A 1$

$1.24 E-A 1$

b. $26 t-02$

6. $36 E-9 \mathrm{C}$

$9.6 j E-A 2$

$9.615-12$

$8,27 E-42$

B. $27 t-1.2$

D.

4.

$7.76 E-12$

$7.7 b E=42$

1. PAF -J2

1. $8.4 F-A i$

in.

i.

1. $85 E-132$

1. $B 5 E-112$

1.

\section{THITONS}

1?.

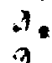

3.

(4)

$a$

3.

.

5)

u.

$\therefore$

a.

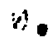

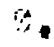

s.

A.

ki.

i)

$\because$.

4. 88E-

$4.88 E-42$

$\therefore$.

$c$

$n$

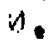

$n$.

i。

1. $17 E-v 1$

1. $17 F-21$

a.

7.

$\Delta$

$d$.

$3.3 E t-k 2$

$5,52 f-42$

i.

$\theta$

9.

i.

0

b.

1. $20 E-42$

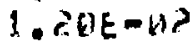

i).

i).

i.
ALPHAS

6. $4 I E+$ DO

$0.4+E+x)$

1. $32 E+\forall 1$

1. $32 E+*^{*} 1$

$7.79 F+116$

$7.79 E+W H$

1. $14 E+0 i$

1. $\mid 4 E+D 1$

$5.48 E+6$

$5.4 B E+A 6$

1. 3 IIE +1

1. $3: t E+x ! 1$

5. $36 E+V U$

$5.36 E+40$

B. $\triangle 3 E+\pi$

$8.83 E+B 14$

9. $D 1 E+W B$

4. $A 1 \mathrm{E}+\mathrm{bn}$

$5.68 E+A B$

$5,63 E+(16)$

2. $11 !+41$

2. $11 E+11$

1.17E+:11

1. $17 E+11$

1. $6 \mathrm{BN}^{4} E+1$

1. $6[E+]$ :

$1.89 E+191$

1. .59E+ 1

1. $54 E+A 1$

1. $54 E+4 i 1$

1. B $1 t+b 1$

1. $V I E+D 1$

$1.91 E+1$

$1.91 E+D 1$

1. $89 E+21$

1. $89 E+11$

$1.75 E_{0}+41$

1.75E+ ind

1. $12 E+\Delta 1$

1. $12 E+d 1$

9. $81 E+x$

9. 8$\rfloor E+U w$

1. $47 E+41$

1. $47 E+111$

$2.24 E+b 1$ 
IINITIAL SPECTRUM AT M, 4R-6, OAT CH. FOR ICPU MUSCLE TISSUE 12 MEV P+ ON HF UEUTRON SPECTPUIPA

ENERGY

4.24 MEV

4.40 MEV

4.6U MEV

4. 90 MEV

5. MV MEV

5,50 MEV

6. 01 M MEV

$6.50 \mathrm{MEV}$

7. 9 MEV

$7.50 \mathrm{MEV}$

8. $\triangle$ IV MEV

8,50 MEV

9. VTI $M E V$

$9,50 \mathrm{MEV}$

10.DO MEV

$11.00 \mathrm{MEV}$

12. QO MEV

13.06 MEV

14. TQ MEV

15. $\triangle 9$ MEV

$16.00 \mathrm{MEV}$

17. $O Q$ MEV

18. HQ MEV

19.0 MEV

20.00 MEV

21.00 MEV

22.00 MEV

23. BO MEV

$24, D$ D MEV

$25,00 \mathrm{MEV}$

$26,00 \mathrm{MEV}$

$27.0 \mathrm{MEV}$

$28.0 V M E V$

$29, U 5$ MEV

30,00 MEV

32.90 MEV

34.90 MEV

36.00 MEV

3B. AB MEV

40, DO MEV

42. DG MEV

44.0O MEV

46. MU MEV

48, AO MEV

$50, G B$ MEV

GAVE. E(MEV)

\section{PROTJNS}

$3.34 E+11$

$8.76[+190$

$2.87 E+01$

$1.53 E+b 1$

1. $5 U E+B] 1$

5. $4 ? E+B 1$

5. $\triangle 2 E+11$

4. $44 E+11$

$4.43 E+11$

$3.5 A E+1$

2. $5 \Pi E+D 1$

2. $5 A E+21$

2. $n>E+1$

2. $37 E+1$

3. 5 OE + 11

$6.50 E+W 1$

$2.935+11$

3,0 , E + 1

S. (UTE + DI I

2. GME 91

2. WOE+

2. $5 \square E+\square 1$

1. $5 a E+B 1$

?. BOE $+B 1$

2. $\triangle A E+01$

5. $) A E+\triangle D$

1. $5 A E+D 1$

U.

5. $\triangle A E+D D$

1. ANE + 1

1. $B O E+1$

4.

1. $30 E+51$

V.

1. $\triangle A E+D 1$

5. $) E+D C$

5. $\triangle 0 E+k 1 G$

5. $0 A E+D a$

$5,0,0 E+60$

1.

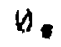

v.

o.

B.

is.

\section{DELITERONS}

is.

in.

$7 . B S E-U 2$

$7.85 E-132$

4. 8टE- 3

$4.82 E \cdot 03$

.

o.

A.

F!

a.

(t)

it.

$\because$

$n$.

4

a.

n.

$\stackrel{4}{4}$

.

?.

n.

D.

c.

9

a.

a.

1.

6

$a$

0.

a.

a.

b.

i.

6.

a)

a.

B.

i)

b.

6

$\theta$

$\theta$

1.69
TRITUNS

4.

A.

宛。

b).

H.

0.

a.

n.

a.

0

$\theta$

b.

a.

$n$

a.

0

n.

n.

$\theta$.

S.

2 ,

B.

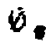

a.

0

6)

e.

s.

$b$

a.

0.

i)

a.

3

!,

t).

(i).

G.

i.

4

D.

in

is.

b.

o.

1.94
ALFIIAS

2. $24 E+H I$

1. $95+41$

$1.95 E+11$

1. $6 B E+11$

$1.68 E+V 1$

3. BUE+ 11

3. $B H E+$ it 1

$1.55 E+\Delta 1$

$1.5 S E+11$

$9.78 t+i j E$

$9.79 E+4 G$

1. $4 D E+U 1$

1. $4 B E+11$

$5.43 E+4 n$

$5.43 E+46$

1. $59 \mathrm{E}+1$

1. $59[+\cdots 1$

$5.79[-x) 2$

$5.996 .-32$

4.

is.

S. BNE +14

5. Uf $E+b$ k

1.

a.

a.

$\theta$.

a.

b.

v.

is.

i.

b.

a.

i).

a.

s.

i.

u.

i1.

v.

D.

a.

$\theta$

4

6.4 is 
1INITIAL SPECTRUM AT $3.60-1,66 \mathrm{CM}^{\mathrm{M}}$. FOR ICRU MUSCLE IISSUE 42 MEV P + DN BE IIEUTRON SPFCTRUM

\section{ENERGY}

D. 25 MF.V

0.16 MEV

3.15 MEV

0.20 MEV

2.25 MEV

D.3U MEV

0.35 MEV

0.40 MEV

D. 45 MEV

$0.50 \mathrm{MEV}$

$0.55 \mathrm{MEV}$

0.61 MEV

0.65 MEV

$0.70 \mathrm{MEV}$

0.75 MEV

O. 80 MEV

0.85 MEV

$0.90 \mathrm{MEV}$

0.95 MEV

1. DU MEV

$1.10 \mathrm{MEV}$

1. 20 MEV

$1.30 \mathrm{MEV}$

1.40 MEV

1.50 MEV

$1.60 \mathrm{MEV}$

1.70 MEV

1.80 MEV

$1.90 \mathrm{MEV}$

2.00 MEV

2.10 MEV

2. 20 MEV

2,30 MEV

2. 40 MEV

2. $50 \mathrm{MEV}$

$2.60 \mathrm{MEV}$

$2.70 \mathrm{MEV}$

2,80 MEV

2.90 MEV

3.DO MEV

3.20 MEV

3. 40 MEV V

$3.60 \mathrm{MEV}$

3 BO MEV

4.00 MEV

\section{PROTONS}

b.

$0.95 E+3$ ?

3. MIE+ 2

$2.215+42$

2. $09 E+122$

1. $23 E+02$

1. $2 a r+v 2$

1. $11 E+102$

7. $50[+1$

8. $43 E+01$

5. $16 E+611$

$5.96[+2] 1$

6. $84 E+D 1$

6. $11 E+01$

$2.73 E+11$

3.08E + 01

2. $76 E+11$

5. BDE + H1

$3,33 E+D 1$

3. $16 E+11$

$7.85 E+11$

$7.17 E+11$

$6.87 E+11$

$6.26 E+B 1$

$3.97 E+101$

S. $12 E+01$

4. $20 E+21$

$5.18 E+d 1$

$2.74 E+11$

4. $71 E+11$

$4.27 E+01$

3. $21 E+11$

3. 3RE + 1

3. $12 E+B 1$

2. $4 a E+01$

1. $15 E+1$

$2,73 E+d]$

1. $25 E+61$

3. $2 A E+B 1$

1. $7 \mid E+B 1$

2. $\triangle 1 E+B 1$

4. $16 E+61$

4. $21 E+\square 1$

$2.50 E+01$

$1.99 E+181$
VIEUTERONS

1. I DE $O Q$ ?

1. 1 G $F-O$ ?

3. उOE-viz

3. $89 E-12$

$2.65 F-02$

$2.65 F-102$

2. 6 ? $20-2$ 2

$2.62 \mathrm{~F}=\mathrm{il} 2$

5. UQE-Az

5. DAE - DI

4. AGE - I1?

$4.86 F-192$

$4.99 E-1 \mathrm{a}$

$4.99 F-1.2$

$3.80 E=P 2$

$3.804-02$.

$6 . B G E=02$

6. BOE - D2

7. $\triangle 1 \mathrm{~F}-02$

7. $\triangle 1 F=\mathrm{BL}_{2}$

1. $125=41$

1. ACE-DI

8. BQE 02

8. $89 \mathrm{~F}=\mathrm{H}^{2}$

1. $27 E-H 1$

1. $27 \mathrm{~F}-61$

1. $28 \mathrm{E}-1$

1. $28 E-8.1$

$7.4 G E-B 2$

7. $46 F=0.2$

3. BOt $=\mathrm{AZ}_{2}$

3. $805-02$

$a$.

i:

1. $15 E-02$

$1.15 E-A C$

2. $72 F-\$ 3$

$2.72 E=03$

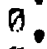

$B$

ด.

$3.2 B E=02$

3. $28 F-02$

$3.45 f=03$

\section{TRITONS}

V.

$r$

i)

0.

6.

C.

is.

i.

$\ddot{2} .68 t-02$

$2.68 E-42$

Q.

k.

B.

vi.

2. $49 E-192$

$2.47 t-.12$

$2.04 E-32$

$2.64 E=02$

$2.63 t-1) 2$

$2.63 t-t: 2$

b.

b.

$n$.

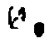

$2.74 t-62$

2.74E-A2?

a.

w.

a.

n.

b.

9.

$7.41 E-5.3$

$7.41 E-W 3$

$2.33 E-112$

$2,33 E-42$

B.

$\Delta$

c.

b.

$7.41 E-03$

$7.41 E-03$

$4.89 t=52$

$4,897 .-132$

0.
ALFHAS

4. $1.4 E+H H$

4. $1+E+11$.

$4.55 F+b 0$

$4.55 E+D u$

4. $76 . E+64$

4. $7 k t+t a$

3. $5: E+B i$,

3. 5 MLt

b. 1 Q $E+n k:$

b. $16, E+1) b$

b. BEE+OU

b. $82 E+19$

$1.33 t+111$

$1.33 E+111$

4. $13 E+414$

$4.13 E+A G$

$6.34 E+\Delta k$

$6.34 E+D H$

$6.12 E+1)$

6. $121+10$

1. $24 E+61$

1. $24 E+\pi 1$

1. $91 E+11$

1. $91 \mathrm{E}+\mathrm{U1}$

1. $48 E+11$

1. $4 B E+\square !$

1. $68 \mathrm{BE}+1.1$

$1.68 E+01$

$1.54 E+81$

1. $34 E+61$

1. $10 E+1$

1. $\mid 0 E+01$

1. $82 E+11$

1. $82 E+B 1$

1. $57 E+121$

1. $57 \mathrm{E}+11$

$9.74 E+14$

$9.74 E+1) b$

1. $44 E+11$

1. $44 E+11$

2. $42 E+31$

2. $42 E+1$

1. $30 E+11$

1. 3UE +1

B. $34 E+B E$ 
IINITIAL SPECTRUM AT Q.6B-1, GO CM. FDR ICRU MUSCLE TISSUF.

42 MEY P+ ON RE NEUTROOS SPLCTRUM

\section{ENERGY}

4.20 MEV

4.40 MEV

4.60 MEV

4.80 MEV

5.010 HEV

5.5 .7 MEV

6. OO MEV

$6.50 \mathrm{MEV}$

7.00 MEV

7.50 MEV

8., $O$ MEV

$8.50 \mathrm{MEV}$

9.00 MEV

9.50 MEV

$10 . D Q M E V$

11,00 MEV

$12,00 \mathrm{MEV}$

13.0. MEV

14.00 MEV

$15,00 \mathrm{MEV}$

16. OU MEV

17. 40 MEV

18.0D MEV

19.0 MEV

20.DO MEV

21.00 MEV

22. DO MEV

23, DO MEV

24. OU MEV

25. 00 MEV

$26.00 \mathrm{MEV}$

27.00 MEV

28. OU MEV

$29.00 \mathrm{MEV}$

$30.00 \mathrm{MEV}$

32. OD MEV

34,DO MEV

36. DD MEV

38. DO MEV

4B,D MEV

$42.00 \mathrm{MEV}$

44.00 MEV

46. 00 MEV

48, OD MEV

SO. BO MEV
PROTONS

3. $44 E+11$

2. $25 E+\pi 1$

2. $76 E+61$

2. 71 Eमd

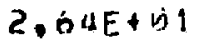

$6.13 E+D 1$

$2.23 E+1$

$3.21 E+61$

4. $71 E+61$

3. ODE + 1

3. 2थE + U1

$2.74 F+D 1$

3. $23 E+11$

$5, A 2 E+2 B$

2. 4RE +01

2. $98 E+01$

$2.24 E+11$

3. $5 B E+D 1$

$3.75 E+11$

2. $50 E+191$

1. $50 E+11$

$2,75 E+11$

2. $09 E+11$

1. $25 E+31$

$7.50 F+00$

1. $5 \pi E+1$

$7.50 t+00$

1. $25 E+01$

1. DRE +D 1

7. $5 A F+U D$

1. $25 E+B 1$

1. BOF $+D 1$

5. DOE

5. CAOE + WOS

7. $50 E+D A$

$7.50 E+B O$

2. $54 E+00$

$2.50 E+190$

5. DNE + BU

a.

in.

0.

E.

i.

1.
DEUTERONS

3. ASF-B 3

$1.31 E+D Q$

1. $31 E+B$

3. $\triangle D E=D 2$

3. 4 QE -32

5. $14 E=$ is 2

5. $14 F-n 2$

$7.935-12$

$7.93 E-62$

$6.94 E-02$

$6.94 E-O Z$

7. $6 A E-O 2$

7. 6 AE - PIZ

a.

is.

a.

$a$

a.

0

2

ต

a.

0

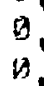

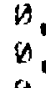

a

a)

$a$

0

0

0

9

1.)

a.

0

a.

a.

4.66
TEITUHS

U.

$3.7 \mathrm{iE}-\Delta 2$

3. $71 \mathrm{E}-\mathrm{n}$ ?

4. $3 H E-4$ ?

$4.3 R E-A 2$

2. $29 E-32$

2. $29 E-W 2$

S. BRE-IS

3. QDE-U?

4.

1

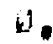

$\Delta$

b.

b.

เi.

b.

b.

b.

i.

b.

$k$

6.

n.

0.

b.

w.

6

$v$.

i.

in,

b.

0.

a.

v.

b.

$\theta$.

4.

n.

b.

b.

$\theta$

6

$\theta$.

4.48
ALPHAS

B. $34 E+30$

1. $155 t+1$

1. $.45 t+151$

$1,2 n E+1$

1. 2 WE + 1

2. जEE + L1 1

2. $\triangle B E+$ ? 1

1. $66 E+1$

1. $66 E+1$

4. $24 E+W 0$

4. $24 E+\cdots ?$

$7.73[+116]$

$7.73 E+A V$

1. $D \perp E+1$

1. $D 1 E+\infty 1$

$9.24 E+A L$

$9.24 t+b v$

5. $\triangle B E+1 J$

5. DBE+ $D$

$3.77 E+U v$

$3.77 E+B G$

2. 5 A E + U1)

2. $5 w E+w b$

1. $25 t+3 n$

1. $25 E+D k$

a.

$\Delta$.

1

a.

$\theta$

$\theta$

e.

i.

6.

0

0.

0.

i.

h.

bi

0

a.

$\theta$

i.

i.

7. 1 
IINITIAL SPECTRUM AT 1.BP-2, BA CM. FOR ICRU MUSCLE TISSUE 42 MEV P+ ON BE NEUTRON SPECTRUIT

\section{ENERLYY}

D. DS MEV

Q.13 MEV

$0.15 \mathrm{MEV}$

0.23 MEV

0.25 MEV

D. 30 MEV

b. 35 MEV

0. 40 MEV

$0.45 \mathrm{MEV}$

4.50 MEV

$0.55 \mathrm{MEV}$

7.6 MEV

0.65 MEV

0.7 MEV

0.75 MEV

D. BN MEV

Q.8S MEV

0.90 MEV

0.95 MEV

1.01 MEV

1.10 MEV

1.20 MEV

1.30 NEV

1.4D MEV

1.50 MEV

1.60 MEV

$1.70 \mathrm{MEV}$

$1.80 \mathrm{MEV}$

$1.90 \mathrm{MEV}$

2. OU MEV

2.10 MEV

2.20 MEV

2.3 MEV

2. 40 MEV

2. 5 B MFV

2.61 MEV

2,70 MEV

$2,80 \mathrm{MEV}$

$2,9 \$ \mathrm{MEV}$

3. OA MEV

3.20 MEV

3. 40 MEV

3.6H MEV

3.80 MEV

4. OO MEV

\section{PROTONS}

B.

Q. 23E+ 32 .

$3.28 E+42$

2. $19 E+122$

$1.74 F+142$

$1.54 E_{-}+42$

1. $21 E+\square 2$

$9.49 E+01$

$9.62 E+b 1$

$8.74 E+1$

$8.59 F+01$

$5.67 E+11$

b. $5 \times E+01$

$5,68 E+V 1$

$0.165+11$

S. $7 \pi E+D 1$

$3.84 E+61$

$4.78 E+11$

3. $73 E+21$

$3.84 E+1$

$7.79 E+61$

$5.76 E+B 1$

$6.14 E+11$

$6.42 E+01$

4. $50 E+61$

$4.57 E+41$

4. $2 A F+B 1$

3. $95 E+11$

$3,15 E+D$

3. $2 ! E+H !$

$3.5 B F+B 1$

$3,50 F+01$

$2.98 E+01$

2. $79 E+B 1$

$2.57 E+61$

$2.53 F+1$

1. $27 E+01$

2. $06 E+\square 1$

1. $11 E+B 1$

1.86E+ 81

3. $11 E+11$

3. $44 E+\square 1$

3. $77 E+01$

2. $98 E+1$

$4,13 E+11$
DFUTEROIVS

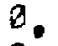

$B$.

3. $13 E-D ?$

3. $1 H E=D ?$

$4.71 E . O^{2}$

4. TIE- IL

$4.71 E-B 2$

4. $71 E-112$

$5.58 E-112$

5. $58 E-B Z$

1. $U E-D 1$

1. DIE-DI

$9.45 E-12$

9. ASE-OZ2

$3.45 E-D ?$

$3.45 E-B 2$

6. $R Q E=112$

$6 . B 9 E-B)$

6. $42 E-12$

6. $42 E-62$

2. $1 E-01$

2. EIE- 1

$1.96 E-D 1$

$1.96 E-01$

$1.62 F=9 i$

1. $62 E-0$ :

1. $21 E-D 1$

1. $2|E-B|$

$3.48 E-12$

$3.48 t=112$

$2.73 E-A 2$

$2,73 \mathrm{~F}-\mathrm{HL}_{2}$

2. $42 E-12$

$2.42 E-12$

2. DOGF $-O 2$

?. QGE $=12$

$1.04 E-A 2$

1. Q प पE-U2

$3.81 F .02$

$3.81 E-02$

2. 1 DE-D?

2. $10 E-02$

$2.17 E-12$

$2.17 F-172$

$3.74 E-62$
IRITUNS

$\theta$

0.

$\theta$.

a.

a.

$B$.

U.

a

i).

6.

a.

6.

$9.77 t-\square 3$

$9.77 E-53$

$1,43 t-132$

$1.43 E-112$

15.

b.

a.

D.

3. $28 E=63$

3. $28 t=I 3$

1. . $7 \mathrm{~T}=-12$

1. $\triangle 7 F-U 2$

a.

is.

(5)

(1.

1. A6E- 62

1. WGE - 62

a.

$\theta$

4. $47 E-192$

4. $47 E-[2 Z$

b.

iv.

i.

w.

$2.5 B E-12$

2. $.5 B E-\triangle 2$

2. 35E- म12

2. 35E-H?

6. $86 t-13$

B. $86 E-43$

$7.75 E-A 3$
ALPHAS

$3.66 F+V 6$

$\left.3.60 F_{-}+4\right)$

4. $1 \in t+(1) k$

4. $15 E+3 k^{4}$

$4.32 E+d W$

$4,32 E+B D$

$5.61 E+D$

$5.61 E+B V$

$5.94 E+0$

$5.94 E+i 1 U$

$6.67 E+3 t$

$6.67 E+.1 \%$

$8.93 E+d k$

$8.93 E+1 \%$

B. $36 E+A V$

$8.36 E+b a$

$6.96 E+B$

6. $96 E+1, j$

6. 3ot + d

$6.3+E+11$

$1.53 k+21$

1. $53 t+11$

$1,2 k E+41$

1. $2 B E+61$

$1.67 E+41$

$1.67 E+01$

1. $57 \mathrm{E}+11$

1. $57 E+61$

1. $51 E+191$

1. $51 E+41$

1. $42 E+61$

1. $42 E+11$

$1.65 E+31$

$1.65 E+41$

1. $24 E+61$

1. $24 E+U 1$

$4.51 E+1 J$

$9,51 E+C u$

8. $98 E+Q U$

3. $98 E+U d$

$1.78 E+41$

1. $78 E+61$

1. $A 4 E+D 1$

$1.84 E+191$

1. G9E+11 
1INITIAL SPECTRUM AT 1.WH-2. BO CM. FDR IRRU MUSCLE TISSUE 42 MEV P+ ON BE NEUTRON SPF.CTRUM

\section{ENERGY}

$4.21 \mathrm{MEV}$

4. 41 MEV

4.60 MEV

4. BO MEV

5. AQ MEV

5,50 MEV

6. UD MEV

6,50 IEE

7. DO MEV

7.50 MEV

8,00 :AEV

$B, 50$ MEV

?.UB MEV

$9.50 \mathrm{MEV}$

10. 610 MEV

11.00 MEV

$12 . M O M E V$

13, AO MEV

14.00 MEV

$15,00 M E V$

16, DO MEV

$17.00 \mathrm{MEV}$

$18.00 \mathrm{MEV}$

19. DO MEV

2D. DO MEV

21. DA MEV

22, HO MEV

23. $\triangle M$ MEV

$24.00 \mathrm{MEV}$

25.00 MEV

26. 10 MEV

$27.00 \mathrm{MEV}$

28,DU MEV

29.01 MEV

30,00 MEV

32, D. MEV

34. DO MEV

36.01 MEV

$38.00 \mathrm{MEV}$

42.06 MEV

प2.DO MEV

44. DU MEV

46.0D MEV

4B, AD MEV

$50 . \triangle D$ MEV

ZAVE, E(MEV)
PROTONS

2. $57 E+D 1$

2. $23 E+01$

2. $43 E+b 1$

1.83E+61

2. $19 E+01$

$6.89 E+61$

$5.92 E+D 1$

$4.13 E+11$

5. 66 G 01

3. $29 E+61$

$2.9(E+6)$

$1.71 F+1] 1$

2. $5 A F+31$

1. $8 \pi E+11$

2. $15 B E+6]$

4. TTE+U1

3. $2 D E+51$

1. $87 E+11$

2. $89 E+191$

2. SOE + U1

1. $8 A E+1$

2. $40 E+01$

1. $90 \mathrm{E}+101$

1. $3 A E+91$

1. $B A E+11$

8. $\triangle A E+B^{3} Q$

1. TUE + Bi

1. $511 E+B 1$

1. $23 E+B 1$

1. $10 E+71$

6. UQE + Uh

4. BOE + DO

8, UQE +DO

5. DAE + DA

5. DAE + DO

7. $D A E+Q B$

6. ODE + ID

$4,0 B E+43$

n.

D.

a.

W.

a.

if.

a.

10.33
TEUTERONS

3.94E- 02

5.11E-OI

5. IIE-RI

$2.75 E-03$

$2.75 F-13$

$4 . A B F-12$

4. $\cap \mathrm{BE}-\mathrm{i}^{2} 2$

5. $73 t-01$

5. $7 \mathrm{AF}-\mathrm{DI}$

$4.39 E-1 \mathrm{C}$

4. MUE-li?

1. UจF-U2

1. $995-112$

$6.81 F-0 s$

$6.81 E=U 3$

4

H.

$1.71 E-1 A 3$

$1.71 E-113$

0

$b$

4

c.

$a$

$\theta$

in.

(1)

0

9

a.

i.

a.

$a$

a.

$a$

9.

1.

$A$

i.

$a$

5. 10
TRITUAS

$7.75 t-k .3$

$2, S 5 E-42$.

$2,55 E=2 c$

4. 2 ? $[-(1)]$

4. $22 t=03$

$3.72 t=k ?$

$3.72 E-J$ ?

2. $17 t=33$

2. $17 E-35$

2. $21 E=3$

E. $21 E=+3$

$u$.

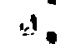

2. $27 E-15$

2. $27 E-4: 3$

3.

a.

d)

4

i.

it

$A$

1).

i)

a.

a.

is

汀

in.

1

b.

$\theta$

$v$

i)

6.

i.

n.

is.

v.

i.

a.

b.

n.

a.

$a$

4.49
ALPHAS

1. BQE + U1

$1.62 t+41$

$1.62 E+\cdots 1$

$1.51 F+41$

$1.51 E+11$

2. $11 E+B 1$

?. ||$E+4 \mid$

$1.33 E+11$

1. $33 F+n !$

1. $0.3 E+i 1$

1. $b V E+A 1$

$4.71 E+W G$

$4.71 \mathrm{~F}+\mathrm{W}$

$9.72 E+A \omega$

7. $72 E+A L:$

2. $74 t+14 n$

$2,74 E+19 j$

$2.55 E+$ UQ

2. $55 E+3$ is

$2.23 E-112$

2. $235-102$

2. 3 kit- -3

2. 3 , t $t-x) 3$

$1.43 E-13$

$1.93 E-4.3$

b.

g.

B.

a.

$\theta$

b.

a.

1.

$\theta$

t).

品.

4.

4

i)

1.

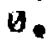

is

H.

n.

G.

5.44 
IINITIAL SPECTRUM AT 2.HA-4. BH CM. FOK ICRU MUSCLE TISSUE 42 MEV P\$ UN BE NEUTRON SPECTPUM

\section{ENERGY}

D. US MEV

D. 10 MEV

D. 15 MEV

$0.20 \mathrm{MEV}$

0.25 MEV

$0.30 \mathrm{MEV}$

0.35 MEV

0.4O MEV

D. 45 MEV

$0.50 \mathrm{MEV}$

0.55 MEV

$0.60 \mathrm{MEV}$

0.65 MEV

$0.70 \mathrm{MEV}$

D.TS MEV

D. 8 MEV

D.85 MEV

0.90 MEV

$0.95 \mathrm{MEV}$

1. DV MEV

1.16 MEV

1.20 MEV

1.35 MEV

1. 4 LEV

$1.50 \mathrm{MEV}$

1.66 MEV

$1.70 \mathrm{MEV}$

$1.86 \mathrm{MEV}$

1.9 MEV

2. OP MEV

2,10 MEV

2,20 MEV

$2.30 \mathrm{MEV}$

2. 40 MEV

$2.50 \mathrm{MEV}$

$2.60 \mathrm{MEV}$

2.76 MEV

2.80 MEV

2.90 MEV

3,00 MEV

3.20 MEV

3.40 MEV

3,60 MEV

$3.8 D$ MEV

4.01 MEV
PRQTONS

a.

8. $6 M E+3$ ?

$3.41 F+10 ?$

2. $29 E+12$

$1.89 E+12$

1. $39 E+B 2$

$1.27 E+\Delta Z$

1. $16 E+62$

1. $32 E+i 2$

$8.53 E+D 1$

7. $4 \Delta E+D 1$

$6.57 E+31$

$5.81 \mathrm{~F}+\mathrm{D} 1$

$6.79 E+B 1$

5. $31 E+31$

$4.79 t+1$

4. $79 E+191$

5. (1) $3 E+11$

4. $97 E+11$

$4.23 E+01$

$0.91 E+.1$

b. $53 E+i j 1$

$0 . H 3 E+111$

4. . $6 E+U 1$

S. (SRE + W I

$4.83 E+D 9$

A. $4 A E+B 1$

if. $\triangle \Delta E+B)$

3. $38 F+B 1$

$3,13 E+b 1$

2. $59 F+1$

3. $47 E+41$

3. $13 E+61$

3. $15 E+01$

2. BDE + 1

2. $56 E+H 1$

2. 6 UE + HA 1

2. $39 E+1$

2. $55 E+01$

Z.

4. $6 B E+61$

3. $Q 4 E+B 1$

4. $05 E+01$

$2.65 E+11$

$2.74 E+61$

\section{DEUTERTNS}

n.

is.

4. $27 E-192$

4. $27 E-02$

2. $93 \mathrm{E}-\mathrm{HZ}$

$2.83 F-112$

$6.25 E-1) 2$

$6.25 E-112$

$4.62 E-D 2$

4. $62 t-1) 2$

7. PQEADI

7.PBE-PC

$6.55 \mathrm{~F}-\mathrm{PL}$

$0.55 E-? ?$

$7.55 \mathrm{ST}=0 ?$

$7.55 F-.2$

6. B 3E-BZ

6. $B 3 E-122$

$6.14 F-D \cdot \bar{E}$

t. $14 \mathrm{~F}=\mathrm{AC}$

$1.29 E-1.1$

1. 2 OF -21

!. $35 E-B 1$

1. 35E- $\triangle 1$

1. $M T E-M 1$

1. $B 7 E-1$

$7,018-02$

$7011-0 ?$

6. ÓGE-DL

$6.66 E-122$

$3.91 E-D^{2} 2$

$3.91 E=6.2$

$2.60 E=1$

2.6AE-VI

3. $31 E-A 2$

3. $31 F-P 2$

2. 571.41

2. $57 E-11$

$4.36 F=93$

$4.36 F-133$

$3.55 E-A C$

$3.55 E-02$

2. $82 E-112$

2. $82 E-W 2$

3.19E-O2
TRITOIS

ज.

b.

H.

b.

n.

is.

id.

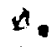

1. BZE-DS

1. $M 2 E-53$

b.

i.

1. $14 E=42$

1. 1 UE - IA?

i.

i.

6

4.

$1.57 t-133$

1. $57 E-\lambda 3$

$1.52 \mathrm{E}-\mathrm{n} 2$

1.5PE-G2

5. $44 t-13$

5. $4 \Delta f-63$

5. $112 E-13$

$3.42 E-03$

$1.44 E-4$ ?

1. $44 E-B 2$

b.

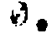

$0.15 E-03$

$0,15 E-W 3$

$5.32 E-03$

$5.32 E=03$

$7.58 E-\square 3$

$7.58 t-13$

8. 4 A E $=03$

8. $4 Q E-B 3$

1.17E- 22

1.17E-ט2

$1.52 E-k 2$

1. $52 \mathrm{E}-\mathrm{LZ}$

2. $0.5 t-1.2$

$2.05 E-02$

2. जGE-U?
ALPHAS

2.57E+ $3 n$

$2.57 \mathrm{~F}+4 \mathrm{~V}$

4. $82 E+4 n$

4. $82 E+30$

0. $19 E+A L$

h. $99 E+d k$

7. B9E 6 .

$7.89 E+36$

$5.62 E+$. WV

$5.62 E+k(k)$

6. $78 E+v$

$6.78 E+116$

6. $28 E+1 E$

6. $2 E E+b G$

8. $56 E+16$

0. $56 E+8 i$

$7.65 E+W 0$

$7.65 E+100$

8. $54 E+b^{3} B$

B. SUE + BU

1. $37 E+11$

1. $37 E+w 1$

1. $41 E+41$

$1.41 E+41$

1. $58 E+C 1$

1. $58 E+1$

$1.37 E+11$

1. $37 E+V 1$

1. $27 E+11$

1. $27 E+11$

1. $19 E+61$

$1,19 E+1$

$1.19 t+31$

1. $19 E+1$

1. $13 E+V I$

1. $13 E+11$

1. $1 \Delta t+v 1$

1. $16 E+91$

1. $29 E+91$

1. $29 E+V 1$

1. $84 E+\square 1$

1. $84 E+111$

1. $.5 E+41$

$1.65 E+01$

1. $78 E+01$ 
IINITIAL SPECTRUM AT 2. UO-4. OA CH. FDR ICRU PUSLLE TISSUE 42 MEV P+ ON BE NEIJTRON SHECTRYUM

\begin{tabular}{|c|c|c|c|c|c|}
\hline \multicolumn{2}{|c|}{ ENERGY } & PROTONS & DEUTERDHS & TRITINS & ALPHAS \\
\hline 4.20 & MEV & 4. $17 E+11$ & $3,19 E-12$ & $2,16 t-132$ & $1.76 E+11$ \\
\hline 4.40 & MEV & $3.07 E+1$ & $1.45 F-193$ & $1,30 t=112$ & $1.33 E+1$ \\
\hline 4.60 & MEV & $2.94 E+11$ & $1.45 F-03$ & $1.39 \mathrm{~F}-112$ & $1.23 t+1+1$ \\
\hline 4.80 & MEV & $3.1: t+19$ & $? .54 \mathrm{E}-1 \mathrm{I}$ & $=45 E-33$ & $S E+b k$ \\
\hline 5,00 & MEV & $2.49 E+61$ & $2.54 E-A 1$ & $5.45 E-.3$ & $8.95 E+16$ \\
\hline 5.50 & MEV & $5, R 7 \bar{E}+B 1$ & $2.71 E=61$ & $1.79 E-122$ & $2.18 E+41$ \\
\hline 6,010 & MEV & $5.95 E+61$ & $2.71 E-91$ & $1.79 t-1.2$ & $2,18 E+d 1$ \\
\hline 6.50 & $M E V$ & $3.44 E+12$ & $4.26 E-12$ & $3.38 E-13$ & $1.38 E+21$ \\
\hline 7 . P & MEV & $3.41 E+31$ & $4.26 F-1.2$ & $3.38 \tilde{E}-\mathrm{x}^{3}$ & $1.36 E+1$ \\
\hline 7.50 & MEV & $3.5 P E+11$ & $2 \cdot 7$ SE - 12 & 2. $17 E=2.3$ & $7.24 E+150$ \\
\hline 8.40 & MEV & $2.7, \mathrm{E}+11$ & $2.73 E=A 2$ & $2.17 E=\sqrt{3}$ & $7.24 E+10$ \\
\hline 8.50 & $M E V$ & $2.73 \mathrm{E}+\mathrm{L}$ & $1.4 G E-12$ & $5.14 E-D 3$ & $4,31 E+0 i$ \\
\hline 9.00 & $M E V$ & $2.76 E+31$ & $140 F-9 ?$ & $5.14 E-633$ & $31 E+30$ \\
\hline 9.50 & $M E V$ & $2.2 P E+11$ & $8,15 E-1+3$ & $2,21 E-13$ & $77 E+14 n$ \\
\hline 10.00 & MEV & $2.33 E+61$ & $8.15 F-13$ & $2.21 E-3$ & $77 E+16$ \\
\hline 11.00 & MEV & $3 . B 4 E+1$ & $8.97 F=03$ & $2.5 A E-33$ & $8.47 E+8$ \\
\hline 12.40 & MEV & $4.3 \cup E+611$ & $8.09 E-13$ & $2 . \operatorname{SAL}=63$ & B. $47 E_{-}+\pi n$ \\
\hline 13,00 & $M E V$ & $3.2 \geqslant E+21$ & 4 & b. & $3.6 A E+4 V$ \\
\hline 14.00 & MEV V & $2.3 W E+b 1$ & 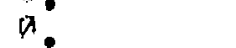 & $i$ & 3. $\triangle A E+v$ \\
\hline 15.00 & MEV & $2.9 P F+101$ & $\eta$ & $\therefore$ & $1.76 f+64$ \\
\hline 16.00 & MEV & $2.50 E+11$ & is. & $a$ & $1.76 E+10 w$ \\
\hline 17.00 & MEV & $1.85 E+1$ & $\dot{n}$ & 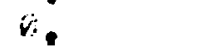 & $7.53 E=t^{2} 1$ \\
\hline 18,00 & MEV & 1,7 ut +41 & $\nabla$ & b. & $7.53 F-41$ \\
\hline 19.00 & $M E V$ & $1.45 E+1$ & $\theta$ & 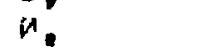 & $7.5 x+2.011$ \\
\hline $20.0 B$ & $M E V$ & $1.35 E+01$ & $n$ & $n$ & $1.5 \cup E-11$ \\
\hline 21.00 & MEV & 1. $60 E+111$ & 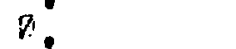 & 4 & $n_{0}$ \\
\hline 22,03 & $M E V$ & 1. $\triangle A B E+D 1$ & 0 & w. & $a$ \\
\hline 23.00 & MEV & $9.50 E+0 \square$ & 0 & b. & 19 \\
\hline 24.00 & MEV & $1.2 a E+11$ & 0 & $d$ & 0 \\
\hline 25.00 & MEV & $1.2 D E+i j 1$ & $a$ & 6 & 0. \\
\hline 26.00 & MEV & $1.69 E+61$ & 0 & $n$ & $n$ \\
\hline 27,00 & MEV & $7.53 E+09$ & $\theta$ & 0 & $a$ \\
\hline 28.00 & MEV & $5.5 \cap E+60$ & $B^{2}$ & $a_{1}$ & 0. \\
\hline 29.60 & MEV & $4.00 E+W 1$ & $a$ & $n$ & 0 \\
\hline 30.01 & MEV & $5.50 E+\Delta 0$ & B. & 0 & 0. \\
\hline 32.00 & MEV & 8. & 0 & 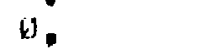 & 0. \\
\hline 34.00 & $M E V$ & 5. & $a$ & 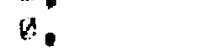 & . \\
\hline 36,00 & $M E V$ & $3,50,+D H$ & 0 & 10 & n. \\
\hline 38.00 & MEV & 1.5 A +14 & !? & u. & a. \\
\hline 40.00 & MEV & $5 . \operatorname{naF}-\square 1$ & H & $n$. & 2 \\
\hline 42.40 & MEV & 4. & 0 & 0 & 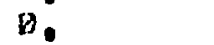 \\
\hline 44.98 & MEV & 0 & $a^{\circ}$ & a. & $B$. \\
\hline 46.00 & $M E V$ & a. & 0 & 0 & B. \\
\hline 48.60 & MEV & 9. & 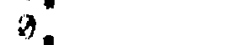 & $b$ & is. \\
\hline 50,00 & MEV & $n$ & a. & 0. & 6. \\
\hline AI & (MEV) & 19.27 & 4.35 & 4.87 & 6.29 \\
\hline
\end{tabular}




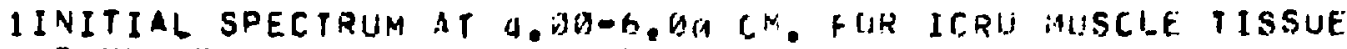
42 MEV P+ ON HE NFUTRON SPFCTRUM

\begin{tabular}{|c|c|c|c|c|}
\hline ENERGY & PFOTONS & DEUTERONS & TRITUHAS & ALPRIAS \\
\hline D. US MEV & $\theta$ & D. & . & $2.77 \mathrm{E}+\therefore 1$ \\
\hline D. IA MEV & $8.06 E+62$ & $\theta$ & ki. & $2.7 i E+n+2$ \\
\hline$D .15 \mathrm{MEV}$ & $3.41 E+3 ?$ & $4.91 E-62$ & $a^{\prime}$ & $15, \ddot{z}+x^{4}$ \\
\hline ZD NEV & $2.26 E+112$ & $915-32$ & & $6 t+.15$ \\
\hline 25 MEV & 1. $8 B E+\Delta 2$ & $5.4 \Delta E-12$ & 9 & $\vec{c} t+k^{4} \mathrm{v}:$ \\
\hline 3U MEV & $1.61 F+12$ & $5 . \times 4 E-11 ?$ & $\because$ & $5.72 E+4$ \\
\hline $35 \mathrm{MEV}$ & $1.27 E+12$ & $4.63 F-02$ & . & $7,36 t+A v:$ \\
\hline 40 MEV & $1.06 E+12$ & $4 ., S E-D z$ & ii. & $7.30 t+1.1$ \\
\hline 45 MEV & $8.99 F+11$ & $5.7 b F-d 2$ & i. & $6.52 E+110$ \\
\hline 5ه MEV & $B .+1 E+(1)$ & $5.76 t-7.2$ & 1. & $6.52 E+36$ \\
\hline $55 \mathrm{MEV}$ & B.62E+L1 & $6.36 F=62$ & $5.26 t-E .6$ & $6.27 t+11 v$ \\
\hline bI MEV & $7.26 E+111$ & $6.36 t-1 ! 2$ & $5.26 E-b 3$ & $6.2 J E+i$ \\
\hline $65 \mathrm{MEV}$ & $6.92 E+111$ & $7.94 F-t^{k} 2$ & $\therefore$ & $7.55 E+4: 1$ \\
\hline 70 MEV & $6.2 A E+x^{3} 1$ & $7.34 E-1 i{ }^{2}$ & $\pi$ & $7.55 E+11.1$ \\
\hline 75 MEV & $5.55 E+0 i$ & $5,0 ? 5-42$ & vi & A. $22 t+110$ \\
\hline IO MEV & $4.3 P E+A 3$ & $5.425=12$ & $\therefore$ & B. $22 E+36$ \\
\hline $35 \mathrm{MEV}$ & $+.13 E+11$ & $8.21 E-0.2$ & $5,12 E-133$ & $0.2 a t_{-}+1 ! n$ \\
\hline MEV & $4.87 E+11$ & $8.21 E-112$ & $5,12 \mathrm{~F}=1: 3$ & $6+2+5+3$. \\
\hline 5 MEV & $4.67 E+11$ & $6.5 \%=02$ & $4.93 E=.3$ & $8.4 x^{2} F+x_{4}$ \\
\hline D Nit.V & $3.75 E+31$ & $0.35 f-i^{3} 2$ & $4.73 t=193$ & $3.4 n E+\pi \omega$ \\
\hline B $M E V$ & $7.66 E+31$ & 1. $\because, E-n 1$ & $5.34 E-\forall 3$ & $1.37 E+11$ \\
\hline 20 MEV & $7.77 E+\square 1$ & $1, k \mathrm{E}=\mathrm{Al}$ & $3.34 E-0.3$ & $1.37 E+81$ \\
\hline $30 \mathrm{MEV}$ & $5.91 F+1$ & $1.2 A F-H 1$ & $5.51 E-53$ & $1.32 E+\triangle 1$ \\
\hline 4D MEV & $5.38 E+11$ & $1.2, \mathrm{~F}-\mathrm{01}$ & $5, S I E-03$ & $1.32 E+11$ \\
\hline $5 a$ MEV & $5.59 E+V 1$ & $9.965-82$ & $1.25 t=112$ & $1.42 E+i$ \\
\hline MEV & $4.41 E+01$ & $9.96 E-02$ & $1.25 t-32$ & $1.42 E+11$ \\
\hline MEV & 4. $16 E+11$ & s. $15 t-1$ & $1.24 E-U 2$ & $1.41 E+11$ \\
\hline Q MEV & $4.74 E+61$ & $05 E-13$ & 1. $.11 E=Q 2$ & $1.41 E+31$ \\
\hline$O$ MEV & $4.22 E+\lambda J$ & $3,14 E-V I ?$ & $0.45 E-.3$ & $1.4 n E+A 1$ \\
\hline GD MEV & $3.83 E+141$ & $3.14 E=C^{2}$ & $0.05 t=03$ & $1.4 v E+41$ \\
\hline $1 G$ MEV & $3: 7 E+\lambda 1$ & $2.075-11$ & 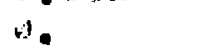 & $1.18 E+1: 1$ \\
\hline MEV & $3.36 E+01$ & $2,671-131$ & 3 & $1.18 E+1:$ \\
\hline MEV & $3.305+01$ & $2.91 E=2$ & $6.17 t-1.3$ & $1.19 E+1.1$ \\
\hline MEV & $2.74 F+01$ & $2.016-42$ & $0.17 t=n 3$ & $1.19 E+11$ \\
\hline 50 MEV & $2.60 E+11$ & $8.74 E-113$ & $2.45 F-42$ & $1.25 E+.11$ \\
\hline SU MEV & $1.82 E+41$ & H. $/ \triangle E=.3$ & $2.65 E-62$ & $1.25 E+11$ \\
\hline $7 D$ MEV & $2.27 E+11$ & $1.10 E-A 2$ & i. & $1.27 E+1.1$ \\
\hline NEV & ?. BSE+ 1 & 1. IAE- 12 & U. & $1.27 E+.11$ \\
\hline MEV & $2,5 Q E+41$ & $9.41 E-13$ & $5,71 E-13$ & $1,19 E+11$ \\
\hline MEV & $1.91 E+21$ & $9,1 E-3$ & $3.91 E-13$ & $1.19 E+11$ \\
\hline $.20 \mathrm{MEV}$ & $5.17 E+b 1$ & $3.53 E-m 2$ & $1.86 t=32$ & $1.64 E+11$ \\
\hline $3.41 \mathrm{MEV}$ & $3.33 E+01$ & $3.53 F-192$ & $1.86 F .012$ & $1.04 E+1$ \\
\hline MEV & $3 .+B E+61$ & $1.3 \Delta E-D^{2} 2$ & $7.16 E-23$ & $1.8 / E+4 \mid$ \\
\hline MEV & $2.7 \Delta E+\Delta 1$ & $1, C+E-C$ & $7.16 t-43$ & $1.87 E+i 11$ \\
\hline MEV & $33 E+11$ & $4 Z E=M Z$ & $2.05 E-05$ & 1.3 ViE + S1 \\
\hline
\end{tabular}




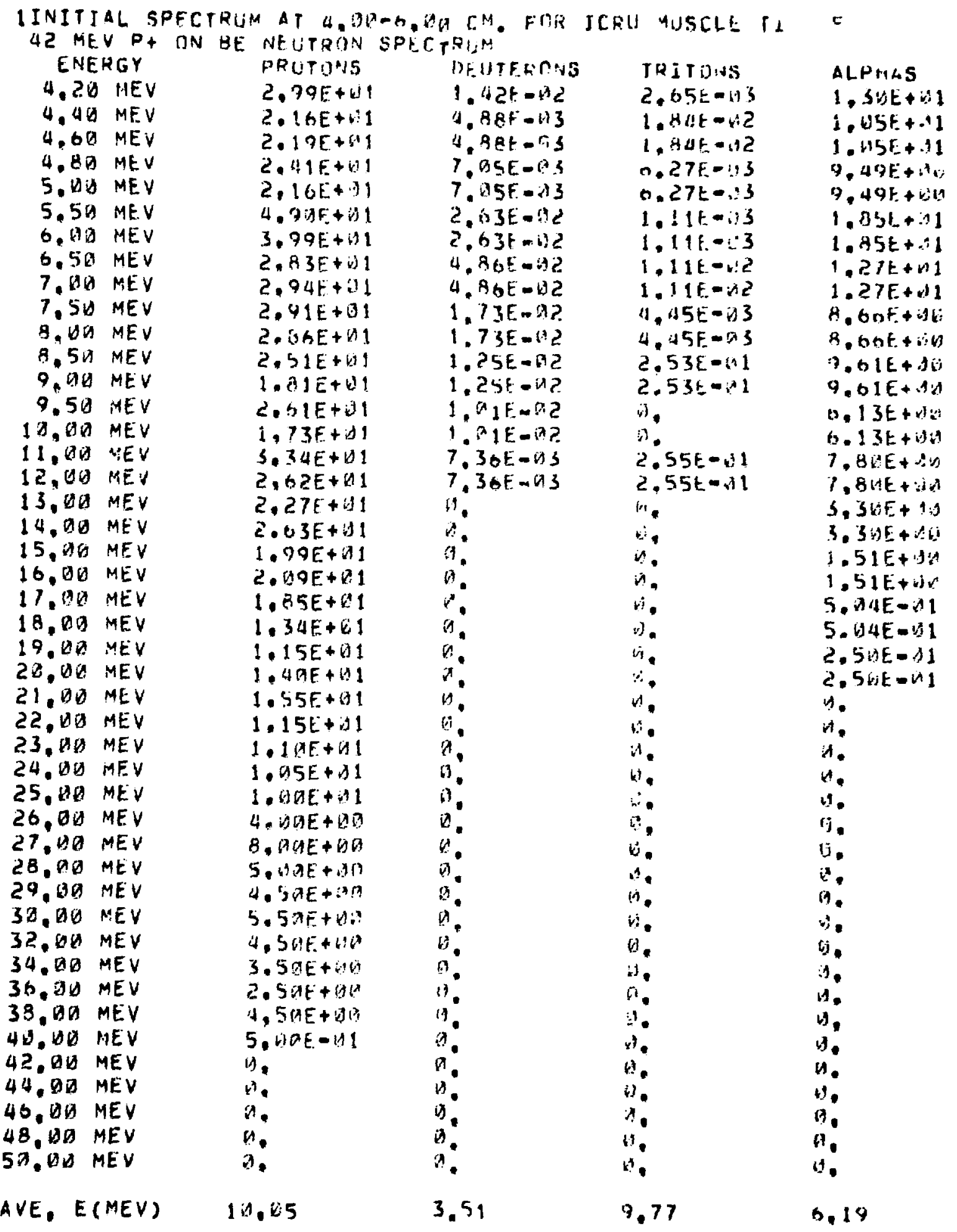


1INITIAL SPECTRUM AT 6.AH-1H. CH. IOK ICRU MUSCLE TISSLE 42 MEV P+ ON BE NEUTROP 5PECTRIM"

\section{ENERGY}

A. USS MEV

0.10 MFV

0,15 MEV

0.20 MEV

0.25 MEV

0.30 MEV

$3.35 \mathrm{MEV}$

0.4 MEV

0.4S MEV

D. 50 MEV

0.55 MEV

$0,60 \mathrm{MEV}$

$0.65 \mathrm{MEV}$

$D, 70$ MEV

0.75 MEV

Q.80 MEV

0.85 MEV

0.9 ME NEV

0.95 MEV

1. OO MEV

1.10 MEV

1.2D MEV

1.30 MEV

$1.4 D$ MEV

1.50 HEV

1.60 MEV

1.70 MEV

1,80 MEV

$1.90 \mathrm{MEV}$

2.00 MEV

2. 1 M MEV

2. 20 MEV

2,30 MEV

2. 40 MEV

2,50 MEV

$2.60 \mathrm{MEV}$

2.70 MEV

2. $80 \mathrm{MEV}$

$2,90 \mathrm{MEV}$

3. WO MEV

3.20 MEV

3. 40 MEV

3.60 MEV

3.80 MEV

4. OU MEV

\section{PRITONS}

4.

$4.19 E+42$

$3.12 E+V^{2}$

C. 1 RE $+\square E$

1. $75 E+62$

1. $36 f+B$ ?

i. $25 E+i ?$

1. $1 E+A 2$

9. $\triangle B E+31$

7. $795+11$

7. $065+41$

$6 . \mid 3 F+01$

5. $89 E+4.1$

5. $: B E+1$

5. $\{7 E+111$

4. $\triangle A E+\lambda 1$

4. $\therefore 5 E+91$

4. $3 R E+? 1$

3. $75 E+1$

4. $\because 1 E+d$ !

$0.71 E+19$

5. $4 R E+B 1$

5. औAE + 1

5.? $1 E+61$

5. $17 E+11$

$4.46 E+01$

$4,|\triangle E+B|$

$3.73 E+1$

3. $2 B E+i 1$

2. $8 R E+11$

3. 2 QE +11

3. $21 E+D 1$

3. $22 E+D 1$

3. 1BE+ 1

2. $49[+1]$

$2.49[+6]$

2. $19 E+k 1$

$1.98 E+61$

1. $9 ? E+01$

2. $12 E+\cdots 1$

4. $29 E+B 1$

2. $92 E+11$

3. $11 E+01$

2. $48 E+W 1$

2. $46 E+21$
DE ITERUHIS

5. 3.4 - 21 ?

5. SaE-11.5

$3.45 E=4^{2} 2$

$3.45 E-12$

A. HAE Q ? ?

$4.9 F-r^{2}$

$4.61 E=-12$

$4.61 E=42$

$3,6, O F-1+2$

$3, \rightarrow O E-1,2$

$5.45 T-102$

$5.45 t=k^{\prime} ?$

4.7 IF -62

$4.74 E-$ IA 2

5. $24 E-1 ! 2$

$5.24 E-V ?$

4. $72 E-42$

$4.72 E-12$

$5.325-62$

$5.32 t-i ? 2$

$9,92 E-15$

$9.92 E-112$

$2,32 E-11$

2. $32 \mathrm{~F}-\mathrm{X}^{2} \mathrm{I}$

$9.19 E=112$

$9.19 E-32$

7. $? \mathrm{I} E-\mathrm{B} 2$

7. $21 E=42$

$4.54 F-02$

$4.54 E-A 2$

1. 7 2E-A2

$1.72 E-132$

1. $35 E-91$

$1.35 E-11$

1. $62 E-D 2$

1. $62 F-22$

$1.12 E-B 2$

1. $12 E-D 2$

$1,17 E=02$

1.17E-DC

$8.82 E-B 3$

B. $325-113$

$1.56 \mathrm{~F}-02$

$1.56 E=02$

$1.42 F=62$

\begin{tabular}{|c|c|}
\hline 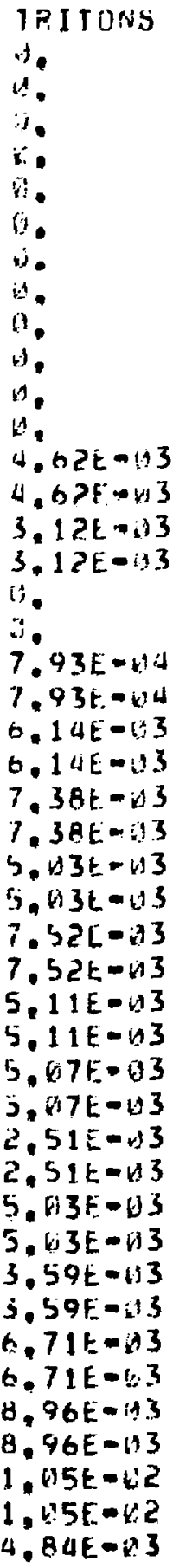 & 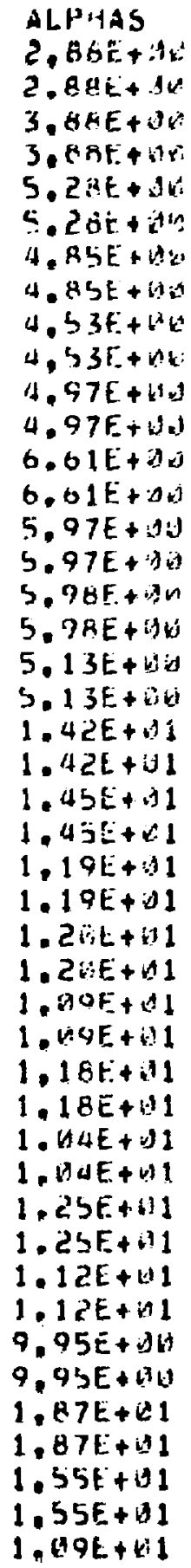 \\
\hline
\end{tabular}


IINITIAL. SPECIRUM AT G,UIA-10,E: CM. FOR ICRU RLISCLE TISSIJE 4? MEV P+ ON HE NFUTROA SFECTEIIN

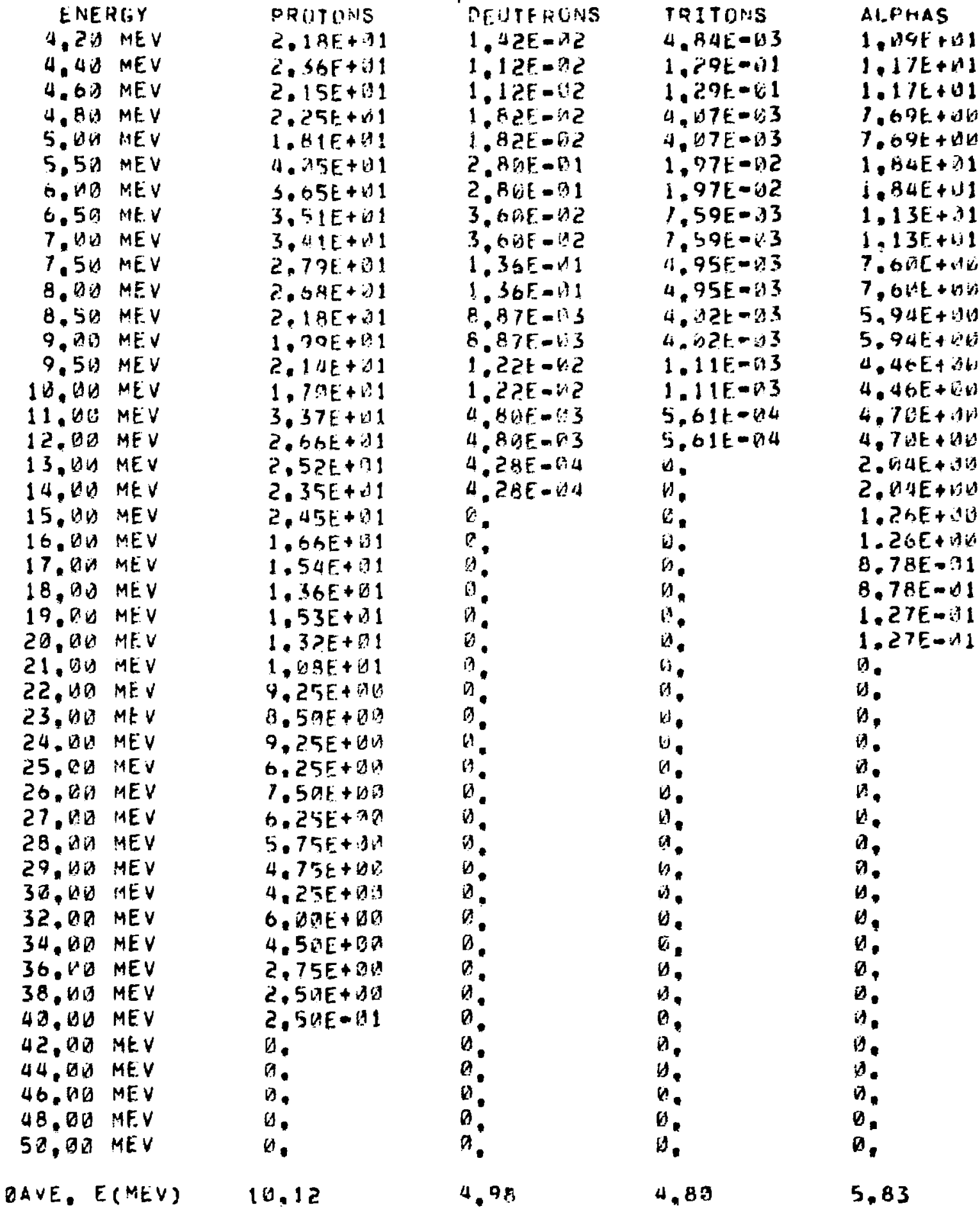


IINITIAL SPECTRUM AT $19,0-14,0$ CM, FOR ICRL MUSCLE TISSUE 42 MEV P + ON BE NEUTHOY SPECTRUM

\section{ENERGY}

3. 05 MEV

D. 10 MEV

0.15 MEV

Q.20 MEV

0.25 MEV

D. 3W MEV

$0.35 \mathrm{MEV}$

0.40 MEV

0.45 MEV

$0.50 \mathrm{MEV}$

$0.55 \mathrm{MEV}$

$0.60 \mathrm{MEV}$

0.65 MEV

Q.70 MEV

0.75 MEV

0.80 MEV

0.85 MEV

$0.90 \mathrm{MEV}$

0.95 MEV

1,00 MEV

1.10 MEV

1.20 MEV

$1.30 \mathrm{MEV}$

$1.40 \mathrm{MEV}$

1.50 MEV

1.60 MEV

1.70 MEV

1.80 MEY

$1.90 \mathrm{MEV}$

2,DU MEV

$2,10 \mathrm{MEV}$

$2,20 \mathrm{MEV}$

2. 3V MEV

2. $40 \mathrm{MEV}$

$2.50 \mathrm{MEV}$

2.60 MEV

2.70 MEV

$2.80 \mathrm{MEV}$

2.90 MEV

3. DU MEV

3.20 MEV

3.40 MEV

$3.60 \mathrm{MEV}$

3.80 MEV

4. OO MEV

\section{PROTUNS}

a.

7. $45 E+112$

$2.64 f .+i) 2$

1. $945+12$ ?

$1.375+192$

$1.15 E+b 2$

$9.63 E+11$

9. $D 9 E+\| 1$

$7.17 E+D 1$

$6.55 F+01$

5. $34 E+11$

4. $99 E+D 1$

$5,12 E+b 1$

4. $65 E+01$

$3.97 E+1$

$4.47[+1$

$3.6 B E+V 1$

$3.16 F+31$

3. $57 E+61$

$3,12 E+U 1$

$4.91 E+141$

$4.76 E+D 1$

$4.7 G E+D 1$

$4.33 E+1$

4. $19 E+D 1$

$3.66 E+D 1$

$2.91 E+01$

$3.64 E+01$

$3.15 E+B 1$

$2.67 E+D 1$

$2.71 E+D 1$

2. 4RE+01

$1.84 E+U 1$

2.13E+DI

2. $03 E+D 1$

2. $U 2 E+\square 1$

$1.5 \Delta E+B 1$

1. $52 E+11$

$1.55 E+101$

2. $94 E+01$

$2.53 E+d 1$

$2,57 E+1$

$2.85 E+01$

2. $24 E+D 1$

$2.27 E+D 1$
DEUPERONS

2. $758=173$

2.75E-US

2. $33 E-V 2$

2. $33 E-1+2$

$1.79 E-32$

1. $99 E-42$

2. $92 E-112$

2. $92 \mathrm{CF}-82$

3. $41 E-62$

3. $11 \mathrm{1r}=2$

4. DGF-OL

$4.010-42$

3. 5.9E- D2

3. 5 म $\mathrm{F}-\mathrm{B2}$

2. $20 E-V 2$

2. $26 \mathrm{E}$.a

1. $5 B E=1$

1. $58 \mathrm{E}=01$

2. $36 E-D 2$

2. $36 E-02$

6. $22 F-02$

$6.22 E=142$

$7.21 E-B 2$

$7.21 E-\Delta 2$

$6.76 F-92$

6.7 GE $D 2$

$3.27 E-192$

$3.27 F-\theta \ddot{C}$

1. $89 E-02$

$1.89 E-102$

1. 9 ? -9 ?

1. 9 ZE - WL

1. $4 B E=41$

1. 4 UE - B 1

1. $34[-3]$

1. $34 E-D 1$

$7.66 E=3$

$7,66 E-D 3$

$6.76 E-13$

$6.76 \mathrm{~F}-13$

$8.1 B E-133$

$8.18 E-23$

$6.96 E-13$

$6.9 i E-33$

1. $32 F-D 1$
TRETONS

a.

a.

v.

a.

(A.

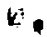

$2.56[-1 d 3$

2. $56 E=\operatorname{Ml} 3$

3. $44 E-03$

3. $44 E-A S$

v.

(ii.

$9.3 n c-04$

$9.39 E-24$

v.

$\theta$

$5.34 E-03$

$5,34 E-B] 3$

b.

$\Delta$,

$1.75 t-13$

$1.75 E-13$

$8.73 E-13$

$8.73 E-03$

2. $38 F=73$

2. $36 E-d 3$

7. 5 DE- 3

7. 5DE- 63

$5.25 E-63$

$5.25 E=23$

2. $39 E-1) 3$

2. $39 E-93$

2. $59 E-113$

2. $59 E-b 3$

4. 1DE-D 3

4. $10 E-D 3$

$2.31 E-33$

2. $3[E \operatorname{Ros} 3$

$1 . \triangle 2 E-\lambda 2$

1. $12 E-12$

4.5 EE- .3

$4.5 R E-13$

$6.53 E-U 3$

$6.53 E=03$

3,3 Ut -13
Al.PriAs

$1.95 E+.1 v$

1.95E+i1V

$3.73 E+4 v$

$5.73 E+A 0$

$3.93 \mathrm{E}+1 \mathrm{n}$

$3.93 E+W 6$

4. 37E+iJ)

4. $37 \bar{E}+1 \mathrm{~V}$

4.16i+tw

A. 1 HE + EA:

$3.7 \mathrm{GE}+\mathrm{WU}$

3.79E+kin

b. $695+00$

$6.64 E+40$

4.7 $A F+b d$

$4,73 t+10$

5.85E+ 40

$3.8 \supset E+d W$

$4.66 E+W 6$

4. E, $6 E+W B$

$9.83 E+26$

$9.635+18$

$9.77 E+4 k$

$9.77 E+U U$

1. $12 \bar{F}+11$

1. $1 \geq E+A 1$

1. $\triangle 3 E+\square 1$

1. $\triangle 3 E+1$

1. $31 E+31$

1. i) $1 E+\lambda 1$

B. $11 E+10$

$A, 11 E+1 D$

1. UAE+.J1

1. $A B E+11$

$7.67 E+6$

$7.67 E+D W$

$7.88 E+14$

$7.88 E+11$

$7.76 E+3 V$

$7.7 E E+U C$

1,4 EE + ل1

1. $46 E+11$

$1.10 E+\Delta 1$

1. $10 E+1$

I. $U 3 E+D 1$ 
IINITIAL SPECIRUM AT 10,B-14.A CM. FOH ICRU MUSCLE TISSUE 42 MEV P+ DN BE NEUTRON SPKCTRUM

\section{ENEKGY}

4.20 MEV

4.40 MEV

$4.60 \mathrm{MEV}$

4.80 in $V$

5. $\triangle 10$ MEV

5,50 MEV

6.60 MEV

6.51 MEV

7. $\triangle$ G MEV

$7.51 \mathrm{MEV}$

8. 10 MEV

8.5 MEV

9. DU MEV

9.50 MEV

10.00 MEV

$11,00 \mathrm{MEV}$

12.00 MEV

13. 10 MEV

14.OV MEV

$15.00 \mathrm{MEV}$

$16,00 \mathrm{MEV}$

$17,0 D M E V$

18. OQ MEV

19. OD MEY

20. DN MEV

21. UR MEV

22. UD MEV

23.00 MEV

$24.01 \mathrm{MEV}$

25. DO MEV

26. DU MEV

$27.00 \mathrm{MEV}$

28. DO MEV

29, HO MEV

$30.0 \square \mathrm{MEV}$

32.DO MEV

$34.00 \mathrm{MEV}$

36. DO MEV

38,0 A MEV

40. UO MEV

$42, H Q \mathrm{MEV}$

$\triangle 4.0$ MEV

46. DO MEV

$48.0 \mathrm{OU}$ MEV

$50.90 \mathrm{MEV}$

DAYE, E(MEV)
PROTONS

2. 3DE+D1

$1.96 E+D 1$

1.97E+ 111

$1.93 E+1$

1. $55 E+D 1$

$4.47 E+31$

3. Q $Q E+D 1$

$2.93 \varepsilon+b 1$

2.80E+U1

$2.44 E+b ?$

2. $25 E+121$

$1.64 E+11$

1. $59 E+D 1$

1. $b 4 E+B !$

2. $34 E+B 1$

2. $83 E+D 1$

$2.25 E+01$

2. $4 R E+\lambda 1$

1. $87 E+D 1$

1. $49 E+11$

1. $52 E+\cdots 1$

1. $|9 E+B|$

1. $17 E+11$

1. $12 E+11$

$1,03 E+01$

7. SAE + a

1. $U D E+\Delta I$

7. $P A E+D D$

7. UABE+ UA

5. $25 E+D a$

7. $25 E+1 D A$

$3.50 E+30$

4. $25 E+D ?$

$3,5 a E+D a$

$3.59 E+D A$

$5.75 t+b 1$

4. $25 E+0.3$

1. OAE + B D

1. OGE + DU

$5, \Delta \lambda E-W 1$

a.

$\theta$.

0.

a.

D.

9.86
DEUTFRONS

$1.32 E-A 1$

$9.265-63$

$9.28 F=3$

1. $35 E-11$

1. 35F-A

$1.56 E=1$

1. 56E-A1

$2.42 E-02$

2. $42 E-\omega 2$

1. $42 E-91$

$1.42 F-b 1$

6. $13 E-1) 3$

$6.13 \mathrm{~F}-\mathrm{W} 3$

$7.96 \mathrm{E}-\mathrm{YI}$

$7.96 E-43$

$2.77 E-93$

2. $77 \mathrm{E}-\mathrm{B}$

3. $4 \Delta E-U 3$

$3.44 E-B 3$

?.

a.

0

0

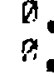

9

b.

0

?

D.

a.

i).

?

n.

a.

5.114
TRITONS

3. 34E-63

$5.60 E=\sqrt{3}$

$5,6 B E=63$

3. $33 E-103$

3. $33 E-13$

8. SUE - V 3

$8.64 E-n 3$

$7.64 E-13$

7. GUE- $\square 3$

$3 ., 3 E-13$

$3.13 t-113$

6. $03 E-03$

6. GIE-U 3

b.

n.

$2.25 E-103$

2. $25 E-113$

$5.27 E-G U$

$5.27 E-v 4$

r.

if.

Ei.

U.

,

i.

(a)

0.

6

$\theta$

$\theta$

v.

H.

b.

b.

6!

$\theta$

b.

a.

i.

a.

v.

b.

ii.

5.72
ALPIAS

1. $33 E+$ W1

7. $6+E+B)$

$7,64 E+(4)$

6. B.3E+JO

6. RSE + Ju

1. $50 k+11$

$1.50 t+111$

1. $15 E+191$

$1.15 E+b i 1$

7.7OE+ SOd

$7.76 E+A D$

5. $46 E+1 d A$

5. $40 E+314$

$2,69 E+d i d$

$2.695+130$

$5.47 E+12$

5. $47 E+x^{7} k$

2. $28 \mathrm{~L}+\mathrm{B})$

2. $2 B E+$ wh

7.57E-VI

7.57E-MI

$7.53 E-n 1$

$7.53 E-41$

2. $51 E-1$

2. $51 E-V 1$

ด.

vi.

n.

n.

n.

D,

A.

n.

b.

,

0.

id.

0.

$\Delta$.

a.

$\theta$

0.

a.

a.

b.

6.13 
IINITIAL SPECTRUM AT 1U, BOAB, CM, FOH ICRU MLISCLE TISSUE 42 MEV P+ ON HE NEUTRON SPECTRIIPI

\section{ENERGY}

D. 05 MEV

0.16 MEV

$D .15$ MEV

D. 2U MEV

D. 2.5 MEV

0.31 HEV

0.35 MFV

D. 40 MEV

0.45 MEV

$0.50 \mathrm{MEV}$

0.55 MEV

$0.60 \mathrm{MEV}$

0.65 MEV

0.70 MEV

$0.75 \mathrm{MEV}$

0.80 MEV

D. BS MEV

$0.90 \mathrm{MEV}$

0.95 MEV

1.00 MEV

$1,10 \mathrm{MEV}$

1.20 MEV

$1.30 \mathrm{MEV}$

1.40 MEV

1.50 MEV

$1.60 \mathrm{MEV}$

$1.70 \mathrm{MEV}$

1.8A MEV

$1.90 \mathrm{MEV}$

2.00 MEV

2. 10 MEV

2.2Q MEV

2,30 MEV

2. UD MEV

$2.50 \mathrm{MEV}$

$2.60 \mathrm{MEV}$

$2.70 \mathrm{MEV}$

2.80 MEV

2.90 MEV

3.00 MEV

3.20 MEV

3.40 MEV

$3.60 \mathrm{MEV}$

3.8U MEV

4. DO MEV
PROTONS

A.

$3.39 E+02$

1. $29 E+4$ ?

$9.59 E+91$

7. $I 2 F+B I$

$5.71 E+11$

5. 1 $4 E+11$

4. $39 E+1$

$3,7 \geq E+b 1$

3. $28 E+1$

$2.97 E+11$

2. $73 E+21$

2. $55 E+1: 1$

2. $S I F+91$

2. $14 E+01$

1. $88 E+1$

$1.93 E+61$

1. 6 . $E+11$

1. $79 E+11$

1. $76 E+11$

$2.33 E+1$

$2,79 E+01$

2. $5 \mathrm{AE}+1$

2. $24 E+01$

1. $86 E+b 1$

$1.82 E+1$

1. $76 F+D 1$

1. $66 E+B 1$

$1.53 E+111$

1.3 $3 E+11$

1. $36 E+E 1$

1. $24 E+B 1$

i. IGE + D!

1. $1 A E+D 1$

1. $10 E+\Delta 1$

1. $13 E+\Delta 1$

$9.71 E+00$

9. JGE+BU

6. $24 E+00$

6. $46 F+$ WI

1. $5\lfloor E+B !$

$1.34 E+31$

$1.53 E+01$

1. $3 A E+B 1$

1. $1 A E+D 1$
DEUTERONS

2. G $2 E-D S$

2. $D E-13$

3. 15E-02

$3.15 E-1: C^{2}$

2. $M 1 E-W 2$

¿ DIE-M

2. 96F-1?2

2. VOE -12

$1.77 E-112$

1. $77 \mathrm{~F}-12$

2. ॠ7F. - B?

2. $U T E-02$

$1.97 \mathrm{E}-12$

1. $77 \mathrm{E}=\mathrm{AC}$

2. $4, E=112$

2. $45 F-12$

$2.37 E-42$

2. $37 \mathrm{E}=192$

2. 35F- 92

2. $35 F-n 2$

$4.94 \mathrm{G}-42$

$4.99 F-112$

$4.42 E-132$

4. 4 CF $-\mathrm{A} 2$

5. $83 F-12$

5. R3F. . .

$2.57 E-62$

2. $57 E-112$

1. 5RE- A2

1. $5 B E-B 2$

2. $9 M F-02$

?. QUE- II?

$6.87 \mathrm{Em} H \mathrm{~S}$

$6.87 E-03$

$2.62 E-62$

2.62E-D2

$5.2 A F=\square 3$

5. 2 \& $=13$

?.23E-Q2

$2.23 E-U 2$

$9.59 \mathrm{~F}-\mathrm{J}$

$9.59 E-U 3$

$4.91 E=03$

4. $91 E-93$

$4.54 E-112$
TRITUNS

n.

i.

t.

.

is

i.

1.95E-k4

1. $95 E-1.4$

$1.17 E-04$

1. $17 F-14$

$7.86 E=x^{4} 4$

$7.86 E-04$

$9.56 E=64$

$9.56 E-U 4$

2. DRE-1.2

2. ARE- 02

7. $12 E=114$

$7.12 E-114$

1. $19 \mathrm{E}-\mathrm{n} 3$

$1.19[-.13$

$2.71 E-v 3$

2. $91 E-1 ; 3$

3. $12 E=13$

$3.12 E=03$

ट. $55 E-\checkmark 3$

$2.55 E-B 3$

$3.29 E=03$

$3,29 E=\square 3$

$3,6 A_{E}=03$

$3.60 E-13$

$2.53 E-D J$

$\therefore .53 t-33$

1. $99 \mathrm{f}-13$

$1.97 F-13$

2. $50 t-i d 3$

2. 5VE-g3

$1.29 E-133$

$1.29 F-13$

1. $21 E-U 3$

1. 2 IE- $D 3$

$4.52 E-1.3$

$4.52 E-13$

$3.98 E=113$

$3.98 E-03$

2. $49 E-43$
ALPHAS

1. $46 . E+46$

1. $44 t+16:$

2. $15 F+40$

2. $15 t+14$

2. $44 E+n n$

2. $4 U E+d i$

2. $15 E+01$

2. $15 E+15 n$

2. $39 E+3 n$

$2.39 E+11$

$2.66 E+4 \mathrm{r}$

2. $66 E+110$

$2.49 E+4 G$

$2,49 t+4 n$

2. $59 E+01$

$2.59 t+41.4$

$2.58 E+\Delta 0$

2. $58 E+\Delta x:$

$2,61 E+4 k$

2. $61 E+4, j$

$5.12 E+4 t$

$5,12 E+d v$

5. $42 E+4$

5. $42 F+H$

$4.97 E+\operatorname{lig} \theta$

$4.97 \bar{E}+0 v$

5. $179 E+619$

5. $09 E+B D$

$11.86 E+60$

$4.8 S E+20$

5.17E+ $U^{2}$

$5.17 E+119$

$4.53 E+3 b$

$4.53 E+(i k)$

4. $26 E+V i v$

4.2BE+

4. $58 E+14$

4. $5 B E+\square U$

$4.03 E+196$

$4.63 E+40$

$7.3 B F+B H$

7. 3RE+UH

$6.38 E+60$

h. $3 A E+E v$

$5,8 B E+h t$ 


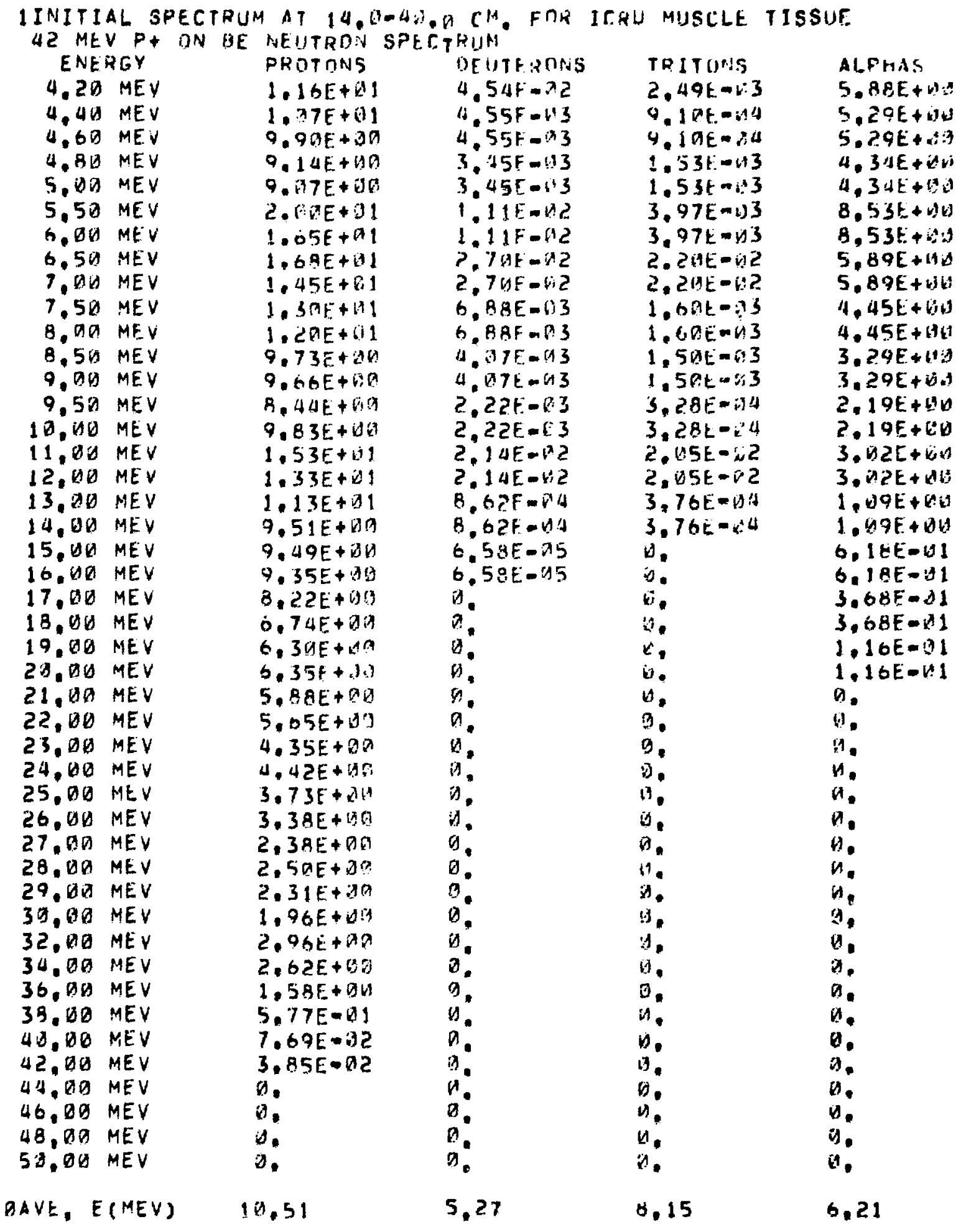




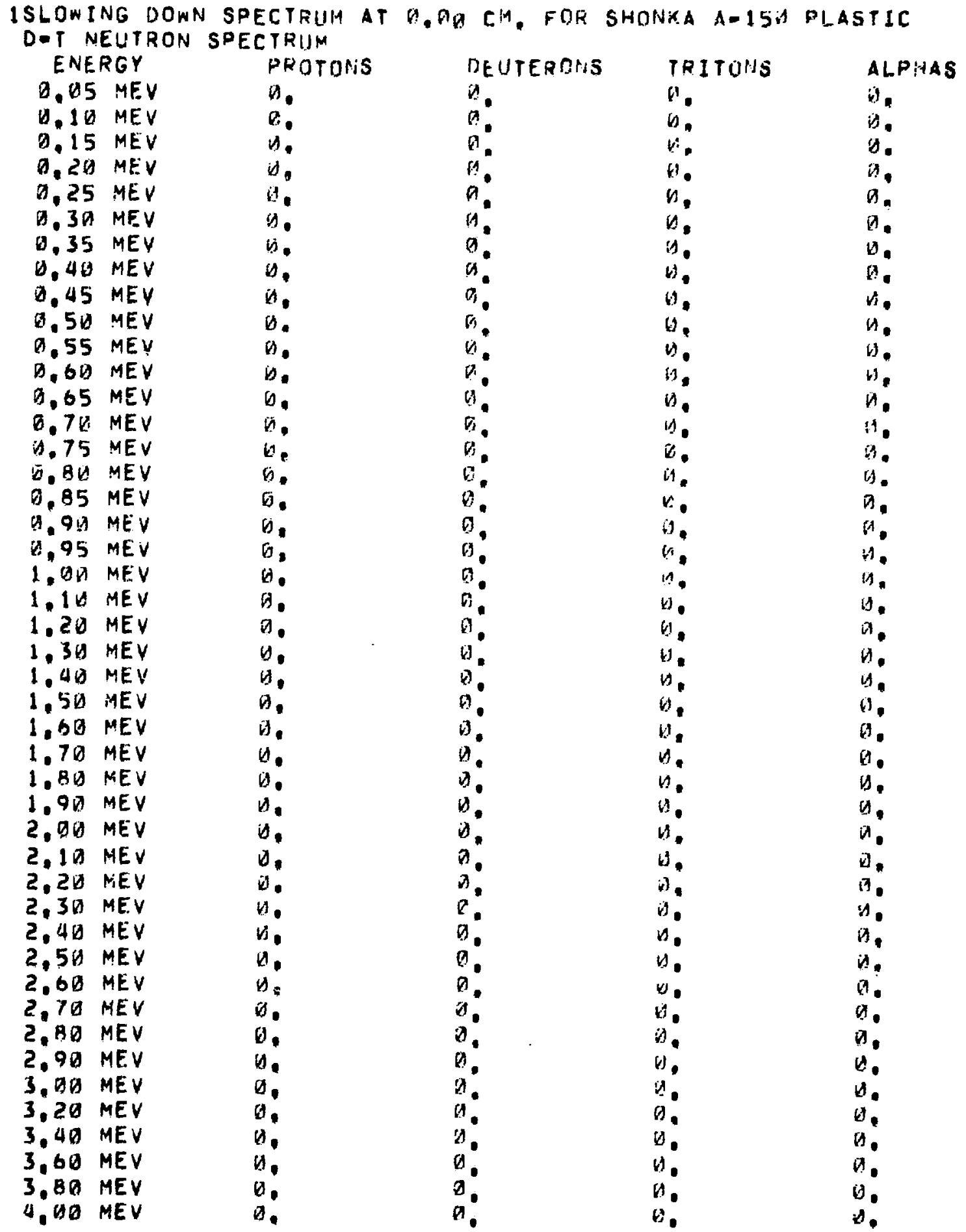


ISLOHING DOAN SPECTRUM AT U. DA CM. FOR SHONKA A-I5H PLASTIC. DOT NEUTRON SPECTRIJM

$$
\text { ENERGY }
$$

4. 2 A MEV

4. 40 MEV

4.60 MEV

4.8D MEV

5. DU MEV

5.50 MEV

6.00 MEV

$6.56 \mathrm{MEV}$

$7.0 D$ MEV

$7.5 \mathrm{II} \mathrm{MEV}$

8. 00 MEV

9.50 MEV

9.00 MEV

$9.50 \mathrm{MEV}$

10 , OD MEV

$11.00 \mathrm{MEV}$

$12,00 \mathrm{MEV}$

13, OO MEV

$14.00 \mathrm{MEV}$

15. DO MEV

16.00 MEV

$17.00 \mathrm{MEV}$

$18.00 \mathrm{MEV}$

19.00 MEV

20. DO MEV

$21.00 \mathrm{MEV}$

2.2. OO MEV

23.00 MEV

24. DO MEV

$25.00 \mathrm{MEV}$

26. OU MEV

27. VIO MEV

28,DO MEV

$29.00 \mathrm{MEV}$

$3 \pi_{100} \mathrm{MEV}$

32,00 MEV

$34.00 \mathrm{MEV}$

$36.00 \mathrm{MEV}$

$38.00 \mathrm{MEV}$

40.00 MEV

42.00 MEV

44.60 MEV

$46.00 \mathrm{MEV}$

48.00 MEV

$50.00 \mathrm{MEV}$

DAVE, E(MEV)
PROTONS

$\Delta$

0

b.

a.

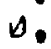

D.

b.

0

$\theta$

i.

0

4

a.

0.

$a$

a.

,

b.

v.

$\theta$

,

i).

D.

$\Delta$

a.

$\emptyset$.

0.

9.

i)

1.

d.

a.

a.

a.

v.

0.

0.

0.

$\theta$.

U.

b.

D.

n.

D.

$\theta$

0
DEUTEEDAS

H.

3

0

a.

$a$

0

0

$a$

b.

0

i.

$n$.

$B$.

7.

0.

$\theta$

1)

is

a.

a

$a$

$\theta$

$a$

a.

0.

a

a

a.

a.

a.

a.

b.

$\theta$

$B$

a.

$\theta$

a.

0.
TRITONS

E.

$s$

0

vi.

ט์.

wi.

$\theta$

1.

0.

0.

a.

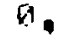

a.

a.

n.

0.

is.

is.

$\theta$

i.

$\lambda$

$B$

$\theta$

$d$.

is.

i)

$D$.

$\Delta$.

5.

1.

v.

if.

a.

i).

b.

i.

in.

v.

v.

U.

b.

0

0
ALPHAS

n.

D.

$\theta$

v.

$A$

$A$

b.

$s$

a.

?

a.

a.

)

9

a.

0

v.

0.

0

b.

$\theta$

$B$

$D$

a.

0

0

i)

0

0

a.

4)

i.

a.

9.

0

D.

(1).

a.

.

b.

a. 
1SLOWING DOWN SPECTRIJM AT D. 15 CM. FOR SHMPH.A A-156 PLASTIC D-T NEUTRON SPECTRUM

\section{ENERGY}

D. B5 MEV

0.10 MEV

7. 15 MEV

a. 20 MEV

0.25 MEV

$0.30 \mathrm{MEV}$

0.35 MEV

$0.40 \mathrm{MEV}$

0.45 MEV

$0.50 \mathrm{MEV}$

$0.55 \mathrm{MEV}$

$0.68 \mathrm{MEV}$

$0.65 \mathrm{MEV}$

$0.70 \mathrm{MEV}$

$0.75 \mathrm{MEV}$

0.80 MEV

0,85 MEV

0.90 MEV

$0.95 \mathrm{MEV}$

$1, \vartheta 0 \mathrm{MEV}$

$1.10 \mathrm{MEV}$

1.20 MEV

$1.30 \mathrm{MEV}$

$1,40 \mathrm{MEV}$

1.50 MEV

$1.60 \mathrm{MEV}$

$1.70 \mathrm{MEV}$

1.80 MEV

$1.90 \mathrm{MEV}$

2, $00 \mathrm{MEV}$

2.10 MEV

2.20 MEY

2.30 MEV

2.40 MEV

2.50 MEV

$2,60 \mathrm{MEV}$

2,70 MEV

2,BO MEV

$2.90 \mathrm{MEV}$

3. DD MEV

3.20 MEV

$3,40 \mathrm{MEV}$

3.60 MEV

3. AO MEV

$4.90 \mathrm{MEV}$
PROTONS

U.

$7,39 E-63$

$4.43 E-13$

$1.25 E-\mathrm{d} J$ ?

$1.35 E-12$

$4.75 E=D 3$

8. B1E- 03

$4.03 E-13$

$3.46 E-03$

$5.86 E-143$

$2.62 E-112$

2. $U 8 E-D ?$

$1.76 E-13$

$3.93 E-02$

$4.82 E=3$

1. $20 E-D 1$

2. $89 E-D 2$

9.5 $4 E=03$

4.7 DE-O3

1. 22E-

$2.44 E-02$

$3.61 \mathrm{E}-12 \mathrm{C}$

$1.83 E-02$

$2,96 E-B 2$

$1.86 \mathrm{E}-\mathrm{E2}$

$3.69 E=12$

$3,9 B E-02$

$2.66 E-02$

$5.26 E-D 2$

$3,61 E=D 2$

$1.70 \mathrm{E} 0 \mathrm{Z}$

1. 5 ZE- -42

7.99E-G2

$4.995-02$

2.80Eल 112

$6.54 E=\emptyset 2$

$3.37 E-62$

$5.95 E=D ?$

$1.74 E=B 2$

$4.73 E-02$

1. $\triangle B E-D 1$

$2.34 E-02$

$4.63 E-12$

$5.22 E-B 2$

$1.26 E-01$

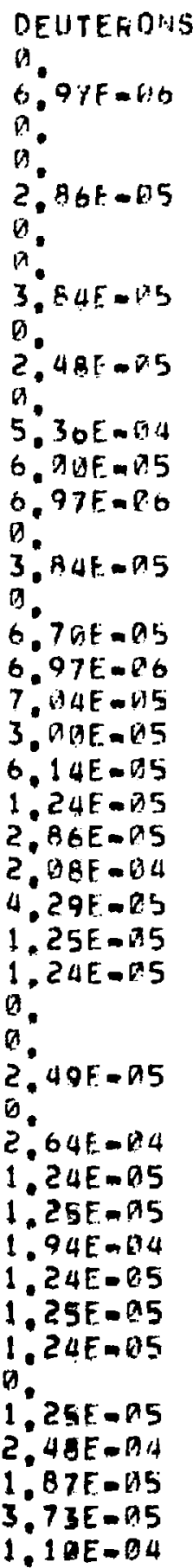

IRITONS

D.

$n$

3. DRE-gS

$n$.

0

D.

$a$.

6. BOE- - 15

$8.17 E-66$

6.2 DE $=35$

is.

b.

b.

$3.0 B E-65$

.

a,

$1.41 E=014$

a.

$\because$.

$6.20 E=05$

$4.9 A E-0.5$

$\theta$.

$1.54 E-Q 5$

$3.43 E-\angle .5$

$4,96 E-1.5$

$7,64 E-65$

4. $645-25$

$4.90 E-05$

$4.64 E=05$

$4,61 E-65$

$6.95 t-1) 5$

$1.23 E-04$

$6.54 E-05$

$3,43 E-125$

$6,5 \Delta E-W 5$

$1.63 E=64$

$3.4 \triangle E-D S$

$3.44 E-D 5$

$1.05 E-24$

$1.03 E-D A$

3.89E-05

3. $52 E-05$

6,3 ME-US

3. $72 E-\lambda 5$

3. 52E $=05$
ALPHAS

1.

$1.29 E-122$

$6.62 E-43$

2. SUE-W?

2. $72 E=\square 2$

2. $.4 E=13$

2. $6 A E-113$

$1.68 E=U 2$

$1 . B 2 E=W 2$

3. $24 E=\$ S$

1. $29 E=\$ 2$

$9.95 E-1.5$

9.67E-AS

3.11Em

1.27E-K2

$5.55 E-G 2$

$1.54 E-6 ?$

$3.51 E-63$

1. BUE-U2

4.74E= 33

B. BSE-W3

$2.62 E-132$

$1.7 E-V_{2}^{2}$

$1.31 \mathrm{EDG}$

2. $80 E-42$

$1.27 F-d 2$

$4.51 E=43$

1. DGE- M2

$1.95 E-A 2$

1. BGE $B D 2$

d.

$4.61 E-62$

$\Delta$.

$1.34 E-52$

$1.44 E-i 32$

2. 11E-UC

A.

1.1 $4 E=B 2$

$3.67 E=112$

$5,37 E-03$

1. $10 E=192$

$1.81 E=12$

1. 32E-32

1. A2E-

$1,15 E-152$ 


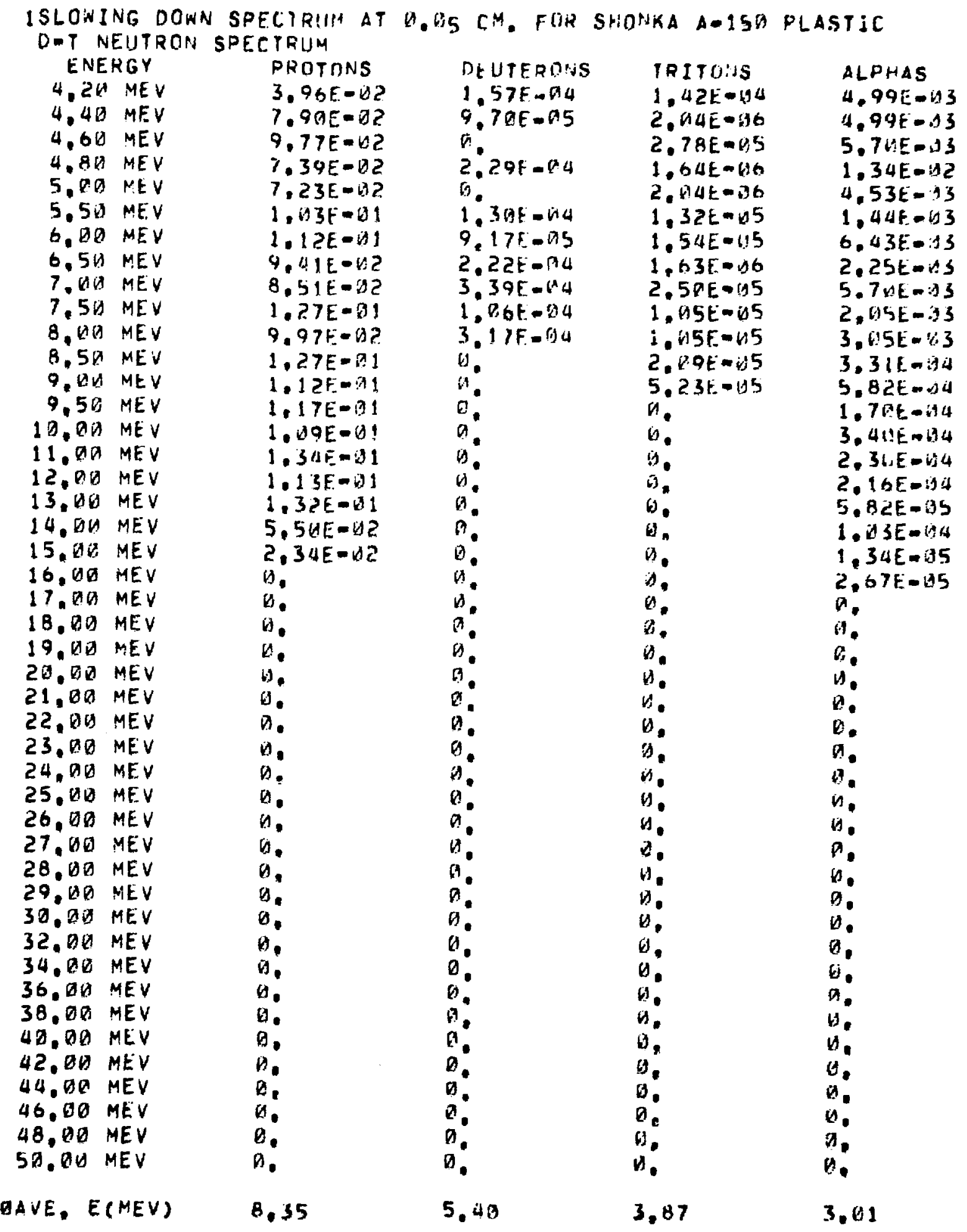


1SLOWING DOWN SPFCTRIMM AT M, IA CM. FOK SHONKA A-15R PLASTIC DWT NEUTRON SPECTRIM

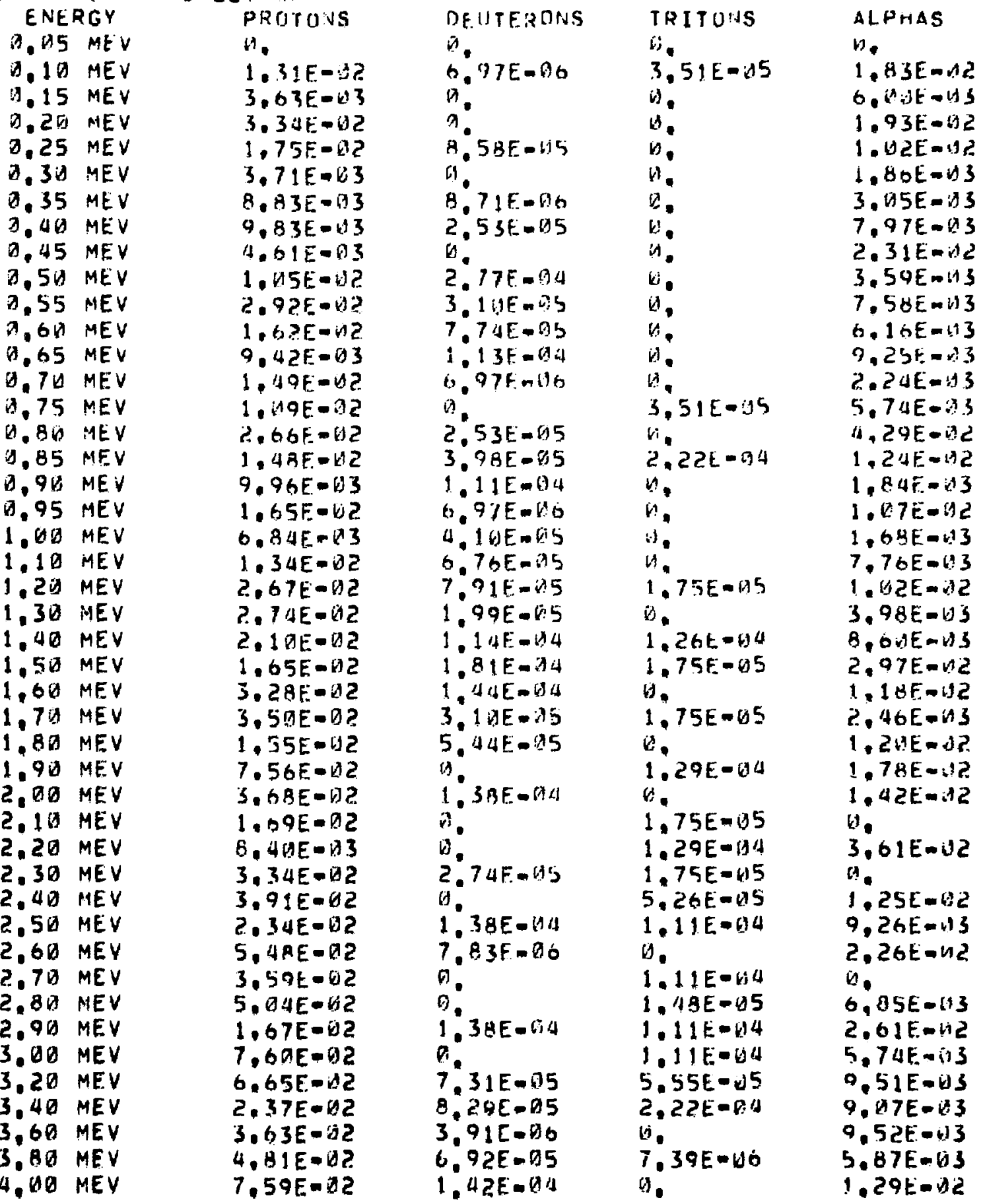


1SLOWING DOWN SPFCTRIHA AT H. IA CM. FOM SHOUKA AOISE PLASTIC D-T NEUTRON SPECTRUM

ENERGY

4. 24 MEV

4. $4 U$ MEV

4.60 MEV

4. BD MEV

5. DO MEV

$5.50 \mathrm{MEV}$

6. OA MEV

6.5 MEV

7. OD MEV

7.5 M MEV

B.DQ MEV

B.SU MEV

9. GO MEV

$9.50 \mathrm{MEV}$

10. DD MEV

11.00 MEV

$12.00 \mathrm{MEV}$

$13, O D$ MEV

14.00 MEV

$15.00 \mathrm{MEV}$

$16.50 \mathrm{MEV}$

17,DO MEV

$18 ., B Q$ HEV

$19.00 \mathrm{MEV}$

20. 10 MEV

21.00 MEY

22. DO MEV

23. 00 MEV

24.00 MEV

25.DO MEV

26.DO MEV

27.00 MEV

28. DO MEV

29.00 MEV

30.00 MEV

32.00 MEV

$34.00 \mathrm{MEV}$

36. DO MEV

$38.01 \mathrm{MEV}$

40.00 MEV

42. DO MEV

44.00 MEV

46.00 MEV

48.00 MEV

50. UO MEV

DAVE, E(NEV)
PROTOHS

2. 99E-112

$6.16 E-42$

$6.53 E-02$

$5.93 E-B 2$

$6.2 .6 E-102$

7. $21 E-02$

8. $82 E-\square 2$

$7.51 E-112$

$7.49 E-0 ?$

$6.65 F-02$

$9.48 E-62$

$6.42 E-1) 2$

$0.17 E-02$

$9.12 F=02$

$0.95 E-D 2$

1. GAE-OI

$7.19 E-B 2$

5.96E-102

1. XUE-BI

$1.17 E-0 ?$

U.

D.

a.

a.

a.

b.

a.

0

D.

it.

U.

a.

a.

b.

a.

0.

0.

b.

1.

$\theta$

0.

0.

0

0

$\theta$

B. 34
DEUTERDNS

8. $29 E-15$

1. 4 ? E- BA

2. $77 \mathrm{E}-114$

1.76E-AS

$3.91 E=00$

1. $92 t-35$

1. $49 F-05$

1.13E- 105

1. $1 d E-A T 5$

1. 1 UE-AS

3. 29E-AS

i)

6

b.

$a$

a

a

ii

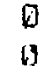

a

a.

H.

a

6

6

$a$

a

$a$

$a$

$a$

a

b

$a$

$a$

$a$

$\theta$

$B$

0

3,31
TR ITUNล

W.

H.

$7.39 E-19 t$

b.

i.

$2.96 E-116$

$2.965-136$

$2.96 E-116$

$5.91 E-156$

$5.91 E-$ is

1. $\triangle B E-B^{2} 5$

w.

b.

i.

b.

b.

$n$.

v.

i.

iv.

H.

i.

a.

is.

6.

(i)

is.

i.

a.

a.

is.

B.

in.

v.

0.

i.

$\theta$.

$\theta$

s.

b.

$n$

$D$

a.

t)

2,90
ALPHAS

$0.91 f-u ! 3$

5. $40 E-93$

$5.49 E-V^{3} 3$

1. $43 E=42$

3. $46 E-H 3$

C. $17 E-03$

7. $62 E=k: 3$

2. $81 E=43$

G. $29 E-13$

2. DXIF. -33

2. $75 E=13$

$5.192 E-114$

7. $815-14$

1. $48 E-614$

$2,95 \mathrm{~F}-34$

$9.72 E-15$

$0.6: E-15$

$3.73 \mathrm{E} .45$

$4.33 E-25$

$3,12 \mathrm{E}-\mathrm{JS}$

$6,24 E-D S$

$\Delta$.

is.

a.

a.

n.

a.

a.

B.

a.

P.

B.

$\theta$.

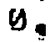

B.

a.

a.

a.

0.

y.

0.

0.

0.

?.

$v$.

3.26 


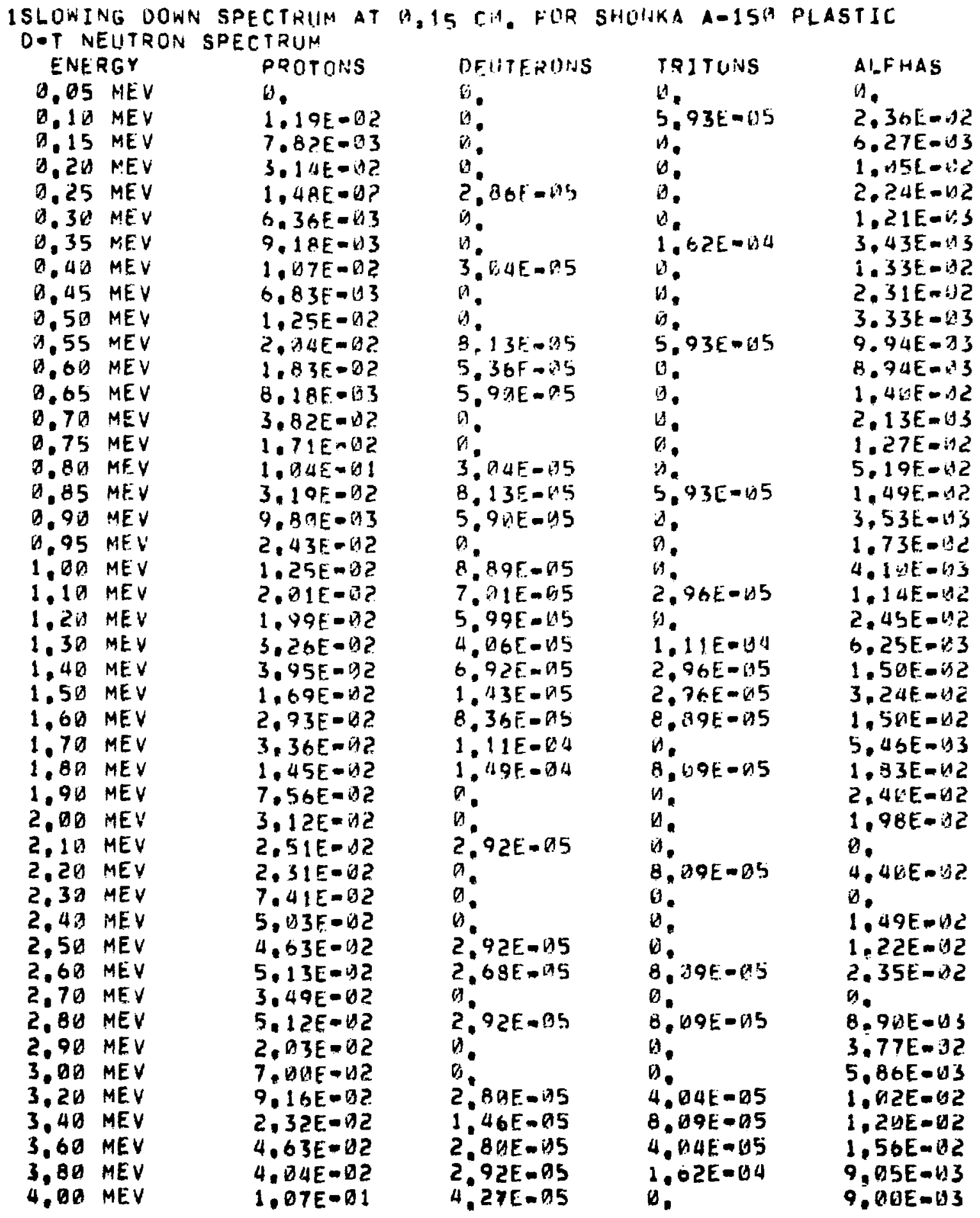


ISLOWING DOWN SPECTRIMM AT M.15 CM. FOR SHONKA A-ISH PLASTIC

D-T NEUTRON SPECTRUM

\section{ENERGY}

4,20 MEV

$4.40 \mathrm{MEV}$

4., i) MEV

4.80 MEV

$5 . \triangle 0 M E V$

5,50 MEV

$6, O 0$ MEV

6.5 MEV MEV

7. U1) MEV

$7,50 \mathrm{MEV}$

$8.00 \mathrm{MEV}$

8.50 MEV

9.,DO MEV

9,50 MEV

10.0ด MEV

$11.0 \%$ MEV

$12.00 \mathrm{MEV}$

$13.00 \mathrm{MEV}$

14.01 MEV

$15.01 \mathrm{MEV}$

16. DS MEV

17.0Q MEV

18.00 MEV

$19.00 \mathrm{MEV}$

20,01 MEV

$21 . K A M E V$

22,00 MEV

23, DD MEV

$24,00 \mathrm{MEV}$

$25,00 \mathrm{MEV}$

$26,00 \mathrm{MEV}$

$27.00 \mathrm{MEV}$

28. DU MEV

$29.00 \mathrm{MEV}$

30.00 MEV

32.0D MEV

34. 40 MEV

$36.00 \mathrm{MEV}$

36,DO MEV

$40,00 \mathrm{MEV}$

42.00 MEV

44.00 MEV

46.00 MEV

48, ME MEV

SO.DO MEV

gAVE, E(MEV)
PROTONS

$3.72 E-D 2$

$7.79 E-B ?$

7. OUE-U2

$5.29 E=\square 2$

$5.59 t-02$

$8.57 E-1.2$

$8.55 \mathrm{~F}-\mathrm{A2} 2$

8. $43 \mathrm{E}-\mathrm{ni}$

$9.32 \mathrm{E}=\mathrm{n2}$

1. ICF- 1

$9.55 E-2$

9. ?. $2 \mathrm{E}-\mathrm{G}$ ?

1. $\triangle 1 E-B 1$

1. $2\{E-19 \mid$

$9.95 E-9$ ?

$1.1 \cap E=A 1$

$1.17 E-D 1$

1. $|B E-B|$

$7.72 E-D ?$

0 .

.

$D$

a.

$\theta$

$\theta$.

$B$.

$\theta$.

0.

0.

$\theta$.

0.

$\theta$

0.

0.

b.

0.

n.

n.

$\theta$

0.

$\theta$.

$\Delta$.

$\theta$.

$\theta$.

0.

0.31

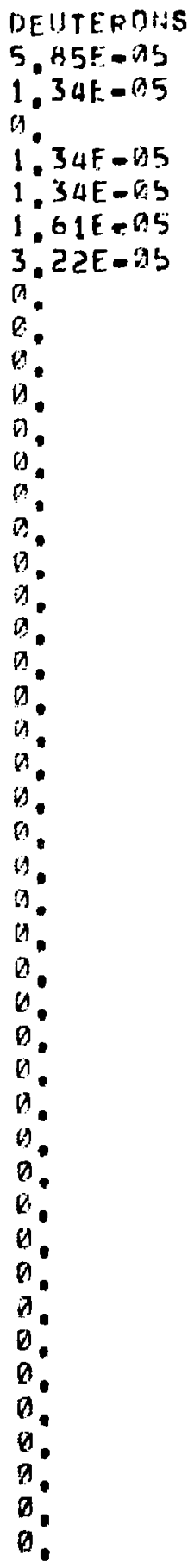

2.69
TR ITONS

is.

i.

in.

,

i.

v.

6

in.

bi:

a.

$n$

$v$

i.

is.

6

1.

n.

$\forall$,

0.

i:

a.

b.

.

0.

(1).

$\theta$.

$\theta$

$n$.

:

S.

2.

1.

2.

o.

.

n.

$\theta$.

G.

ต.

$\theta$.

0.

$\theta$

$\theta$

0.

2.43
ALPHAE

7. 4 GE $D S$

$7, \triangle \Delta E=3$

$4.21 E-33$

$1.22 E-92$

$6.7 v E=-i n$

$1.77 \mathrm{E}-\mathrm{BS}$

7.79E-U3

$2.91 \mathrm{E}-\mathrm{n} 3$

7. $1 \mathrm{BE}=0.3$

2. VIE-T13

$3.2 E E-D 3$

$2.57 E-i) 4$

3. 9kE-EIS

$1,21 E-V i 4$

$2.41 E-64$

$1.17 E-64$

1.17t-74

i.

i.

b.

n.

a.

is.

0.

1.

b.

a.

9.

$\theta$.

a.

$D$.

u.

B.

v.

$\Delta$.

H.

,

b.

0

$\theta$.

3.

v.

0.

.

D.

2.99 
1SLOWING DOWN SPECTRUM AT O. ZR CM. FDR SHONKA AOISU PLASTIC DOT NEUTRON SPECTRUM

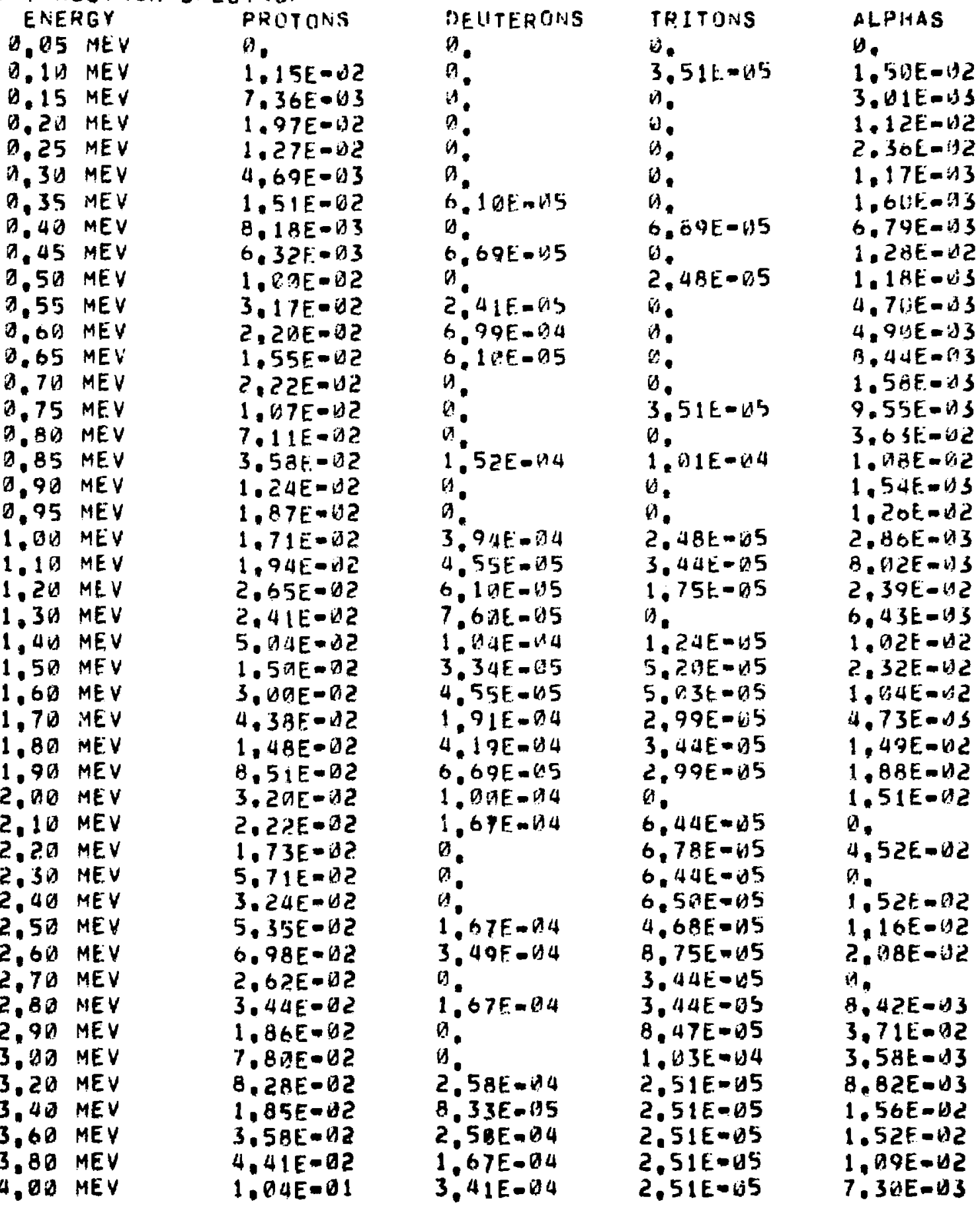


ISLOWING DOWN SPECTRUM AT G, PQ CM, FOR SHDNKA A-15И PLASTIC D-T NEUTRON SPECTRUM

\section{ENERGY}

4.26 MEV

4.40 MEV

$4.60 \mathrm{MEV}$

4.80 MEV

$5.00 \mathrm{MEV}$

$5.50 \mathrm{MEV}$

6. DO MEV

6.50 MEV

$7.00 \mathrm{MEV}$

$7.50 \mathrm{MEV}$

B. $00 \mathrm{MEV}$

$8.50 \mathrm{MEV}$

9.01 MEV

$9.50 \mathrm{MEV}$

10. D0 MEV

$11,00 \mathrm{MEV}$

12, DQ MEY

13,00 MEV

14,00 MEV

15, DO MEV

$16,00 \mathrm{MEV}$

17. DO MEV

1B. OO MEV

19.DQ MEV

20.00 MEV

21.00 MEV

$22.00 \mathrm{MEV}$

$23.40 \mathrm{MEV}$

$24, D G$ MEV

25. $O 5$ MEV

26.01 MEV

$27, O M)$ MEV

$28.00 \mathrm{HEV}$

29.00 MEV

$38.0 B$ MEV

32.00 MEV

34.00 MEV

$36.00 \mathrm{MEV}$

38.00 MEV

40,00 MEV

42.00 MEV

44.00 MEV

46.00 MEV

48,00 MEV

$5 \%, 00 \mathrm{MEV}$

gAVE, $E$ (MEV)
PHOTONS

$2.9 \Delta E-122$

7.7 ZE-02

$7.29 E-142$

$4.90 E-12$

$5.39 E-102$

$7,68 E-102$

$8.24 t-62$

$0.78 E-02$

$6.65 \mathrm{E}=02$

$9,12 E-\Delta 2$

$8.18 E-B 2$

7. 56E-D?

$1,03 E-D 1$

$1.19 E-B 1$

1. $|1 E-b|$

1. $32 E \rightarrow \square 1$

$1,31 E-U 1$

$9.64 E-11$ ?

1. $\triangle G F=B 1$

$\theta$.

0 .

a.

i.

is.

n.

a.

b.

a.

a.

$\Delta$.

g.

u.

y.

n.

O.

0.

$\theta$.

a.

a.

0.

n:

$\theta$

s.

:

8.56
DEUTERONS

$3.33 E-04$

$1.75 \mathrm{E}-\mathrm{GH}$

0.7

$1.75 E-0.4$

$1.75 E=0.4$

2, 1 DE- 4

$4,19 E=64$

a.

is

0

$n$.

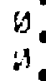

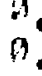

a.

.

9.

a:

a.

4

a.

a.

0.

.

0

:

:

0.

0.

0

a.

:

$0:$

a:

3.82
TRI TUNS

1. $1 E=B 4$

$\omega$.

s.

u.

$v$.

i.

i.

is.

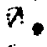

b.

4.

it,

b.

$n$.

is:

b.

0

n.

b.

9

a.

$\theta$

0 ,

n.

19.

i.

b.

.

n.

b.

a.

D.

$\Delta$

0.

$\Delta$

a.

o.

a.

0.

a.

B.

2.53
ALPHAS

$5.45 E-43$

$6.27 \mathrm{t}-\Delta 3$

$4,4 U E-13$

$9.2 U E-d 3$

$1.99 E=\$ 3$

$9.99 E-B A$

$5,31 E-\lambda 3$

2. VUE $=43$

$5,37 E-U 3$

$1.73 E-45$

$3.08 E-43$

$1.23 E-04$

2. $35 E-14$

$3.52 E=115$

7. $x^{15 E-15}$

G. GGE - AS

6. GBE - WS

i.

b.

.

a.

n.

i.

B.

a.

$\Delta$

is

4

0

$a_{0}$

$b$

a.

B.

0

D.

0.

0.

a.

:

:

3.00 
1SLOWING DOWN SPECTRUM AT O. UU CM, FOR SHONKA A-15H PLASTIC D-T NEUTRON SPECTRUM

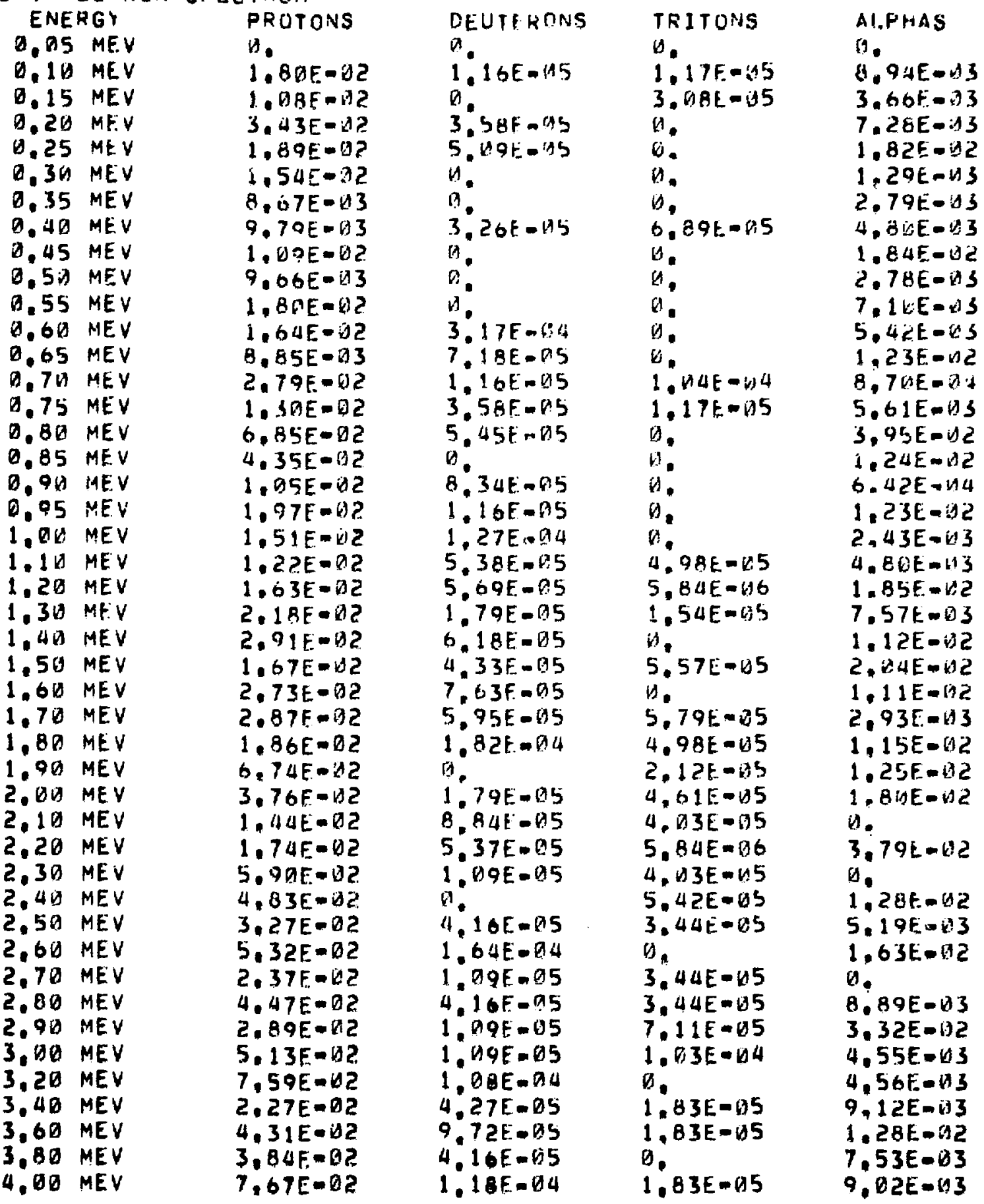




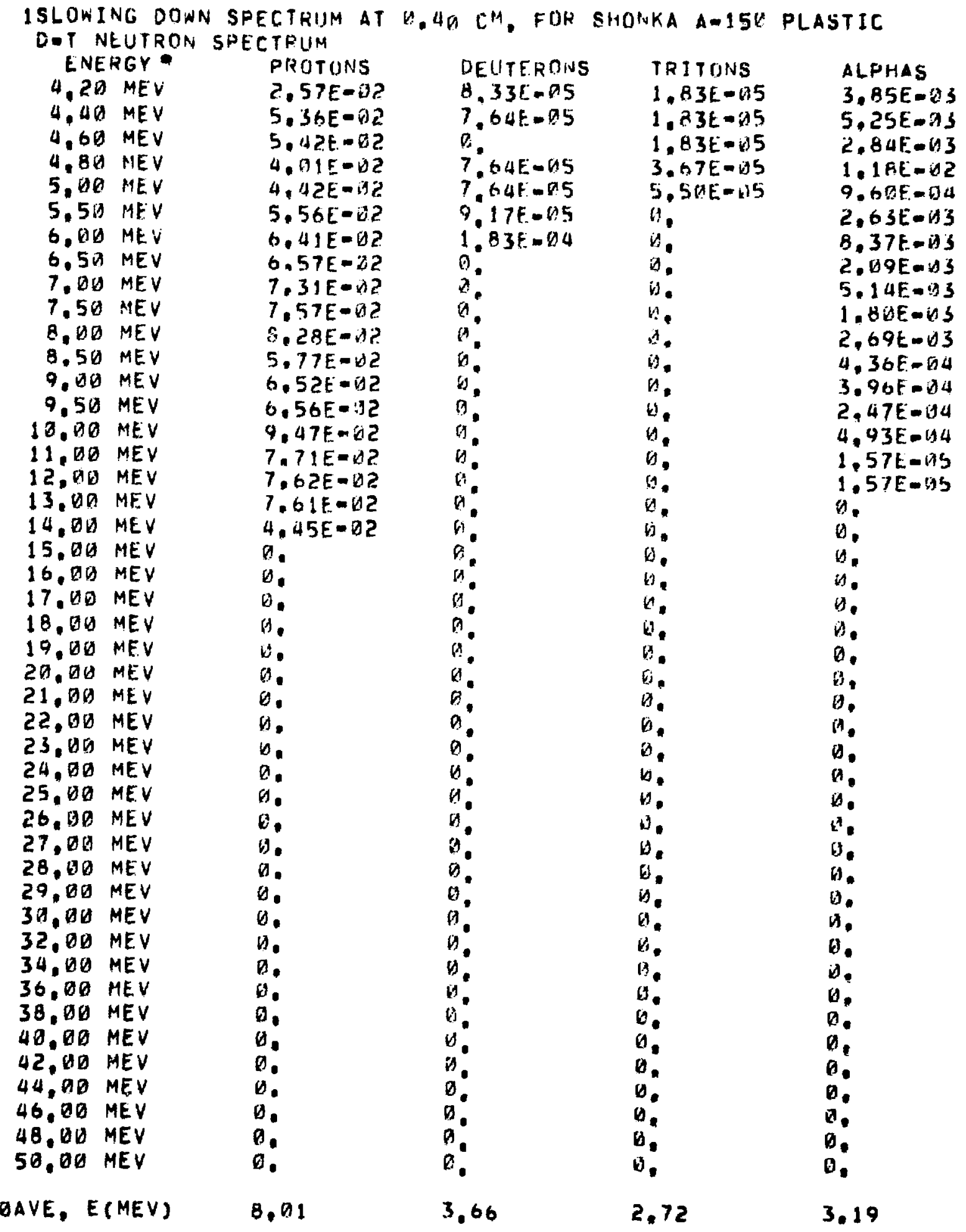


ISLOWING DOWN SPECTRUM AT H, GA CH. FOR SHOHKA A-15O PLASTIC

D-T NEUTRON SPECTRUM ENERGY

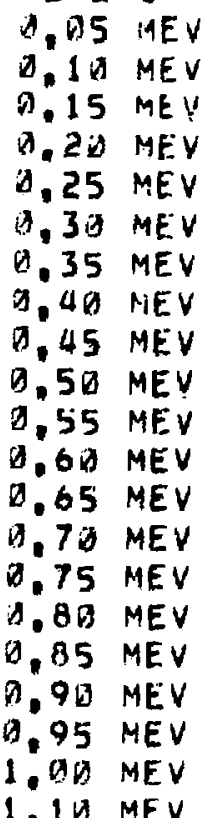

PROTINS

D.

1. $02 E-k 2$

9. $211 E=H 3$

C. 9 A -132

1. $94 \mathrm{E}-\overline{\mathrm{V}} \mathrm{C}$

1.27E-G2

1. $59 E-112$

$1.63 E=0$ ?

$1.07 E-D Z$

1. $49 E=0 ?$

2. $38 E-D 2$

$2.57 E-102$

$1,26 E-2 ?$

3. 4 ?E-D?

$1.33 E-6 ?$

$6.74 E-W 2$

3. $78 E-i \mathrm{D}$ ?

1. $54 E=d 2$

2. $17 \mathrm{TE}=02$

$1.94 E-W ?$

2. $39 E-142$

1.20 MEV

1.30 MEV

1. 4G MEV

$1,50 \mathrm{MEV}$

$1.60 \mathrm{MEV}$

$1,70 \mathrm{MEV}$

1.80 MEV

1.96 MEV

2. QD MEV

2.1 $1 \triangle \mathrm{MEV}$

2.20 MEV

$2.30 \mathrm{MEV}$

$2.40 \mathrm{MEV}$

2.50 MEV

2.60 MEV

$2.70 \mathrm{MEV}$

$2,80 \mathrm{MEV}$

$2.90 \mathrm{MEV}$

3.OU MEV

$3.20 \mathrm{MEV}$

$3.40 \mathrm{MEV}$

$3.60 \mathrm{MEV}$

$3.80 \mathrm{MEV}$

$4.00 \mathrm{MEV}$

2. 52E-v?

$2.81 \mathrm{E}-\mathrm{UL}$

4. $43 E-1 D E$

$1.92 \mathrm{E}-1 \mathrm{~d} 2$

$3,54 E-i ?$

$3.54 \varepsilon-02$

1. $79 E-i$ ?

$6.47 E-12$

$3.13 F-12$

$2.23 E=0$ ?

1.76E-1)?

$5.29 E-02$

$4.5 \& 5-12$

$4,24 E=12$ ?

$5.40 E-b 2$

$3,2 B E-132$

$4.45 E-112$

1.98E-Uद

$5,2 B E-02$

$8,42 E-02$

$2.74 E-92$

$4.53 E-62$

3. 9UE-DZ

9. DAE-U2

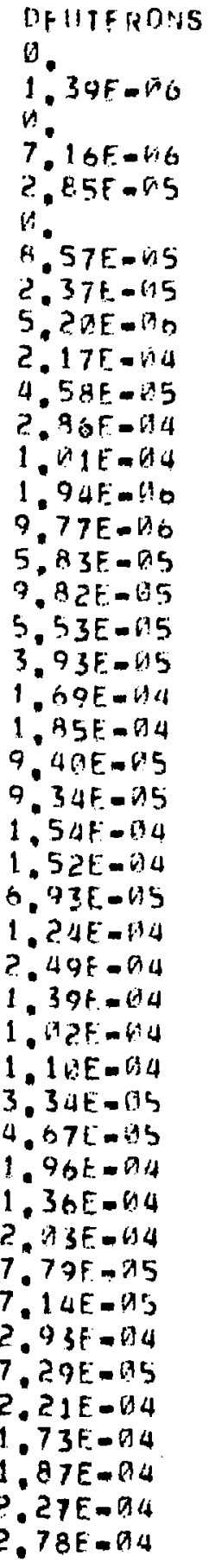

DFHTERDNS

TRITONG

4

4

$3.13 E-196$

V.

$3.13 t=66$

4. R3E- 36

1. $5 M E-144$

2. $25 E-45$

$1.96 E-D 5$

$2,48 E-66$

$n$.

a.

4. $15 E=06$

$7.82 E-65$

0.

$1.55 E-0.5$

$8.67 E-675$

们

2. $45 E=66$

2. $4 E E-636$

9. $36 F=46$

4. $53 E=b 6$

1. $12 E-34$

5, h DEE -5

$0.891-016$

1. $2 t E-55$

A. U3E- IS

B. HSE-OS

$4.43 E-15$

$3,12 F-115$

2. $45 E-15$

1. $2 G E-W 4$

1. $47 E-125$

$4 ., 3 E-.55$

$8.23 \xi=05$

9. $10 \ddot{c}-65$

$4.99 E-65$

1. IGE-DU

1. $20 E-14$

$5.71 t-55$

b. $3 B E-65$

$1.85 E-04$

$7.67 E-U 5$

1. $79 E-114$

$3.82 E-v 5$
ALPHAS

b.

8. $77 E-4 i 3$

3.5/E- $=$ is

1. VIAE-VC

$1,8,1 E-3$ ?

1. $19 E-Z 3$

$1.99 E-B S$

$7.64 E-U S$

1. $64 E-112$

$1.78 E=13$

$7.37 E-3$

4. $63 E-13$

9.69E- 43

$1.37 E-D S$

$6.62 E-13$

$3.97 E-V 2$

$9,81 E-13$

2. $1 \cap E-143$

1. $14 E-i) 2$

2. $15 E-K^{2} S$

$5.5 B E-Z 3$

$1.54 E-\Delta 2$

$6.91 E-13$

$9.54 t-143$

1.91E-पL

$9.74 E-13$

$3.6 .5 t-13$

1. $13 \mathrm{E}-\mathrm{vi2}$

$1.73 E-d 2$

$1.56 E-62$

4.

3.24E-D2

1).

$1.16 \mathrm{~F}-\mathrm{ij}$

8. $43 E-D 3$

1. OUE-U2

a.

7. $89 E-$ त 3

$3 . H 4 E=D 2$

$3.62 E-d S$

$6.56 E=43$

7. $25 E-1 \mathrm{U} 3$

$1,16 t-i j 2$

$6.03 E-13$

$7.83 E-(1) 3$ 
ISLOWING DONN SPECTRUM AT O. HO CM. FOR SHONKA A-15O PLAETIC D-T NEUTRON SPECTRUM

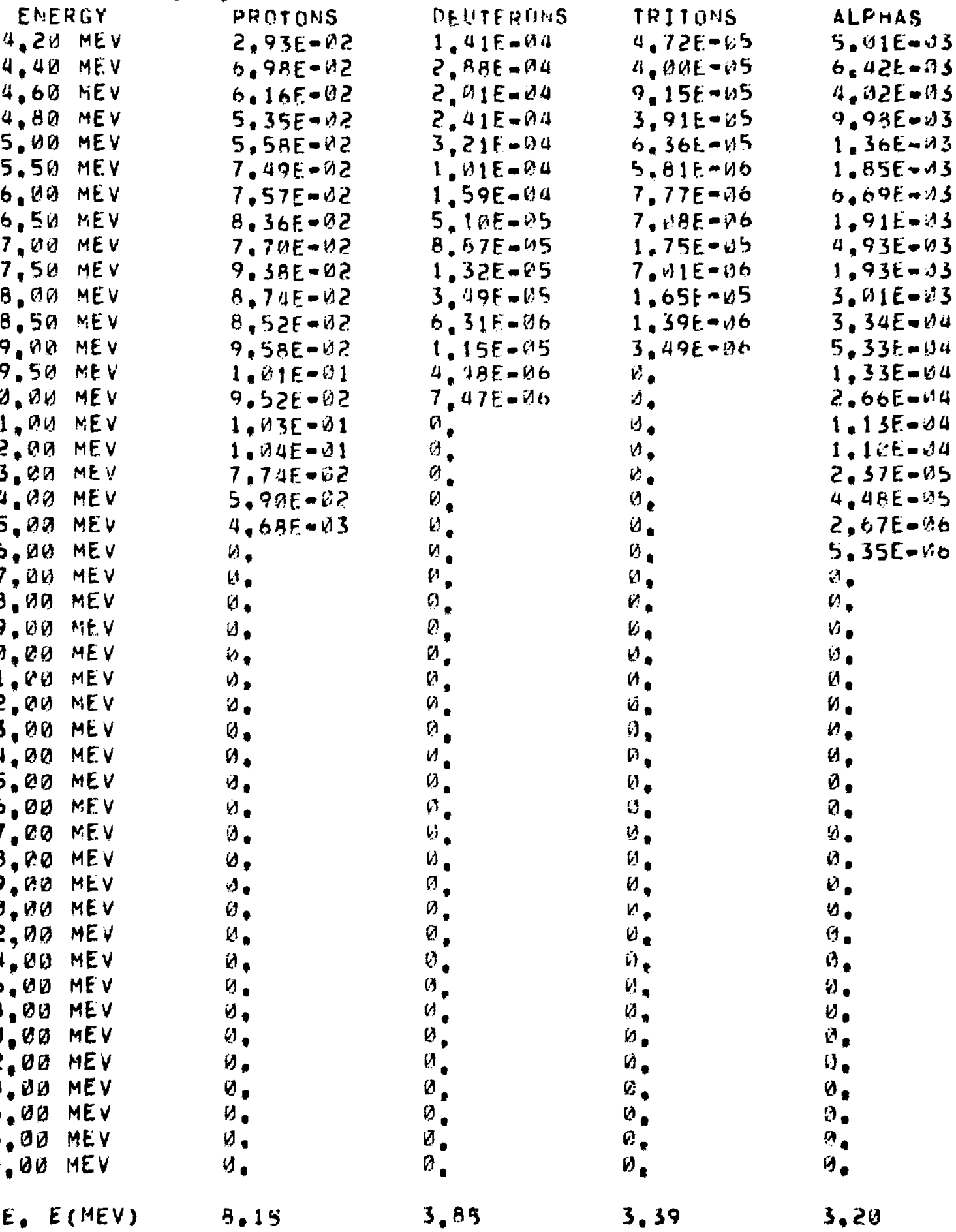


1SLOWING DOWN SPECTRUM AT 1. GO CAI FMR SHCINKA A-1SF FLASTIC D-T NEUTRON SPECTRLIH

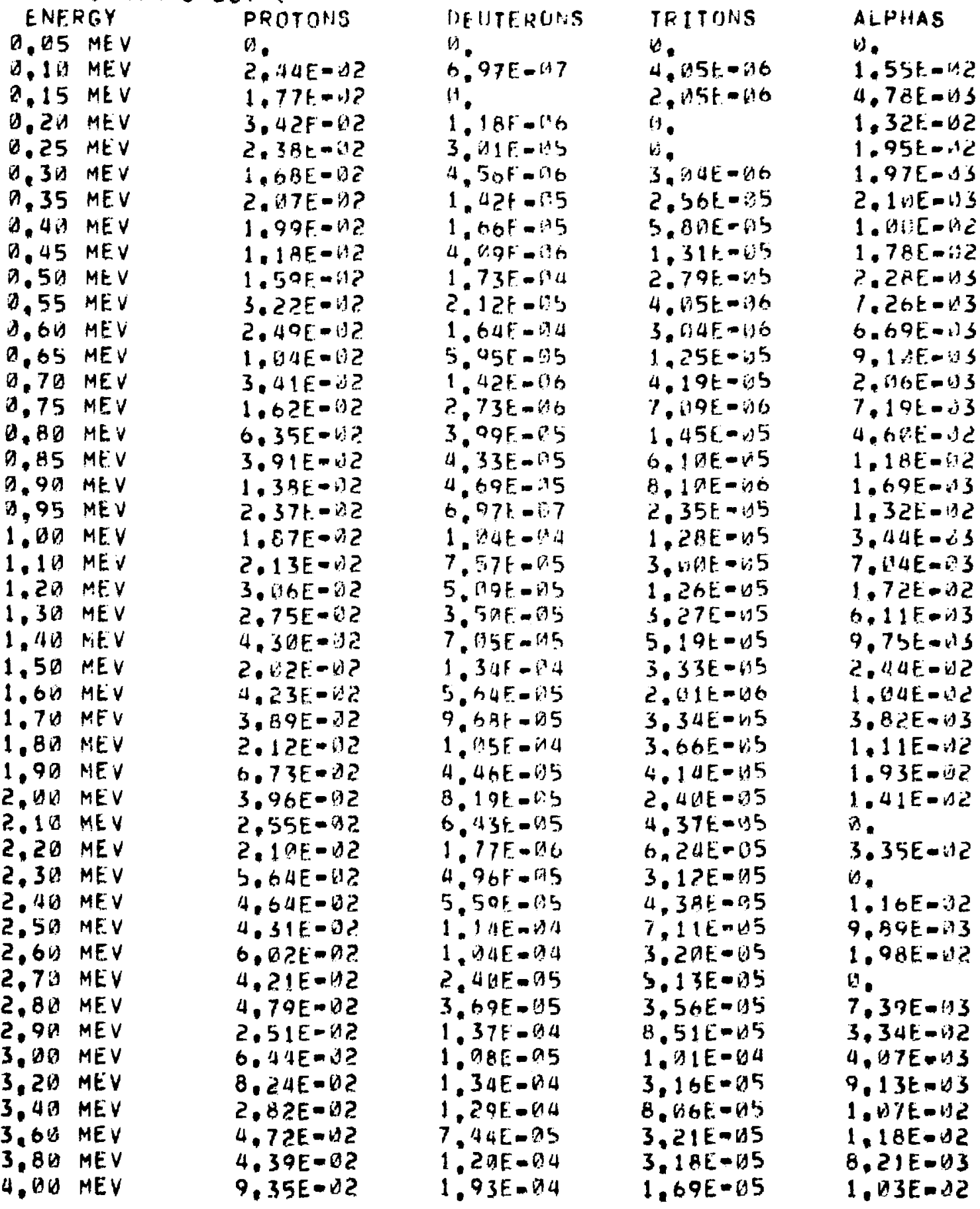




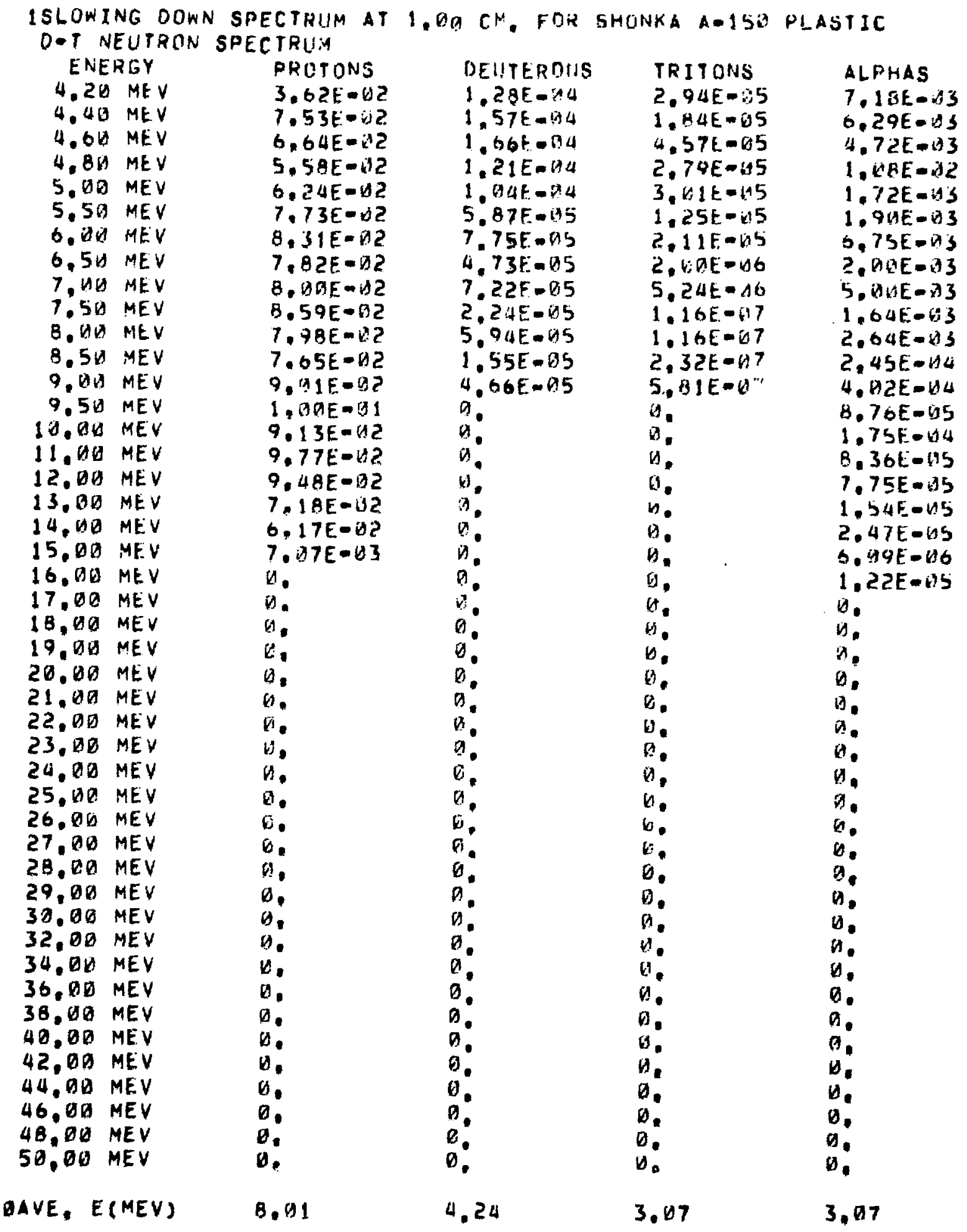


15LOWING DOWN SPECTRII:A AT 2 , BO CM. FOK SHONKA A-15A PLASTJC D-T NEUTRON SPECTHUM

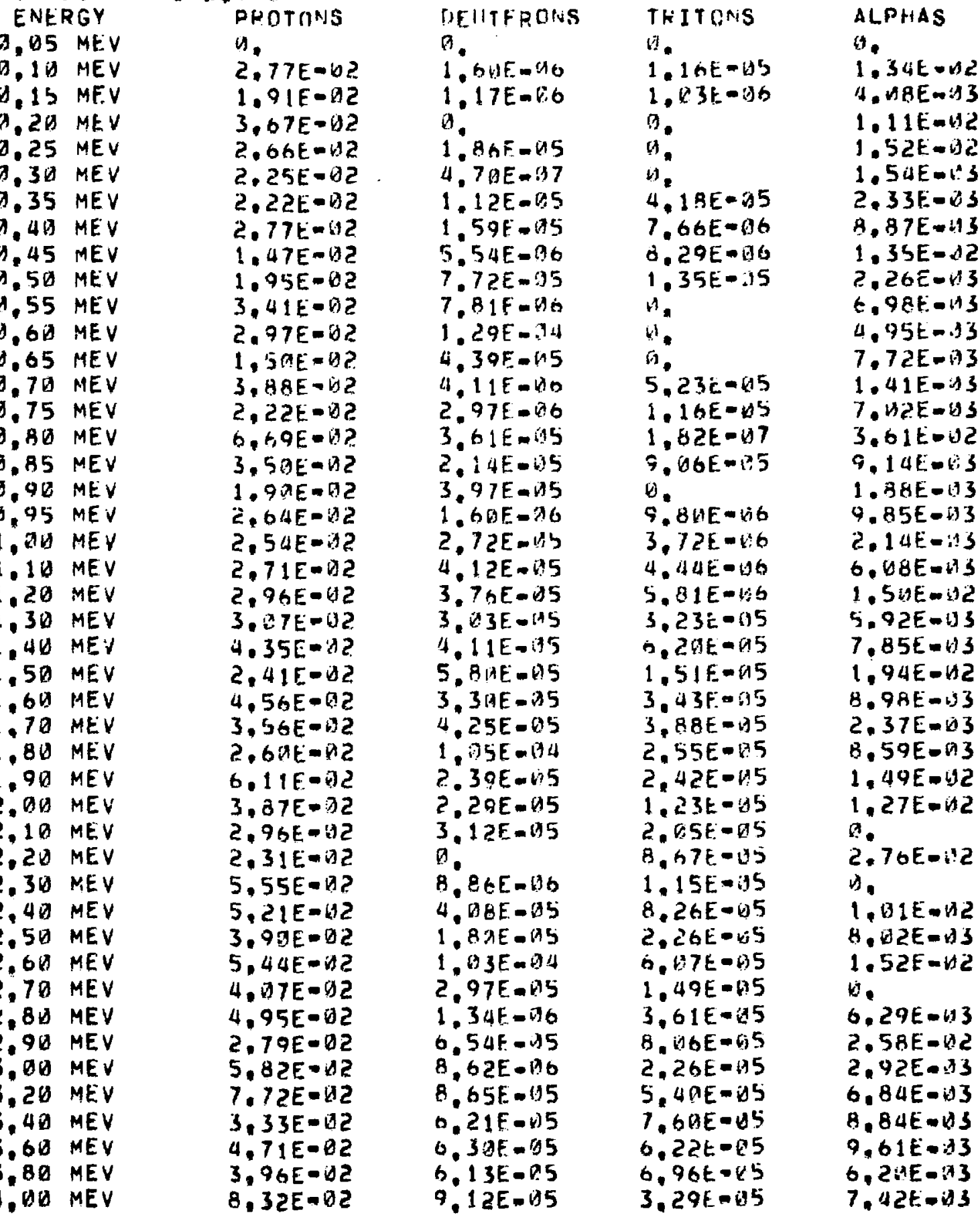




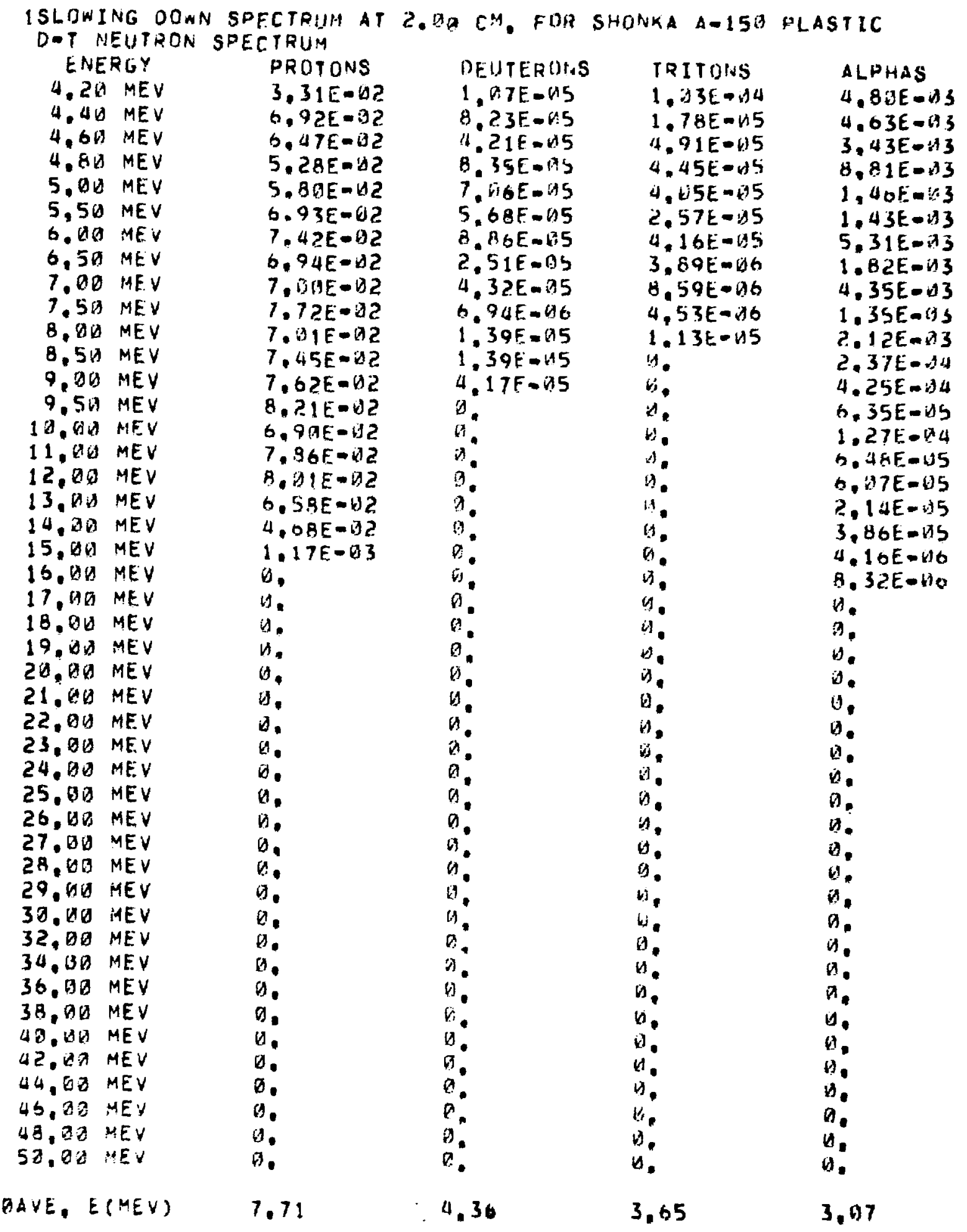


1SLOWING DOWN SPECTRUM AT H.ZA CH. FOH SHONKA A-15U PLASTIC D-I NEUTRON SPECTRUHT

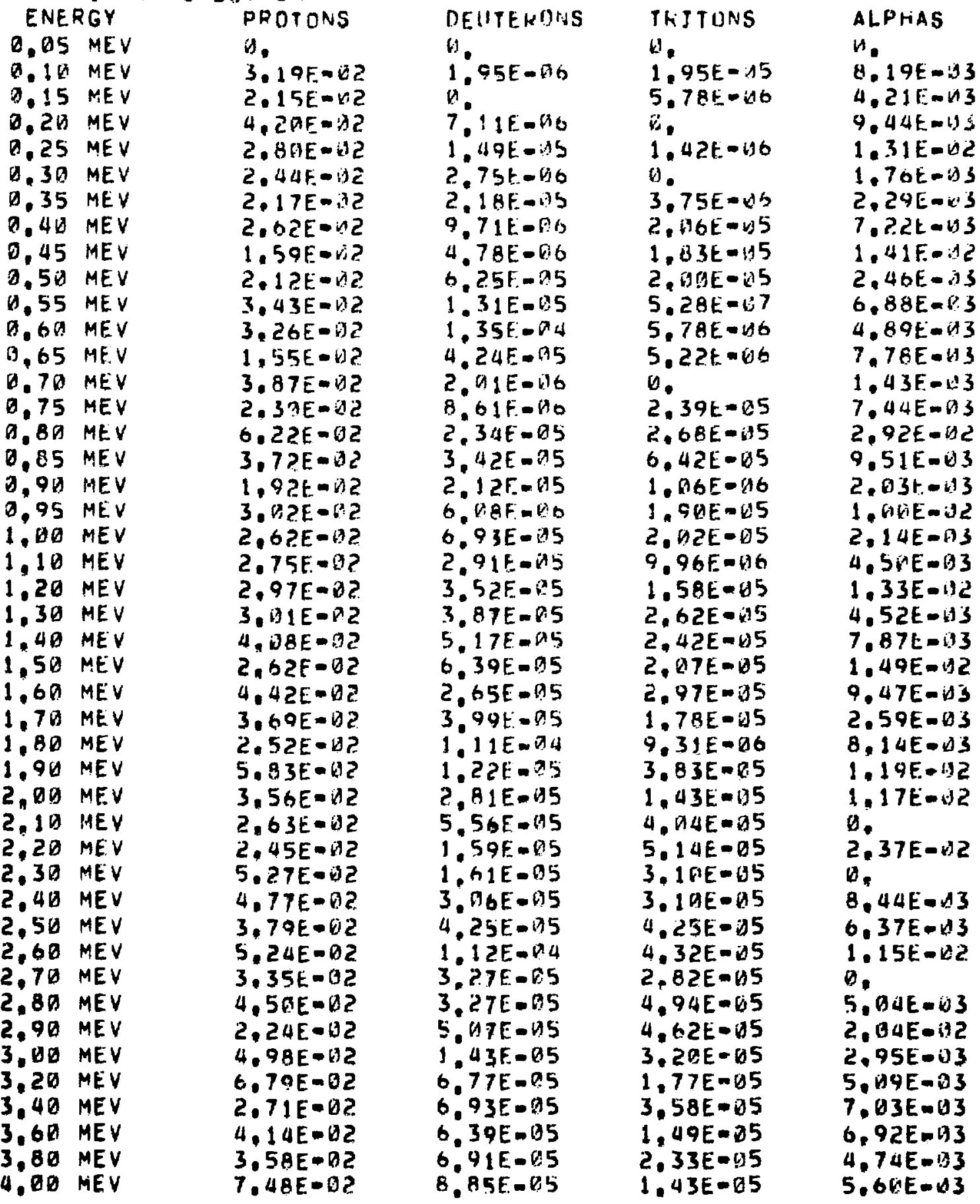


1SLOWING DOIN SPECTRIIM AT 4. QH CM. FDR SHONKA A-15H PLASTIC. D-I NEUTRON SPECTRIIM

\section{ENERGY}

4.2D ME.V

4. 40 MEV

4.60 MEV

4. BD MEV

5. DO MEV

$5.50 \mathrm{MEV}$

6. 00 MEV

$6.56 \mathrm{MEV}$

$7.00 \mathrm{MEV}$

$7.50 \mathrm{MEV}$

8. O MEV

8.50 MEV

$9.00 \mathrm{MEV}$

9.5B MEY

10. DU MEV

11. $\triangle O D$ MEV

12.90 MEV

$13.00 \mathrm{MEV}$

14.00 MEV

15, DO MEV

16.DO MEV

$17.00 \mathrm{MEV}$

$18.00 \mathrm{MEV}$

19.00 MEV

2Q. DO MEV

$21.00 \mathrm{MEV}$

22, $D O M E V$

23,00 MEV

$24.00 \mathrm{MEV}$

25.OM MEV

$26.010 \mathrm{MEV}$

$27.00 \mathrm{MEV}$

28. 06 HEY

29.0O MEV

$30.00 \mathrm{MEV}$

$32.00 \mathrm{MEV}$

34.00 MEV

36. AO MEV

$38.00 \mathrm{MEV}$

40.00 MEV

42,00 MEV

$44 . O D$ MEV

46.06 MEV

48,00 MEV

53. DU MEV
PRDT DHS

3. B) $4 E=02$

$5.95 E-02$

$5.67 E-x^{1} 2$

4. $45 E-62$

$5,14 E-192$

$0.14 F-02$

$6.43 E-1.2$

$6,152 E-W ?$

6. $5 \cap E-O ?$ ?

$6.97 E-192$

$0.54 E-b$ ?

$6.93 \mathrm{E}-1 \mathrm{H}$ ?

$7.27 E-12 \ddot{E}$

$7.26 E-12$

$6.32, E-D 2$

7. $21 F-02$

$6.83 E-02$

$5,78 E-W$ ?

4. ?7E- तT?

2. $34 E-13$

$\forall$.

i.

a.

a.

E.

D.

0.

$\theta$.

B.

$\theta$

O.

$\theta$

$B$

$\theta$

0.

D.

b.

:

$\theta$

0

a.

b.

$\theta$

a.

e.

ZUE, E (MEV)

7.67
DEUTEROALS

$5.34 E-U 5$

6. $49 E-A 5$

2. ABE $=135$

6. G9F-AS

4.71E-AS

$5.1 B E-1 A 5$

B. $15 E-05$

2. $43 E-4.5$

$4.5 B E-05$

2. $81 E-06$

$7.28 E-196$

1. $24 E-16$

1. $.5 E-06$

1. DAE $D O$

2. $.7 E-86$

a.

0

$\theta$

a.

$a$

a.

a.

i)

$a$

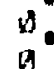

a.

.

0.

a.

a.

$a$

$a$

0

$\theta$

0

0

a.

0

$a$

0

9

D.

3.91
TPITONA

4.25E-DS

$7.5 B E-136$

$2.46 \mathrm{E}-75$

$1.64 \mathrm{E}-\Delta 7$

$2.57 E-66$

3. $18 E=6.6$

$4,24 E-706$

$3.91 E-16$

$8.8 \triangle E-116$

$3.71 E-k 6$

$9.27 E=06$

i.

a.

0.

v.

0

(1).

0.

b.

0

D.

b.

$B$

$\Delta$

a.

$D$

$v$

D.

i).

b.

0

i)

$\theta$

0

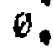

a.

$a$

0

$b^{\circ}$

0.

in.

b.

a.

0

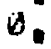

3,29
ALPRAS

3. $14 E-143$

3. $17 E-13$

2. $25 E-63$

$6.48 E-\triangle \Delta 3$

1. DICE $\triangle \triangle 3$

$1.17 E-1.3$

4. $09 E-E_{1} 3$

1. $19 E-V 3$

2. $96 E=113$

$9.55 E-d 4$

1. 55E- 13

1. $48 E=114$

$2.49 E-34$

4. B9E- 15

$9.78 E-d j$

$4.113 E-115$

3. $66 t=65$

$1.73 E-0 S$

3.10E-145

3. beE - 16

7. 32E $=40$

U.

$u$.

4

i.

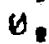

$\theta$.

U.

3.

0

0

$\theta$.

$n$.

a.

a.

a.

0

0.

$b$.

0.

b.

b.

b.

a.

a

2.96 
ISLOWING DONH SPECTRIIM AT G. DQ CM. FOR SHIONKA A-ISUI PLASTIC D-T NEUTRON SPECTRUA!

\section{ENERGY}

D. BS MEV

0.11 MEV

0.15 MEV

$0,20 \mathrm{MEV}$

$0.25 \mathrm{MEV}$

$0.36 \mathrm{MEV}$

$0.35 \mathrm{MEV}$

$0.40 \mathrm{MEV}$

0.45 MEV

$0.50 \mathrm{MEV}$

$0.55 \mathrm{MEV}$

0.60 MEV

$0.65 \mathrm{MEV}$

D.7D MEV

Q.75 MEV

0.8 MEV

$0.85 \mathrm{MEV}$

$0.90 \mathrm{MEV}$

$0.95 \mathrm{MEV}$

1, $\triangle 0$ MEV

1,10 MEV

$1,26 \mathrm{MEV}$

1,3 MEV

1. 4 U MEV

1,5 W MEV

$1.60 \mathrm{MEV}$

1,70 MEV

$1.81 \mathrm{MEV}$

$1.90 \mathrm{MEV}$

2,00 MEV

$2.10 \mathrm{MEV}$

2.20 MEV

2,30 MEV

2.40 MF. V

$2.50 \mathrm{MEV}$

2,60 MEV

2,70 MEV

$2.80 \mathrm{MEV}$

2.90 MEV

3. UQ MEV

3.20 MEV

$3.40 \mathrm{MEV}$

3.6D MEV

$3.80 \mathrm{MEV}$

4. 00 MEV

\section{PROT ONS}

I.

3. ज3E-42

$2,14 E-1] 2$

$3,98 E-J ?$

2. $96 E-02$

$2.425-3$ ?

$2.44 E-.12$

$2,77 E-4$ ?

1. $5 B E-19$ ?

2.11E-D?

$2,9 B E-3$ ?

$2.34 E-32$

$1,73 E-3 ?$

3.61E-B?

1. $85 E-D ?$

5. DAE- BAE

$3.64 E-32$

1. $69 E=0$ ?

$2.97 \mathrm{E}-\mathrm{k}$ ?

$2.67 t-02$

2.77E-0?

$2.95 E-\Delta 2$

$2,74 E-D 2$

$3.65 E-3$ ?

2. $27 E-102$

4. UTE-D2

3.53E -13 ?

$2.02 E-112$

$5.34 E-112$

$3,34 E-U$ ?

$2,49 E-42$ ?

$2.22 E-0 ?$

$4.45 E=02$

$4.03 E-\square ?$

3.41E-D?

$4.91 E-92$

$3.26 E-112$

$4.41 E-112$

2. $49 E-U$ ?

$5,21 E=\square 2$

$6.4 B E-6$ ?

$2.37 E-\Delta 2$

$3.91 E-b ?$

3. 5BE $-\dot{x} \cdot 2$

$6.55 E=02$
DEUTEROAS

.

$2: 32 E-1,7$

:

B.

1. A AE-GS

$5.59 E-D_{6}$

$8,08 E-176$

1. 1 AF-AS

$4.69 E-56$

$8.78 E=135$

9.4 DE $=176$

1. $47 E-$. 4

3 . M OF - 15

$4.13 E-697$

1. U1E- -96

$1.73 F=05$

$2.76 \mathrm{E}-05$

2. $34 E-D 5$

$2,32 E-47$

7.61E-0.5

$4,72 E-175$

$2,90 E-05$

$2.39 \mathrm{~F}-1 \mathrm{BS}$

$2.90 \mathrm{E}-\mathrm{O}) 5$

$6.52 E-65$

2. $44 \mathrm{E}=125$

$3.97 E-$ AS

$6.83 E-A S$

$3.64 E=05$

$4.760 .-25$

4. $98 E-115$

$2.57 E-D 6$

5.96E-95

$4.711-05$

$6.75 E-415$

$6.87 E-05$

1.2AE-OS

$2.76 E-95$

$7.63 E-0.05$

$3.28 T=06$

7. DQE-AS

$7.6 .7 E=05$

$5.21 E-A 5$

$7.71 E=05$

I.ATE-04
TRITINS

V.

$6.53 E=4.6$

0.

$k^{2}$

vi.

$\therefore$

4. 31E- 1.5

2. POE-AS

4. F $9 E=$ - Ih

1. MGE- 5

$6.53 E=116$

i.

$\because$.

n.

in.

a.

$5.97 E-65$

4.

$1.10 k-196$

$9.52 E-i 6$

1. $43 E=115$

i.

$3,54[-65$

$4,65 t-1,5$

1. $4 B E-65$

2.69E- T5

$5,31 E-36$

$3,26 E-14$ 's

1. $\triangle B E-115$

1, VGE-DS

$1,64 E-155$

$4.9 B E-D 5$

$1.58 E-U 5$

2.52E-AS

$3.53 E-65$

$5.29 E-135$

2. $05 E-115$

$3.51 E-B S$

$4.77 E-U 5$

4.26E-05

$3,53 E-15$

$5.42 E-135$

$3.46 E-05$

$5.89 t-15$

$1.36 E-05$
ALPHAS

a.

7. 3 IE $E-13$

4.8RE-H3

1. $13 E-132$

1. $59 \mathrm{E}-62$

1.69E-DS

$2.99 \mathrm{E}-\mathrm{W} 3$

$7.5 \mathrm{dE}-\mathrm{A} 3$

1. $43 E-62$

3. $106 E-13$

$5,97 E-13$

$5.43 F-13$

9. $\triangle 8 E-13$

$1.41 \mathrm{t}-\mathrm{d} 3$

$0.25 E-43$

2. 9 HE- $1 \%$

7. HOE - BS

$1,74 \mathrm{E}$ - BIS

$9.9150-13$

2. $D 2 E-$ in 3

$4.23 E-i) 3$

$1.20 E-a)$

$5,35 t-d s$

$8.22 \mathrm{E}-13$

$1.54 E-.32$

6. $82 E=33$

2. $88 E-13$

$8,33 E-\dot{U} 3$

$1.13 E-42$

$1.11 E-k 12$

0.

$2.29 t-192$

3.

$7.96 E=: 33$

$6.62 E=$ is 3

$1.29 E=192$

0.

$5.55 E-U 3$

2. 14E-i+2

$2.30 k=33$

$5,68 E-a 3$

$7.79 E-B 3$

$7.39 E-113$

5.7 GE -1$) 3$

$6,60 E-1) 3$ 
1SLOWING DOWN SPECTRIM AT 6. CIA. FOK SHONKA A-15H PLASTIC D-T NEUTRON SPECTRUM

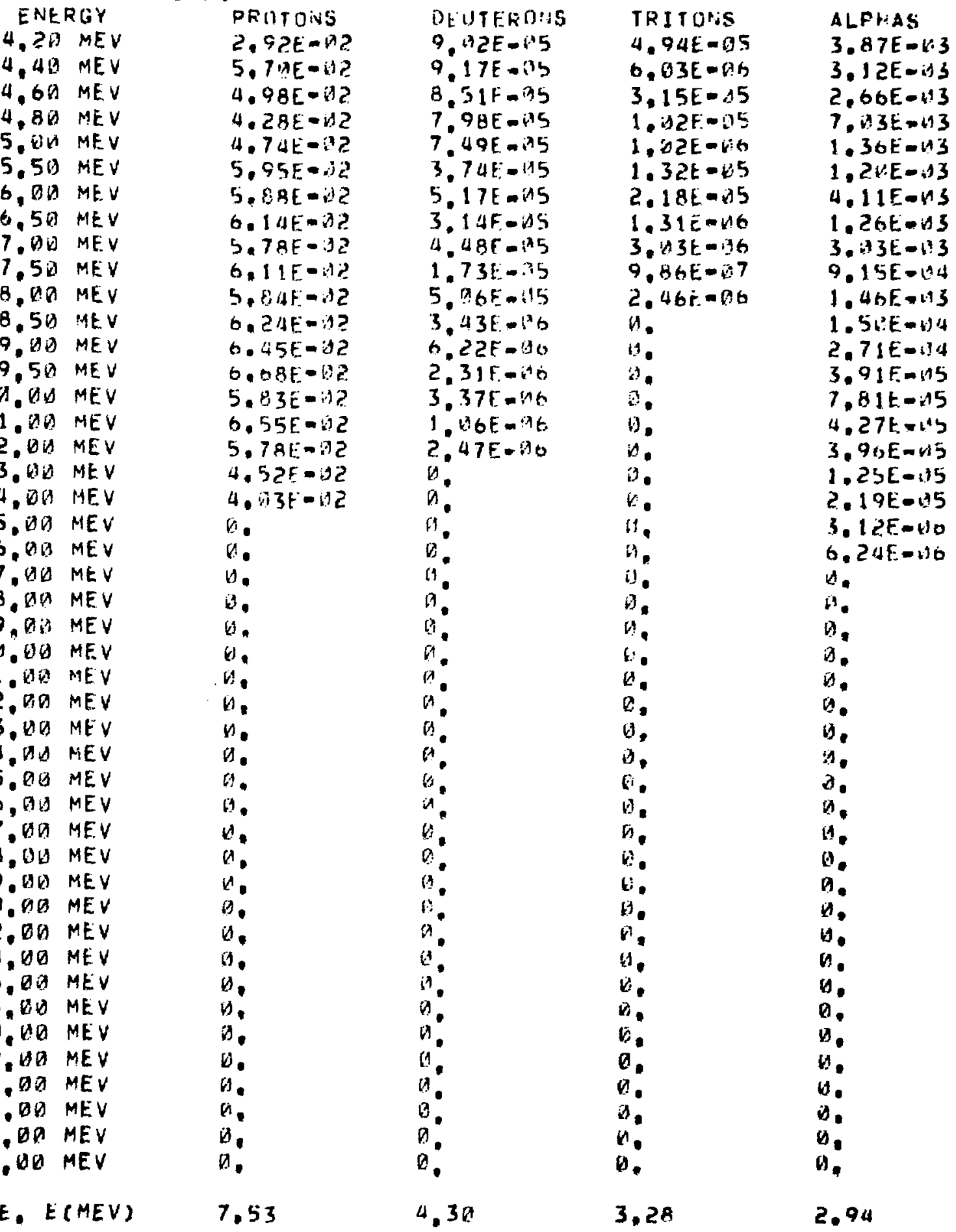




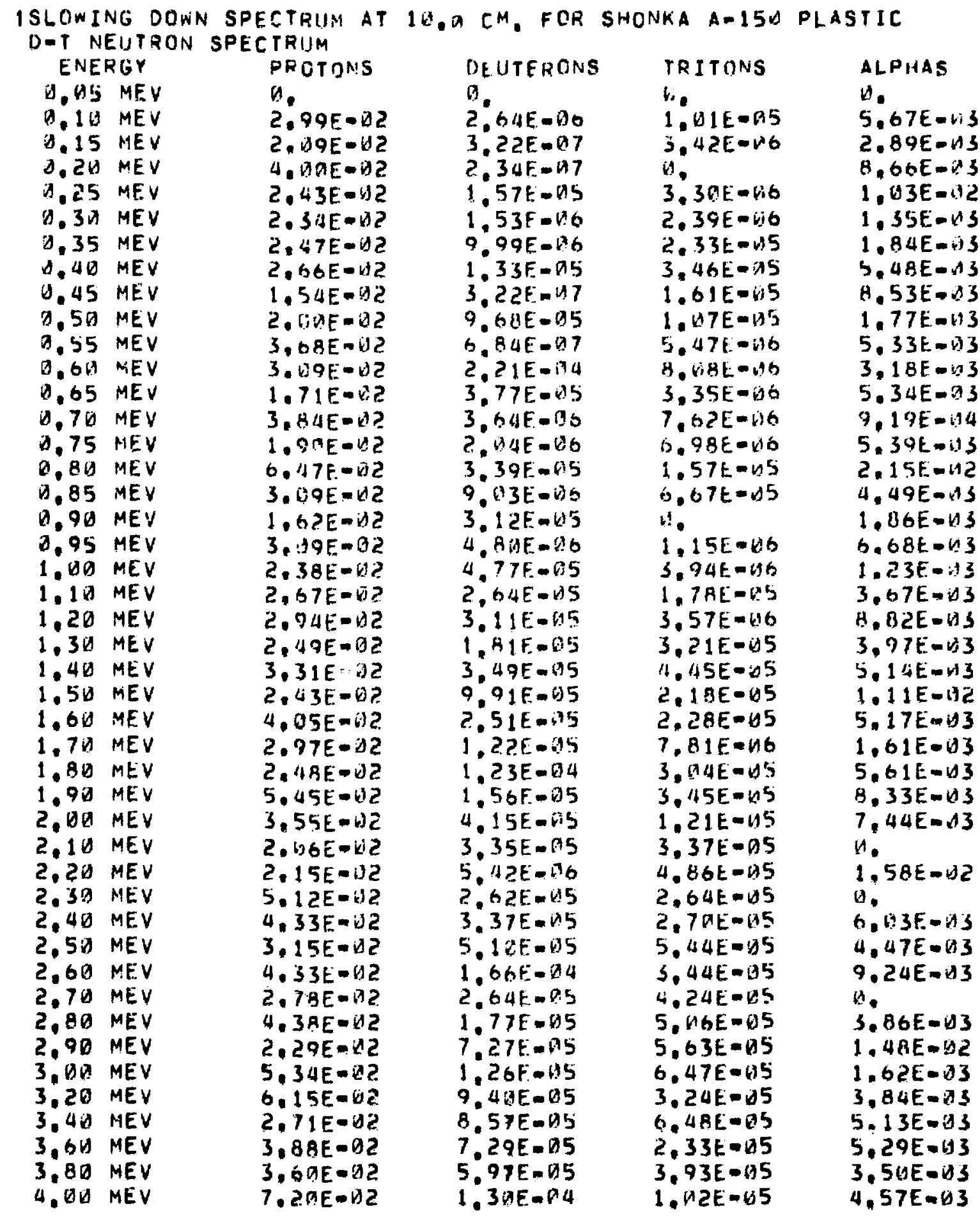




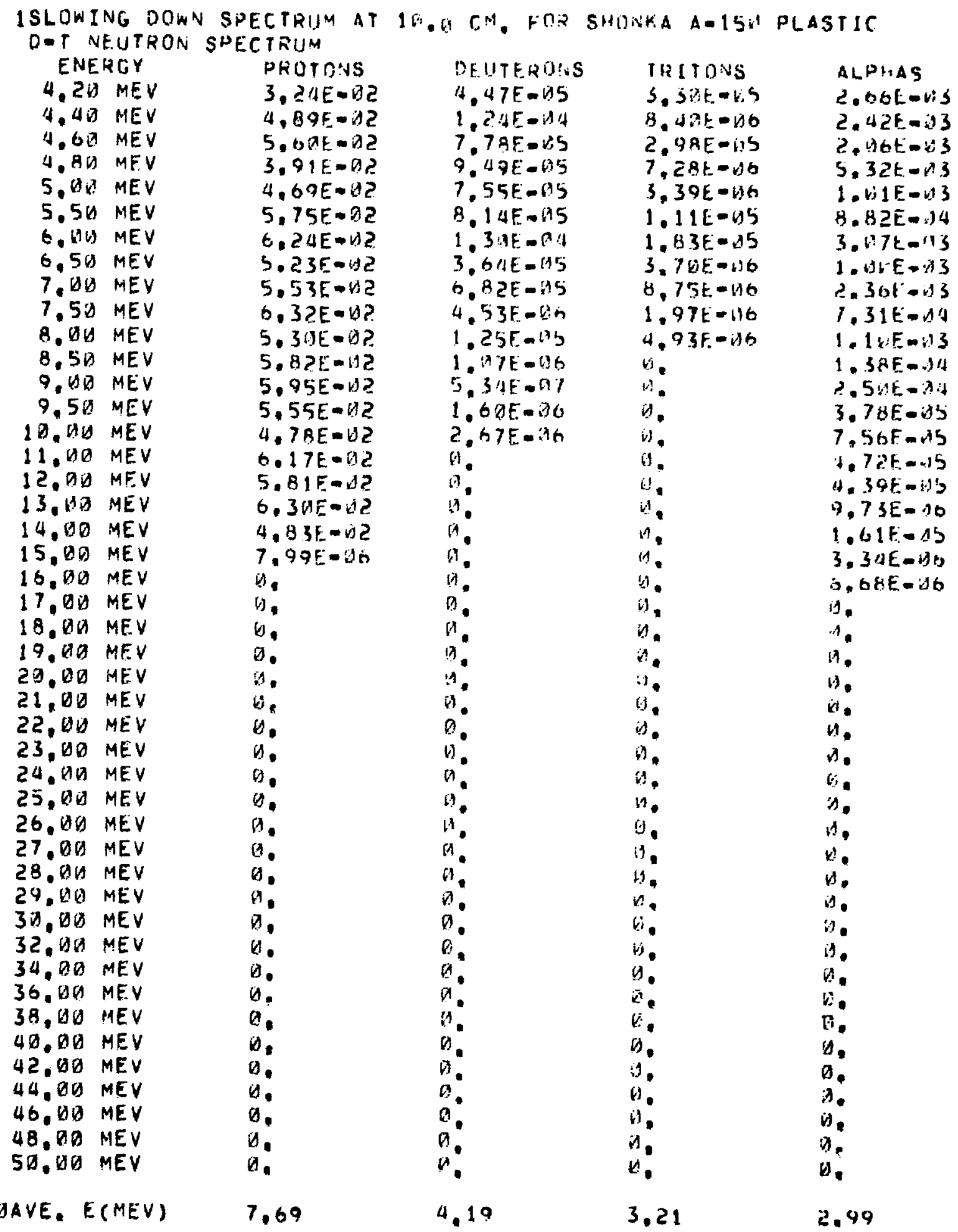




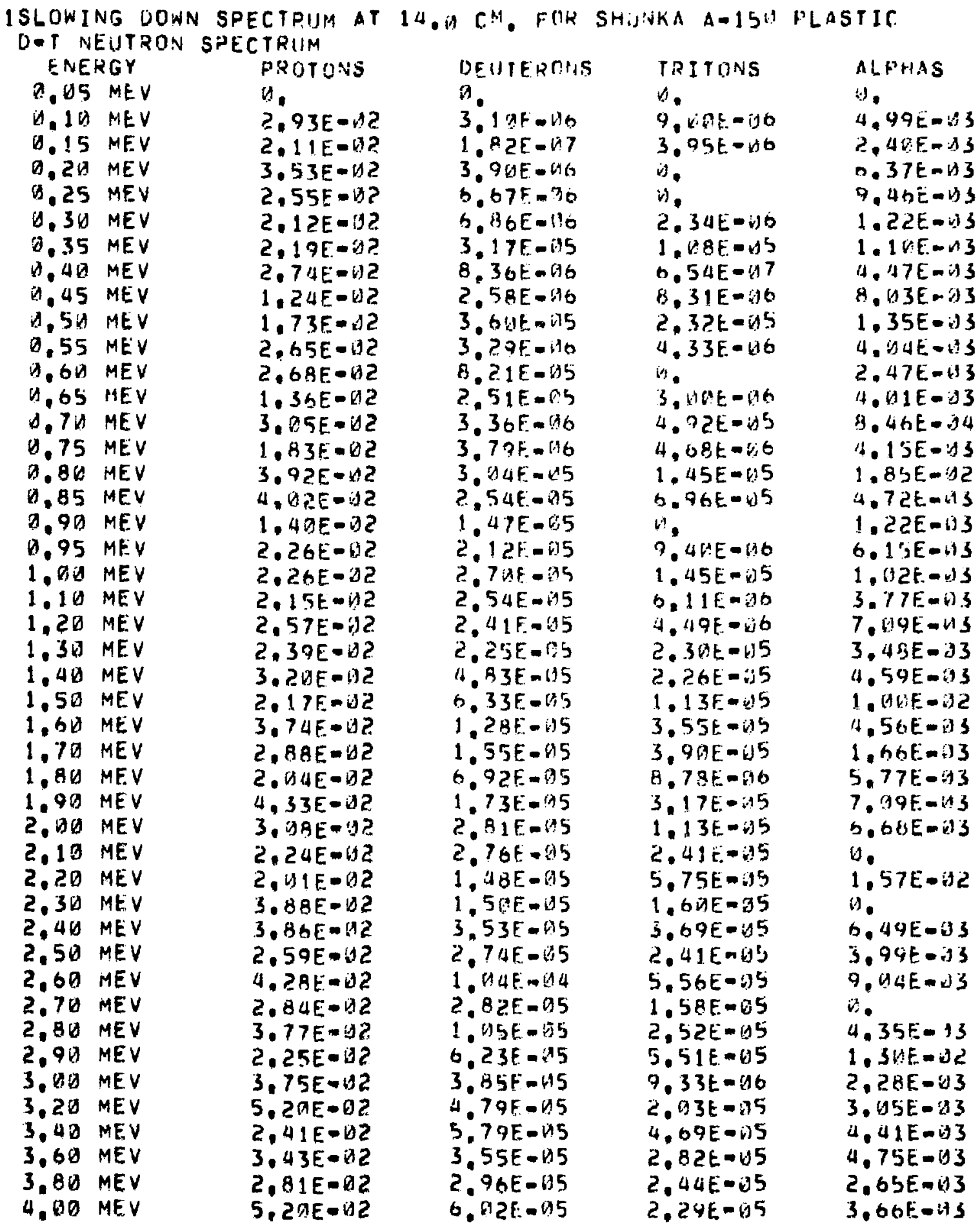




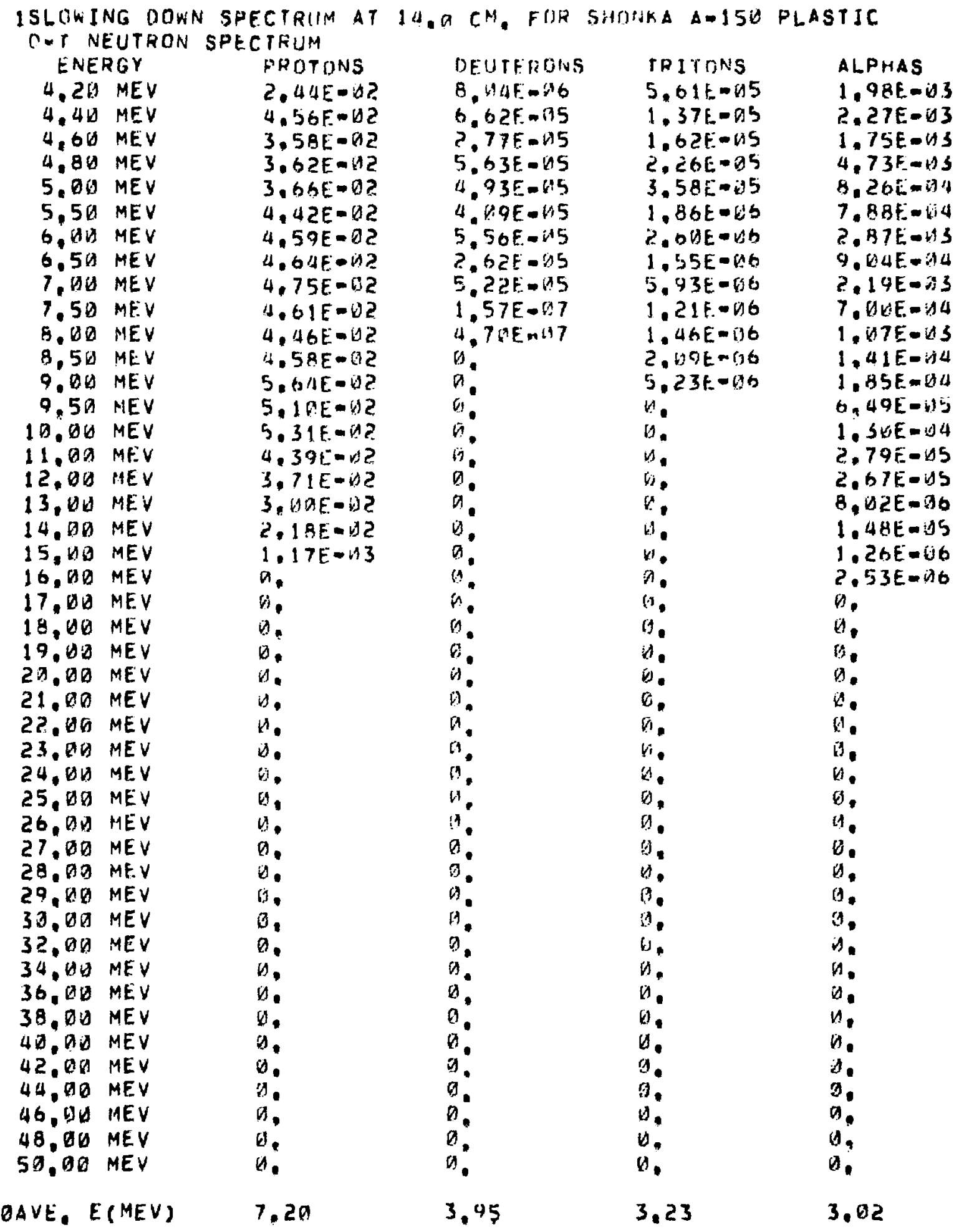


1SLOWING DOWN SPECTRUM AT M. WA CM. FOR IRRU MISCLL TISSUE D-T NEUTRON SPECTRUM

\section{ENERGY}

D. 05 MEV

0.10 MEV

0.15 MEV

$0,20 \mathrm{MEV}$

0.25 MEV

0.3 3 MEV

0.35 MEV

$9.40 \mathrm{MEV}$

$0.45 \mathrm{MEV}$

$0.50 \mathrm{MEV}$

$0.55 \mathrm{MEV}$

$0.60 \mathrm{MEV}$

$0.65 \mathrm{MEV}$

0.70 MEV

$0.75 \mathrm{MEV}$

9.80 MEV

$0.85 \mathrm{MEV}$

$0.90 \mathrm{MEV}$

0.95 MEV

1. DO MEV

$1.10 \mathrm{MEV}$

$1.20 \mathrm{MEV}$

1.30 MEV

1.40 MEV

$1,50 \mathrm{MEV}$

1.60 MEV

1,70 MEV

1.80 MEV

1.94 MEV

2, DO MEV

2,1 D MEV

2.20 MEV

2.31 MEV

2, 4U MEV

2.50 MEV

$2.60 \mathrm{MEV}$

2.7 И MEV

2.81 MEV

2,90 MEV

3.00 MEV

3.20 MEV

3,40 MEV

3.60 MEV

$3.80 \mathrm{MEV}$

4,00 MEV

\section{PROTUNS}

U.

).

$i$

in,

b.

a.

6.

o.

a.

b.

v.

n.

$n$

a.

$n$

$n$.

n.

a.

$\theta$,

a.

$\theta$.

in.

i.

b.

b.

b.

B.

$B$

$\theta$

0.

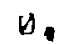

b.

b.

,

0.

D.

a.

1.

$B$.

D.

$n$.

a.

6.

B.

0.

\section{DEUTERONS}

ด.

a.

a.

(1).

n.

.

:

i.

a.

$n$

$\because$

11.

n.

$\theta$

ค.

i.

(4.

n.

$n$

:

:

a.

a.

:

9.

.

:

3

9

$\theta$

0

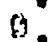

n.

9

a.

0.

a.
TRITONS

n.

n.

6.

M.

,

b.

$n$

a.

$\Delta$

b.

$\theta$.

$n$

in.

k.

a.

is.

b.

0

$\theta$.

a.

$\theta$.

v.

a.

a.

v.

$n$

$\theta$.

r.

i.

I,

$v$

a.

0.

n.

4

di.

B.

$n$

k.

a.

(3.,

$\Delta$.

i.
ALPHIAS

U.

$n$

s.

0

b.

is.

a.

4.

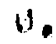

0.

i.

8

in.

H.

$\therefore$

U.

n.

14.

$B$

$\theta$.

a.

i.

$\therefore$

a.

a.

a.

a.

4.

D.

n.

a.

$a$.

D.

ด.

b.

\section{k.}

a.

$B$.

U.

$\theta$.

B.

0.

$\theta$.

$\theta$

$\theta$ 
ISLOWING DOWN SPECTRUM AT M. GM CM, FOR ICRU MUSCLE TISSUF DOT NEUTRON SPECTRUM

\section{ENERGY}

4. 20 MEV

4. 40 MEV

$4,60 \mathrm{MEV}$

4.8D MEV

5. $02 M E V$

$5.50 M E V$

6. 50 MEV

$6.50 \mathrm{MEV}$

7 . BU MEV

$7.50 \mathrm{MEV}$

8. VD MEV

8.5\% MEV

$9.00 \mathrm{MEV}$

$9,50 \mathrm{MEV}$

10. DO MEV

11. DO MEV

12. OU MEV

13. DO MEV

14. US MEV

15.0O MEV

16. OD MEV

17.00 MEV

18.01 MEV

19.00 MEV

$20 . \triangle Q M E V$

21. DU MEV

22. DO MEV

23. OO MEV

$24.00 \mathrm{MEV}$

25, OU MEV

26. HU MEV

27. DU MEV

28. QU MEV

$29 . \triangle D M E V$

$30.00 \mathrm{MEV}$

$32,00 \mathrm{MEV}$

34.DO MEV

$36,00 \mathrm{MEV}$

3B.DQ MEV

40.00 MEV

42.00 MEV

44.01S MEV

46.06 MEV

4B. OD MEV

50, OO MEV

GAVE, E(MEV)

\section{FROTONS}

v.

$y$.

U.

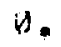

4.

3.

0.

$\theta$

n.

v.

3

a.

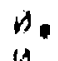

b.

a.

v.

U.

is.

0

,

U.

0

b.

b.

b.

b.

a.

D.

b.

0.

i.

U.

$a$

a.

b.

0

as

a.

0

0

a.

b.
DE UTERONS

a.

a.

a.

7.

a)

$\sqrt{n}$

a.

in.

A.

,

9.

?.

a.

$\nabla$.

a.

is

$\theta$

i.

i.

$\theta$

i.

$\theta$

?.

$\theta$

$\theta$

$\theta$.

$\theta$.

D.

a.

4

n.

$\theta$

0

$\theta$

6.

i)

a.

(3).

a.

0

$a$

a.

0

0

a.
TRITONS

0

b.

i.

$\therefore$

$n$

i.

H.

0.

$\Delta$.

in.

$r$

,

is.

$\theta$

a.

\&?

o.

$k$

4

6.

$n$

V.

n.

is.

a.

w.

6

v.

i.

d.

in,

b.

U.

0.

a.

0

$\theta$

4.

0.

0.

b.

i.

0.

B.

v.

b.
ALPHAS

U.

is.

$d$.

is.

$n$.

is.

H.

b.

$\therefore$

$\Delta$

n.

i.

v.

$n$.

$b$

v.

i.

0.

H.

,

b.

a.

b.

i.

0.

a.

b.

b.

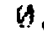

vi.

$\theta$

$\theta$

0.

b.

U.

ט.

D.

L.

B.

B.

b.

D.

0.

D.

v.

0 
1SLOKING DOWN SPECTRUM AT D.05 CM. FOR ICRIJ MUSCLE TISSUE DIT NEUTRON SPECTRUM

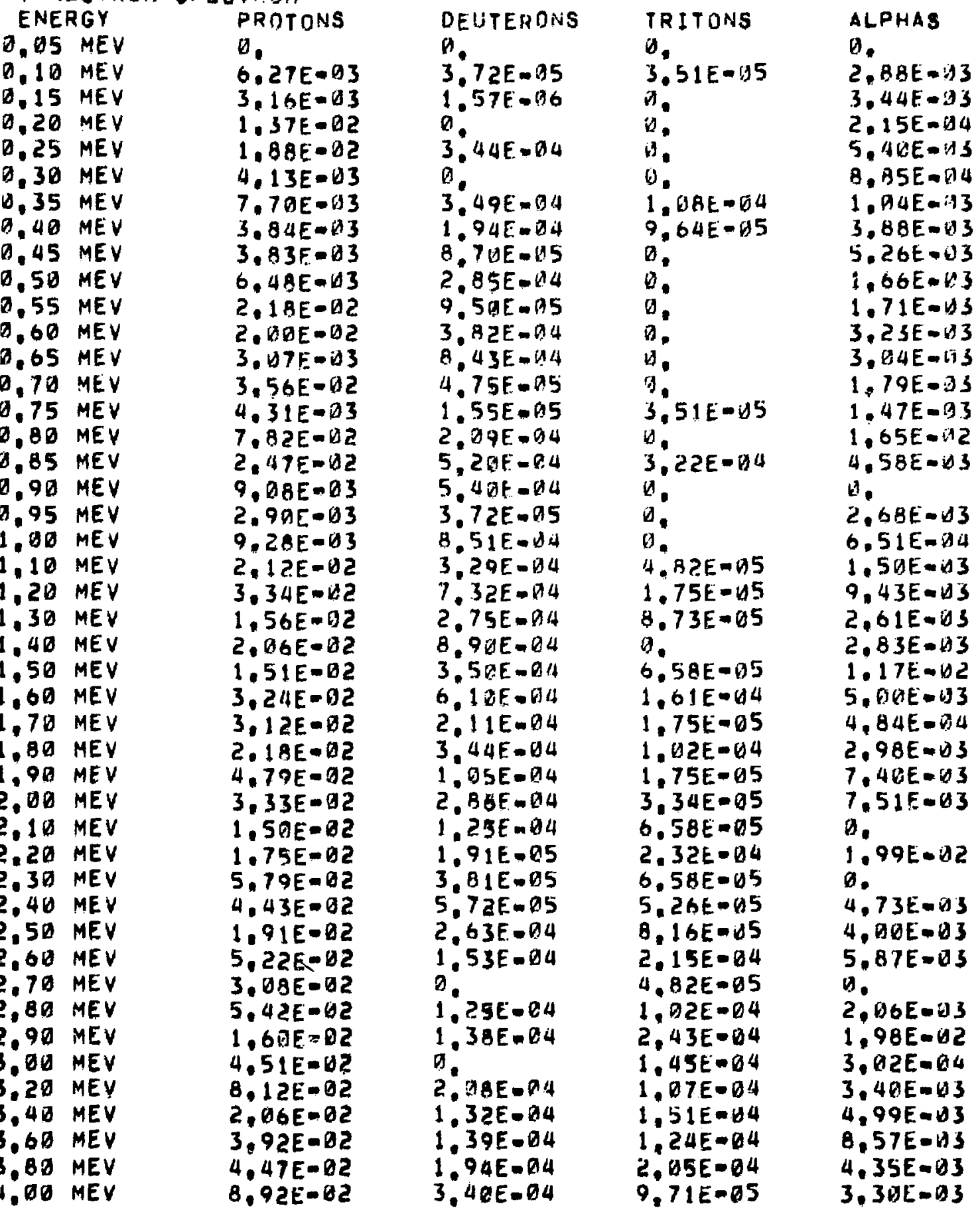




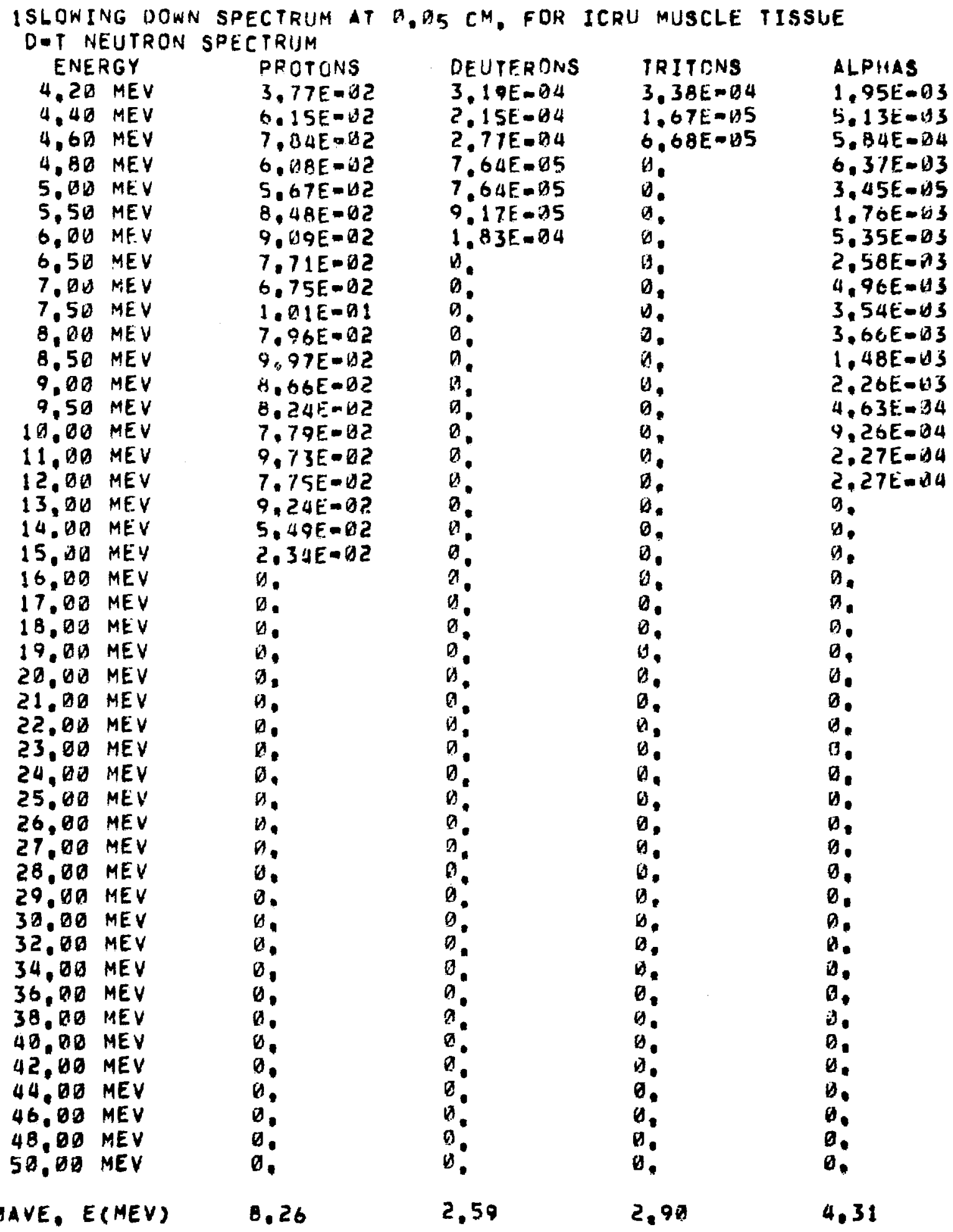




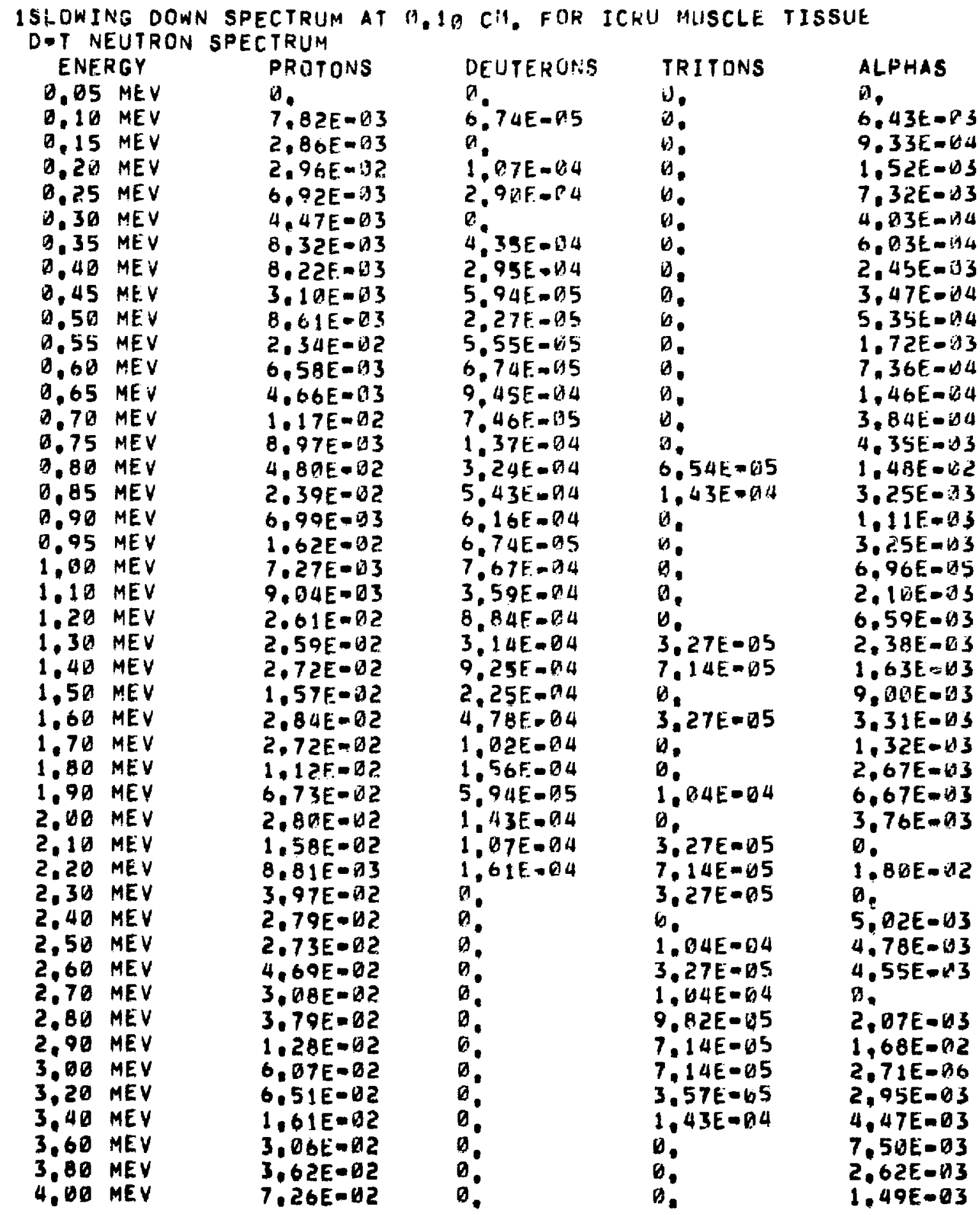




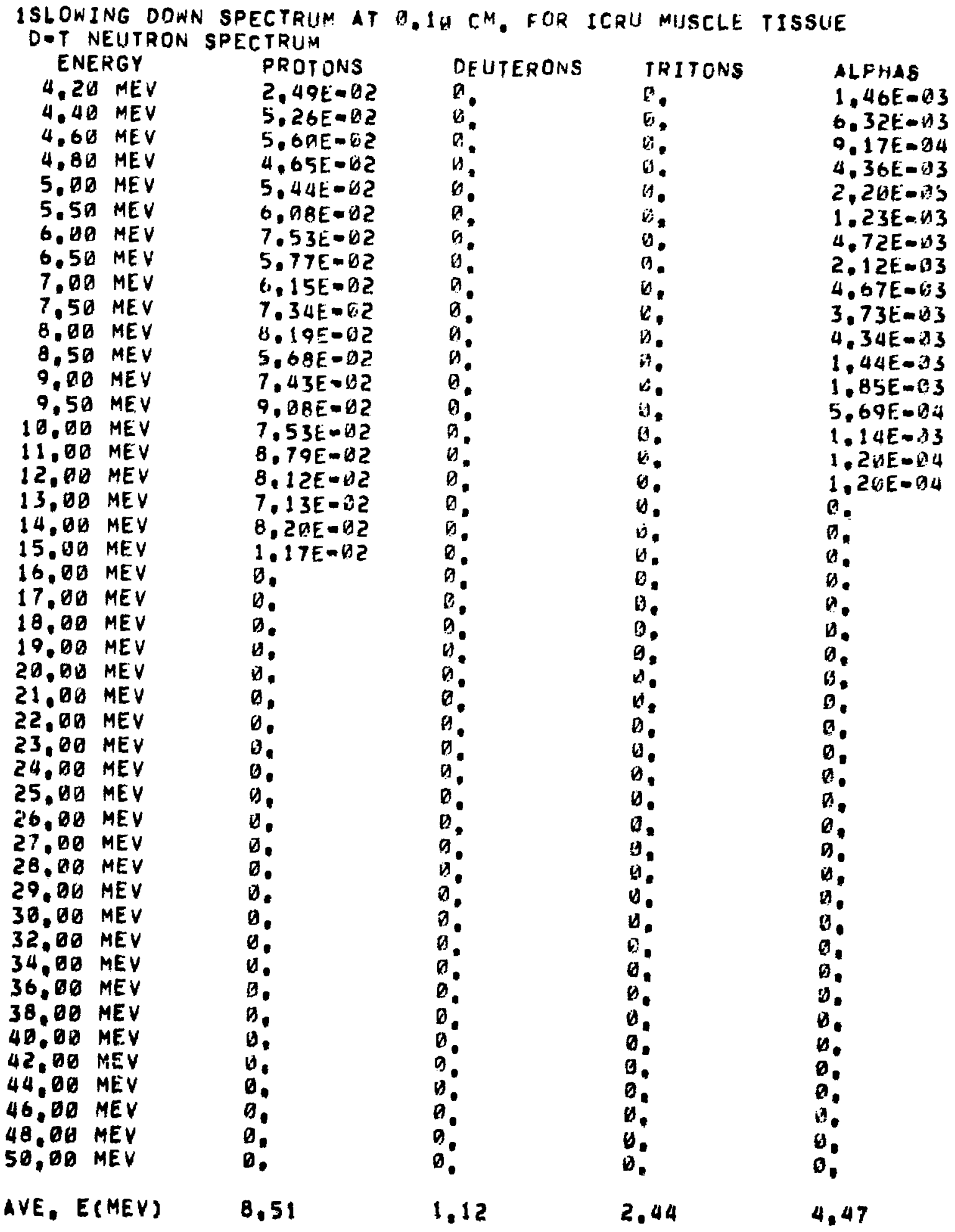


1SLOWING DOWN SPECTRUM AT $9.15 \mathrm{cM}$, FOR ICRU MUSCLE TISSUE DIT NEUTRON SPECTRUM

\section{ENFRGY}

D. U5 MEV

Q. $10 \mathrm{MEV}$

Q.15 MEV

$0.20 \mathrm{MEV}$

D. 25 MEV

Q.30 MEV

$9.35 \mathrm{MEV}$

9,40 MEV

Q, 45 MEV

0.50 MEV

0.55 MEV

0.60 MEV

0.65 NEV

$0.70 \mathrm{MEV}$

$0.75 \mathrm{MEV}$

Q,BD MEV

0.85 MEV

0.90 MEV

0.95 MEV

$1,00 \mathrm{MEV}$

$1.10 \mathrm{MEV}$

$1.20 \mathrm{MEV}$

1,3 औEV

$1.40 \mathrm{MEV}$

$1,50 \mathrm{MEV}$

$1.60 \mathrm{MEV}$

1.70 MEV

$1.80 \mathrm{MEV}$

$1.90 \mathrm{MEV}$

$2.00 \mathrm{MEV}$

2.10 MEV

2.2. MEV

$2.30 \mathrm{MEV}$

2.40 HEV

$2.58 \mathrm{MEV}$

2.60 MEV

2.7 MEV

2,80 MEV

$2.90 \mathrm{MEV}$

3. Q1 MEV

3.20 MEV

3.4D MEV

$3,6 \mathrm{~g}$ MEV

$3,80 \mathrm{MEV}$

4,DO MEV
PRTTOLS

$\theta$.

1. $1 \Delta E-92$

$5.10 E-03$

2. $38 E-U 2$

$9, B 1 E-D 3$

B. $26 E-03$

$5.98 E-33$

$7.16 E-03$

$3.79 E-143$

$9.54 E-\Delta 3$

1.92E-U2

1. $54 E-0$ ?

$3.0 \% E-03$

$1,43 E-92$

$1,18 E-192$

D. $96 E-U$ ?

2. $46 E-152$

$4.46 E-13$

1.2YE-D2

$7.82 E-63$

$1.10 E-\square 2$

$1,43 E-12$

2. 15E-12

$2.6 B E-A 2$

$9 . B B E-B 3$

$1,89 E-02$

$1,82 \mathrm{E}-02$

$1,3 D E-\Delta ?$

$7.92 E=02$

$1.51 F-02$

$1,71 E-02$

$1.21 E-42$

$4.63 E=02$

2,96E-D?

3.13E-02

$2.79 E=02$

$2.25 E-42$

$3.53 E-112$

$1,60 E-02$

$7.55 E-02$

$6 ., B D E-Q 2$

$1.27 E-02$

3. $24 E-02$

$2.64 E-02$

$8,66 E=02$
DEUTERONS

A.

3.23E-AS

$\because$

$1 . \square 7 E-\angle 4$

$2,79 E=H_{4}$

3.

2. AGE-DA

1. BDE-OA

$n$.

9.24E-196

$6,57 E-05$

$4.25 E-A 4$

$6,52 E-0.4$

$3.61 E-65$

$1,19 E-M 4$

$2.58 E-A 4$

2. $71 E-94$

$4.89 E-34$

$3.25 E-115$

7. $42 E-64$

3. DSE-DI

$5.64 E-94$

$1.85 E-94$

$6.64 E-194$

2. $\triangle 2 E-A 4$

$4.75 E-114$

?.82E- -3

3.7. E- (T)

8.

5. $37 E=115$

$3.07 E-D 4$

1. $.1 E-04$

3. $285=0 \mathrm{BS}$

D.

1. $A 7 E=94$

2. $29 E-214$

3. 28E= 05

$1.67 E-D 4$

3. 2 QE-DS

$3.28 E-(15)$

2. 1 UE- 94

1. $49 \mathrm{ED} 94$

$1.825-04$

$1,67 E-04$

$2.65 E=$ is 4
TRITONS

i.

A. 27E-3S

.

b.

iี.

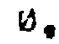

$4,27 E-65$

$9.64 E-05$

0.

2 .

$4.27 E-195$

i.

4. $27 E-U 5$

i.

$4.27 E-45$

$6.51 f .=-15$

1. $22 E-1) 4$

$8.55 E-45$

b.

6.

4.8 ? $E-1) 5$

a.

3. $27 E-115$

3. $96 E-65$

4. $82 E-15$

$3,27 E-65$

$\theta$

$4.82 E=155$

$7.24 E-k 5$

$\theta$.

8. W9E-WS

$3.96 E-155$

8. DQE-25

a.

1. $21 E-34$

$3.27 E-05$

1. $21 E-64$

$1.46 E=04$

$8.79 k-V 5$

$1.84 E-04$

$1.98 E-E 5$

$7.93 E-0.5$

0.

i).

0.
ALPHAS

4.

$0.22 t-13$

$1.29 E-13$

$2.43 E-013$

$1.5 b E-142$

5. $82 E-114$

$1.51 E=03$

$2.83 E-113$

1. $47 E=-12$

1. $29 \mathrm{E}-\mathrm{Di} 3$

5. $199 E-113$

$1,76 t-13$

$5,25 E-113$

9.

$5,36 E-3$

$1.81 E-32$

$6.46 E-33$

3. $34 E-B 4$

7. 3OE- 13

$9.93 E-:^{2} 4$

$2.9,1 E=3$

1. $19 E-V 2$

7.5 EE-US 3

$5.88 E-63$

$1.42 E=12$

$0,47 E-63$

1.55E- -1$) 3$

5.93E-IS 3

1. MOE- IIC

$1,38 E-A 2$

1).

$2,53 E-12$

$\theta$.

$1.03 E-42$

$5,6,7 E-43$

$1,21 E=42$

a.

$8.83 E-(1) 3$

$1.92 E-42$

3. $46 E-$ - 33

3.4 KIE- 13

5.1 1 EEA 3

$7,74 E-A 3$

2.BUE-O3

$1.77 E=03$ 
ISLOWING DOWN SPECTRIIM AT O.1S CH. FOR ICRU :4USCLE TISSUE DET NEUTRON SPECTRIJM

$$
\text { ENERGY }
$$

4.2O MEV

4. $4 \mathrm{~B} M E V$

4.6D MEV

4.87 MEV

5.BO MEV

5. $5 U$ MEV

6. HO MEV

6.50 MEV

7. AO MEV

7 . $D$ MEV

8. DO MEV

8.5D MEV

9. DO MEV

$9.50 \mathrm{MEV}$

$10,0 \mathrm{~V}$ MEV

11. OU MEV

12. DO MEV

$13, D O M E V$

14.00 MEV

$15.60 \mathrm{MEV}$

16. HO MEV

17.00 MEV

18.OO MEV

19.DU MEV

2Q.BU MEV

21. VO MEV

22. Dด MEV

23. DO MEV

24. OO MEY

25. OO MEV

26.00 MEV

$27.0 B$ MEV

2B. AO MEV

29. 10 MEV

30.00 MEV

$32.00 \mathrm{MEV}$

$34.00 \mathrm{MEV}$

36. OU MEV

38.00 HEV

4O. OD MEV

42.0V MEV

44.06 MEV

46.90 MEV

48.00 MEV

50.00 MEV
PROTONS

P. $67 E-0$ ?

$5.23 E=0 ?$

$5,73 E-6 ?$

2. $B B E=\square 2$

$3.63 E=02$

$5.65 E-112$

b. 35E- -12

S. ITE-GZ

6. $1 A E-H 2$

$7.7 a E-\Delta 2$

7.6GE-D2

$6.72 E-92$

$7.97 E-V 2$

8.87E-42

7.2WE-पZ

$7.15 E-O ?$ ?

9.3 $4 E-D 2$

$9.63 E-D 2$

$4.9 D E-62$

b.

1

b.

b.

4

b.

a.

b.

0

0

a

$\theta$

$\theta$.

$\theta$

0.

.

$\theta$.

a.

n.

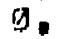

$\theta$

D.

i.

$\theta$

$\theta$.

$\theta$
DEUTERONS

$3.33 E=04$

9. $82 E-05$

4.

9.82F- 05

$9.82 F-A 5$

$1.18 E-B 4$

2. $36 E-W 4$

0

a.

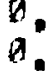

a.

n.

a.

3

:

9

ด.

a.

:

a:

:

a.

a.

a.

a.

:

$a$

$a$

:

is.

is:

v.

a.

:.

:.

0 :

8.72

2.74
TRITUNS

a.

$\theta$

a.

$\theta$.

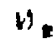

i)

$\theta$.

a.

$\Delta$.

s.

$a$

v.

is.

3

u.

6.

s.

a.

a.

U.

a.

in.

$\because$

v.

a.

0.

H.

B.

$B$,

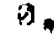

9

$n$

b.

b.

b.

0

13

is.

$\forall$

B.

0.

$B$

$\theta$

b.

2.13
ALPHAS

$1.47 E-U S$

$5.92 E-43$

9.91E-nu

$5.31 f-23$

B. $88 E-145$

$1.44 E-03$

4. $74 E-W 3$

2. $47 E-03$

$4.96 E=13$

$3.53 F=03$

4. $\triangle 2 E-U 3$

$1,36 E-33$

$2.15 E-13$

$3.63 E-64$

$7.27 E=114$

1. $67 E-14$

$1.67 E A 14$

a.

$n$

g.

n.

is.

$\theta$

$\theta$.

9.

4.

3.

A.

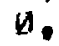

b.

b.

.

o.

0.

$\theta$.

a.

0.

0

,

i.

$D$.

.

a.

D.

D.

3.77 


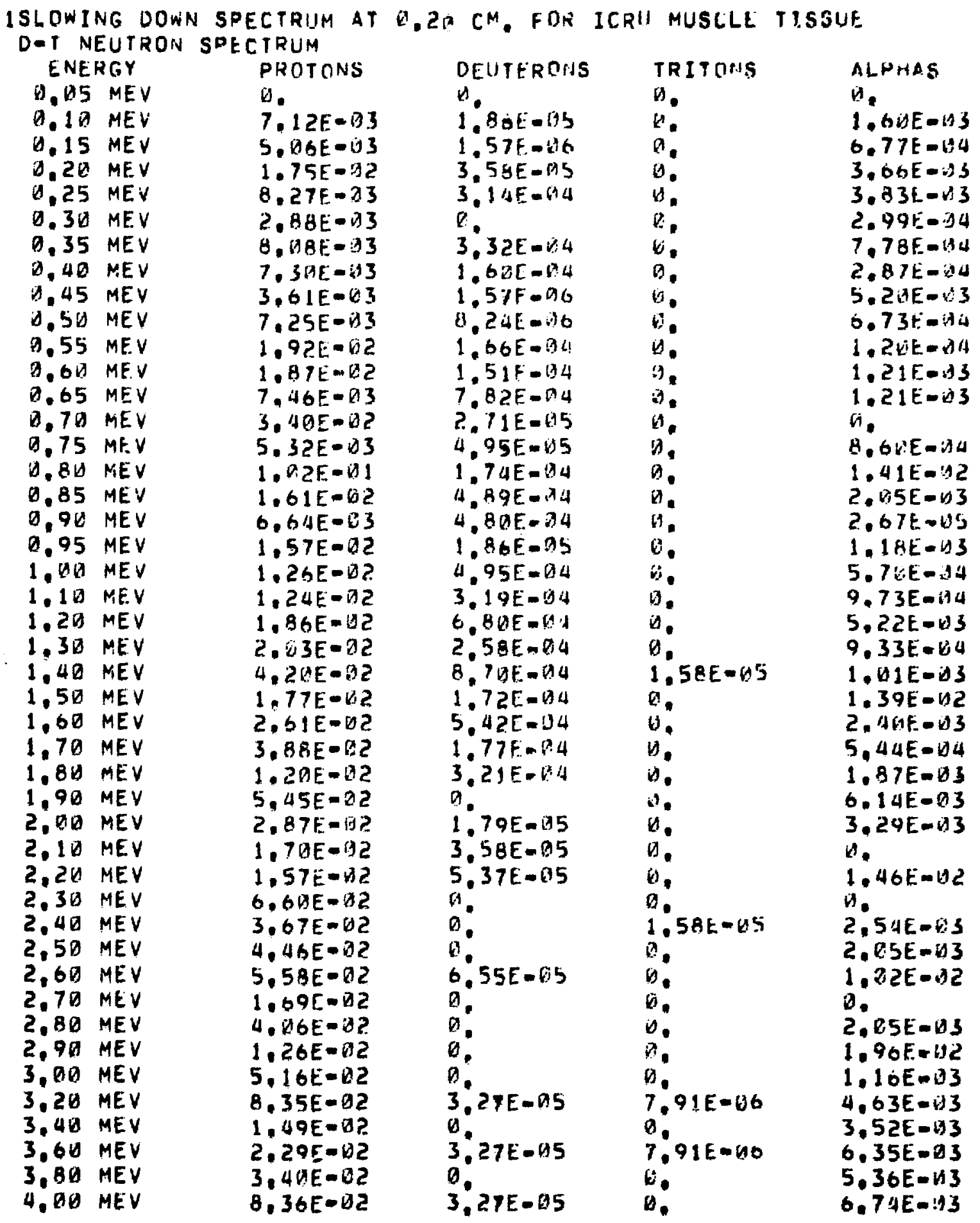




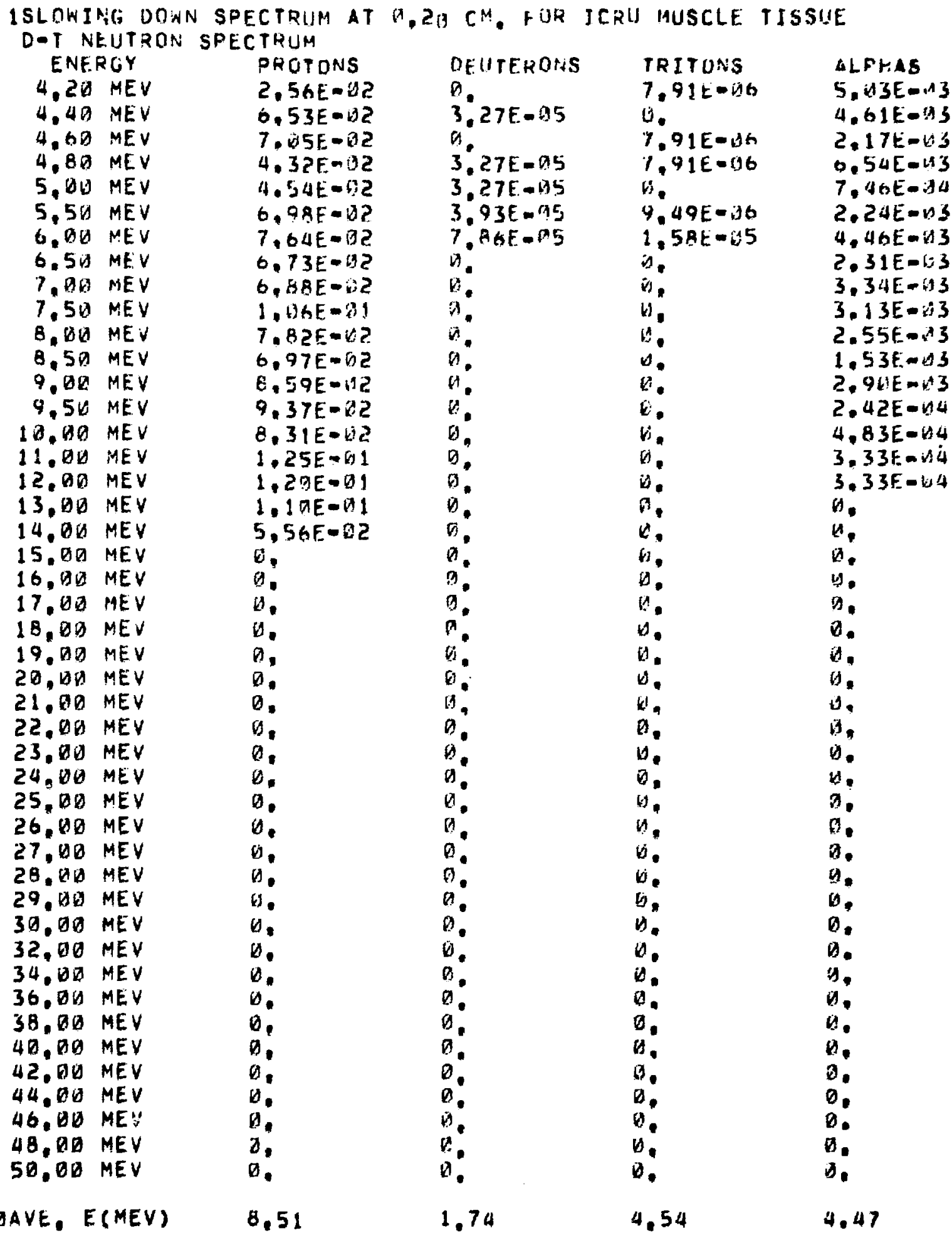




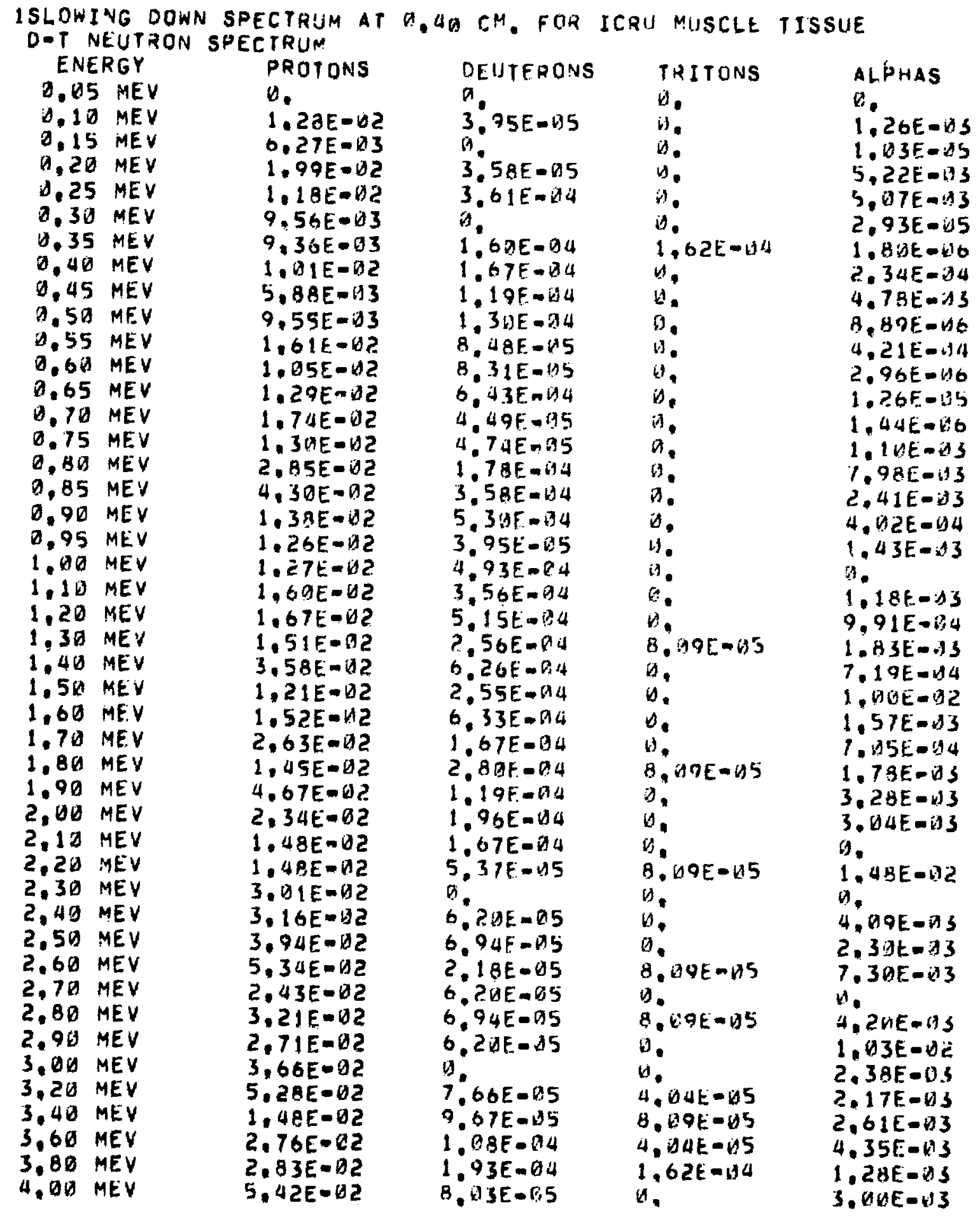


ISLOWING DOWN SPECTRUM AT G, UU CM. FOR ICRU MUSCLE TISSUE DUT NEUTREN SPECTRUM

ENERGY

4.20 MEV

4.40 MEV

4.60 MEV

4.80 MEV

5,00 MEV

5,50 MEV

$6.00 \mathrm{MEV}$

6.50 MEV

7. DQ MEV

7.50 MEV

B. 10 MEV

$8,50 \mathrm{MEV}$

$9.06 \mathrm{MEV}$

$9,50 \mathrm{MEV}$

10,00 MEV

11.00 MEV

$12,00 \mathrm{MEV}$

13.00 MEV

14,06 MEV

$15.00 \mathrm{MEV}$

$16.0 B$ MEV

17.00 MEV

18.00 MEV

$19.00 \mathrm{MEV}$

20.00 MEV

21. DO MEV

22. DO MEV

23. $U D$ MEV

24. OO MEV

$25.00 \mathrm{MEV}$

$26.00 \mathrm{MEV}$

$27.00 \mathrm{MEV}$

$28.00 \mathrm{MEV}$

$29,00 \mathrm{MEV}$

30,OW MEV

32,01 MEV

34.UV MEV

36.00 MEV

$38.00 \mathrm{MEV}$

$40.00 \mathrm{MEV}$

$42.01 \mathrm{MEV}$

44.00 MEV

$46.00 \mathrm{MEV}$

48.00 MEV

$50.00 \mathrm{MEV}$

DAVE, E(MEV)
PROTONS

$1.64 E-62$

$3.93 E-62$

$2.43 E-02$

2.8.3E-0́2

$3.36 F=02$

$3.74 E-1 D 2$

$3.87 E=12$

$3.92 E-02$

$3.68 E=02$

$4.16 E-62$

$4.93 E-6 ?$

$3.99 \mathrm{E}-02$

$4,87 E=02$

$6.45 E=02$

$7.99 E-02$

$6.5 B E-\triangle 2$

$6.51 E=02$

$3,21 \mathrm{E}-02$

$3,34 E=02$

0.

$\theta$

b.

0.

i.

7.

n.

0.

a.

0.

$\theta$.

Q.

t.

$b$.

b.

$v$.

0

$\theta$.

0

0

i.

is.

$\Delta$

b.

0.

0.

B. 0?
DEUTERDNS

1. 39E-AI

1. DOE-OS

$\theta$

1. 99E-05

1. $D 9 E-0.5$

1. $31 E-05$

2. U2E- U5

$a$

a.

0

a.

vis.

D.

b)

9

(1)

$a$

ด

0

0

0.

a.

a.

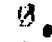

a.

a.

a

$a$

i)

$n$

a.

a.

$b$

a.

$\theta$

0

$b$

a.

0.

1,87
TRITUNS

G.

0.

0.

D.

it.

D.

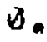

$\theta$

b.

b.

is.

d.

$\theta$

b.

6.

$a$

H.

0

$c$

v.

0.

U.

b.

u.

a.

b.

bs

n.

0

b.

b.

is

6.

ה.

b.

0.

b.

0

$\theta$

k.

0

b.

G.

2.77
ALPHAS

$1.5 B E=15$

4. $) B E=W 3$

1. 6BE- $\triangle 3$

$5,15 E-113$

$1,2 A E-B 3$

1. $31 E-\Delta 3$

$3,51 E=13$

2. $05 E-13$

$3,55 E-13$

3. () $4 E-1) 3$

2. 8 BE -15

$1.38 E-b 3$

2., $21 E-d 3$

$3.87 E-04$

$7.75 E-14$

2. $13 E-134$

$2,13 E-1) 4$

a.

i.

ts.

a.

$\theta$

,

v.

$B$.

$\theta$

a.

4

$\theta$

$\theta$

$n$

$\theta$

0.

a.

$\Delta$.

$\theta$

a.

0

0

(S)

$\theta$.

a.

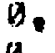

0

e.

4.71 
ISLOWING DOWN SPECTRUM AT O. GG CM. FOR ICRU MUSCI.E TISSUE. DOT NEUTRON SPECTRUM!

\section{ENERGY}

0.05 MEV

0.10 MEV

$0.15 \mathrm{MEV}$

D. 20 MEV

0.25 MEV

$0.30 \mathrm{MEV}$

0.35 MEV

0.40 MEV

0.45 MEV

0.50 MEV

$0.55 \mathrm{MEV}$

0.60 MEV

$0.65 \mathrm{MEV}$

$0.70 \mathrm{MEV}$

0.75 MEV

0.80 MEV

0.85 MEV

0.90 MEV

0.95 MEV

1. DO MEV

1.10 MEV

1,20 MEV

$1,30 \mathrm{MEV}$

1.40 MEV

$1.50 \mathrm{MEV}$

$1.60 \mathrm{MEV}$

1.70 MEV

1,80 MEV

1.90 MEV

2. $\triangle Q$ MEV

2.10 MEV

2.20 MEV

2. 30 MEV

2. 40 MEV

2.50 MEV

2.60 MEV

$2.70 \mathrm{MEV}$

2,8D MEV

$2.96 \mathrm{MEV}$

3.DO MEV

3.20 MEV

3.40 MEV

3.60 MEV

3.80 MEV

$4.00 \mathrm{MEV}$
PKOTONS

0.

$1.33 E-42$

$7 ., 2 E-D 3$

2. $47 E-D 2$

$1.73 E-\triangle 2$

1. $21 E-1.2$

1.21E-a2

1. $36 E-k 2$

8. 33E-13

1. $27 \mathrm{E}=02$

2. $19 E-B 2$

2. $23 E-D 2$

$9.41 E-113$

$2.91 \mathrm{E}-102$

1. $17 E-D 2$

7. $02 E-14$ ?

3. 3AE-62

1. $\triangle 1 E-B 2$

$1.64 E-1) 2$

1. $72 E-D 2$

2. $03 E-112$

$2,31 E=132$

2. $47 E-D 2$

$3.95 E-02$

$1.75 E-D$ ?

$3.19 E=\square$ ?

$3.14 E-02$

$1.61 E-1) 2$

$5.3 A E-32$

$2.75 E=12$

2. $96 E-9$ ?

$1.62 E-02$

$5.23 E-02$

$3,62 E-U 2$

$3.66 E-4 ?$

4.65E-D2

$2.91 E-02$

$3.66 E-02$

1. B IE-D2

4. 35E- 12

$7.53 E-102$

2. 1 DE- 12

3. $73 E=42$

3. $21 E-B 2$

$7.76 E-02$
DELITERONS

a.

$2.65 E-05$

.

1.99F-15

$2,56 t=04$

7

2. $44 E-04$

$1.74 E-24$

$5.35 E-95$

1. $\triangle 3 E-D 4$

$1.19 \mathrm{E}-\mathrm{AL}$

1. $84 \mathrm{~F}-\mathrm{A} 4$

6. $52 E-0.4$

$3,19 E-0.5$

$4.27 E-25$

$1.96 F=614$

$4.11 E=04$

4. $53 E-04$

$2.65 E-135$

$4.49 E-04$

2.9 VE - D 14

$5.70 E-04$

$2.295-14$

$6.47 E-D 4$

$2.16 E=04$

$4.59 E-94$

1. 38E-W 4

$3.13 E-A 4$

$5.35 E-115$

1.17E-O4

$5.53 E-25$

3. $50 E-05$

5.13E- 06

$2.15 E-0.5$

$4 . D 5 E-A S$

1.11E-DA

$3.18 E-1) 5$

2.93E-05

$4.31 E=05$

a.

$6.73 E-05$

$5.28 E-135$

6.7.E- .55

$6.77 E-15$

8.83E-OS
TRITONS

A.

u.

6.

$\theta$.

$B$.

$\theta$.

1. DGE $=(1) 4$

1. $3 B E=05$

$1.59 E-136$

2. $13 E-05$

$\theta$

9.

b.

b.

b.

1.99E-G 5

0.

$\theta$.

$6.59 E=186$

1. $48 E-95$

$7.69 E-196$

v.

$6.61 E=05$

7.3 BE-D6

1. 1 DE- 15

$9.96 E=06$

1. $15 E=05$

$6.21 E=05$

$1.93 E-05$

$3.29 E-166$

2.67E- 05

6. $27 F-35$

$2.34 E-B 5$

$7.38 E-66$

2. $34 E-U 5$

B. $41 E-05$

$1.61 E=05$

8.72E- 35

$6.89 E-26$

2. Q7E-D5

2. $64 E-65$

5. ?. BE- 15

2.64E-105

1. Q6E 04

4.
ALPIIAS

U.

2. $\triangle 4 E=D 3$

1. $24 E-B 3$

$4,2 B E-3$

$3, B 7 E=3$

4.6BE-OA

7. $23 E=\square 4$

1.9BE-D3

4. $D B E=\square 3$

$7.29 E=144$

$1.27 E-03$

1. 5UE 143

$9.59 E-B D$

4. VZE-CA

$1.14 E-13$

9. 1 UE-US

$2,56 E=03$

$4.81 E=04$

$1.34 E-133$

7. 3OE-U 4

1. $14 E-B 3$

3. 1 UE $=13$

1. $45 E=3$

$8.45 E-64$

1. $14 E-42$

$1.66 E-U 3$

2. 7 OE-BA

$1.13 E-G 3$

$3.92 E-103$

$2,57 E-\square 3$

0.

$1 . \square 2 E=\Delta 2$

0.

$2.64 E-103$

1. $94 E-103$

$7,42 E-\triangle 3$

$\theta$.

$2,38 E-133$

1. $21 E-A 2$

$1,32 E-03$

$3.16 E-03$

$2.63 E=113$

$5.42 E-B 3$

$3.13 E=03$

3.81E-U3 


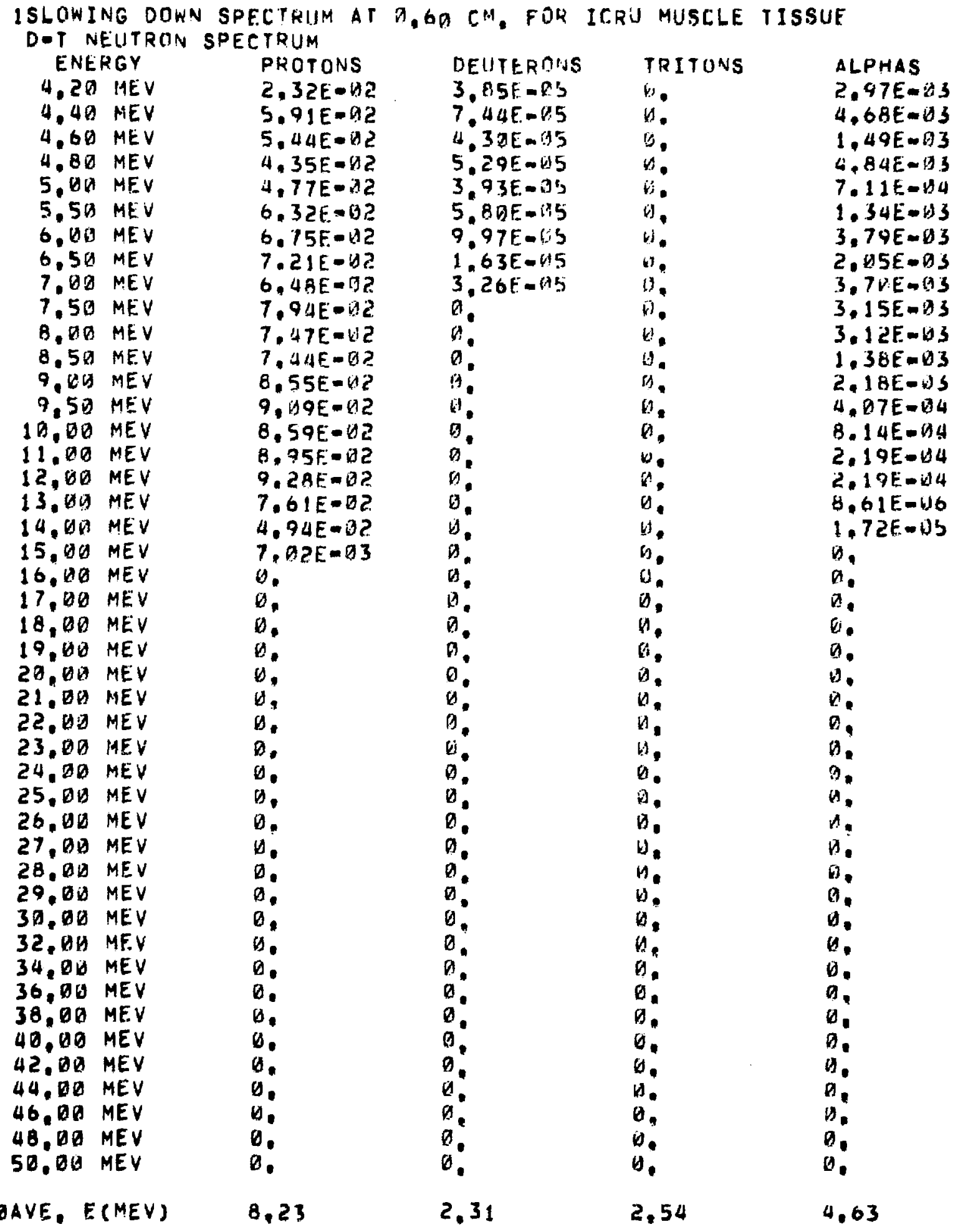




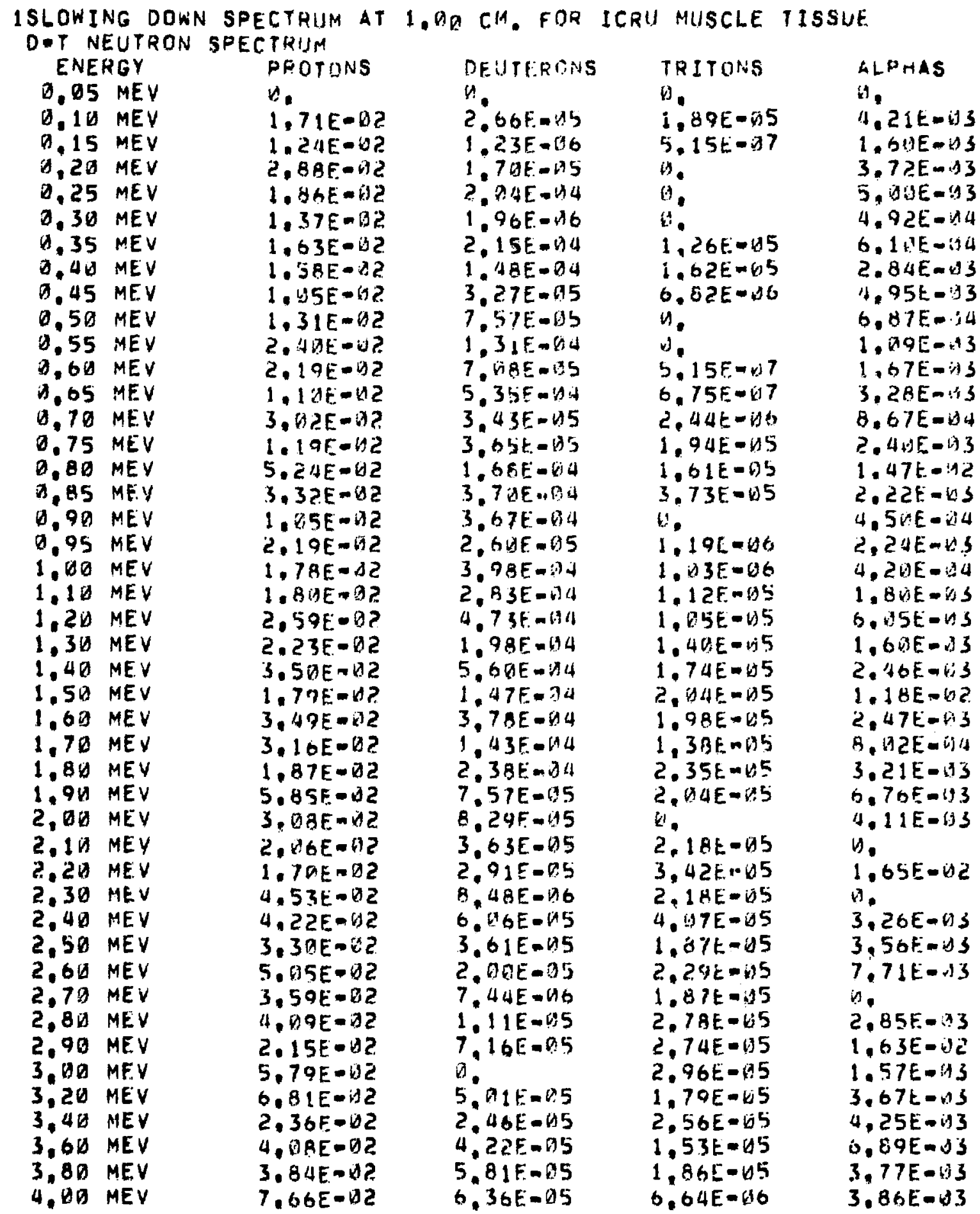


ISLOWING DOWN SPECTRIIM AT 1.DA CH. FOR ITRU MUSCLE IISSIJE. D-T NEUTRON SPECTRUM.

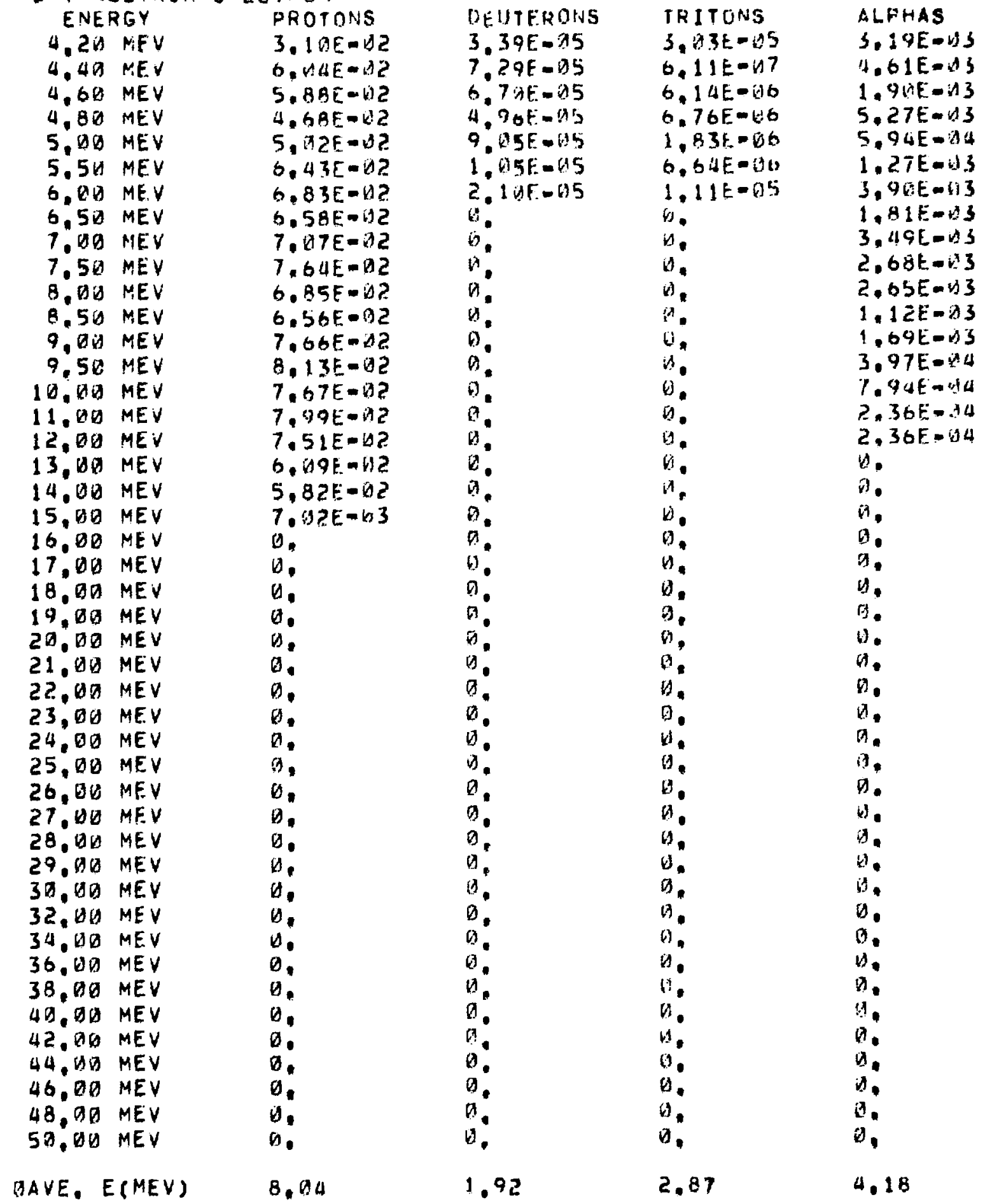


ISLOWING DOWN SPECTRIIM AT 2 , HO CM, FOR ICRU MUSCLE TISSUF.

D-T NEUTRON SPECTRUM

ENERGY

D. O5 MEV

D. 10 MEV

D. 15 MEV

0.20 MEV

0.25 MEV

B. 3D MEV

$0.35 \mathrm{MEV}$

$0.40 \mathrm{MEV}$

0.45 MEV

0.50 MEV

0.55 MEV

0.60 ME.V

$0.65 \mathrm{MEV}$

$0.70 \mathrm{MEV}$

0.75 MEV

$0.80 \mathrm{MEV}$

0.85 MEV

0.90 MEV

0,95 NIEV

1,00 MEV

1.10 MEV

1.20 MEV

$1.30 \mathrm{MEV}$

$1.40 \mathrm{MEV}$

$1.50 \mathrm{MEV}$

$1.60 \mathrm{MEV}$

$1.70 \mathrm{MEV}$

1.BO MEV

$1.90 \mathrm{MEV}$

2. $O B$ MEV

$2.10 \mathrm{MEV}$

$2.20 \mathrm{MEV}$

2. 30 MEV

$2.40 \mathrm{MEV}$

$2.50 \mathrm{MEV}$

$2.60 \mathrm{MEV}$

$2.70 \mathrm{MEV}$

2.8U MEV

2.90 MEV

3.00 MEV

3.20 MEV

3. 40 MEV

3.60 MEV

$3,80 \mathrm{MEV}$

4.DO MEV

\section{PROTONS}

O.

$2.05 F-132$

1. $52 E-42$

$2.75 E-02$

2. $17 E-02$

$1.45 F-32$

$1.68 E-12$

$2.26 E-32$

1. $) 5 E-112$

1. $54 E-132$

$2.75 F .02$

$2.56 E-6) ?$

1.19E $\rightarrow 2$ ?

3. $11 E-b 2$

$1.8) F-42$

6. $26 E=12$ ?

$2.32 E-A ?$

$1.5 \Delta F=02$

$2.11 E=\Delta 2$

$2.19 E-112$

2. $16 E-62$

$2.59 E-62$

$2,62 E-12$

3. $8 ?[-02$

2. U1E-A2

$3.9 P, E-3 ?$

$3.3 \cap E-V I C$

$2.13 F-2$ ?

5. $2 O E-62$

$3.34 E-132$

2. $66 E-112$

7. $33 E-62$

$5.31 E=02$

$4.4 A E-W 2$

$3.67 E-02$

4. $46 E-62$

3. $59 E-02$

4. $1 \mathrm{BE}-02$

$2.46 E-\Delta 2$

$5,19 E-122$

$6.72 E-132$

$2.87 E-02$

$3.67 E-D 2$

3. 31E-102

$7,16 E-02$
DEUTERORS

Q.

$2.57 F-B b$

1. $27 E-5,6$

$7.16 E=116$

$1.81 \mathrm{E}=\mathrm{B}$ is

a.

2. $14 E-114$

1. $35 E-34$

4. $356-65$

1. A $3 E$ - И 4

1. $15 E-134$

2. 3UE - A 4

4. $925-64$

3. $48 E-1 S$

2. $77 E-15$

1. $58 E-14$

3. $53 E-14$

$3.30 E-04$

3. DQF -15

$4.29 E-\square 4$

2. $45 F-174$

$4.35 E-G 4$

1. $95 E-14$

5. $23 E-14$

2. $2 B E=F 4$

$3.3 D E=014$

1. $55 E-04$

2. $79 E-A \triangle A$

7. 1OE-D 5

9. 9VE-AS

6. $40 E-015$

1. $53 E-65$

$3.44 E-135$

5. ?.2E- 15

$6.92 E-05$

$1.59 E-64$

2. $\triangle \in E=05$

4. 15E-O5

7.79E-05

$9.07 \mathrm{E}-06$

$9.12 E-95$

$1.11 E-D 4$

8.4TE-DS

$9.98 E-105$

$1.59 E-04$
TRITONS

S.

7. DIE-DO

$1.03 E=16$

Q.

औ.

5. $12 E-016$

5. 39E- -5

2. $\triangle D E-\triangle 5$

$\theta$

$2.43 E=05$

6.

4.

$5.12 E-46$

1.03E- 36

7. O1E-DO

1. B3E-US

1. $21 E-1 B 4$

b.

5. $49 E-116$

1. $89 E-105$

1. $36 E=05$

6. $171 E-46$

$5,31 E=05$

$5.32 E-35$

1.72E- 65

3. 1 JE- 5

$1.62 E-45$

3. BQE-DS

6. $21 E-V S$

$1.54 E-65$

$3,53 E-15$

$9.65 E-105$

3. 26E- 155

$1.99 E-115$

$7.69 E-65$

$8.55 E-05$

$5.64 E-05$

$6.40 E-05$

$7.95 E-15$

$6.82 E-65$

$4.24 E=65$

1. $17 E-04$

2. $96 E-65$

7. DOE- 5

$1.61 E-45$
ALPHAS

4.

$2.77 E-.35$

$9.41 E-14$

$3,43 E-A S$

4. $B Q E=-133$

$4.32 E-B 4$

a. $3 B E-34$

1. $82 E-\square 3$

$3.39 E-33$

$5.99 E-34$

1. $56 E=\mathrm{ub}^{\mathrm{s}} \mathrm{s}$

1. $19 E-W 3$

$1.78 E-13$

$2.85 E-174$

1. $27 \mathrm{E}=133$

1. $48 E-112$

2.17E- 15

4. $12 E-$ iJ 4

$2.27 E-113$

$0.41 E-64$

$1,5 \mathrm{BE}=\mathrm{AS}$

$4,17 E-1 \mathrm{~S}$

$1.95 \mathrm{E}$. 3

$1,83 E-B 3$

$9.63 E-E 3$

1. $56 E-13$

$5.63 E-04$

$2.24 E-63$

$6.13 E=163$

$3.2 D E-O B$

i).

1. $4 \Delta E-i t 2$

i).

$3.13 E-1,3$

$3,87 F-03$

$6.65 E-03$

0.

2. $52 E-193$

$1.47 E-132$

1. $1.4 E=33$

$3,51 E-13$

$3,97 E-13$

$5,94 E-33$

$3.00 E-U 3$

3. $59 E-O S$ 
1SLOWING DOWN SPECTRUM AT 2,09 C.M. FOR ICRU MUSCLE TISSUE DET NEUTRON SPECTRUM

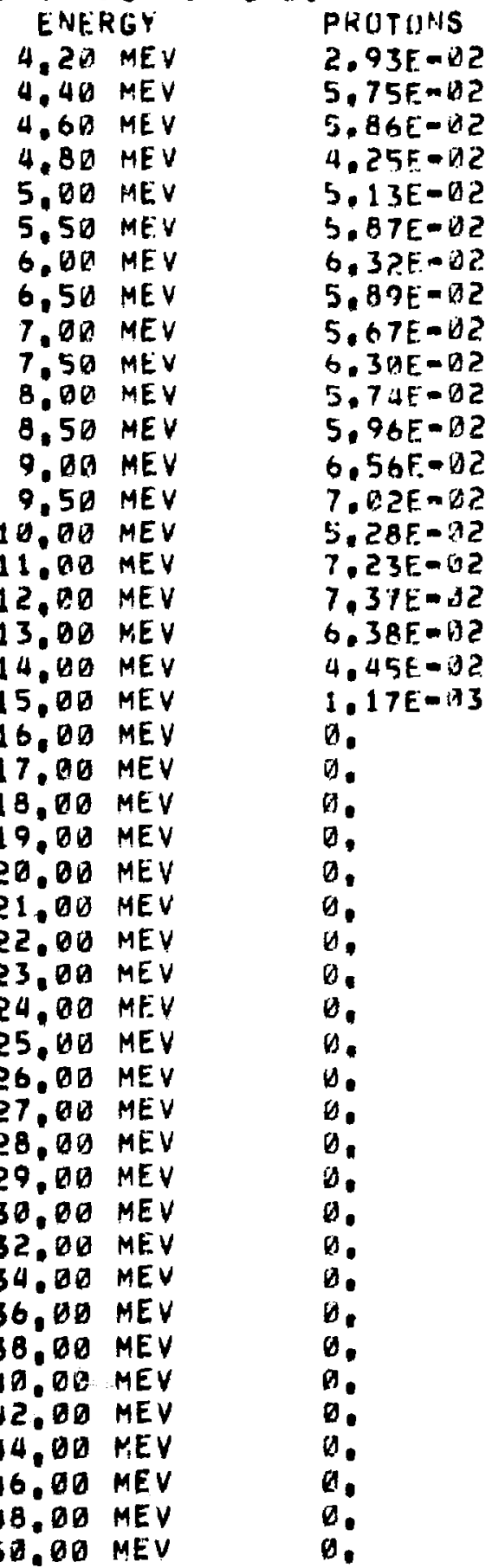
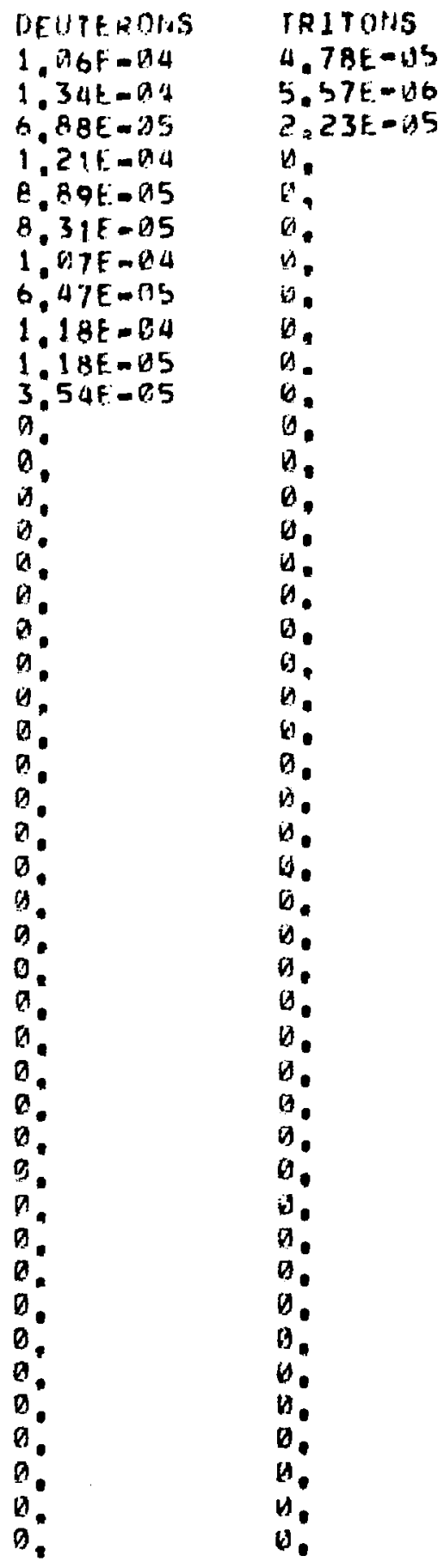

ALPHAS

$4.11 E-35$

1. $56 E=113$

$5.159 E-1 B S$

$6.05 E-24$

$1.22 E-113$

$3.82 E-03$

$1.63 E-D .3$

$3.23 E-113$

2. $\rightarrow 1 E-B 3$

$2.53 E-33$

$1 . U \cap E-03$

$1,55 E-D 3$

$4,41 E-04$

$8.83 E-14$

$2.47 E=34$

$2.47 E-104$

0.

b.

(1).

b.

4.

$\theta$

n.

0.

0.

a.

$\theta$.

a.

H.

0.

$\theta$.

0.

a.

D.

9.

a.

b.

b.

0.

D.

0.

0.

D.

$\theta$

GAVE. E(MEV)

7.84

3,13

2.63

4.34 


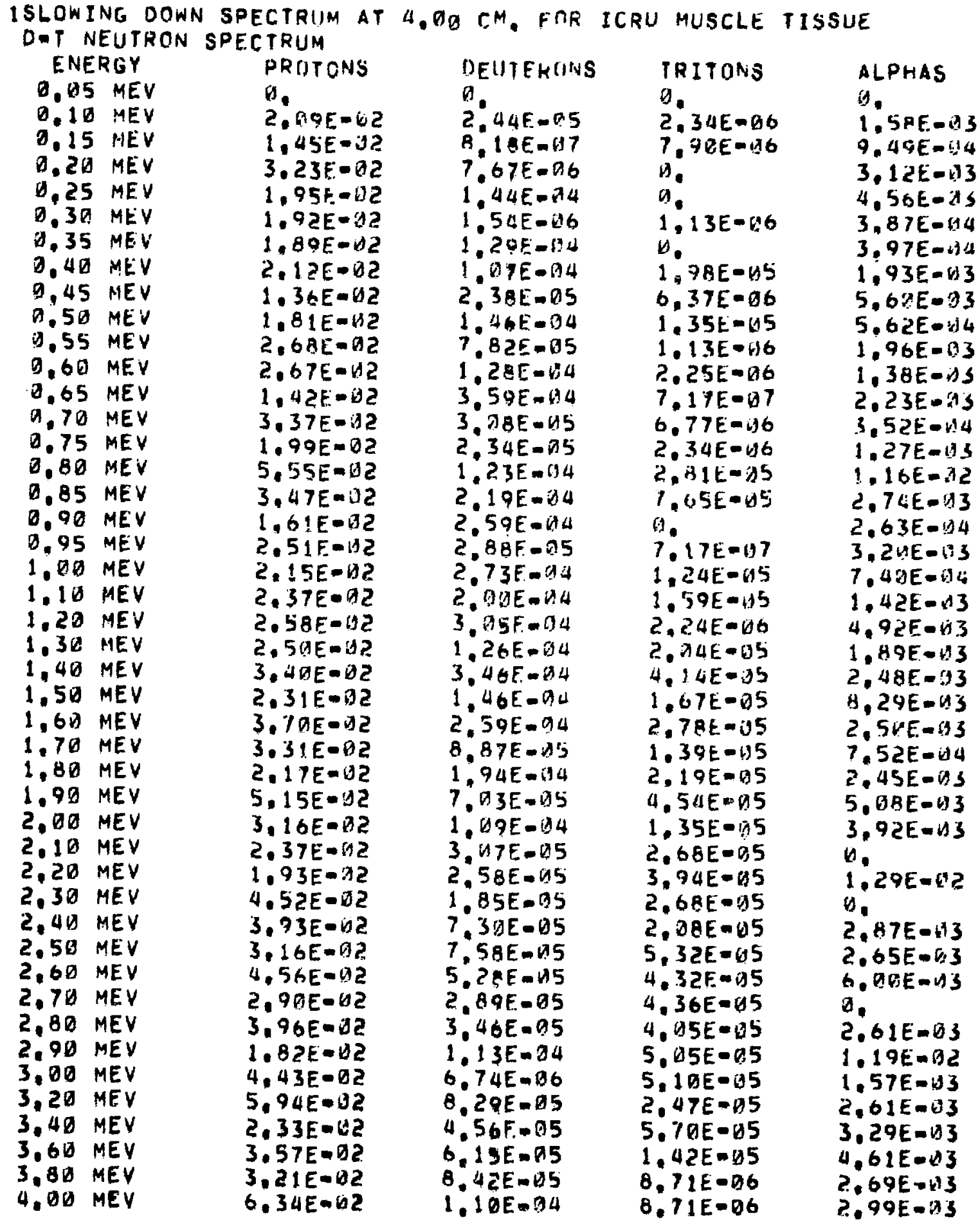




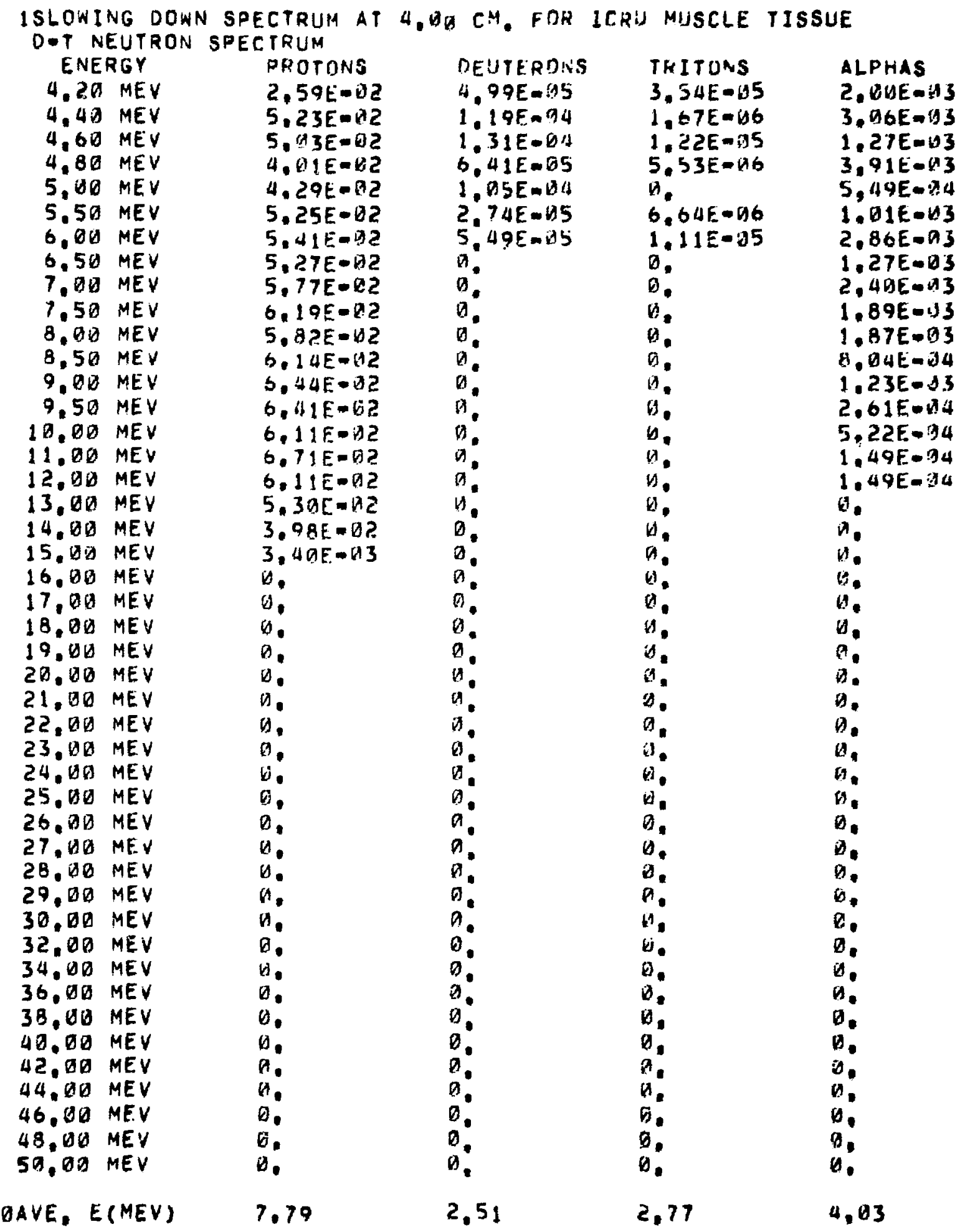




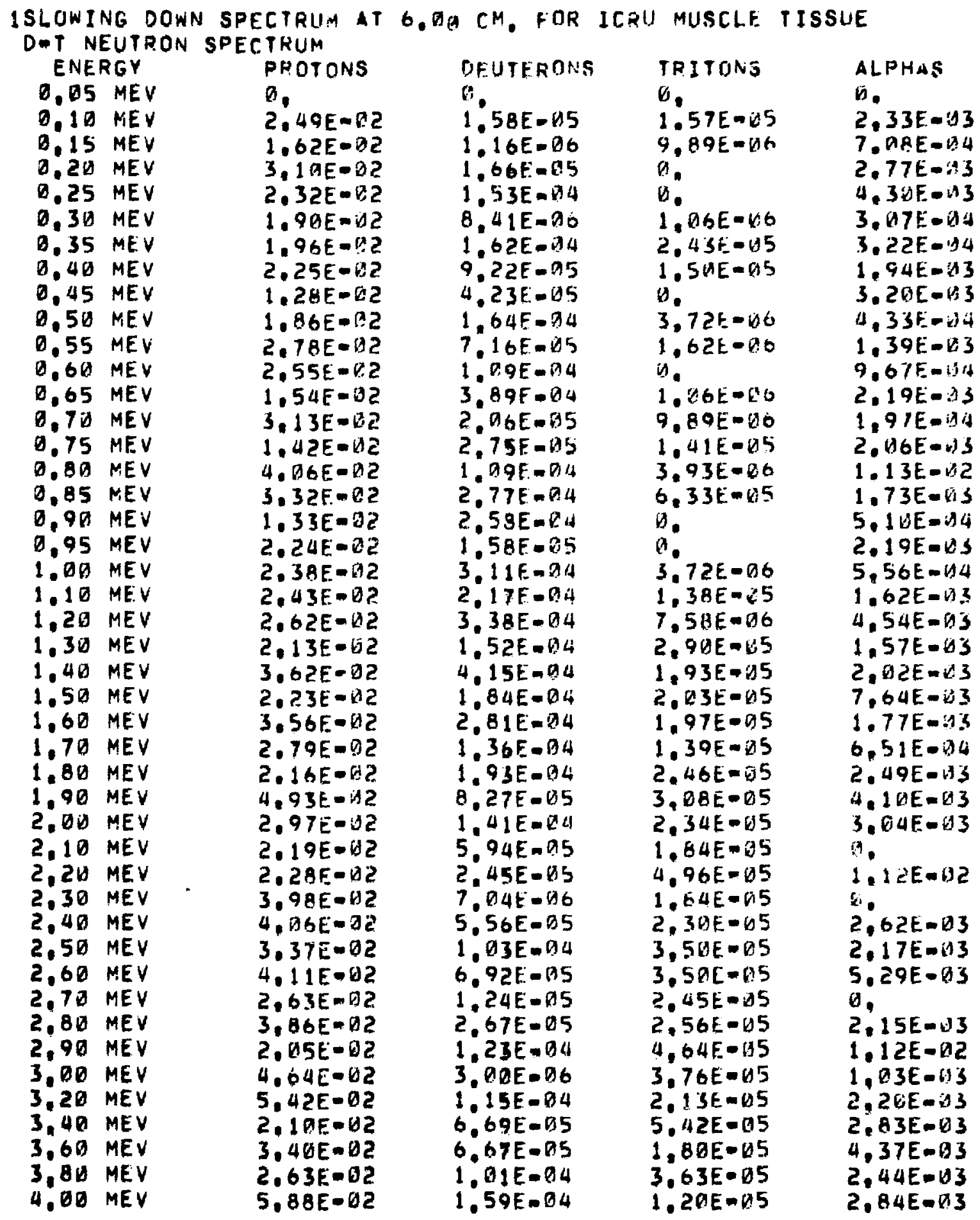


ISLOWING DOWN SPECTRUM AT 6.0A CM. FOR ICRU MIISCIE TISSUE DET NEUTRON SPECTRUM

\section{ENERGY}

4.2D MEV

4. 40 MEV

$4.60 \mathrm{MEV}$

4.80 MEV

5. OQ MEV

5,50 MEV

$6.06 \mathrm{MEV}$

$6.50 \mathrm{MEV}$

7. BD MEV

$7.5 B$ MEV

8. DO MEV

$8.50 \mathrm{MEV}$

9.00 MEV

9.50 MEV

10.00 MEV

$11,00 \mathrm{MEV}$

$12.00 \mathrm{MEV}$

$13.00 \mathrm{MEV}$

14.00 MEV

15. BO MEV

16. BO MEV

17.00 MEV

18.00 MEV

$19.00 \mathrm{MEV}$

$20.00 \mathrm{MEV}$

$21.00 \mathrm{MEV}$

22. $00 \mathrm{MEV}$

$23.00 \mathrm{MEV}$

$24.00 \mathrm{MEV}$

$25.00 \mathrm{MEV}$

26.00 MEV

$27.00 \mathrm{MEV}$

$28.00 \mathrm{MEV}$

$29.00 \mathrm{MEV}$

30.00 MEV

$32.00 \mathrm{MEV}$

34.00 HEV

36.00 MEV

38,00 MEV

40.00 MEV

42,00 MEV

44. DO MEV

46,00 MEV

48,00 MEV

50.00 MEY

DAVE, E(MEV)
PROTONS

2. $41 E-B 2$

4. $82 E-B 2$

$4.2 B E=02$

3.5 CEE $=12$

$3.93 E-122$

$4.94 E-152$

5.68E-D?

$4.81 E=02$

4.5BE-OZ

5.DIE-Lं2

$4.54 E-02$

5. B6E-B2

5. $24 E=12$

$5.30 E-D 2$

$5,35 E-02$

$4.93 E-152$

$5.36 E=b 2$

$3.73 E-12$

3.5 QE- $Q 2$

0.

0.

0.

0

0.

a.

0.

$\theta$

0.

$\theta$

D.

0.

$\theta$

0.

0.

0.

B.

B.

0.

0.

D.

b.

D.

B.

$\theta$

.

7.52

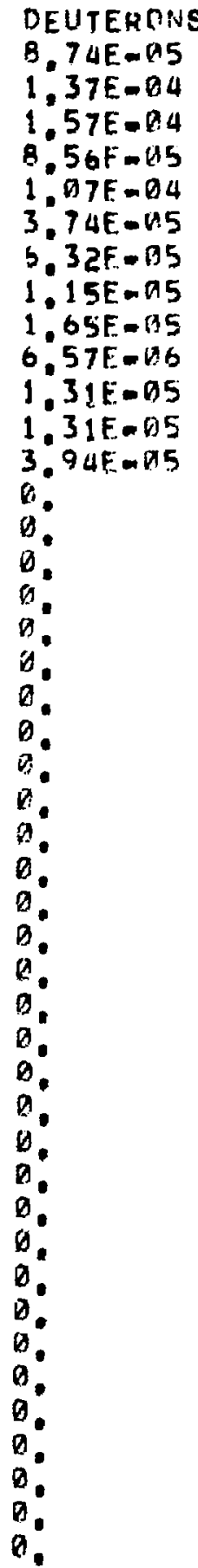

2.97
TRITOUS

3. $49 E-55$

4. 3AE-DO6

$1.72 E-155$

0.

$B$

0

0

0

$\theta$

$b$

$\theta$

b.

i.

0.

$\Delta$

$\theta$.

$\theta$

0

$\forall$.

.

b.

a.

0.

0.

.

c.

e.

0.

0.

0.

0.

Q.

0.

G.

$\theta$.

1.

0.

0.

b.

0.

b.

2.62
ALPHAS

1.87E- 13

2. $80 E-63$

$1,18 E-103$

4. $07 E-B 3$

4. $21 E-1) 4$

9. T5E $=44$

$2, B O E=W 3$

1. $18 \mathrm{E}=\mathrm{dOS}$

2. $29 E-33$

1. TUE-B3

1. $68 E-D 3$

0. $45 E-1) 4$

$1.12 E-103$

$1.92 E-M 4$

3. $B 3 E=64$

2. $1 \triangle E=\triangle 4$

2. $14 E-44$

0.

$D$

0.

a.

D.

a.

b.

a.

B.

0.

.

$\theta$.

0.

0.

D.

0.

$\theta$.

0

0.

0.

1.

b.

0.

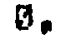

0.

b.

a.

4.11 


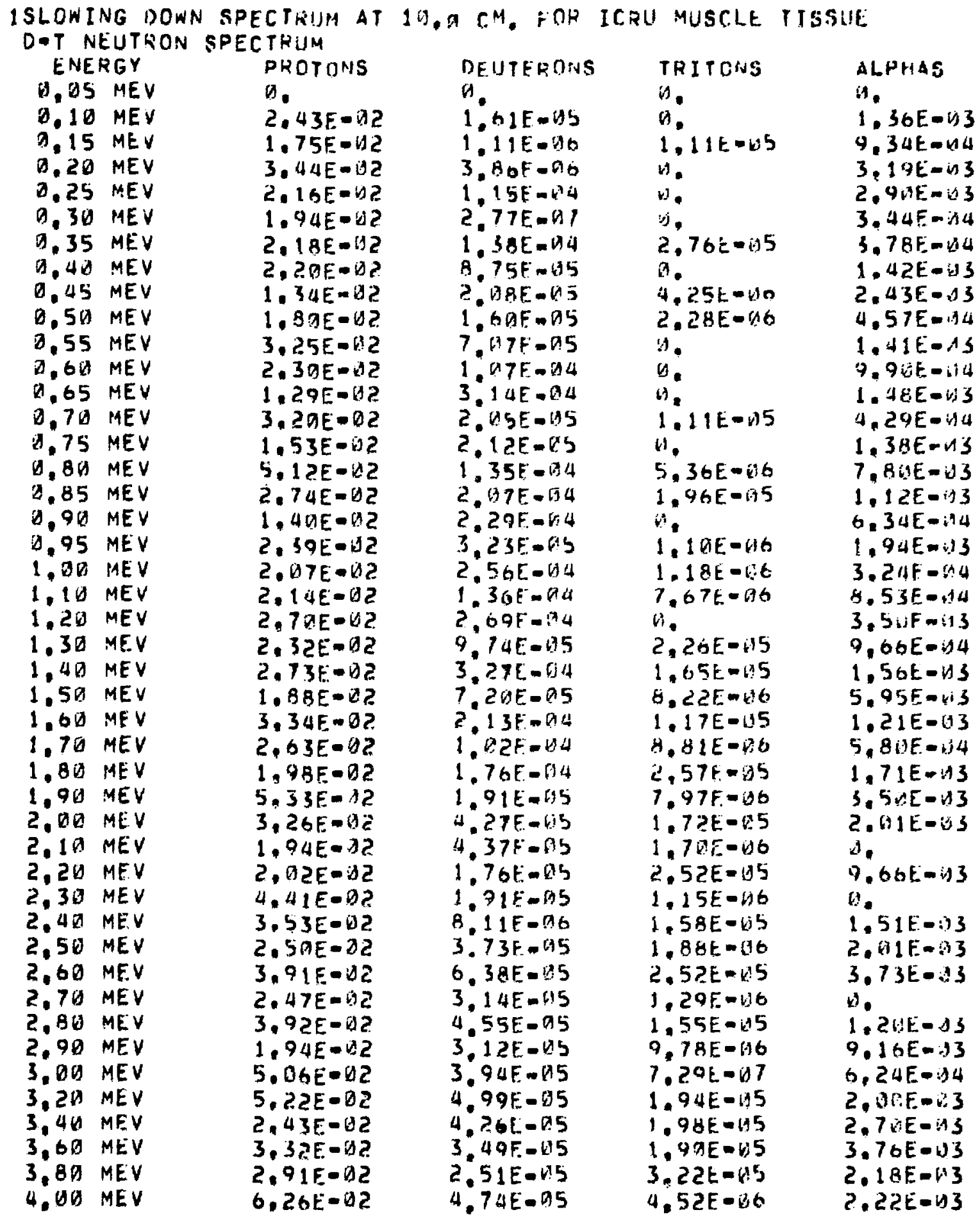




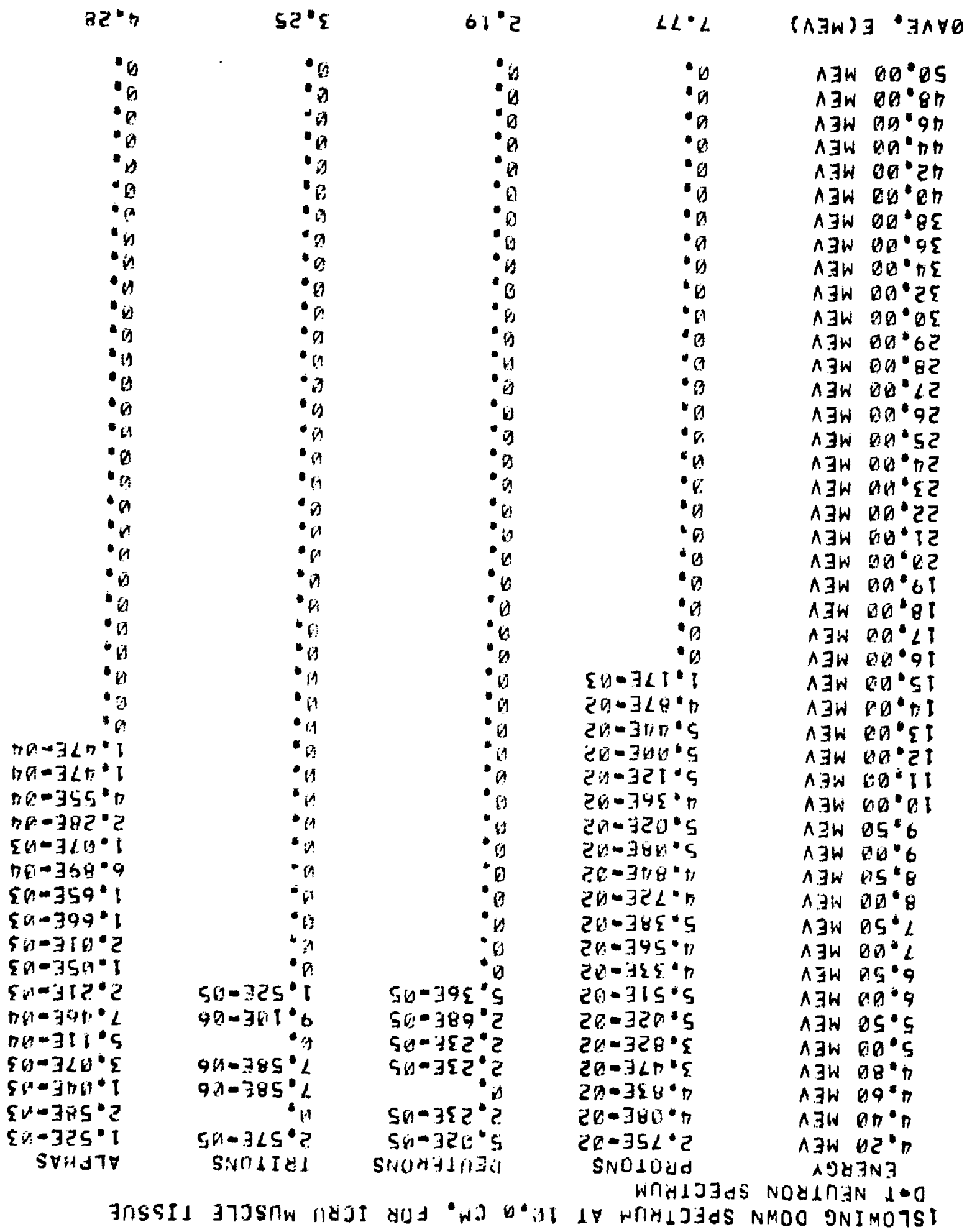




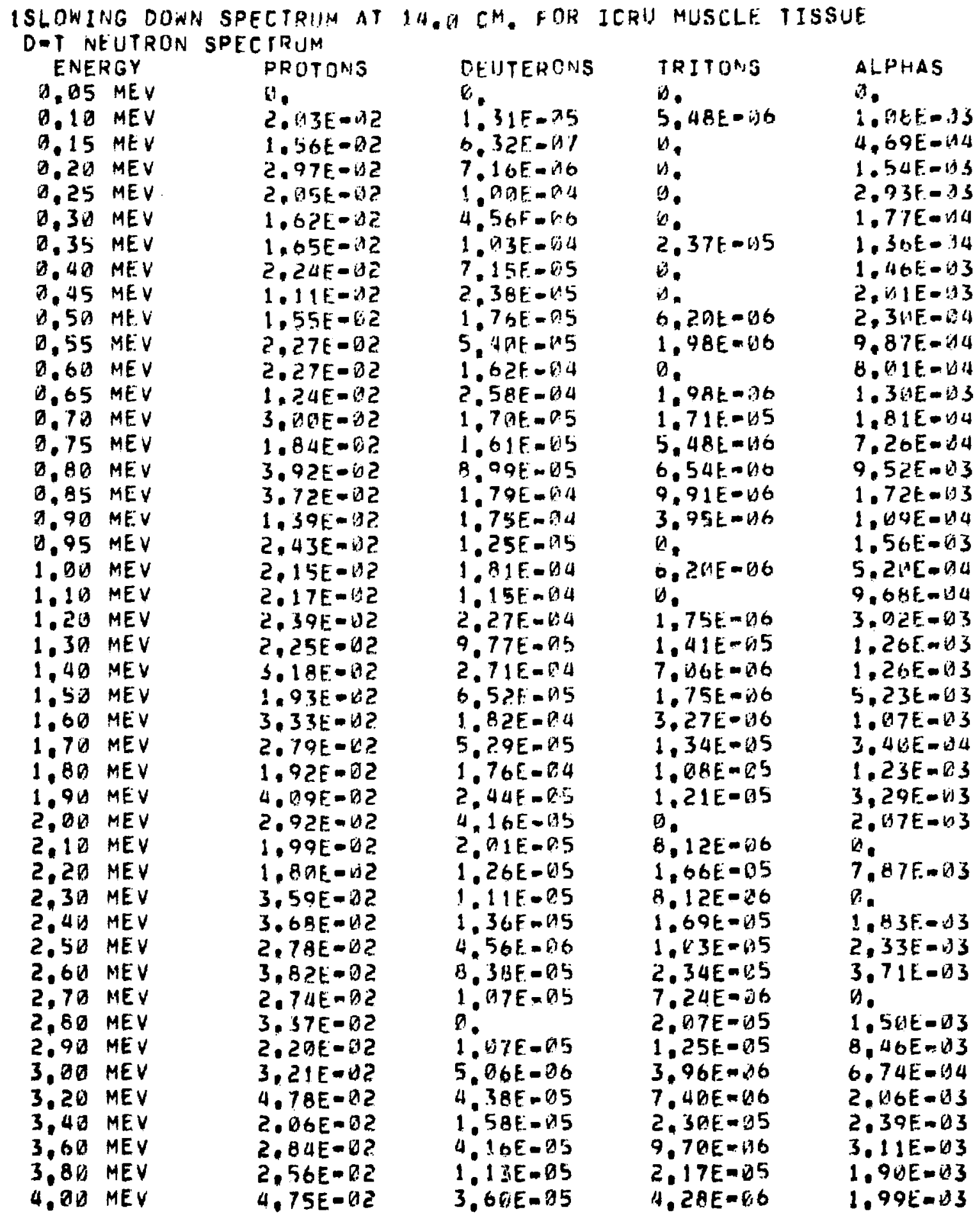




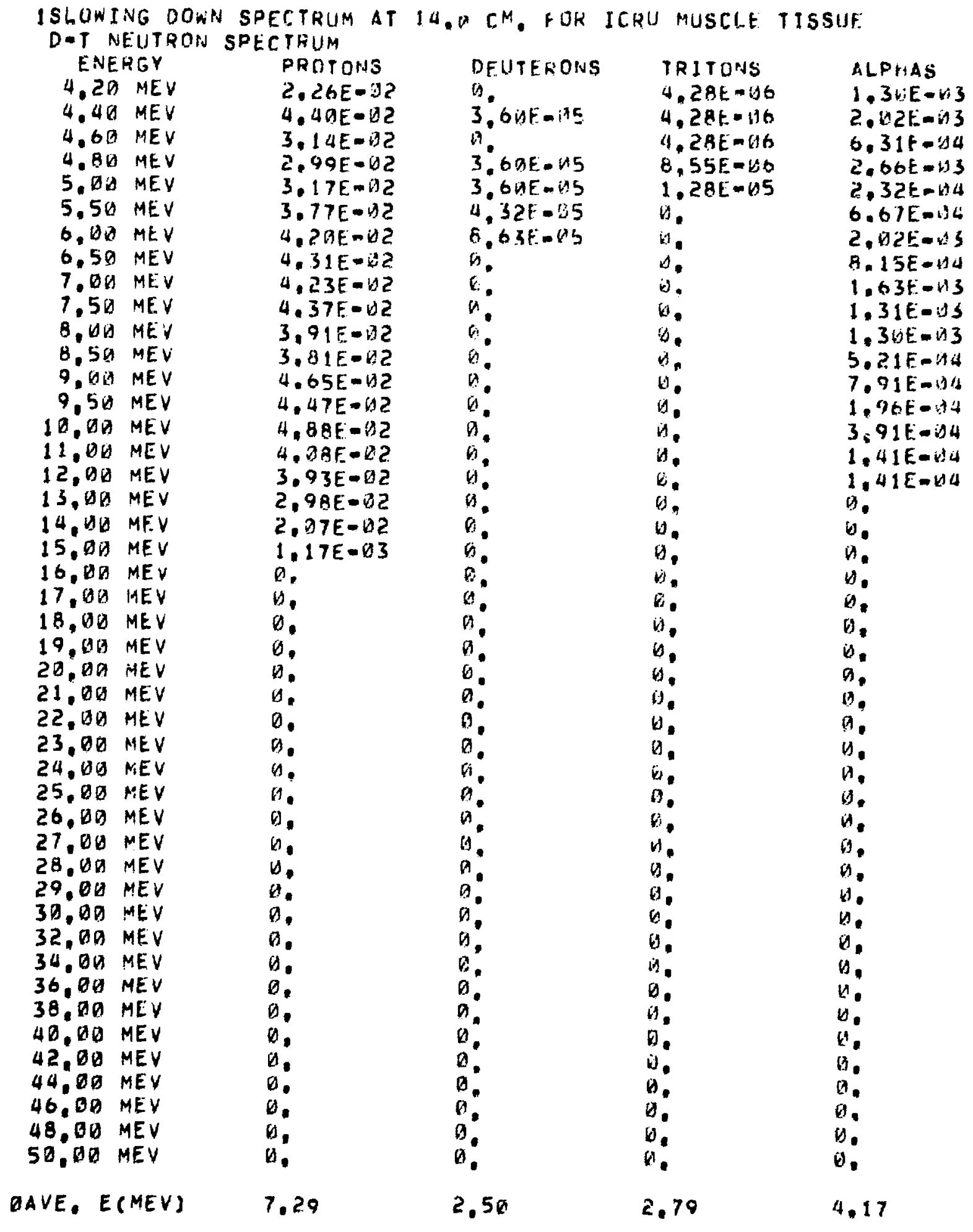


IINITIAL SPECTRIJM AT H. HAOW, D5 CN. FOK SHOHKA A-15H PLASTIC DWT NEUTRON SPECTRUUM

\section{ENERGY}

D. D5 MEV

i) 119 MEV

D.15 MEV

3.20 MEV

D. 25 MEV

$0.31 \mathrm{MEV}$

$0.35 \mathrm{MEV}$

D. 19 MEV

$0.45 \mathrm{MEV}$

1.5 MEV

$0.55 \mathrm{MEV}$

$1.60 \mathrm{MEV}$

0.65 MEV

$D .7$ V MEV

D. 15 MEV

$0.80 \mathrm{MEV}$

0.85 MEV

$0.91 \mathrm{MEV}$

0.95 MEV

1. 90 MEV

$1.10 \mathrm{MEV}$

1.20 MEV

1,30 MEV

1.40 MEV

1.5 1.4 MEV

$1.60 \mathrm{MEV}$

$1.70 \mathrm{MEV}$

1.80 MEV

$1,90 \mathrm{MEV}$

2.DO MEV

2.10 MEV

2.2Q MEVV

2.3A MEV

2. 4O MEV

2. 50 MEV

$2,60 \mathrm{MEV}$

$2.70 \mathrm{MEV}$

$2.86 \mathrm{MEV}$

$2.9 W$ MEV

3. DU MEV

3.20 MEV

3. $46^{*}$ MEV

3.60 MEV

$3.80 \mathrm{MEV}$

4. $\triangle O$ MEV
PROTONS

i.

1. $D Q E+Q 2$

2. $B R E+U 1$

2. 3AE + HI

9. $4[E+J b$

3.? $\{E+B \mid$

2. $41 E+J 1$

4. $53 E+314$

2. $33 E+A 1$

1. $75 E+31$

1. ?רE + $)$ !

$5.62 E+4 ! 4$

$8.95 E+30$

$1,45 E-1)$ ?

$2.91 E-\lambda$ ?

$9.53 E+10$

1. $4.4 E+31$

$9.63[+.18]$

5. $11 E+114$

4. $72 E+3 H$

1. $37 E+11$

$6.37 E+10$

$1,14 E+11$

1. $\triangle A E+O H$

$4.45 E+414$

$2.96 E+11$

4. $44 E+d 1$

$1.23 E+11$

9. $52 E+D H$

1. $11 E+11$

5. URE+ DA

1. $47 E+Q 1$

4. $8 \Delta E+B A$

i.

v.

$3.82 E-D ?$

$3.82 E-12$

4. $7 D E+D A$

4. $74 E+D O$

v.

1. $39 E+B 1$

$4.77 E+30$

4.7 $A E+10$

$4.78 E+1)$

2. $39 E+D 1$
DEUTERONS

a.

1. $396-32$

1. $395-192$

7.

(i)

(n)

$\therefore$

1. $A 4 E-92$

1. $R 3 E-12$

19

1.1.

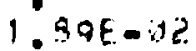

1. A9E-I?

9. $44 E-12$

$9.44 F-12$

$1.59 \mathrm{~F}-\mathrm{il} \mathrm{C}$

1. $395=\omega 2$

$1.89 E-112$

1. 39E-J2

a.

.

A.

a.

$\theta$

if.

tr.

1.

in.

7. 19E- -2

7. IOF-AC

in.

a.

n.

in.

$7.19 E-B 2$

7 1 $9 E-W 2$

1. ISE

\begin{tabular}{|c|c|}
\hline 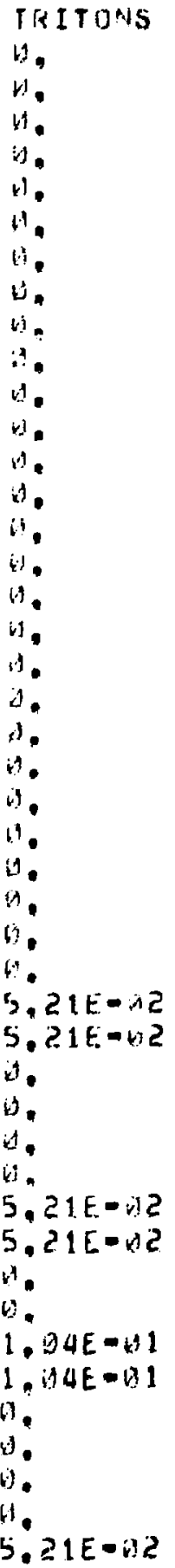 & 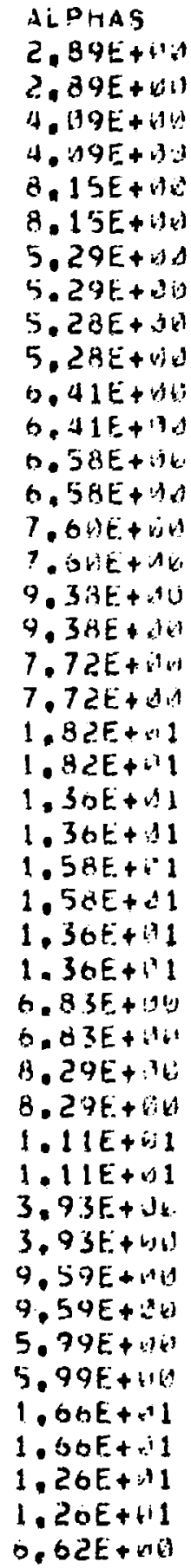 \\
\hline
\end{tabular}




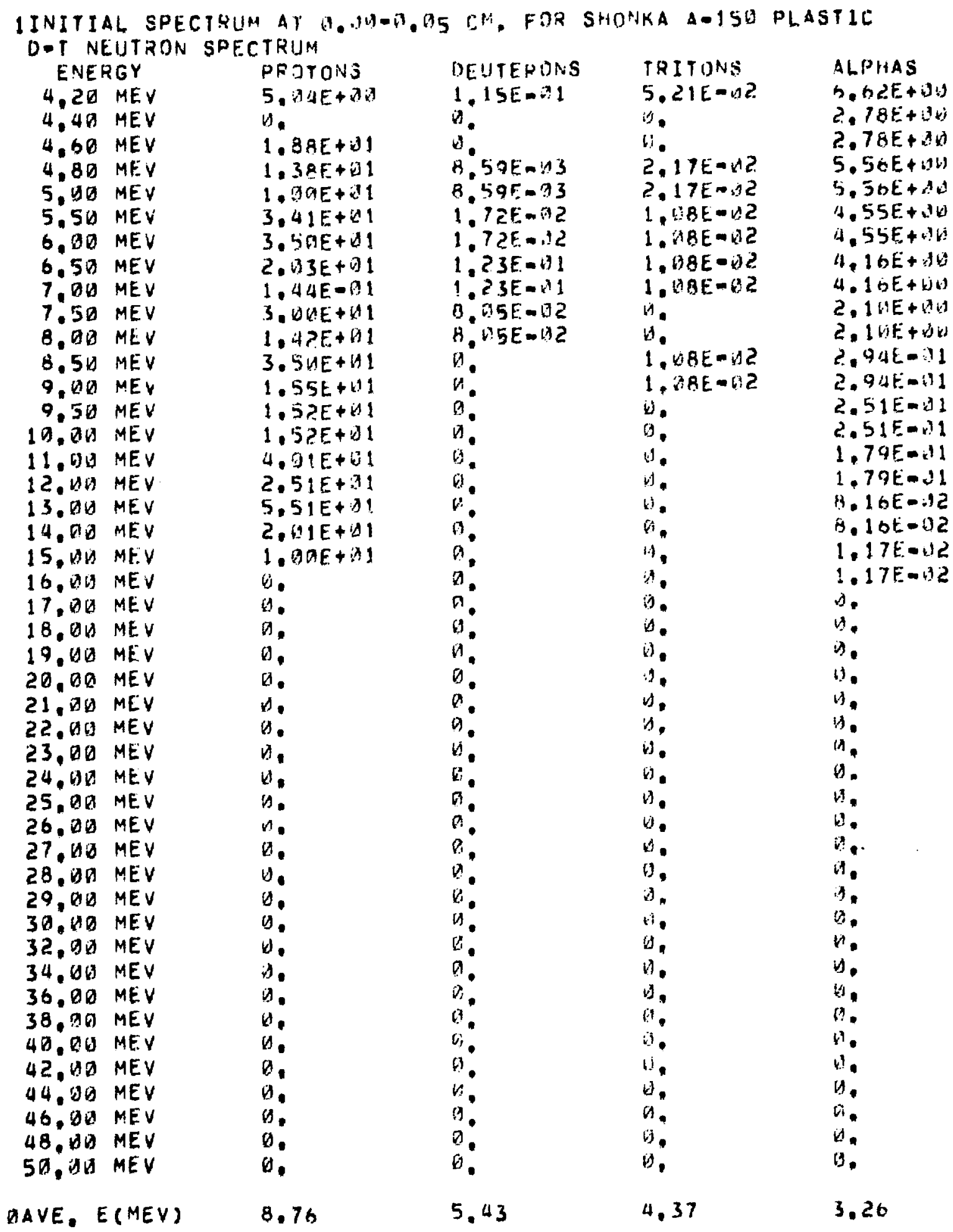


IINIIIAL SPECTRUM AT D, D5-1, IA CH. FOK SHONKA A-15\% PLASTIC D-T NEUTRON SPECTRUM

\section{ENERGY}

Q. $\triangle 5$ MEV

0.10 MEV

0.15 MEV

0.20 MEV

0.25 MEV

0.30 MEV

0.35 MEV

D. $\triangle D M E V$

0.45 MEV

$0.50 \mathrm{MEV}$

0.55 MEV

0.60 NEV

0.65 MEV

0.71 MEV

0.75 MEV

$0.8 \%$ MEV

$0.85 \mathrm{MEV}$

$0.90 \mathrm{MEV}$

0.95 MEV

1. DO MEV

1,10 MEV

1.20 MEY

1.SO MEV

1.40 MEV

1.5W MEV

$1.60 \mathrm{MEV}$

$1.70 \mathrm{MEV}$

1.9A MEV

1.9 $\because M E V$

2. OO MEV

$2,1 D$ MEV

$2.20 \mathrm{MEV}$

2. 30 MEV

2.4U MEV

$2,56 M E V$

2,61 MEV

$2.70 \mathrm{MEV}$

2,80 MEV

$2.90 \mathrm{MEV}$

3. $60 \mathrm{MEV}$

3.2U MEV

3. 4H MEV

$3.6 \mathrm{~V}$ MEY

3,80 MEV

4. DIS MEV
PROTONS

B.

1. $77 E+192$

2. $82 E+A 1$

$3.38 E+1$

3. $6 A E+D 1$

$1.865+41$

$7.23 F+16$

1. $41 F+3$ !

$4.04 \mathrm{~F}+4 \mathrm{~m}$

2. $47 E+W 1$

1.94E+11

$9.75 F+61$

2. $3 A E+1$

4. $25 E+n A$

4. $72 E+i 14$

1. $M D E+1$

$9.34 E+211$

9. $42 E+U 1)$

5. OIE +UF?

$9.4 \geq E+30$

$5.44 E+D D$

1. $44 E+11$

1. $58 F-01$

1. $205-01$

$9.71 E+D 13$

5. WOE+QU

3. $82 E-142$

$1,0 I F+31$

5. $11 E+913$

1.9 $9 d E+B 1$

5. DAE + BOA

5. DOSE+ DA

4. $5 A E+D G$

0.

$7.65 E-0 ?$

4. $8 ?[+1)$

5. $\triangle B E+10 Q$

$9.7 A E_{-}+19$

3. $B 2 E=02$

$4.82 E+b i$

1. $48 E+1$

1. $4 R E+61$

3. $82 F=6$ ?

1. $\triangle D E+D 1$

5. AOE +00

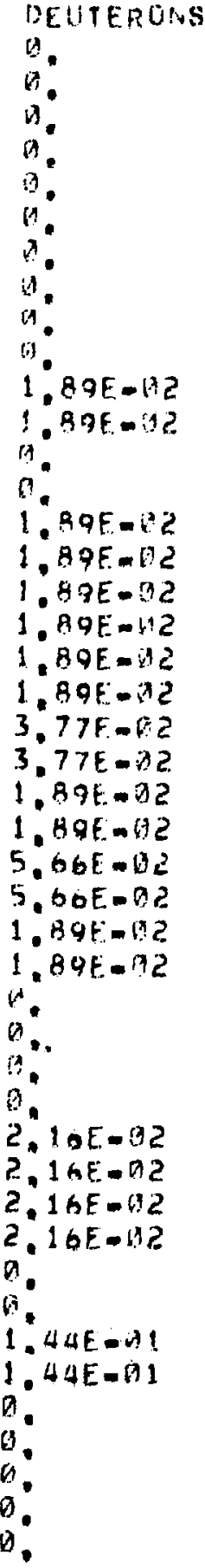

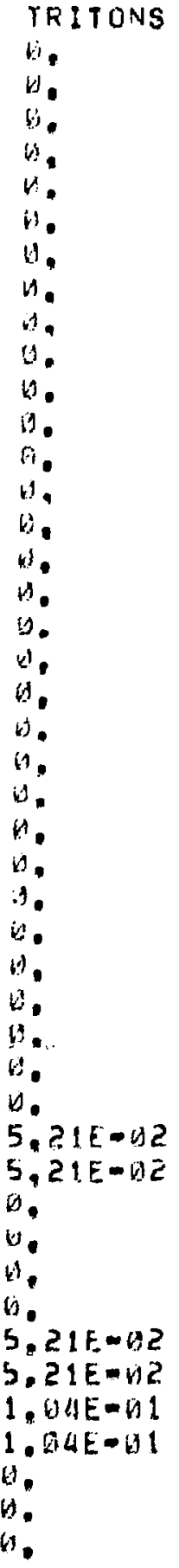

ALPHAS

5. $12 E+124$

5. $12 E+\Delta U$

5. ARE + Di

5. $W \partial E+U D$

5. 2WE + $\triangle 13$

5.2ut+w

9.3BE+.Mv

$9.38 E+\cdots$

Q. $23 E+196$

B. $23 E+10$

$7.6 \perp E+D U$

7.6IE+JU

$7.66 E+30$

$7.66 E+W 10$

$8.93 E+U W$

$\$ .93 E+3 G$

$4.08 E+00$

$4 . B^{3} 8 E+i=n$

2.4 LE + UJ

2. $4 A E+100$

$1.23 t+.1$

1. $23 E+1$

$1,14 E+31$

1. $14 E+d 1$

$6.48 E+8 k$

$0.48 E+D !$

$1.01 E+O I$

1. DIE +1

1. $19 E+11$

1. $19 E+11$

$5.54 E+1 W$

$5,54 E+11$

A. $15 E+114$

3. $15 E+40$

8. $13 E+130$

8. 1 UE + WA

$3.51 E+D 13$

$3.51 E+00$

$7.72 E+b V$

$7.72 E+W B$

$1.12 E+1$

1. $12 E+11$

$3.83 E+60$

3.83E+UV

$7.82 E+d b$ 


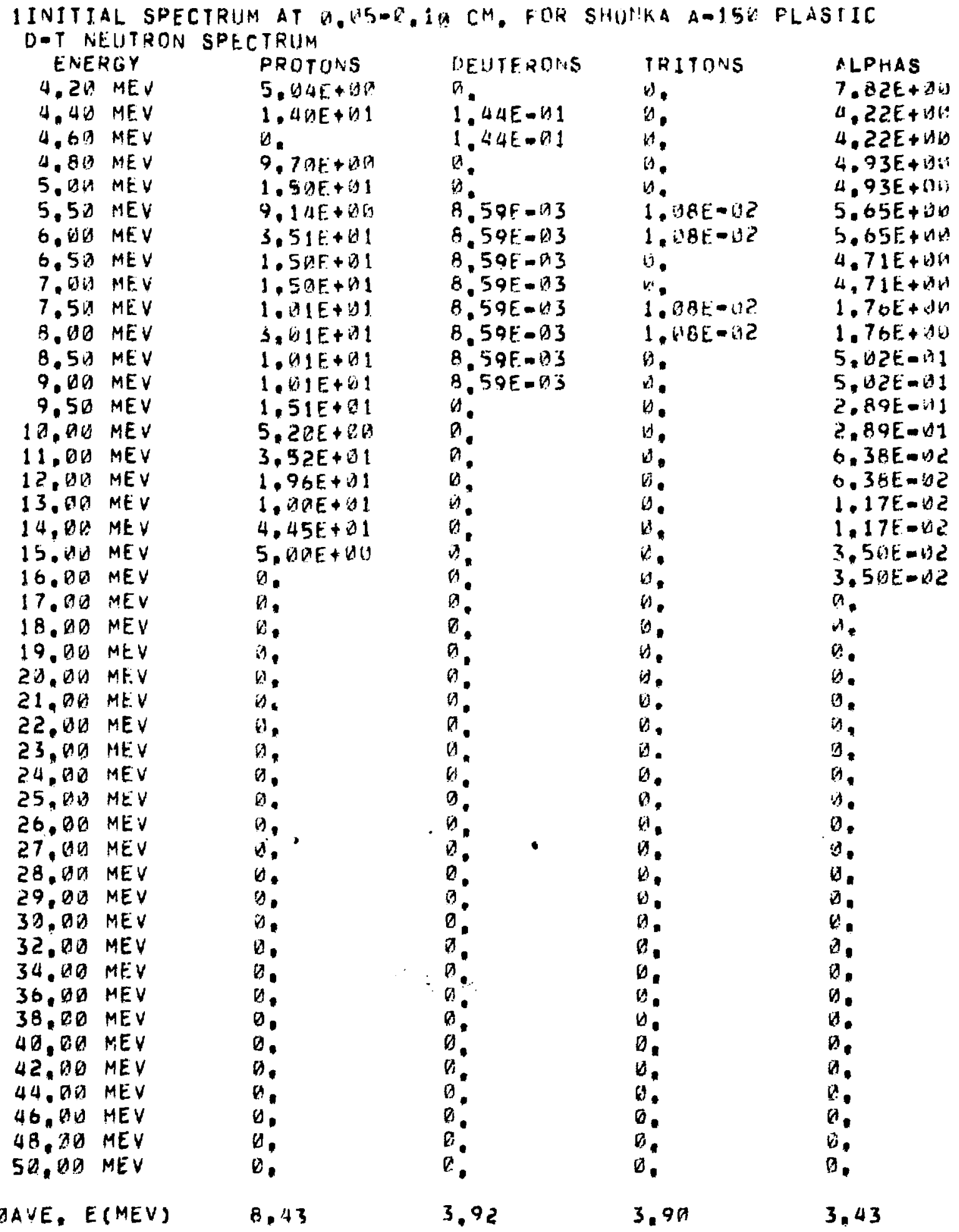


IINITIAL SPECTRUM AT 9.10-3.15 CM. FOR SHUNKA A-ISG PLASTIC D-T NEUTRDN SPECTRUM

\section{ENERGY}

औ. US MEV

2. II MEV

0.15 MEV

0.2 I $M E V$

$0.25 \mathrm{MEV}$

$\triangle .30 \mathrm{MEV}$

0.35 MEV

0.4 MEV

0.45 MEV

0,50 MEV

D. 55 MEV

$0.60 \mathrm{MEV}$

0.65 MEV

$0.70 \mathrm{MEV}$

Q.75 MEV

$0.80 \mathrm{MEV}$

$0.85 \mathrm{MEV}$

0.90 MEV

$0.95 \mathrm{MEV}$

$1,00 \mathrm{MEV}$

1,10 MEV

1.20 MEV

1.36 MEV

$1.40 \mathrm{MEV}$

$1.50 \mathrm{MEV}$

$1.60 \mathrm{MEV}$

1.79 MEV

$1.80 \mathrm{MEV}$

$1.90 \mathrm{MEV}$

2. $\triangle D M E V$

$2,10 \mathrm{MEV}$

2.20 MEV

$2.30 \mathrm{MEV}$

2.40 MEV

2.5D MEV

2.60 MEV

2.70 MEV

2. BU MEV

2.90 MEV

3. $O Q M E V$

3.20 MEV

$3.40 \mathrm{MEV}$

$3.60 \mathrm{MEV}$

$3,80 \mathrm{MEV}$

4. $D 0$ MEV
PROTONS

$\theta$.

$1.31 E+02$

3. $33 E+01$

$\left.6,24 E_{-}+1\right] 1$

1. $94 E+01$

$2,11 E+11$

2. $75 E+11$

2. $84 E+01$

5. 1$) 2 E+O B$

$2,29 E+1] 1$

1.5OE+DI

$4.12 E+00$

$9.42 E+9$

$8.99 E+180$

1. $5 D E+W 1$

1. $41 E+01$

$4.73 E+61$

$4.74 E+B$

1. $47 E+01$

7. $46 E+D 0$

5. $33 E+\partial U$

9. $81 E+O A$

$3.19 E+B 1$

1. $45 E+1$

1. $94 E+131$

$4.24 E+100$

$9,44 E+10$

5. DQE $+\triangle O$

1. $15 E-1$

$4.79 E+10$

3. $82 E-02$

$5.15 E+00$

$9.74 E+O D$

6,

1. $D 2 E+Q 1$

5. ACE + UO

$3,82 E-02$

1. $199 E+61$

5.11E+QO

5. B $\angle E+B Q$

9. $2,2 E+D O$

2. $14 E+D D$

5. $38 E+O B$

$6.67 E+00$

$1,41 E+B 1$

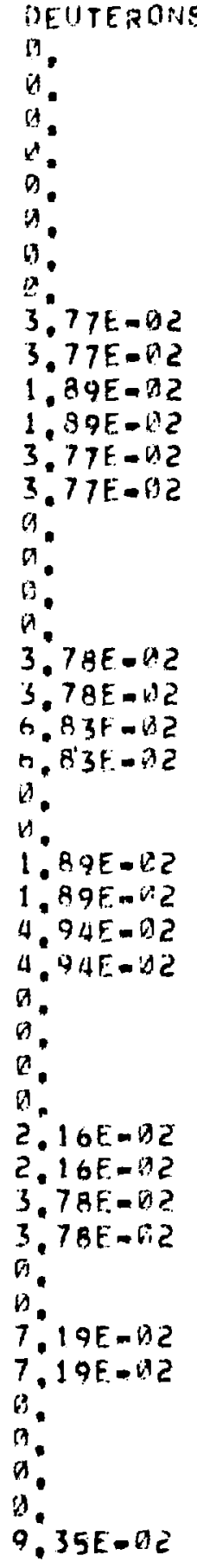

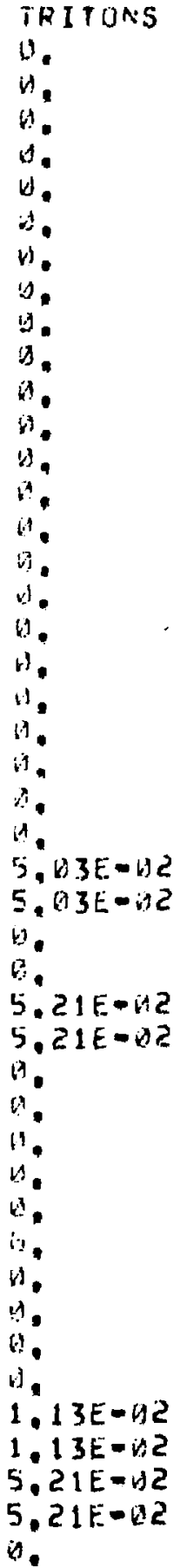

ALPHAS

1.76E+ 36

1. $76 E+b u$

9. $4[4 E+11]$

9. $4 W E+W W$

$5.3 B E+116$

5. $88 E+\lambda U$

7. $) 5 E+W U$

7. $125 E+\angle C$

5. $29 E+W$

$5.29 t+14$

1. $29 E+k^{3} 1$

$1.29 E+11$

$5,76 E+14$

$5.76 E+46$

$4.64 E+16$

$4.64 E+14$

1. $115 E+111$

1. $95 t+1$

B. $23 E+d v$

8. $23 E+b 0$

$1.77 E+d 1$

$1,77 E+01$

$1.18 E+41$

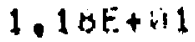

$1.35 E+b 1$

1. $35 E+01$

$1.11 E+1]$

1. $11 E+11$

1. $11 E+U 1$

1. $\mid 1 E+1$

$1.17 E+111$

1. $\mid 7 E+11$

$9.76 E+D U$

$9.70 E+W U$

7. $5 B E+11 \mathrm{~B}$

$7.58 E+46$

$5.43 E+d v$

$3,43 E+b)$

A. $61 E+b 0$

$8.61 E+30$

$1.62 E+D 1$

$1.62 E+11$

7.71E+OD

$9.71 E+U A$

$7.36 E+136$ 


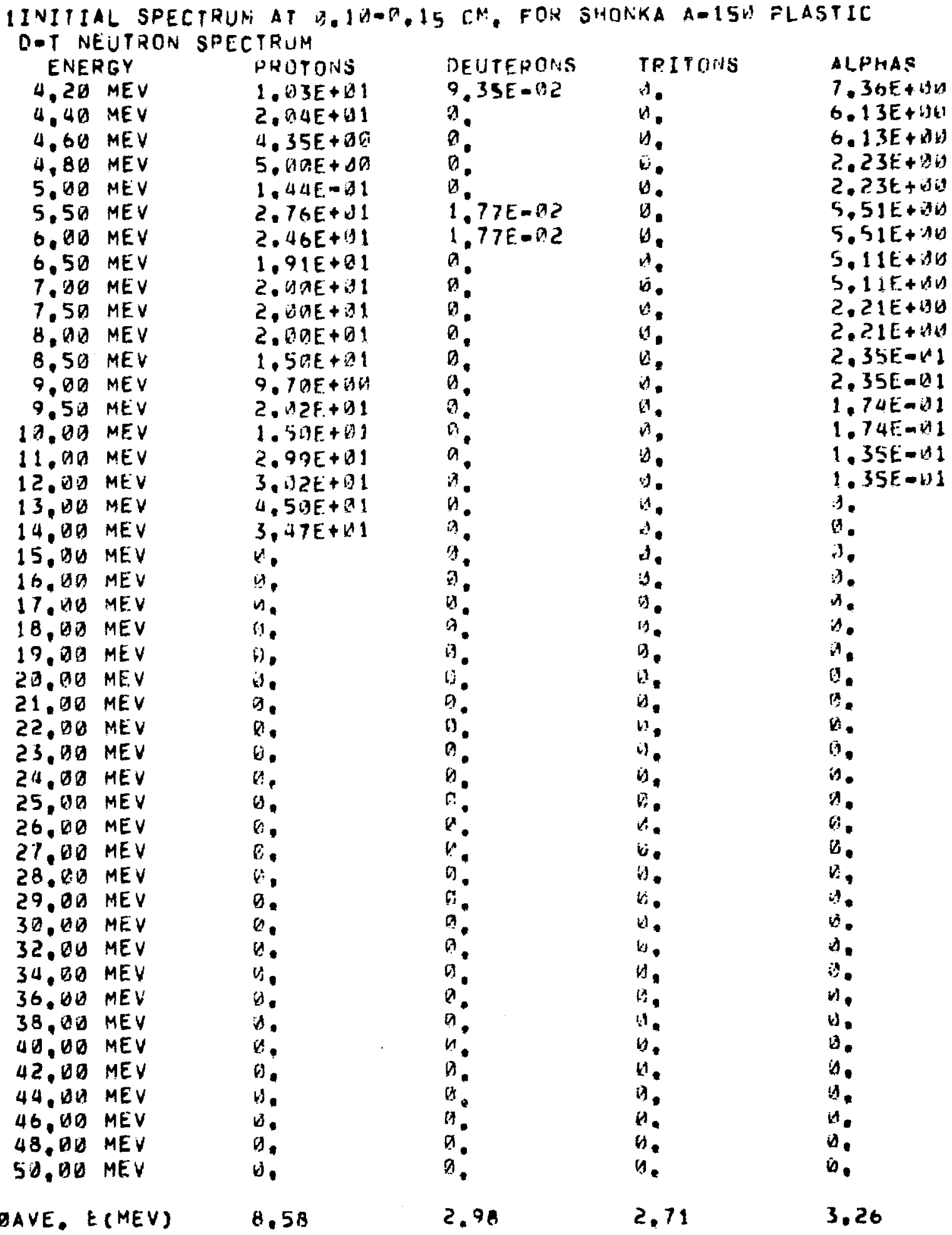


1INITIAL SFECTRUM AT A, 15-6.2A CH. FDR SHUNKA A-15" PLASTIC D-T NEUTTRON SPECTRLIMT

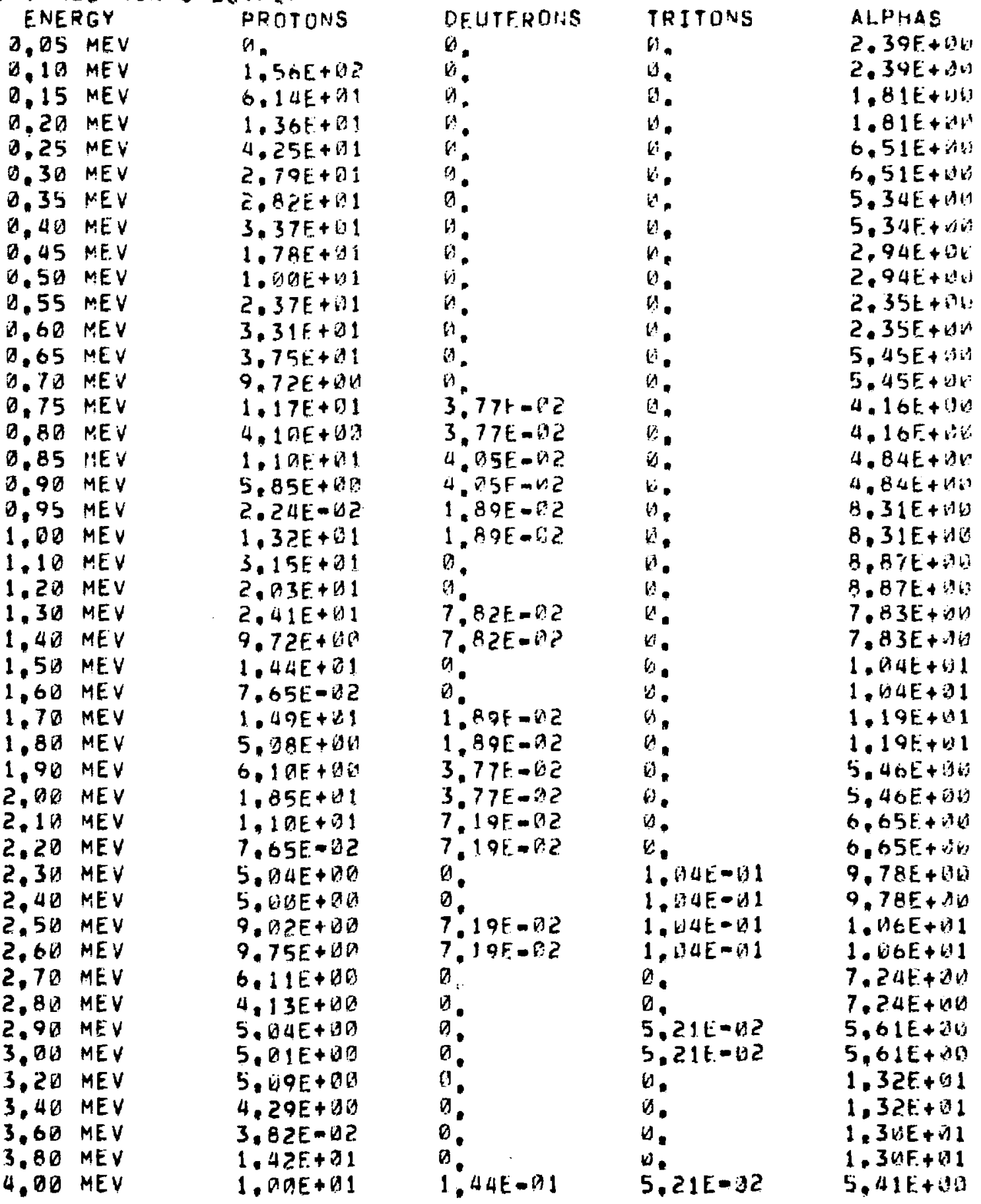




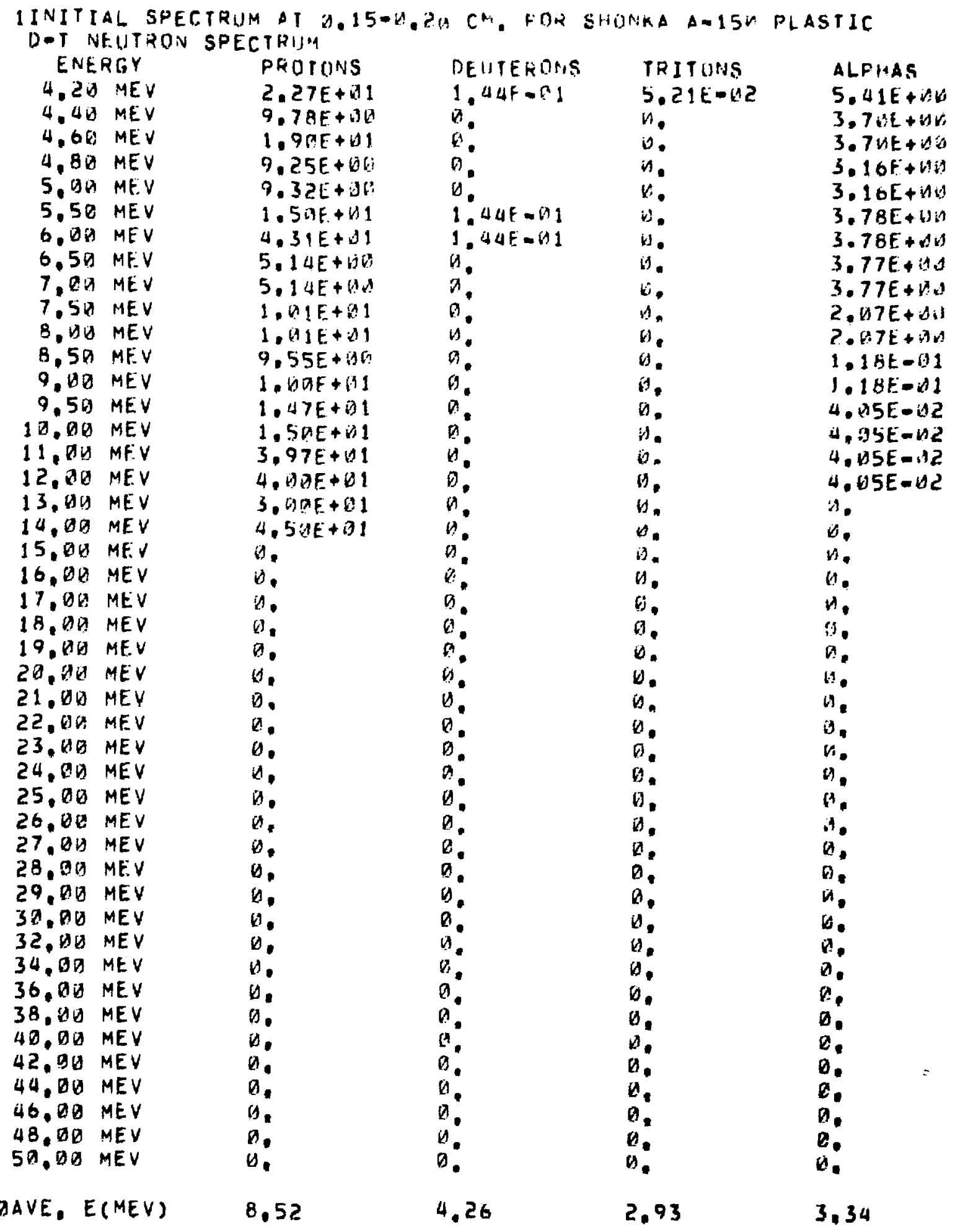




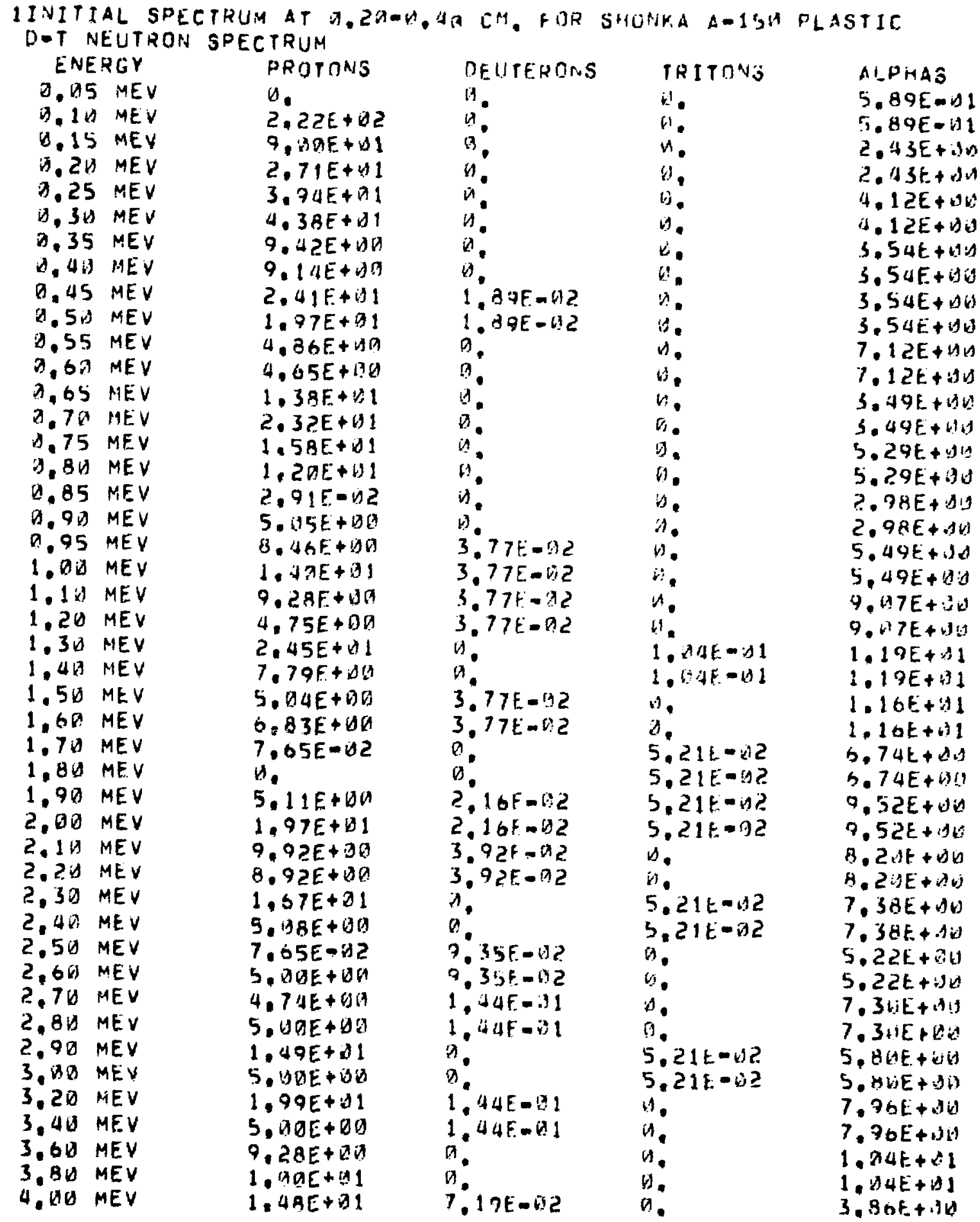




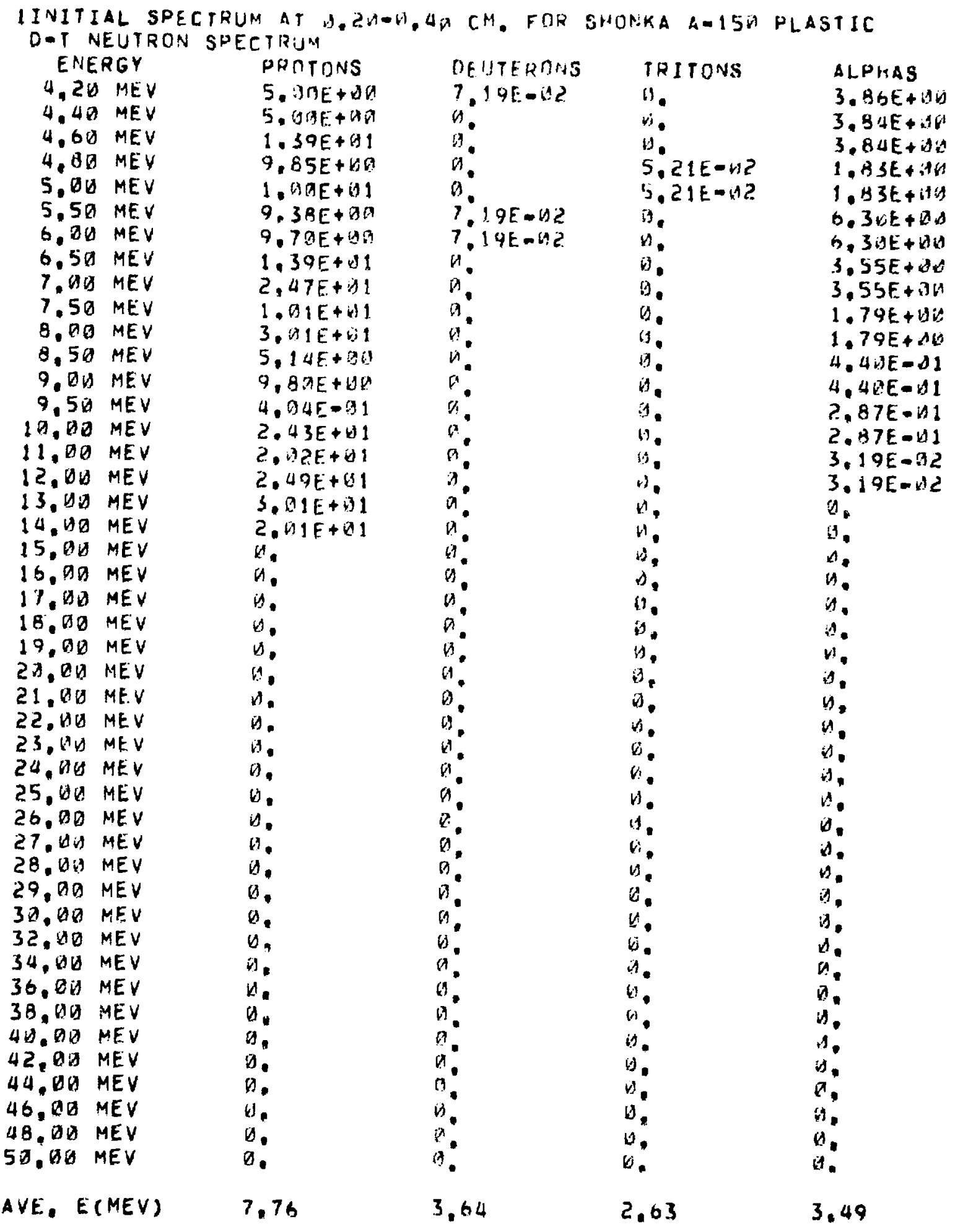


IINITIAL SPECTRUM AT G.4G-3.6R C.H. FOK SHONKA A-15H PLASTIC DAT NEUTRON SPECTRUH

\section{ENERGY}

0.05 MEV

D. 10 MEV

0.15 MEV

$0.2 D M E V$

1.25 MEV

0.30 MEV

0.35 MEV

0.40 MEV

0.45 MEV

0.5 MEV

$0.55 \mathrm{MEV}$

D. 6U MEV

1.05 MEV

$0.70 \mathrm{MEV}$

$0.75 \mathrm{MEV}$

D. BO MEY

$0.85 \mathrm{MEV}$

$0.90 \mathrm{MEV}$

0.95 MEV

1.00 MEV

1. IU MEV

1.20 MEV

1.30 MEV

1. 40 MEV

1.50 MEV

1.6月 MEV

1.70 MEV

$1.86 \mathrm{MEV}$

$1.90 \mathrm{MEV}$

2. $O A$ ME.V

2.1D MEV

2.20 MEV

2.30 MEV

2. $40 M E Y$

2.5D MEV

$2.60 \mathrm{MEV}$

2.70 MEV

2. BU MEV

2,90 MEV

3. DO MEV

3.20 MEV

3.4D MEV

3,60 MEV

3. 80 MEV

$4,0 D$ MEV
PROTONS

b.

1. $83 E+12$

$6.52 E+B 1$

5. $136 E+3$ ?

3. GGE + U1

$2.35 \mathrm{~F}+31$

2. $99 E+61$

$2.50 E+61$

$1.73 E+B 1$

1. $93 E+111$

$1.77 E+11$

1. $53 E+\partial 1$

1. $32 E+11$

1. $47 E+B 1$

1. $15 E+b 1$

i. $26 E+101$

$9.9 \lambda E+A D$

1. $2 A E+31$

1. $26 E+6$ :

$1.61 E+B 1$

$1.66 E+01$

1. $35 E+01$

$1.05 E+0]$ ?

$7.92 E+B O$

1. $40 E+101$

$9.77 E+80$

1. DRE +1

$8,5+E+U G$

$6.86 E+139$

6. $18 E+D Q$

$1.37 E+B 1$

$7.77 E+00$

$7.96 E+10$

$7.27 E+10 A$

$6.74 E+B a$

$4.87 F+B P$

$2.89 E+00$

5. $21 E+D \theta$

$5.21 E+00$

5.11E+ 10

$7.6 A E+\Delta 0$

$1.33 E+11$

$1.28 E+01$

$6.51 E+90$

1.16E+D1
DEUTEROUS

1

i.

H.

$\theta$

a.

$3.77 E-93$

$3.77 \mathrm{~F}=03$

$3.77 \mathrm{~F}-\mathrm{B} 3$

$3.77 \mathrm{~F}=113$

3.77E- 3

$3.77 t-23$

3. 38F m11?

$3.38 F=92$

$1.91 E-D: 3$

1. $91 \mathrm{E}=3$

$1.51 \mathrm{E}-8 \mathrm{C}$

$1.51 E-A 2$

$7.55 \mathrm{E}$ - 3

7 .5SE- 3

5. b2F-192

$5.62 F-172$

$4.88 E-142$

$4 . A 8 F-\triangle A$

2. $26 E-142$

2. $26 \mathrm{E}-02$

$3.71 F-1.2$

$3.71 \mathrm{E}-02$

$3.77 E-\backsim 3$

3. $77 E-A 3$

$1.82 E=A 2$

$1.82 E=02$

7. $92 E-D 3$

$7.72 t-03$

1.87E-12

1.87E-O2

$2.62 F-12$

2. $62 \mathrm{E}-02$

$1.93 E-A ?$

1.93E-GZ

$4.38 E-12$

$4,3 R F-22$

5.75F-112

$5.75 F=02$

$7.62 E-02$
TRIT THS

H.

6.

$s$.

a.

$\theta$

$\therefore$

9. $\triangle 7 E-$ is 3

$9.47 F-43$

a.

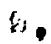

a.

is.

n.

b.

$9.29 t-83$

$9.29 E-63$

b.

$b$.

b.

i.

1. $24 E=192$

1. $24 E-H Z 2$

2. $12 E-42$

2. $12 E-B 2$

1. OUE W ?

1. $.44 F-42$

$1.93 E-62$

$1.93 E-\{3$

1. $b 4 t=b 2$

1. $U 4 E-B 2$

$n$.

$\theta$.

1. $\triangle 4 E-\triangle 2$

1. $\triangle \triangle E E-D ?$

2. $6 t-D 2$

2.,$B E-12$

3. $12 E-V 2$

3. $12 E-D 2$

1. $\triangle \triangle E=D 2$

1. $\because 4 E=02$

$6,25 E-\square 2$

$6.25 E=\square 2$

$6.25 E=0 ?$

$6.25 E-02$

1. $26 E-D 2$
ALPHAS

3. HTE+HE

3. $67 E+16$

2. $47 \mathrm{~F}+\mathrm{B} D$

$2.47 E+3 V$

$3.53 F+1$

$3.53 E+14$

$4.52 E+1913$

4. $52 E+11 k$

$4,53 E+46$

$4.53 E+9 k^{4}$

$5.26 E+44 i$

5. $26 E+134$

$5.96 E+4 k^{i}$

$5.96 F+4 x^{\circ}$

5. $28 E+i v$

$5,28 E+\cdots$

6. O1E+ $1 V$

b. $U 1 E+A V$

S. O9E +

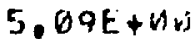

$9.75 E+i v$

$9.75 E+65^{\circ}$

9. $11 E+16$

9.AIE+WE

8.4IE+

8. $41 E+B$

7. $59 E+1 A k$

$7.89 E+13 E$

$7.61 E+d b$

$7.61 E+\partial v$

$7.58 F+v_{k 1}$

$7.58 E+W H$

$6.36 E+i v$

$6.36 E+i 3 v$

$6.3 U E+\angle E$

$6.30 E+D V$

$6.83 E+100$

$6.83 E+11.1$

5. $18 E+A U$

5. $\triangle 8 E+\triangle B$

$9.61 E+6)$

$7.61 E+\Delta U$

$7.29 E+196$

$7.29 E+D D$

$4.53 E+b(t)$ 


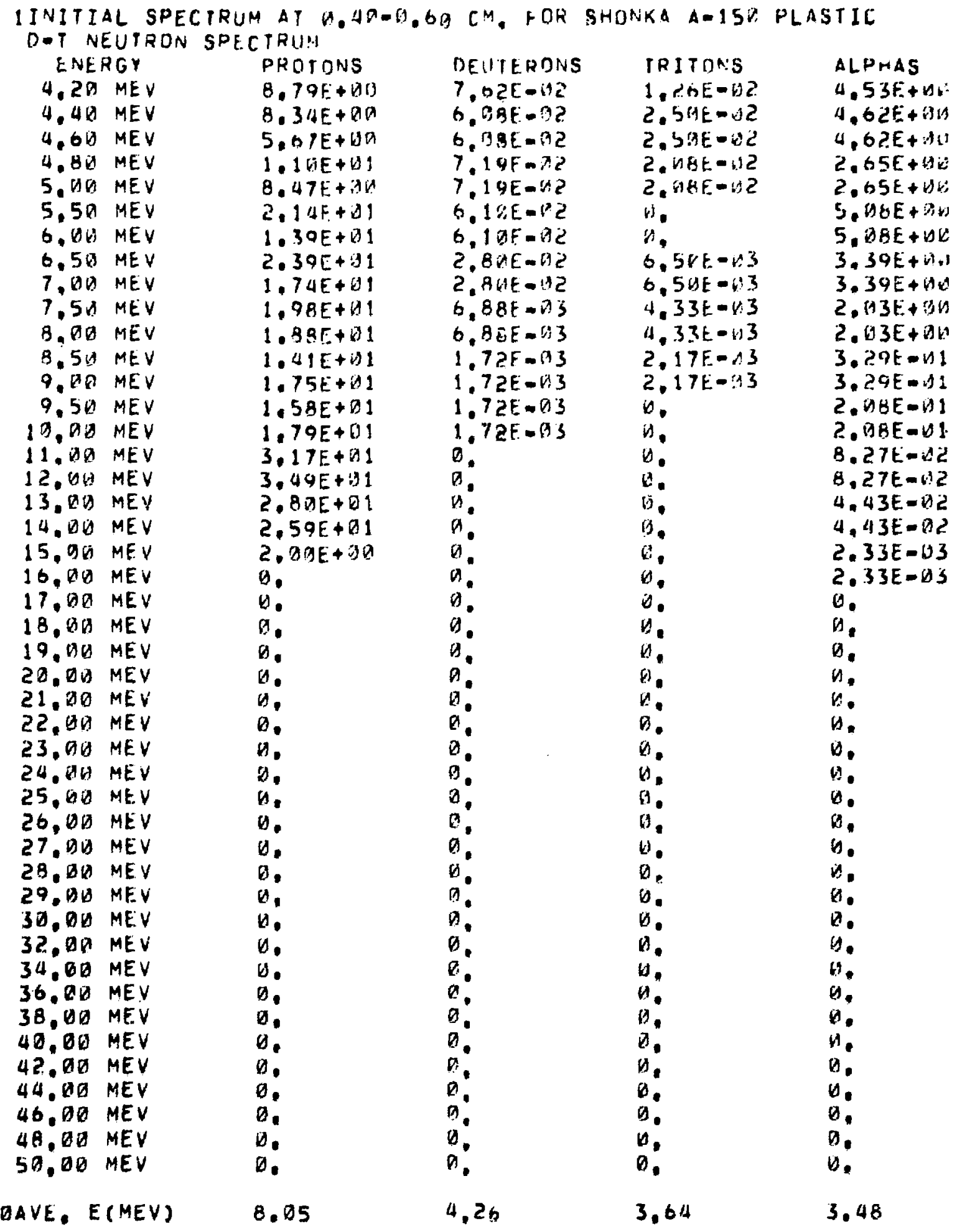




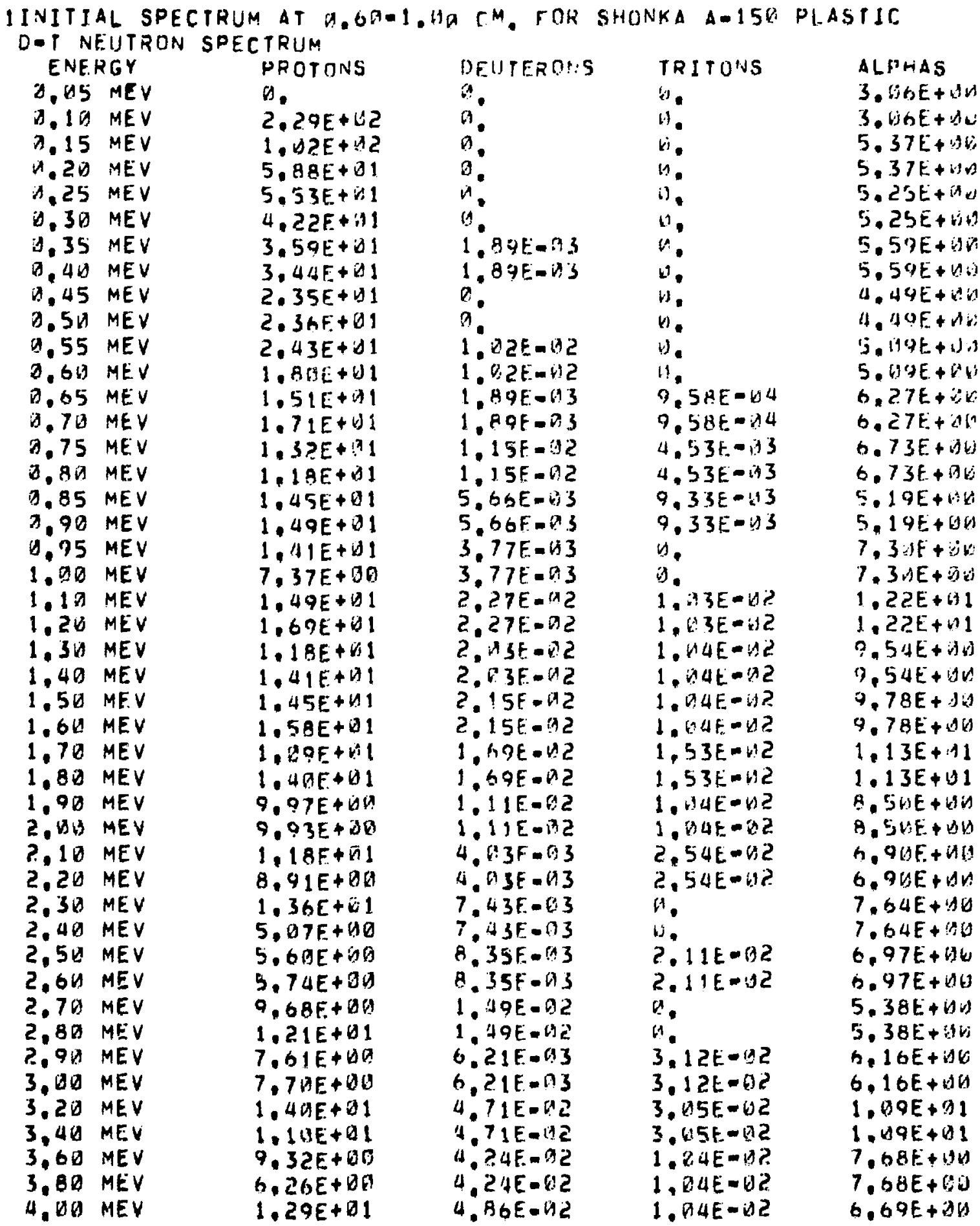


1INITIAL SPECTHUM AT D.6A-1, DU CM. FUJH SHUMKA A-15Ó PLASTIC DIT NELITRON SEECTRUM

\section{ENERGY}

4.2: MEV

$4,40 \mathrm{MEV}$

4.60 MEV

$4.80 \mathrm{MEV}$

$5,00 \mathrm{MEV}$

$5,50 \mathrm{MEV}$

6.DO MEV

$6,50 \mathrm{MEV}$

7. OD MEV

7.5B MEV

8,00 MEV

A. 50 MEV

$9.90 \mathrm{MEV}$

$9.50 \mathrm{HEV}$

10.00 MEV

$11.00 \mathrm{MEV}$

$12 . \triangle 0 \mathrm{MEV}$

13,00 MEV

14.00 MEV

15, UD MEV

16. HO MEV

17. DO MEV

$18.01 \mathrm{MEV}$

19.418 MEV

$20,00 \mathrm{MEV}$

21. DO MEV

$22.00 \mathrm{MEV}$

$23.00 \mathrm{MEV}$

24. DO MEV

25, ME MEV

$26.00 \mathrm{MEV}$

$27.00 \mathrm{MEV}$

$28.00 \mathrm{MEV}$

$29.00 \mathrm{MEV}$

3D.DO MEV

$32.00 \mathrm{MEV}$

$34.00 \mathrm{MEV}$

36.01 MEV

38. MV MEV

$4 A .00 \mathrm{MEV}$

42. DD MEV

44,06 MEV

46.00 MEV

$48.00 \mathrm{MEV}$

50.01 MEV

gAVE, E(MEV)
PFOTONS

1. $17 E+01$

1. $k 4 E+B)$

$1.23 E+\Delta 1$

$9,40 E+100$

$1,65 E+81$

$2,31 E+11$

$2,68 E+01$

$1.79 E+11$

$2.17 E+D 1$

$1.63 E+91$

$1.51 E+01$

$1.31 E+b 1$

$1.314 E+11$

$1.04 E+61$

$2.8 B E+B 1$

$3.11 E+11$

3. $92 E+61$

2. $\triangle B E+U 1$

$20 \Delta E+\Delta 1$

3. DRE + U

$\theta$.

0.

$\theta$.

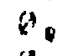

G.

$n_{0}$

b.

a.

0.

s.

$v_{0}$

i.

i.

n.

H.

w.

i.

i.

o.

:

i.

is.

$\theta$,

7.59
DEUTERUMS

$4.86 E-112$

$6.198-9$ ?

6. $1 B E-92$

3. OSE-E

$3, c 5 E-a^{4}$ ?

$4,76 E-192$

$4,7 b E-17 C$

$2.33 E-0.2$

?. $33 E-A 2$

$1.15 E-B ?$ ?

$1.15 E-92$

H. VISE-LI 3

B. PSE-CIS

11 .

!)

:

n.

i.

:

:

$n$

a.

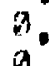

a.

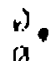

a.

$n$

$n$

D.

.

9

.

.

.

9

0

$a$

0

i.

$\theta$

a.

0

0

4.63
IEITONS

1. $14 E-62$

$1.75 E-62$

$1.75 E-62$

$9.72 t=63$

$9.72 E-B 3$

1. $S B E=B 2$

$1.58 E=n 2$

$4.36 E-1) 3$

$4.36 \mathrm{~F}-03$

i.

v.

1. $\forall B E-13$

1. $\triangle B E E-13$

$\because$.

a.

b.

w.

b.

$\Delta$

n.

b.

i.

0.

0

A.

b.

D.

ii.

$n$

s.

r.

a.

i.

0 .

$\forall$.

$H_{1}$

o.

0

is.

.

$\theta$

$a$.

$\theta$

$\theta$

4.

3.73
ALPMAS

6. $69 E+D K$

$4.69 E+1041$

$4.64 E+20$

$2.79 E+40$

$2.74 E+B A$

$5.19 E+D B$

5.1 $1 E+10$

3. 5 LIE + DD

$3.5 P E+10$

$3.76 E+116$

$1.7 \mathrm{BE}+\mathrm{BH}$

?.A AE-U1

$2.87 E-11$

1. 3GE=A1

$1.3 G E-11$

$3.04 E=32$

B. 64E-WI2

$1.98 E-D 2$

$1.96 E-\Delta C$

$5.33 E=03$

$5.83 E=1) 3$

औ.

,

D.

.

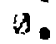

$b$,

a.

$\theta$,

$\theta$.

in.

0.

$a$

$\theta$

D.

n.

B.

0

0

a.

H.

.

.

9.

$\Delta$.

3.32 


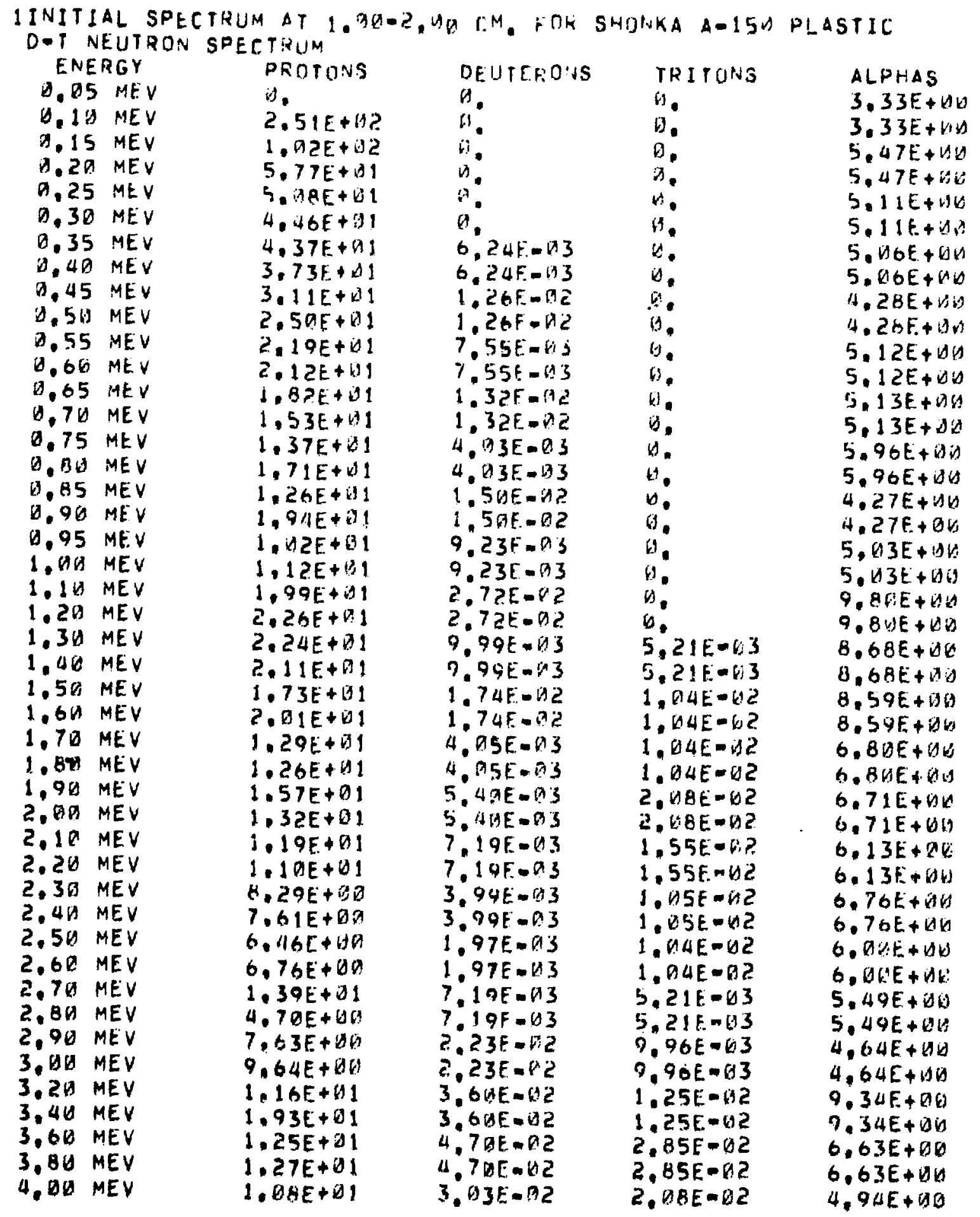


IINITIAL SPECTRUH AT 1.OD-2,DG CM. FOH SHUPKA A-ISIA PLASTIC DAT INEUTRON SPECTRIJM

$$
\text { ENERGY }
$$

4.20 MEV

4.46 MEV

4.6D MEV

$4.80 \mathrm{MEV}$

5. OD MEY

5,50 MEV

6.00 MEV

$6.50 \mathrm{MEV}$

7.00 MEV

$7.5 W$ MEV

B. OU MEV

$8.5 B$ MEV

9. D) MEV

9.50 MEV

10.00 MEV

11.00 MEV

12.00 MEV

13, 10 MEV

$14,00 \mathrm{MEV}$

15,06 MEV

16. 9 O MEV

$17.00 \mathrm{MEV}$

18,00 MEV

19.00 MEV

2月. OB MEV

21.00 MEV

22. DO MEV

23. $10 \mathrm{MEV}$

24.00 MEV

25. DO MEV

$26,0 D M E V$

27. OQ MEV

2B, DO MEV

29. BO MEV

30. UQ MEV

32,00 MEV

34.00 MEV

$36.00 \mathrm{MEV}$

38.00 MEV

4D. OD MEV

42,0 MEV

$44.00 \mathrm{MEV}$

46.00 MEV

48, DO MEV

50.00 MEV

DAVE. E(MEV)
PROTONS

8.51E+ 90

$1.16 E+11$

$1.27 E+\square 1$

1. $11 E+01$

$9,54 E+\triangle A G$

Z, $B 2 E+D 1$

$2.56 E+11$

1.98E+ 11

$1.99 E+61$

$1.67 \mathrm{E}+01$

1. $4 A E+Q 1$

$1.69 E+D 1$

$1.41 E+11$

$1.62 E+101$

$1.29 E+D 1$

$2,32 E+11$

2. $55 E+131$

2. $53 E+1$

2. DBF + DI

5. GOE - LI!

a.

a.

$\theta$

$\Delta$.

9.

0.

0.

0.

$b$.

0.

h.

n.

$\theta$

$\theta$

$\Delta$.

0.

$\theta$.

0.

0.

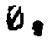

$\theta$.

0.

0

0.

7.96
DEUTEROAS

3. $) 3 F-02$

?. ИTE I II 2

$\therefore .87 E-192$

b. $11 t-A 2$

$6.11 E-B 2$

5. $4 B E=C^{4}$

$5.48 t-02$

1. $24 \mathrm{~F}-\mathrm{BC}$

1. $24 E-B 2$

$1.62 E=63$

$1.62 E=43$

$7.19 E=\pi 3$

$7.19 E-13$

$n$

a

n

?

0

is.

a.

$\theta$

a.

a

$a$

a

0

6

a.

0

0

0

0

0

0.

4.48
TRITUNS

2. $38 E-112$

$6.56 \mathrm{k}=\mathrm{ki}$

$6.56 E-2,3$

$1.66 E-112$

$1.66 E-112$

2. BOE-B?

2. $\triangle O E-\square 2$

2, $14 E=-13$

2. $14 E=13$

$5.35 F-03$

$5,35 f: 0.3$

n.

b.

a.

6

b.

b.

0.

0

i.

i.

b.

0.

0.

3

v.

b.

a.

b.

$\theta$.

0

$B$

is.

9.

$\theta$

0.

b.

0.

0

$\theta$

a.

a.

4,18
ALPHAS

$4.94 E+6 I$

3. $46 E+9 n$

3. $46 E+i 00$

2. $41 E+010$

2. $41 E+O Q$

$4.13 E+6 v$

$4.13 E+Q V$

$3.25 E+v i$

$3.25 E+W V$

1. $45 E+b V$

$1.45 E+46$

$2,3 E E-01$

$2.38 E-61$

$1.15 E=41$

1. $15 E-B 1$

$5.28 E-142$

$5.28 \varepsilon-\Delta 2$

$2,44 E-102$

$2,44 E=02$

$4.66 E-163$

$4.665-03$

b.

n.

b.

a.

H.

0.

(1).

a

$\theta$

v.

e.

a.

D.

$\theta$

$\theta$

i.

U.

n.

b.

$\theta$.

$\theta$

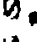

.

$b$.

3,31 


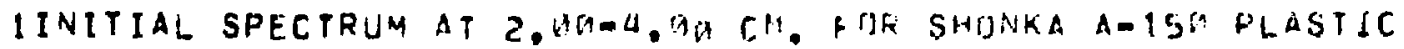
D-T NEUTRON SPECTRUIA

$$
\text { ENERGY }
$$

D. OS MEV

$0.18 \mathrm{MEV}$

0.15 MEV

ग. 2 W MEV

0.25 MEV

D. 3U MEV

0.35 MEV

0. 40 MEV

0.45 MEV

0.50 MEV

Q.55 MEV

$0.00 \mathrm{MEV}$

0.65 MEV

$0.70 \mathrm{MEV}$

0.75 MEV

0.80 MEV

0.85 MEV

0.90 NEV

Q.95 MEV

1. DO MEV

$1.10 \mathrm{MEV}$

$1.26 \mathrm{MEV}$

1.3O MEV

1,40 MEV

$1.50 \mathrm{MEV}$

1,60 MEV

1,70 MEV

1. RD MEV

1.90 MEV

$2.00 \mathrm{MEV}$

2.10 MEV

2. 2У MEV

2,30 MEV

2.4 MEV

2.50 MEV

2,60 MEV

2.70 MEV

2.8 A MEV

2,90 MEV

3.OU MEV

3.2D MEV

3. 4D MEV

3,60 MEV

3.60 MEV

4. 19 MEV
PROTOHS

O.

द. $O P E+9 ?$

1. $10 E+B 2$

B. OOE + DI

6. WUE 1 D 1

$4.79 E+01$

4. $13 E+1$

$3.05 E+41$

2. $82 E+01$

3. $\{$ SE + 11

2. $25 E+W 1$

2. $23 E+11$

$1.91 F+1]$

2. $12 E+1$

$1.92 E+11$

$1.8 M E+131$

$1.34 E+O 1$

1. $82 F+1$

$1.64 E+61$

1. $2 B E+\angle 1$

2. $\mid 2 F+\Delta 1$

$1.93 E+91$

2. $34 E+11$

1. $78 E+101$

$1.96 E+B 1$

$1.75 E+1$

$1.36 E+11$

$9.75 E+10$

1. $53 E+1$

$9.92 E+66$

$8.36 E+09$

1. $2 A E+B 1$

1. DQE + D1

$9.06 E+00$

7.12E+DD

1. $) 3 E+B 1$

3. $86 E+39$

$6.42 E+40$

$7.6 \Delta E+U A$

7. $23 E+110$

1. $11 E+11$

1.17E+D1

1. 1 1 EE+B1

$9.23 E+00$

$1.20 E+41$
DEITERITHS

1

$\pi$

$n$

(iv)

ii.

2. ALE-VI

?. OUE $-l^{2} 3$

3. $77 F-0.3$

$3.77 E-13$

R. OQE- 3

5. $29 F=3$

2. MUE $=12$

2. $M U E-\lambda 2$

6. OIF-HS

6. DUF-in 3

1. R.RE-R3

7. 5 ? -53

$3.776-53$

$3.77 E-43$

$1,33 E-9 ?$

$1.33 E-A 2$

2. $72 E-112$

2. $72 E-4$ त?

1. $8 B E-W 2$

1. ARE-VI

1. OSE - HL

1. $\triangle S E-112$

1. $17 \mathrm{E}=\mathrm{AC}$

$1.17 E-i) 2$

6. $B 6 E=13$

6. BGE-D3

$9.35 E-193$

$9.35 E-13$

2. $\triangle 1 E-12$

2. $\triangle 45-B 2$

$3.33 E-172$

$3.33 E-02$

1. $54 \mathrm{~F}=0.2$

$1.54 E-1) 2$

$5.43 E-D 2$

$5.43 E-$ ज)

$3 . \%$ UE -D2

3.7GF. - 12

2. $47 E-12$
IRITORS

b.

i.

$\therefore$

4)

1.

i.

n.

b.

4. $R 3 E-43$

$4.83 t-43$

$\therefore$

6

(s)

b.

1.

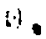

$4.83 E-43$

$4.83 E-4$

$0.31 E-4.3$

6. $S 1 E=E .3$

$1,47 E-j 2$

$1.47 \mathrm{E}-4 \mathrm{t}$

1. $\square 4 E-42$

1, GUE- 12

$1,56 E-V \overrightarrow{2}$

$1.56 E-632$

$4.57 E-W 3$

$4.57 E-63$

$5.21 E-U 3$

$5.21 E-413$

$1.54 E-1.2$

$1,54 E-i 2$

2. $62 E-12$

2. $62 E-11$ ?

2. $V T E-H Z$

2. $И T E-\triangle 2$

?. $P A E-B 2$

2. VUE-UL

1. HSE-VIZ

1. $15 E-W 2$

1.56E-D?

$1.56 E=42$

5. $21 E-U 3$

$5.21 E-0.3$

$1.6 \| E-B 2$
ALPRAS

2. UIE+ DiU

2. $11 E+i g$

3.5 HE + DU

$3.54 E+4 \pi$

$4.86 E+106$

$4.86 E+i j W$

4.76E+140

4. $76 E+46$

$4.27 E+4 B$

4.2. $7 \mathrm{E}+\mathrm{U} \mathrm{k}$

4. SSE +A H

4. ASE + A

4. $A 9 E+B C$

4. $\triangle 9 E+30$

$5.15 E+i t)$

$5,16 E+15$

A. $66 E+4 V$

$4.60 E+\omega n$

$4.77 E+\Delta V$

$4,77 E+M E$

7. $6 B E+10$

$7.68 E+20$

8. $33 E+46$

$9,33 E+i 9 w$

$6.99 E+18$

$6.39 E+1 \%$

7. $4 / E+d W$

7. $47 \mathrm{E}+4 \forall$

b. $81 E+B$ H

$6.81 E+U 6$

$5.73 E+B D$

$5.73 E+0 v$

b. $23 E+\Delta y$

$6.23 E+10$

4. $B E E+A Q$

4. $B D E+$ b

3. $5 B E+B A$

3. $58 E+114$

$4.36 E+16$

$4.36 E+166$

7. I $2 E+\Delta D$

$7.72 E+16$

5. $19 E+36$

$5.49 E+A w$

$3.3 B E+C B$ 
IINITIAL SPECTRUM AT 2.HO-4, MA CM. FOR SHDHKA A-1SR FLASTIC DIT NEUTRON SPECTRUM

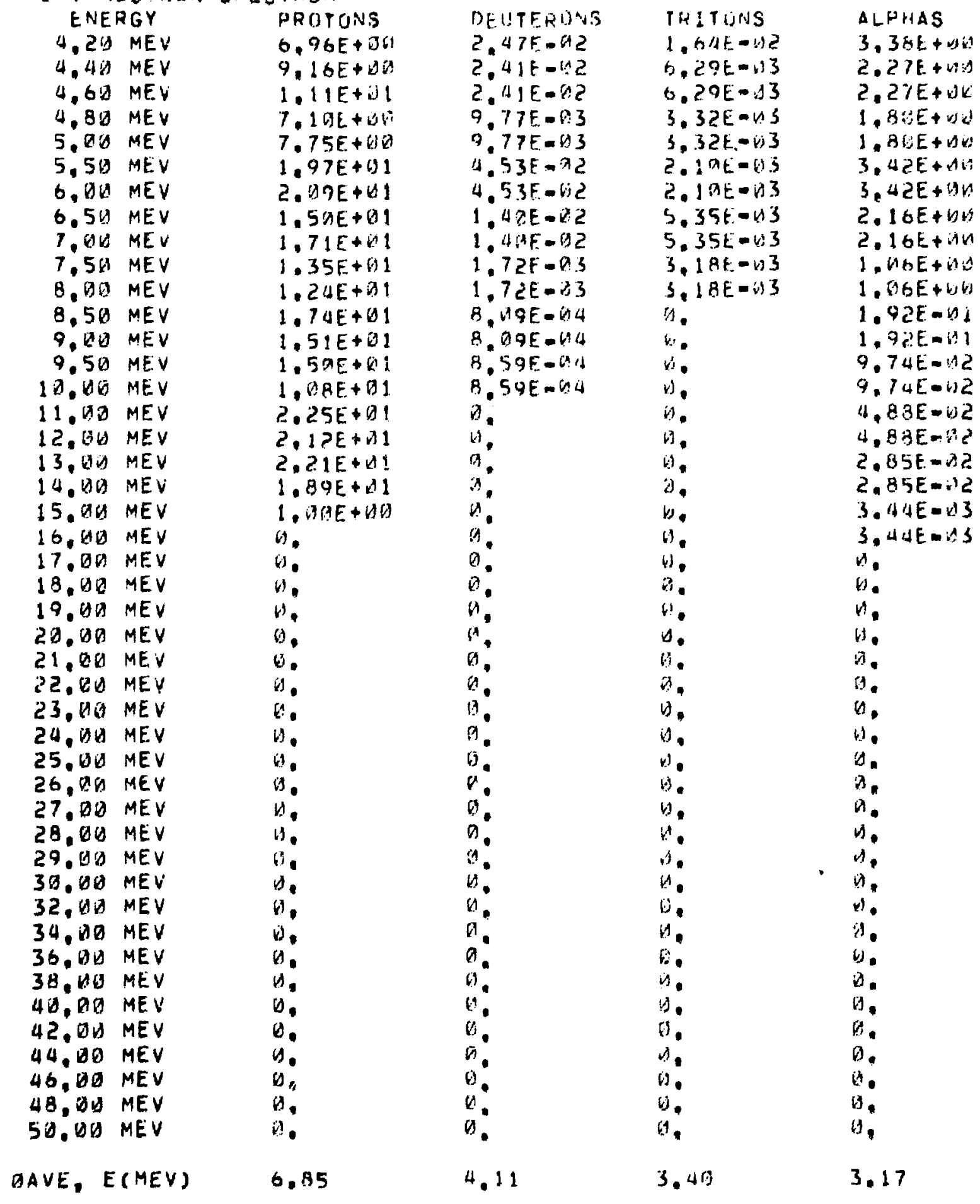




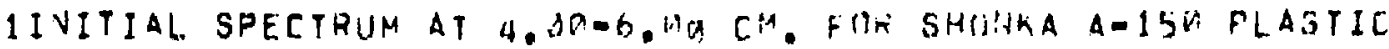
D-T NEUTRON SPECTRUM

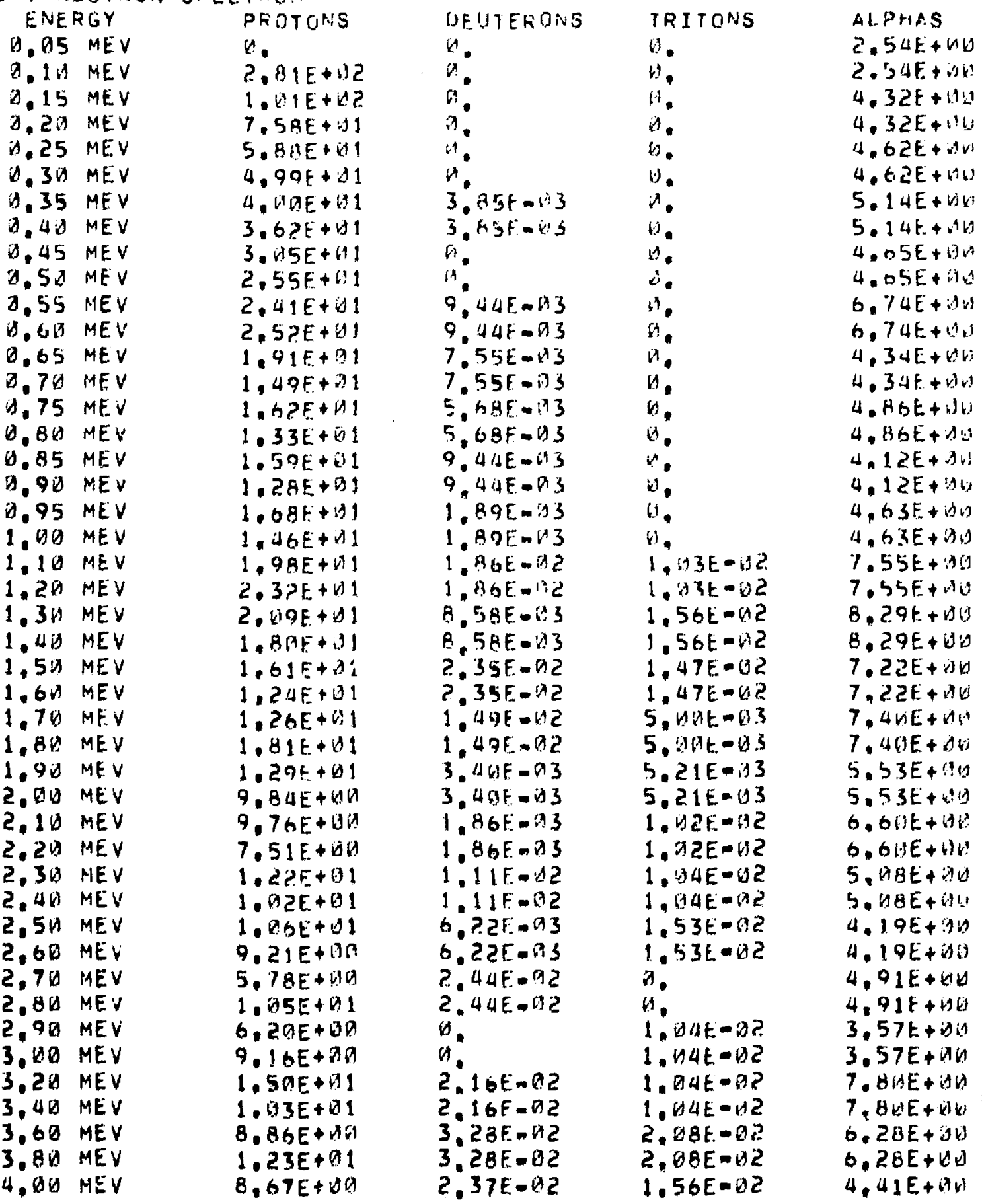




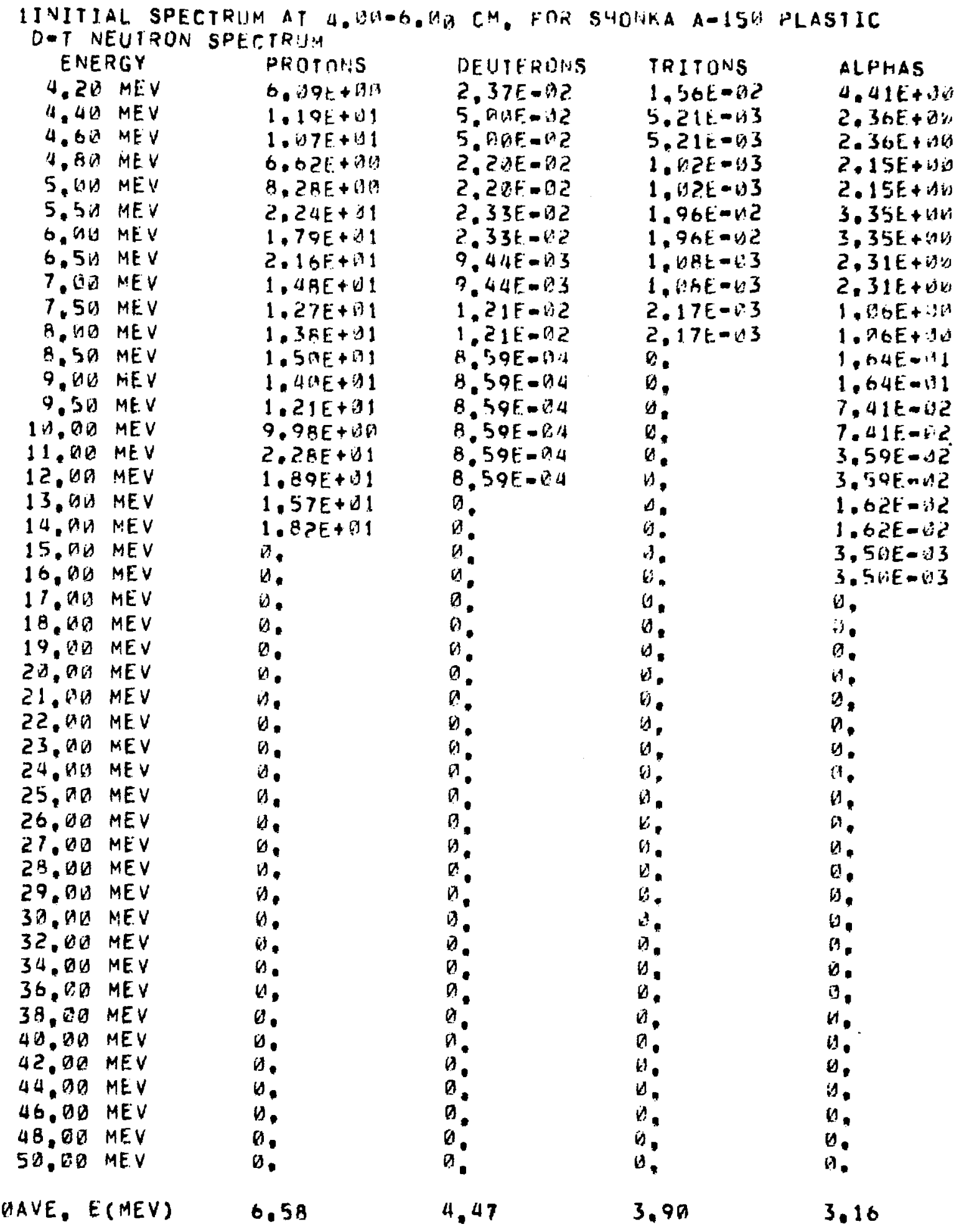


IINITIAL SPECTRUM AT 6.BO-10, CM. FOR SHONKA AO15B PLASTIC D.T NEUTRON SPECTRUM EPERGY

T. 05 MEV

D.10 MEV

D. 15 MEV

0.20 MEV

D. 25 MEV

D. 3D MEV

D. 35 MEV

D. 40 MEV

0.45 MEV

0,50 MEV

0.55 MEV

$0.60 \mathrm{MEV}$

0.65 MEV

$0,70 \mathrm{MEV}$

0.75 MEV

0.80 MEV

0.85 MEV

$0.90 \mathrm{MEV}$

$0.95 \mathrm{MEV}$

1.OD MEV

$1.10 \mathrm{MEV}$

$1.20 \mathrm{MEV}$

$1.30 \mathrm{MEV}$

$1.40 \mathrm{MEV}$

$1.50 \mathrm{MEV}$

$1.60 \mathrm{MEV}$

$1.70 \mathrm{MEV}$

$1.80 \mathrm{MEV}$

$1.90 \mathrm{MEV}$

2.01 MEV

$2.10 \mathrm{MEV}$

2.20 MEV

$2,30 \mathrm{MEV}$

$2.40 \mathrm{MEV}$

$2.50 \mathrm{MEV}$

$2.60 \mathrm{MEV}$

2.70 MEV

2.80 MEV

$2.90 \mathrm{MEV}$

3.DO MEV

$3.20 \mathrm{MEV}$

3.40 MEV

3.60 MEV

$3.80 \mathrm{MEV}$

4. OD MEV

\section{PROTONS}

$D$.

$2,59 E+\forall 2$

$1.84 E+112$

$7.11 E+\Delta I$

$5,45 E+101$

$4.67 E+71$

$3,71 E+01$

$2.76 E+21$

$2.4 B E+81$

$2,2 b E+01$

$2.69 E+101$

$2.39 E+21$

$1.95 E+21$

2. $) \triangle E+01$

1.59E+ 31

1. $40 E+01$

$1.26 E+B 1$

$1.53 E+1$

$1.82 E+11$

$1,43 E+101$

3. $07 E+21$

2. $10 E+Q 1$

$1.73 E+01$

$1.69 E+01$

$1.92 E+111$

1. $52 E+01$

$8,51 E+D 0$

$1.34 E+B 1$

$1.31 E+D 1$

1. $O Q F+a 1$

$1.13 E+1$

$8.57 E+00$

$7.63 E+02$

$7.65 E+10 B$

$1.09 E+101$

$6.07 E+10$

$8.83 E+100$

$6.05 E+0 D$

$6.03 E+B 0$

$3.6 B E+00$

1. $\triangle B E+Q 1$

1. $22 E+01$

$9.18 E+Q D D$

$8.71 E+B 0$

$1.19 E+B 1$
DEUTERONS

D.

2 .

$\theta$

$\theta$

0

0.

$1.895 .=13$

$1.89 \mathrm{E}-\mathrm{OB}$

$6.12 F-D 3$

$6.12 E-D 3$

$7,77 E-.93$

$7.77 t .03$

$5.76 E-A 3$

$5,76 E-D 3$

$5.66 E-D 13$

$5.66 E-113$

1.17E-DC

$1.17 E-1) 2$

1. RQE-G2

1. $39 E-192$

2. $34 E=92$

$2.34 E-92$ ?

$9.8 B E-23$

9 BBE-G3

$1.31 E-U 2$

$1.31 E-12$

3. QSE-A3

$3.95 E-0.3$

1. $2 T E-12$

1.2TE-O2

$6.61 E=03$

6. GIE-OB

$2.72 E-02$

2. $72 E-172$

3. B?E-D3

$3.32 E=133$

$4.33 E-03$

$4.33 E-D 3$

$9.10 E-1) 4$

$9.10 E=B 4$

$4.19 E-132$

$4.19 E-192$

2. $49 E-92$

$2,49 E-112$

$1.63 E-02$
TRITUNS

D.

$D$

a.

,

G.

$\theta$.

$\theta$.

$\Delta$

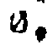

0.

$4,83 E=03$

$4.83 E-03$

$4.41 E-03$

$4.01 t-03$

n.

.

$\forall$

a.

$\Delta$.

0.

$5.58 E-B 3$

$5,5 B E=113$

$4,65 E-d 3$

$4.65 E-03$

$3.66 \mathrm{E}-13$

$3.66 E-D 3$

$9.91 E-\theta 3$

$9.91 E-03$

1. $\triangle \triangle E=A 2$

$1.04 E-42$

$1.04 E-52$

$1.04 E-12$

$0.22 E-03$

$6.22 E-03$

$1.52 E-\theta 2$

$1.52 E-B 2$

$1.04 E-(1) 2$

1. OUE DOZ

2. 57E- $\{$ ?

2.5TE-BC

2. $11 E=02$

$2.91 E-B 2$

$1.53 E=02$

$1.53 E-\Delta 2$

$9.43 E=\square 3$
ALPHAS

2.39E+13

$2.39 E+B V$

2. $B O E+14 D$

2. BOE + OD

4. $10 E+90$

4. 16E+CZ

$3 \cdot 54 E+96$

3. $54 E+2 W$

3. $58 E+06$

$3.58 E+B$

3. $49 E+10$

3. $49 E+19 E$

3. $19 E+\Delta 4$

3. $19 E+\Delta b$

$4.29 E+116$

$4.29 E+4 D$

$3,85 E+101$

$3.85 E+10 b$

2. $B T E+Z U$

E. $87 E+$ D.

$5.62 E+20$

$5,62 E+1) 6$

$6.01 E+b 0$

S.DIE+ BD

$5.48 E+7 \theta$

5. $4 E E+d \square$

5. $4 B E+1 D$

$5.48 E+1)$

$4.32 E+D O$

4. $32 E+$ iH 10

$3.96 E+00$

3. $9 \Delta E+\theta \|$

$3.42 E+40$

3. $42 E+19$

3.77E+ 19

$3.77 E+\triangle 6$

$3.72 E+30$

$3.72 E+01$

2. $45 E+90$

$2.45 E+6 \theta$

$5.62 E+b 10$

$5.62 E+00$

$3.89 E+B V$

$3.89 E+40$

$3,0 u E+d U$ 
IINITIAL SPECTRUM AT 6.BO-10.H CH. FOR SHONKA A-15B PLASTIC DIT NEUTRON SPECTRUM

ENERGY

4.20 MEV

4. 40 MEV

4.60 MEV

4. 80 MEV

$5.90 \mathrm{MEV}$

$5.50 \mathrm{MEV}$

$6.00 \mathrm{MEY}$

$6.50 \mathrm{MEV}$

7.00 MEV

$7.50 \mathrm{MEV}$

Q.00 MEV

B.50 MEV

9.00 MEV

$9.50 \mathrm{MEV}$

10.00 MEV

$11.90 \mathrm{MEV}$

$12,00 \mathrm{MEV}$

13,00 MEV

14.010 MEV

15,09 MEV

$16.00 \mathrm{MEV}$

$17.00 \mathrm{MEV}$

18.00 MEV

19.00 MEV

$20.00 \mathrm{MEV}$

$21.00 \mathrm{MEV}$

22. DO MEV

23. DO MEV

24,00 MEV

$25.00 \mathrm{MEV}$

26,00 MEV

27. DO MEV

28.00 MEV

29.01 MEV

$30.00 \mathrm{MEV}$

32.00 MEV

$34, D E$ MEV

$36,00 \mathrm{MEV}$

$38.00 \mathrm{MEV}$

$40.00 \mathrm{MEV}$

42.99 MEV

$44.00 \mathrm{MEV}$

46.00 MEY

$48.50 \mathrm{MEV}$

$50.00 \mathrm{MEV}$

DAVE. E(MEV)
PROTONS

1. $21 E+11$

7.1 $1 \cap E+00$

$8.37 E+\square D$

5. $28 E+10$

$8.49 E+D 0$

$2.11 E+11$

$2.41 E+31$

$1.49 E+B 1$

$1.52 E+01$

$1.27 E+1$

1. $14 E+B 1$

1. $50 E+01$

$1.23 E+B 1$

$8.69 E+00$

$5.92 E+109$

$1.87 E+01$

$1.37 E+101$

2. $45 E+01$

2.17E+ 11

1. $\triangle 2 Z E=02$

B.

a.

D.

0.

0.

$\theta$

a.

a.

$B$.

$a$.

a.

1.

a.

0.

0.

.

D.

0

0.

0

0.

0.

0

0

0

6.77
DEUTERONS

$1.63 E-92$

3. $77 E=U$ ?

3. $77 E-\triangle 2$

1. $44 E-02$

$1.44 E-72$

$4.84 E-02$

4. BUE-OL?

$1.94 E-B 2$

1. $94 E-02$

4. $3 \triangle E-03$

$4.34 E-B 3$

a.

0.

$8.59 E-04$

$8.59 E-04$

9

a.

9

a.

9

0.

0.

d)

0

0

0.

0

$a$

$a$

$a$

0

0

0

0.

4.47
TRITONS

9. $43 E-03$

$6.17 E-\Delta 3$

$0.17 E=03$

2. $25 E=03$

2. 25E-103

8. 39E- 03

8.39E-OS

$4.41 E-03$

4. $41 E-03$

2. $17 E-03$

2. $17 E=03$

0

$\theta$

a.

b.

9.

0.

$\theta$

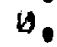

$\theta$.

0

$\theta$

0

0.

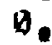

0.

4.

b.

0

D.

is.

0

6.

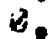

6.

0

$\theta$.

$\Delta$.

$\theta$.

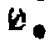

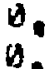

0.

0

3.75
AlPHAS

3. DUE +UB

$1.95 E+110$

$1.95 E+00$

$1.64 E+i 0$

$1.64 E+30$

$2.66 E+\Delta B$

$2.66 E+100$

1. $86 E+00$

$1.86 E+i J 6$

7.7OE-G1

7.7UE= DI

$1.77 E-41$

$1.77 E-U 1$

5.97E-62

$5.97 E-42$

$5.22 E-\Delta 2$

5. ग2E- ID

$1.16 E-\square 2$

1. $16 E-162$

$3.43 E-03$

$3.43 E-13$

a.

3

a.

Q.

i.

0.

0.

$\theta$

.

a.

6

n.

a.

i.

b.

0.

$\theta$

$\theta$

13.

$\Delta$

0.

0.

0

b.

3,23 
IINITIAL SPECTRUM AT 1H. D-14.D CM. FOR SHONKA A-15B PLASTIC DWT NEUTRON SPECTRUM

\section{ENERGY}

D. 5 MEV

$0.10 \mathrm{MEV}$

0.15 MEV

9.2U MEV

0.25 MEV

$0.30 \mathrm{MEV}$

0.35 MEV

0.40 MEV

0.45 MEV

$0.50 \mathrm{MEV}$

$0.55 \mathrm{MEV}$

Q.60 MEV

0.65 MEV

0.70 MEV

0.75 MEV

$0.80 \mathrm{MEY}$

$0.85 \mathrm{MEV}$

0.90 MEV

0.95 MEV

1. 00 MEV

$1,10 \mathrm{MEV}$

1.20 MEV

$1.30 \mathrm{MEV}$

1.40 MEV

$1,50 \mathrm{MEV}$

1,60 MEV

1.10 MEV

$1, B 0$ MEV

1.90 MEV

2.00 MEV

2,10 MEV

2,20 MEV

2.30 MEV

2.40 MEV

2,50 MEV

$2.60 \mathrm{MEV}$

2,70 MEV

2.BO MEV

2,90 MEV

3.00 MEV

3.29 MEV

3.40 MEV

3.60 MEV

3.80 MEV

4.00 MEV
PROTONS

$D$.

$2.64 E+52$

1. $\triangle 4 E+B 2$

7. $26 E+B 1$

5. $79 E+\angle 1$

5. $02 E+\Delta 1$

3. $27 E+61$

$3.32 E+Q 1$

2. $48 E+01$

2. $17 E+1$

2. $27 E+01$

1. $74 E+101$

$1.62 E+01$

1. GAE+01

$1.91 E+101$

$1.55 E+11$

$1.5 a E+D 1$

$9.87 E+D D$

1.20E+ 11

$9.53 E+180$

1.76E+ 1

$1.47 E+11$

$1.61 E+01$

1. $51 E+B 1$

1. $34 E+D 1$

1. $4 B E+D 1$

1. $D 7 E+B 1$

1.39E+D1

8.1 $14 E+D E$

$9.64 E+180$

$9.55 E+00$

1. BBE+ $B 1$

7. $26 E+10$

1. $24 E+11$

7. $46 E+00$

$6.50 E+Q 0$

7. $45 E+H B$

1. $\triangle E+D 1$

$6,8 S E+D D$

Q.51E+BO

$9.77 E+00$

1. $50 E+01$

Q.7AE +00

$9.17 E+B A$

B.94E+DQ
DEUTERONS

a.

a.

Q.

6.

0.

0.

$2.26 E-193$

2. 26E-D 3

8. QUE-OI

8. DUE-OB

7. $40 E-D 3$

$7.46 E-03$

4. DaE -03

4. BaE $=03$

3.75E-D3

3.75E-DS

0.

0

$6.37 E-03$

$6.37 E-03$

$9.47 E-93$

$9.47 E-D 3$

2. $D D E-02$

2. QUE-OL

7. $59 E=03$

$7.59 E-105$

$2.56 E-03$

2. 56E-03

$4.06 E-03$

4. MOE-A3

1. $44 E-D 2$

1. $44 E-D 2$

$9.03 E-D 3$

$9.3 E-03$

6. 2ZE-D3

$6.22 E-63$

$2.05 E-02$

2. Q5E-02

2. $59 E-12$

2. $59 E-02$

4. DSE $=02$

$4.05 E-02$

$4.94 E-B 2$

$4.94 E-02$

$2.08 E-02$
TRITONS

0.

H.

$\Delta$,

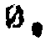

,

$\theta$.

H.

$\Delta$.

id.

c.

1. $Q 3 E-D 3$

1. $2 E-23$

$\theta$.

i.

0.

$\theta$

a.

a.

a.

0.

1. $.22 E-02$

1. $D 2 E-D 2$

$1,52 E-B 2$

1. $52 E-102$

$5.14 E-103$

5. $14 E-W 3$

1. $84 E M Q 2$

1. $04 E-U 2$

1. D2E- $\triangle 2$

1. $D 2 E-D 2$

2. $\triangle B E-42$

2. $\triangle B E=\mathrm{W} 2$

1. $04 E-B 2$

1. BपE-02

1. $02 E-92$

1. $D 2 E-B Z$

$2.01 E-62$

2.01E-02

0.

$\theta$.

$1.02 E-B 2$

1. D2E -02

4. $46 E-D 3$

$4.46 E .03$

2.15E-Q2
ALPHAS

2. $2 \mathrm{GE}+6 \mathrm{JB}$

2. 2UE+ 10

2.39E+ $4 U$

2.39E+130

3. $29 E+i b)$

3. $29 E+0.4$

2. $7 B E+D 0$

2. $78 E+Q 19$

2. $86 E+16$

2. $86 E+Q D$

2. $81 E+\Delta W$

2.81E+

$2,81 E+60$

2. $81 E+B i 3$

$2.47 E+00$

$2.47 E+66$

$2.55 E+1$

$2,55 E+3 v$

$2.41 E+D$

$2.41 E+\Delta B$

$4.91 E+\Delta d$

$4.91 E+44$

5. OIE + DQ

$5.61 E+B 6$

$5.03 E+U()$

$5,03 E+00$

4. $22 E+11 D$

$4.22 E+14$

$3.64 E+00$

$3.64 E+U D$

$4.20 E+\Delta U$

4. $20 E+636$

$3.52 E+60$

3. $52 E+B D$

4. $38 E+U V$

$4.38 E+O G$

3. $13 E+B U$

$3.13 E+00$

$3.37 E+B U$

$3.37 E+B D$

$4.05 E+130$

$4.85 E+40$

$3,25 E+00$

$3.25 E+Q 0$

$2.41 E+U 0$ 


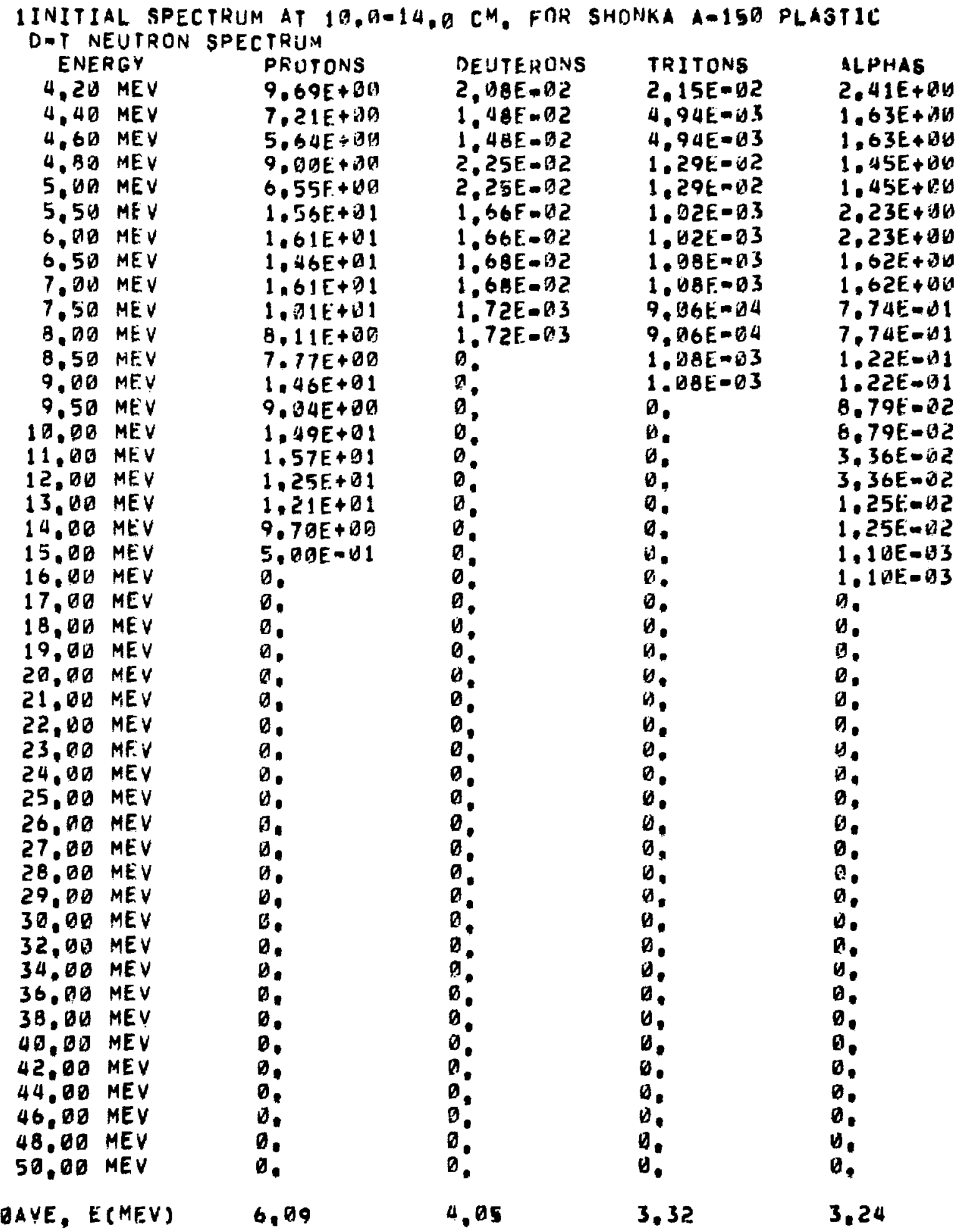


IINITIAL SPECTRUM AT 14, GHAO.O CM. FOR SHONKA A-15H PLASTIC D-T NEUTRON SPECTRUM

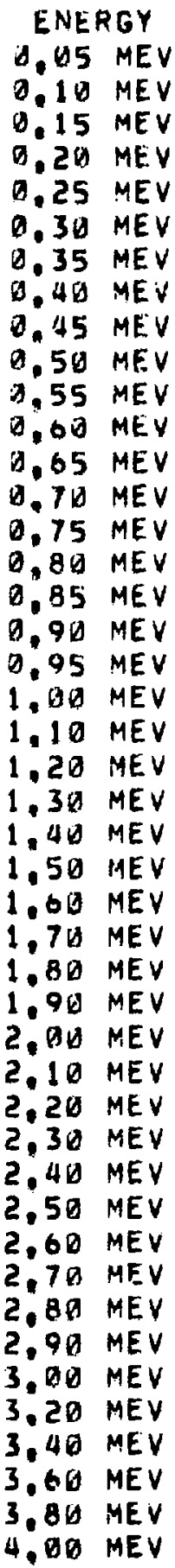

PROTONS

(1)

1.16E+Q2

4. $53 E+01$

3. $H B E+Q 1$

2. $5 D E+Q 1$

2. $D T E+\nabla 1$

$1.72 E+01$

$1.53 E+101$

$1.28 E+\Delta 1$

$1,14 E+B 1$

1. $\triangle B E+D 1$

9. $46 E+09$

$8,55 E+1 D$

B, 3ดE+00

$7.91 E+B D$

$6.87 E+0.9$

$7.07 E+136$

$6.57 E+B D$

4. $86 E+00$

$5.75 E+B B$

$1.03 E+01$

$8,60 E+\Delta B$

$B, 98 E+D G$

7.81E+DO

$6,58 E+O B$

$6,10 E+80$

$6.57 E+\Delta B$

5. $39 E+\Delta B$

$6.01 E+00$

5. $) 9 E+D 0$

$4,65 E+60$

$4.07 E+00$

3. $37 E+\theta U$

$4.13 E+\Delta \theta$

$4.07 E+00$

$3.36 E+00$

$3,30 E+00$

$3,50 E+B O$

$2.80 E+120$

$2.94 E+10$

$4.96 E+\square 0$

5. $16 E+B 0$

4. $06 E+100$

$4.60 E+00$

$4.62 E+00$
DEUTERONS

D.

:. OAE -0.4

2. $94 E-134$

$\theta$.

a.

B. Q UE DU 4

8. BUE-D4

$1.13 E-03$

$1,13 \mathrm{E}-03$

2. B2E-D3

2. $82 E-A 3$

1. TIE-H3

$1.91 E-03$

3.!OF-OS

3. $10 E-\{3$

2. $\triangle B E=A 3$

$2 . \triangle 8 E-\triangle 3$

3. $7 Q E=03$

$3.7 D E-03$

$5,13 E-63$

$5,13 E-W 3$

$4,2 \Delta E-\nabla 3$

4. $2 A E-A 3$

$4.06 E-03$

4.06E-03

$2.27 E-03$

$2.27 E-03$

2. DOE-Q3

2.00E-A3

$3.98 E-13$

$3.98 E-13$

$2.48 E-A 3$

2. 4 BE $\triangle 13$

$3.75 E-03$

$3.75 E-03$

$3.48 F-03$

$3.48 E-03$

$4.0 S E-03$

$4.05 E-B S$

1. OTE-D2

$1.07 E-D 2$

1. 1 BE-O2

$1.18 E-02$

$6,38 E-23$
TRITONS

H.

D.

.

$D$

B.

D.

$6.43 E-05$

$6.43 E-B 5$

$4.18 E-04$

$4.18 E-04$

$1.74 E=-14$

$1.74 E-94$

6.

A.

$6.72 E-104$

8. $72 E=04$

$7.76 E-\angle 5$

$7.76 E-115$

$8.01 E-B 4$

8. VIE- 4

$4.19 E-113$

4. $19 E-193$

3. $32 E-103$

$3.32 E-03$

$4.05 E=63$

$4.05 E-133$

$2.43 E-03$

$2,43 E-D 3$

3. $24 E=03$

$3,24 E-143$

$\exists .7 E-\Delta 3$

3. $07 E-03$

$1.56 E-03$

$1.56 E-03$

$3.91 E-B 3$

$3.91 F=63$

$4.19 E-03$

$4.19 E-B 3$

$1,98 E=113$

$1.98 E-03$

$4.60 E-03$

$4,60 E-03$

2. $89 E-03$

$2,8 Q E-B 3$

$1,80 E-03$
ALPHAS

6.72E= 01

6. $72 E-1$

9.91E-O1

$9,91 \mathrm{E}-\mathrm{H}$

1.17E+M

1. $17 E+v G$

1. $13 E+n v$

1.13E+ $13 H$

1. $22 E+\Delta Q$

$1 \cdot 22 E+A\}$

$1,19 E+4 k$

$1.19 E+\Delta D$

1.17E+ 13

$1.17 E+B O$

$1.25 E+40$

1. $25 E+4 W$

$1.26 E+H y$

1.26E+dV

1. $22 E+16$

1. $22 E+\Delta U$

2. $45 E+\Delta()$

$2,65 E+40$

2. $03 E+B D$

2. $D 3 E+D B$

1. $97 E+B a$

$1.97 E+B Q$

1. $82 E+B d$

1. $82 E+D i$

$1.56 E+B A$

$1.56 E+U D$

$1.55 E+D V$

1. $55 E+100$

$1.42 E+\forall G$

$1.42 E+H D$

$1.3 .3 E+36$

$1.33 E+106$

1. $13 E+40$

1. $13 E+D B$

1. $13 E+\Delta B$

1. $13 E+0 B$

$1.99 E+90$

$1.99 E+b U$

$1.47 E+60$

$1.47 E+10 \theta$

$1,21 E+00$ 


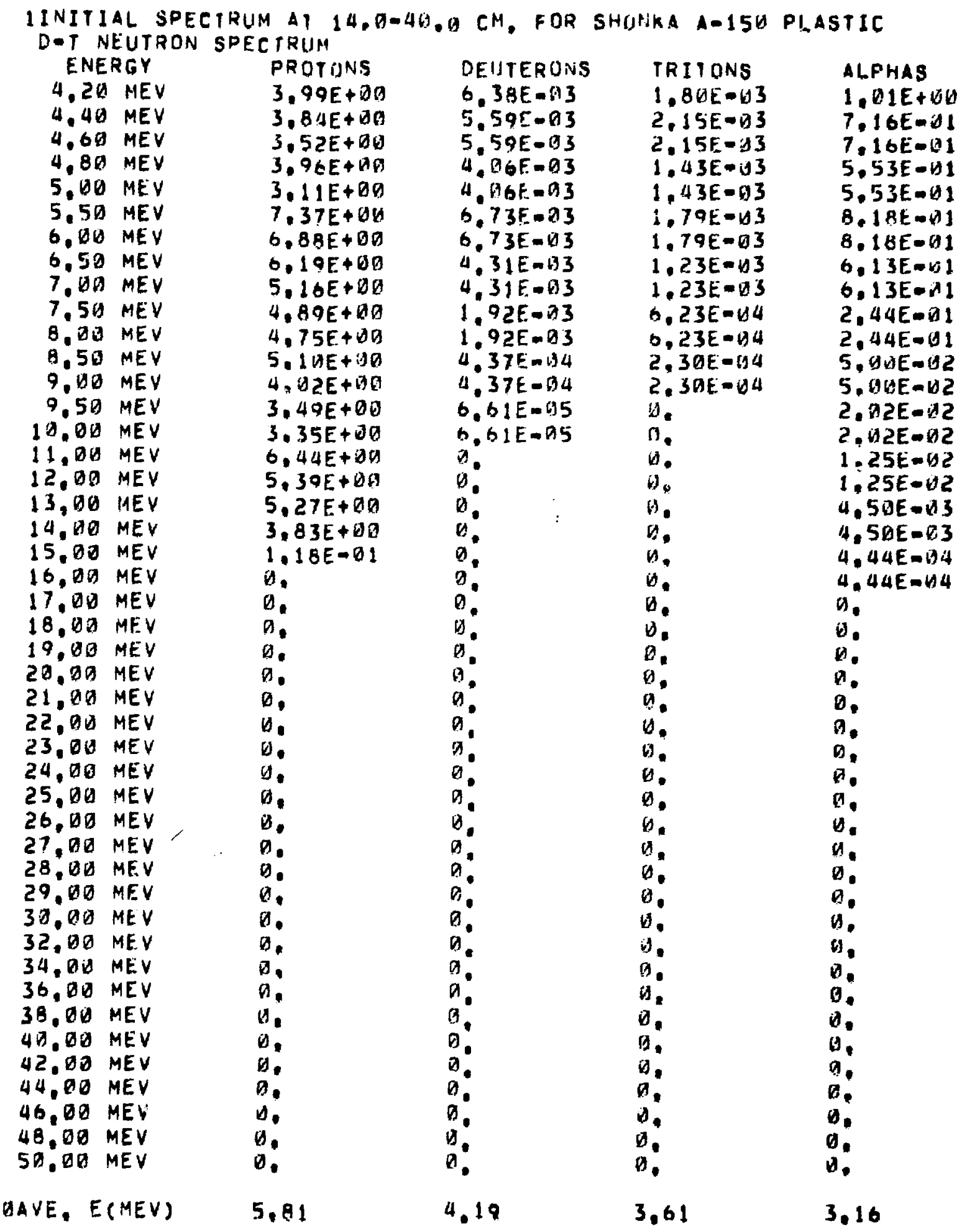




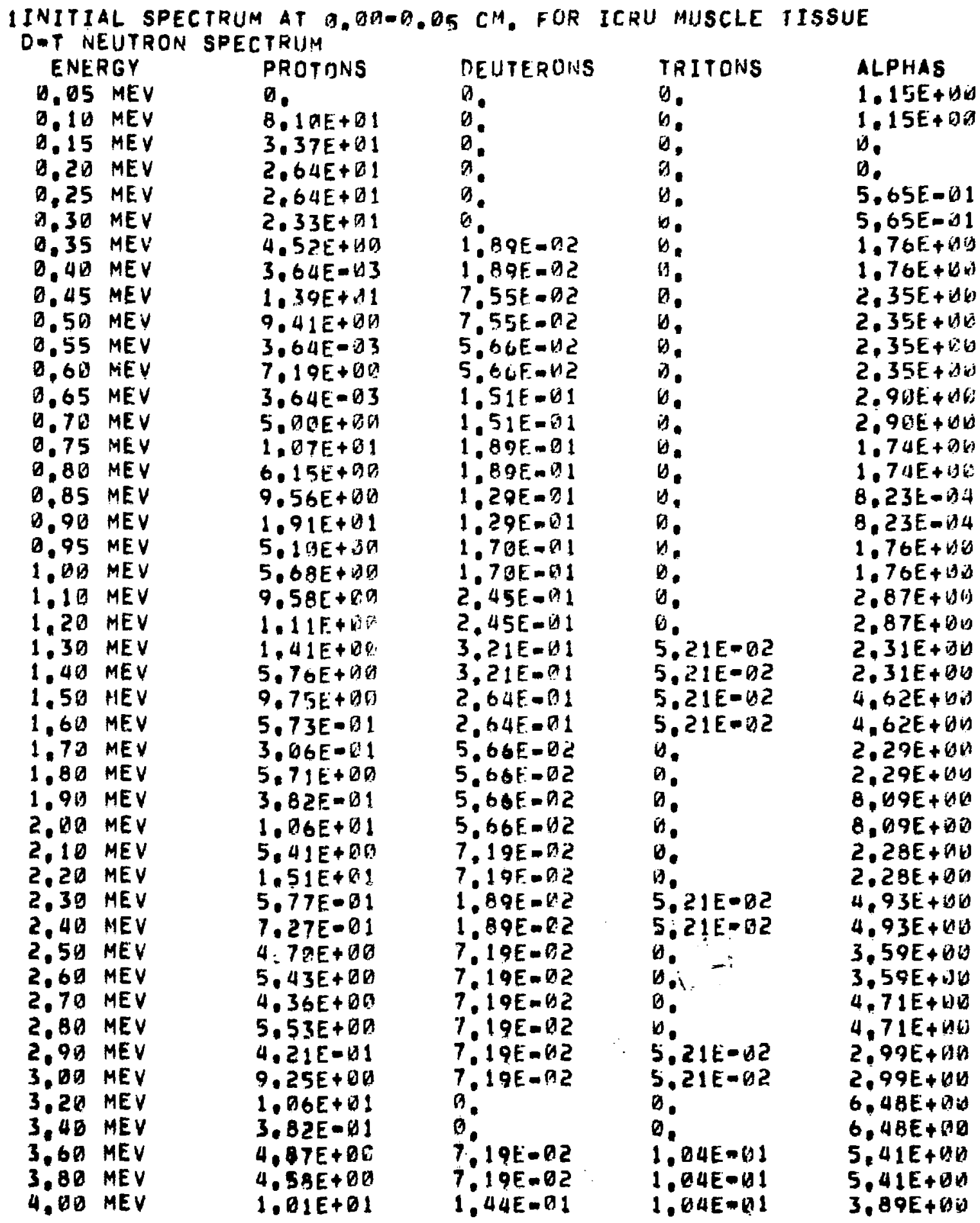




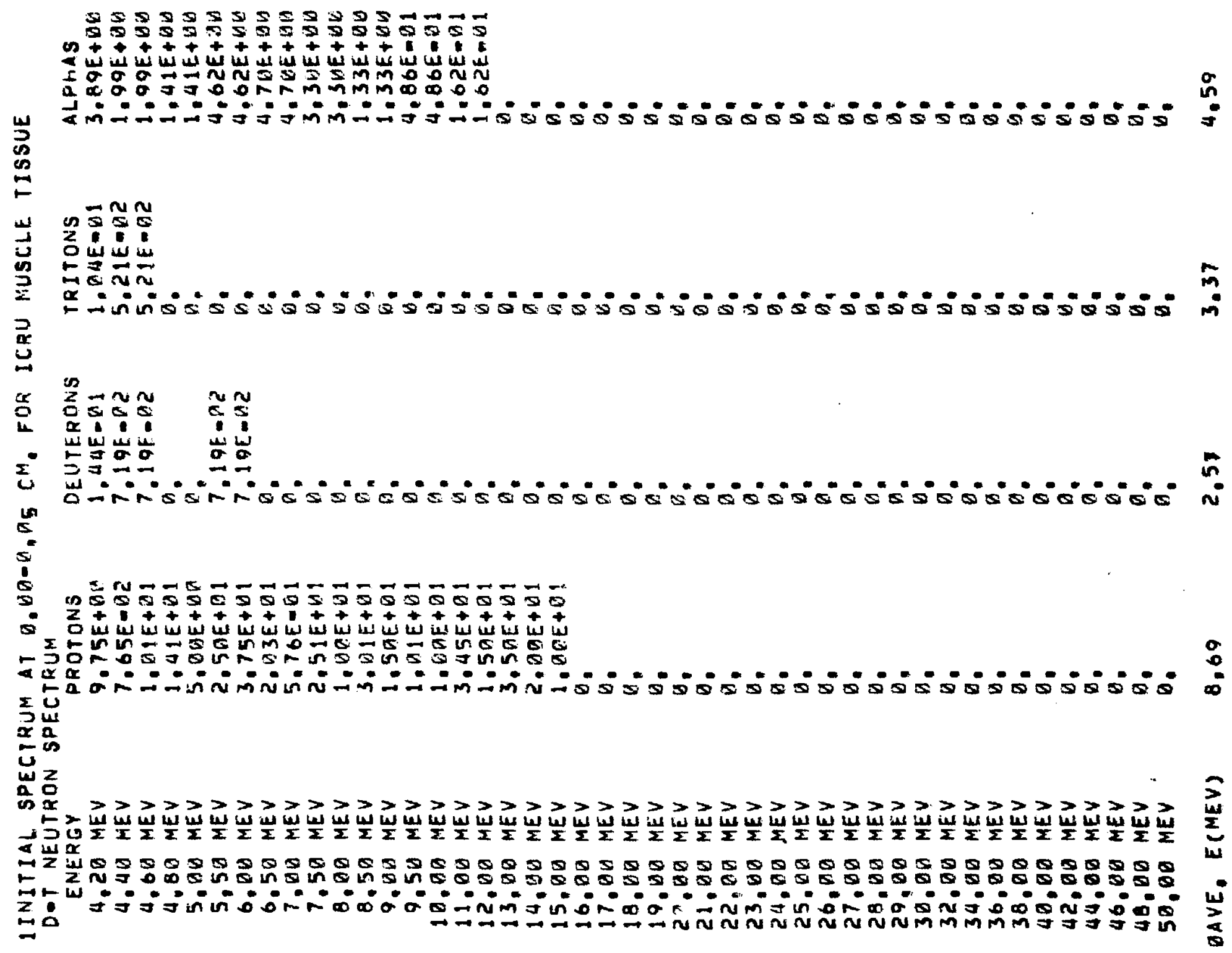


IINITIAL SPECTRUM AT 0.65-G.10 CM. FOR ICRUI HUSCLE TISSUE. D-T NEUTRON SPECTHUM

\section{ENERGY}

๑. D5 MEV

0.10 MEV

0.15 MEV

0.20 MEV

D.25 MEV

D. 3Q MEV

0.35 MEV

0.40 MEV

0.45 MEV

0.50 MEV

0.55 MEV

0.60 MEV

0.65 MEV

0.70 MEV

0.75 MEV

$0.80 \mathrm{MEV}$

0.85 MEV

0.90 MEV

0.95 MEV

1.00 MEV

$1,10 \mathrm{MEV}$

1.20 MEV

1.30 MEV

1.40 MEV

$1.50 \mathrm{MEV}$

$1.60 \mathrm{MEV}$

$1.70 \mathrm{MEV}$

1.80 MEV

$1,90 \mathrm{MEV}$

2. OO MEV

2.10 MEV

2.20 MEV

2. 30 MEV

2. $\triangle 0$ MEV

$2.50 \mathrm{MEV}$

2.60 MEV

2.70 MEV

$2.80 \mathrm{MEV}$

$2.90 \mathrm{MEV}$

3.00 MEV

$3.20 \mathrm{MEV}$

3. 40 MEV

3.60 MEV

3.80 MEV

4.0 MEV
PROTONS

G.

$7.8 G E+\Delta 1$

$1.62 E+b 1$

$4.21 E+01$

1. $29 E+i J$

$1.83 E+11$

2. $76 E+01$

4.7AE+ $\triangle B$

9.11E+ 13

1. $45 E+61$

1. $35 E+101$

i.

5. AAE+UQ

4. $53 E+10$

$3.64 E-63$

5. $\triangle D E+\square D$

$5.07 E+00$

4. $69 E+010$

$1.37 E+11$

7.8DE+

1. $58 E+D 1$

$1,51 E+109$

1. $D 4 E+1$

$3.07 E-11$

5. $23 E+k i B$

$1.96 E+01$

$1.56 E+\Delta a$

7. 1 AE $\triangle 1$

1. $15 E+31$

$1,57 E+d 1$

$5.77 E+80$

$6.86 E+6 a$

5. $29 E+190$

$4.90 E+\Delta A$

6. $\triangle A E+\square G$

$6.611 E-D 1$

$5.50 E+D Q$

5. $46 E+D ?$

4. $94 E+110$

$4.17 E+90$

1. $50 E+D 1$

$5.46 E+D a$

$1.91 E-01$

5. $19 E+10$

$5.19 E+60$
DEUTEROINS

a

a

$\theta$

0.

$a$.

$\theta$

$9.44 E-92$

$9.44 t-122$

$3.77 E-42$

$3.77 E-112$

$1.13 E-H 1$

$1,13 E-1$

1. $13 E-91$

$1,13 E-M 1$

$1.32 \mathrm{~F}-\mathrm{AI}$

1. $32 E=A$

2. OAE-A1

2. BBE-II

3. $21 F-A 1$

3.21E-171

3.77E-U1

$3.77 \mathrm{E}-\mathrm{N} 1$

2. $8 E-1$

2. $\triangle 8 E-1$

$3.77 E-12$

3. $77 \mathrm{E}-12$

$3.77 E-12$

$3.17 E=02$

5.66t- И2

5.6GE-A2

0

a.

7. $19 E-W 2$

$7.19 E-B 2$

$a$

$\theta$

0

b.

1).

3

a.

$\theta$

B.
TRITONS

$\forall$.

b.

$v$.

a.

b.

$n$

i.

i).

a.

$n$,

w.

in.

b.

$\theta$

it.

4.

ib.

is.

i).

i.

in.

4.

i.

6

i.

4.

$\because$.

0.

b.

b.

v.

,

$\theta$

$\Delta$

in.

4.

$5.21 E-1) 2$

' $.21 E-12$

$\Delta$.

is.

5.21E-02

$5.21 E-42$

$\mathfrak{B}_{4}$

$1)$

$\theta$
ALPHAS

$1,53 t-42$

$1.53 E-42$

$1.65 E+61$

$1.65 E+46]$

$5.93 E-11$

$5.93 E-N^{n} 1$

1. $7 \mathrm{~B} F+A \mathrm{~B}$

1. $7 W E+W \omega$

1. $14 E+6 G$

1. $1+1 E+d v$

$2,26 E+H i s$

2. $26 E+10$

1. $O \Delta E+U 1$

1. $64 E+v w$

1. USE + US

1. $D 6.1 E+B D$

$1.03 E+6 V$

$1.63 E+140$

1.7.AE+UV

1. $7 U E+W U$

2. $23 E+6 v$

$2.23 E+16$

1. $16 E+24$

$1.16 E+: 16$

$4.66 E+463$

$4.66 E+D V$

3. $42 E+0 V$

$3.42 E+\partial E$

$6.34 E-B 1$

$6.34 E=J 1$

1. $.59 E+60(0)$

$1.69 E+11+1$

$2.89 E+U U$

$2.89 E+1) W$

3. OUE + W6

3. DEE +Dn

3.14E+Dis

$3,14 E+B$

$6.76 E-A 1$

$6.76 E-111$

$5.47 E+0 V$

$5.47 E+H B$

4. $95 E+4 U$

4. 95E+

2. $19 E+U 1$ 


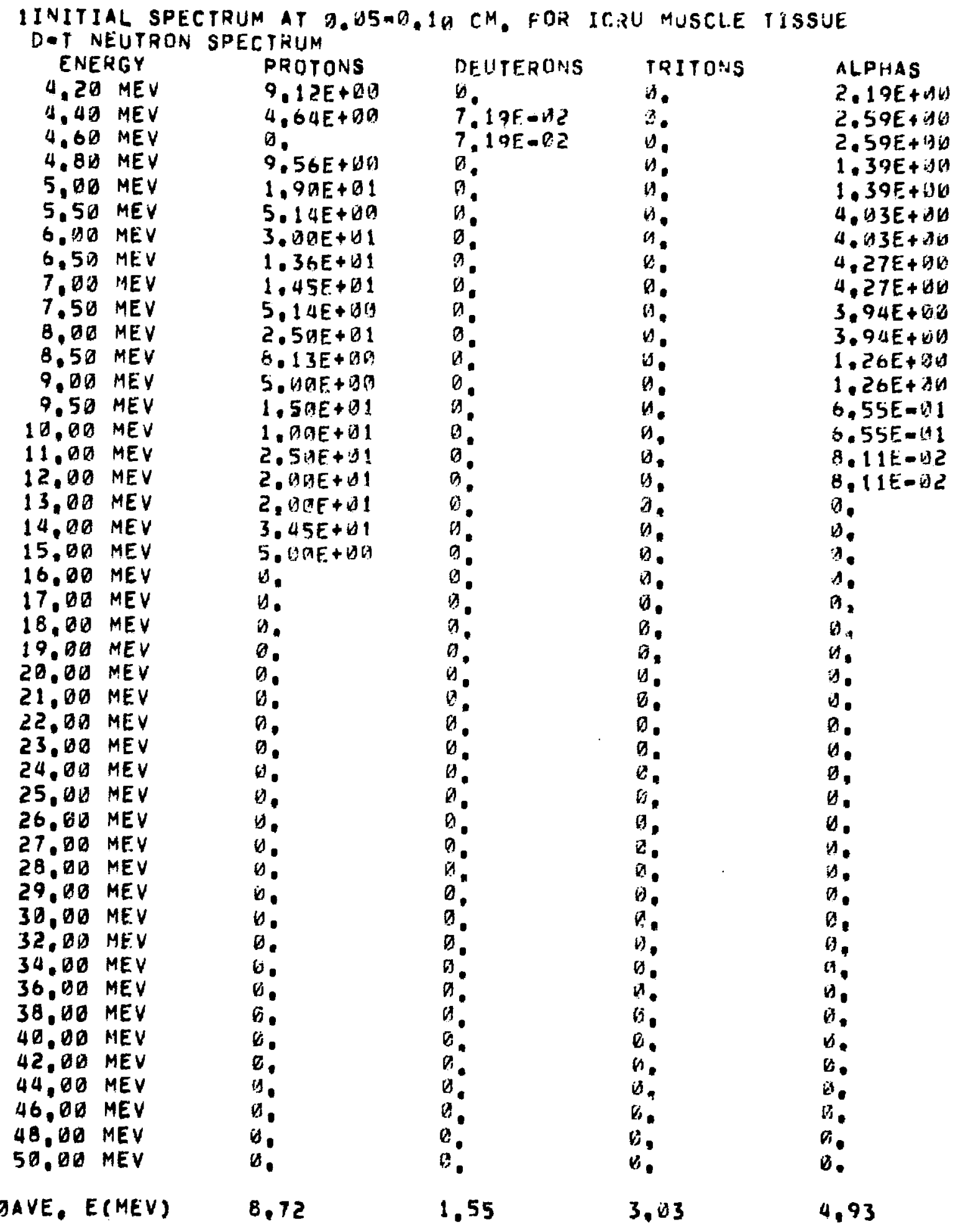




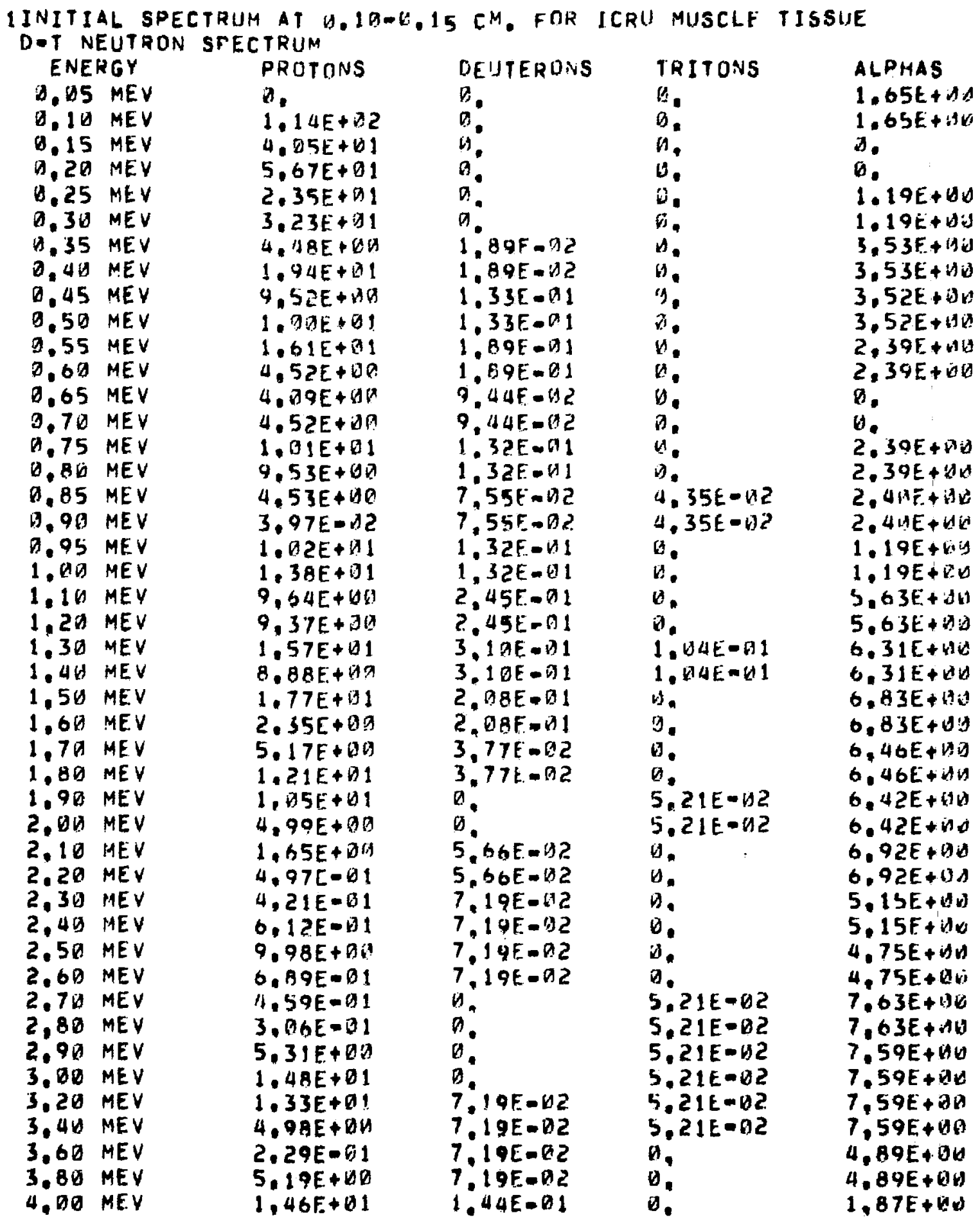


IINITIAL SPECTRUM AT U.1O-U.15 CM. FOR ICRU MUSCLE TISSUE DOT NEUTRON SPECTRUM

\section{ENERGY}

4. 2 ? $M E V$

4.40 MEV

4.60 MEV

4.80 MEV

5,00 MEV

5,50 MEV

6,00 MEV

6.51 MEV

7.00 MEV

7.50 MEV

3.DI MEV

8.50 MEV

Q.0 MEV

$9.50 \mathrm{MEV}$

$10.00 \mathrm{MEV}$

11,00 MEV

12. QO MEV

13.OD MEV

14.00 MEV

$15.00 \mathrm{MEV}$

$16.0 \mathrm{MEV}$

$17.00 \mathrm{MEV}$

$18.00 \mathrm{MEV}$

19.00 MEV

$20,00 \mathrm{MEV}$

$21.00 \mathrm{MEV}$

22. OO MEV

$23.00 \mathrm{MEV}$

24.DO MEV

$25.00 \mathrm{MEV}$

26,00 MEV

$27.00 \mathrm{MEV}$

$2 \$ .00 \mathrm{MEV}$

$29.00 \mathrm{MEV}$

$30.00 \mathrm{MEV}$

32.00 MEV

34.DO MEV

$36.00 \mathrm{MEV}$

$38.00 \mathrm{MEV}$

$40.00 \mathrm{MEV}$

42.00 MEV

44. DO MEV

46.01 MEV

48. OO MEV

$50.00 \mathrm{MEV}$

DAVE. E(MEV)

\section{PROTONS}

$1, \partial Q E+B 1$

2. $01 E+01$

$3.65 E+00$

$3,82 E-B 2$

$\Delta$.

1. $12 E+131$

$1.93 E+11$

4. $4 B E+100$

1. $43 E+1$

$5,07 E+0 \%$

2. $03 E+01$

1. $45 E+01$

$9.52 E+09$

1. $5 A E+B 1$

1. $O A E+B 1$

1. $45 E+01$

2. $D O E+31$

3. $D 1 E+D 1$

$4.45 E+01$

0.

$\theta$.

a.

b.

a.

b.

0.

D.

a.

$\theta$.

0.

0

0.

0.

$\theta$

0.

ต.

a.

is.

0.

$\theta$

$\theta$

0.

$\theta$.

D.

8.77

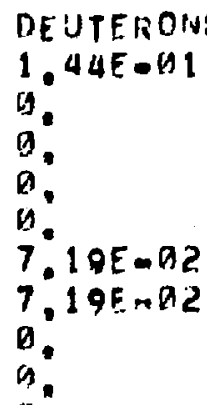

$a$

0.

0

0.

0

0

0

v.

0.

0

0

$a$

$a$

0

$a$

:

a.

0

0

0.

0.

0.

0

$a$

0.

a.

0

0.

2,53
TEITONS

D.

0.

6.

$5.21 E-02$

$5.21 E-D 2$

ï.

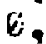

$\theta$

1.

19.

0.

$\Delta$

$\Delta$.

$\theta$

0.

0.

n.

a.

1.

0.

$v_{n}$

0.

g.

D.

a.

v.

!

b.

0.

D.

v.

0.

b.

0.

0.

b.

D.

b.

0.

$\theta$

9

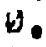

6.

2,81
ALPHAS

$1.87 E+W$

$2.7 W E+V Q$

2. $76 E+B D$

$1.13 E+B .6$

$1.13 E+H$

4. $3 B E+12$ is

4. $38 E+B G$

$3.96 E+19 H$

$3.96 E+114$

$3.52 E+0 \mathrm{f}$

$3.52 E+B D$

1. $35 E+b$ W

$1,35 E+64$

3.70E-U1

$3,70 E=1$

$1.22 E=01$

1. $22 E=U I$

D.

0.

$\theta$.

$\theta$.

a.

0.

0.

9.

b.

B.

b.

a.

0

0

B.

0.

0.

b.

v.

a.

D.

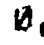

0.

0

0.

9.

4.13 
1INITIAL SPECTRUM AT $11.15-9,2 Q$ CM. FOR ICRU MUSCLE TISSUE D.T NEUTRON SPECTRUM

\section{ENERCY}

$0.0 S$ MEV

Q.16 MEV

ด.15 MEV

0.20 MEV

$0.25 \mathrm{MEV}$

$0.30 \mathrm{MEV}$

Q.35 MEV

0.40 MEV

0.45 MEV

$0.50 \mathrm{MEV}$

0.55 MEV

0.60 MEV

$0.65 \mathrm{MEV}$

0.70 MEV

0.75 MEV

$0,80 \mathrm{MEV}$

0.85 MEV

0,90 MEV

$0.95 \mathrm{MEV}$

1.DO MEV

1.10 MEV

1.20 MEV

$1,30 \mathrm{MEV}$

$1.40 \mathrm{MEV}$

$1.50 \mathrm{MEV}$

$1.60 \mathrm{MEV}$

$1.70 \mathrm{MEV}$

$1,80 \mathrm{MEV}$

$1.90 \mathrm{MEV}$

2.00 MEV

2.10 MEV

2.29 MEV

$2,30 \mathrm{MEV}$

2.4 MEV

2.50 MEV

2,60 MEV

2.70 MEV

2,80 MEV

$2,90 \mathrm{MEV}$

3. DO MEV

3.20 MEV

$3.40 \mathrm{MEV}$

3.63 MEV

3.8D MEV

4.00 MEV
PROTONS

B.

$5.98 E+11$

$4.75 E+01$

$1,76 E+D 1$

$2.90 E+01$

$1,82 E+01$

$1.47 E+D 1$

$1.22 E+D 1$

$2.25 E+131$

$4.52 E+\Delta D$

$1,45 E+D 1$

$1,50 E+D 1$

$3.21 E+01$

1. $47 E+1$

b.

$5.36 E+\Delta A$

$3.73 E-02$

$5.65 E+010$

$6.25 F+00$

$4.71 E+00$

1. $45 E+D 1$

$1.07 E+01$

$1.96 E+01$

$4.92 E+010$

$7.65 E-D 2$

$1.39 E+11$

$9.49 E+109$

1. DUE 401

$1.05 E+01$

1. $95 E+11$

$5.61 E_{-}+00$

$8,23 E+00$

B. 95E- 11

$11.94 E+101$

$5.50 E+10$

$9.97 E+90$

$4.59 E-B 1$

$4.83 E+10$

$4.73 E+40$

$9.58 E+100$

$9.72 E+D 0$

$3,82 E-\Delta 1$

$4.81 E+\Delta 0$

$3,5, E-1) 1$

$1.89 E-01$
DEUTERONS

U.

$\because$

:

0.

i:

a.

$1.89 F=02$

$1.89 E-112$

1.8 GE. H2

$1.89 E-112$

$7.55 E-12$

$7.55 E-D 2$

1. 32E-B1

1. 32E-BI

$1.32 E-31$

1. $32 E-O 1$

$9.44 E-D ?$

$9.44 E-A 2$

$5.66 E-112$

$5.66 E-112$

$2,45 E-31$

2. $45 E-B 1$

3. $72 E$ mit 1

$3.72 E-01$

$2.78 \mathrm{E}-91$

$2.78 E-41$

$1.13 \mathrm{E}-1) 1$

$1.13 E-01$

$\theta$.

:

$1.89 E-A 2$

$1.89 F-12$

a.

$0:$

a.

a.

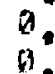

a.

a.

9.

$\theta$

$\theta$.

a.
TRITOHS

W.

a.

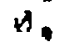

a.

$v$.

$\theta$

$\theta$

a.

9.

$\Delta$.

a.

6

,

i.

i.

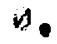

$\Delta$.

a.

a

a.

$\theta$

$B$.

H.

in,

$3.21 E-1) 2$

$5.21 E=92$

.

n.

1.

0.

v.

0

a.

.

i..

a.

$\theta$

a.

$\forall$.

$n$

v.

.

0.

$\theta$

9.
Al.PHAS

1. $18 E+40$

$1.18 E+\Delta x$

$3.51 E-\triangle 2$

3. $51 E-n 2$

2. 4.ME + WA

2. $4 A E+G Q$

S. $13 E-H 1$

5. $13 E-V 1$

$5.65 E-W 1$

$5.65 E-01$

1. $1 S E+D U$

$1,13 E+b y$

$5, B B E-1) 1$

$5.88 E-111$

$\therefore, 2 E E+30$

I, $20 E+v E$

4. $41 E-B C$

$4,4\{E-\square 2$

$1.17 E+10$

1.17E+ 130

$1.19 E+h b$

1. $19 E+110$

$2.39 E+46$

2. $39 E+46$

2.92E+

$2.92 E+d \omega$

$3.7 U E+6 U$

$3.70 E+U M$

2. $5 \omega E+d v$

2. $5 u E+(3)$

$2.52 E+Q 6$

$2.52 E+d D$

$3.96 E+1313$

$3,96 E+D E$

$1,86 E+2 i$

$1.86 E+46$

$1.46 E+i d E$

1. $46 E+196$

2. $16 E+14 n$

$2, B O E+U n$

$3,8\} E+U \mathfrak{J}$

3. $B Q E+66$

$4.04 E+\Delta b$

$4.04 E+310$

$5.57 E+i J d$ 


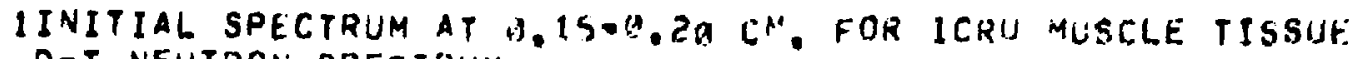
D-T NEUTRON SPECTRUM

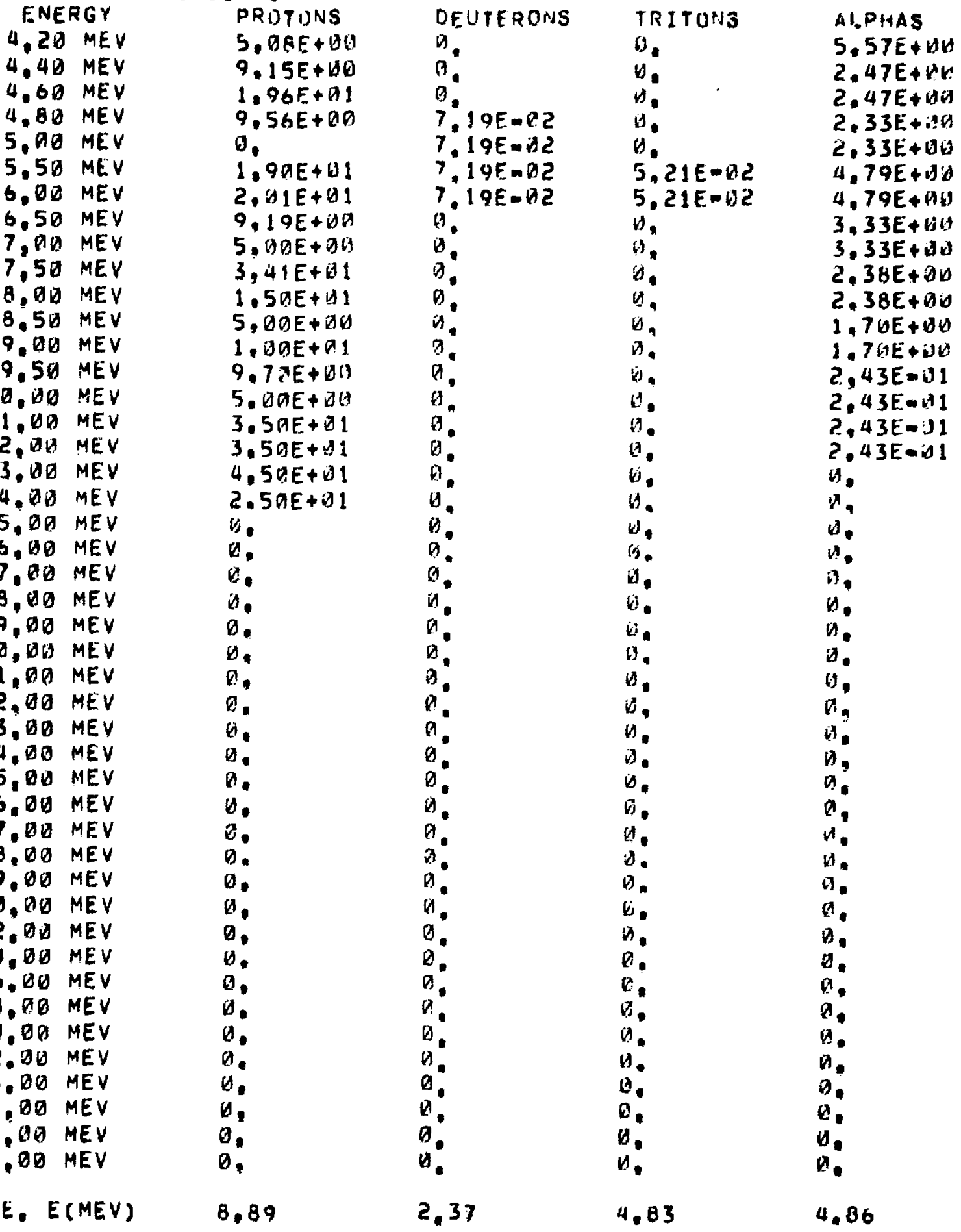




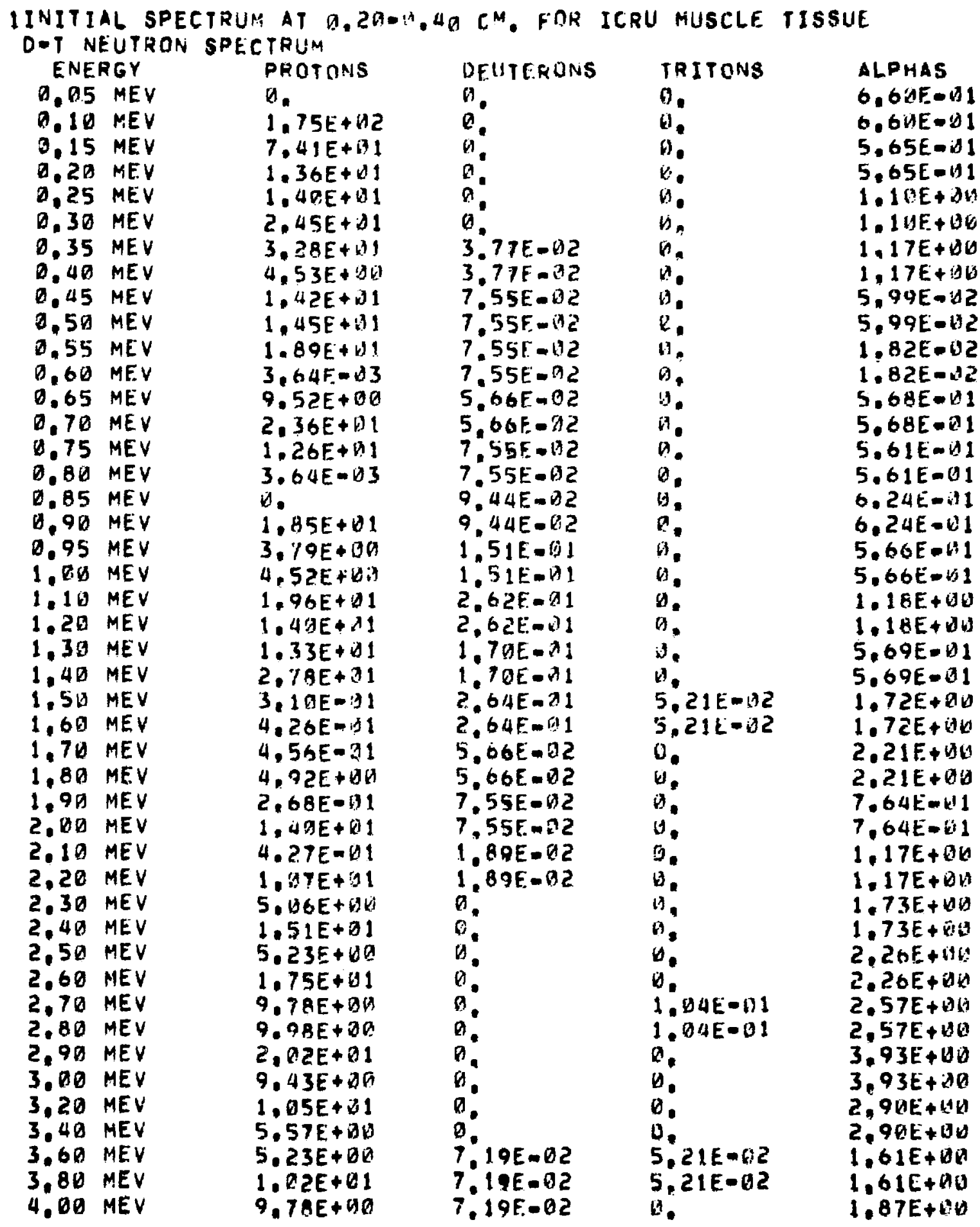


IINITIAL SPECTRUM AT 0.2 . DAT NEUTRCN SPECTRUM ENERGY

4.20 MEV

4.40 MEV

4.60 MEV

4. BO MEV

$5.00 \mathrm{MEV}$

$5.50 \mathrm{MEV}$

6.00 MEV

$6.5 W$ MEV

7. $O D$ MEV

7.5D MEV

8. DO MEV

$8.50 \mathrm{MEV}$

$9.00 \mathrm{MEV}$

$9.50 \mathrm{MEV}$

10.00 MEV

$11.00 \mathrm{MEV}$

$12.00 \mathrm{MEV}$

$13,00 \mathrm{MEV}$

$14.00 \mathrm{MEV}$

15. $\triangle 0$ MEV

16. ME MEV

$17.00 \mathrm{MEV}$

$18.00 \mathrm{MEV}$

19.00 MEV

$20.00 \mathrm{MEV}$

$21.00 \mathrm{MEV}$

22,00 MEV

23. $00 \mathrm{MEV}$

24,00 MEV

$25,00 \mathrm{MEV}$

26. $00 \mathrm{MEV}$

27. $00 \mathrm{MEV}$

28. DQ MEV

$29.00 \mathrm{MEV}$

30.09 MEV

32. UD MEV

34,00 MEV

36, BO MEV

$38, D B \mathrm{MEV}$

$40,0 D$ MEV

42.00 MEV

44.00 MEV

46,DO MEV

48, DO MEV

50,00 MEV
PROTONS

1. $46 E+B 1$

4. $22 E+\Delta a$

$4.23 E+d H$

a.

1. $B G E+Q 1$

S. DAE + UA

1.83E+ 1

5. $14 E+O A$

$5.14 E+D C$

4. $1 b E+b 0$

1. $M A E+H 1$

4. 5?E+ DU

0.

5. $Q 19 E+b)$

2. GUE + UI

2. MOE+ DI

2.97E+01

1. WOE+DI

1. $50 E+D 1$

$\theta$.

o.

0

0.

v.

n.

b.

0.

$\theta$.

$\theta$

D.

b.

b.

$\theta$.

1.

$\theta$.

a.

$\Delta$

$B$

$\ddot{\theta}$

$D$.

b.

$\theta$.

0.

$D$

0.

7.46
DEUTERONS TRITDNS

b.

7. 19E-OD?

a.

$7: 19 E-192$

$7.191-62$

$7.19 F-4 ?$

$7.19 E-V 2$

0

i.

it.

b.

b.

$B$

a.

0

a.

B.

$a$

a.

a.

0

0

$a$

0

0.

0

0.

0.

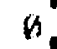

0

a.

0

0

a.

0

0

$\theta$

0.

a)

3

$\theta$

2.68 o.

b.

i.

4.

a.

a)

i)

0

D.

a.

in.

b.

b.

a.

i.

b.

$\theta$

4

a.

a.

E.

is.

$H$

is

0.

v.

ii.

i.

(b).

0,

6

a.

c.

$n$

จ.

.

$\theta$

b.

b.

a.

a.

G.

(19.

0.

2,82
ALPHAS

1.87E+vis

1. BUE $+D$

1. $86 E+0,1$

2. $\triangle 2 E+D \Delta$

2. $02 E+4 \square$

3. $25 E+\Delta v$

$3.25 E+(4 b)$

3. $72 E+b V$

$3,72 E+\omega n$

$2.63 E+1) 0$

$2,63 E+136$

1. $42 E+110$

1. $42 E+B d$

$4.46 E-U 1$

4. $46 E-61$

$1.62 E-111$

$1.62 E-101$

i).

b.

,

$\theta$

i.

i)

$B$

$b$

b.

0.

$\theta$

Q.

(1).

0

0.

4.

$\theta$.

4.

6

n.

a.

$\theta$.

i.

$\theta$

b.

0.

0.

n.

5.28 


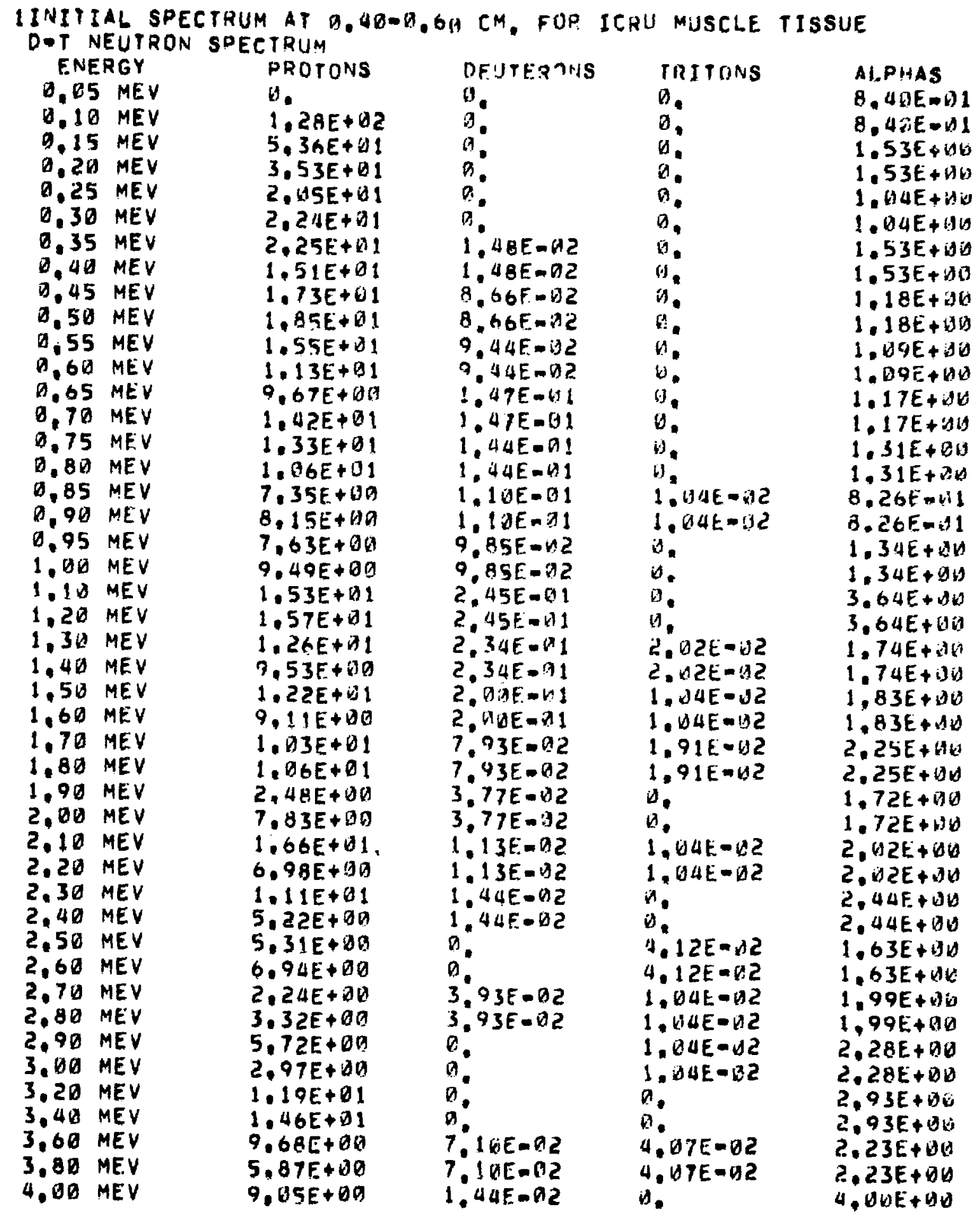




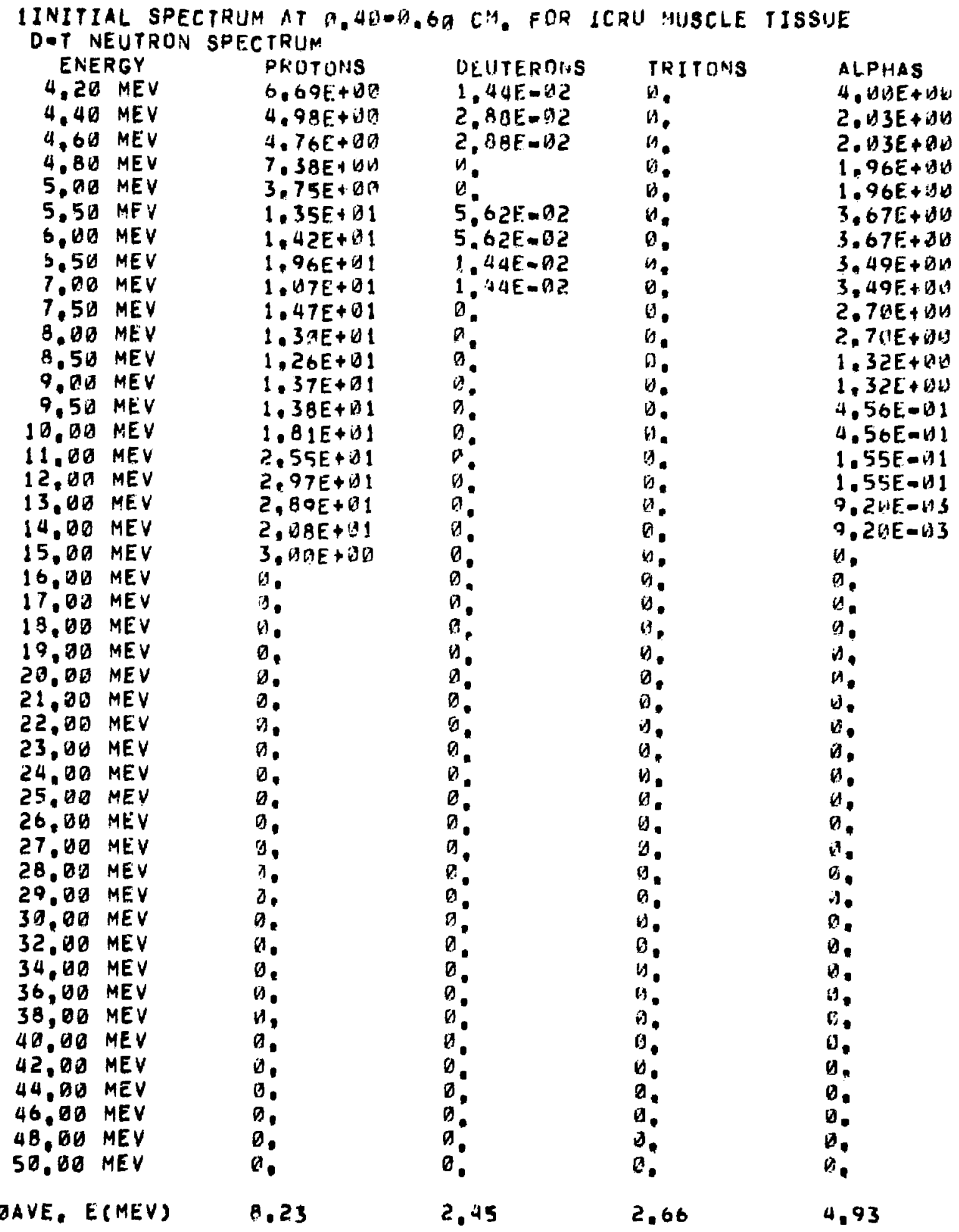




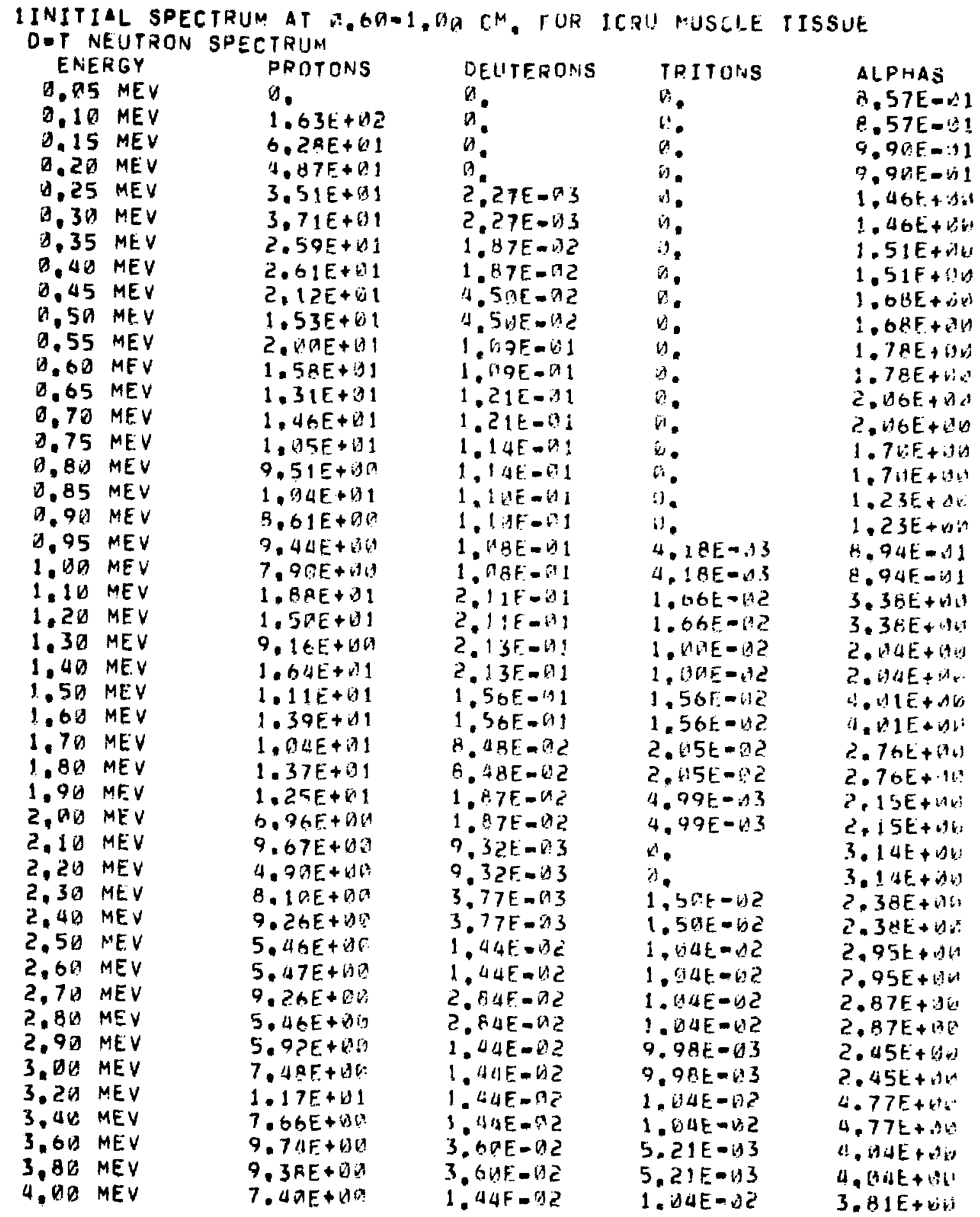


IINITIAL SPECTRUM AT Q.60-1, OG CM. FOR ICRU MUSCLE TISSUE DEI NEUTRON SPECTRUM

ENERGY

4.20 MEV

4.40 MEV

4.61 MEV

4.80 MEV

5. $D Q$ MEV

5.50 MEV

6.00 MEV

$6.50 \mathrm{MEV}$

7. OU MEV

7.50 MEV

8. $\triangle B$ MEV

8.50 MEV

9.DQ MEV

9.50 MEV

10. OO MEV

$11.00 \mathrm{MEV}$

12. OO MEV

13,00 MEV

14.00 MEV

15.00 MEV

$16,00 \mathrm{MEV}$

$17,00 \mathrm{MEV}$

$18,00 \mathrm{MEV}$

$19.00 \mathrm{MEV}$

20.0D MEV

$21.00 \mathrm{MEV}$

22.00 MEV

23. DO MEV

$24.00 \mathrm{MEV}$

25. DU MEV

$26.00 \mathrm{MEV}$

27. DO MEV

28. 0 B MEV

$29.00 \mathrm{MEV}$

$30.00 \mathrm{MEV}$

$32.00 \mathrm{MEV}$

$34.00 \mathrm{MEY}$

$36.00 \mathrm{MEV}$

$38.00 \mathrm{MEV}$

$40.00 \mathrm{MEV}$

42.00 MEY

44.00 MEV

46.00 MEV

48.00 MEV

50,00 MEV

OAVE, E(MEV)
PROTONS

1. $15 E+101$

$6.82 E+19 n$

$9.65 E+0.1$

$5.93 E+00$

8. $13 E+C 4$

$1.75[+01$

1.90E +01

$1.32 E+01$

$1.77 E+B 1$

$1.62 E+01$

$1.36 E+111$

1. $18 E+\| 1$

1. $34 E+D 1$

1. $27 E+\square 1$

1. $48 E+11$

$2,42 E+01$

2.1BE+ 11

2. $03 E+D 1$

2. $49 E+\square 1$

3. ODE +00

$\theta$.

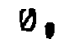

in.

id.

0.

$\theta$.

0.

b.

b.

$\theta$.

b.

$\theta$

0.

0.

0.

D.

0.

0.

D.

0.

0.

0.

D.

0

7.69
DEUTERONS TEITONS

1. $44 E-02 \quad 1.04 E-02$

4. BIE-VI

$4.89 E-B C$

2. $14 E-\square 2$

2. $19 E-0.2$

1. $44 E-P 2$

$1.44 E-192$

0

a.

10

0.

0

(1)

a

0

a.

a.

0

0

0

a.

0

0

0

0

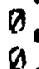

0

a.

0

0

0

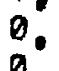

0

a.

0

0

a.

2.60 $\theta$

b.

5.21E-U3

5. $21 E-B$

5. $21 E-\| 3$

$5.21 E-Q$

0

$\theta$

U.

0.

b.

b.

0.

i.

0.

0.

D.

$\theta$

0.

0

$\theta$

$\theta$

a.

$B$.

a.

t.

a.

0.

v.

$\theta$

i.

$\theta$.

0.

D.

O.

0.

0.

0.

b.

$B$.

b.

v,

0.

$\theta$.

2.94
ALPHAS

3. $1 E+U+9$

2.7BE+ 14

$2.78 E+913$

1. $75 E+i v$

$1.75 E+4 V$

$3.82 E+06$

$3,82 E+145$

$3,54 E+0 V$

$3.54 E+4 n$

$2,37 E+U n$

$2.37 E+66$

1. $05 E+01$

1. $95 E+b 6$

4. 47E-181

4. $47 E-01$

$1.71 E-01$

$1.71 E=01$

b.

$\theta$.

v.

$\theta$.

$\Delta$.

D.

0.

$b$.

0.

0.

o.

o.

n.

0.

0.

$\theta$.

B.

$\Delta$.

$B_{8}$

D.

0.

0.

b.

D.

0.

$\theta$.

b.

4.59 
1INITIAL SPECTHUM AT 1.DFO2, BG CM, FOR ICRU MUSCLE 1 ISSHE D-T NEUTRON SPECTRUHA

\section{ENERGY}

0. DS MEV

0.10 MEV

0.15 MEV

$0.20 \mathrm{MEV}$

0.25 MEV

0.30 MEV

0.35 MEV

2,40 MEV

$0.45 \mathrm{MEV}$

$0.50 \mathrm{MEV}$

$0.55 \mathrm{MEV}$

$0.60 \mathrm{MEV}$

$0.65 \mathrm{MEV}$

0.70 MEV

$0.75 \mathrm{MEV}$

$0.80 \mathrm{MEV}$

0.85 MEV

$0.90 \mathrm{MEV}$

$0.95 \mathrm{MEV}$

1. DD MEV

$1.10 \mathrm{MEV}$

1.20 MEV

1.30 MEV

1.40 MEV

1.50 MEV

1.60 MEV

$1.70 \mathrm{MEV}$

1.8@ MEV

1.90 MEV

2,00 MEV

2.10 MEV

2.25 MEV

$2.30 \mathrm{MEV}$

$2,40 \mathrm{MEV}$

2.50 MEV

$2.60 \mathrm{MEV}$

$2.79 \mathrm{MEV}$

2.80 MEV

2.90 MEV

3.00 MEV

$3.26 \mathrm{MEV}$

3.40 MEV

3.60 MEV

3,80 MEV

4. OD MEV

\section{PROTONS}

0.

$1.93 E+132$

$7.59 F_{0}+11$

4. $D B E+D 1$

4. $14 E+11$

2. $72 E+01$

2. $5 A E+B 1$

3. $2 E+W 1$

2. $02 E+01$

$2.17 E+61$

1. $78 E+91$

$1.22 E+01$

$1.56 E+\square 1$

$1,4 D E+B 1$

1. $4.3 E+01$

1. $23 E+01$

$9.74 E+09$

1. $29 E+11$

9. $41 E+D G$

$6.54 E+10$

1. $57 E+01$

1. $60 E+01$

$2.23 E+01$

$1.32 E+01$

1. $34 E+1$

1. $13 E+1$

1. $26 E+D 1$

$9.39 E+110$

1. $49 E+D 1$

$9.2 .3 E+U D$

$9.20 E+00$

$1.24 E+01$

1. $24 E+101$

$9.5 A E+D A$

$8.41 E+10$

$4.39 E+100$

$1.37 E+\Delta 1$

$7.07 E+d Q$

$6.53 E+00$

8. $28 E+00$

1. $22 E+B 1$

$1.67 E+01$

1. $\triangle Q E+D 1$

B. $19 E+O A$

$8.46 E+90$

\section{DEUTFRONS}

O

a.

a.

:

A.

1.89F-DZ

1. $39 E-62$

5. DUE- 12

5. VUE $D$ R2

9. $\triangle 4 E-D 2$

9. UAE- 12

1. $\triangle 5 E-D 1$

1. DSE- 1

8.68F-

8. GE-DZ

9. $1 A E=Q ?$

9. ADE $D 2$

1. OSE-A1

1. MSF-O 1

$1.83 E=01$

1. B $3 E-O 1$

2. $\triangle उ E-A 1$

2. $035-01$

1. $41 E-\Delta 1$

1. it $1 E-O 1$

7. BREDW

7. $8 B E=\square 2$

3. $86 E-12$

3. $86 E-32$

1. $14 F-02$

1. $1 \triangle E-\cap 2$

2. $00 E-12$

2. GDEMDZ

$7.19 E-A 3$

$7.19 E-103$

$3.64 E=13$

$3.04 E=03$

2. $11 E-A 2$

2. 11E-ti2

2. $89 E-32$

2. $89 E-1) 2$

$3.53 E-12$

$3.53 E-92$

$7.13 E-02$
TRITONS

U.

n.

b.

a.

i.

b.

b.

a

b.

$\theta$.

$4.37 E-03$

4. $37 E-63$

b.

$D$

0

$D$

v.

0

5.21E-n 3

5.21E-03

1. $\triangle 1 E-B 2$

1. $41 \mathrm{E}-\mathrm{E} 2$

$9.96 E-63$

$9.96 E=\square 3$

5. ट1E-U 3

2. $21 E M U$

a.

a.

$2.60 \mathrm{E}-1 \mathrm{Z}$

2. $60 E-112$

3. 1 DE $W 2$

3. IDE $=4$ ?

2. $08 E-02$

2. $\triangle 8 E-112$

$1.52 E-62$ ?

1. $52 E-\bar{y} 2$

$1.54 E-1) 2$

$1.54 E-G \mathrm{C}$

2. $19 E-02$

2,1 DE- OS

3. $45 E-112$

3. $46 E-62$

$2.60 E-H 2$

$2.69 E-02$

$1.56 E-102$
AL.PHAS

3. DIE-U1

3. BIE- id 1

$6,29 E=W 1$

6. $29 E-11$

1. $04 E+\Delta \theta$

1. $\triangle \Delta E+D Q$

9. $45 E-1$

$9.45 E-D 1$

1. $15 E+i d$

1. $15 E+14$

1. $16 E+116$

$1.16 E+60$

$9.92 E-$ b 1

$9.92 \mathrm{E}-\mathrm{Ul}$

1. $49 E+116$

1. $49 E+40$

1. $35 E+V E 1$ :

1. $35 E+E Q$

1. $10 E+B Q$

1. $16 E+B G$

2. $B 3 E+Q 6$

2. $D 3 E+D B$

2. $00 E+6 A$

2. $\triangle 3 E+D E$

$=28 E+C D$

2. $28 E+06$

2. $43 E+D i$

$2.43 E+69$

$1.93 E+\triangle B$

$1.93 E+100$

2. $62 E+6 B$

$2.62 E+40$

$2.28 E+i b$

2. $28 E+W 6$

2. $34 E+130$

2. $34 E+H G$

$2,61 E+14$

$2.61 E+119$

$1.91 E+D i d$

1. $\rightarrow 1 E+14 V$

5. 36E+ $D U$

$5,36 E+B D$

3. $42 E+B U$

3. $42 E+b U$

3. $21 E+U E$ 
IINITIAL SPECTRUM AT 1.AG-2.BB CM. FOR ICRU MUSCLE TISSUE DOT NEUTRON.SPECTRUM

\section{ENERGY}

4.2O MEV

4.40 MEV

4.613 MEV

$4.80 \mathrm{MEV}$

5. OE MEV

$5,50 \mathrm{MEV}$

6. $D Q$ MEV

$6.50 \mathrm{MEV}$

7. OO MEV

7.50 MEV

$8.00 \mathrm{MEV}$

8.50 MEV

9.DU MEV

9.50 MEV

10,00 MEV

11.00 MEV

12,00 MEV

13. DO MEV

14.00 MEV

15,00 MEV

$16.00 \mathrm{MEV}$

17.09 MEV

$18.00 \mathrm{MEV}$

$19.80 \mathrm{MEV}$

26,00 MEV

21,00 MEV

22,00 MEV

23.00 MEV

24.00 MEV

25. BD MEV

$26.00 \mathrm{MEV}$

27.00 MEV

26:DO MEV

29.DO MEV

30.00 MEV

$32.00 \mathrm{MEV}$

34.00 MEV

36.00 MEV

$38.00 \mathrm{MEV}$

40.00 MEV

42.00 MEV

44.0U MEV

46,00 MEV

48,DO MEV

52.00 MEV

\section{PROTONS}

B. $16 E+Q B O$

1. UAE +O1

1. $2\{E+D$ I

8. $24 E+0$ ?

$1, \square 2 E+\forall 1$

$1.84 E+01$

$1.92 E+1$

$1.66 E+01$

$1.62 E+01$

$1.12 E+11$

$9.41 E+00$

1. $29 E+01$

1. $40 E+01$

1. $45 E+B 1$

4. $59 E+199$

2. $15 E+01$

2. $41 E+(1) 1$

2. 56E+ $B 1$

1.9AE+Q1

5. $19 E-B 1$

0.

0.

0.

$\theta$.

$\theta$.

(n.

$\theta$

$\theta$

O.

$B$.

$\theta$

Q.

$\theta$

0

0.

0.

$\theta$

$\theta$

$\theta$.

n.

0.

0

0.

0.

7.34

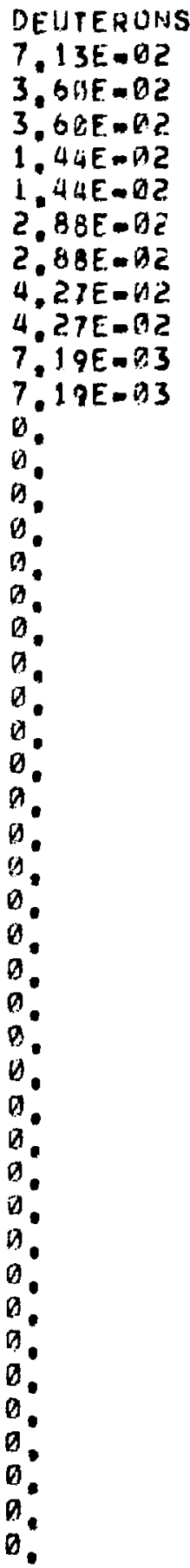

2.99
TRITONS

1. $56 E-62$

1. OUEDD?

1. $\triangle 4 E-B 2$

n.

is.

i.

i.

a.

$b$.

i.

in

v.

$\theta$

$\theta$

b.

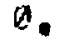

i.

$\theta$

a.

$\theta$

$\theta$

$\theta$

3.

is,

9 ,

a.

0

0.

b.

$b$

0

a.

a

:

0

$\theta$

$\theta$

is:

:

$\theta$

$\nabla$.

2.90
ALPHAS

3. $21 E+Q U$

$2.12 E+190$

2. $12 E+B G$

$1.74 E+9 D$

1. $T A E+U Q$

$3.69 E+00$

$3.09 E+00$

3. $23 E+Q V$

$3.23 E+4 \theta$

2. $17 E+U B$

$2.17 E+019$

$9.15 E-1) 1$

$9.15 E=1$

$4.94 E-U 1$

4. 94EनIA 1

1. B3E- I1

$1.83 E-01$

1).

a.

$\theta$

0.

a.

$\Delta$.

0.

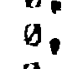

D.

$\theta$

0.

$\theta$.

n.

0.

0.

0.

D.

$\theta$

0.

ด.

$\theta$

i.

0

$\forall$.

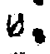

is.

0

0.

4.74 


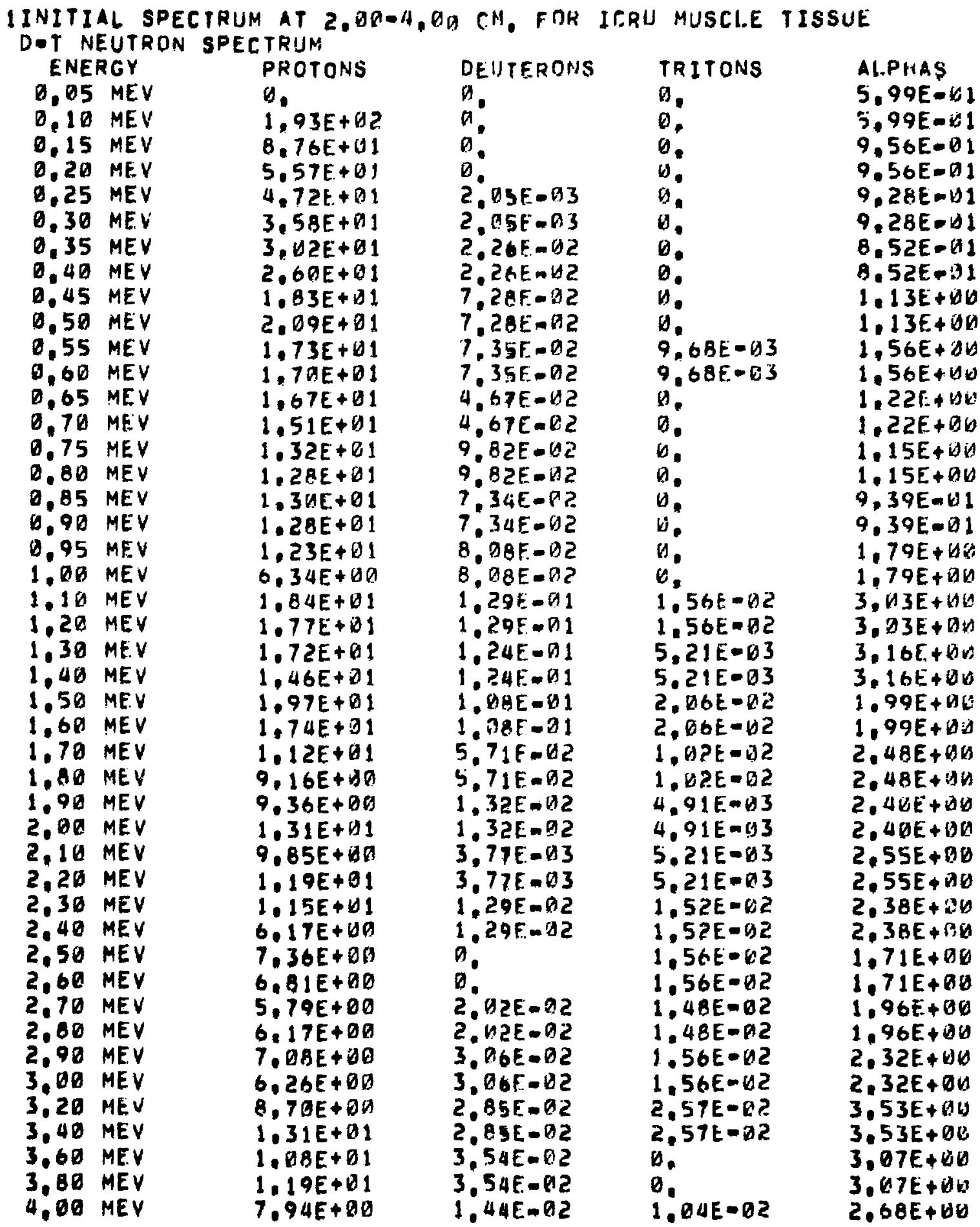


IINITIAL SPECTRUM AT 2.0A-4. BD CM. FOR ICRIJ MUSCLE TISSUE DOT NEUTRON SPECTRUM

ENERGY

4.20 MEV

4.40 MEV

$4.60 \mathrm{MEV}$

4.80 MEV

5,00 MEV

5,50 MEV

$6.00 \mathrm{MEV}$

$6.50 \mathrm{MEV}$

7.DD MEV

7.50 MEV

8,00 MEV

$8.50 \mathrm{MEV}$

9.00 MEV

9,50 MEV

$10,00 \mathrm{MEV}$

11.00 MEV

12. BO MEV

$13.00 \mathrm{MEV}$

$14,00 \mathrm{MEV}$

15.00 MEV

$16.00 \mathrm{MEV}$

$17.00 \mathrm{MEV}$

18,00 MEV

$19,00 \mathrm{MEV}$

20.00 MEV

$21.00 \mathrm{MEV}$

22. DO MEV

23. OO MEV

24.01 MEV

25, DO MEV

26, DO MEV

27,00 MEV

28.DO MEV

29, 0 O MEV

30,DO MEV

$32,00 \mathrm{MEV}$

$34.00 \mathrm{MEV}$

$36.00 \mathrm{MEV}$

38,00 MEV

$40,00 \mathrm{MEV}$

$42,00 \mathrm{MEV}$

44,00 MEV

46, OD MEV

$48,00 \mathrm{MEV}$

$50.00 \mathrm{MEV}$

DAVE, E(MEV)
PROTONS

$5.56 E+Q P$

$9.38 E+\theta E$

$8.11 E+D D$

$7.40 E+D O$

$6.79 E+D O$

$1.67 E+01$

$1.51 E+61$

$1,17 E+B 1$

1.5OE+ 01

1. $16 E+B 1$

1. $09 E+Q 1$

1. $36 E+01$

$1.32 E+01$

$9.23 E+00$

$1.23 E+01$

$2.30 E+B !$

1. $B O E+D 1$

2. D1E+ DI

$1.76 E+01$

$1.45 E+20$

0.

D.

0.

$\theta$

$\theta$

$\theta$

.

$\theta$

$\theta$.

a.

a.

a.

0.

0.

b.

0.

a.

0.

0.

b.

0.

0.

a.

.

7.13

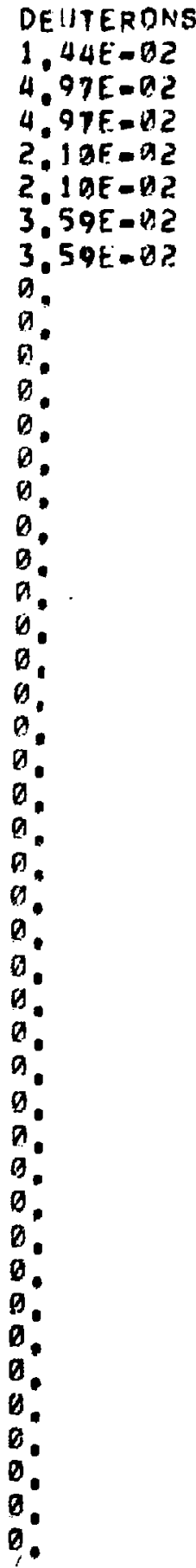

DEUTERONS

TRITONS

$\therefore .04 E-92$

ALPHAS

$2.68 E+16$

$1.89 E+18 d$

$1 . B 9 E+B D$

$1.38 E+38$

$1,38 E+0 E$

$2.84 E+100$

$2.84 E+0.1$

$2.42 E+5 B$

$2.42 E+D M$

$1.74 E+60$

$1.74 E+00$

$7.69 E-111$

$7.60 E-131$

$2.91 E-01$

2.91E-O 1

$1.09 E-D 1$

I. $D 9 E-D 1$

$B$.

0.

0.

B.

8.

0.

0.

0.

$\theta$.

0.

0.

B,

a.

8.

a.

0.

$\theta$

0.

B.

H.

.

$\theta$.

0.

0.

a.

v.

,

D.

4.44 


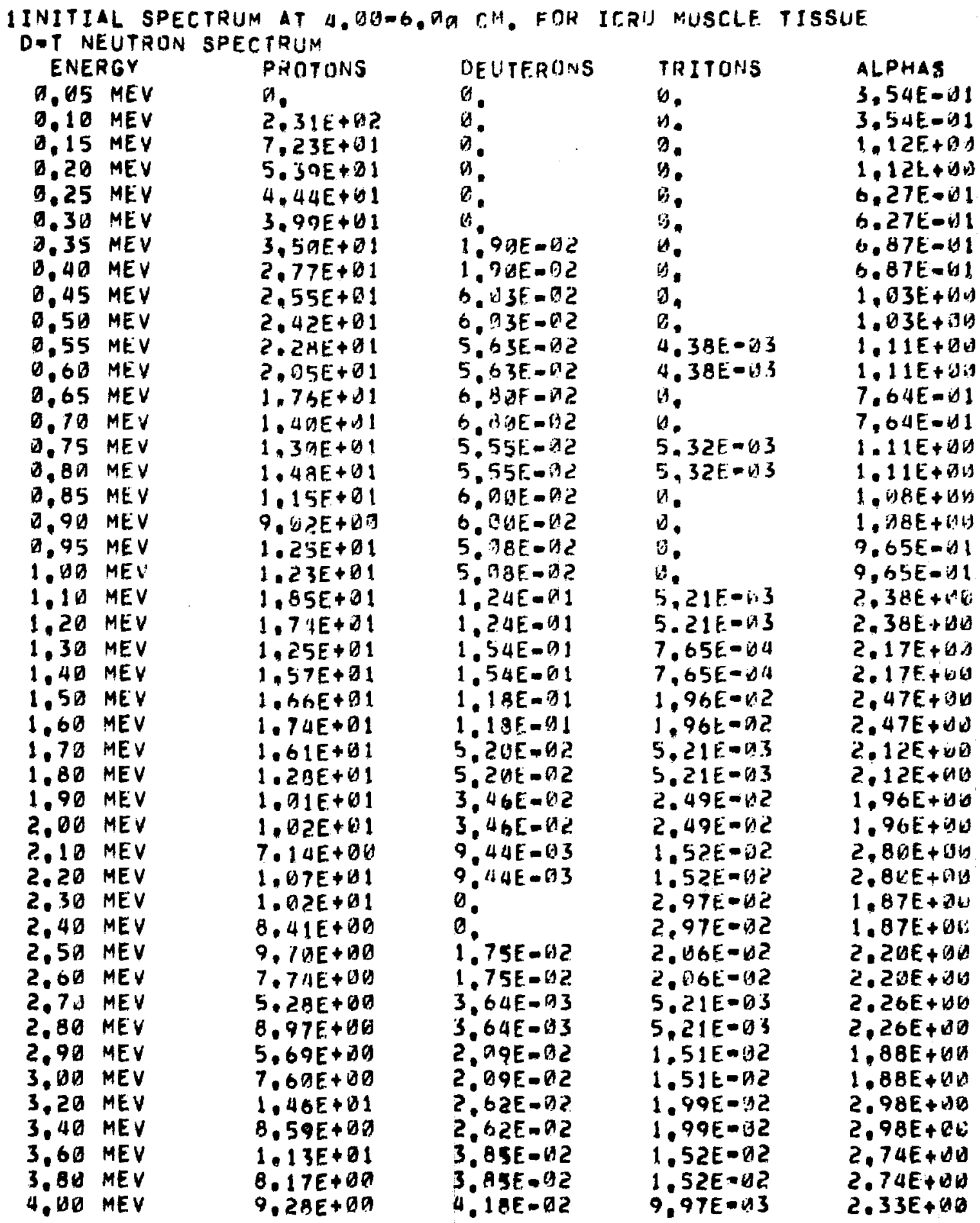




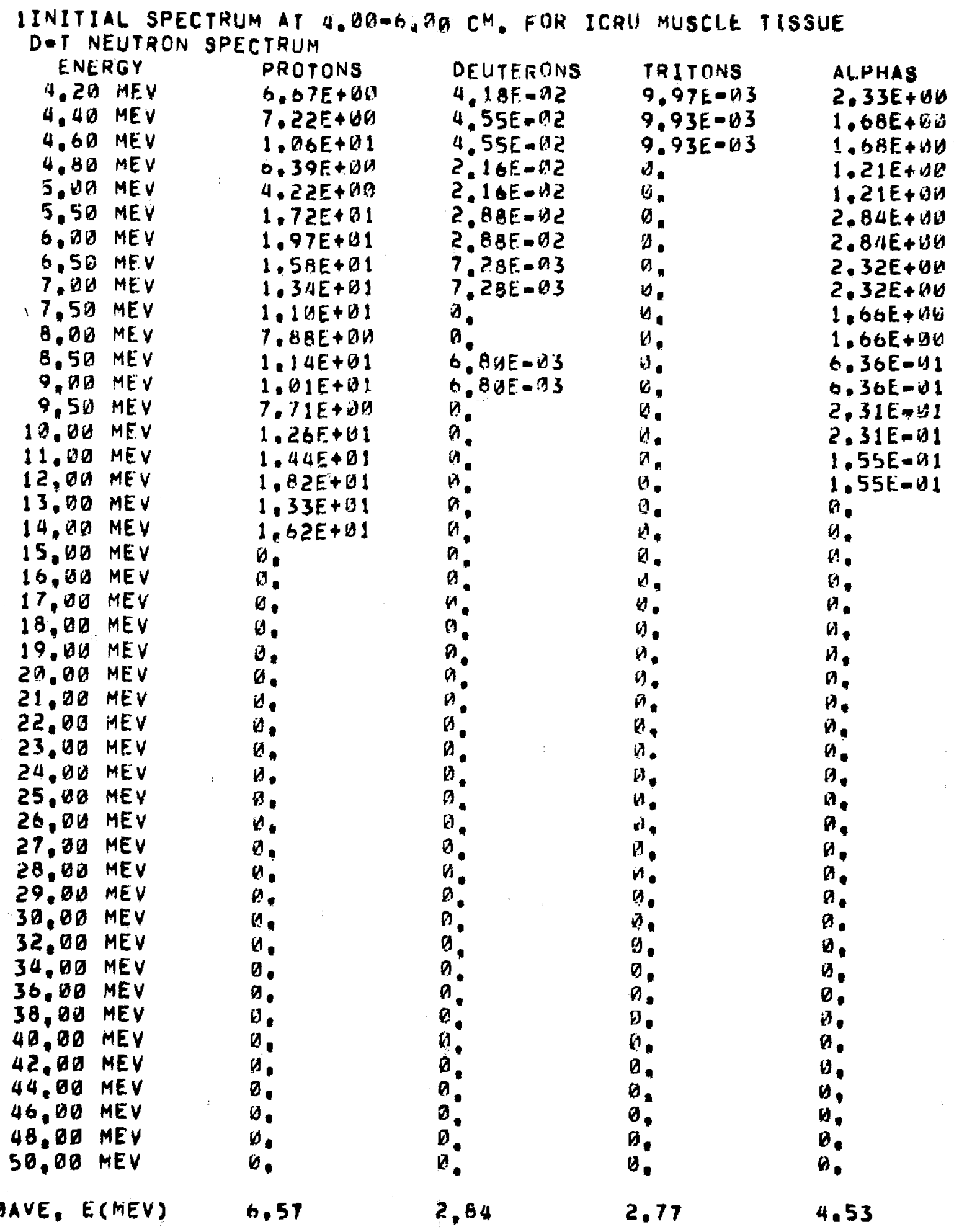


IINITIAL SPECTRUM AT $6.0 H-19.0$ CM. FOR ICRU MUSCLE TISSUE D-T NEUTRON SPECTRUM

ENERGV
$0.05 \mathrm{MEV}$
$0.10 \mathrm{MEV}$
$0.15 \mathrm{MEV}$
$0.20 \mathrm{MEV}$
$0.25 \mathrm{MEV}$
$0.30 \mathrm{MEV}$
$0.35 \mathrm{MEV}$
$0.40 \mathrm{MEV}$
$0.45 \mathrm{MEV}$
$0.50 \mathrm{MEV}$
$0.55 \mathrm{MEV}$
$0.60 \mathrm{MEV}$
$0.65 \mathrm{MEV}$
$0.70 \mathrm{MEV}$
$0.75 \mathrm{MEV}$
$0.80 \mathrm{MEV}$
$0.85 \mathrm{MEV}$
$0.90 \mathrm{MEV}$
$0.95 \mathrm{MEV}$
$1.00 \mathrm{MEV}$
$1.10 \mathrm{MEV}$
$1.20 \mathrm{MEV}$
$1.30 \mathrm{MEV}$
$1.40 \mathrm{MEV}$
$1.50 \mathrm{MEV}$
$1.60 \mathrm{MEV}$
$1.70 \mathrm{MEV}$
$1.80 \mathrm{MEV}$
$1.90 \mathrm{MEV}$
$2.00 \mathrm{MEV}$
$2.10 \mathrm{MEV}$
$2.20 \mathrm{MEV}$
$2.30 \mathrm{MEV}$
$2.40 \mathrm{MEV}$
$2.50 \mathrm{MEV}$
$2.60 \mathrm{MEV}$
$2.70 \mathrm{MEV}$
$2.80 \mathrm{MEV}$
$2.90 \mathrm{MEV}$
$3.00 \mathrm{MEV}$
$3.20 \mathrm{MEV}$
$3.40 \mathrm{MEV}$
$3.60 \mathrm{MEV}$
$4.80 \mathrm{MEV}$
$.00 \mathrm{MEV}$

PROTONS

a.

2.11E+02

$0.33 E+B 1$

5.7EE+ 01

$4,71 E+191$

$3,98 E+101$

3. $14 E+01$

$2.53 E+01$

$2.53 E+11$

1. $77 E+1$

2. $18 E+01$

2. $1 E+1 E+1$

1. $49 E+11$

$1,68 E+101$

1. $.55 E+1$

1. DQE + 1

8. $26 E+B \theta$

$7.47 E+B 0$

$1.22 E+01$

1. $D O E+\square 1$

$1.93 E+101$

$1.61 E+B 1$

$1.82 E+B 1$

1. $48 E+B 1$

$1.20 E+01$

$1.11 E+01$

$9.88 E+100$

1. $06 E+B 1$

1. $26 E+01$

1. DIE+OI

1. $\triangle 2 E+B 1$

1. $05 E+31$

9. $D 3 E+\angle A$

5. $12 E+B A$

$5.91 E+100$

$5.3 T E+\theta 0$

7. $19 E+20$

$6.15 E+D O$

$8,39 E+D O$

3. $79 E+00$

8. $51 E+00$

$1,31 E+01$

6. $42 E+B E$

$5.92 F .+10$

$9.49 E+00$

\section{DEUTERUNS}

0.

a.

H.

$D$

$a$

0

2.2.6E-102

$2.26=-32$

$3.93 E-O 2$

$3.93 \mathrm{E}-02$

$4.7 G F-M 2$

4.70E-Da

3. $4 D E-12$

3. $40 E-D 2$

$6.975-12$

$6.99 E-B 2$

$8.39 E-02$

B. 3QE- 12

$5.91 E-92$

$5.91 E-02$

1. $27 F-01$

$1.27 E-D 1$

1. 13E- 01

1. $13 F-01$

8.78E-D2

B. $78 E=02$

4.82F. 82

$4.82 E-122$

1. $12 E-92$

$1.12 E-02$

$9.08 F-113$

$9 . D B E=D 3$

0

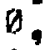

1.98E-O2

1. $98 E-02$

$3.63 E-03$

$3,63 E-03$

1. $2 Q E-B 2$

1. $20 E-D 2$

4. 3QE-OZ

4. 39E- H2

1. OSE - RZ

1. DEE 02

$2.5 \leftrightarrow F M D Z$
TRITUHS

b.

G.

1.

a.

b.

a.

0.

a.

$a$

6.

B)

0.

4.83E- $\square 3$

4. $83 E=03$

$5.45 E=03$

$5.45 E-03$

0.

(8)

0

0

a.

4

2.99E- 12

2.99E-U2

5. $21 E-03$

$5.21 E=03$

$4.65 E-163$

$4.65 E-133$

$1.55 E-62$

$1.55 E-02$

$5.21 E-13$

$5.21 E-A 3$

0.

0.

1. 92E- 02

1. $02 E-02$

4. $43 E-D 3$

$4.43 E-93$

0.

0.

4.79F-G3

$4.79 E-B 3$

$9.95 E-U 3$

$9.95 E-B 3$

5.21E- 53
ALPHAS

3. $8 B E-U 1$

$3,88 E-111$

$6.66 E-01$

$6.66 F=01$

$9.12 E-1$

$9,12 E-H 1$

$0.41 E-01$

$9.41 E-101$

$0.21 E-D 1$

$0.21 E-B 1$

$9.63 E-\triangle 1$

$9, \triangle 3 E-D 1$

$9,14 E-11$

9.14E-B1

B. IIE-DI

8.11E-1O1

$1,18 E+00$

1. $18 E+40$

$6.17 E-1$

$6.17 E-11$

1. $59 E+40$

$1.59 E+40$

$1.59 E+610$

$1.59 E+0$ ?

$1.77 E+00$

$1.77 E+00$

2. $A 3 E+B(3)$

$2.03 E+00$

1. $65 E+20$

$1.65 E+0.0$

1.92E+ 00

1.9UE + OD

$1.36 E+06$

1. $36 E+90$

1. $58 E+\angle B$

$1,5 B E_{-}+U B$

$1.27 E+00$

1. $27 E+D B$

$9.84 E=01$

$9.84 E-B 1$

2.57E+ 56

2. $57 E+00$

$2,59 E+30$

2.59E+6G

2. $02 E+06$ 


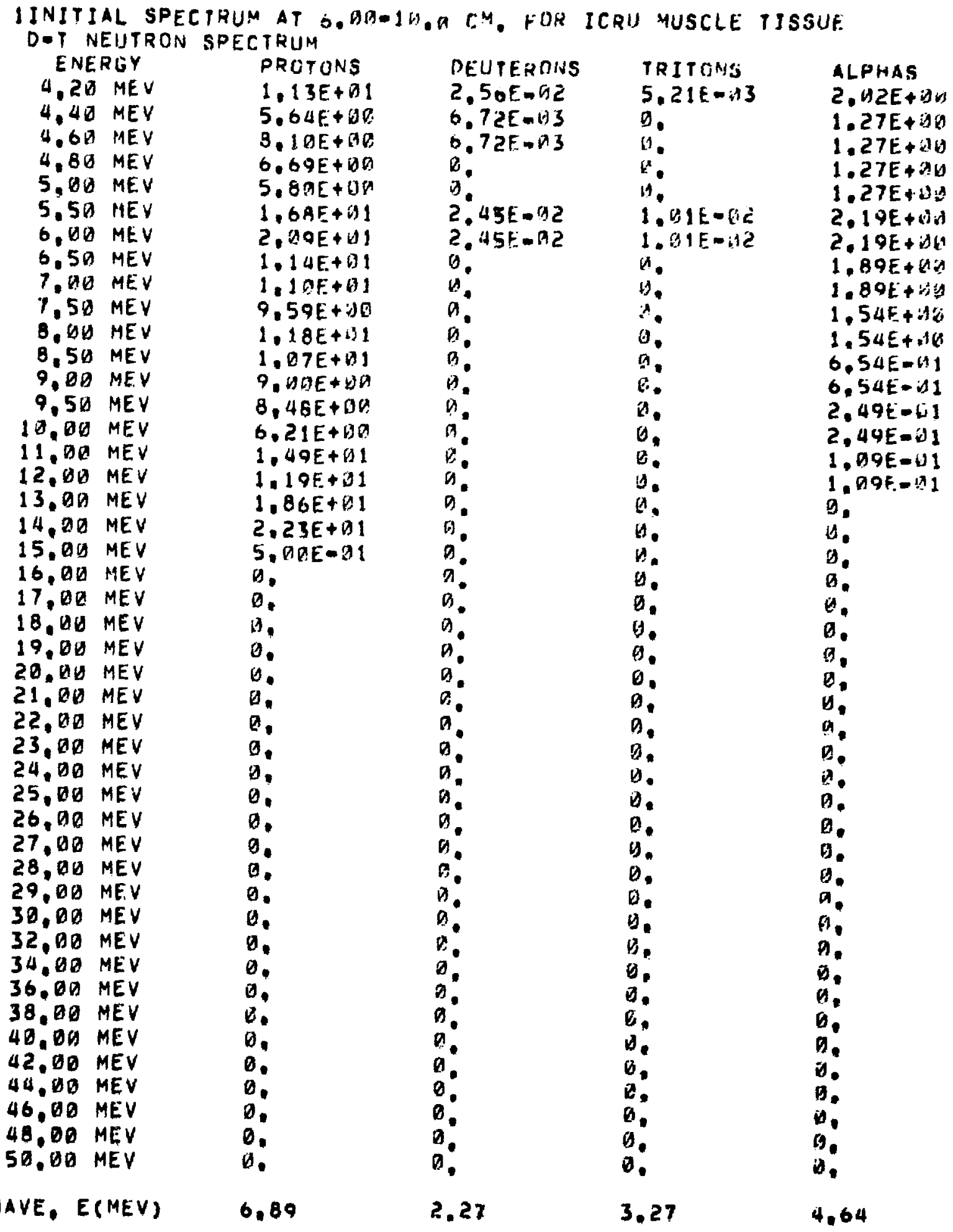




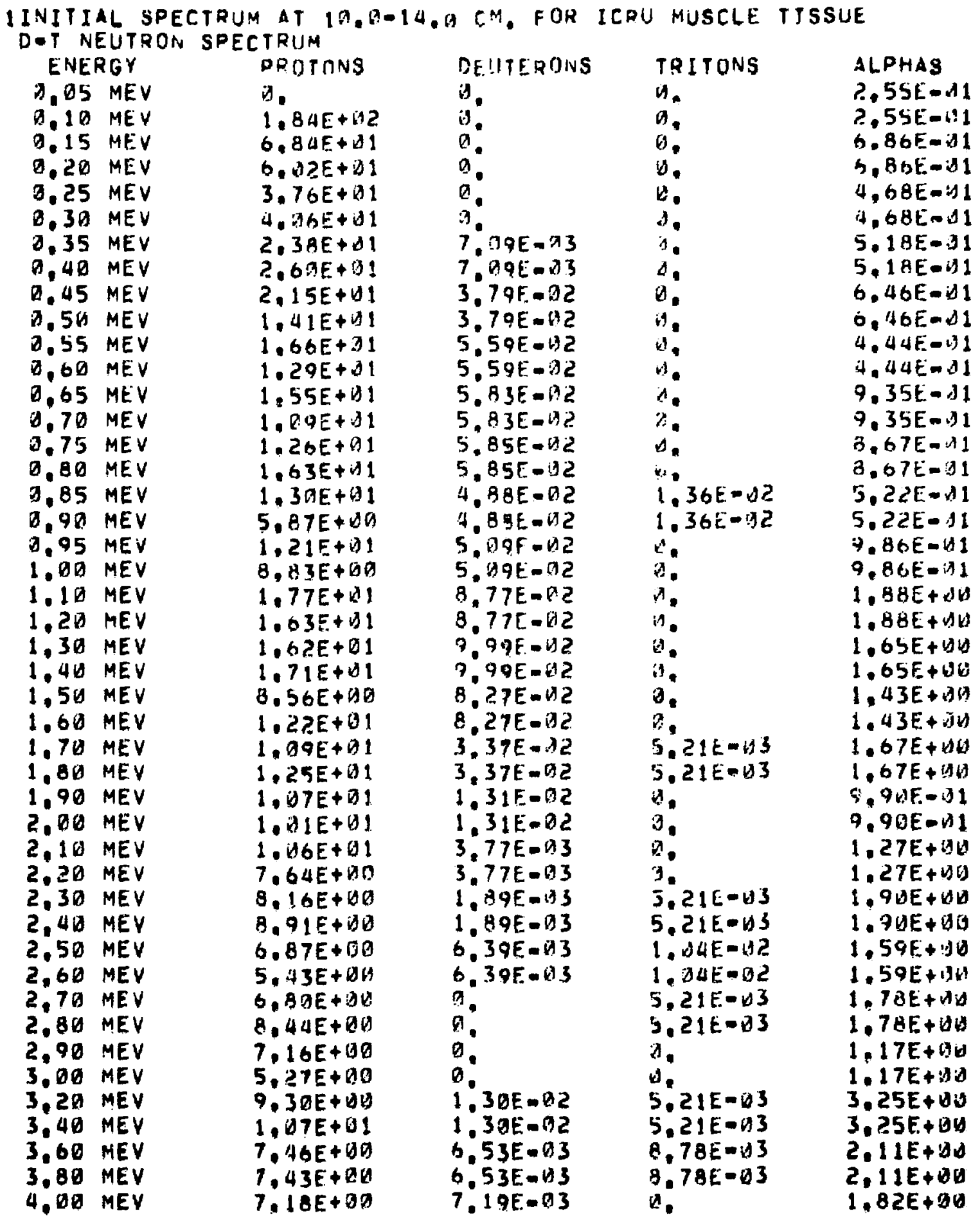


IINITIAL SPECTRUM AT 19.0-14.0 CM. FOR ICRU MUSCLE TISSUE DET NEUTRON SPECTRUM

\section{ENERGY}

4.20 MEV

$4.40 \mathrm{MEV}$

$4.60 \mathrm{MEV}$

4.80 MEV

5. DO MEV

5.50 MEV

$6.00 M E V$

$6.50 \mathrm{MEV}$

7.00 MEV

7.56 MEV

8.0B MEV

0.50 MEV

Q. OU MEV

$9.50 \mathrm{MEV}$

10.00 MEV

$11,00 \mathrm{MEV}$

12,00 MEV

13. DO MEV

14,00 MEV

$15.00 \mathrm{MEV}$

16.00 MEV

$17.00 \mathrm{MEV}$

18.00 MEV

$19,00 \mathrm{MEV}$

$20.00 \mathrm{MEV}$

21,00 MEV

$22.00 \mathrm{MEV}$

$23.00 \mathrm{MEV}$

24. DO MEV

25,00 MEV

26,00 MEV

$27.00 \mathrm{MEV}$

28.0D MEV

29.00 MEV

3月. $00 \mathrm{MEV}$

32.0D MEV

34.00 MEV

36.00 MEV

38. DO MEV

$40,00 \mathrm{MEV}$

42.00 MEV

44, DO MEV

46, DO MEV

48,00 MEV

$50.00 \mathrm{MEV}$
PROTONS

$8.75 E+19 B$

1. 19E+U1

A. $24 E+0 M$

$6.39 E+00$

$4.66 E+00$

1. $Q \geq E+O 1$

$1.47 E+01$

1. $49 E+01$

$1.31 E+01$

$1.2|E+D|$

$0.92 E+B E$

$6,76 E+3$ G

$9.75 E+\theta 0$

$6.45 E+30$

1. $26 E+01$

1. $25 E+01$

1. $4 A E+1$

1. $19 E+\square !$

9. $05 E+\Delta$.

5. OOE $=1$

0.

d.

a.

D.

0.

D.

B.

a.

0.

4.

B.

$\theta$.

(1)

0.

$\theta$

a.

$\theta$

v.

G.

b.

0.

D.

0.

0.

.

6. 36

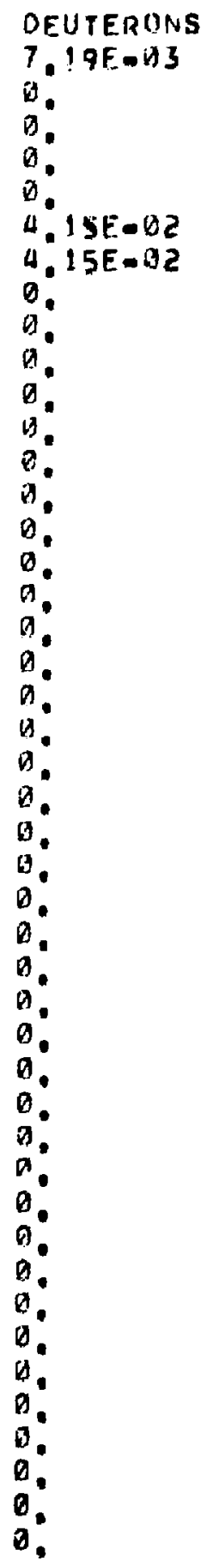

2.54
THITONS

0

0

$5.21 E-B 3$

5. $21 E-B 3$

0 .

0

a.

0.

(3.

3

$n$

0.

a.

0

3

0.

a.

i.

b.

a.

.

$D$

$B$

i.

D.

3.

b.

0

a.

D.

1).

i.

b.

0.

3.

0.

0.

0.

0.

0.

8

a.

2,99
ALPHAS

1. $82 E+d 6$

1. $.55 E+d V$

1. $15 E+11 V$

B. 64E-31

8.64E-01

$2.19 E+130$

2. $19 E+D 0$

1. $64 E+101$

$1.64 E+140$

1. 29E+ +00

1. $29 E+6 U$

4. 69E- 11

$4,69 E-1) 1$

2.10Eनด1

2. 1 (T) - DI 1

1. $D 5 E-01$

$1.85 E-141$

a.

0.

b.

0.

0.

0.

0.

n.

b.

b.

b.

a.

D.

a.

a.

a.

D.

v.

0.

.

0.

a

B. .

6.

a.

0.

0.

0.

4.55 


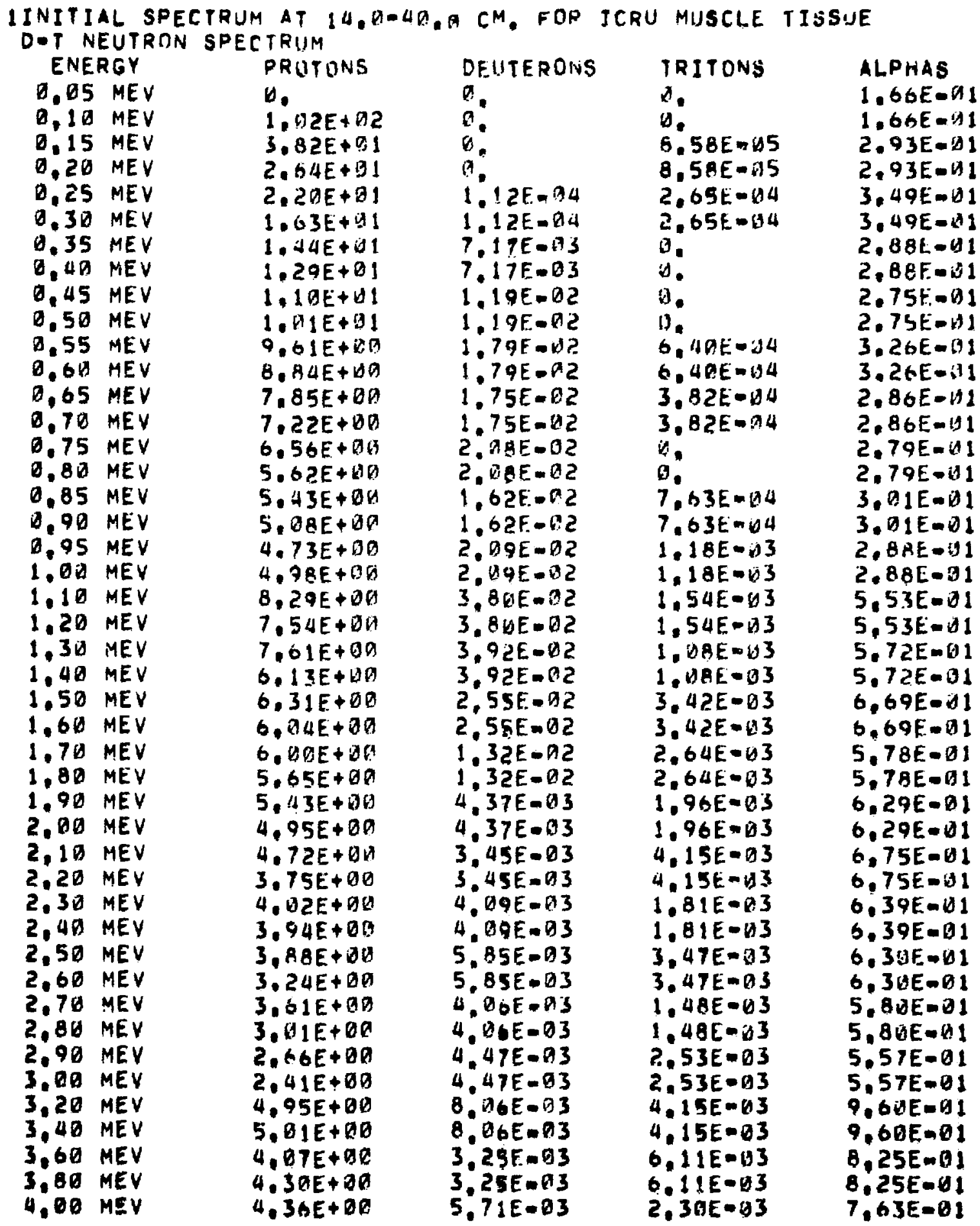


IINITIAL SPECTRUM AT 14.A-4I.,A CM. FOR ICRU MIJSCLE TISSUE. D-T NEUTRON SPECTRUM

\section{ENERGY}

4.2U MEV

4.40 MEV

4.60 MEV

4.80 MEV

5.6Q MEV

$5.50 \mathrm{MEV}$

6,80 MEV

$6,50 \mathrm{MEV}$

7. OU MEV

7.50 MEV

8. OO MEV

8.50 MEV

$9.00 \mathrm{MEV}$

$9.50 \mathrm{MEV}$

$10.00 \mathrm{MEV}$

$11.00 \mathrm{MEV}$

$12.00 \mathrm{MEV}$

$13.00 \mathrm{MEV}$

14.DB MEV

15.0D MEV

$16.00 \mathrm{MEV}$

17.Rด MEV

18.00 MEV

$19.00 \mathrm{MEV}$

$20.00 \mathrm{MEV}$

$21.00 \mathrm{MEV}$

22,00 MEV

23,00 MEV

24.00 MEV

25.00 MEV

$26,00 \mathrm{MEV}$

27.00 MEV

28.00 MEV

$29.00 \mathrm{MEV}$

$30.00 \mathrm{MEV}$

32,00 MEV

$34.00 \mathrm{MEV}$

$36.00 \mathrm{MEV}$

$38.00 \mathrm{MEV}$

40.00 MEV

$42.00 \mathrm{MEV}$

$44.00 \mathrm{MEV}$

46,00 MEV

$48.00 \mathrm{MEV}$

$50.00 \mathrm{MEV}$
PROTONS

$3.5 B E+B Q$

$2.93 E+a A$

$3.61 E+W O$

3.5口E+ $B A$

3. $D T E+W D$

$6.68 E+10$

$6.74 E+V 0$

$5.43 E+80$

$4.80 E+d$,

4. $28 E+U A$

$4 . O 3 E+0 O$

$3.88 E+C$

$3.92 E+3 A$

3. $2 A E+2 Q$

3. $D O E+A D$

$5.47 E+20$

5. $16 E+1.7$

4. $89 E+B 0$

4.17E+ HE

$1.15 E-61$

0.

$\theta$

0.

$B$.

u.

$\Delta$.

a.

0.

0.

0

b.

$D$.

0.

$\theta$

$\theta$

b.

0

0

0.

i.

D.

0.

0.

b.

0

DAVE, E(MEV)

5.92
DEUTERDIS

5.71E-H3

5. $\partial Q E-13$

5. $B$ QIE -53

$1.50 F .113$

1. $596-193$

B. $\triangle A E-13$

B. $M E E-03$

2. DIf-M3

2. $0110-03$

$5.53 E-04$

$5.53 E-D A$

$1.11 E=13$

$1.11 E-93$

4

$a$

$a$

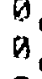

a.

0

a

$\theta$

a

b

$a$

$a$

a

9

0

$\theta$

$D$

a

0

0

0

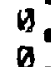

0

0

2.61
TRITONS

2. 3AE $=33$

1. $55 E-23$

1. $55 E-D 3$

B. D) $1 E=04$

8. $61 E-U 4$

$7.66 \mathrm{E}-\mathrm{AS}$

$7.66 E-14$

i.

U.

b.

D.

ij.

$\theta$.

b.

4

0

$v$

的。

.

b.

v.

U.

E.

9.

B.

0.

6

0

i.

0.

a.

0

b.

0.

$b$

$\theta$

E.

0.

0.

0

0

b.

0

$\theta$

0

3,06
ALPHAS

7.63E-b1 1

5. $42 E-D 1$

$5.42 E-31$

$4.68 E=01$

$4.65 E-11$

8.5BE-11

B. SUE-U1

$6.94 E-4$

6. 94E-A1

4.67E-BI

$4.67 E-1) 1$

?. G6E $=1$

2. $U 6 E-1$

7.6 . $E=U 2$

$7.6 A E-42$

$2.03 E-D 2$

2. . $3 E-U 2$

$3.5 U E=134$

3. 54E- 4

0

0.

a.

n.

a.

0.

6.

$\theta$

0.

b.

0.

0.

0

0

0.

$\Delta$

B.

a.

0

9

b.

0.

0

b.

4.51 